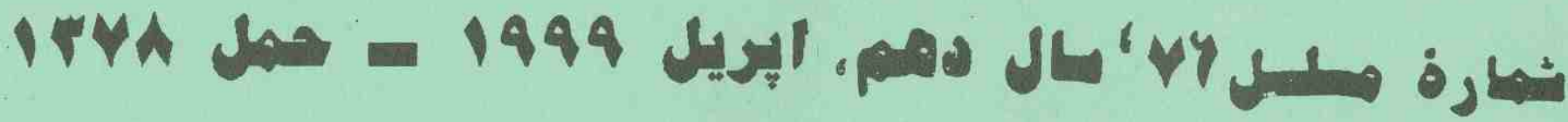
SERIAL NO.76,VOLUME 10,APRIL 1999 - HAMAL 1378

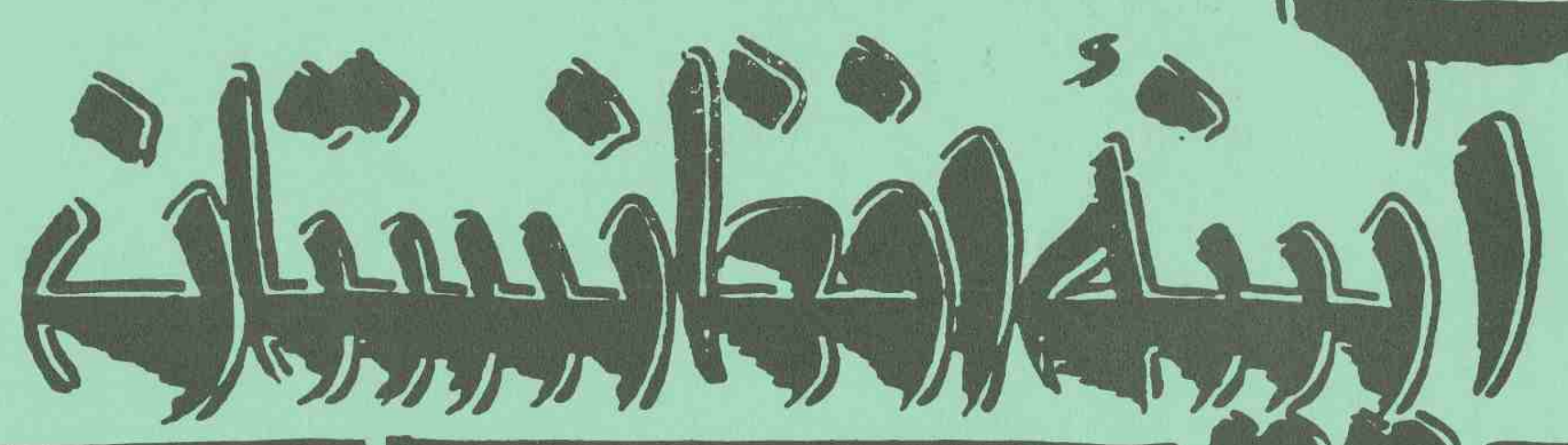

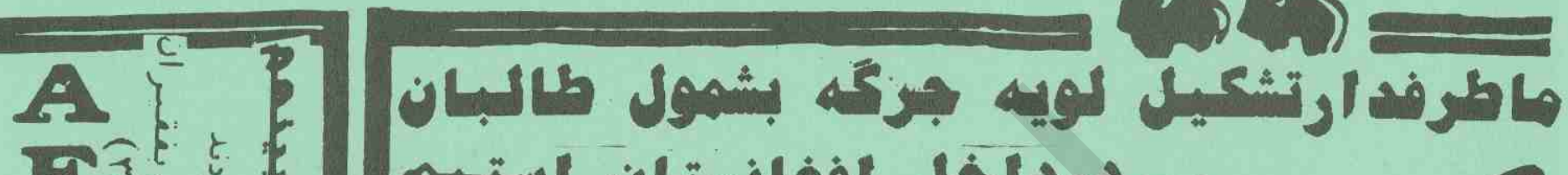

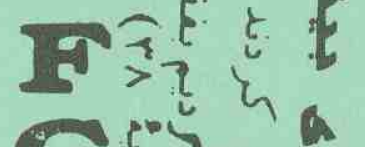
G. I I I

है

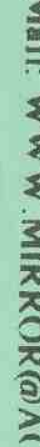

$\mathbf{A}$
$\mathbf{N}$
$\mathbf{I}$
$\mathbf{S}$
$\mathbf{T}$
$\mathbf{A}$

$\frac{3}{3}$

$\mathbf{N}$ हो?

$\mathbf{M}$

I

$\mathbf{R}$

政

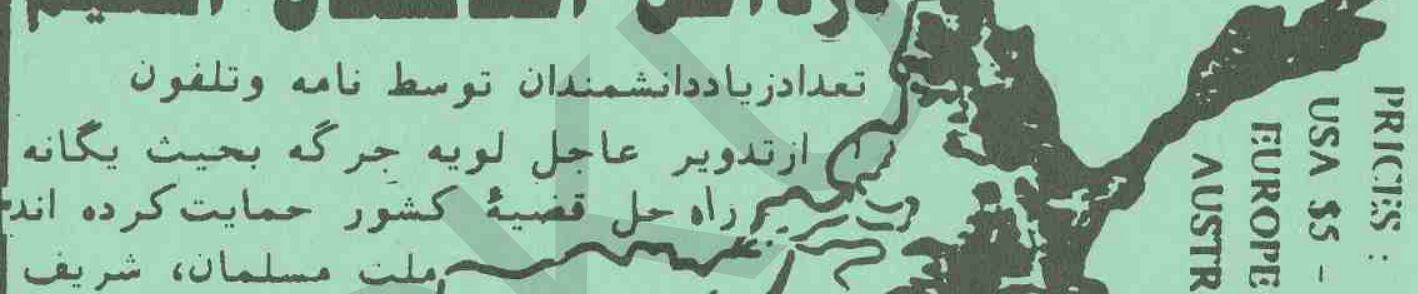

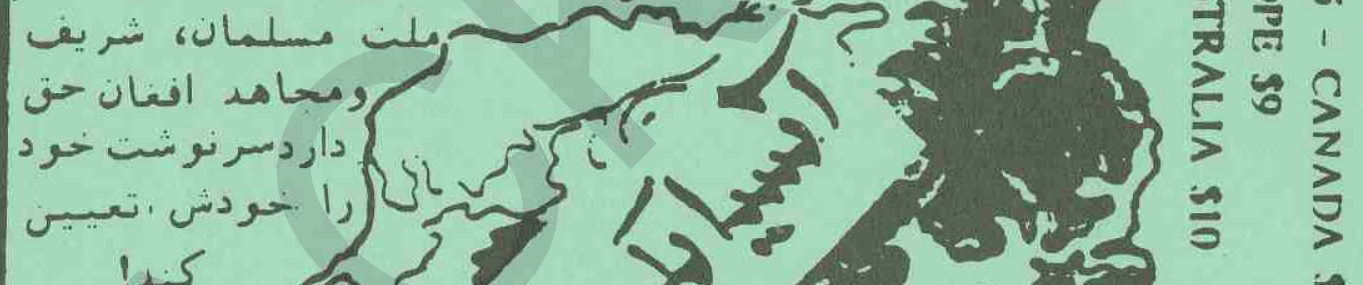
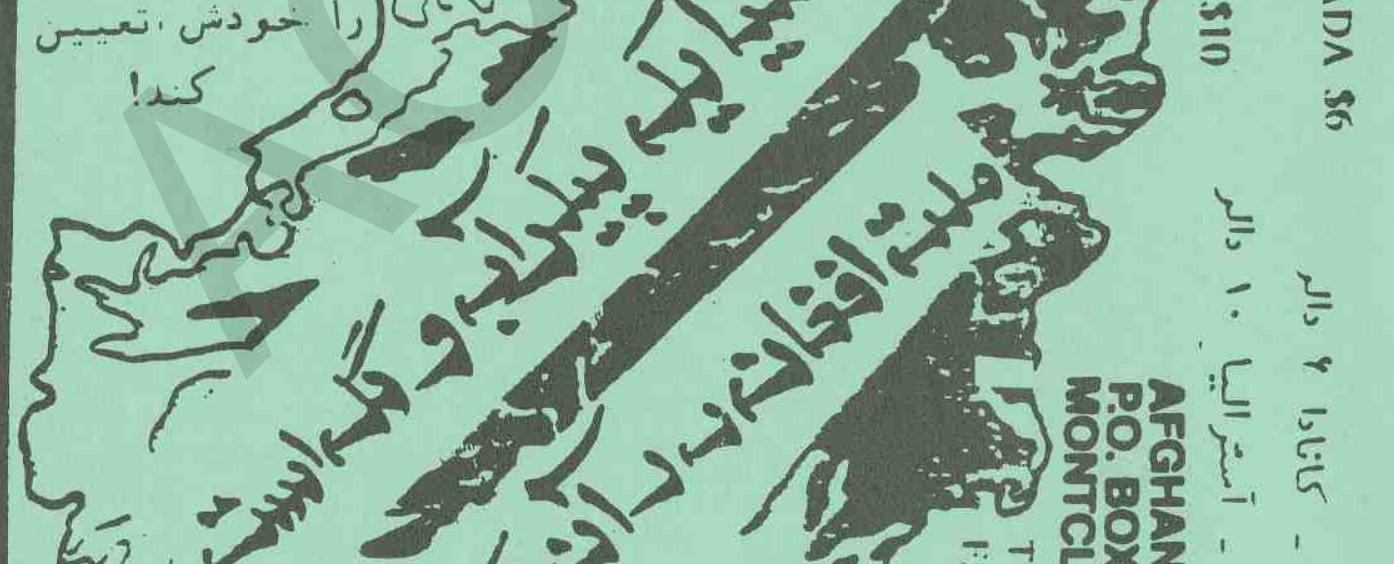

F- 
AFGHANISTAN VY JL O gN

نان

أفغانـنـان
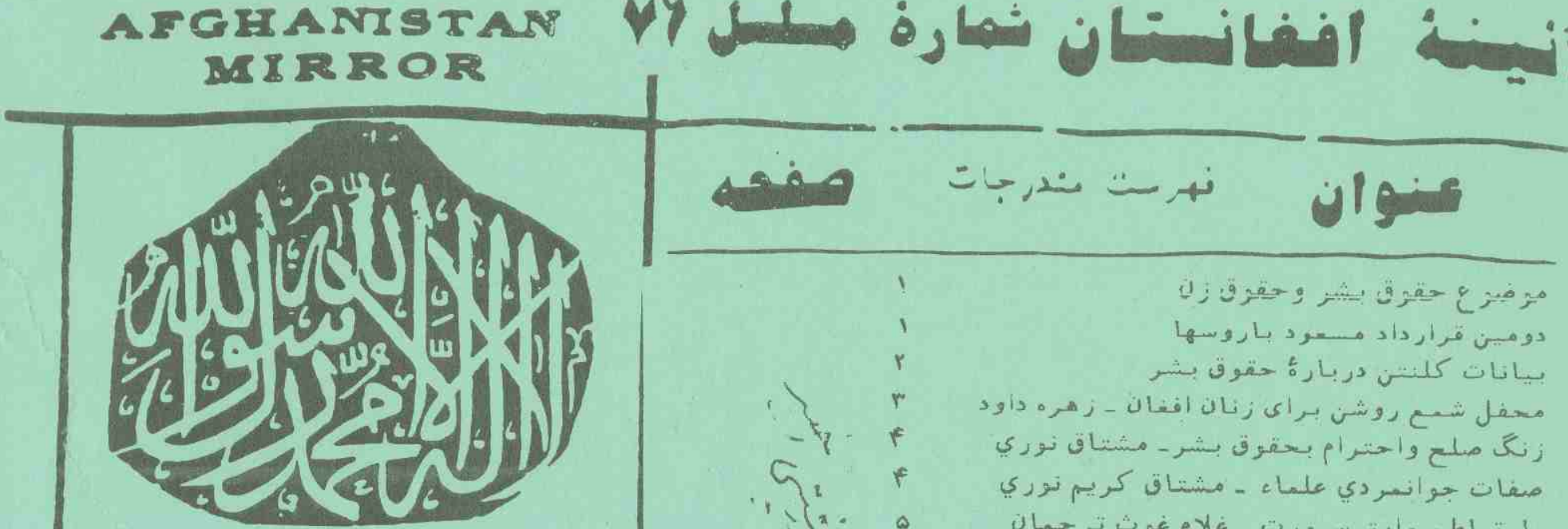

$=\longrightarrow$

if $\mathrm{Pin}$
(ن)
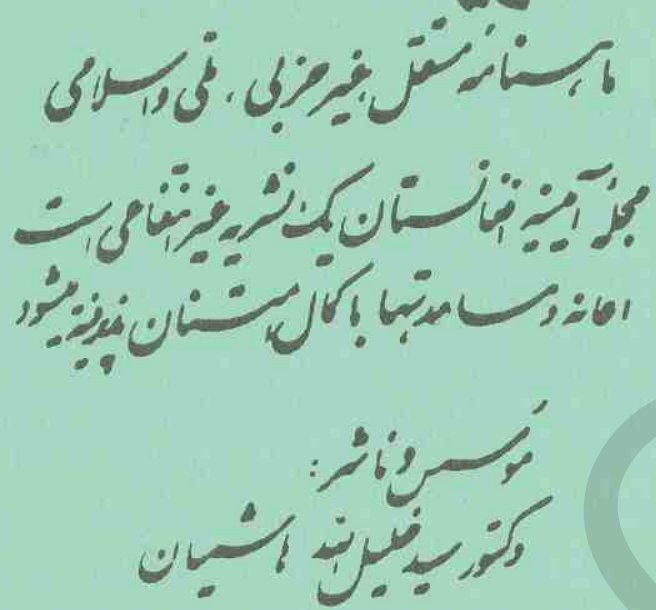

1105 as

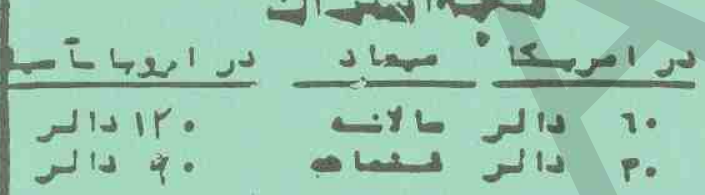

\&

AFGHA.VISTA.V MIRROR

P.O.BOX 418

MONTCLAIR, CA $91763^{2}$ यो

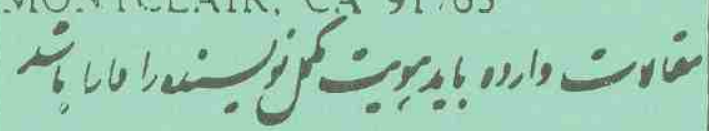

TEL. (909) 6268314

FAX (9n9) 625 675!

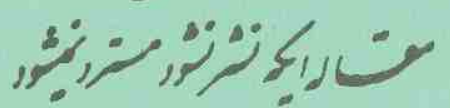

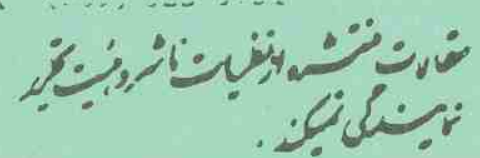

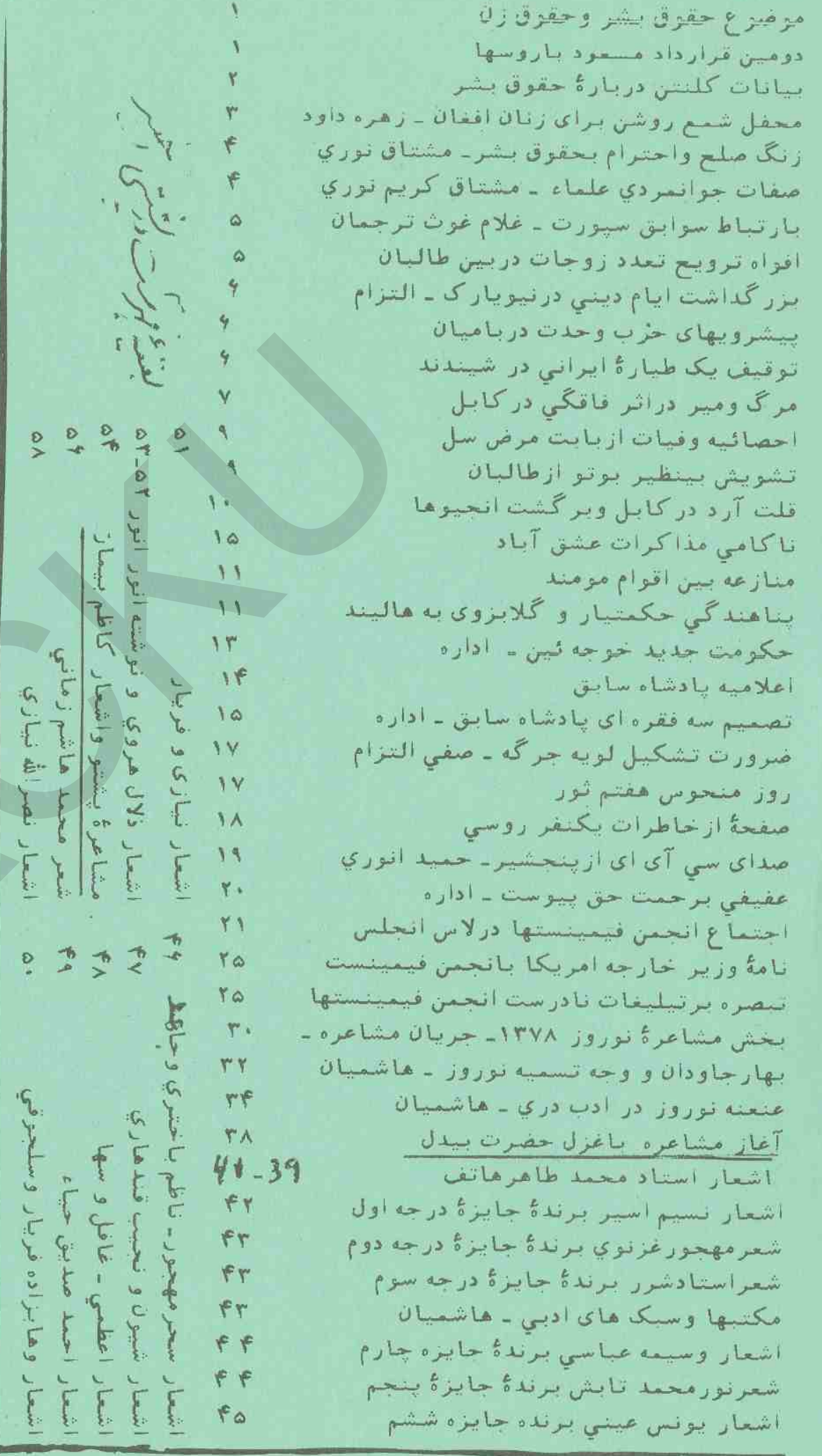

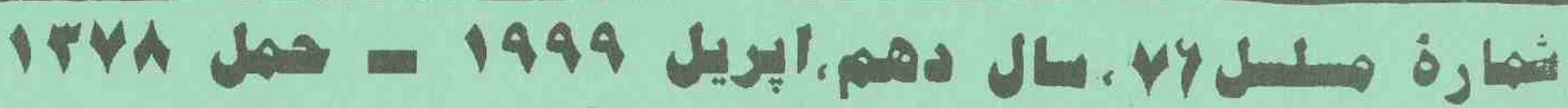

SERIAL NO.76,VOLUME 10,APRIL 1999 - HAMAL 1378 


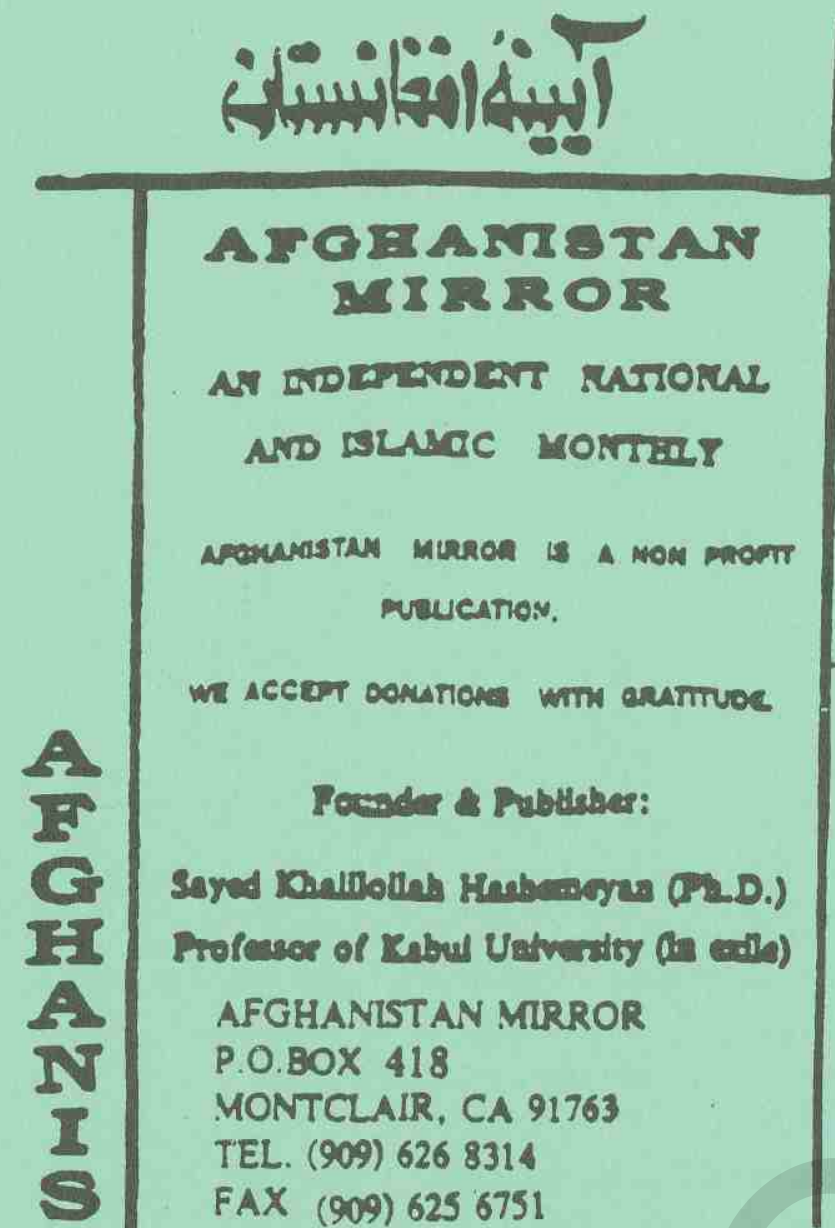

T Our E Ma11: WWW.MIRROR@AOL.COM

- Ever erters b reseadble fer

N

M

Arrieles for publiendion abould

bes a full ldeatry of the writes.

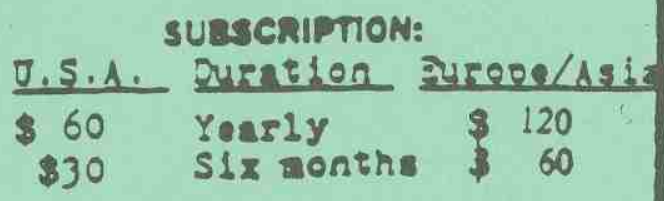

\$ sper lasue la U.S.A.

$\$ 6$ per issue in Canada

59 der issue in Europo

510 por issue in Australia

Ardese ior proulused

chall aos be recurad.

Ariele sobltated bere do soc roprecens the viows of the publiabe of the beard of edirese.

\section{AFOEANITIN MIRIOR}

\section{Tite}

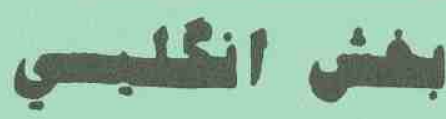

Pase

Editorial: Comments on the Letter of

Feminist Majority to President Clinton

1-7

Feminist Majority Letter to President Clinton 8

Madame Albright Letter of 3/29/99

10

Voice of Afghan Women

Open Letter to Afghan People

by Bruce Richardson

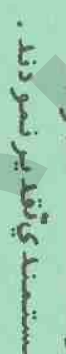

के

t.

द

h.

$\vdots$

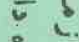

i. :

i.

G

ह.

\&.

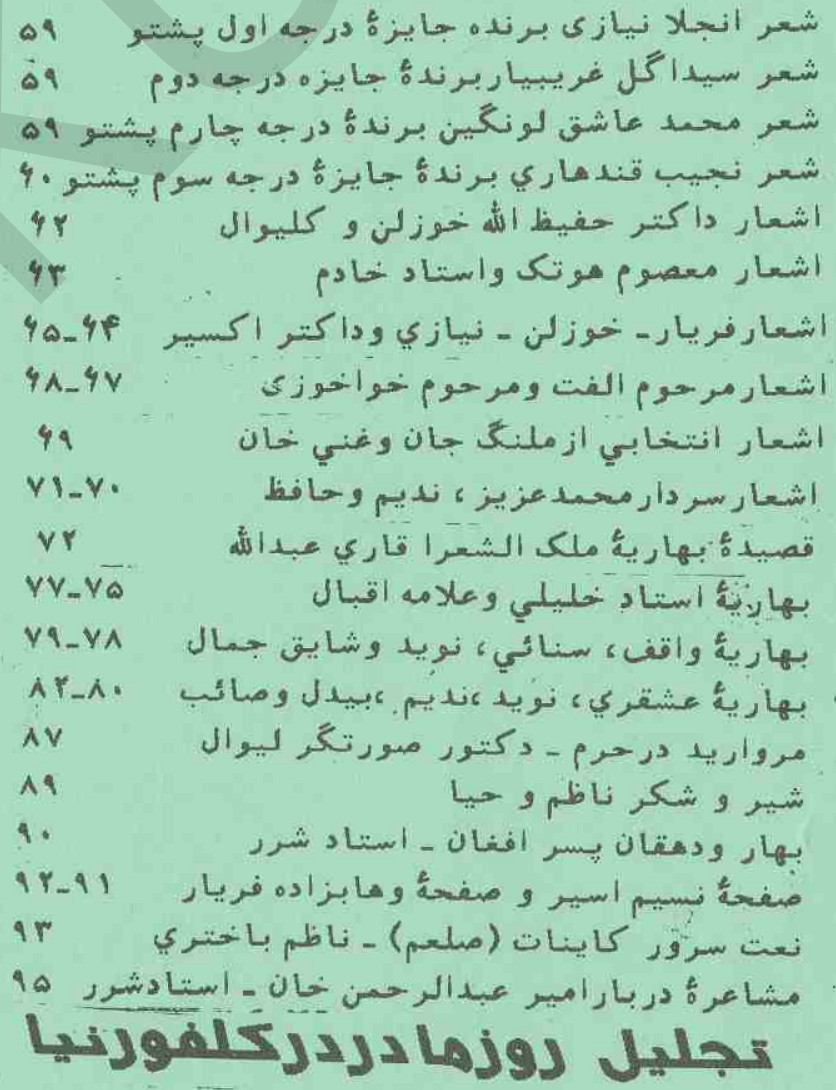

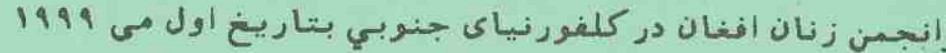

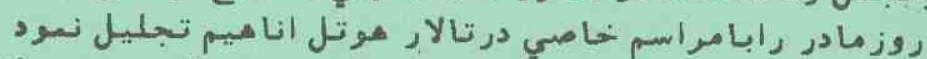

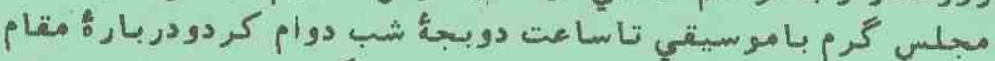

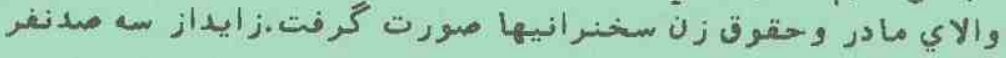




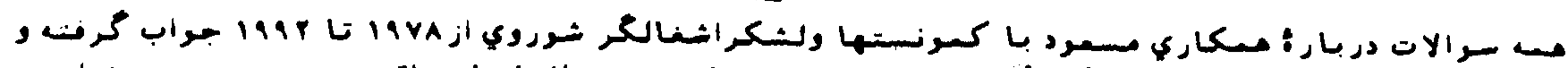

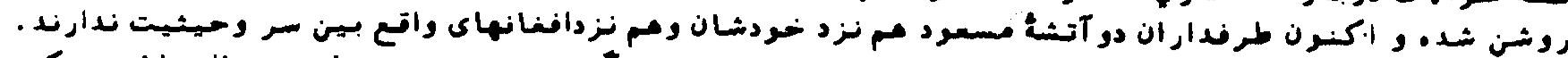

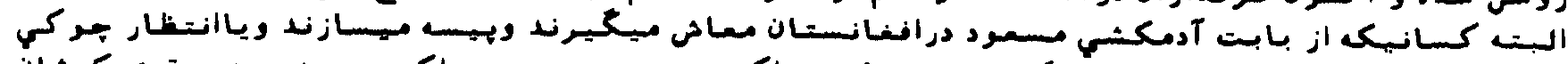

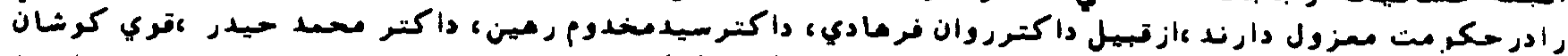

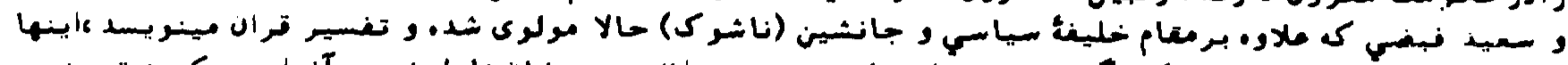

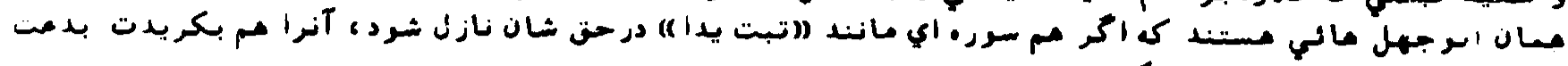

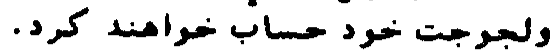

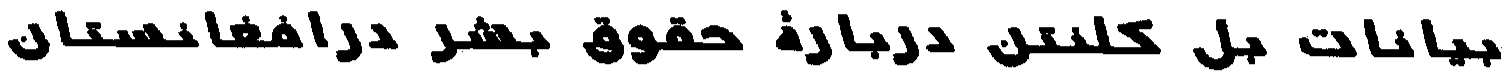

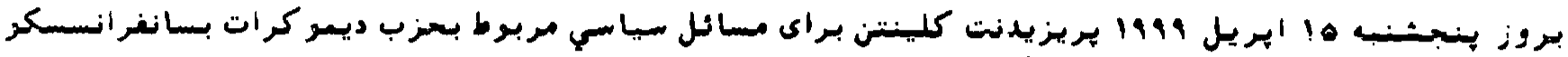

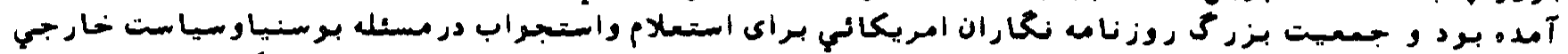

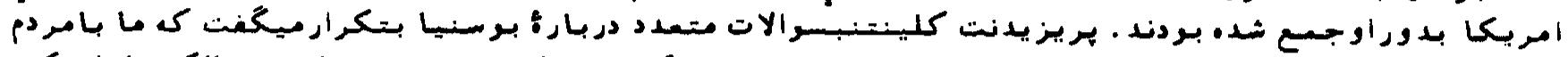

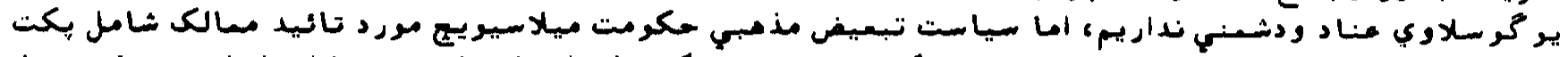

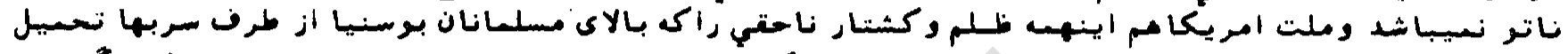

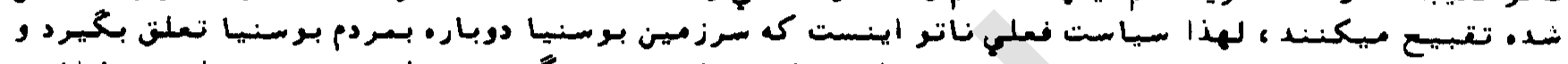

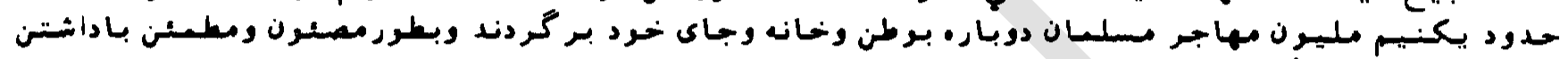

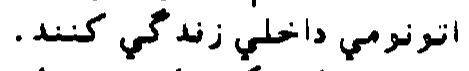

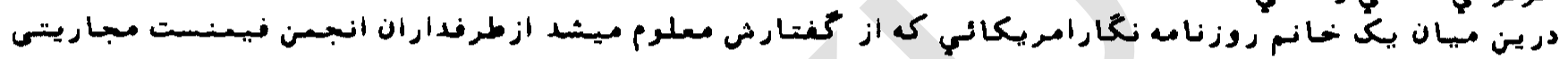

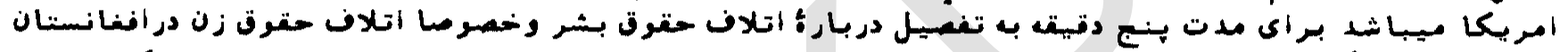

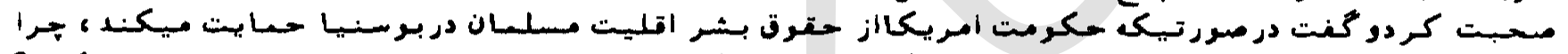

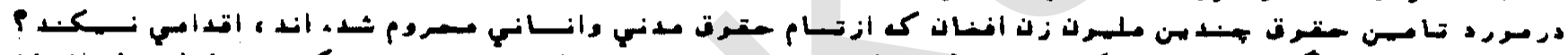

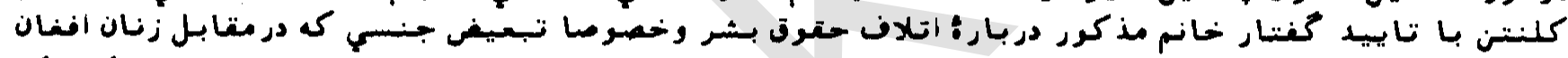

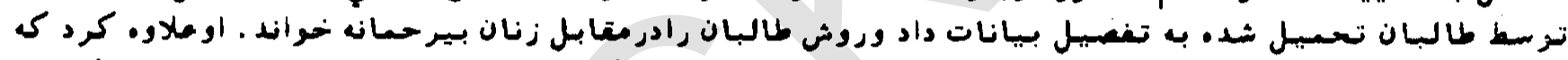

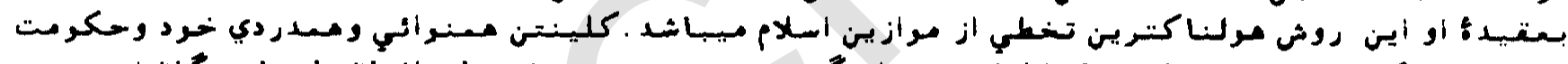

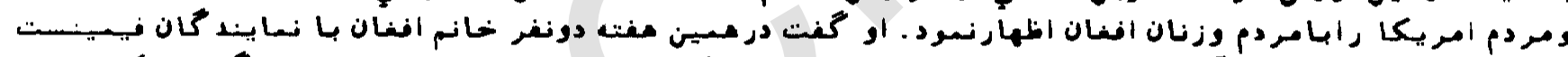

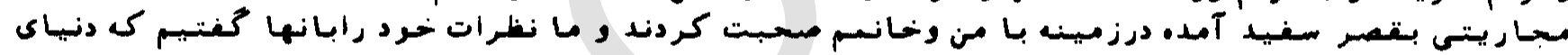

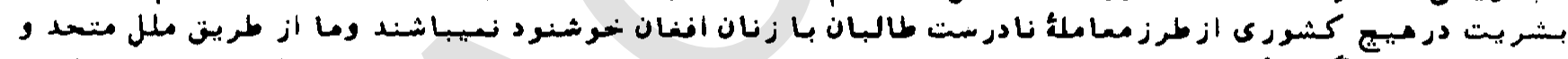

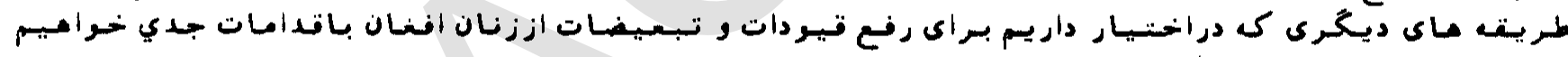

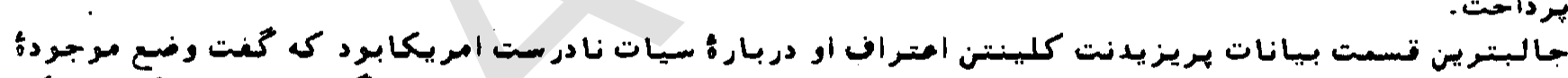

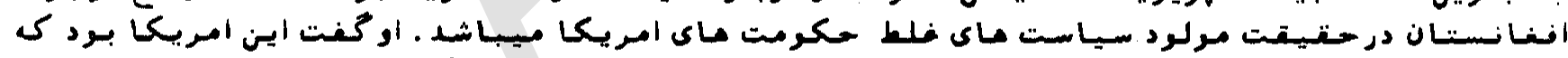

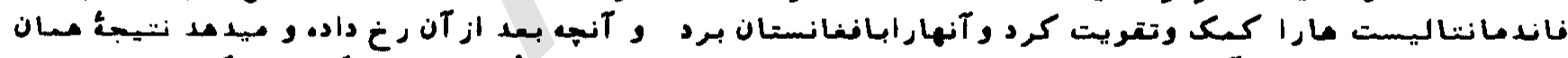

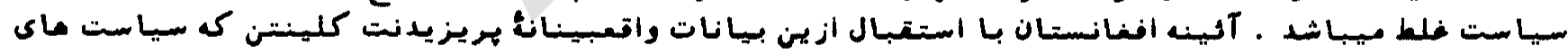

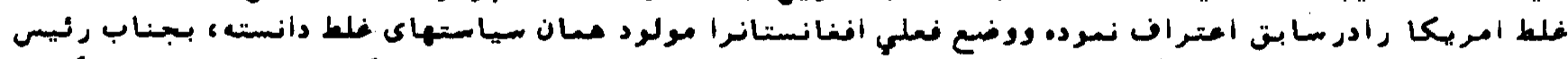

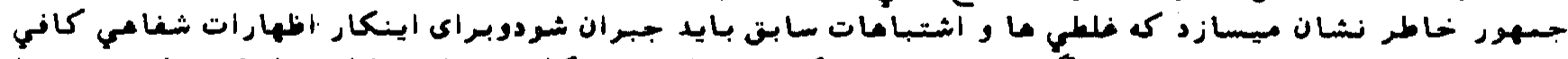

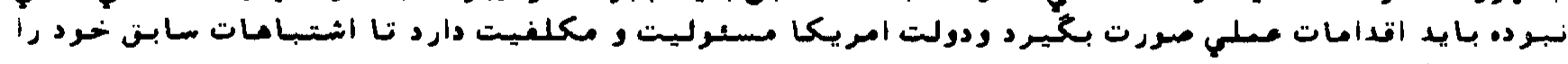

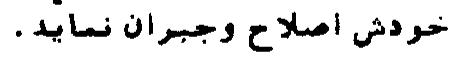

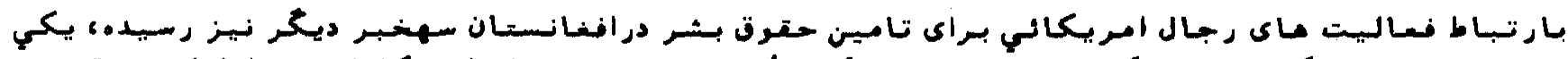

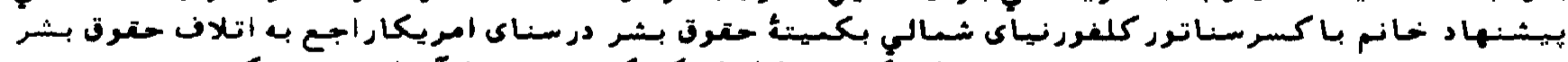

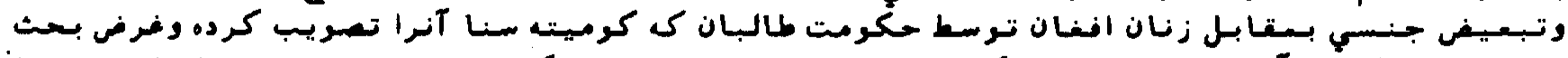

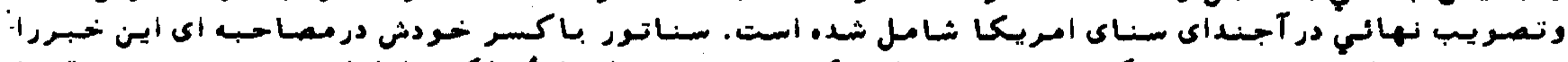

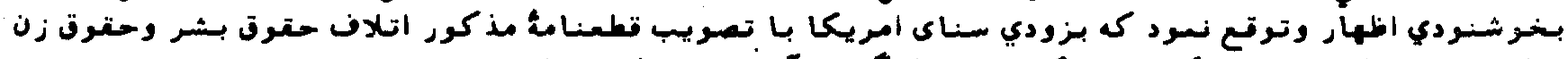

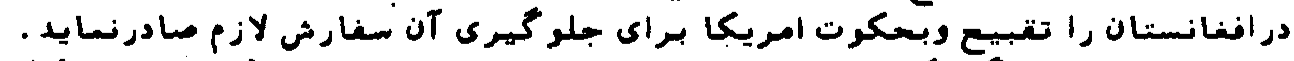

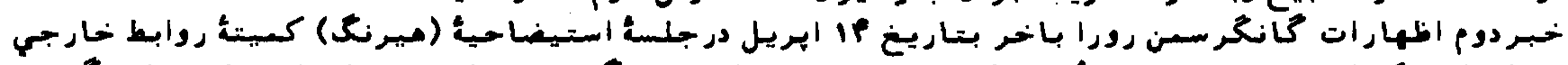

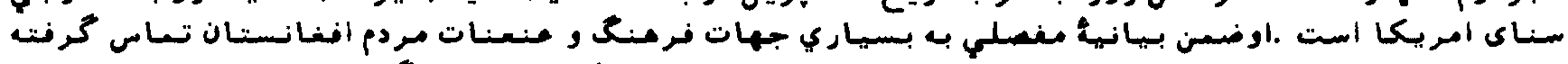

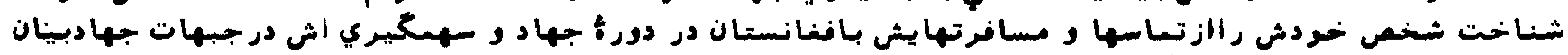

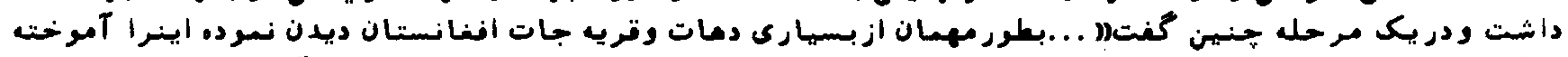

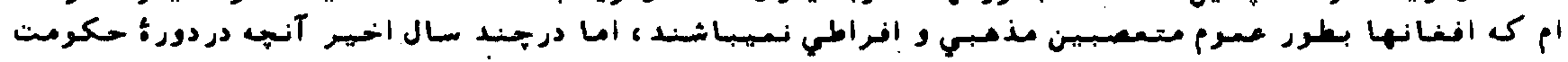




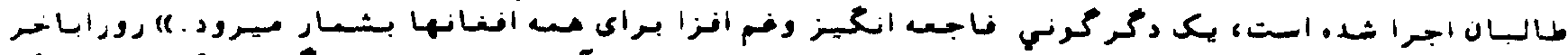

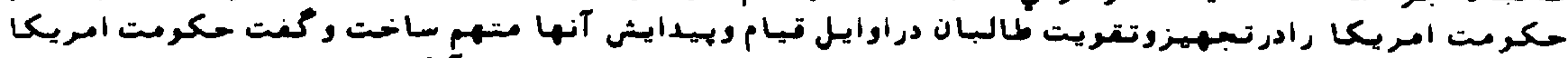

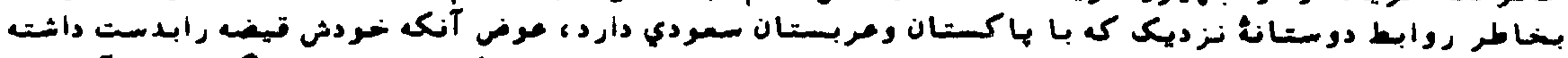

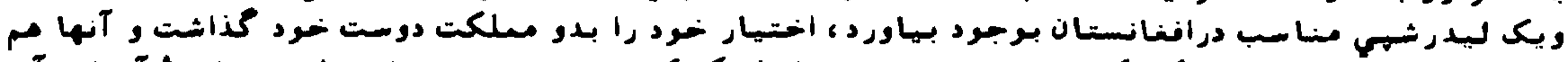

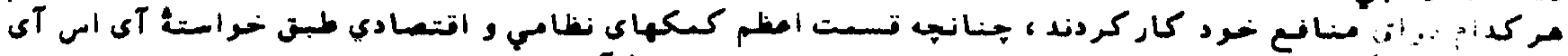

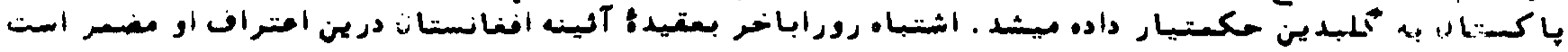

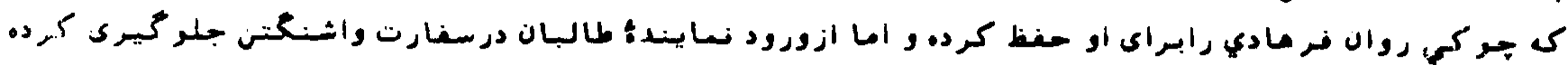

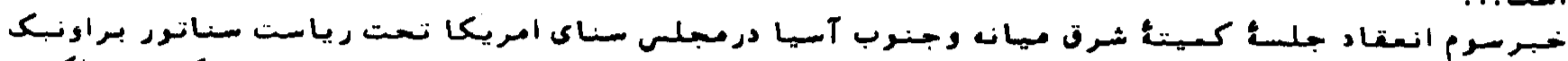

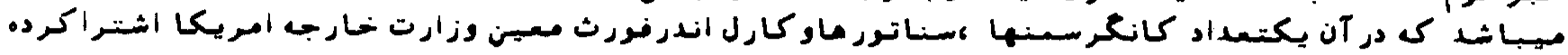

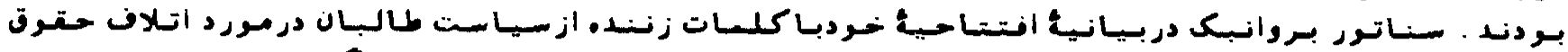

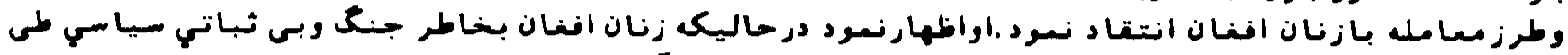

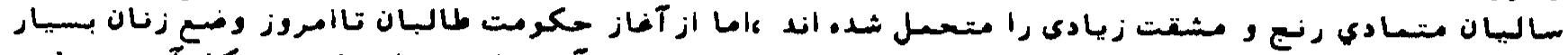

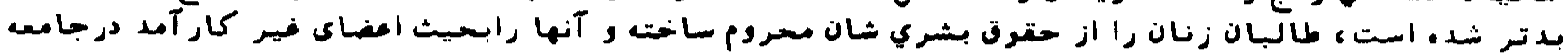

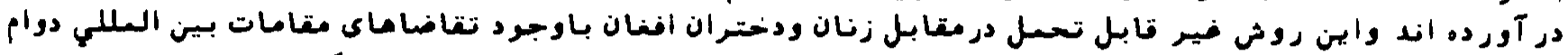

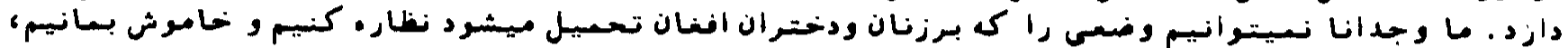

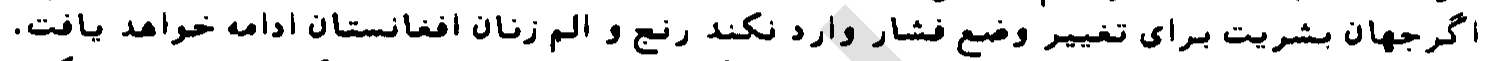

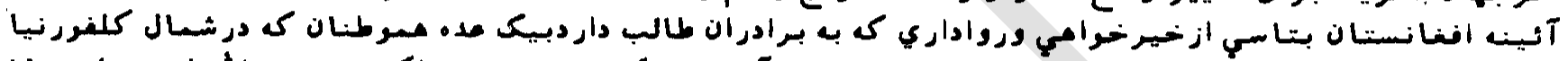

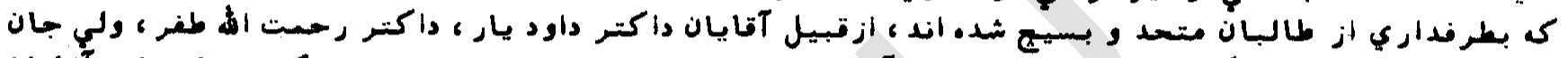

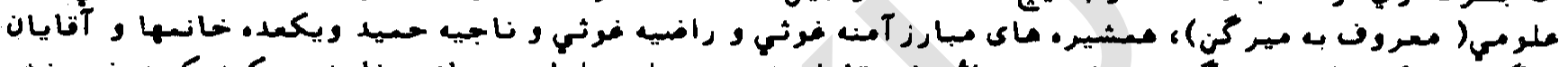

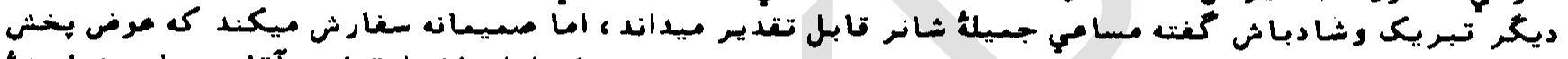

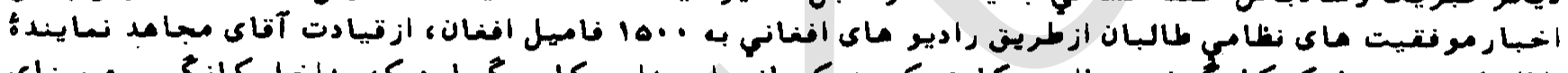

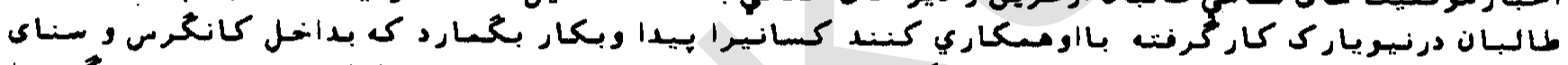

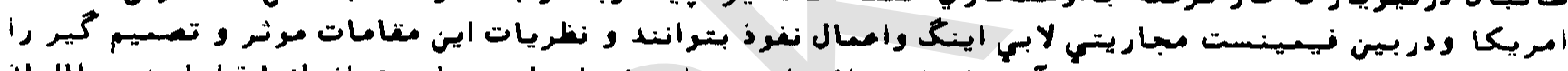

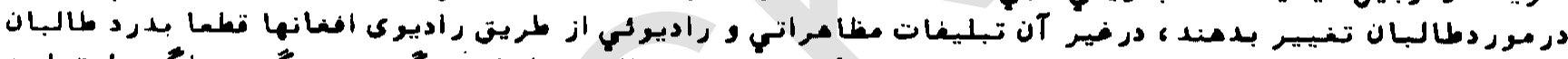

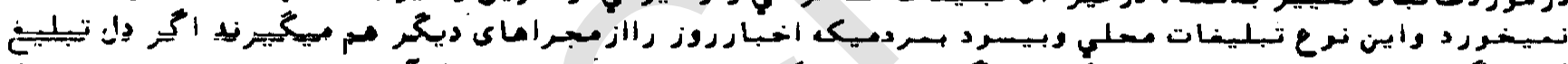

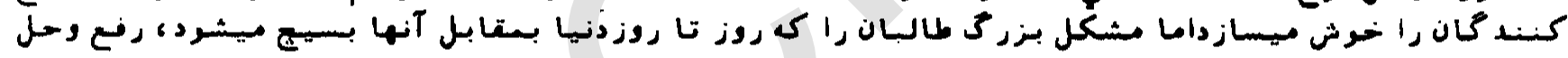

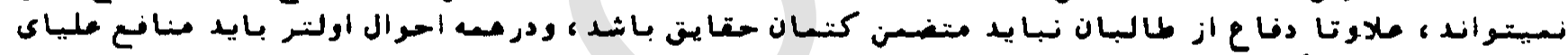

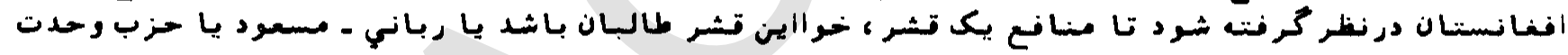

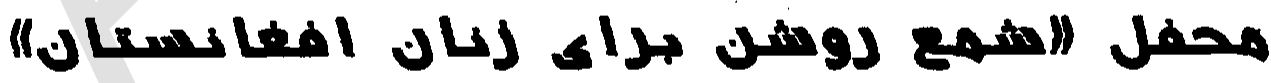

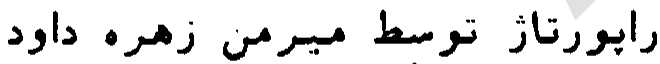

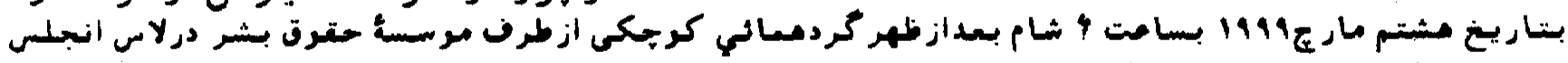

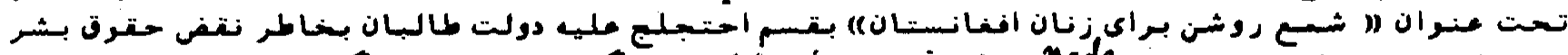

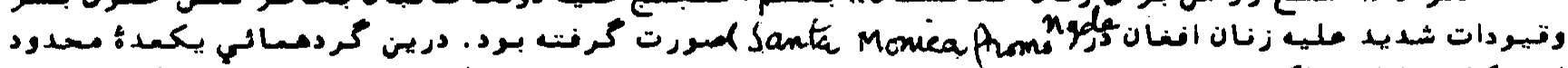

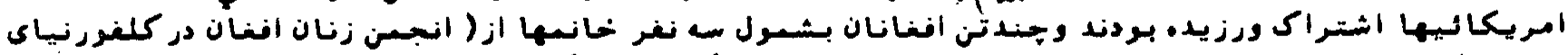

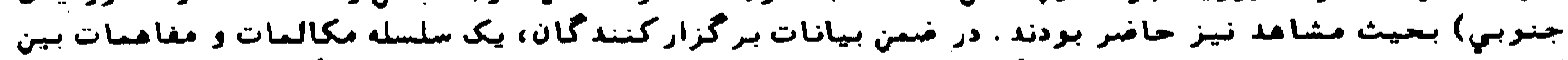

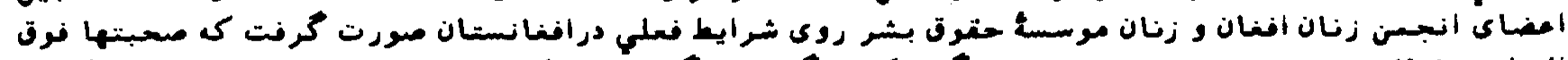

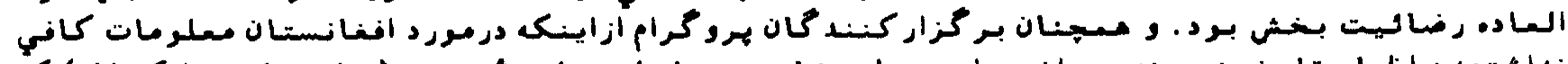

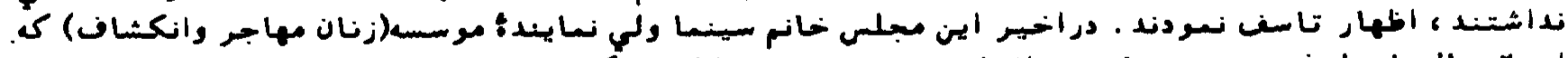

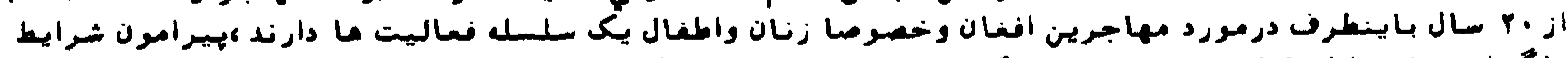

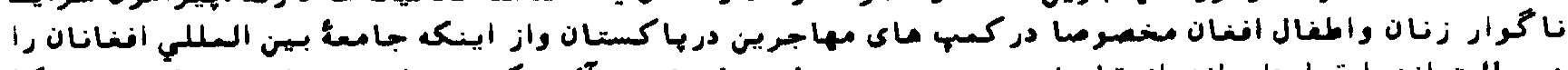

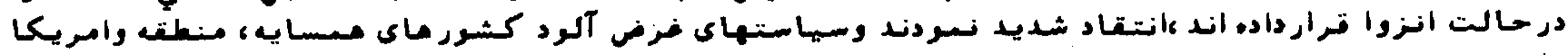

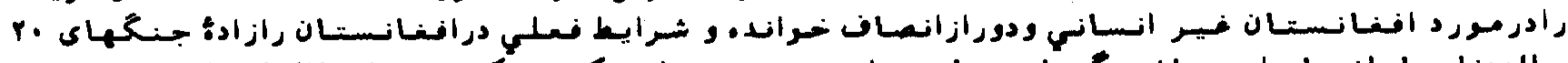

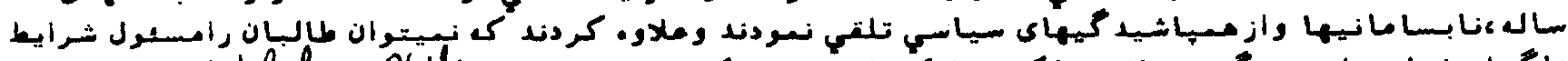

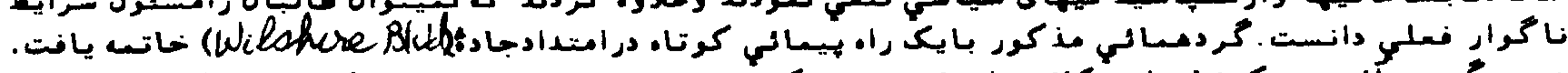

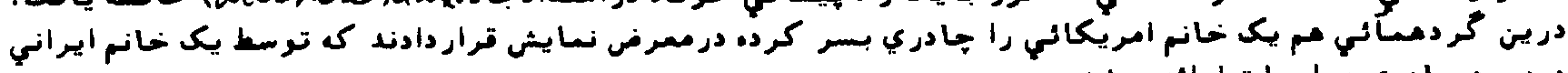

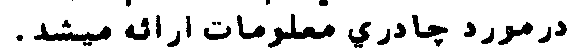




\section{4

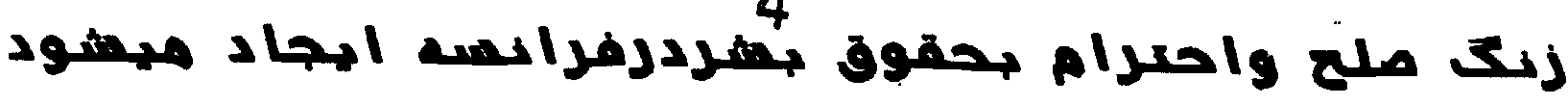

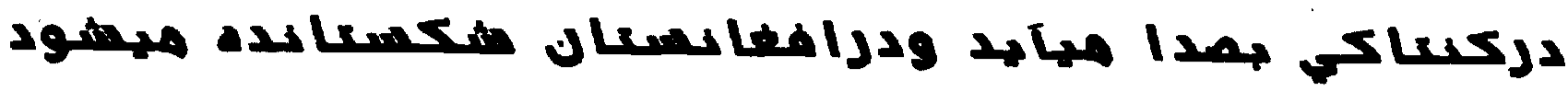

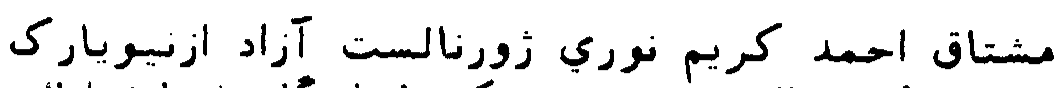

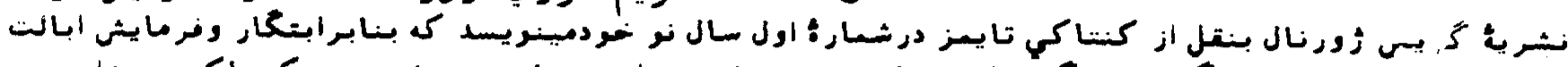

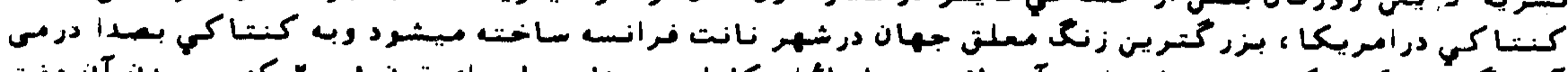

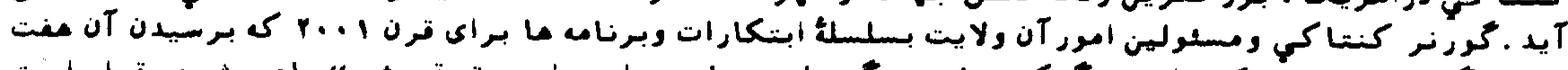

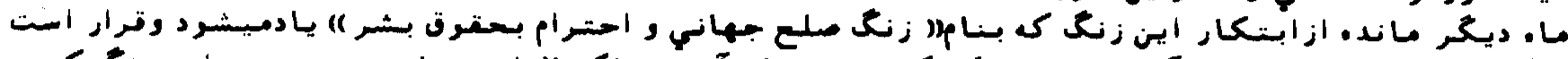

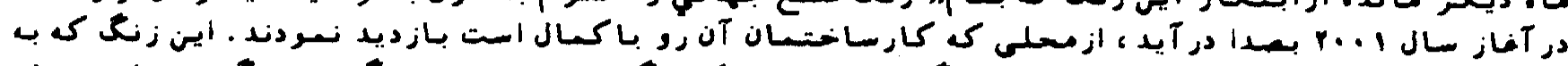

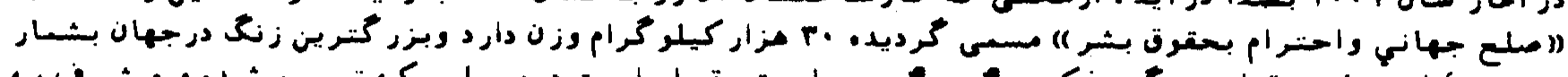

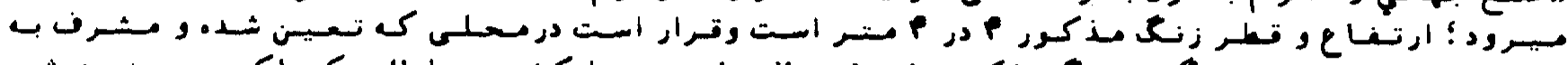

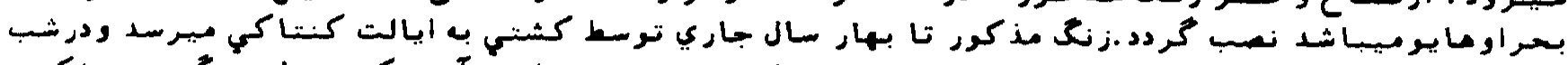

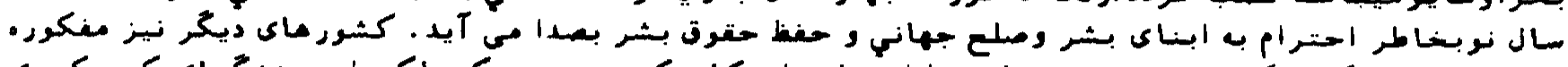

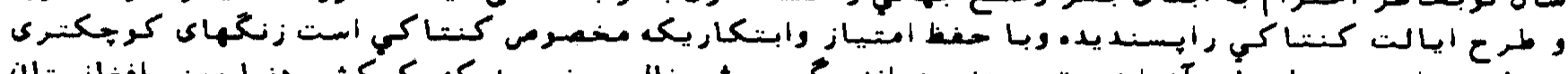

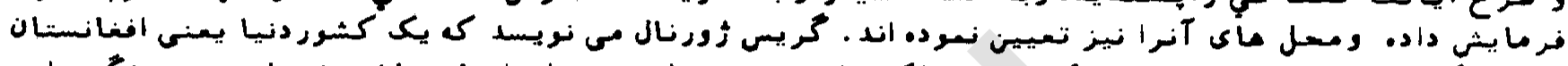

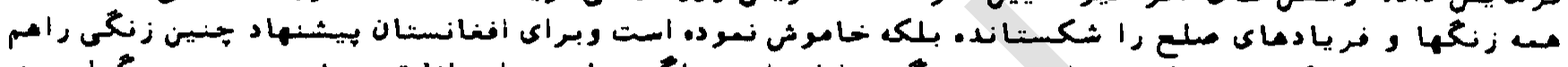

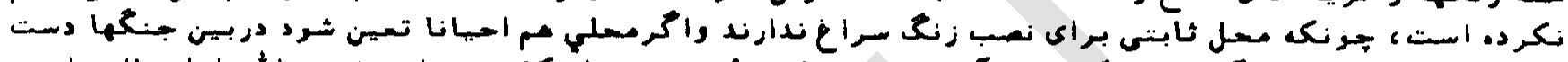

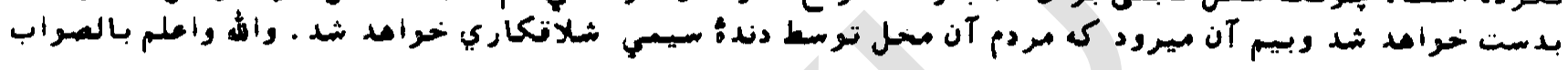

\section{صفات جوالخهرده علها.}

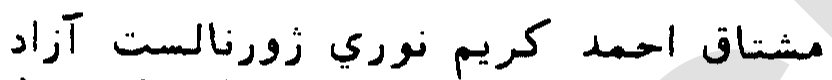

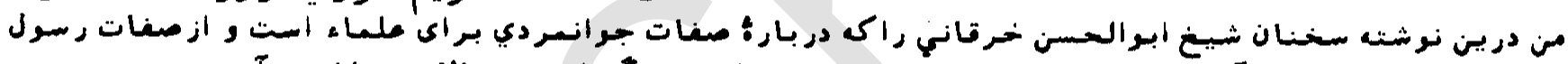

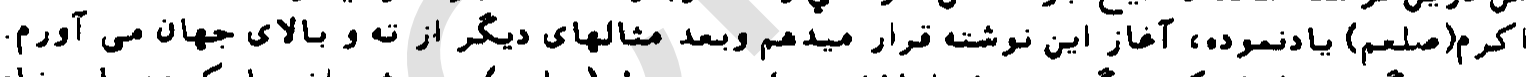

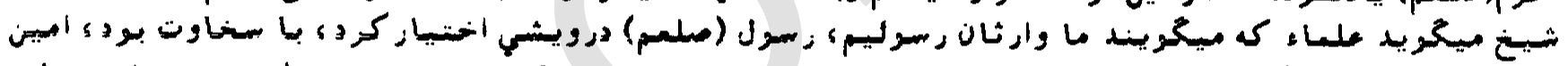

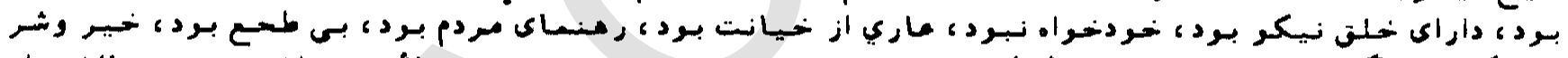

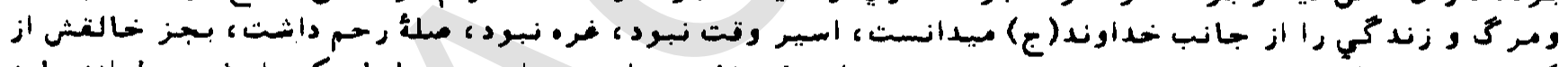

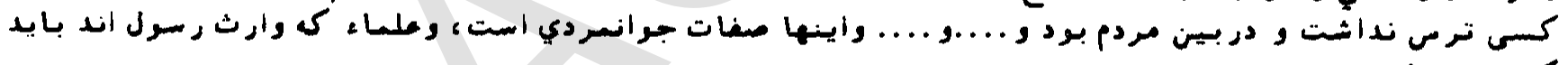

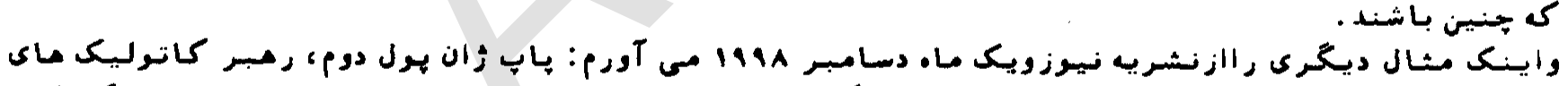

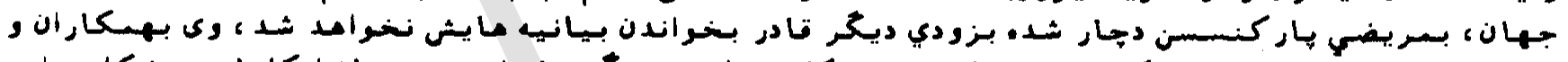

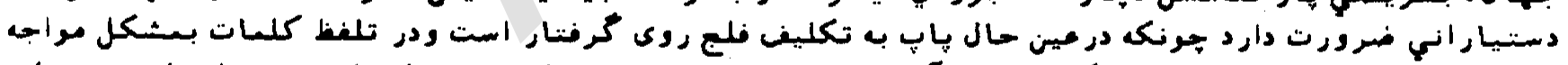

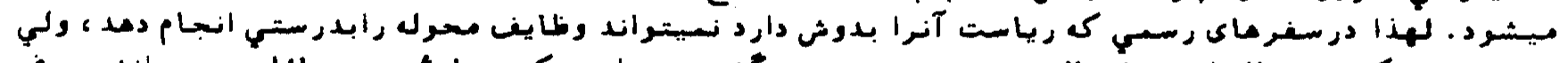

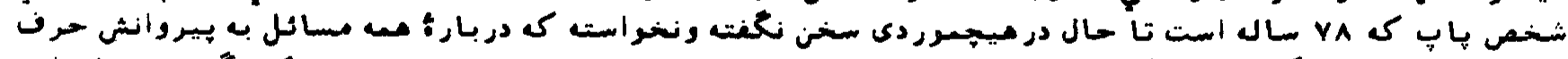

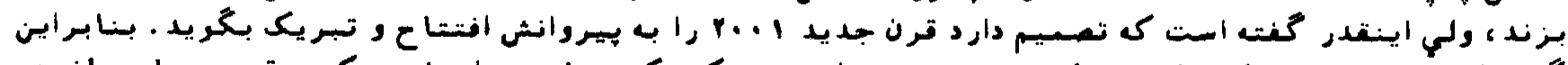

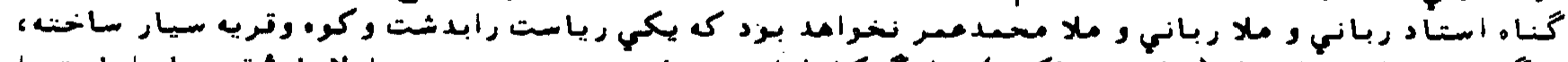

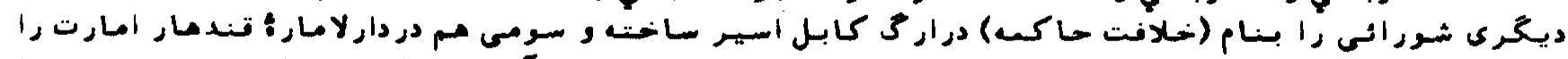

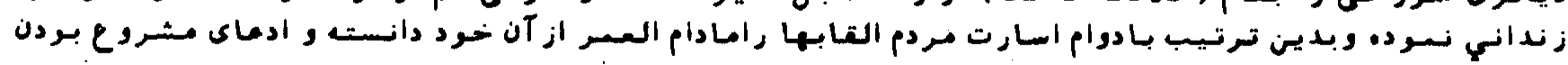

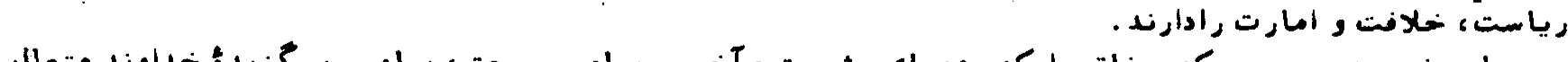

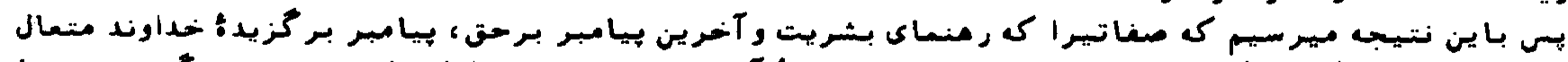

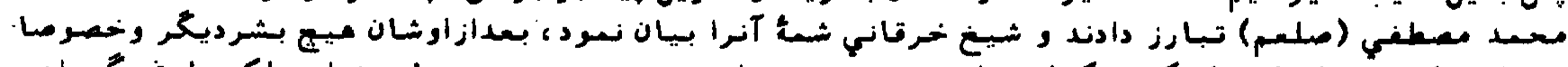

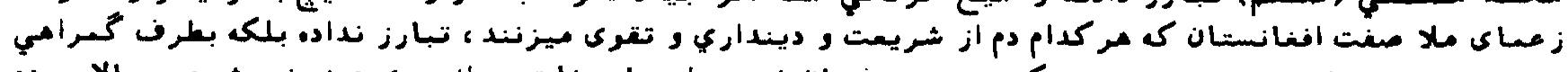

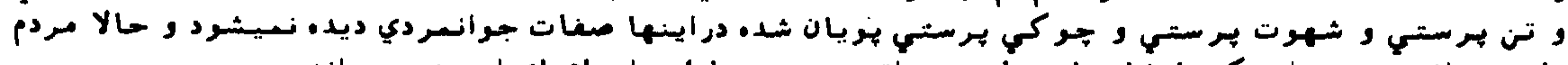

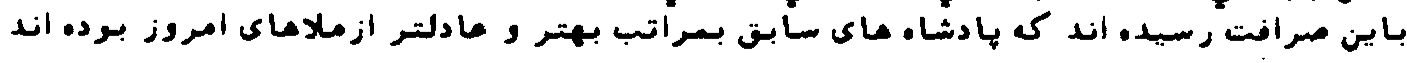

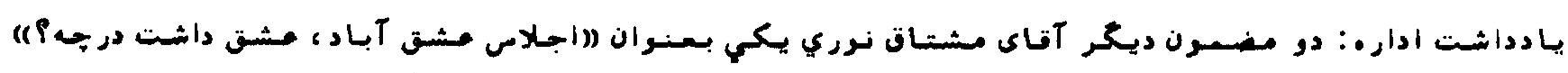

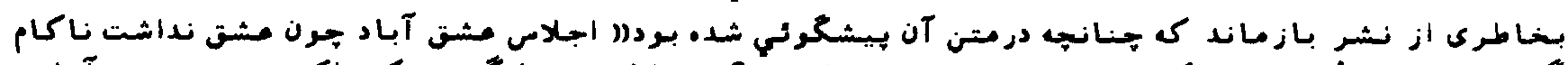

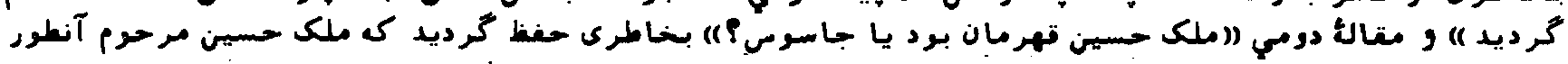




\section{5}

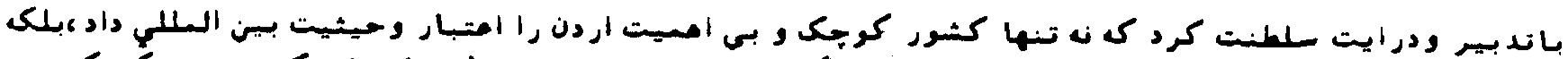

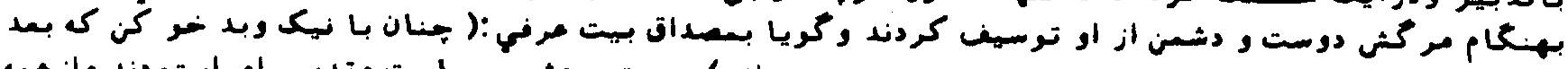

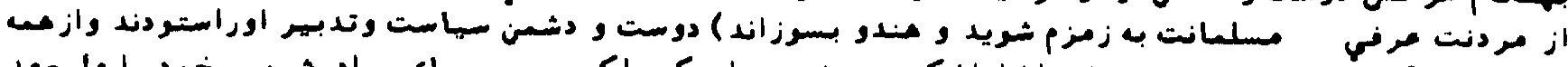

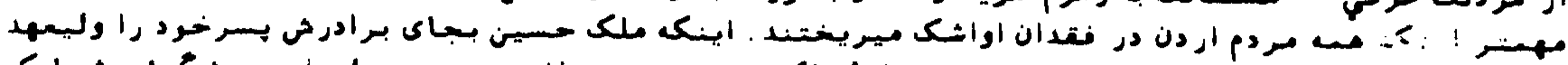

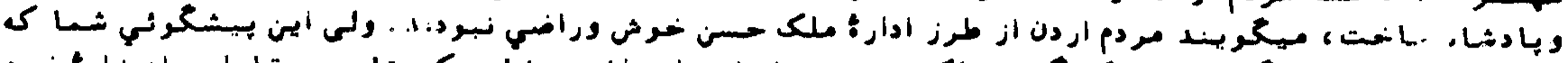

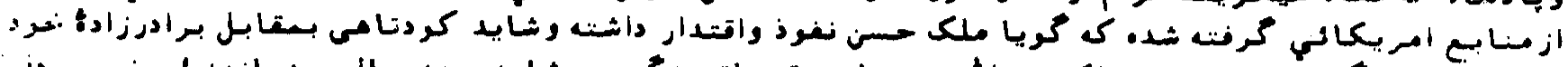

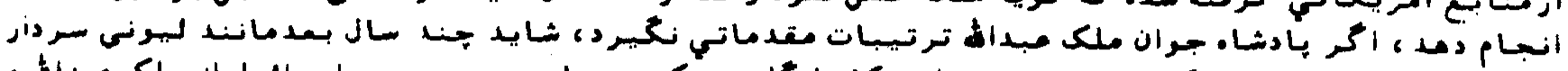

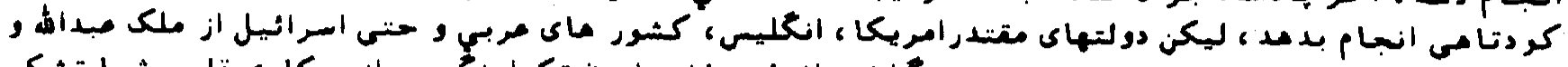

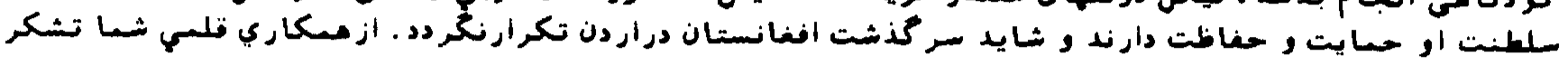
وامتنـان دار يمبر

\section{JLm}

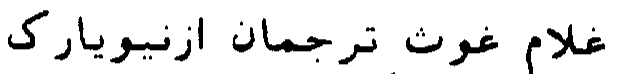

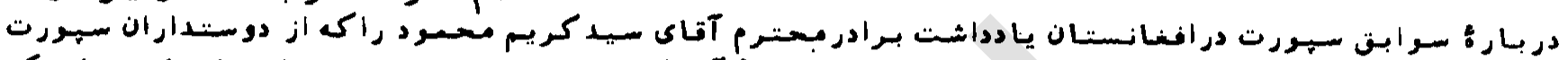

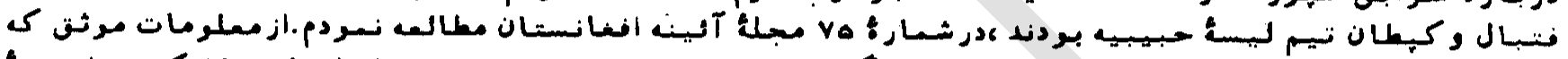

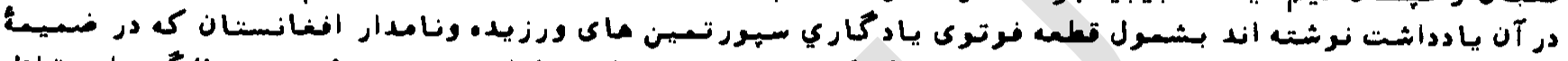

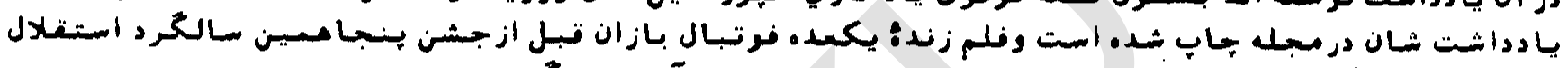

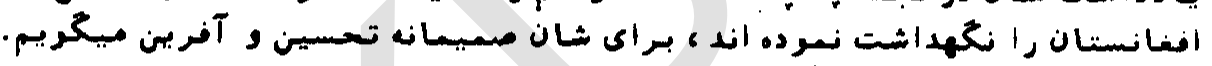

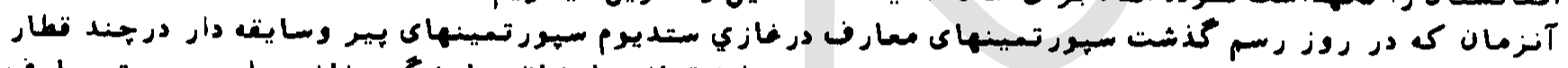

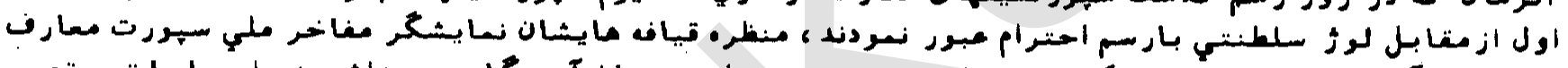

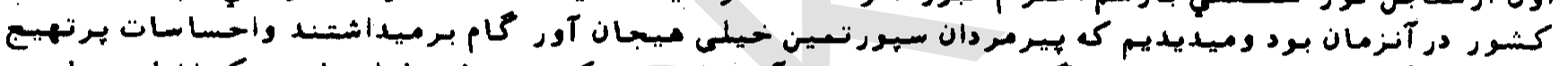

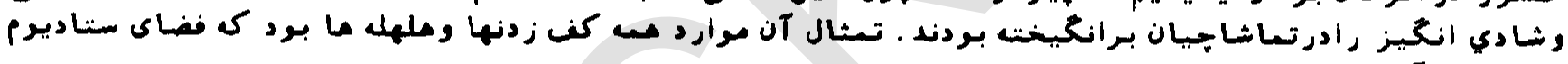

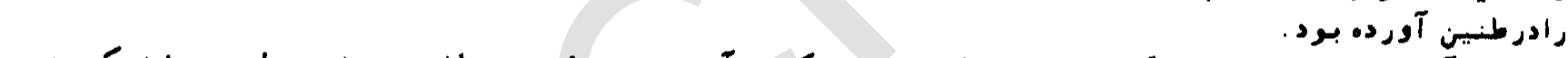

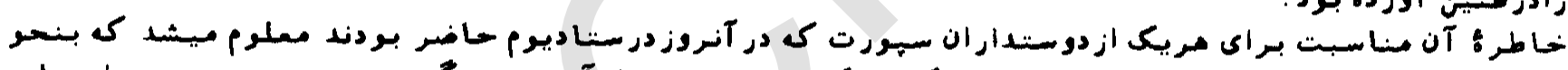

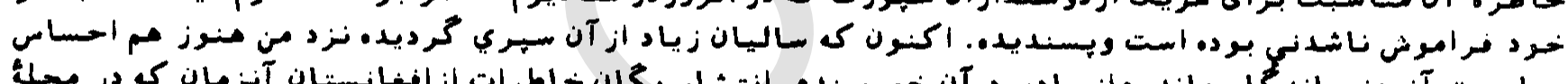

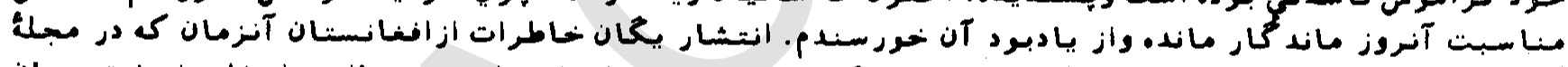

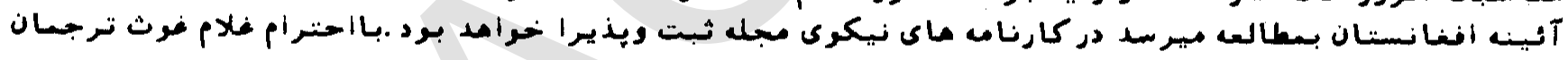

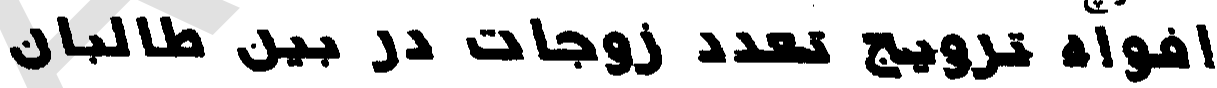

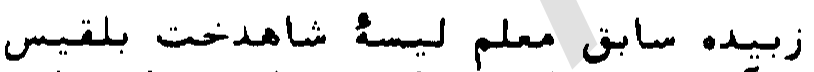

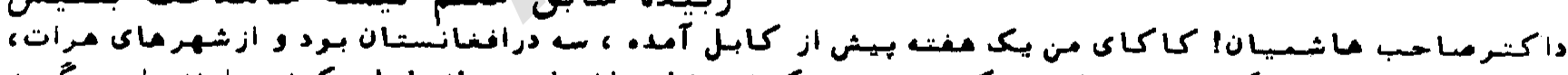

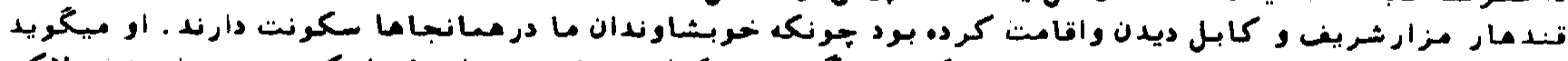

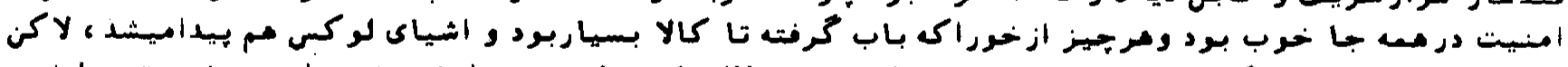

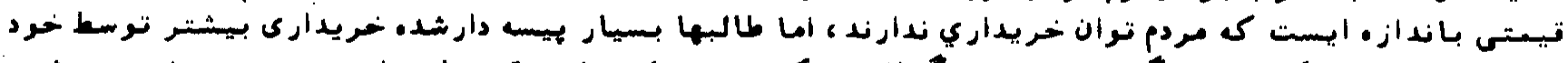

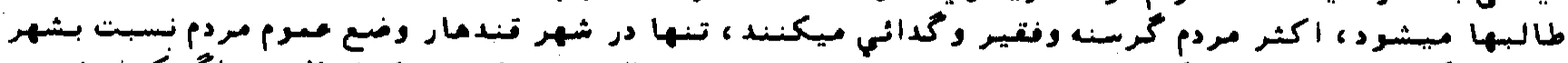

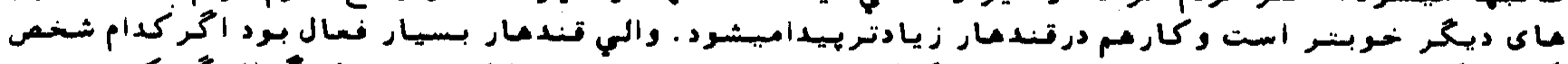

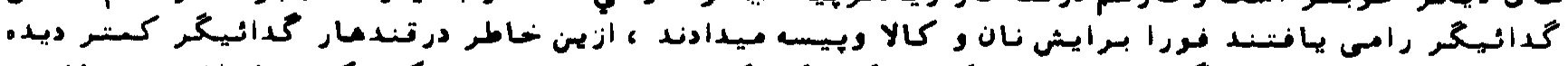

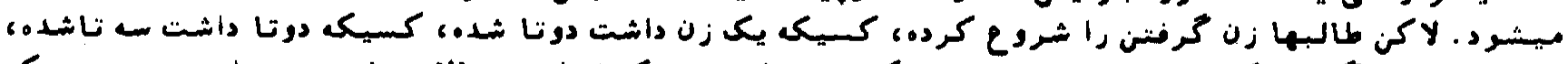

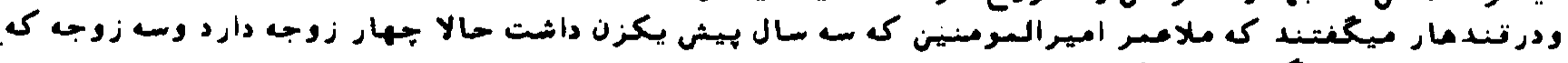

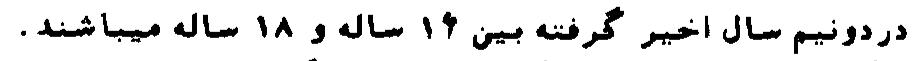

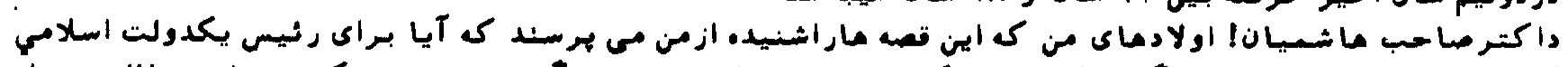

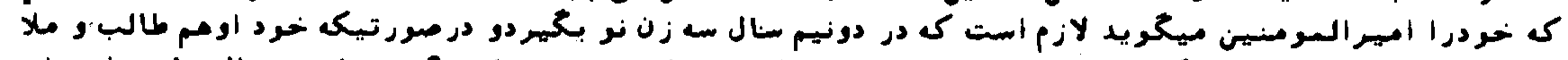

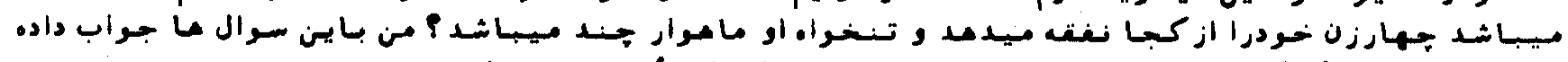

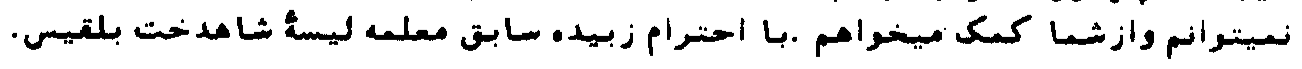

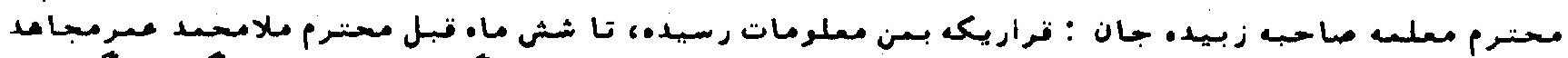

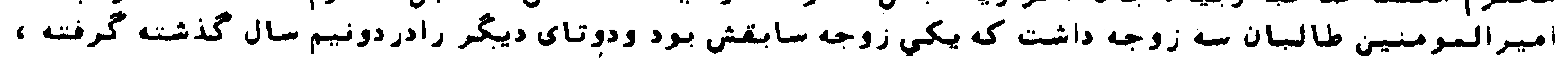




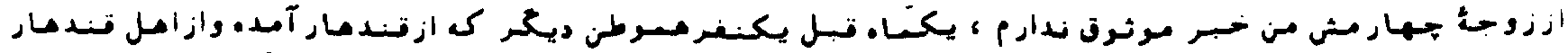

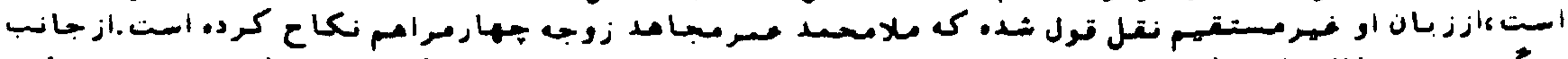

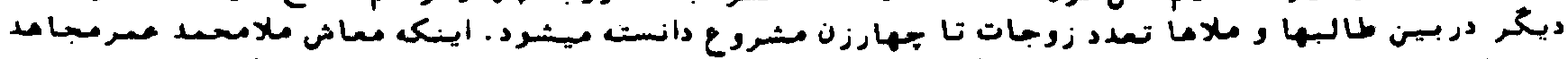

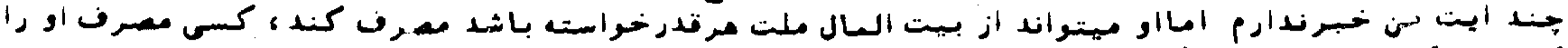

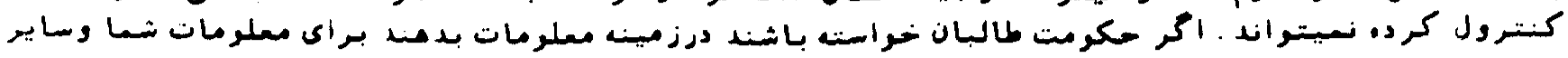

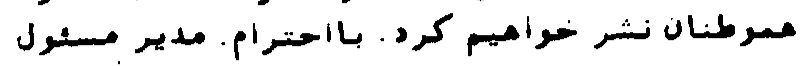

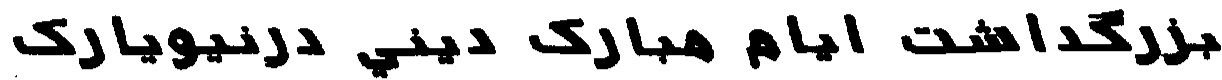

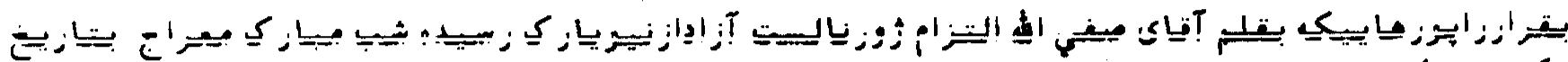

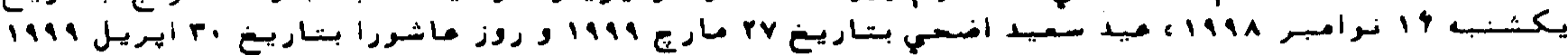

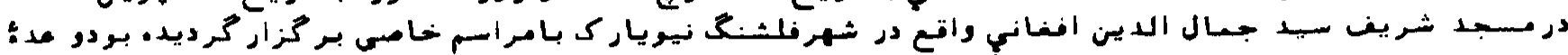

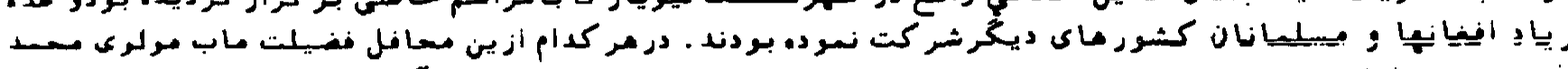

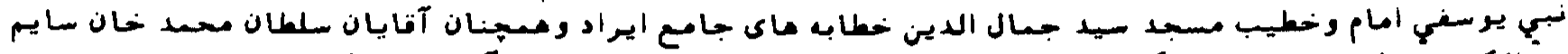

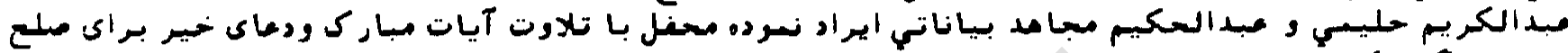

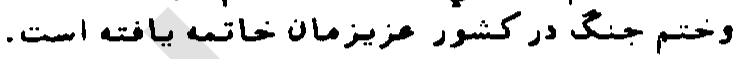

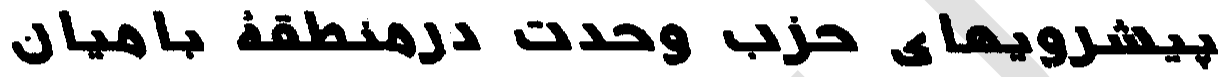

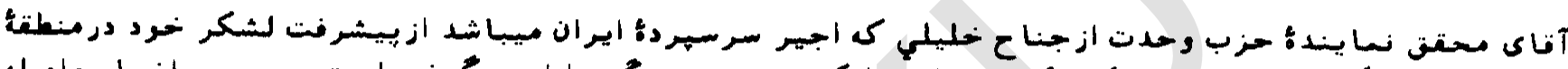

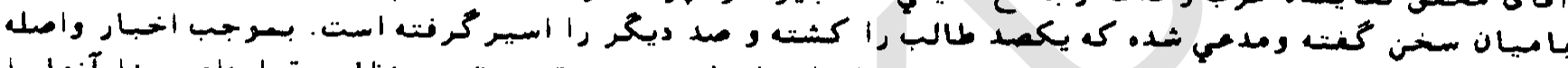

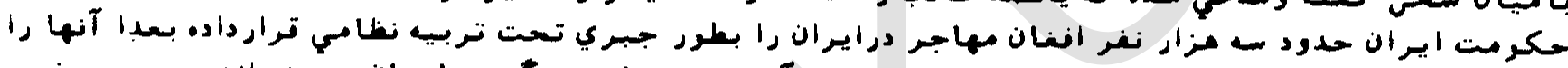

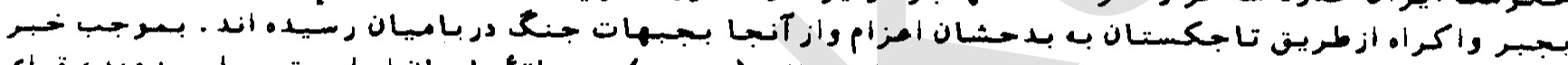

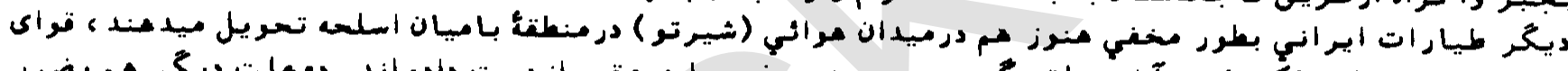

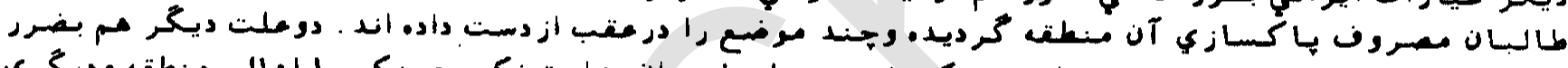

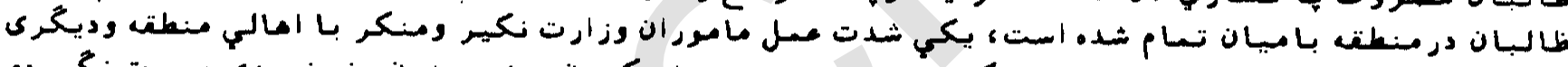

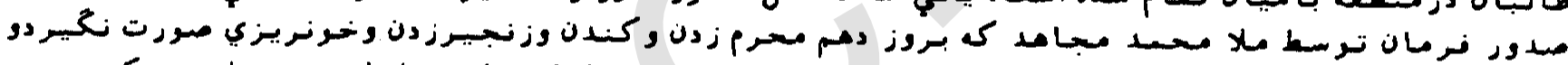

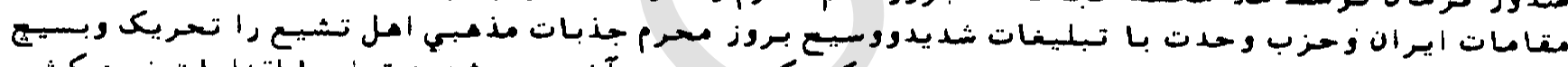

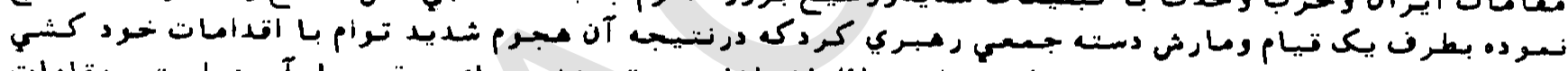

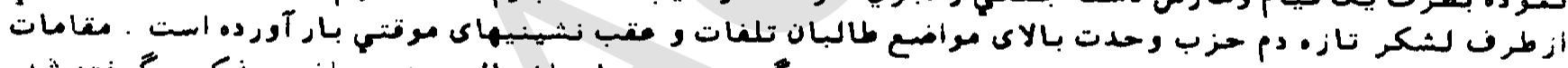

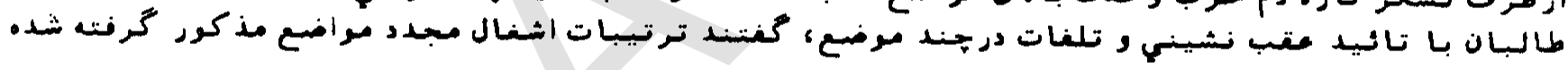

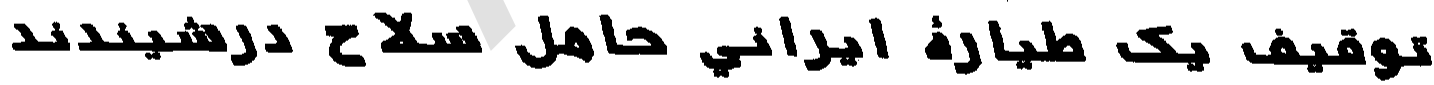

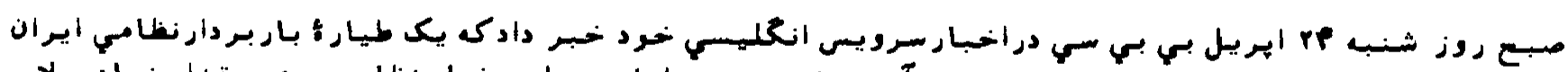

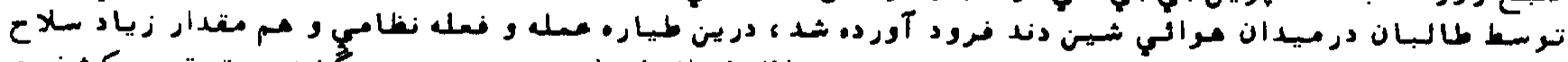

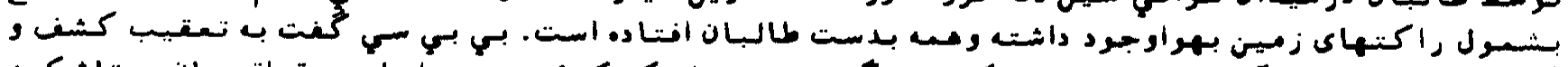

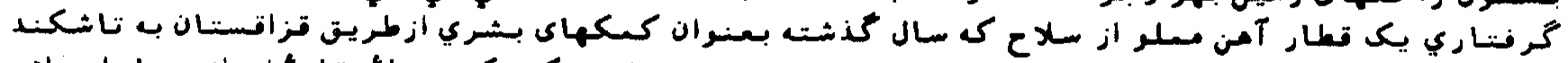

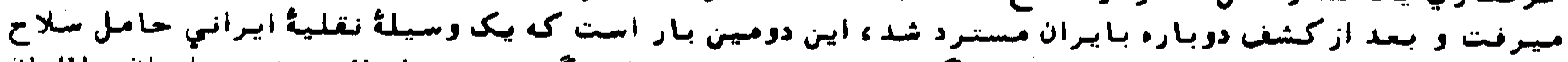

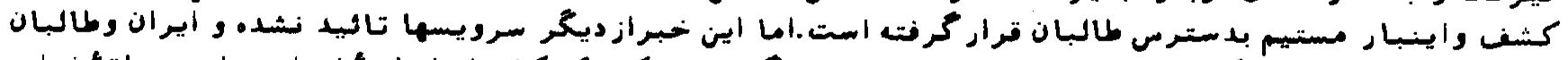

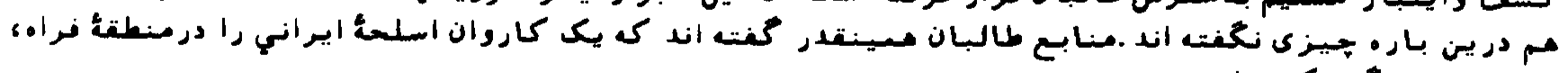

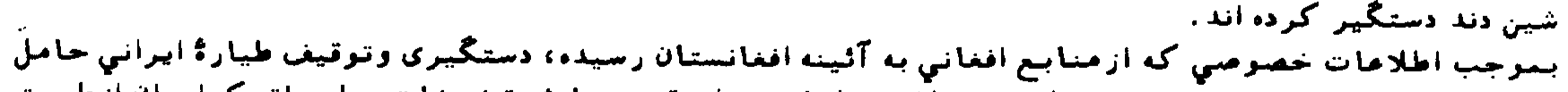

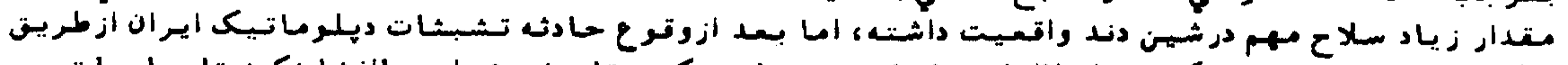

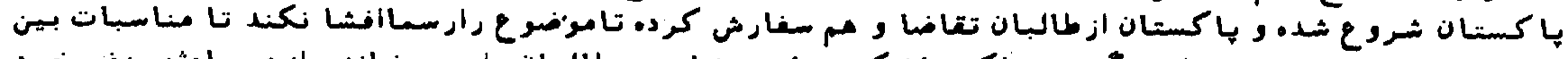

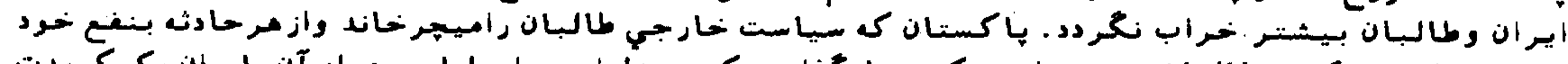

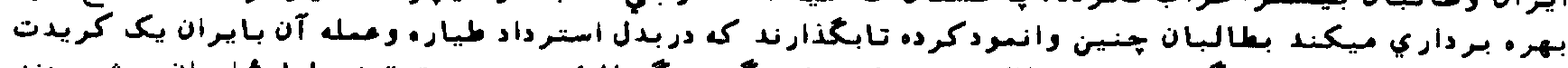

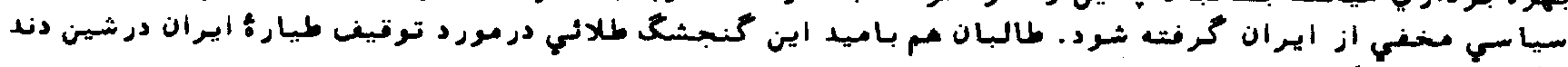




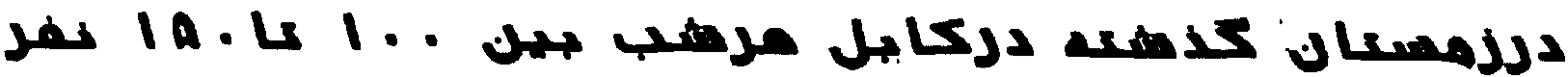

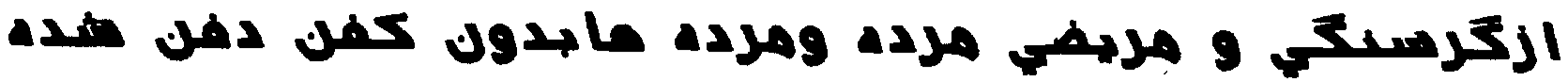

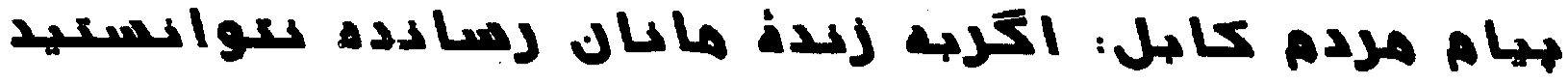

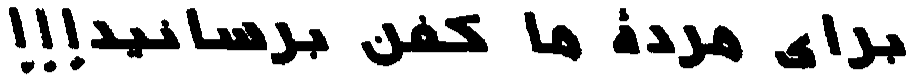

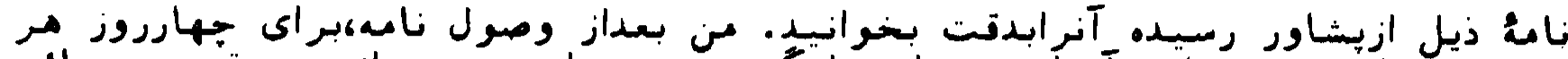

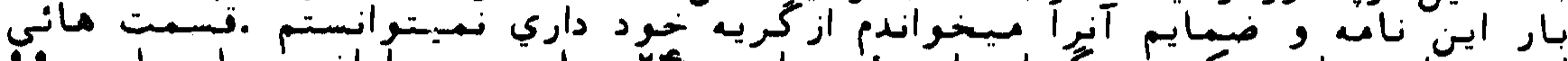

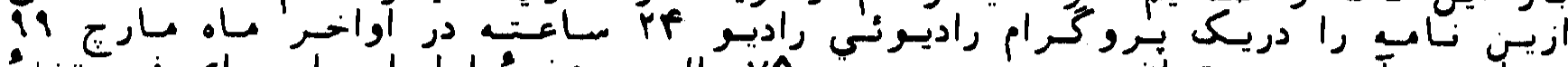

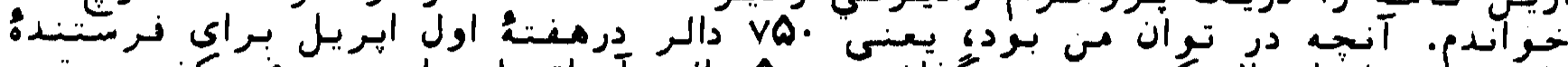

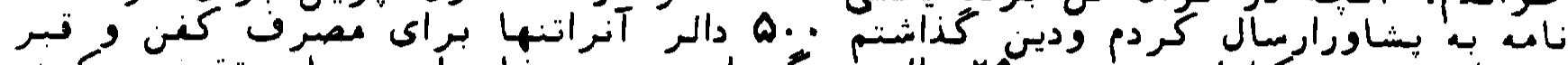

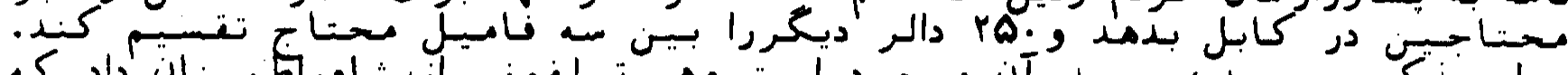

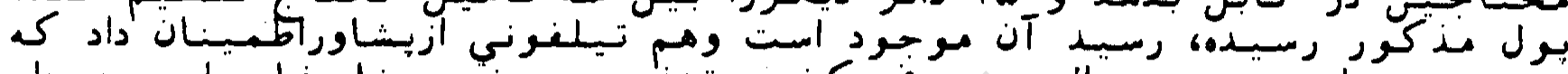

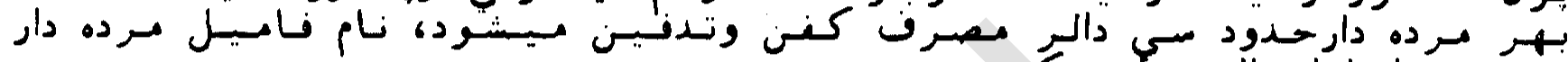

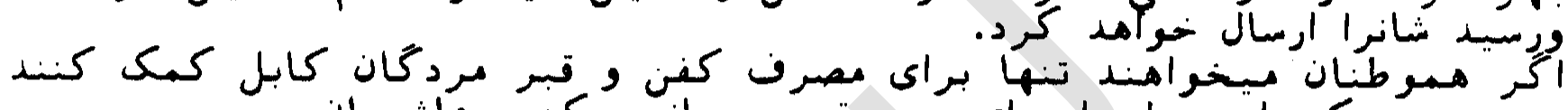

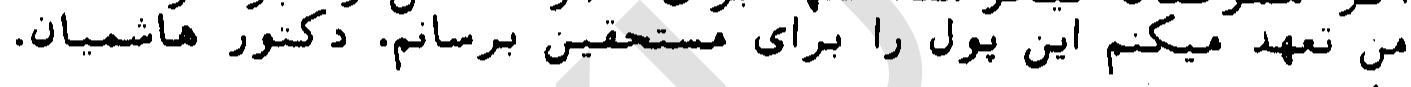

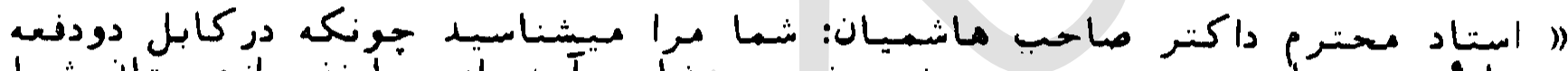

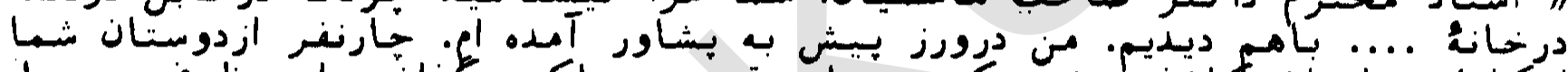

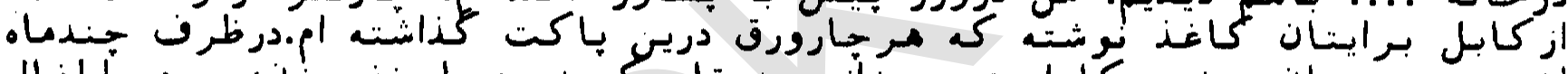

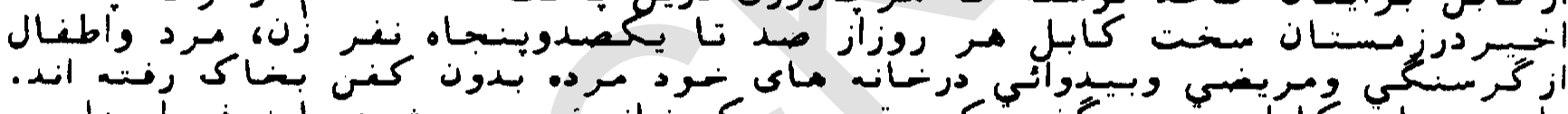

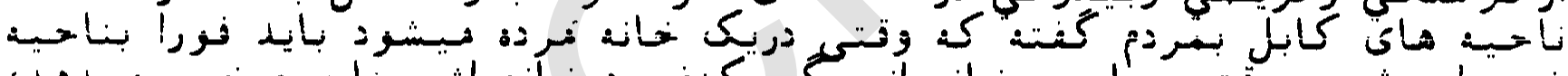

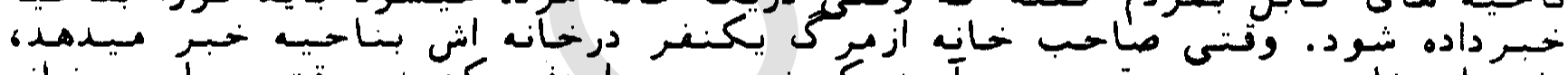

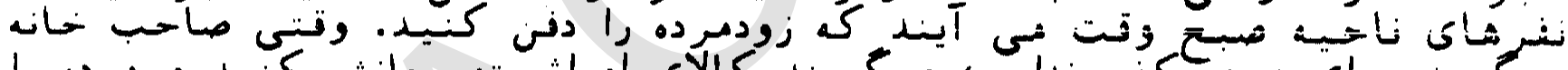

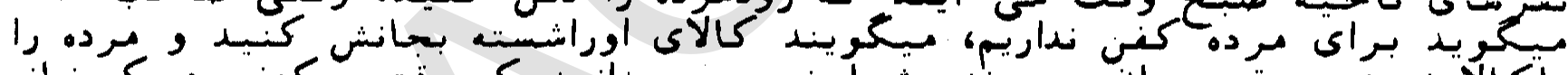

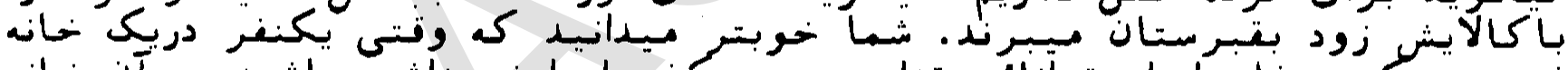

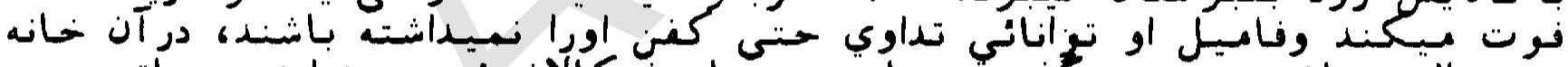

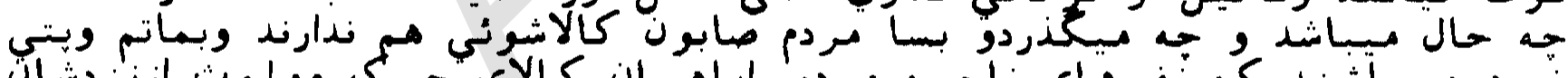

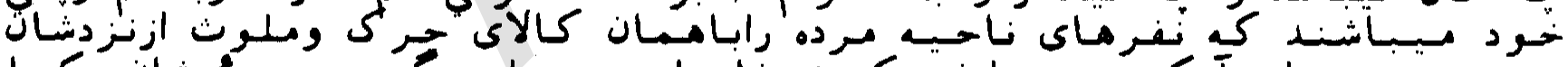

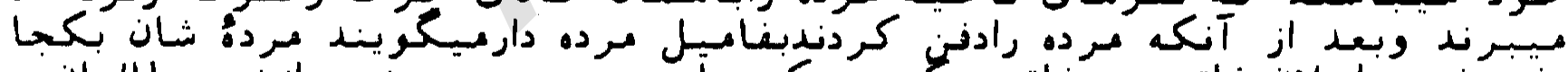

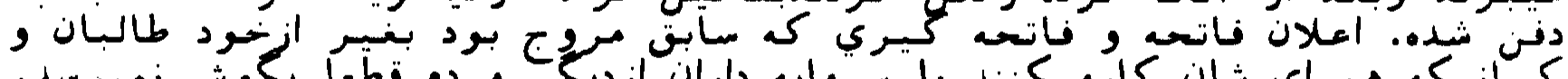

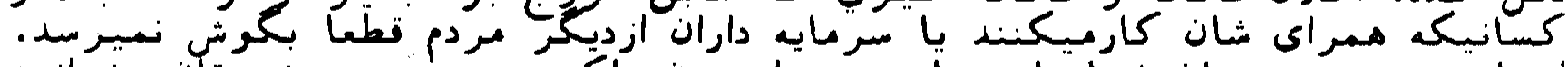

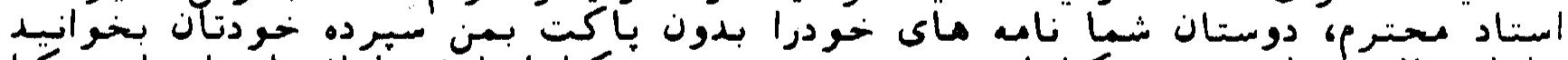

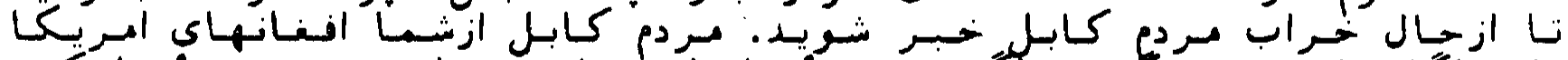

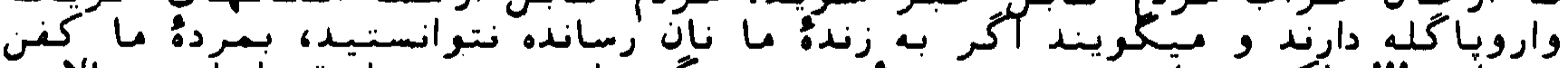

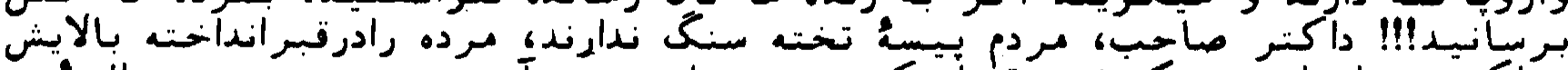

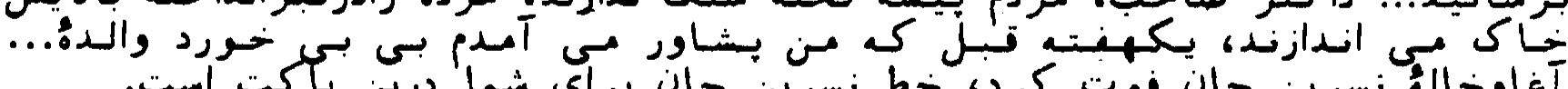

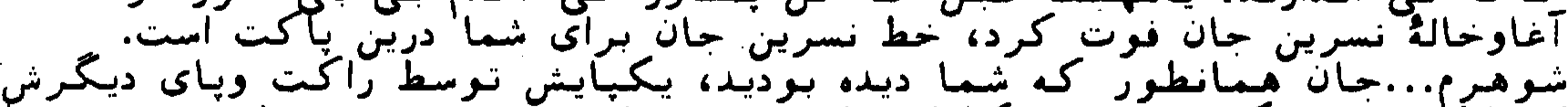

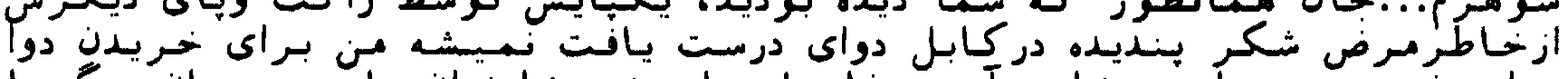

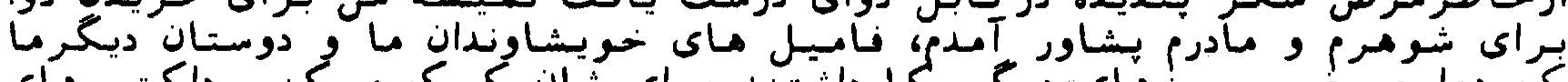

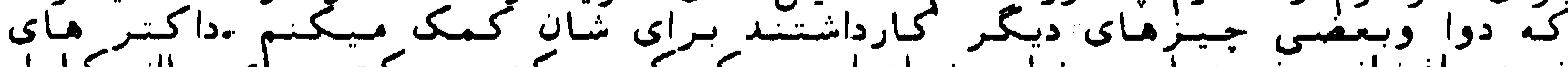

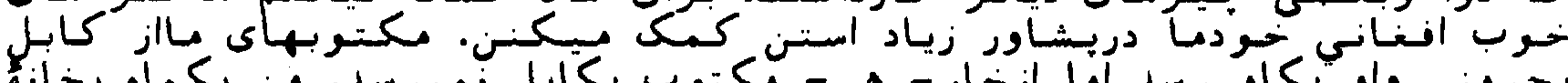

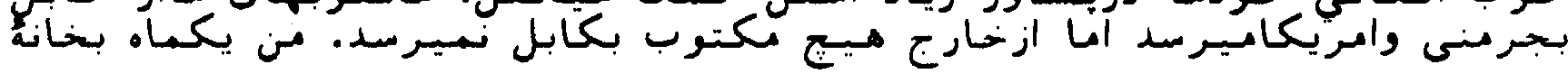




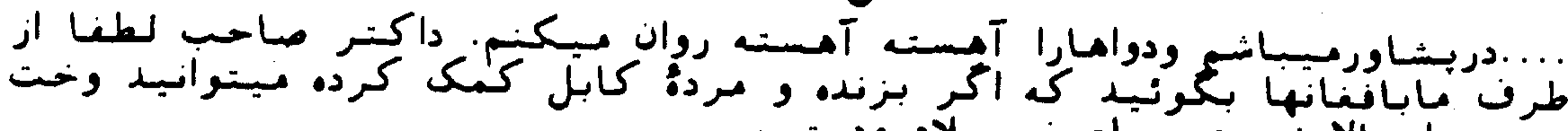

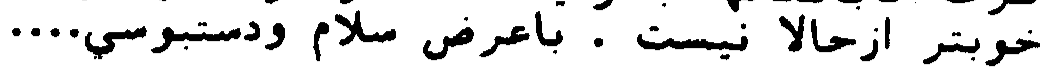

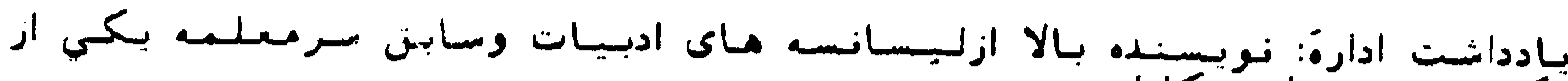

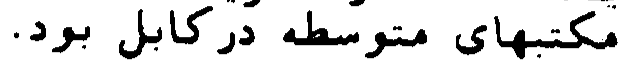

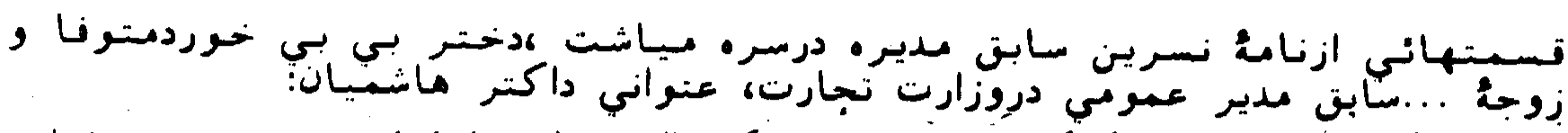

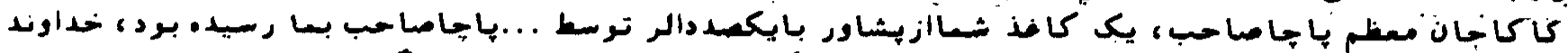

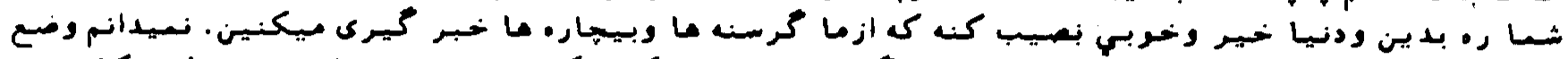

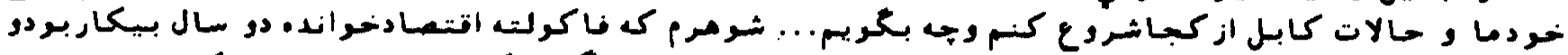

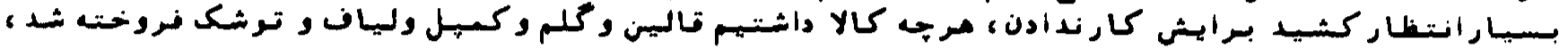

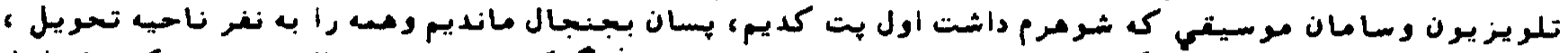

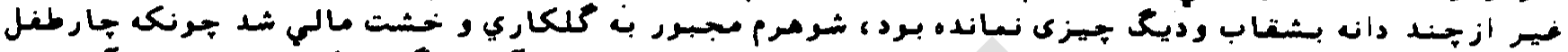

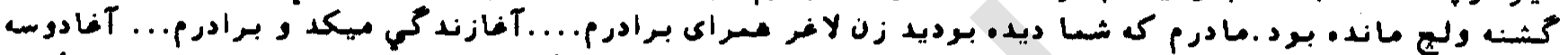

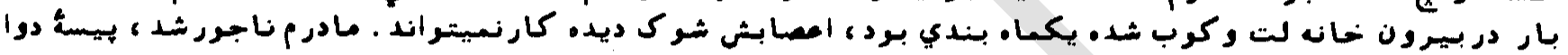

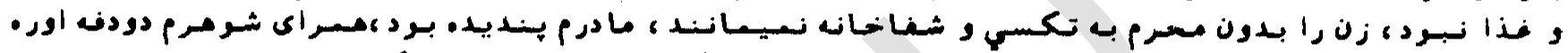

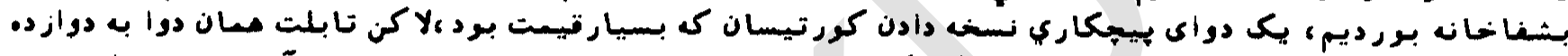

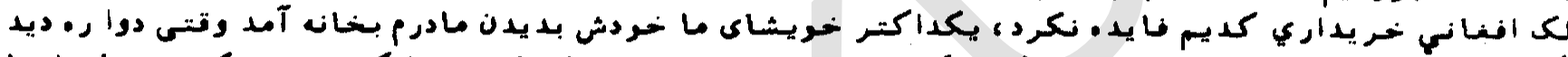

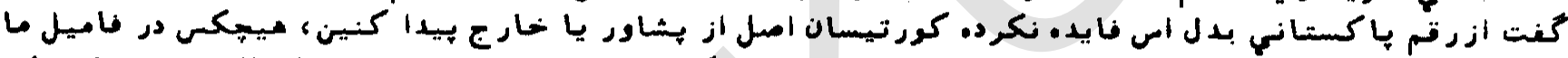

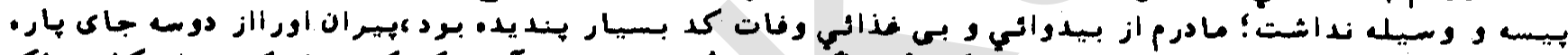

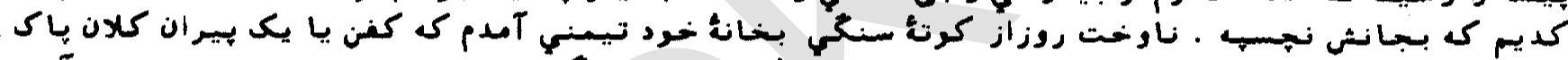

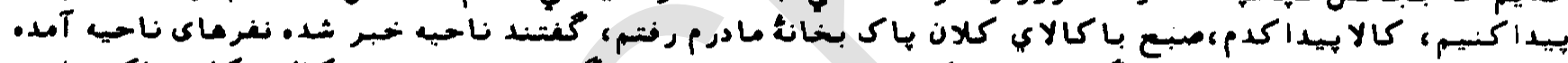

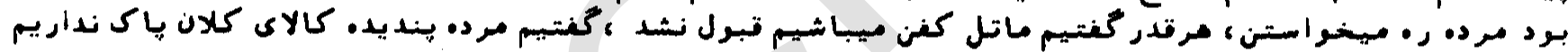

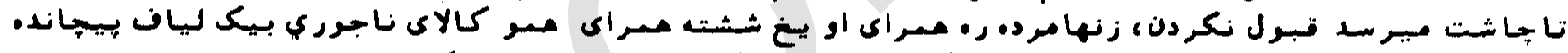

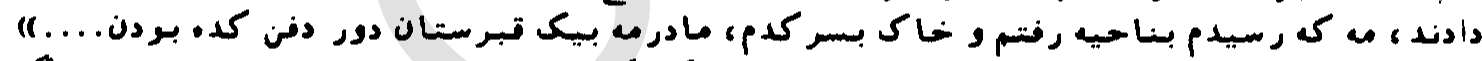

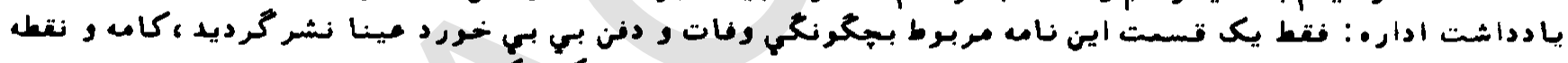

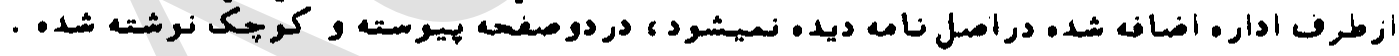

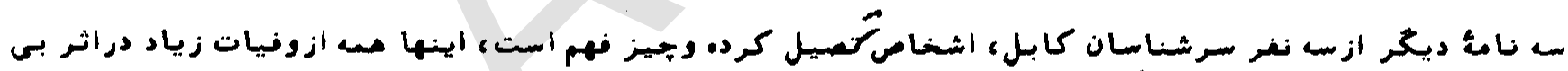

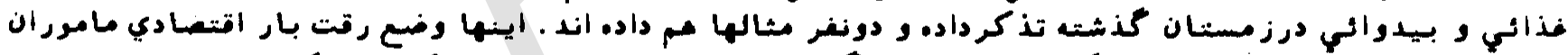

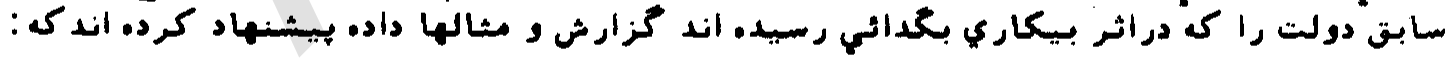

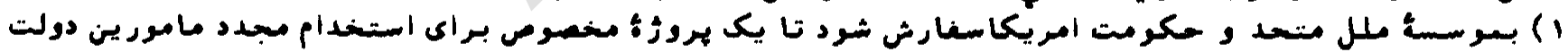

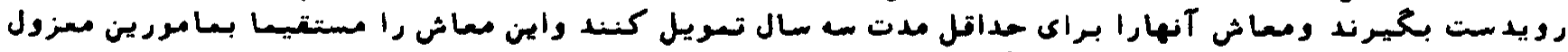

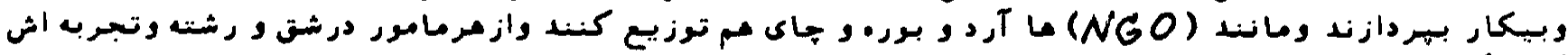

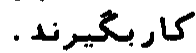

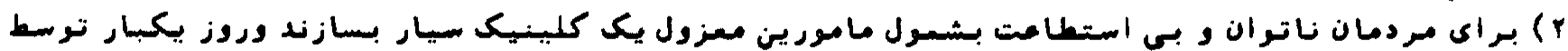

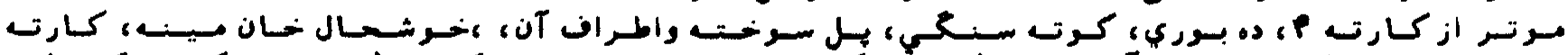

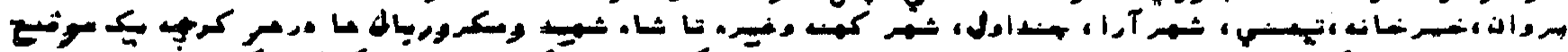

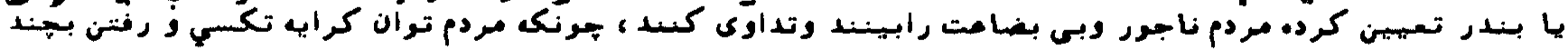

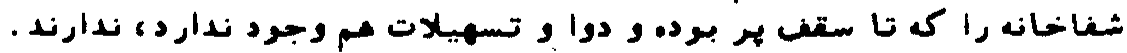

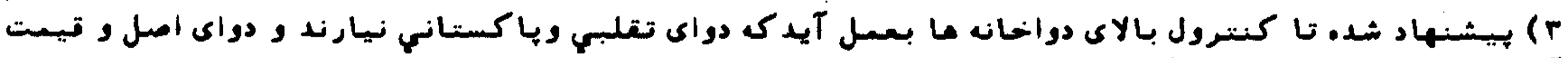

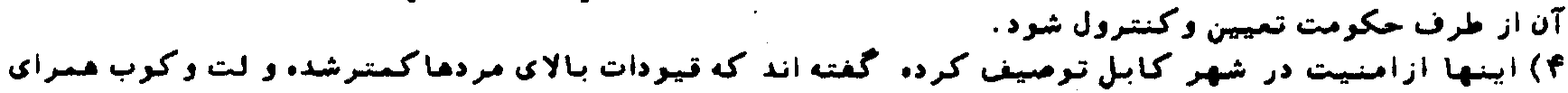

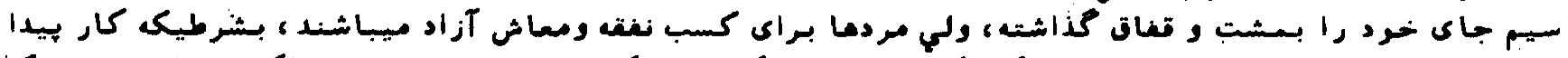

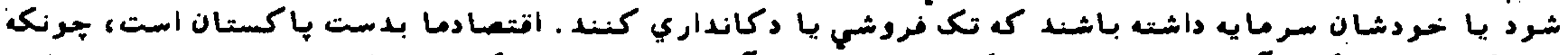

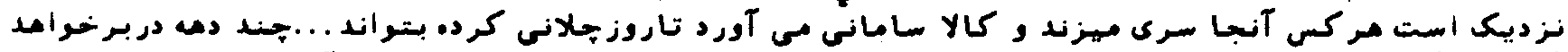

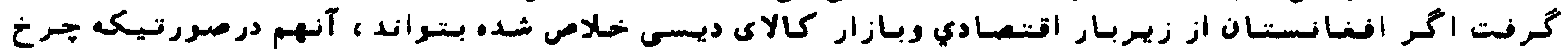




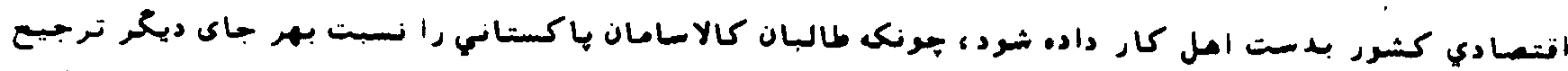

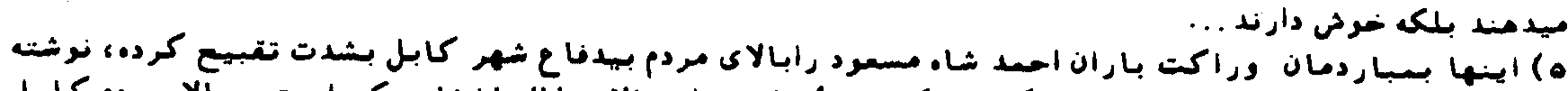

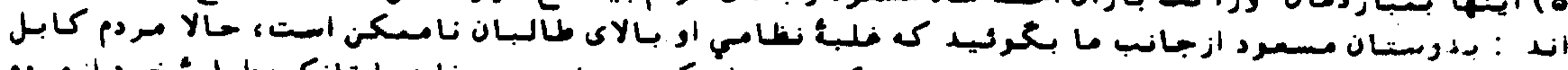

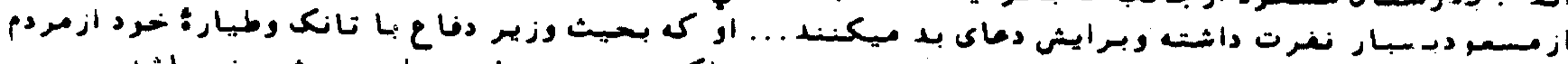

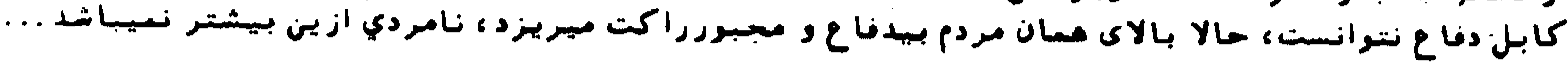

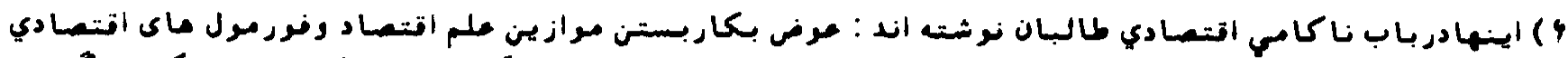

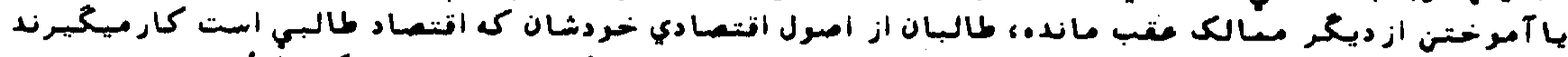

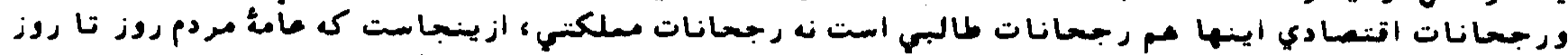

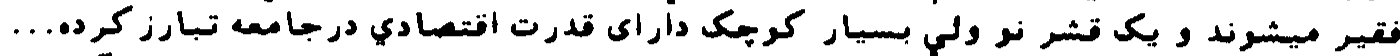

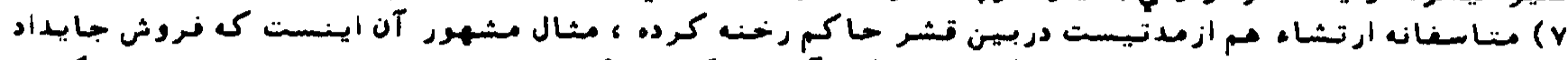

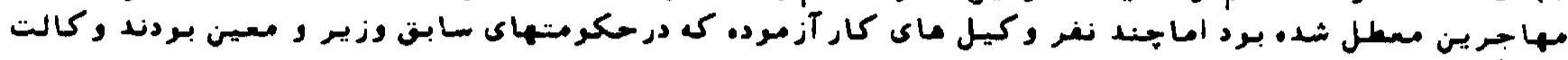

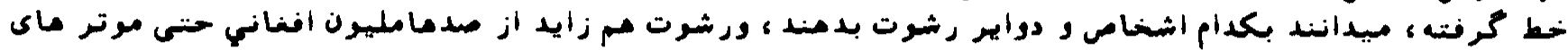

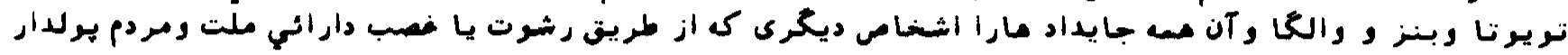

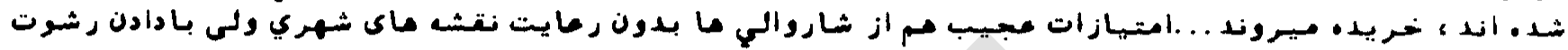

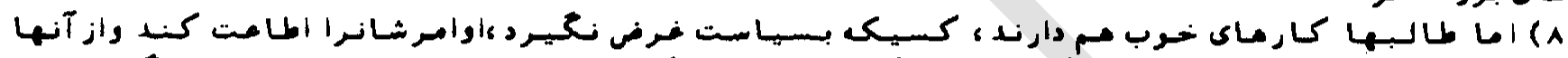

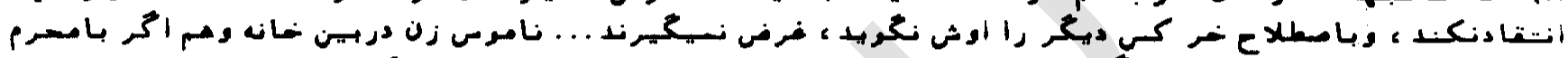

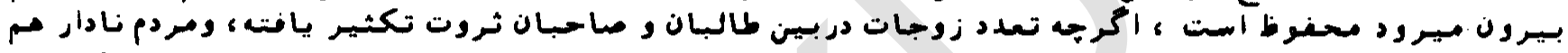

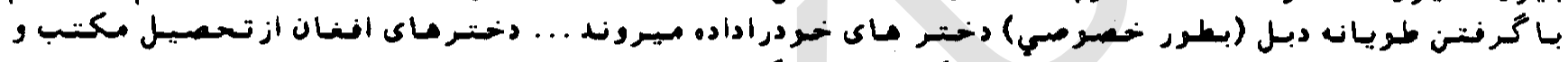

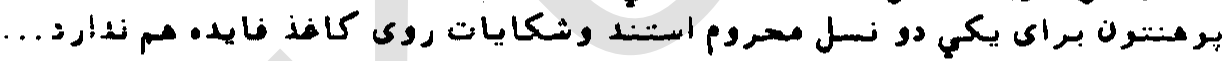

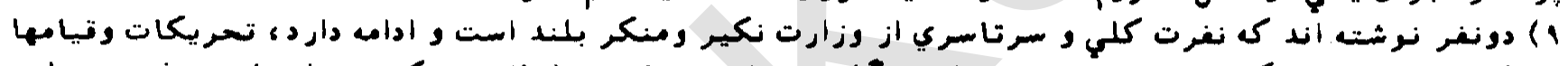

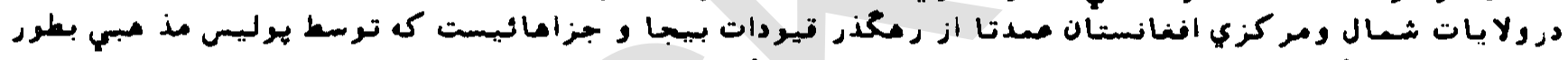

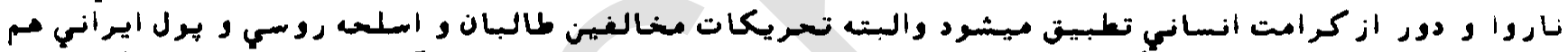

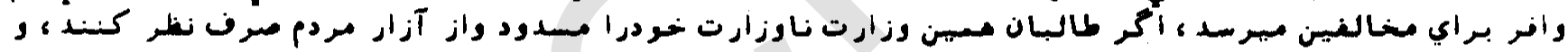

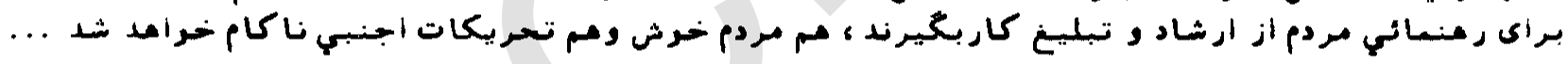

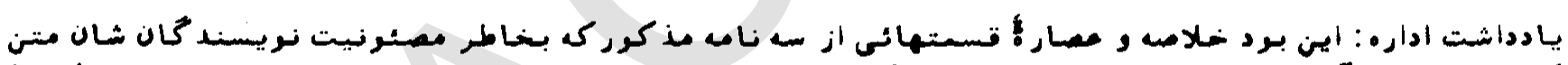

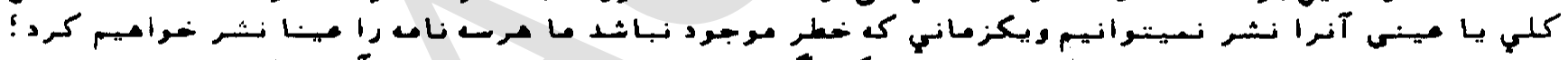

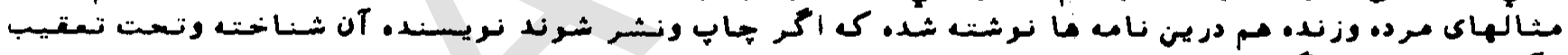

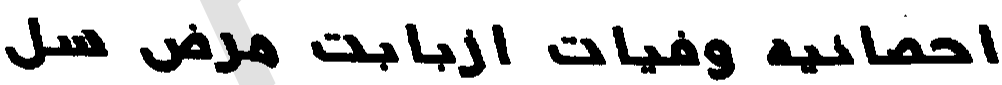

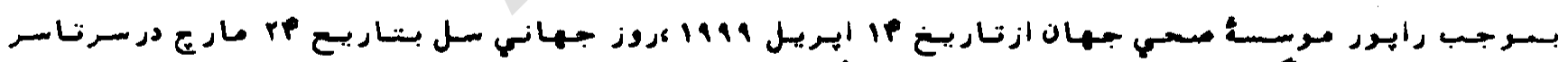

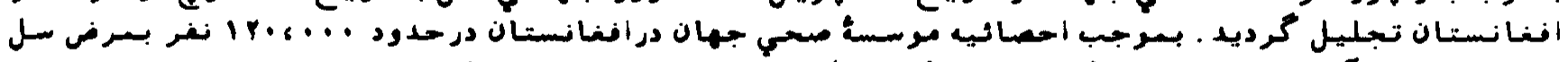

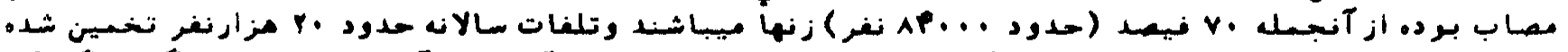

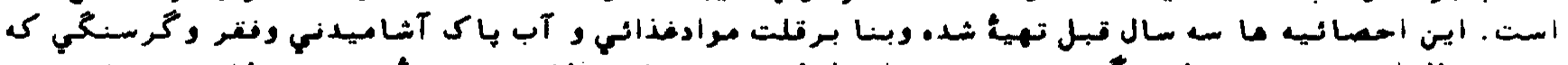

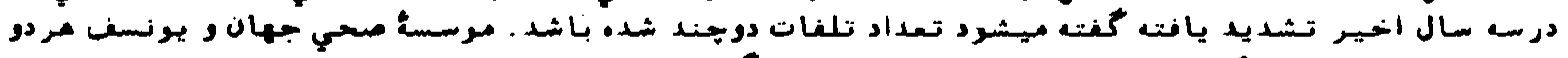

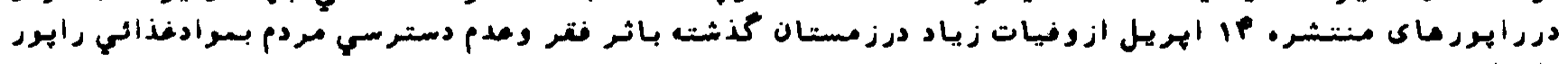

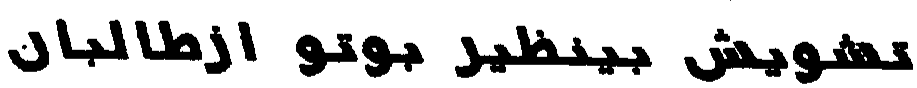

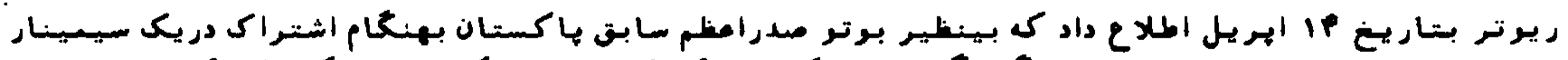

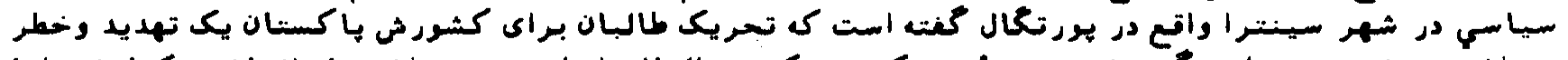

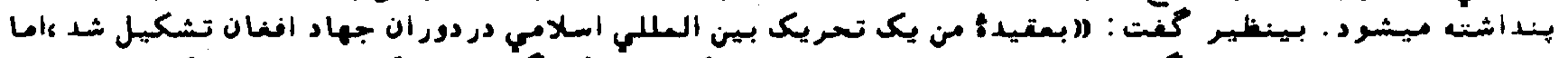

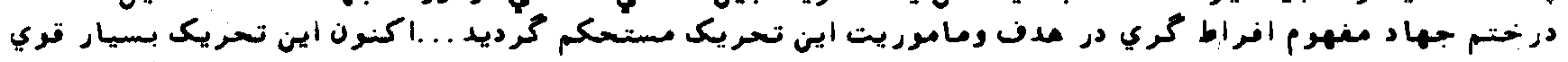

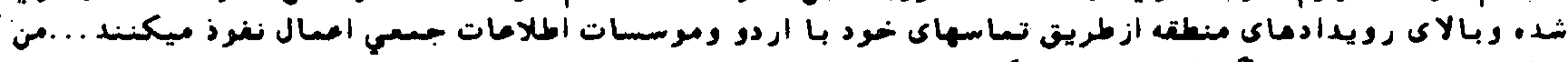

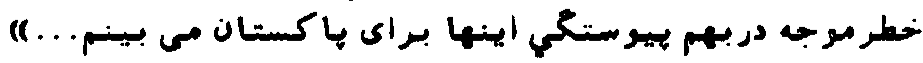




\section{هلت الرد دركاهل 10}

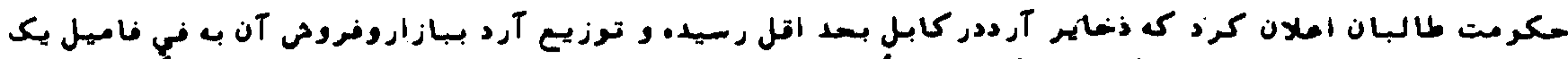

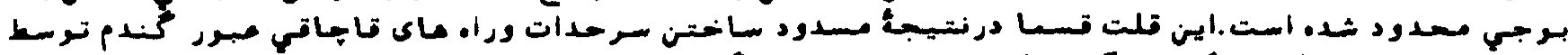

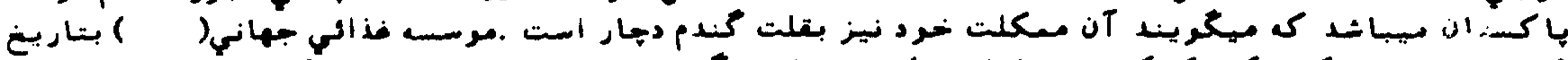

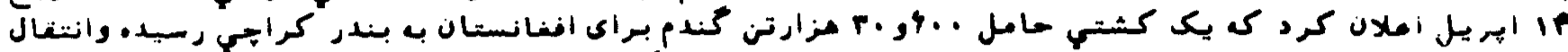

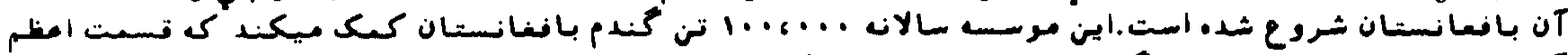

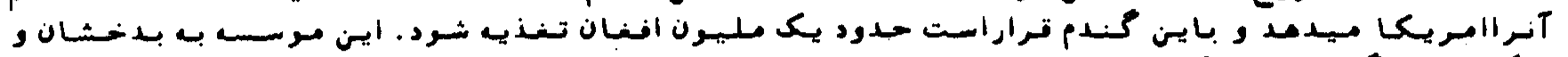

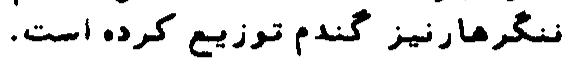

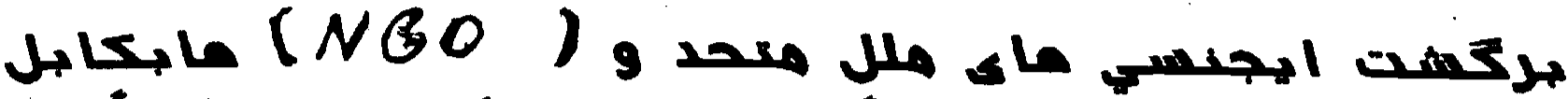

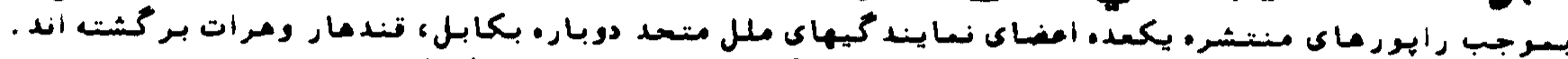

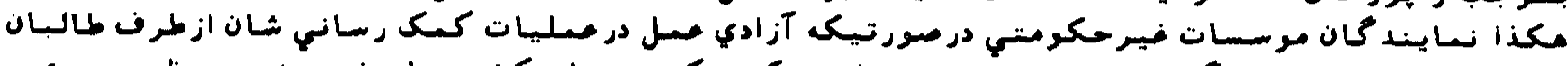

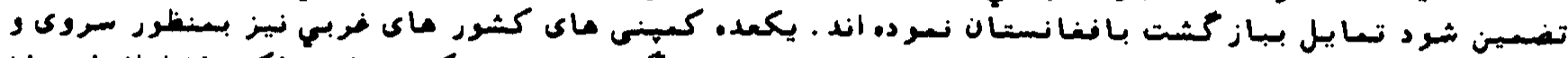

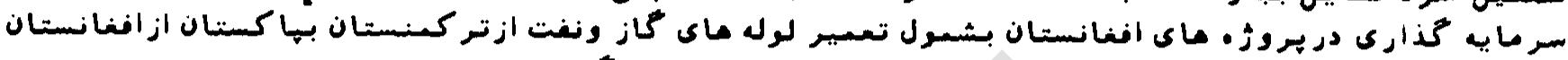

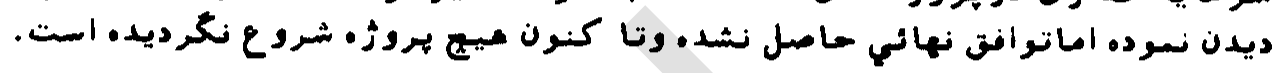

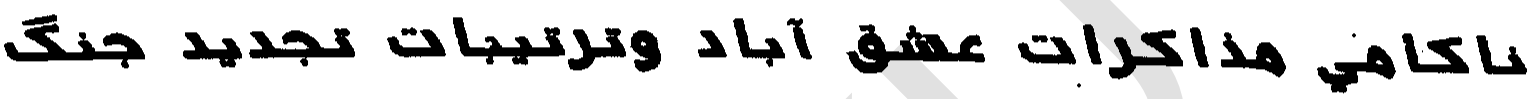

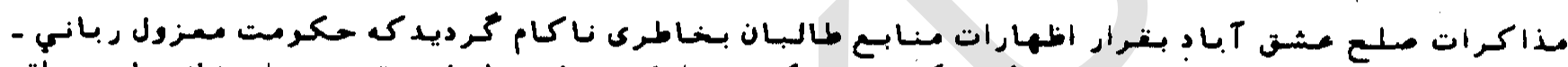

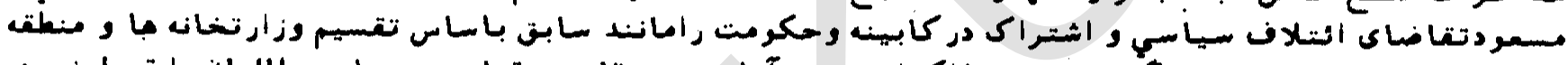

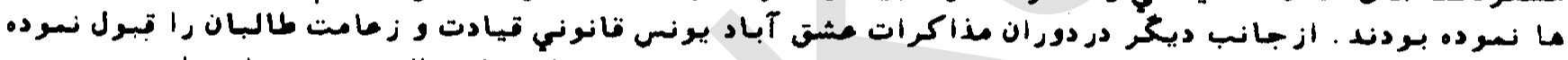

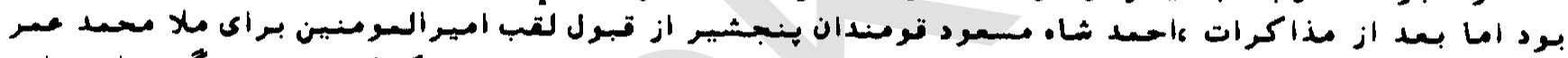

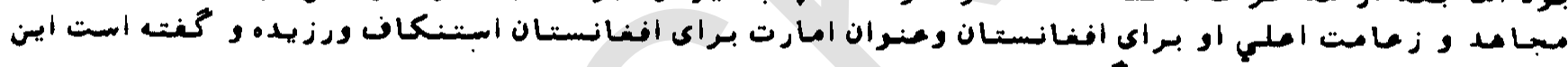

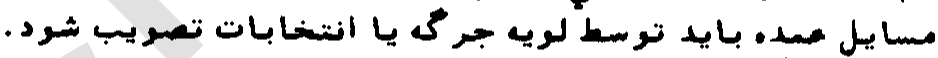

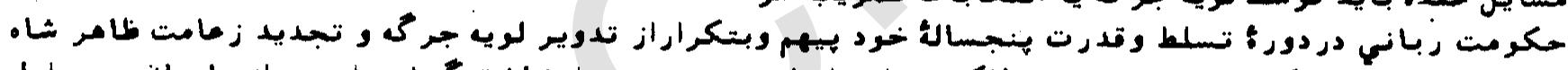

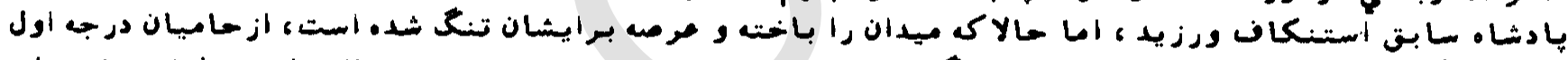

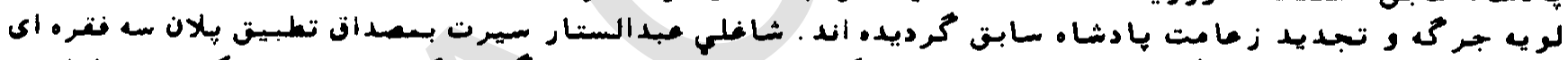

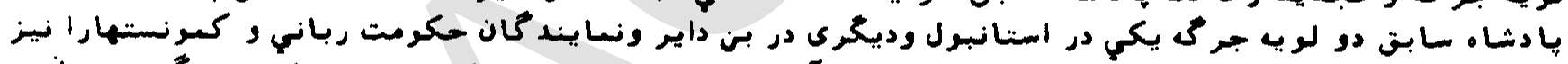

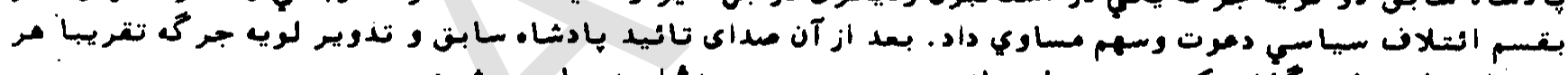

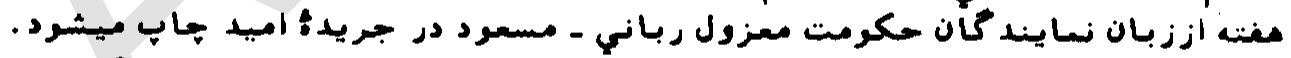

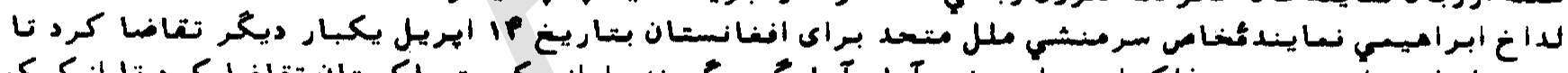

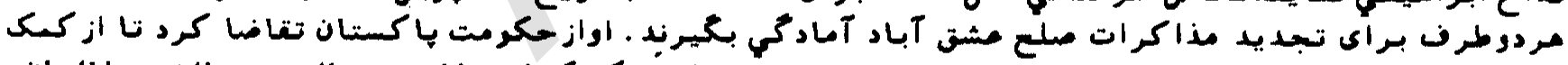

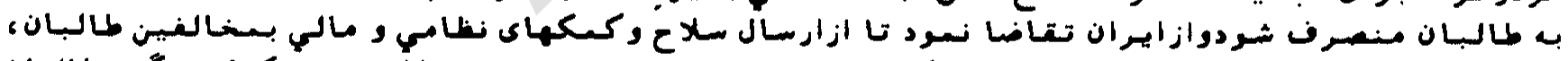

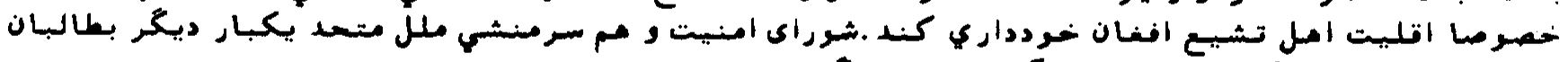

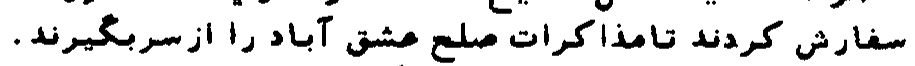

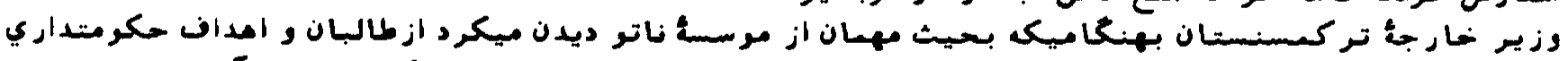

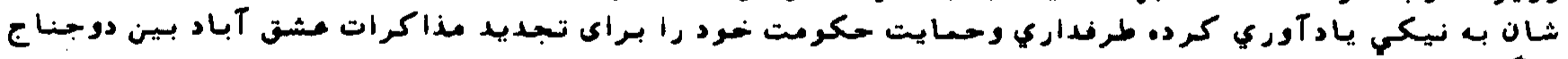

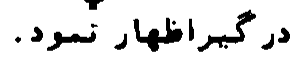

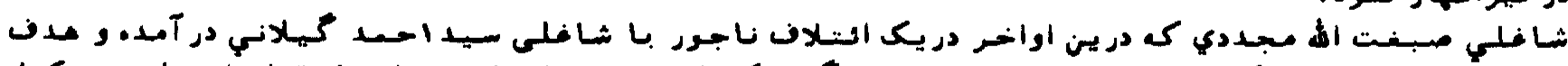

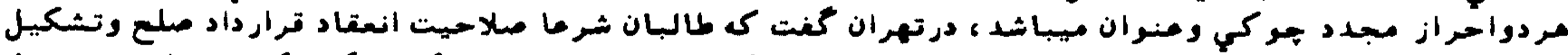

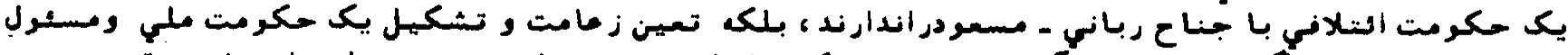

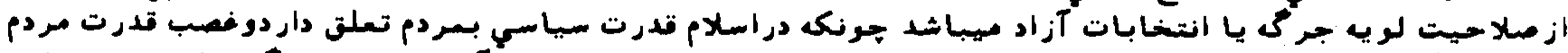

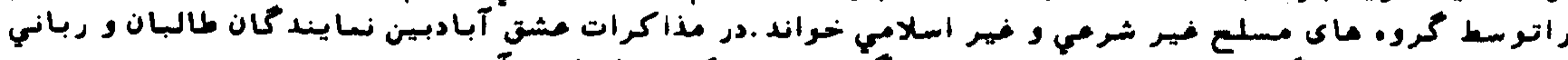

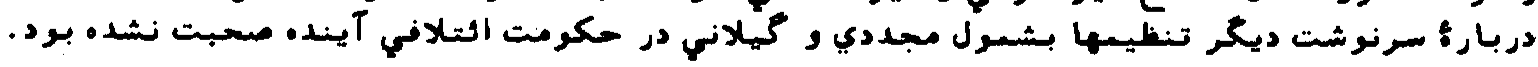

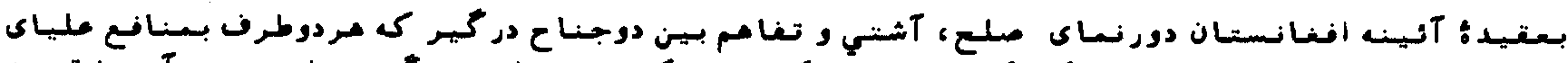

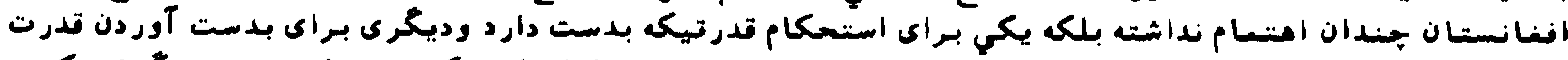

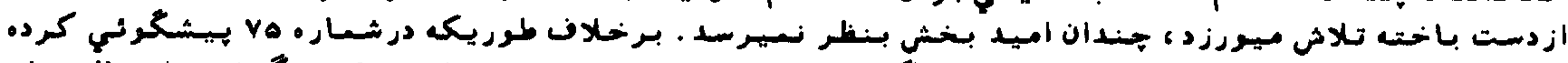

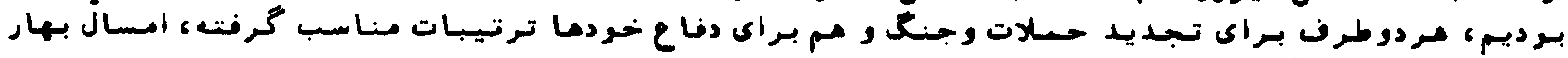




\section{1

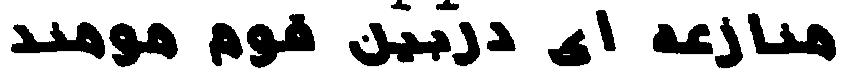

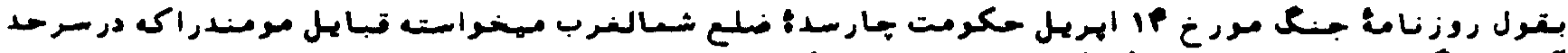

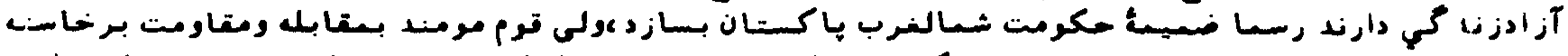

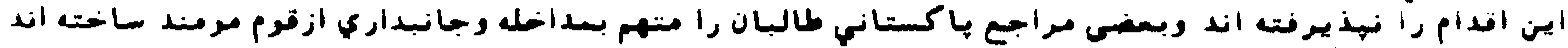

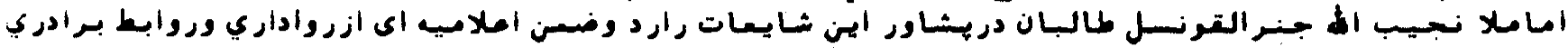

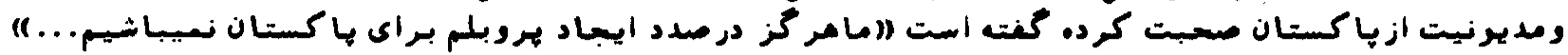

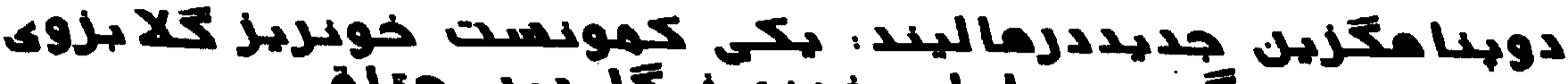

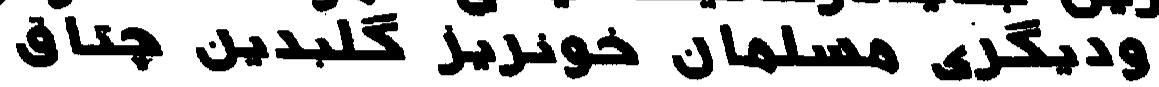

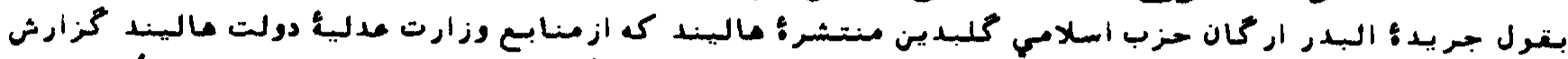

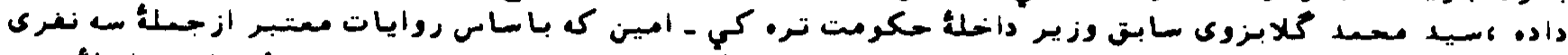

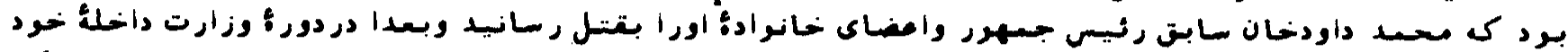

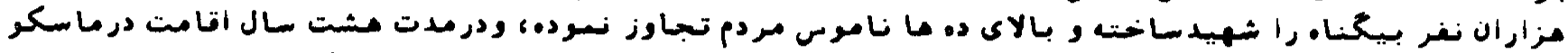

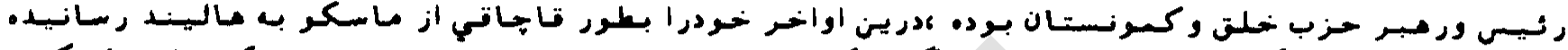

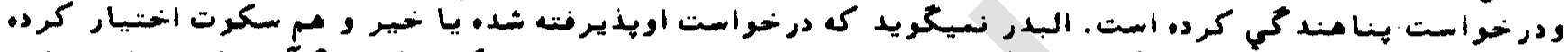

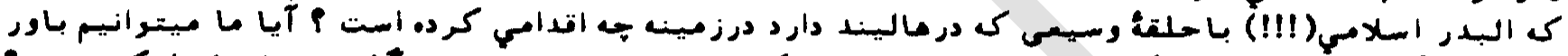

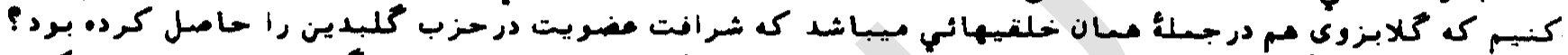

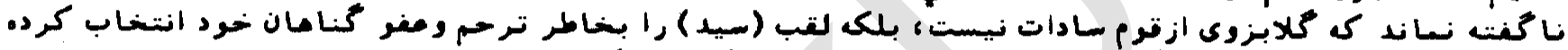

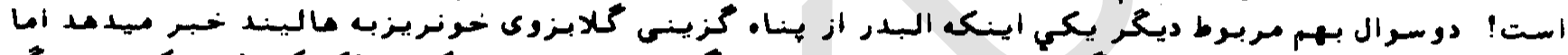

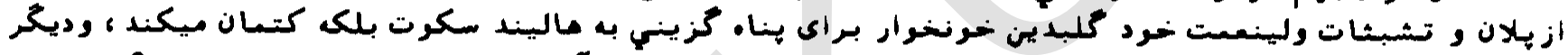

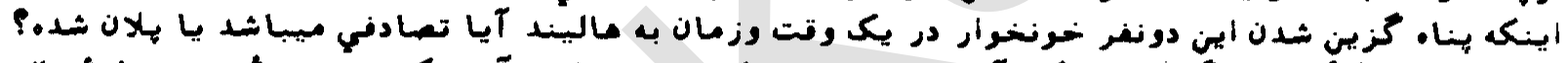

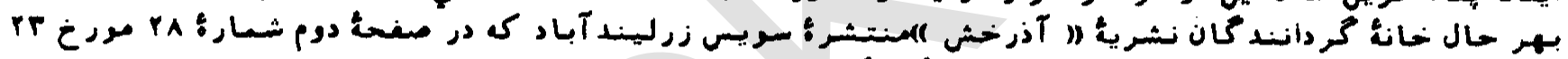

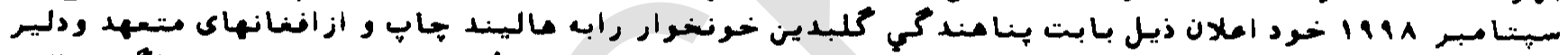

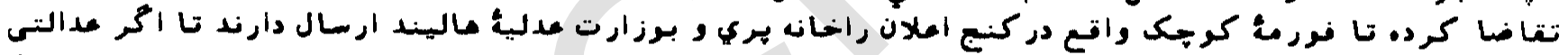

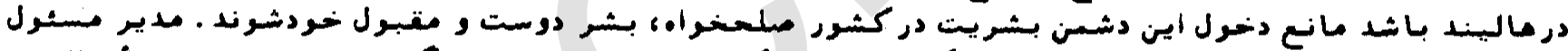

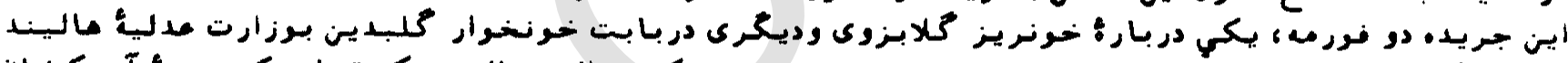

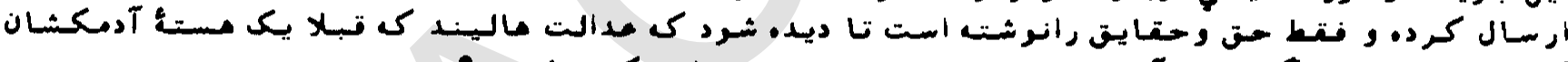

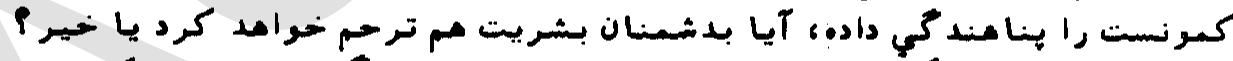

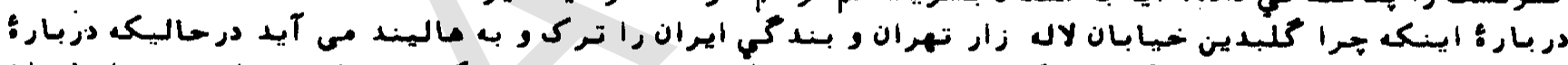

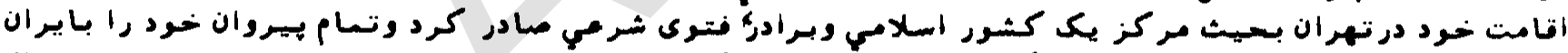

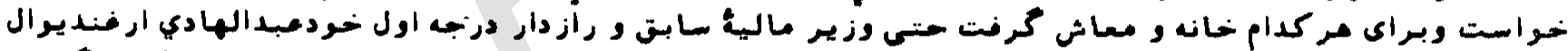

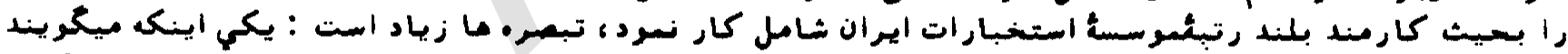

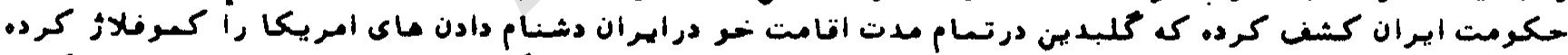

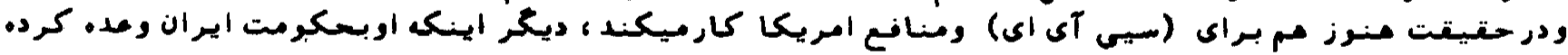

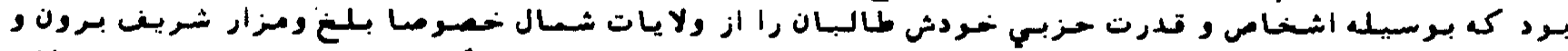

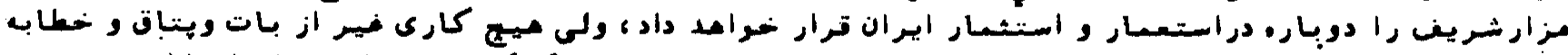

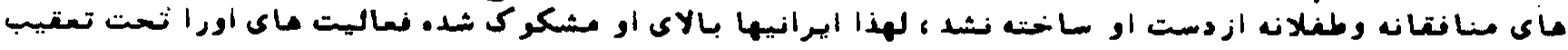

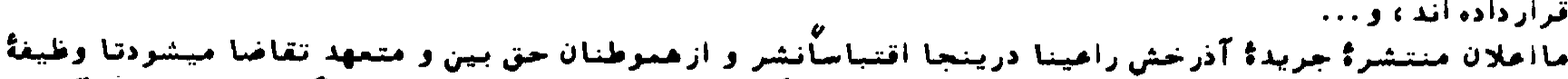

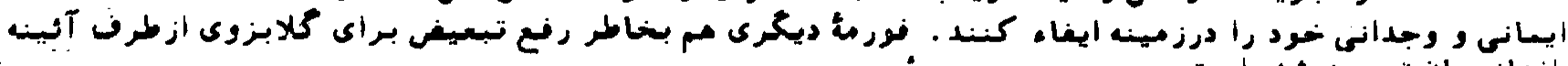

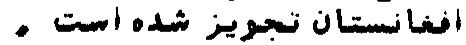

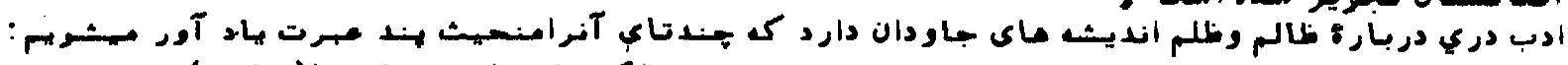

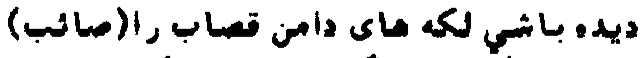

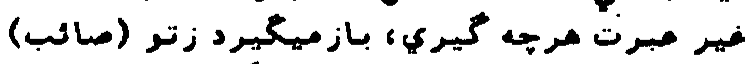

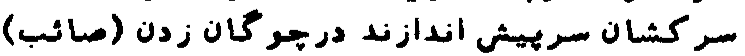

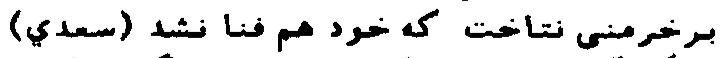

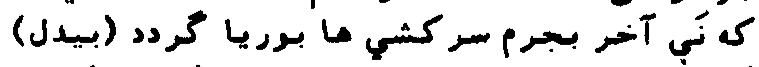

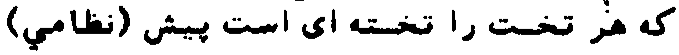

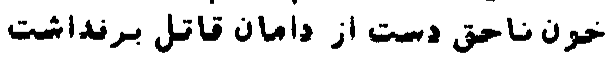

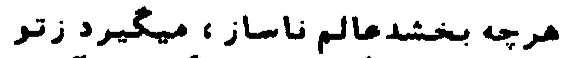

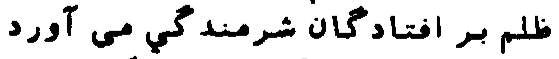

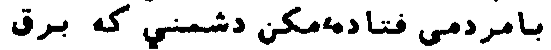

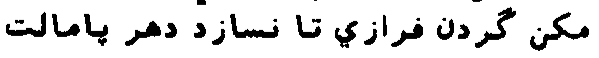

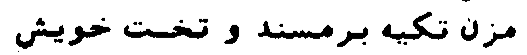



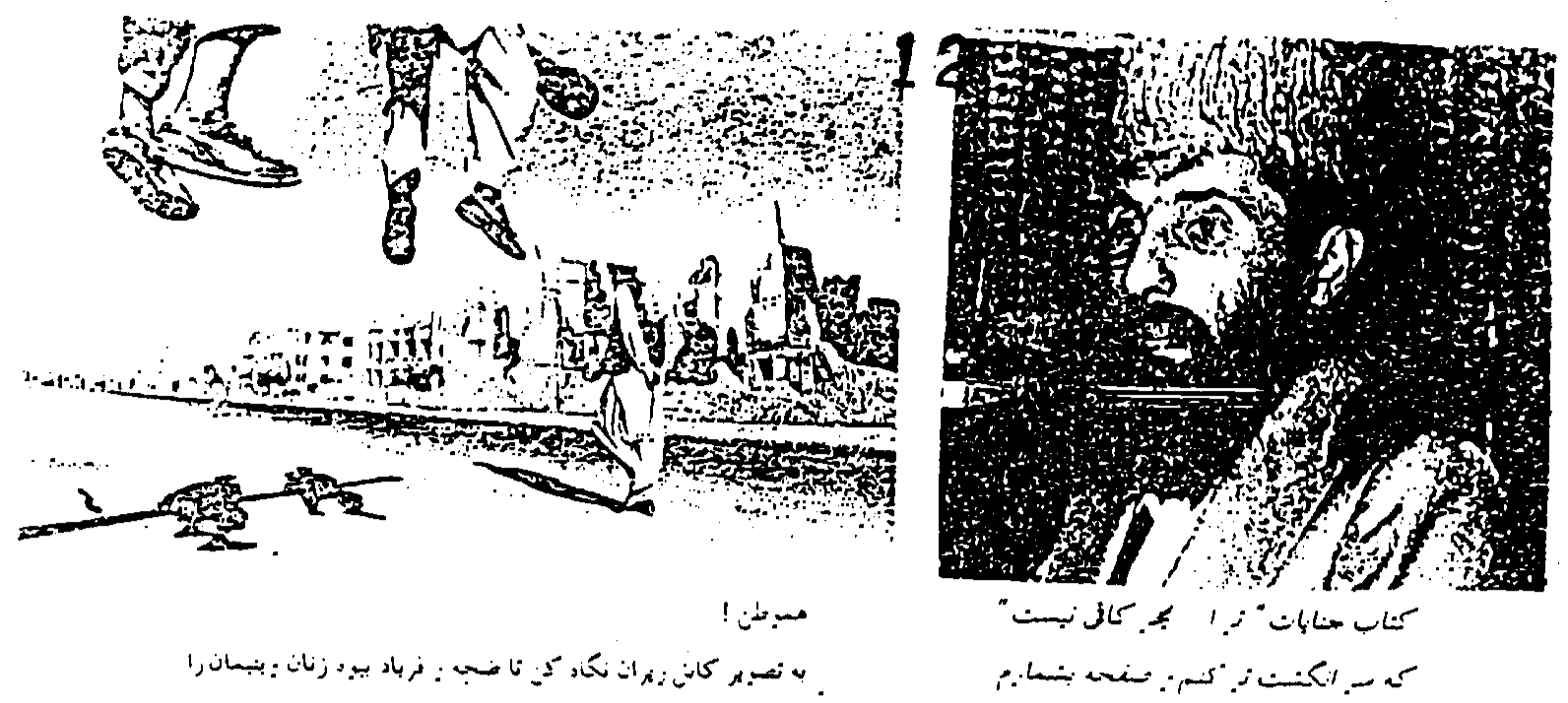

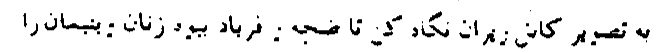

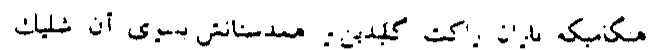

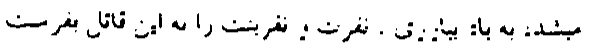

مسبطن ع.يزا

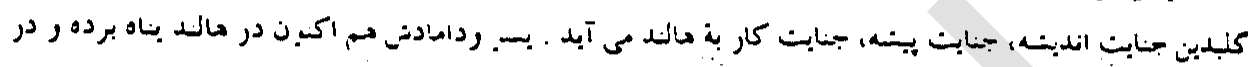

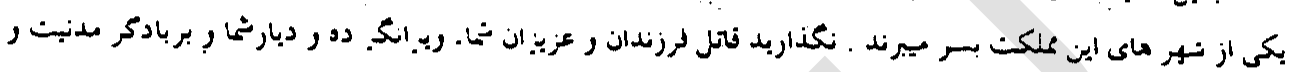

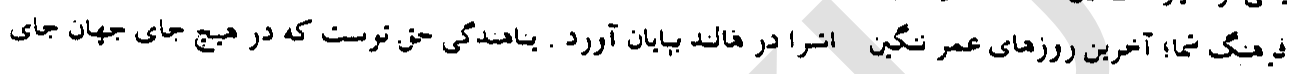

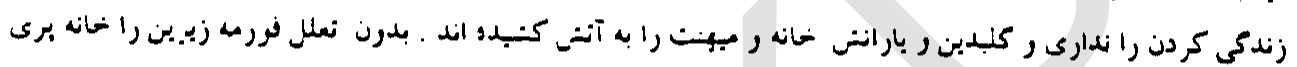

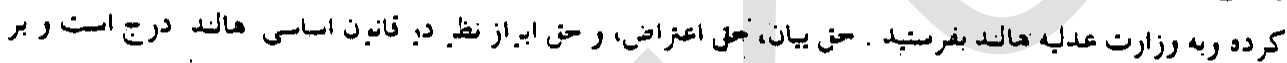

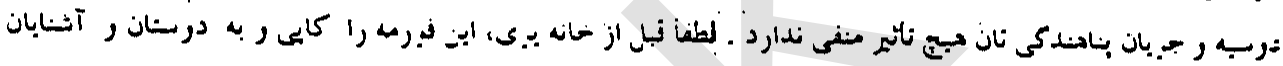

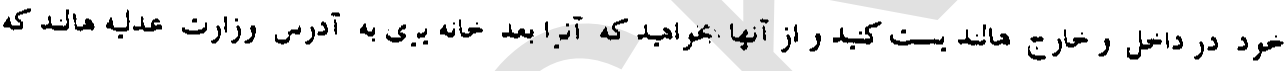

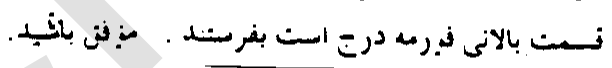

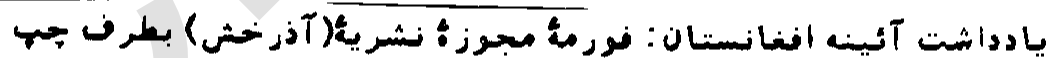

Ministrie van Justitie

t. .J. v. Afdeling IND

Schedeldoekhaven 100

2511 Ex Holland.

Here by I protest against granting asylum to Gulbuddin Hekmatyar by the government of the Netherlands. He has committed crime against humanity and there is no place for him in any democratic society.

Name

lnitials :

Address:

Country:

Signature. D:IL.

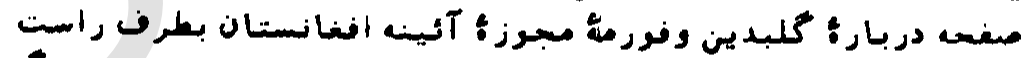

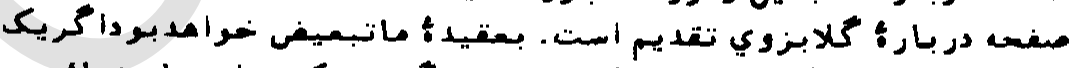

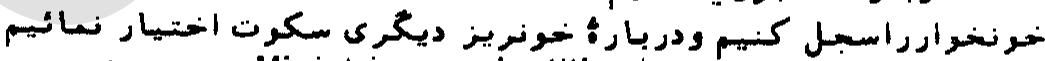
Ministrie van Justitie

t. . . v. Afdeling IND

Schedeldoekhaven 100

2511 EX Holland.

Hereby I protest against granting asylum to Said Mohammad Gulabzoy by the Government of Netherlands. He has committed crime against humnaity and there is no place for such a criminal in any democratic society.

First Name:

Last Name.

Address.

Position in Afghanistan

Telephone ( )

Signature

Date 


\section{3

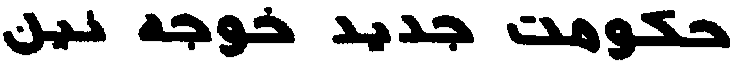

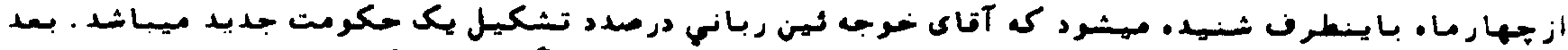

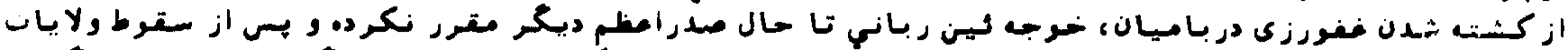

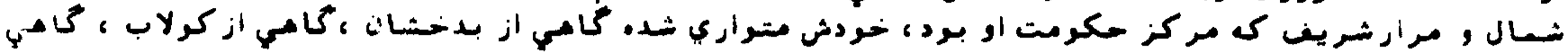

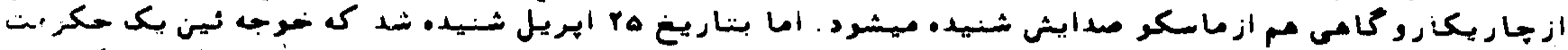

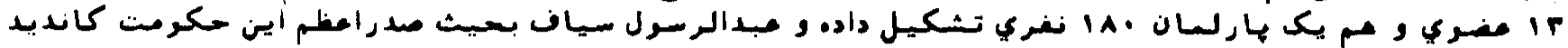

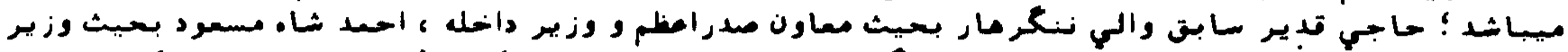

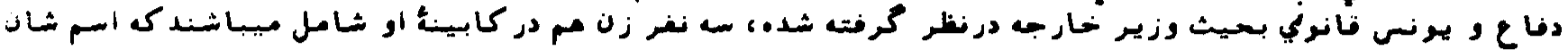

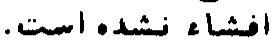

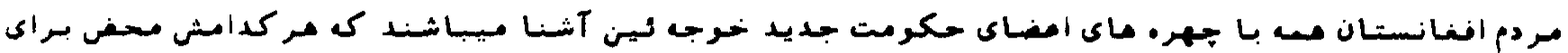

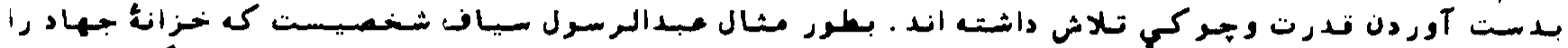

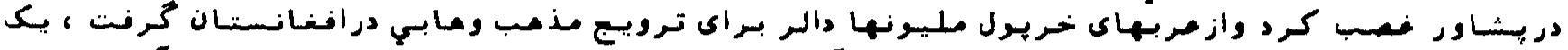

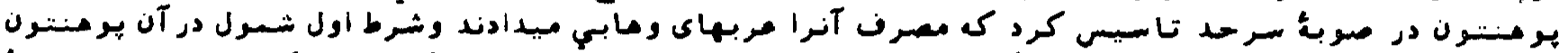

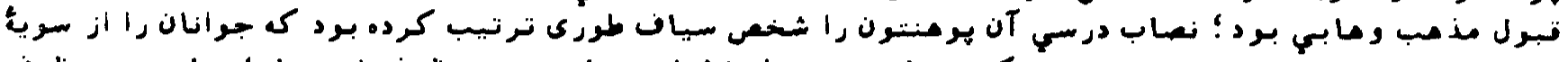

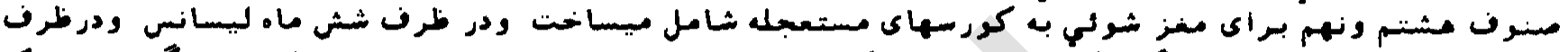

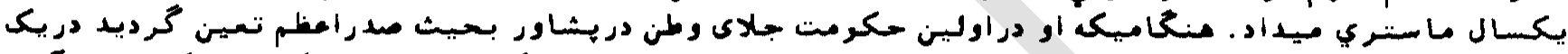

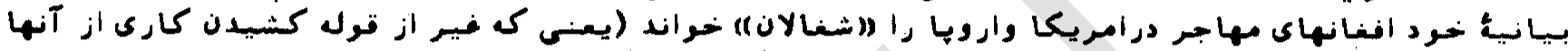

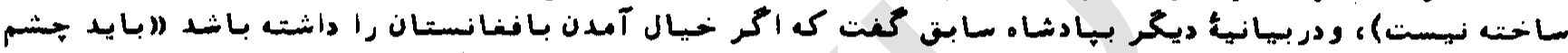

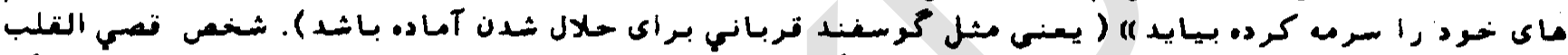

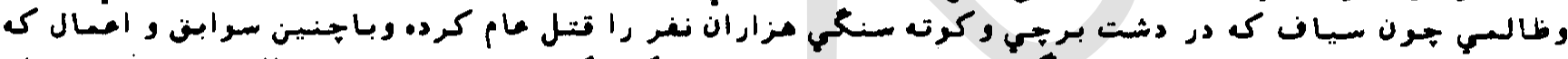

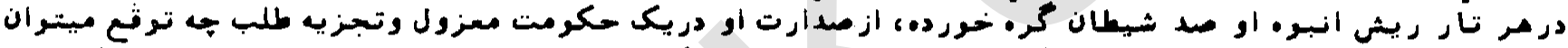

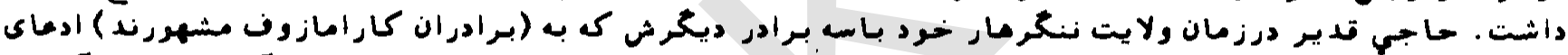

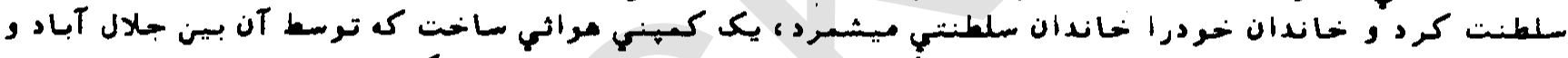

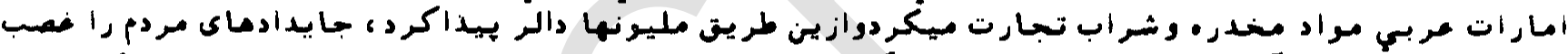

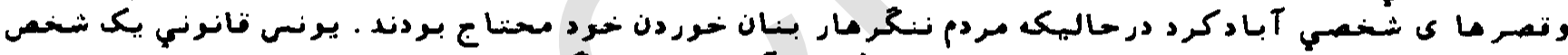

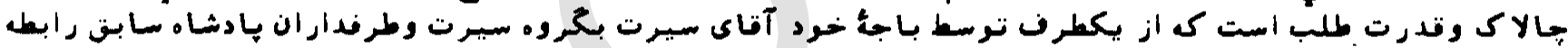

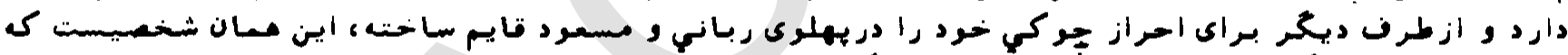

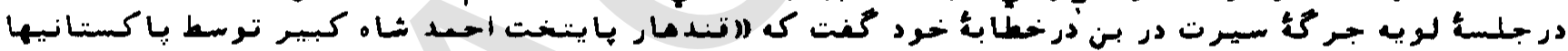

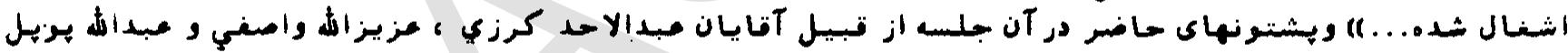

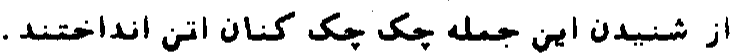

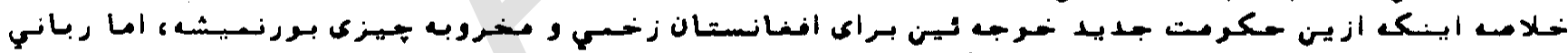

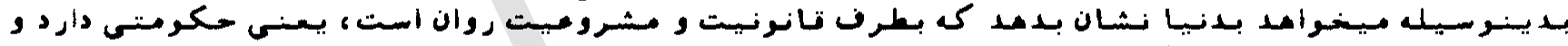

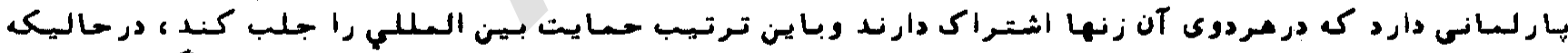

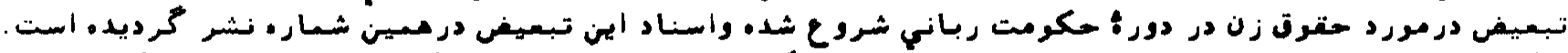

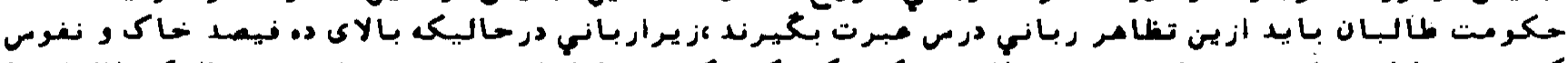

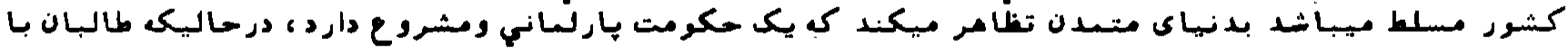

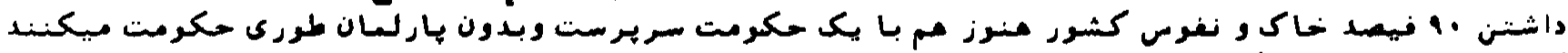

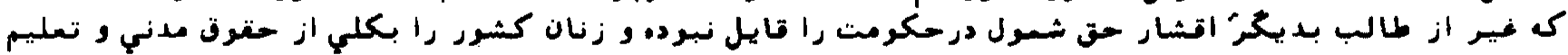

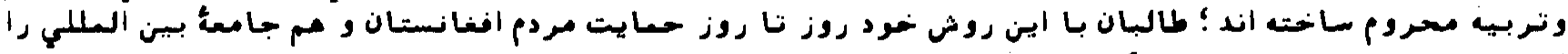

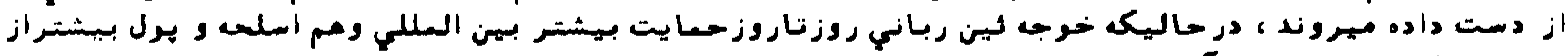

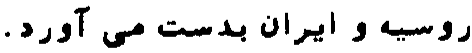

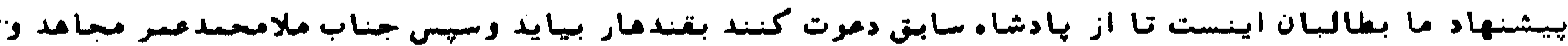

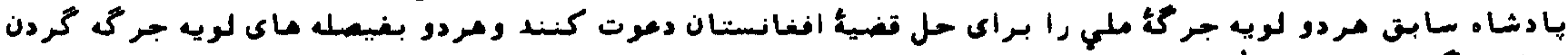

$$
\text { ( تبعرث: مدير مسكرل }
$$




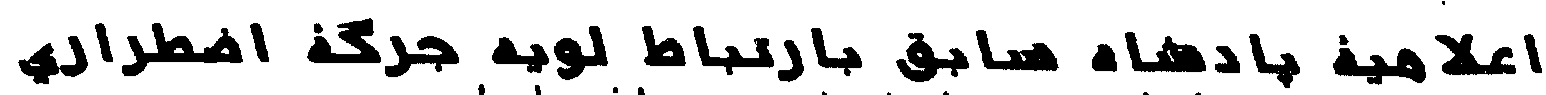

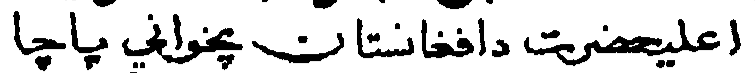

14

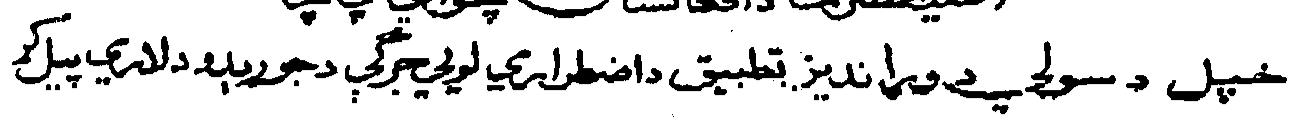

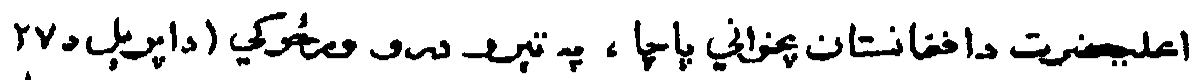

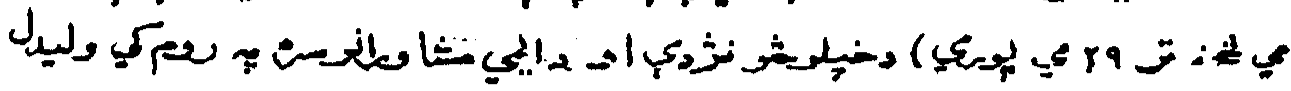

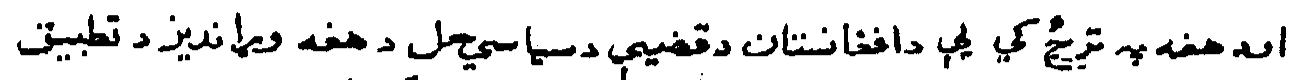
.

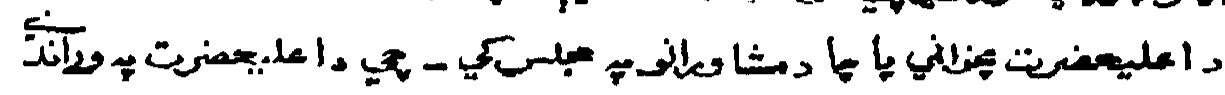

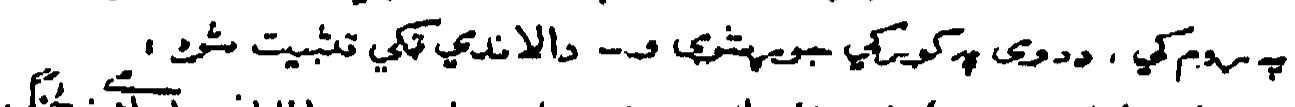

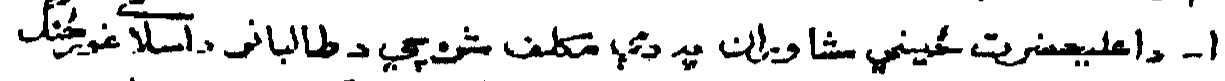

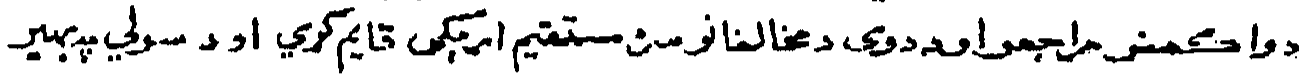

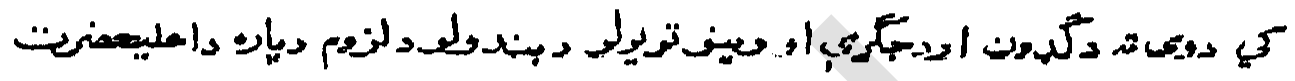

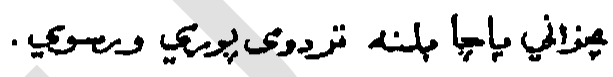

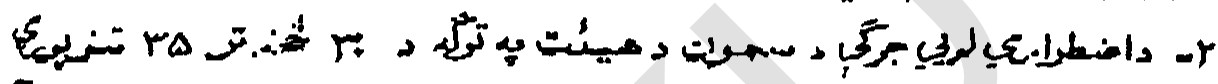

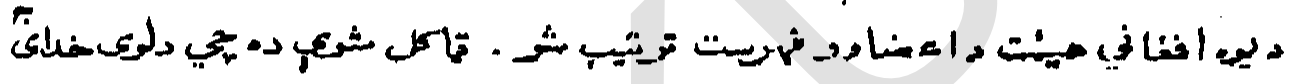

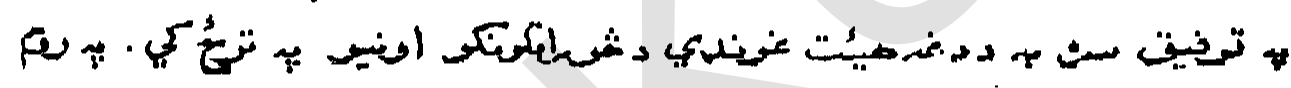

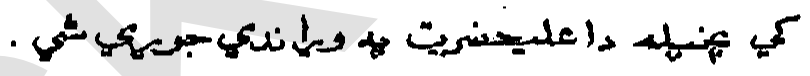
.

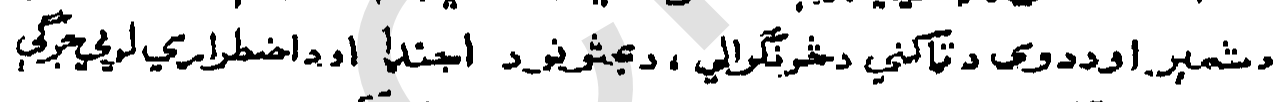

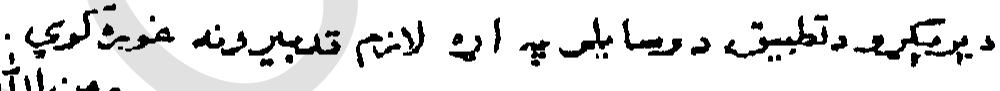

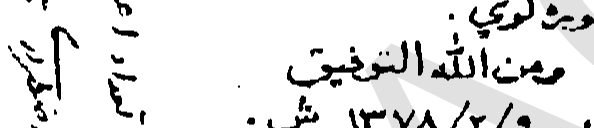
.

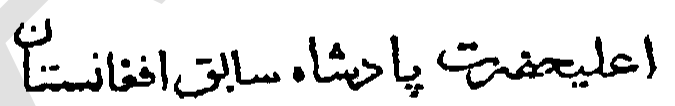

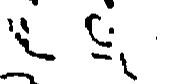

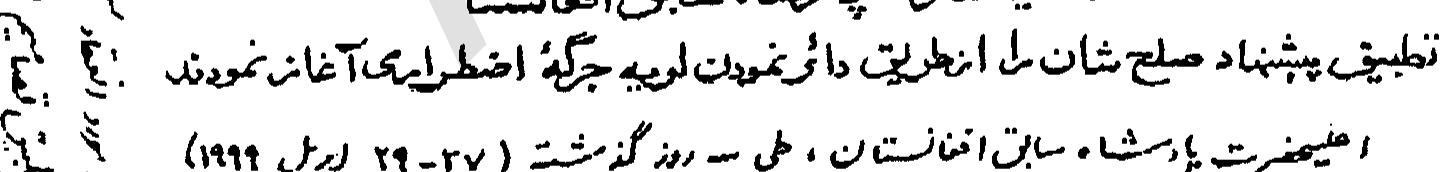

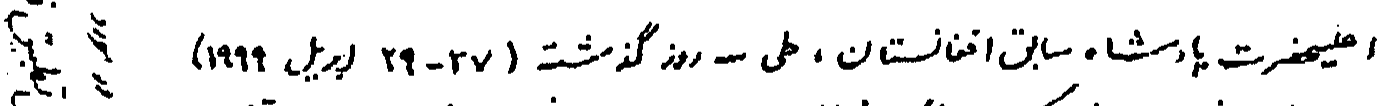

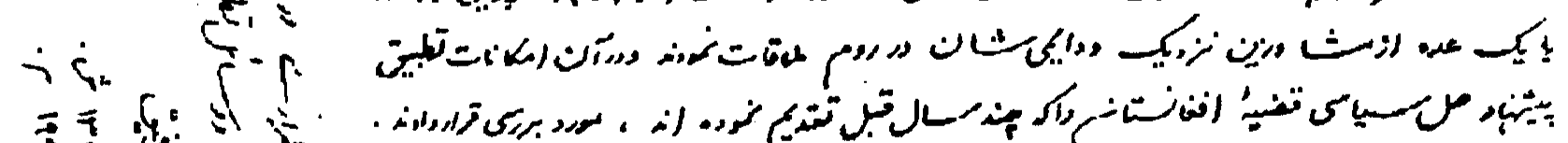

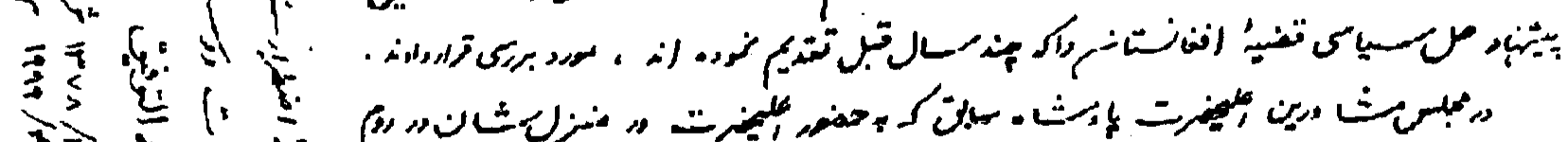

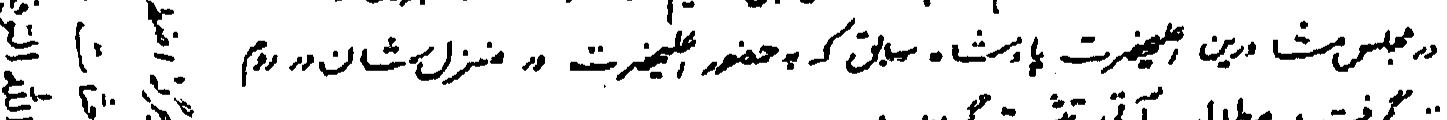

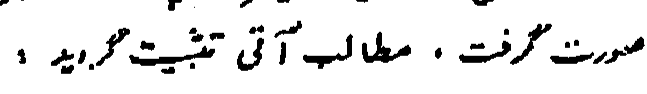

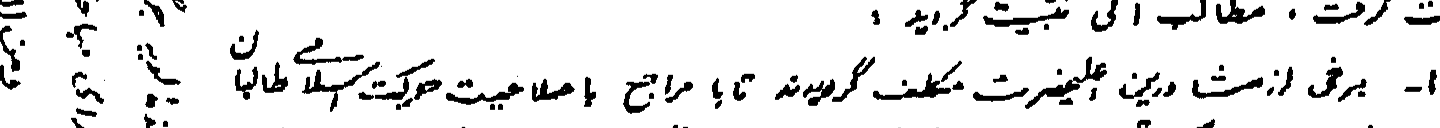

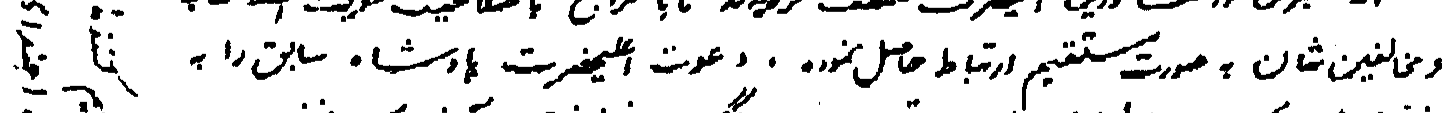

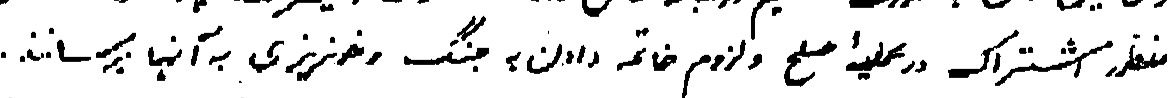

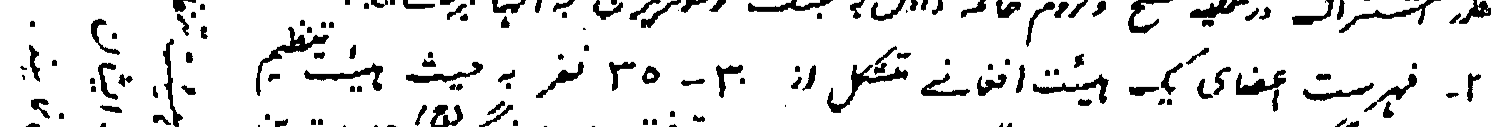

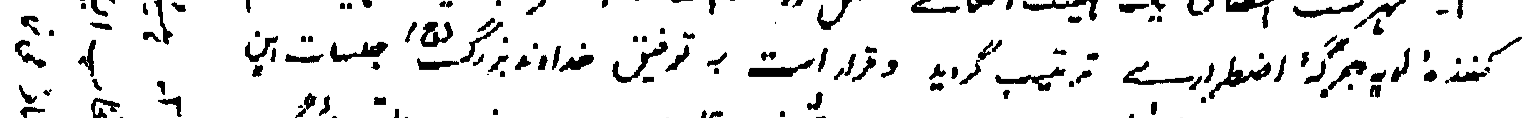

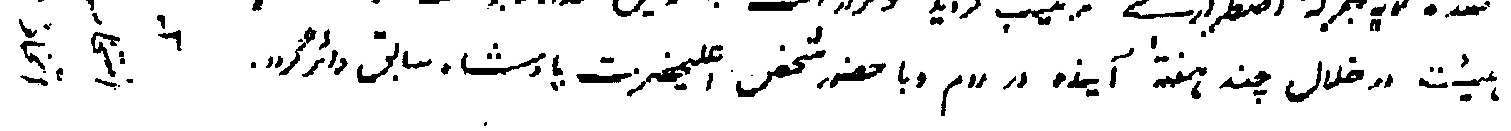




\section{تسمسيمسيم سه فقره أي بادشاه سابق 15}

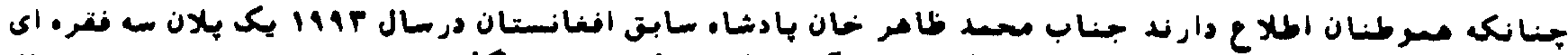

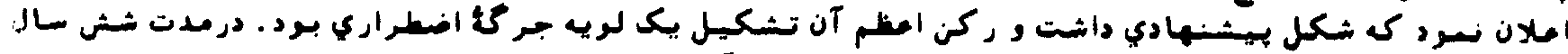

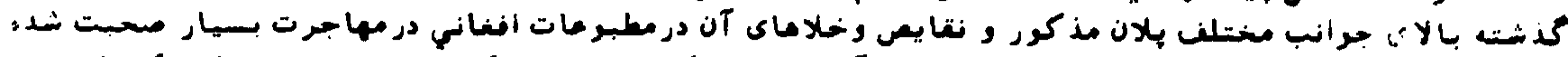

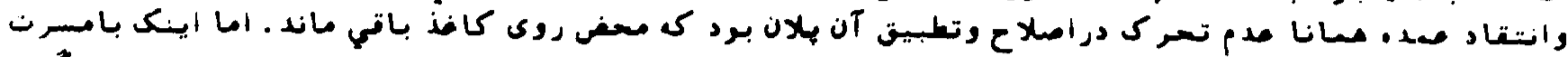

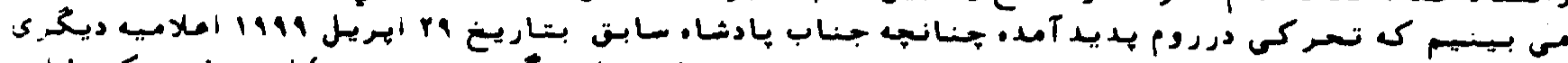

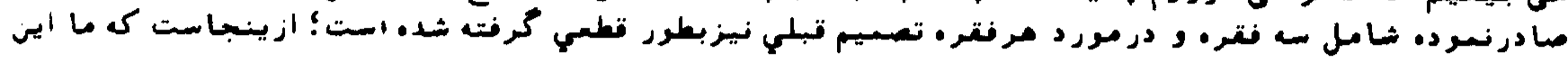

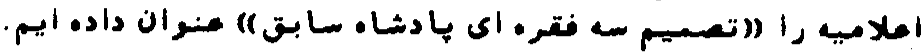

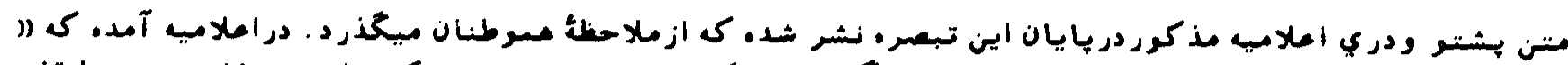

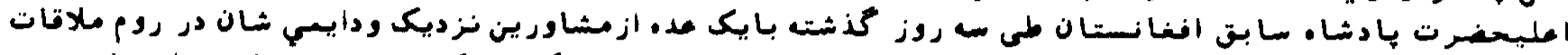

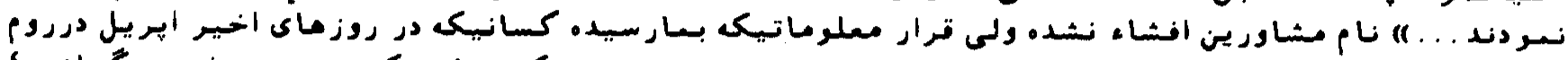

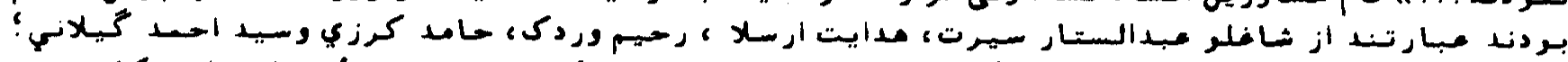

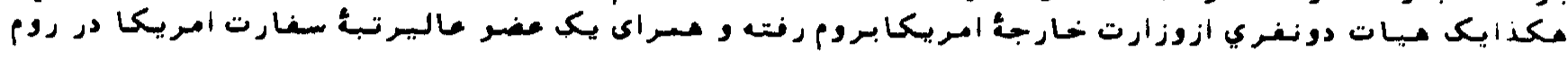

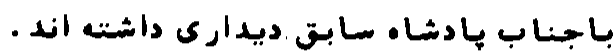

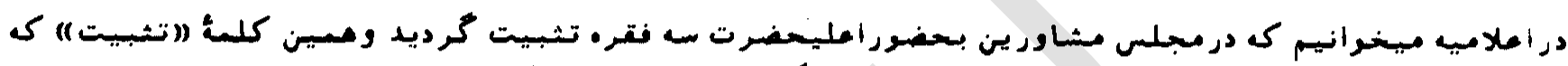

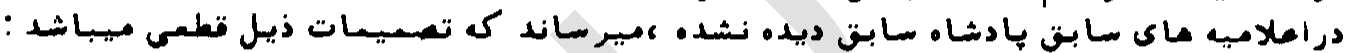

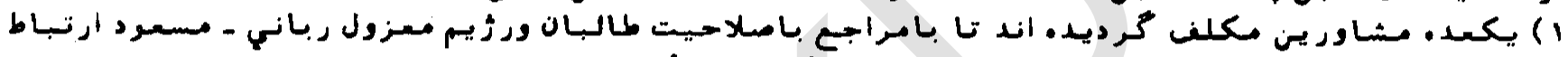

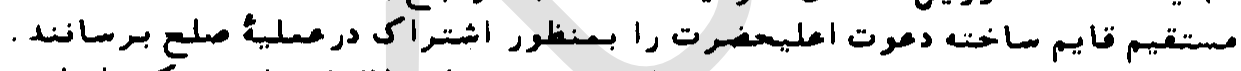

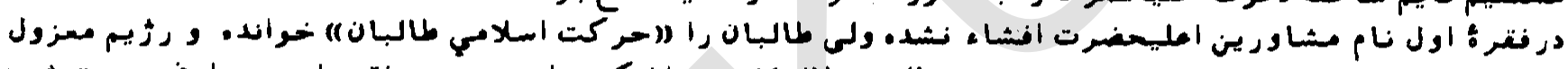

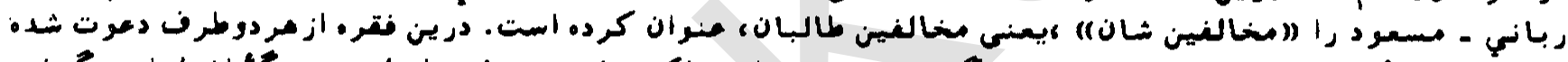

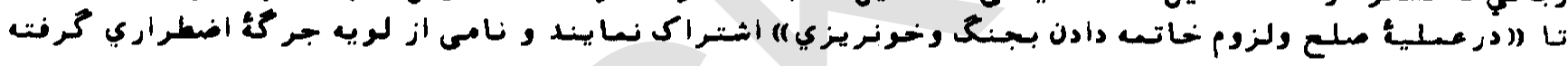

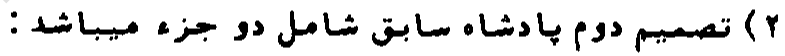

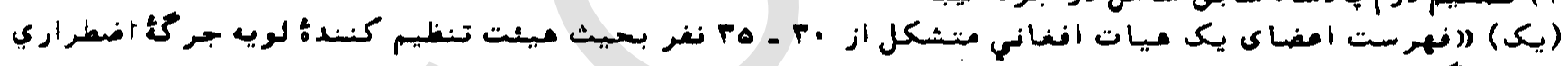

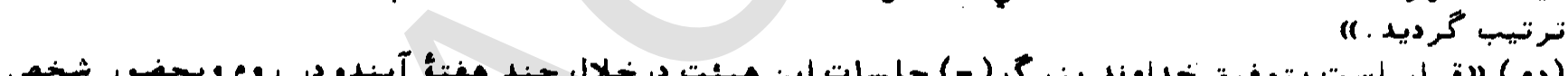

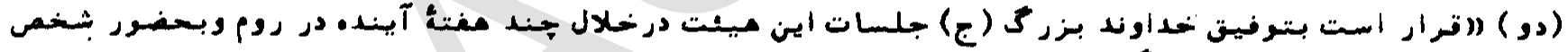

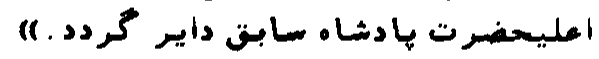

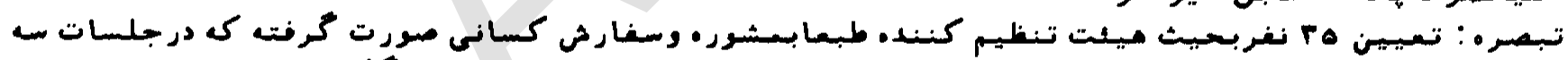

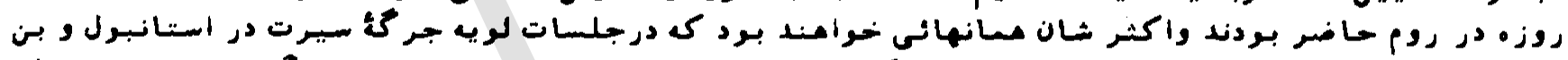

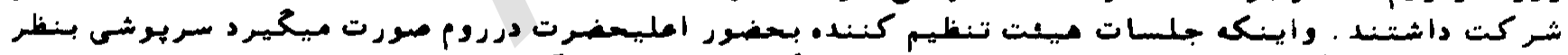

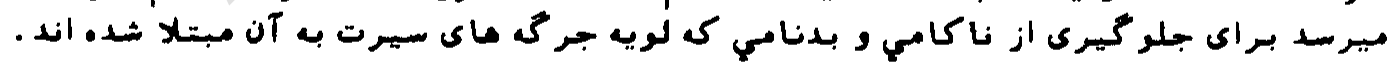

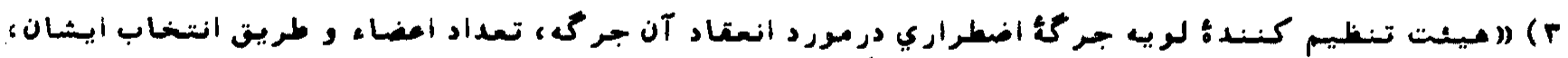

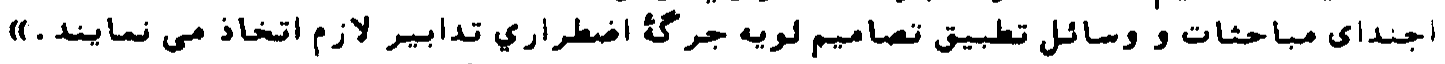

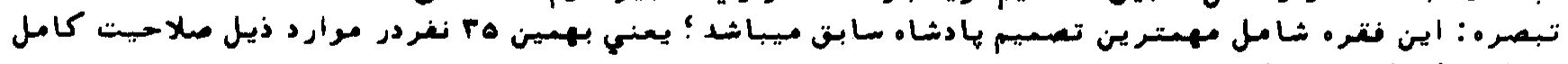

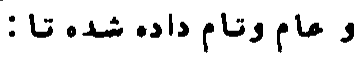

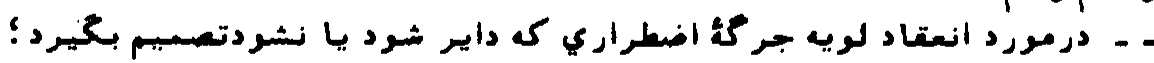

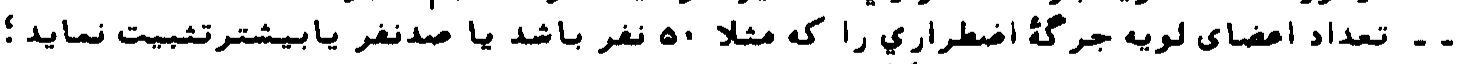

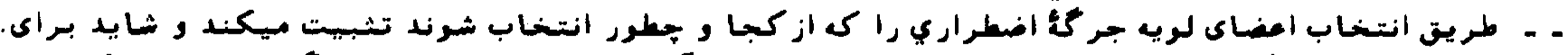

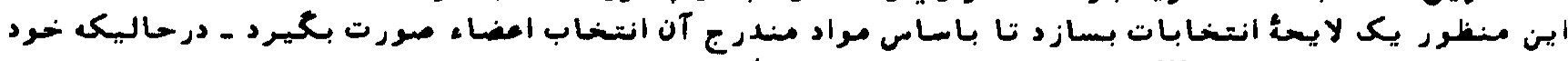

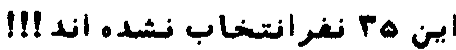

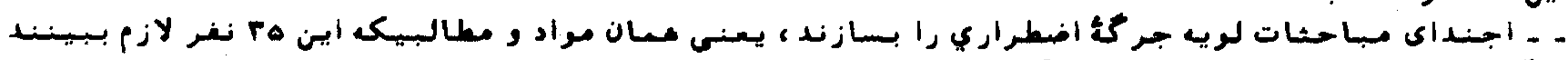

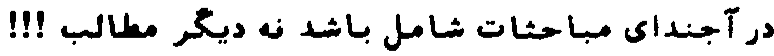

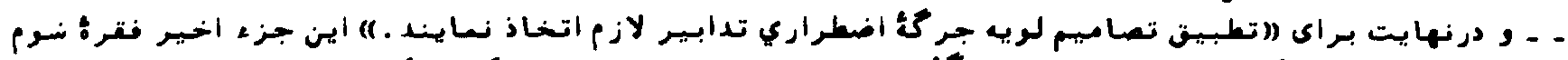

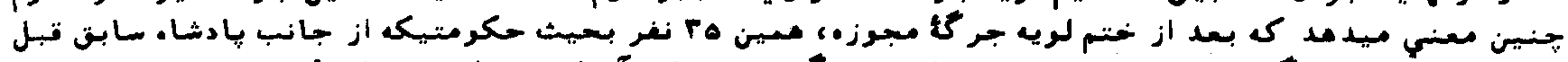

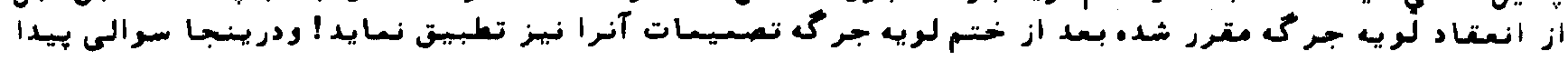




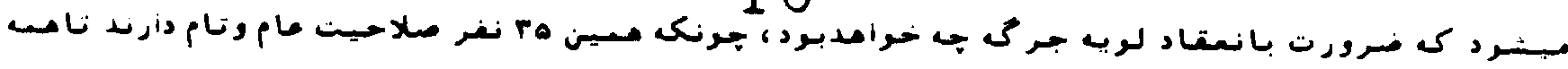

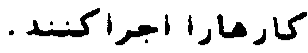

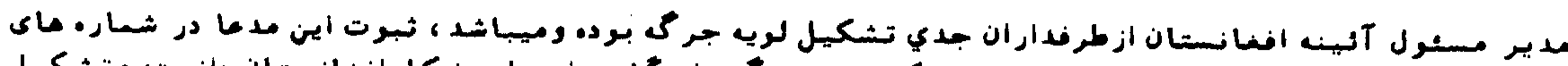

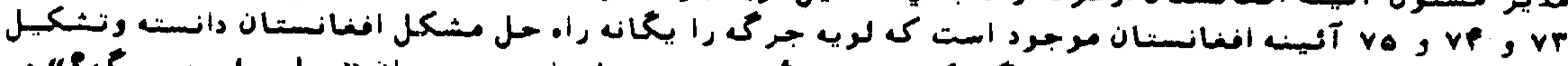

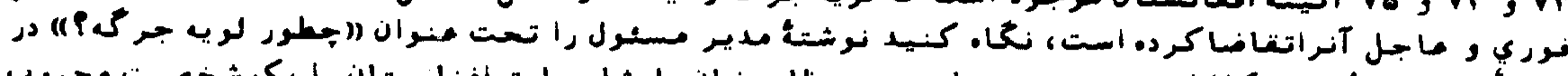

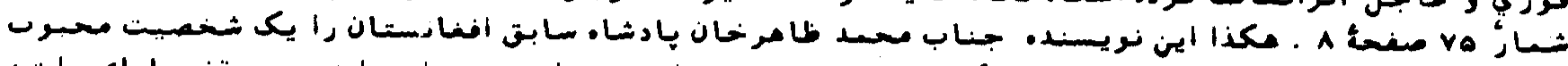

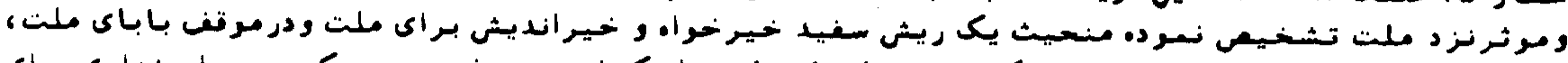

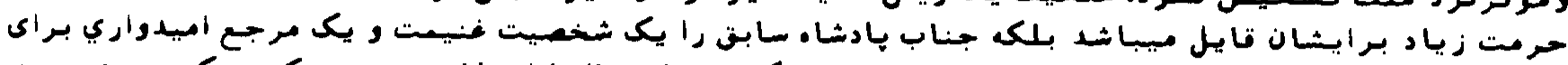

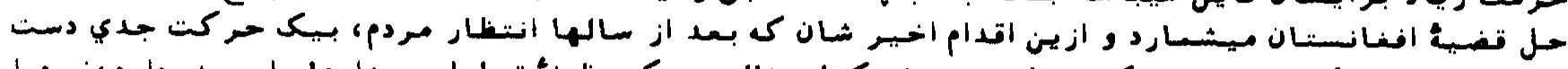

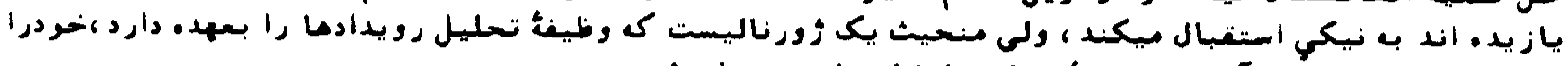

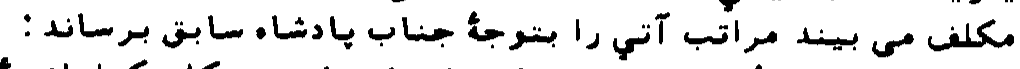

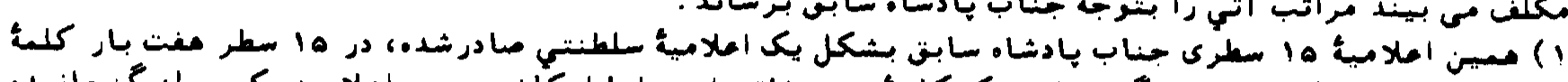

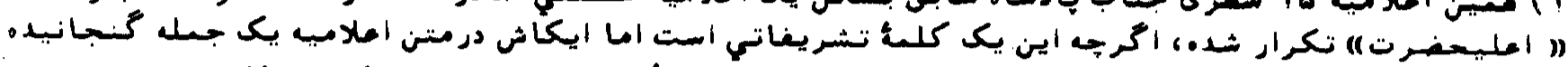

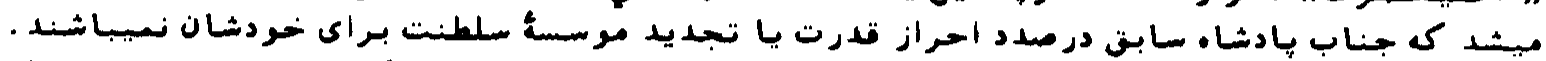

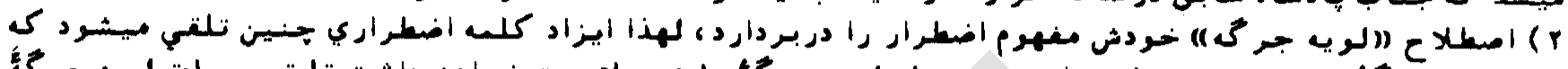

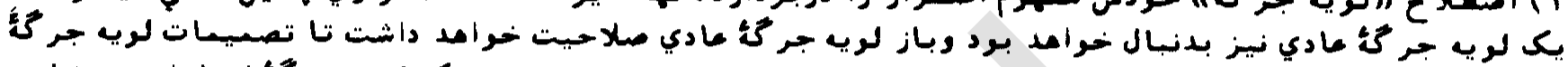

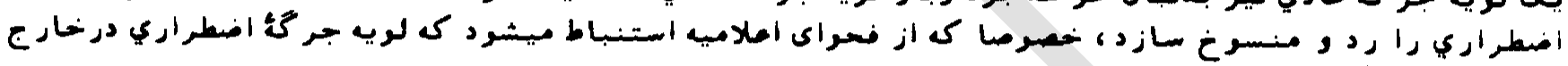

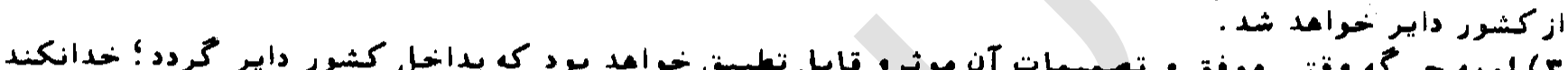

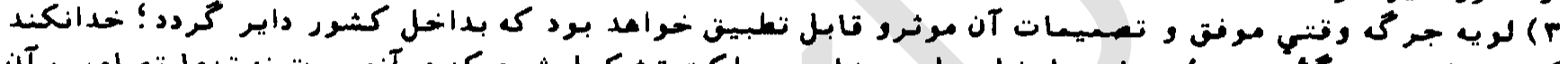

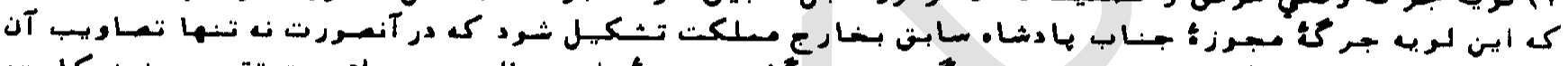

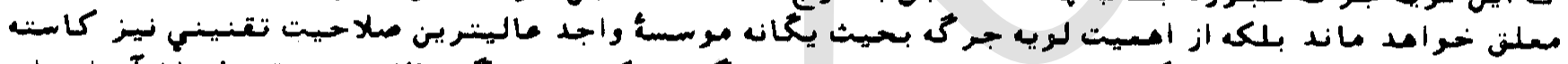

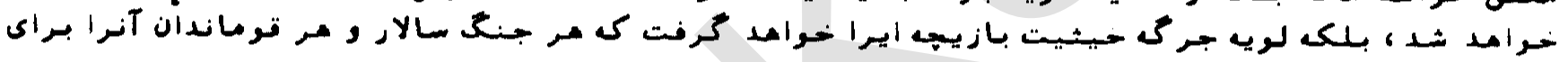

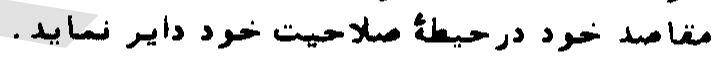

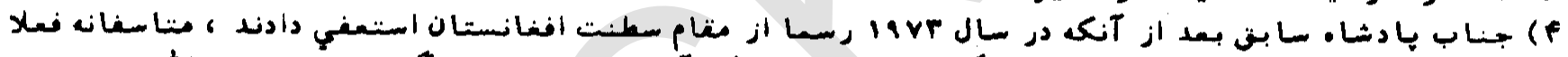

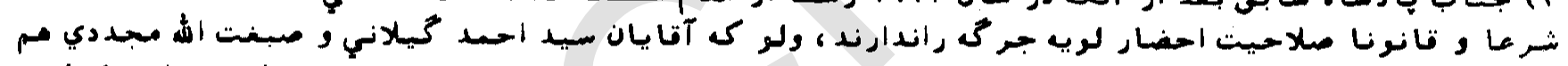

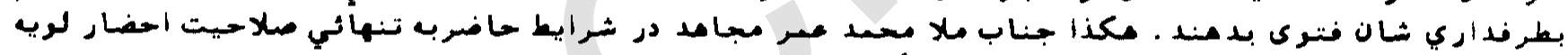

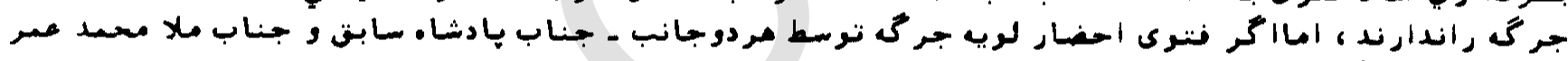

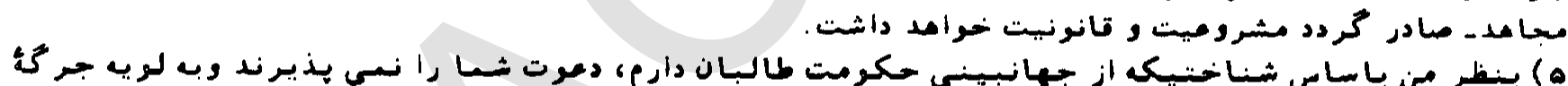

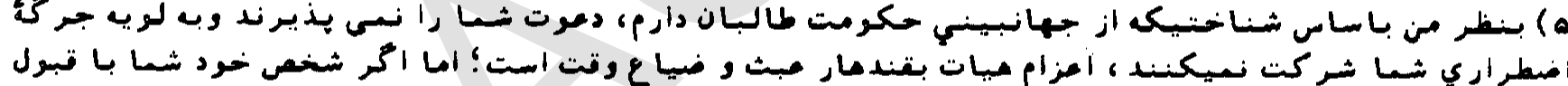

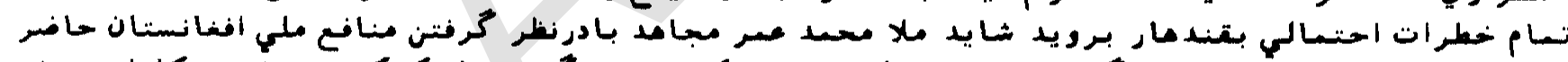

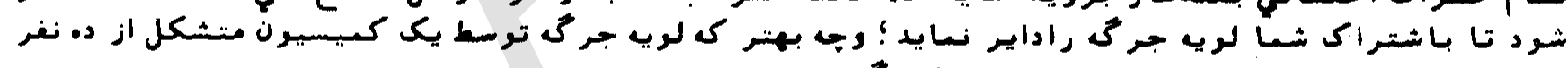

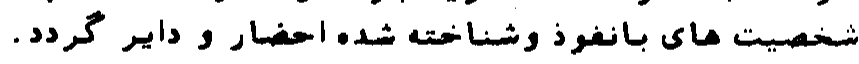

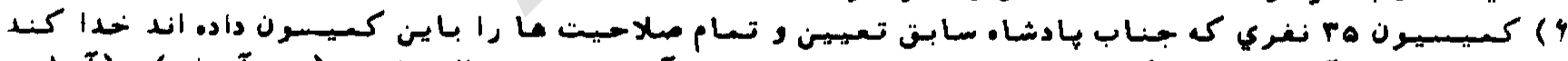

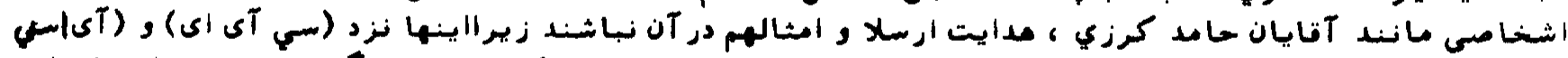

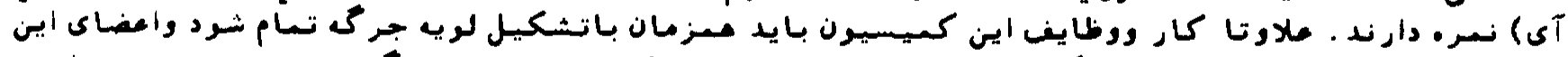

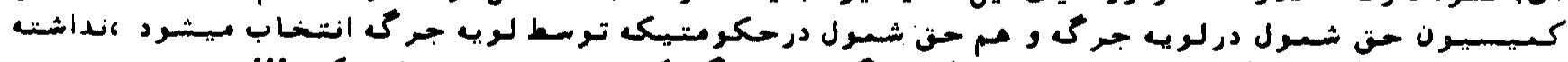

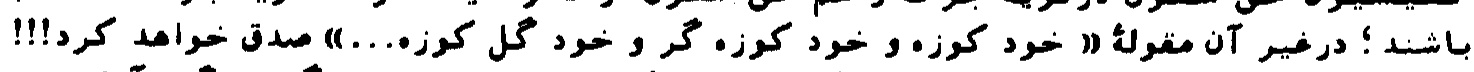

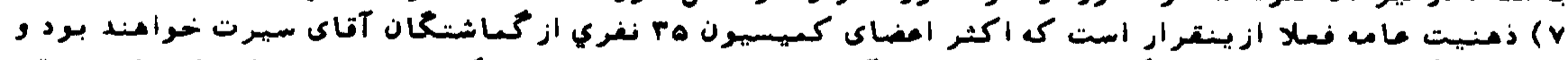

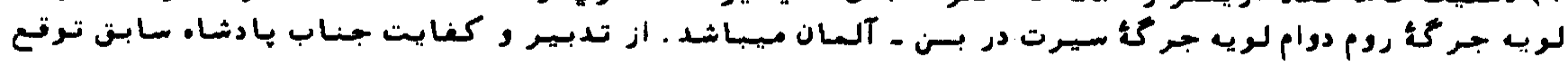

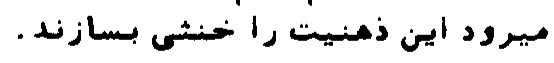

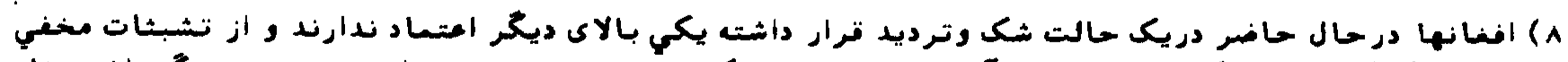

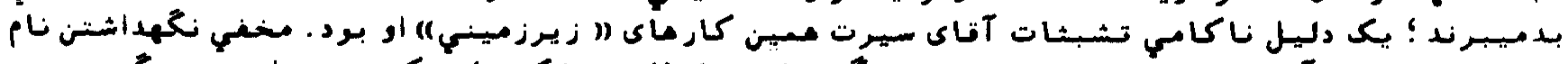

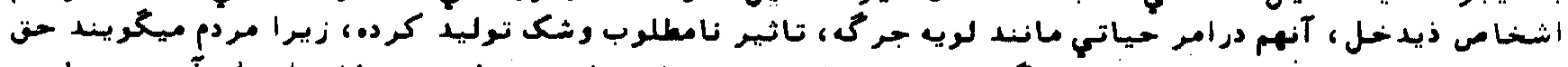

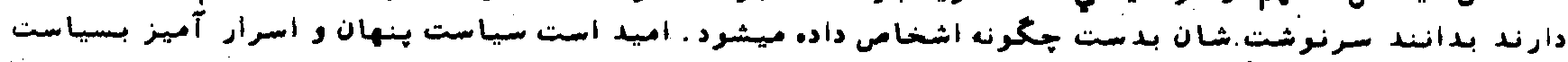

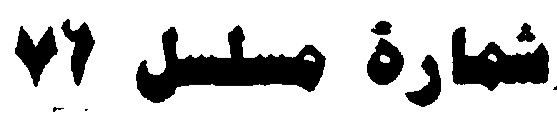

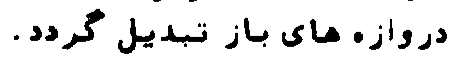

نامُ مر كشادة بروس ريساردبس رادربخش انخليسي بخوانيد 


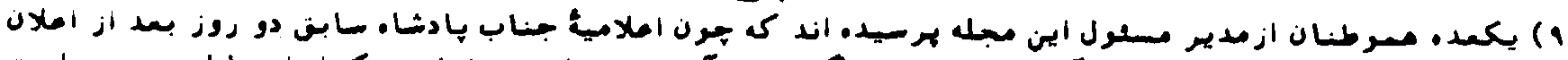

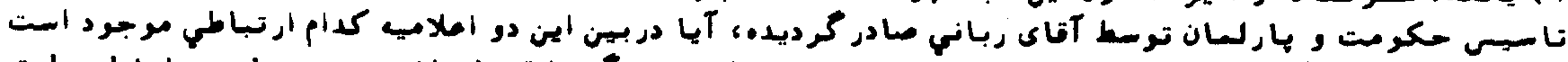

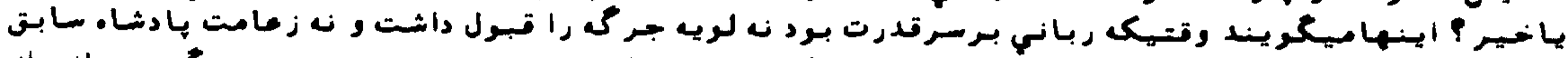

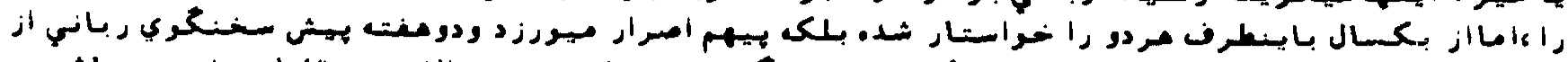

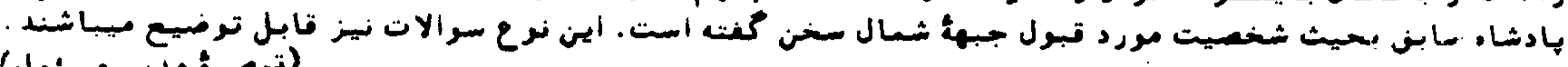

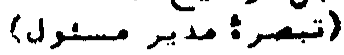

\section{كميل يك لوهي جركه يك هرورت عاجل}

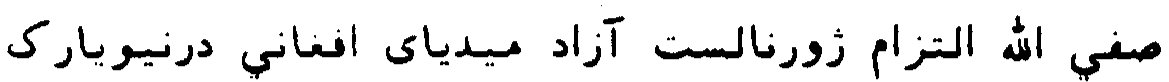

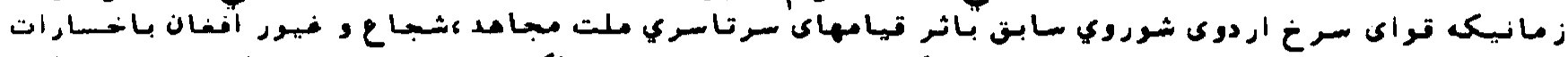

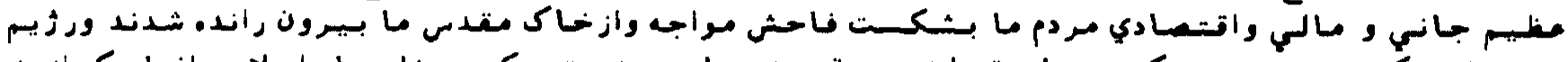

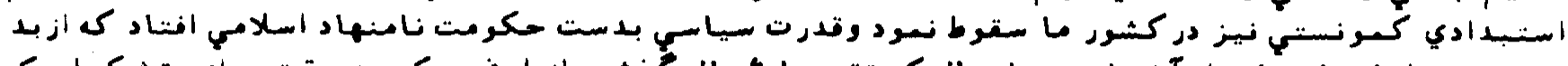

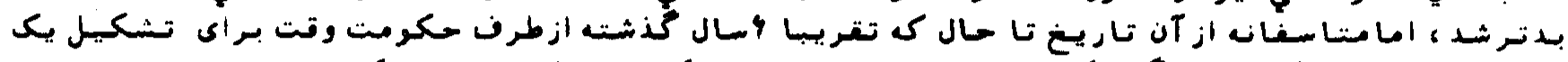

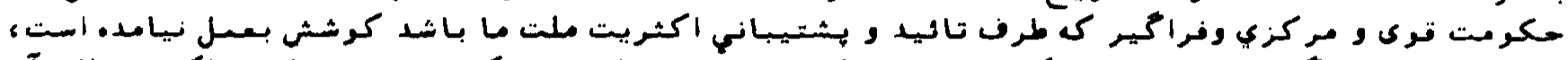

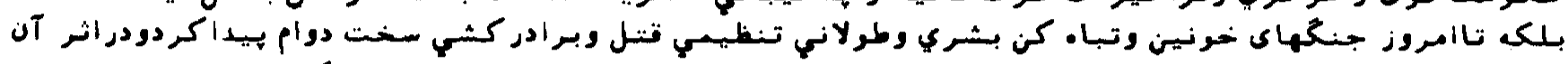

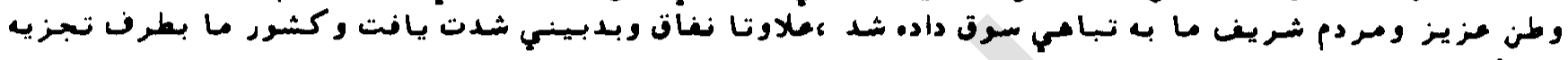

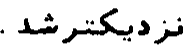

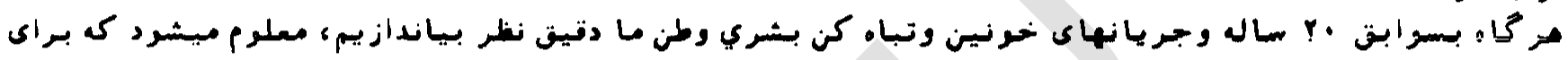

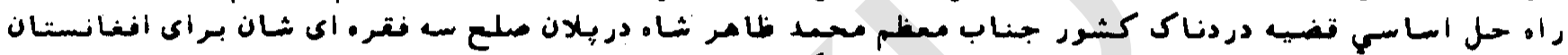

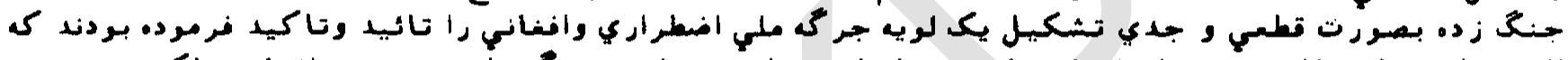

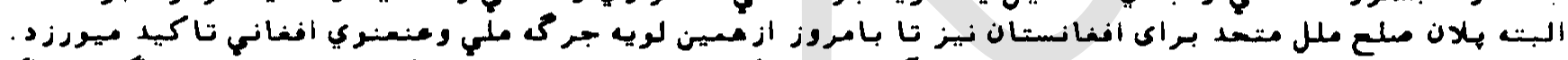

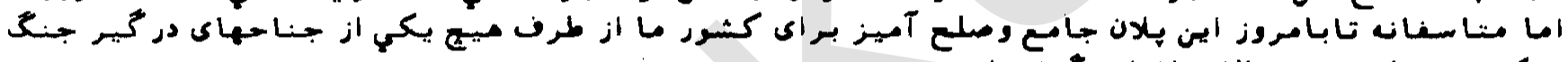

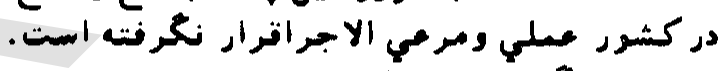

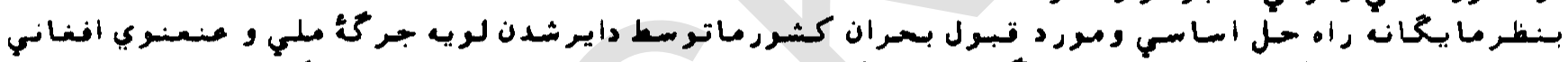

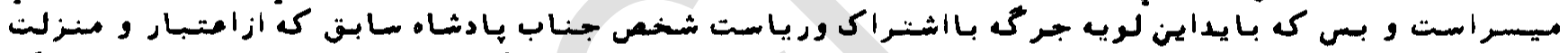

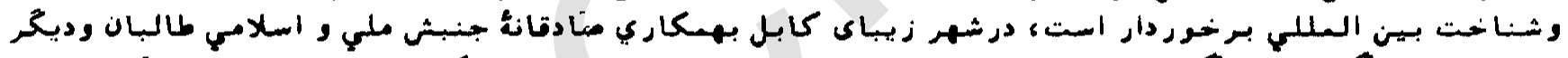

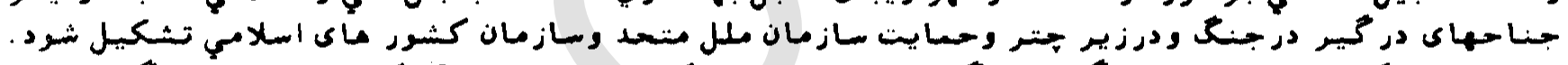

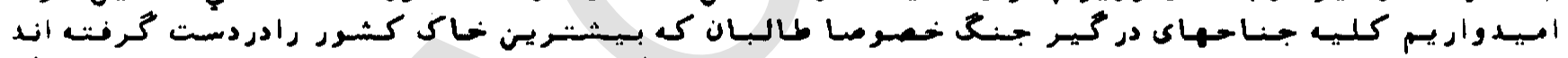

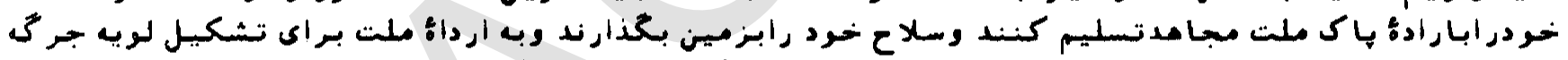

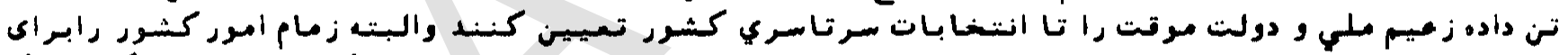

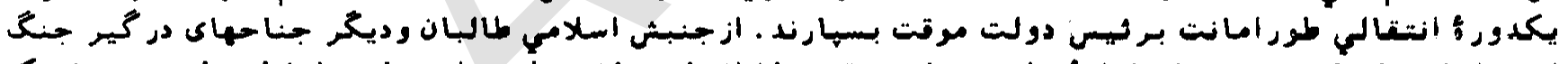

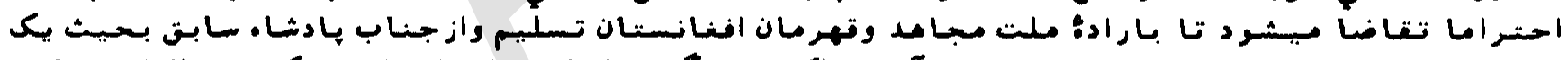

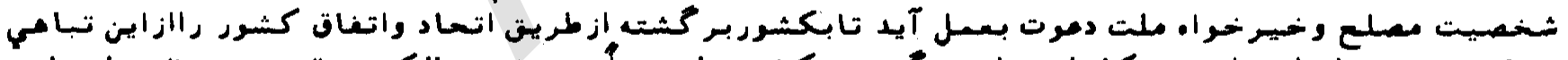

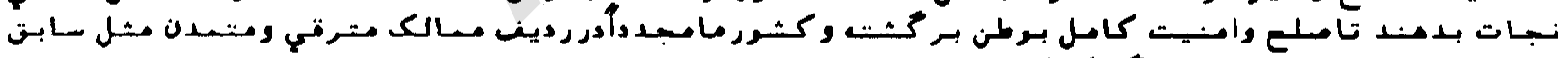

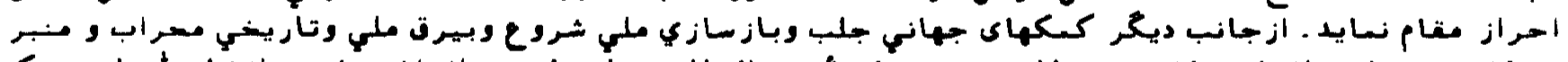

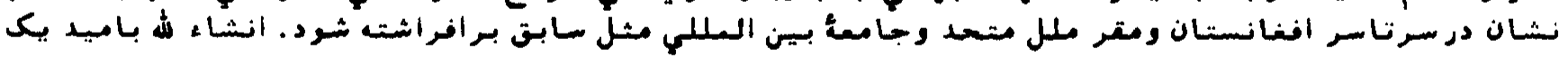

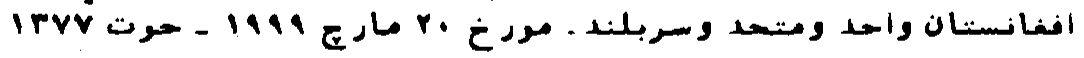

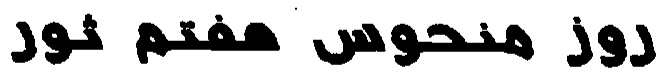

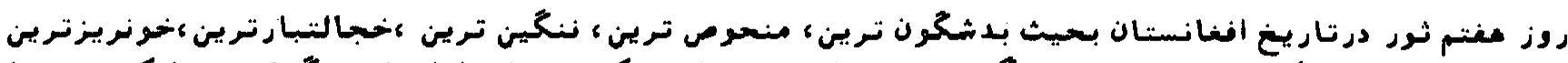

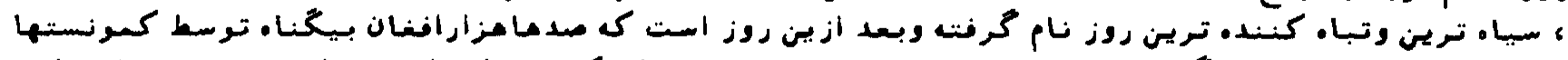

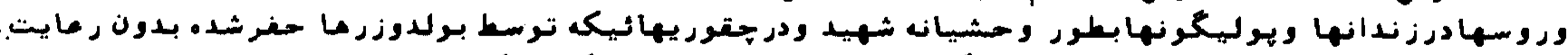

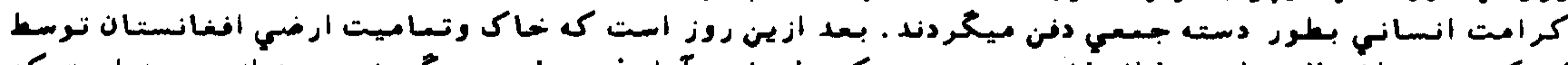

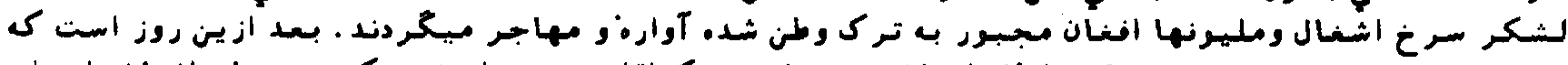

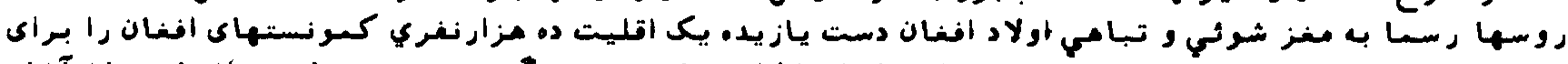

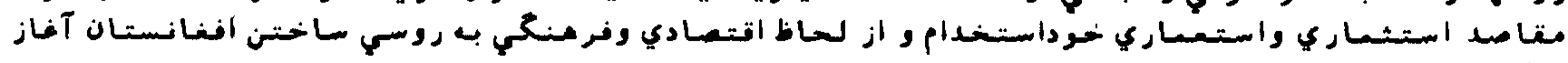

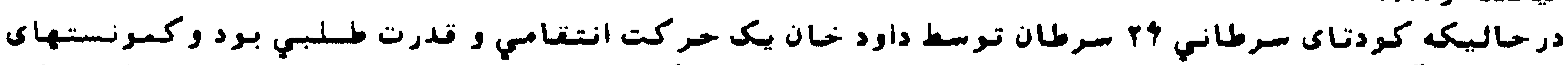

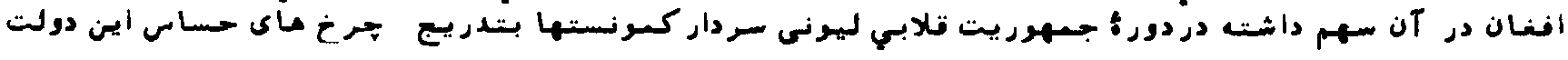




\section{8}

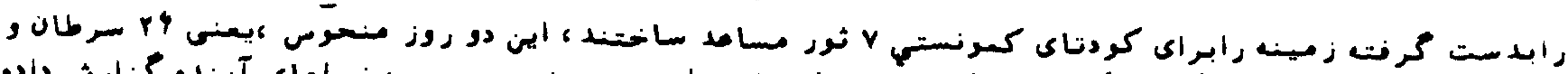

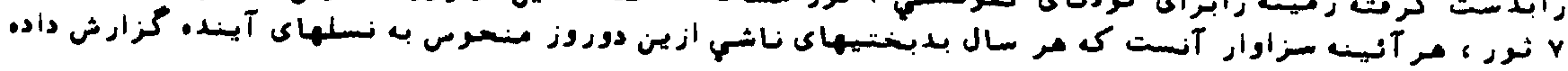

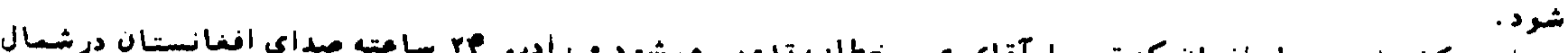

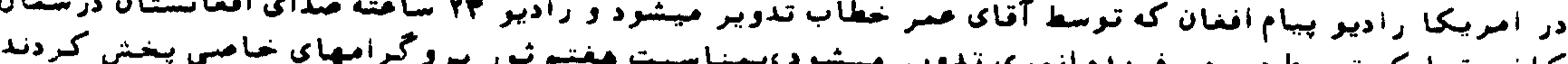

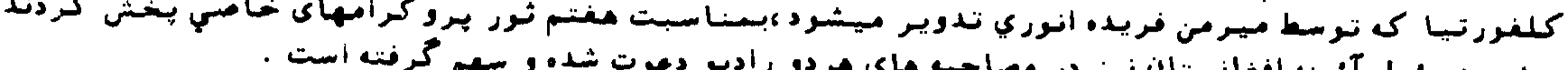

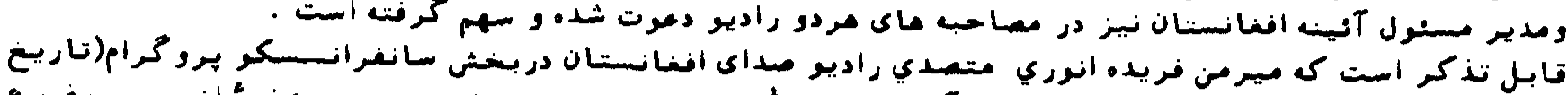

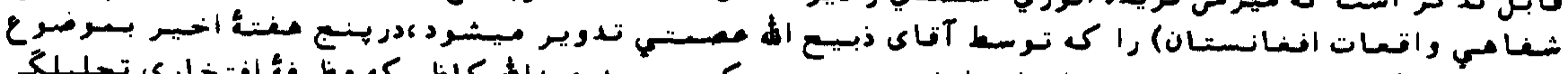

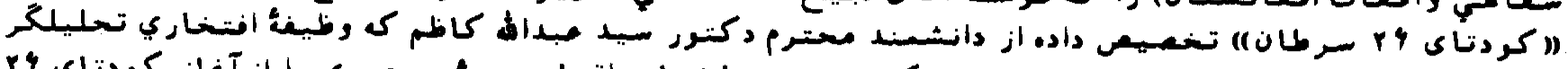

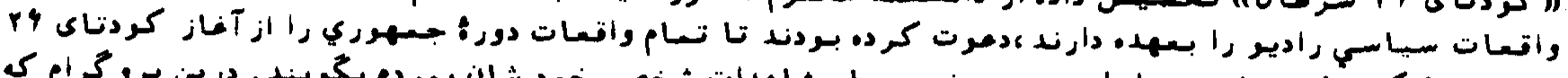

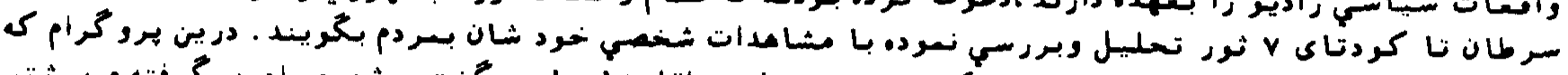

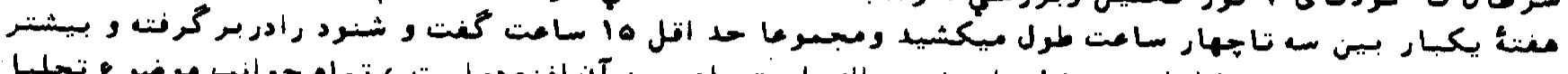

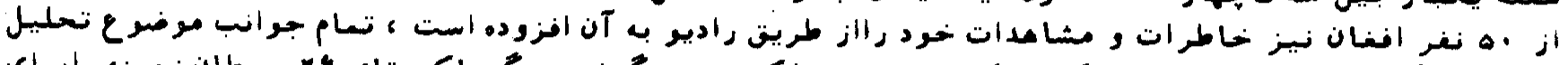

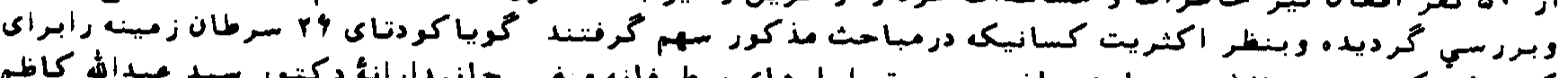

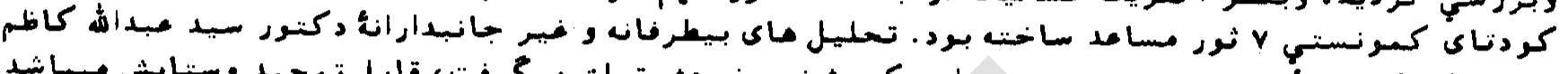

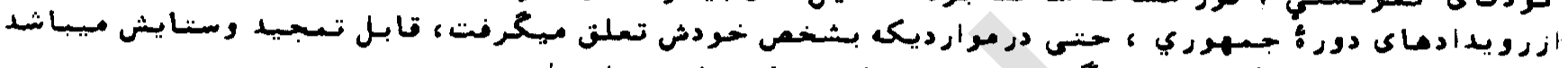

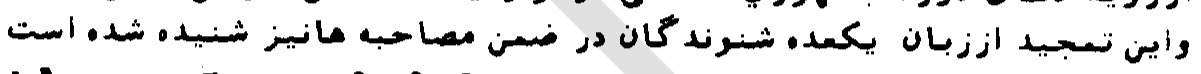

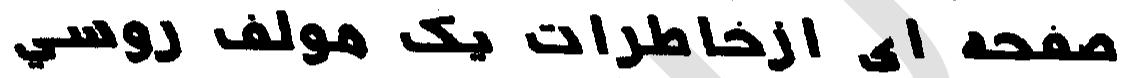

\section{هAly}

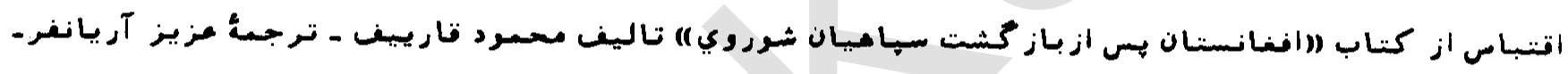

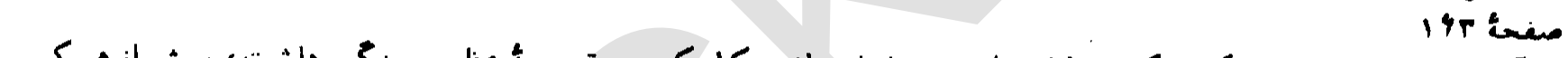

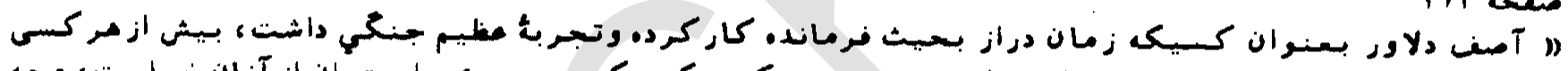

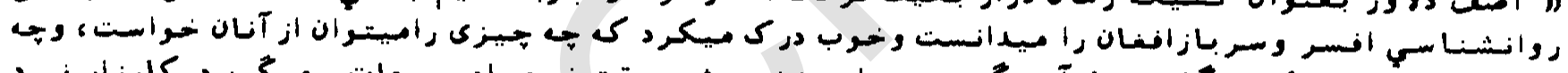

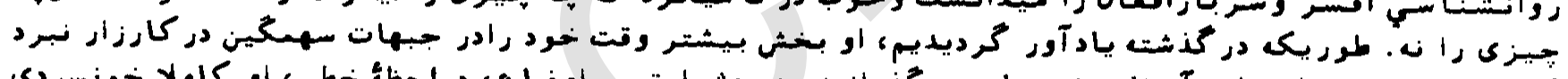

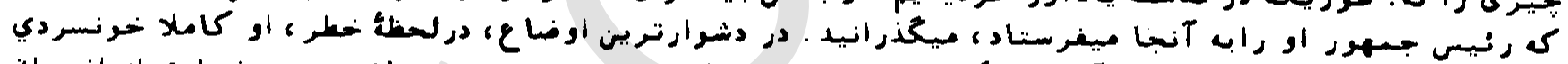

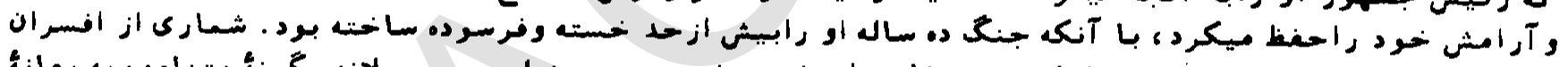

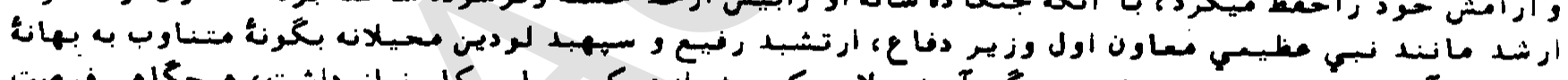

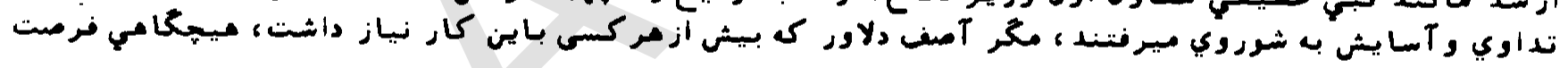

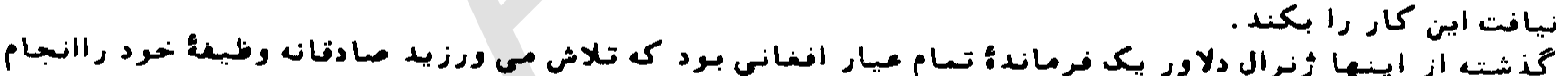

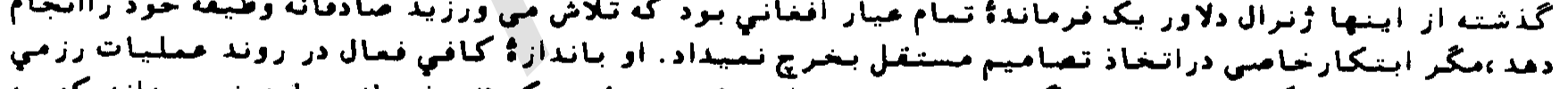

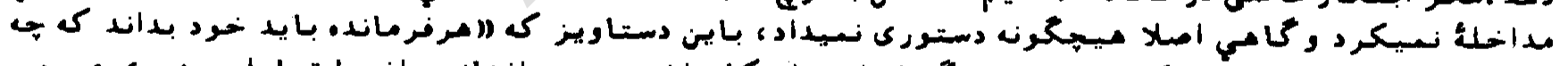

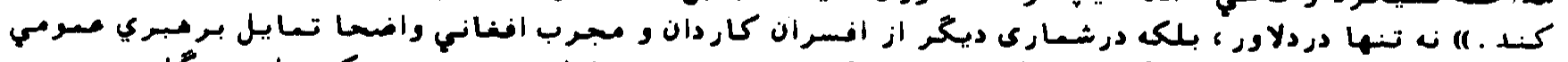

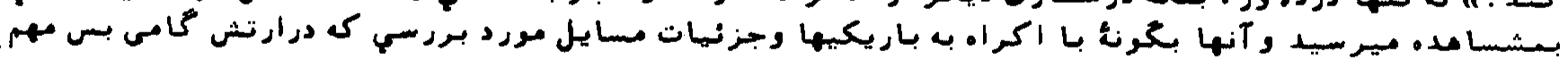

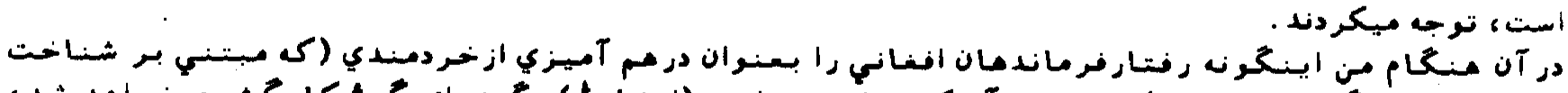

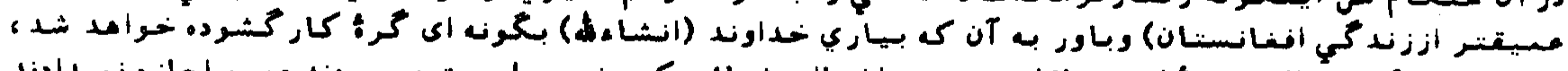

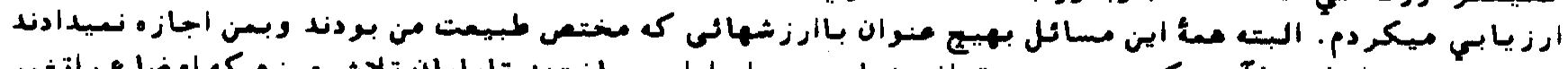

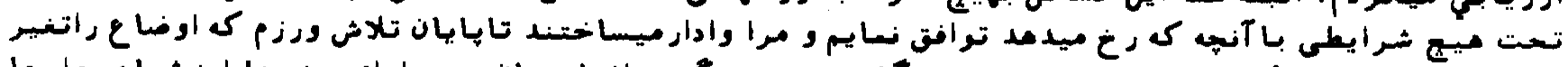

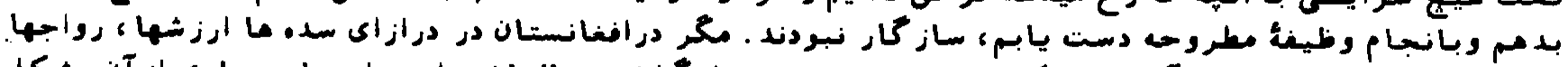

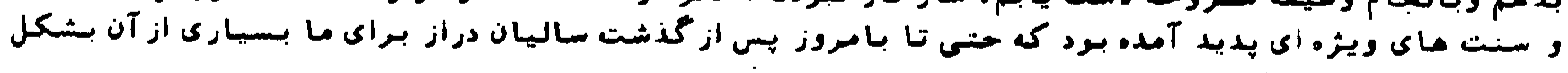

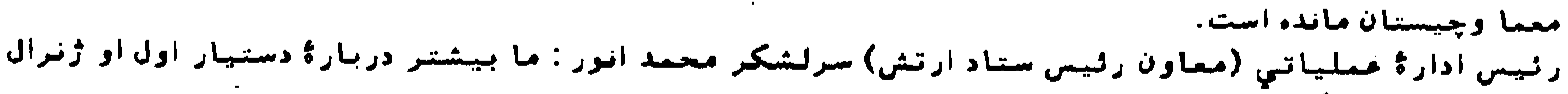

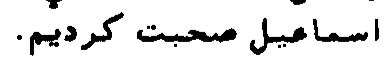

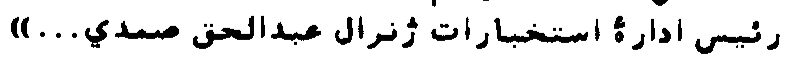

ناهه سر كشادة: بروس ريهاردسن رادربخش انظليسي بخرانيـ 


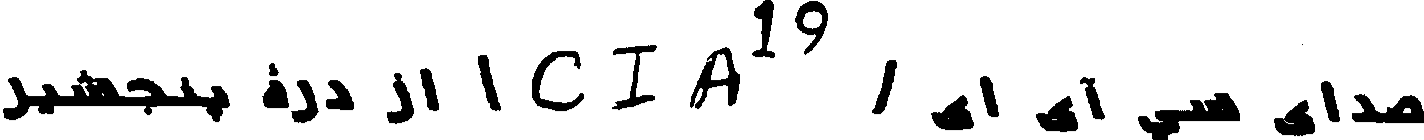

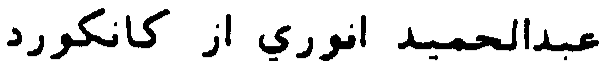

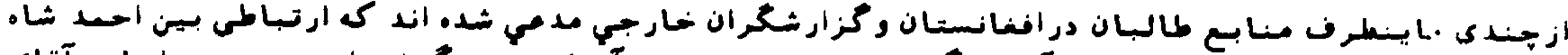

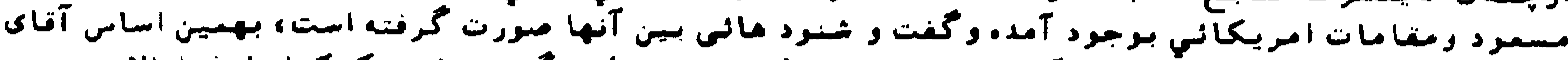

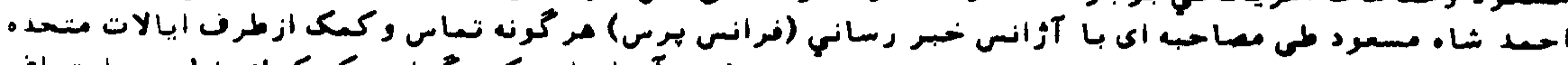

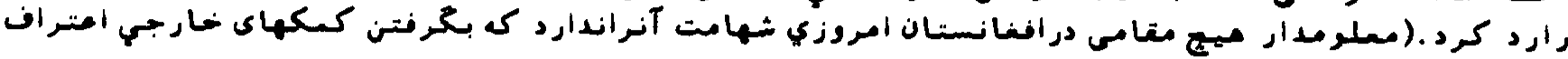

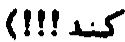

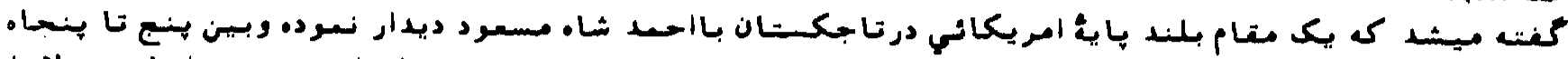

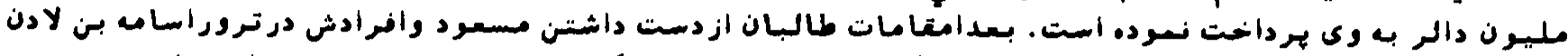

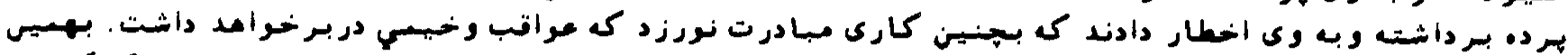

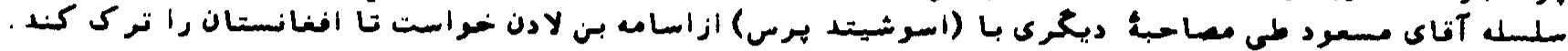

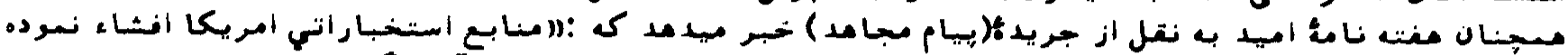

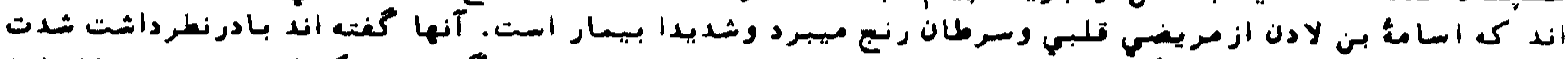

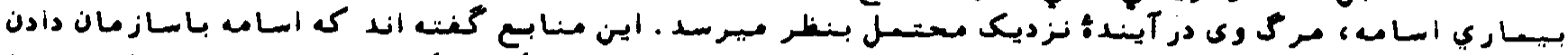

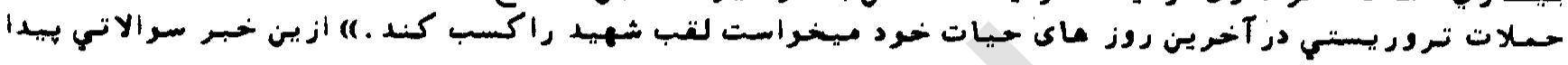

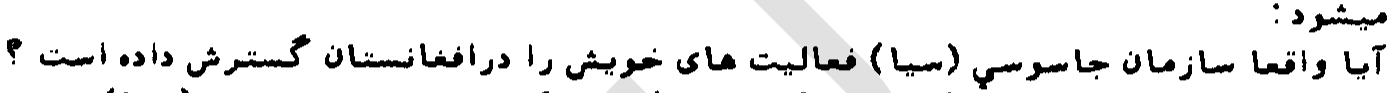

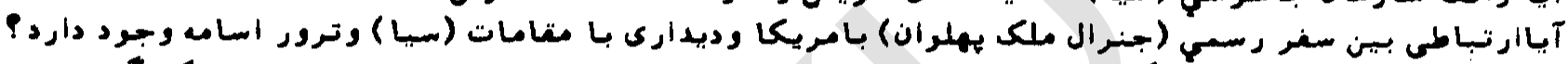

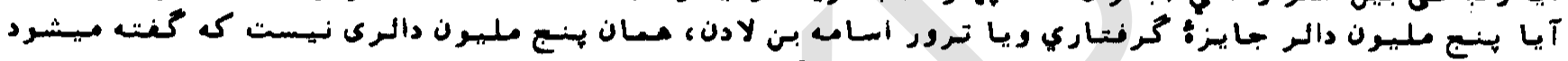

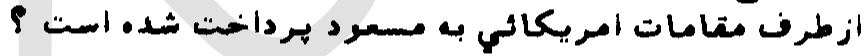

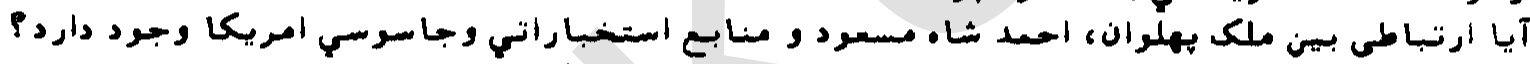

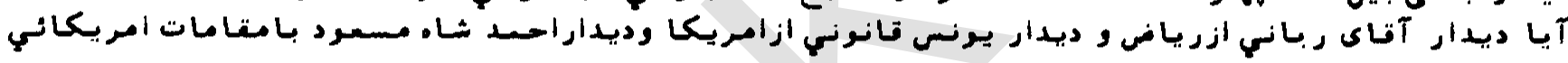

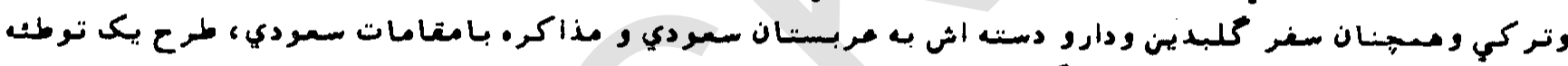

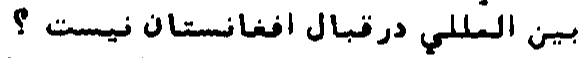

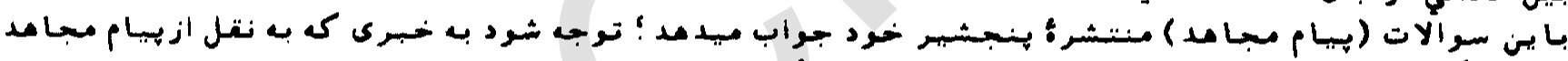

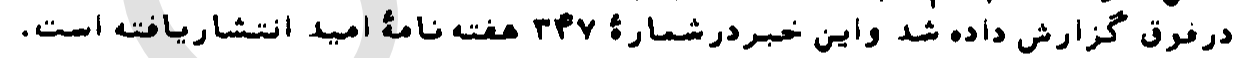

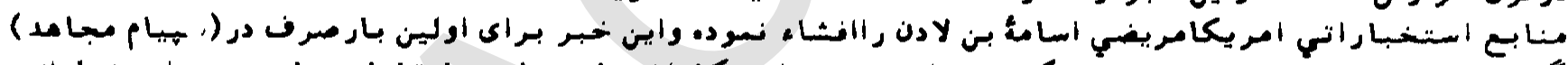

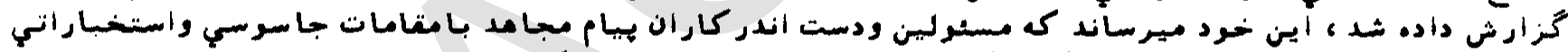

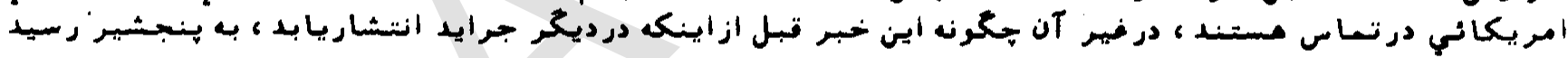

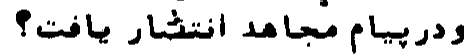

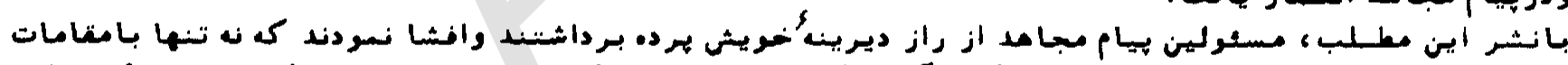

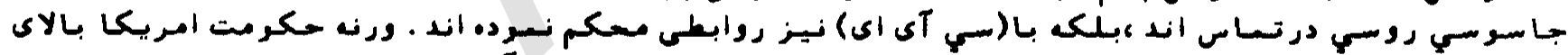

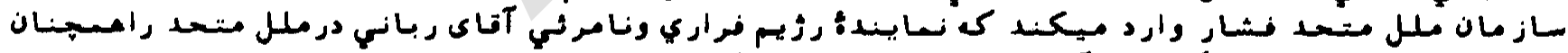

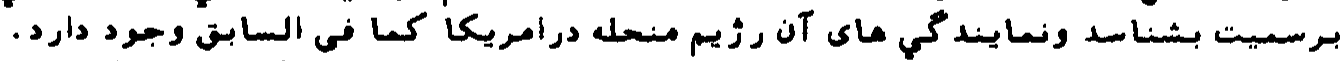

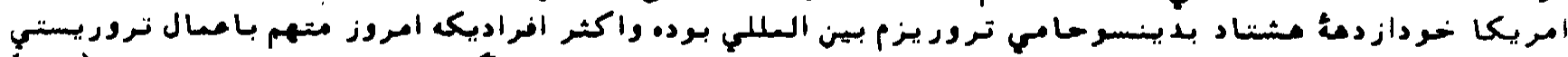

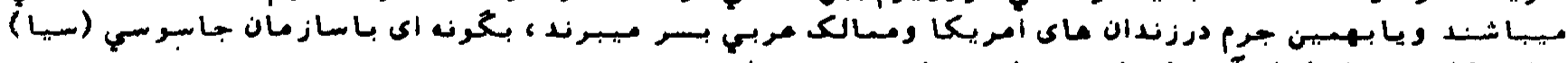

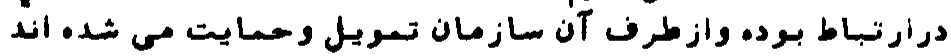

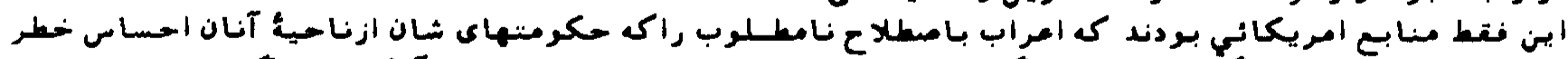

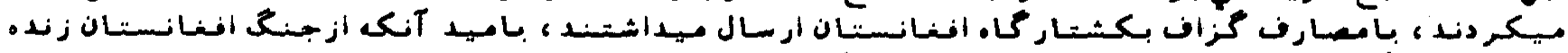

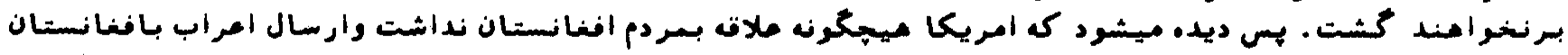

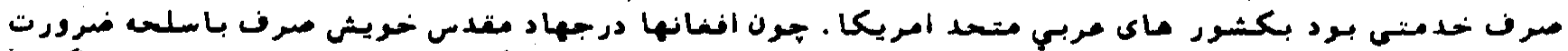

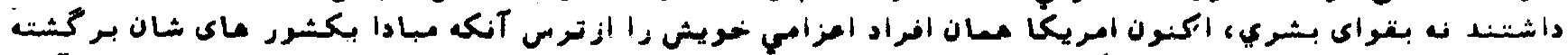

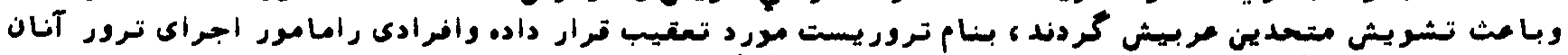

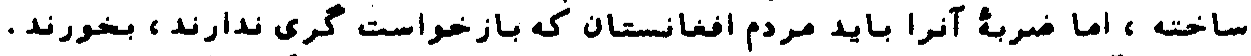

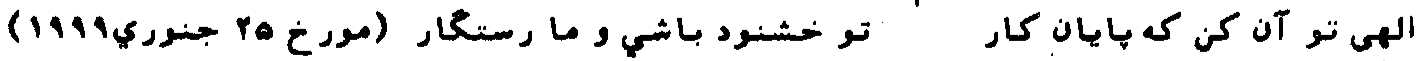


20

\section{عفهف هرحهت حق}

\section{Aومت}

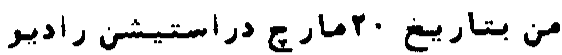

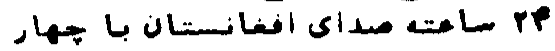

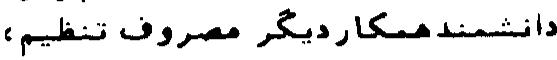

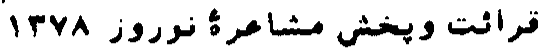

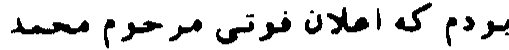

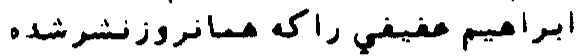

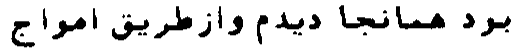

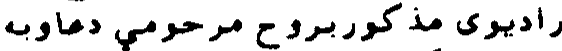

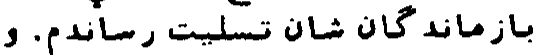

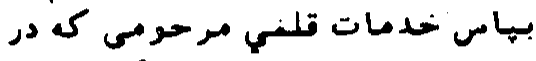

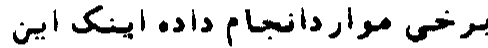

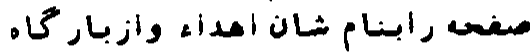

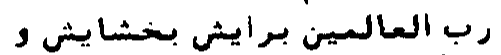

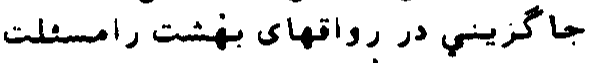

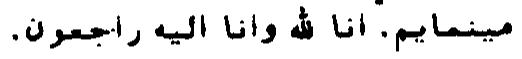

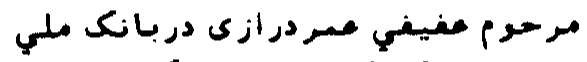

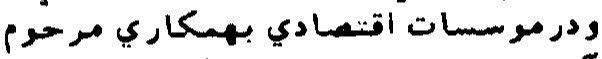

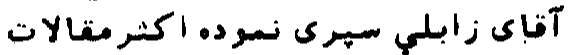

ونغار شهاى زابلمي زادة فكر و قلم

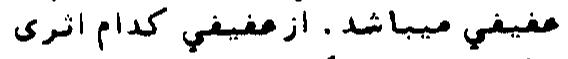

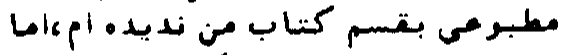

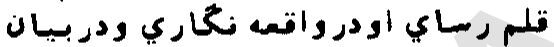

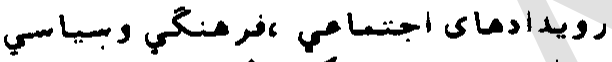

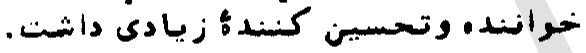

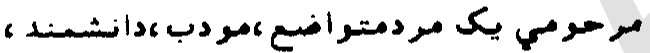

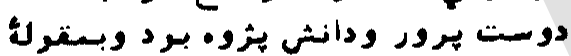

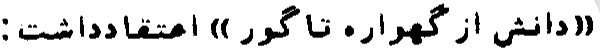

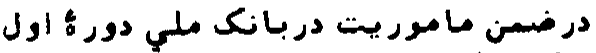

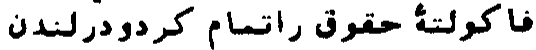

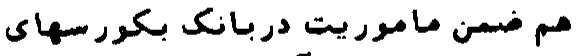

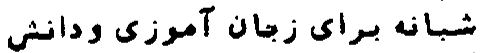

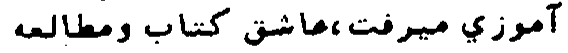

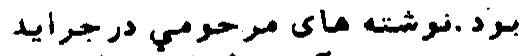

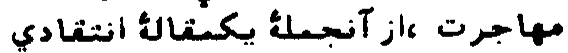

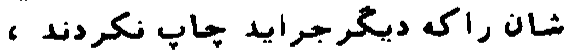
باثرخرامش شان در آثبنه أفنانستان.

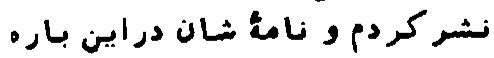

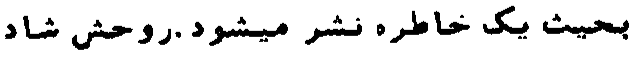

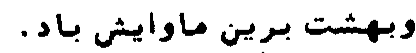

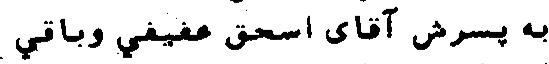

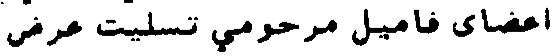

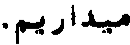

M. 5.A.

36616 Malar Com.

Iremont ea. 94536.5634

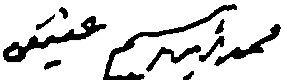

IA

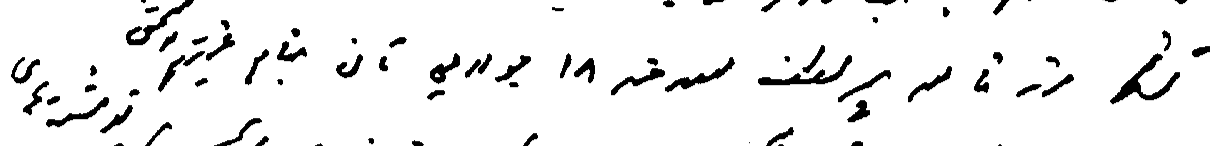
质 "Vs

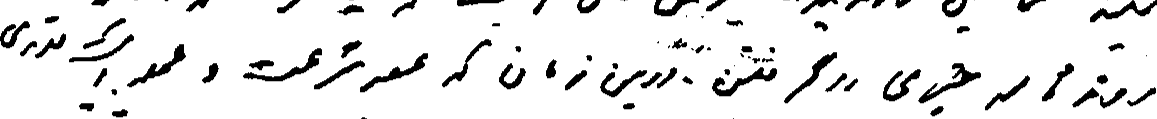

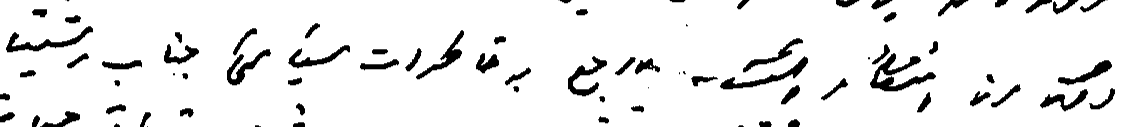
تصرمد

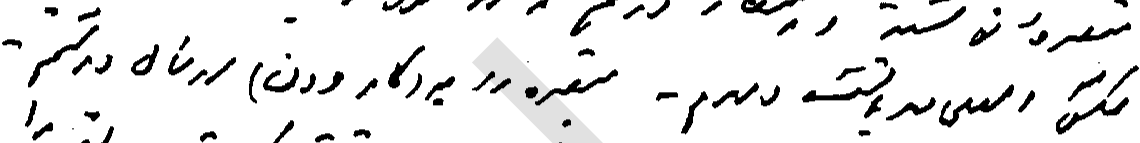

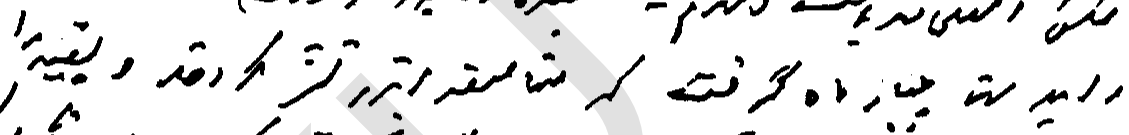

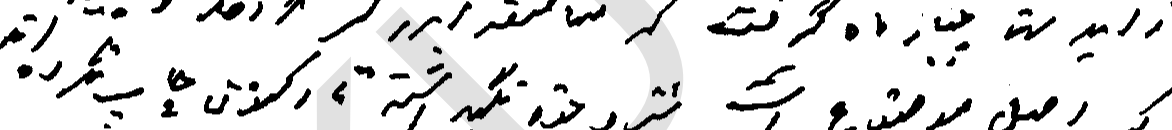

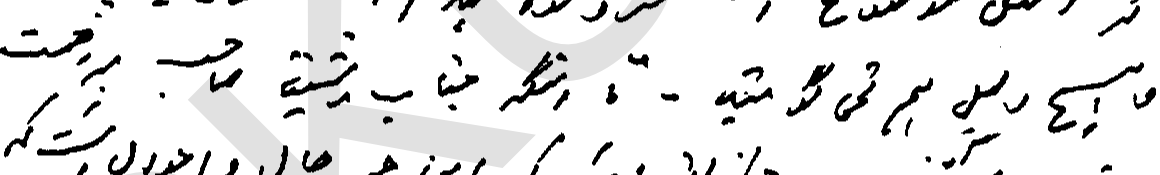

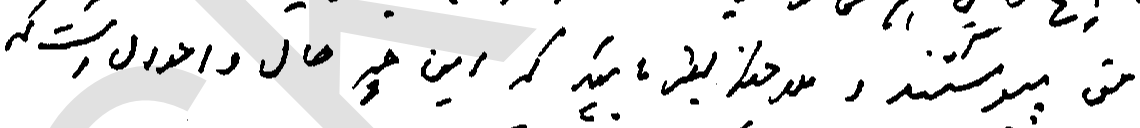
> : :

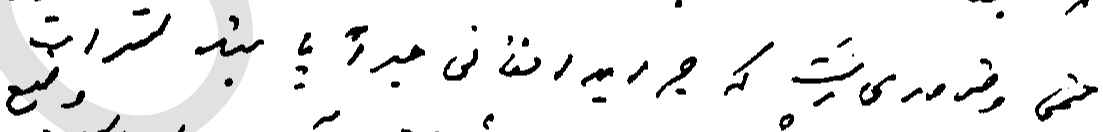
(2)

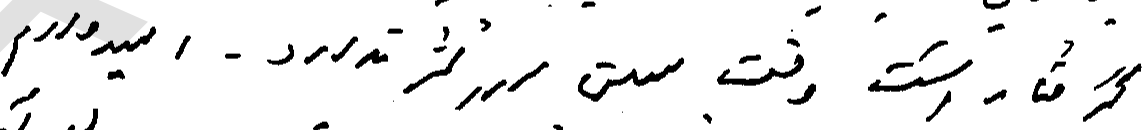
"

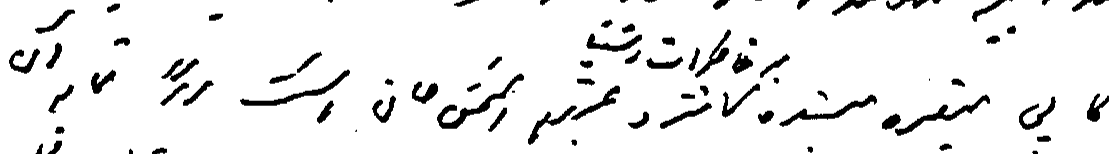

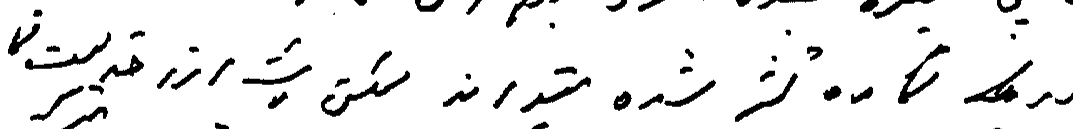

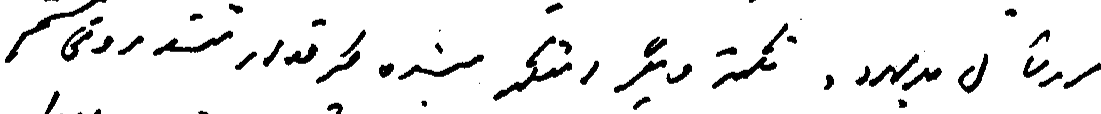

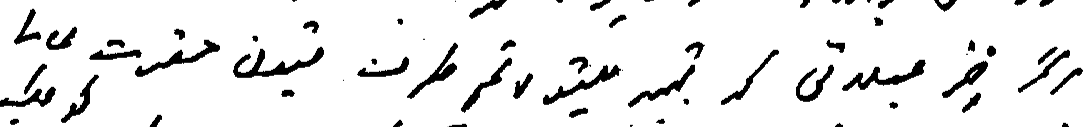
"خ هـ

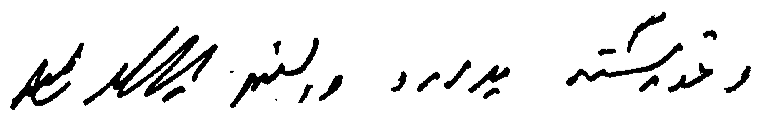




\section{1

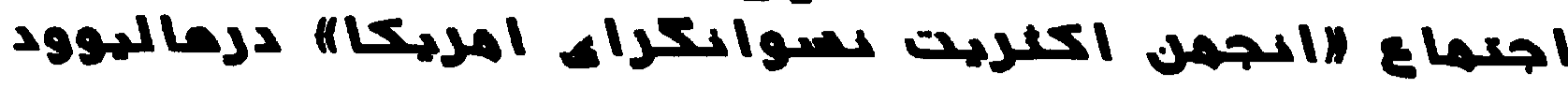

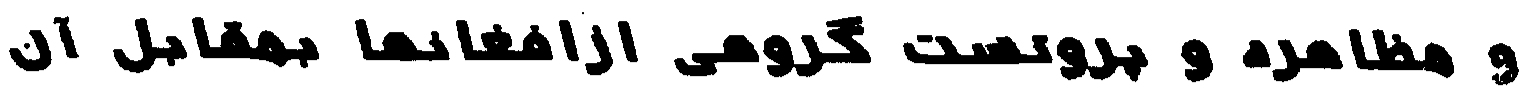

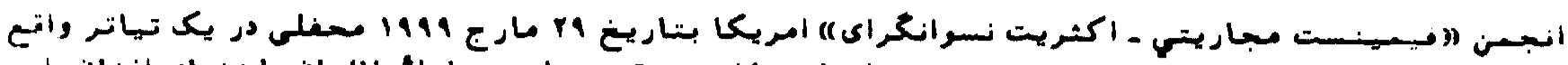

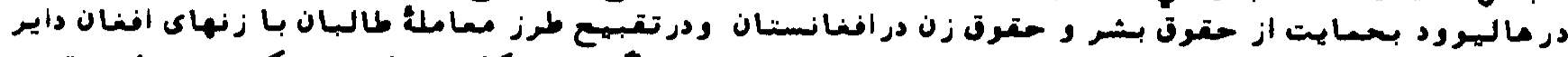

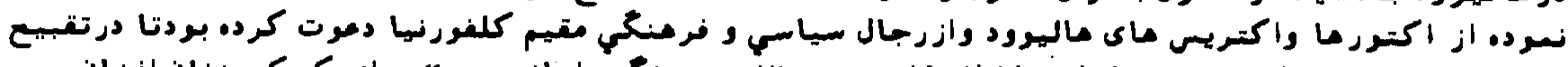

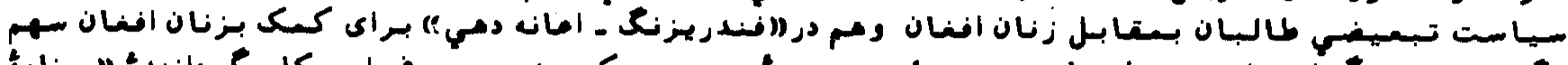

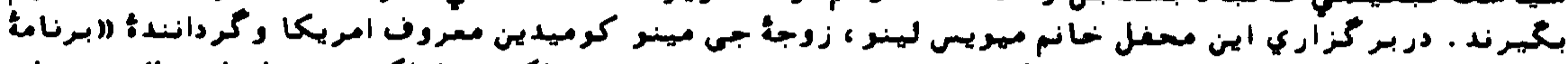

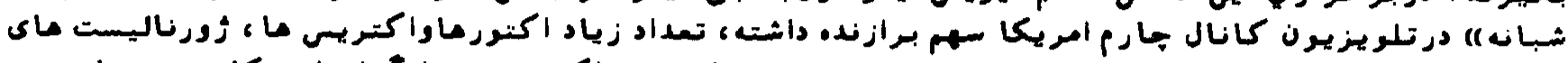

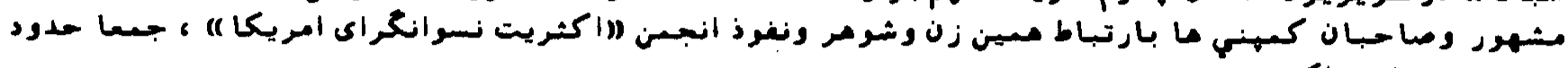

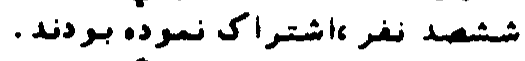

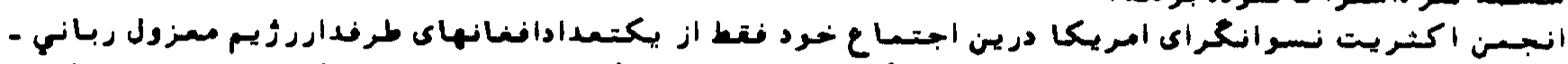

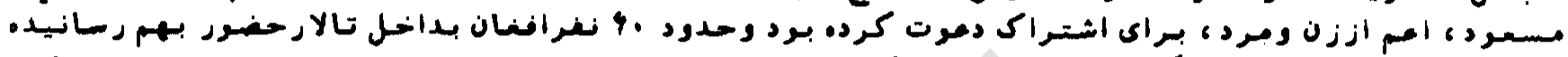

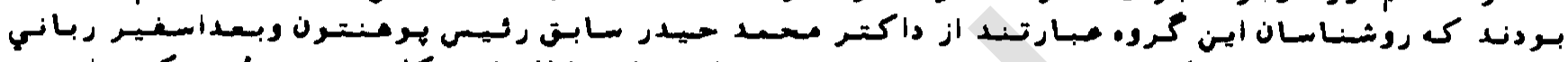

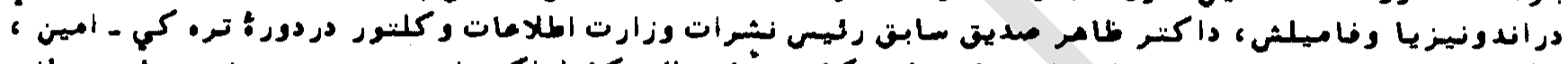

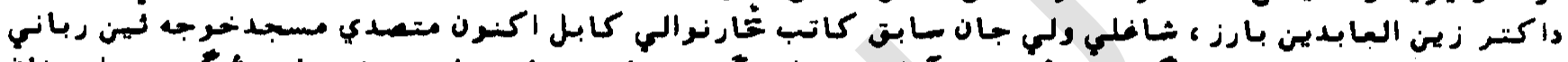

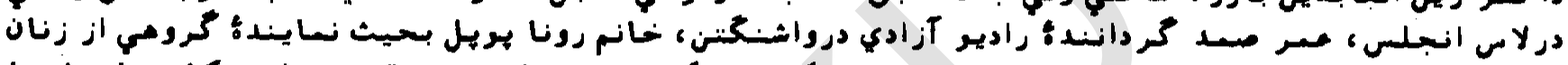

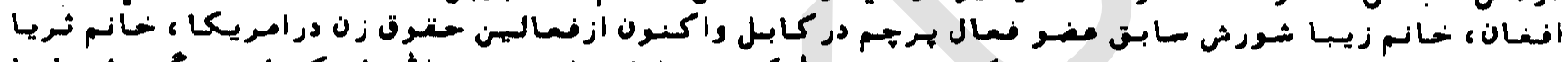

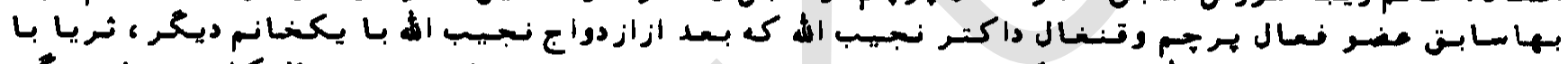

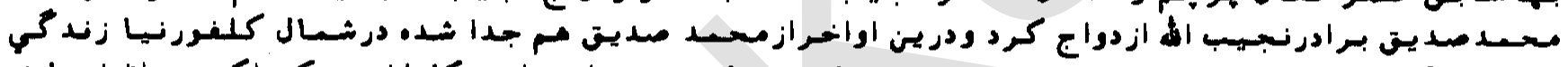

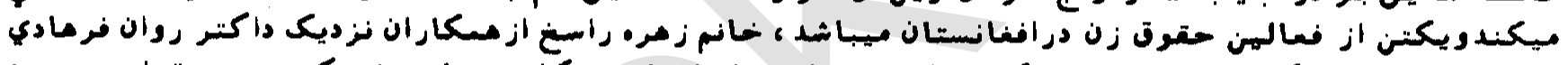

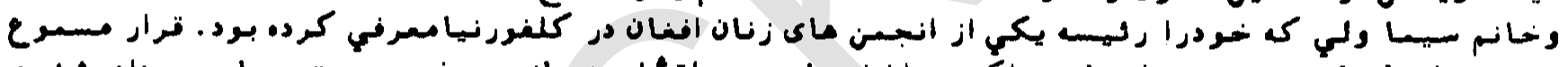

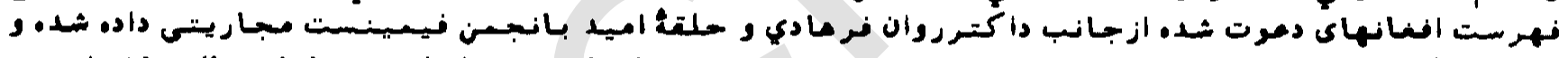

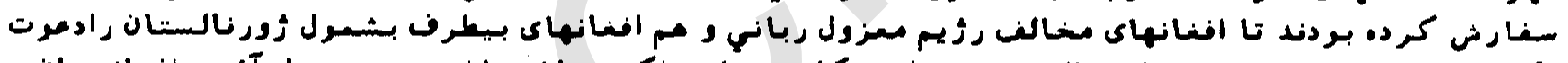

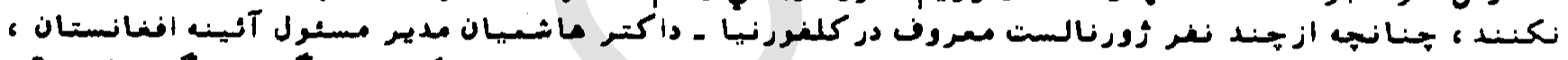

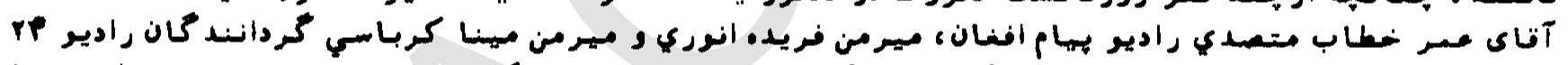

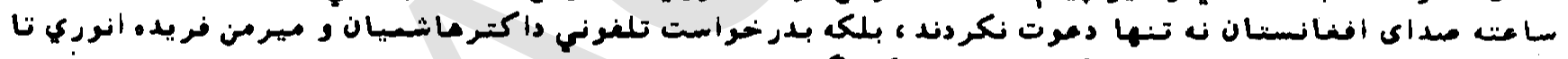

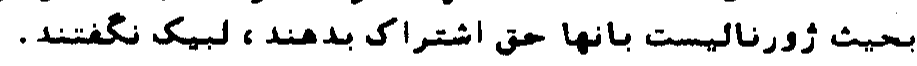

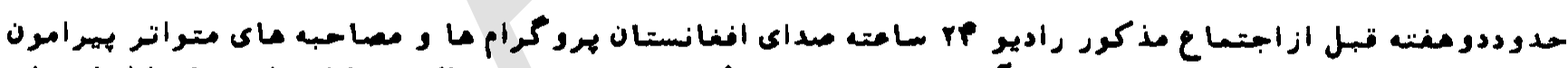

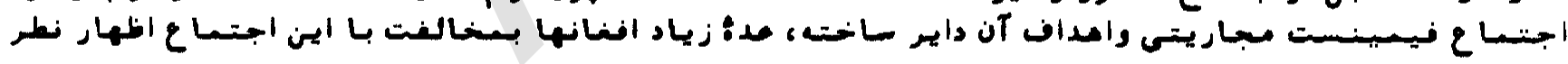

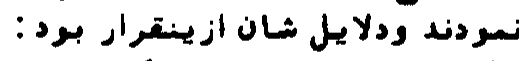

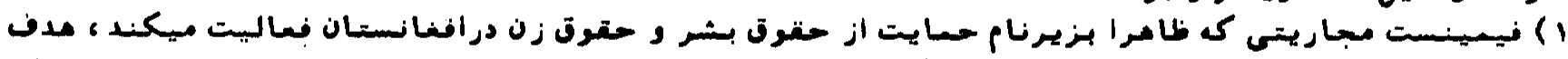

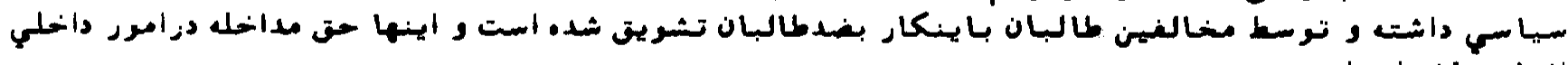

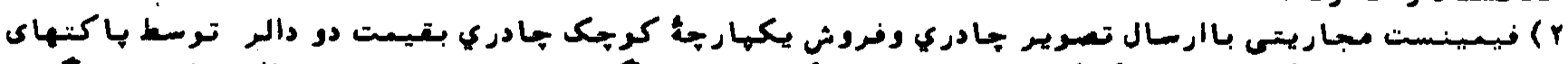

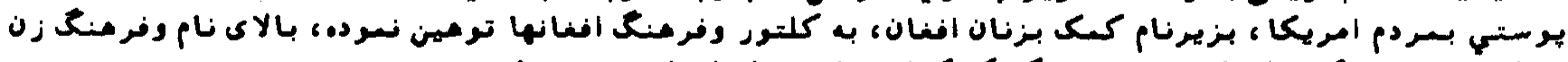

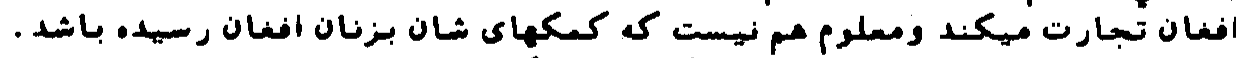

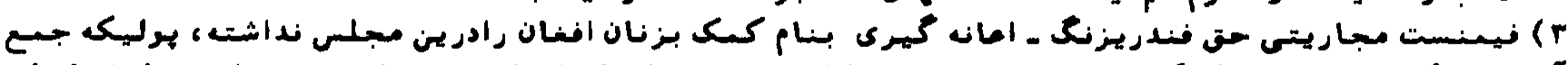

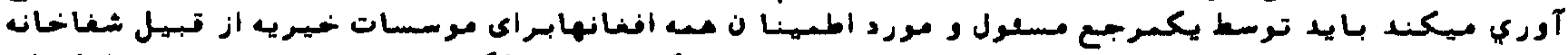

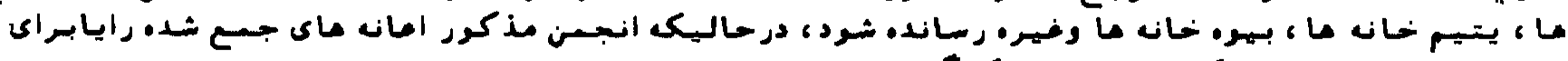

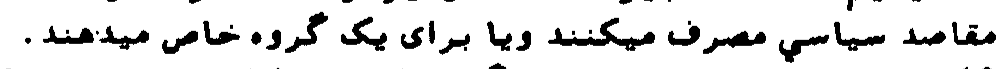

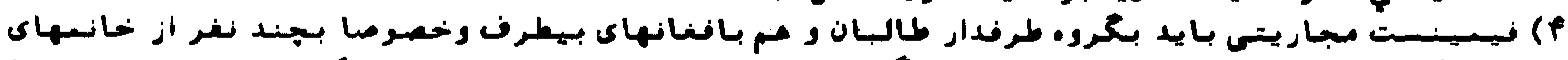

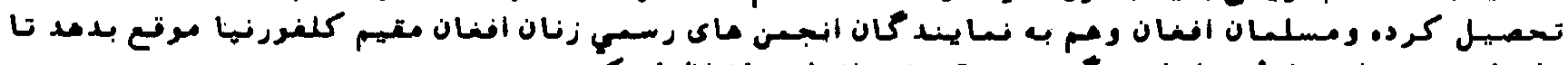

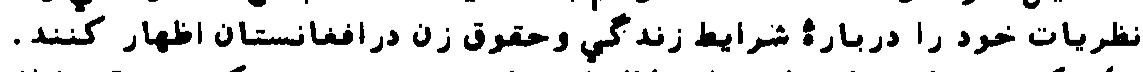

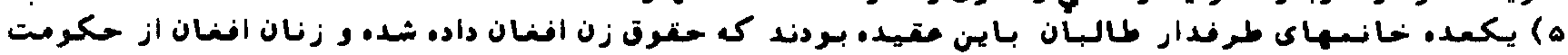

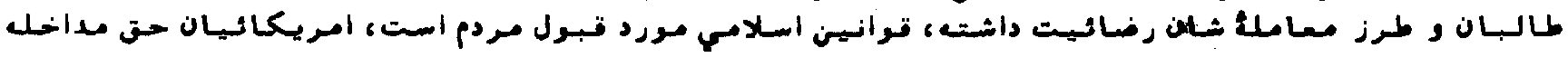

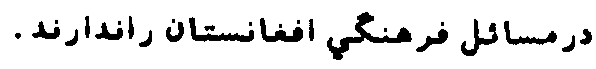




\section{2}

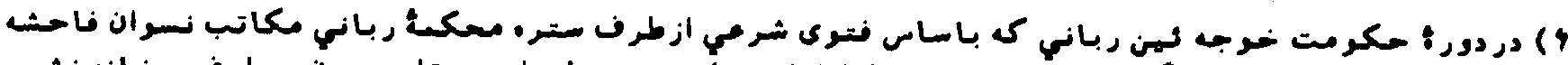

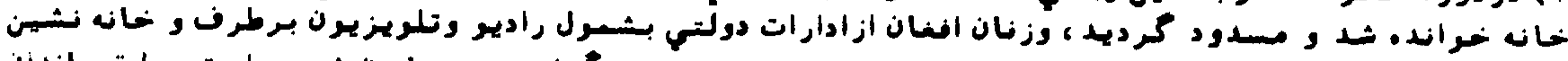

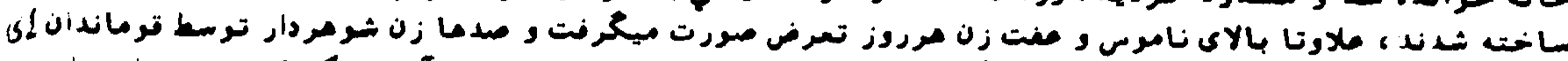

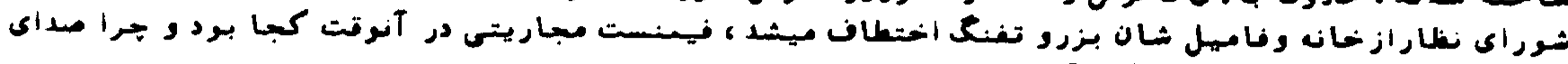

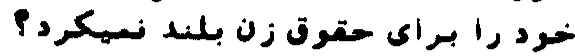

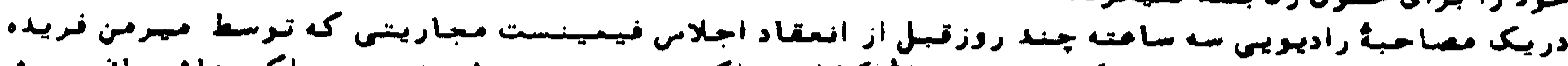

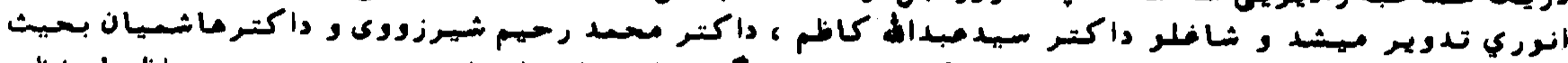

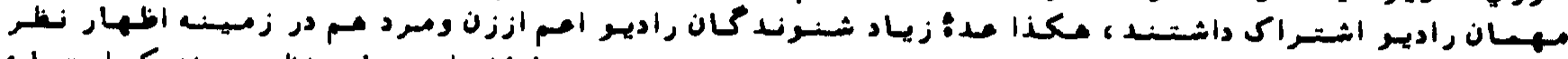

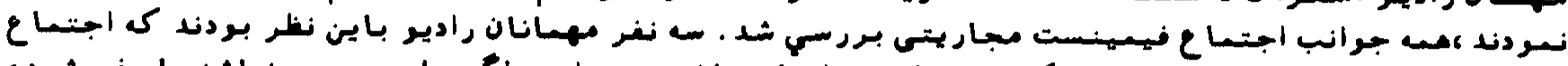

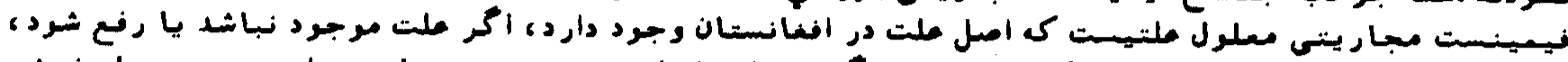

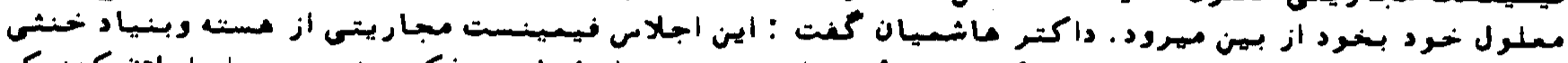

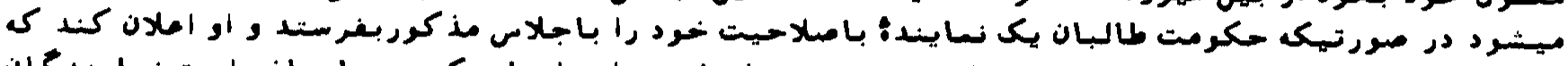

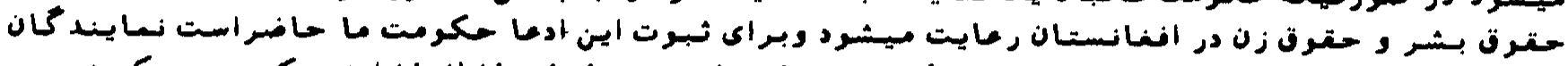

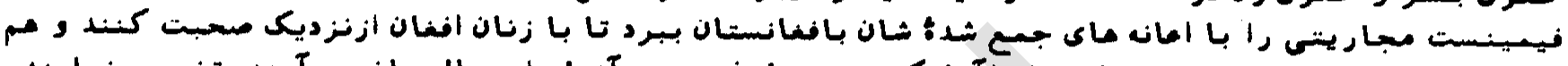

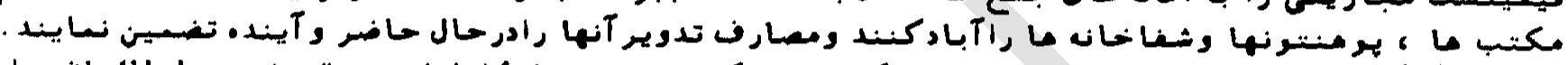

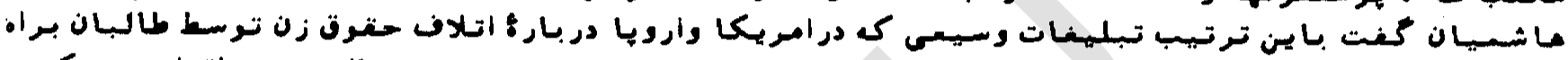

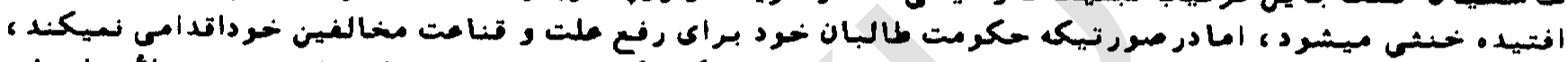

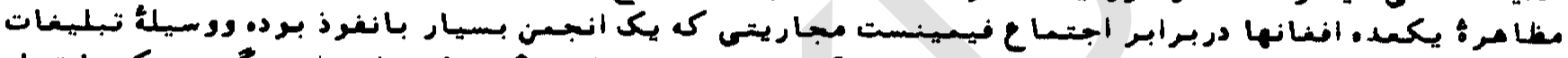

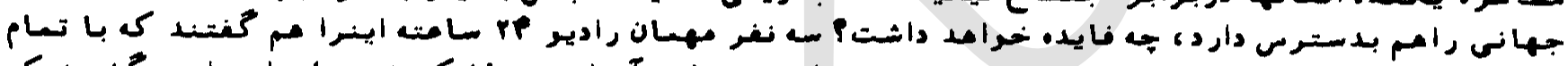

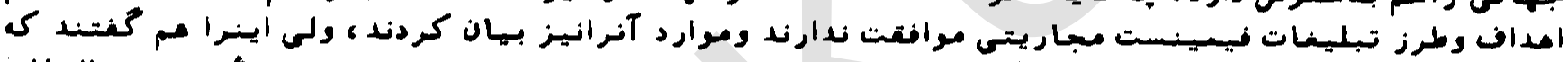

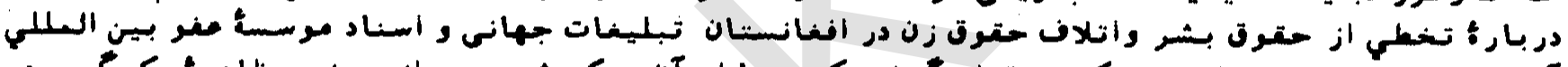

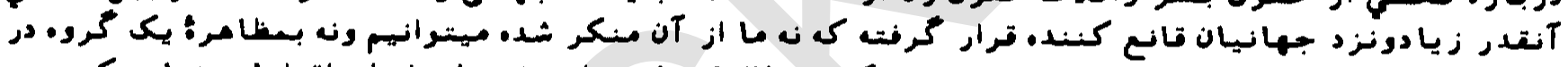

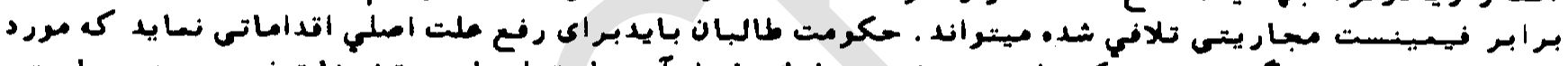

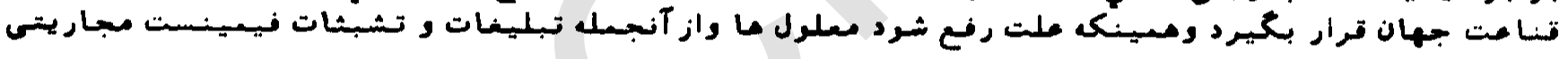

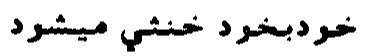

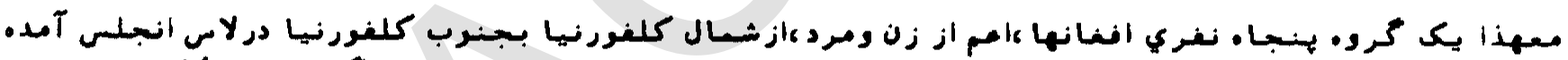

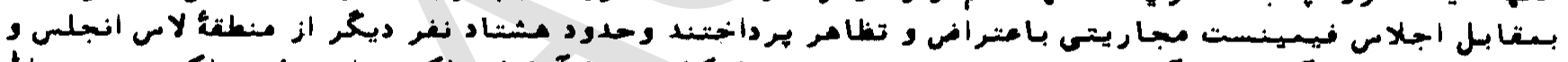

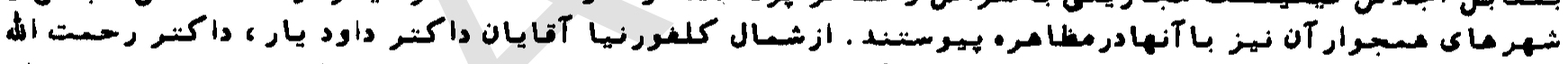

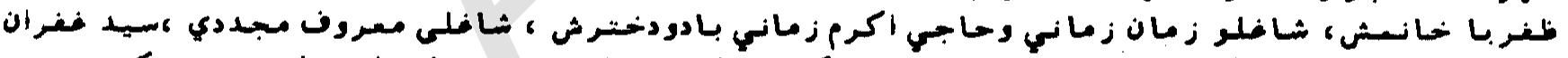

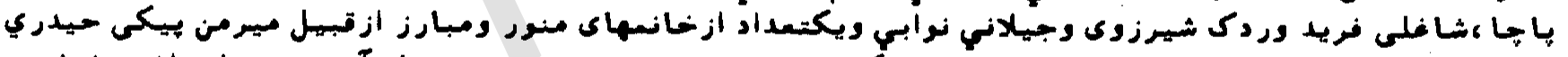

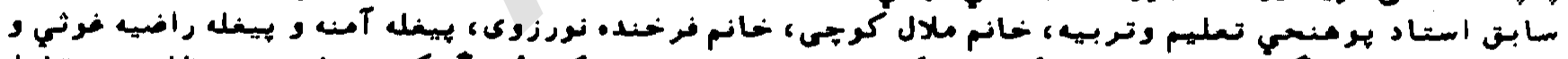

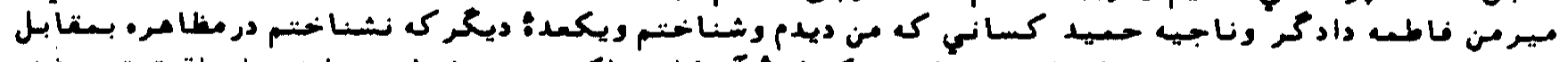

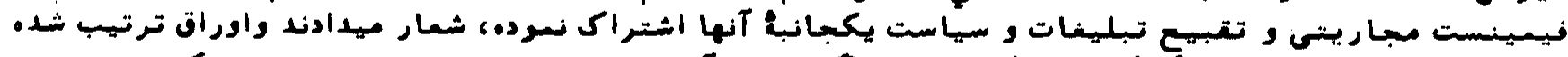

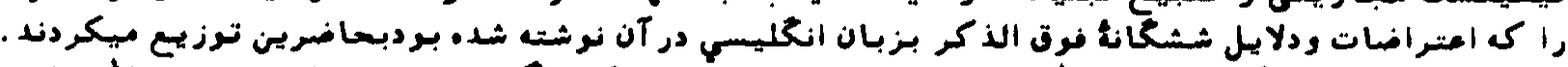

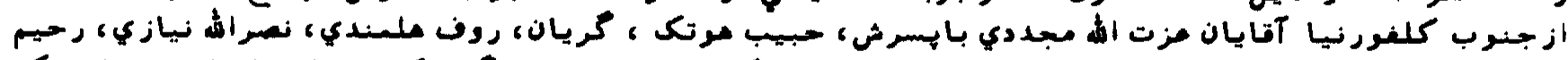

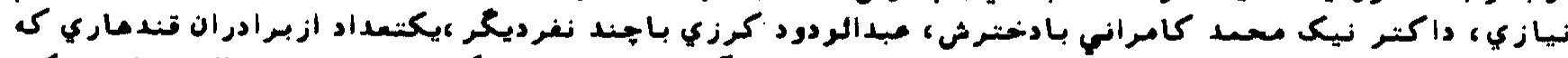

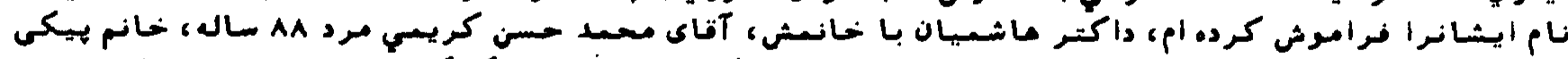

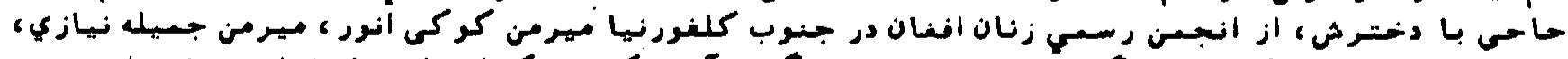

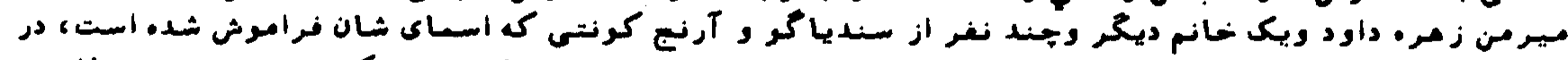

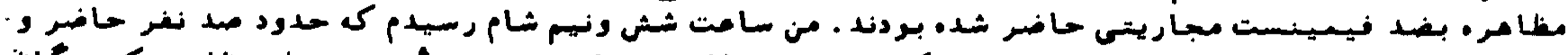

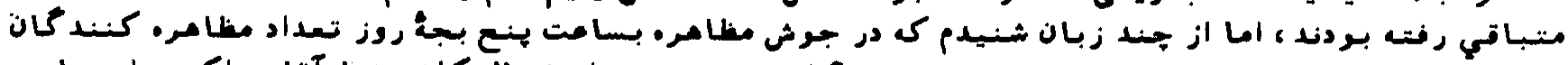

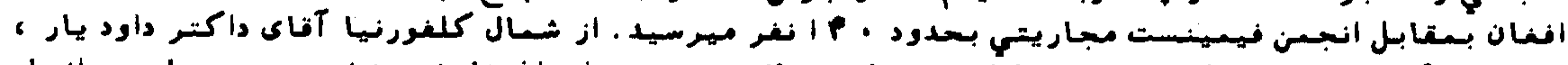

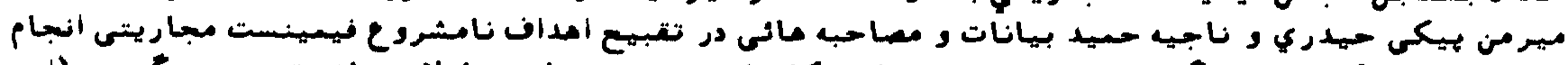

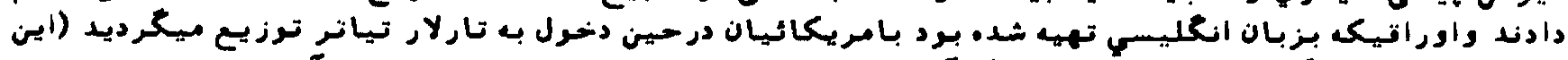

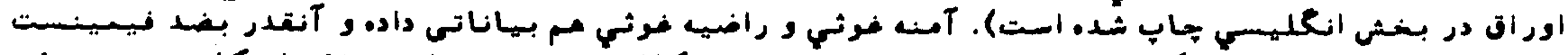

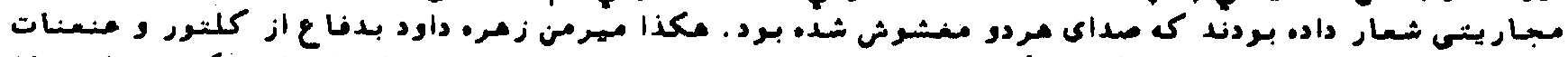

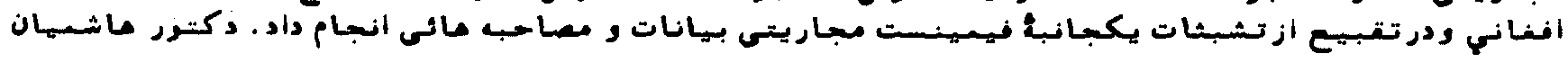




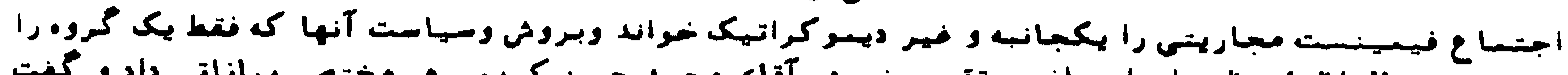

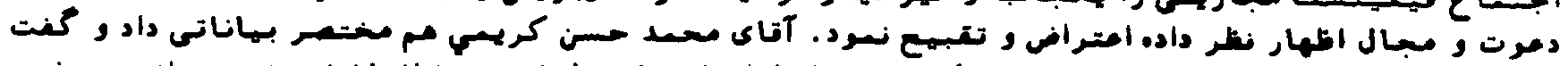

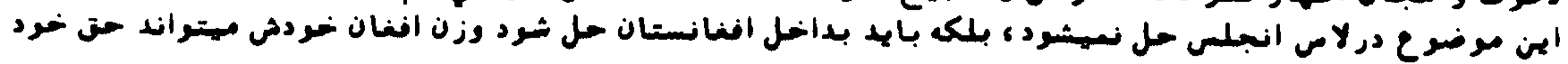
را بحهير د فرو

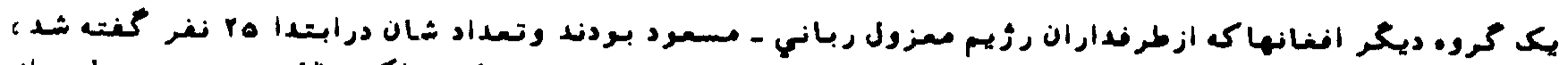

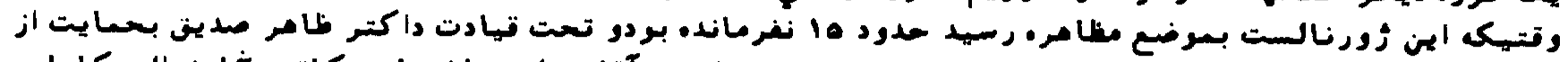

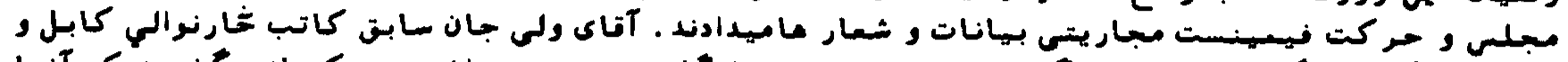

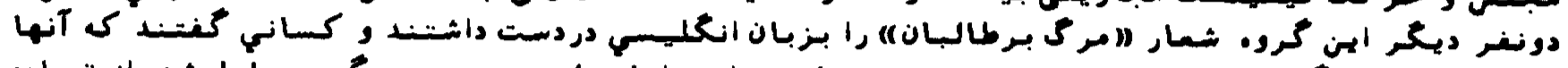

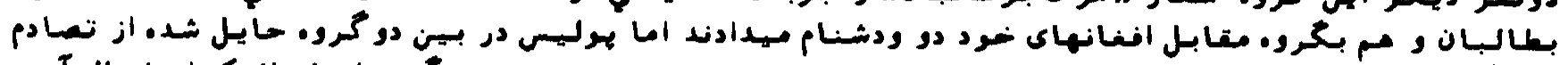

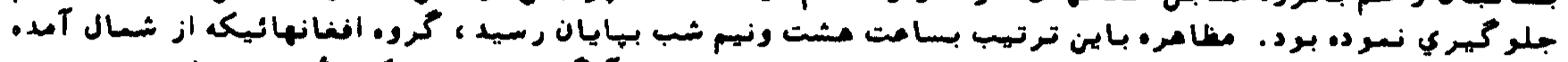

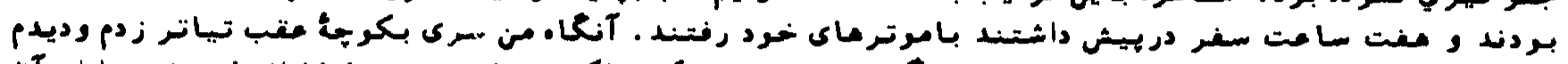

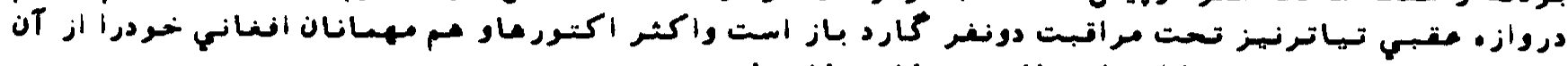

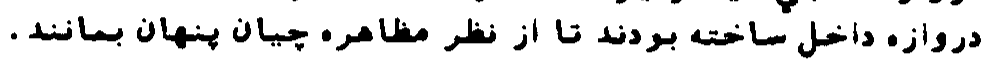

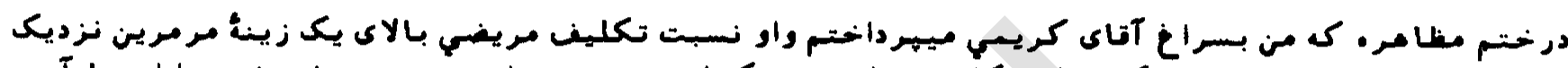

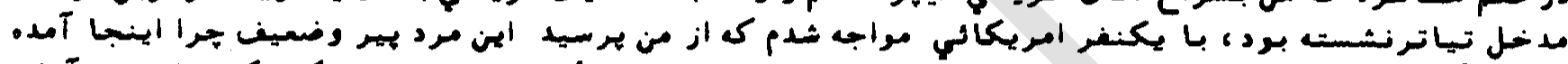

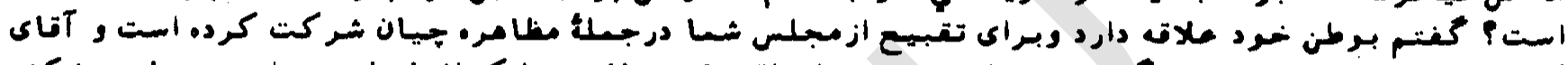

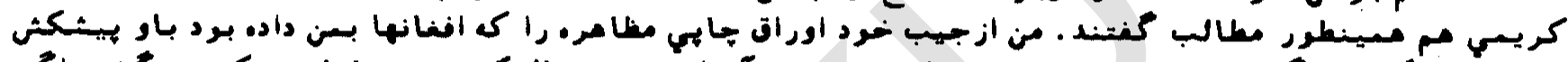

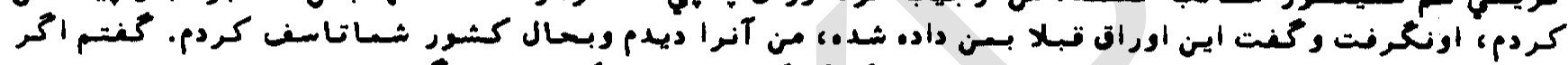

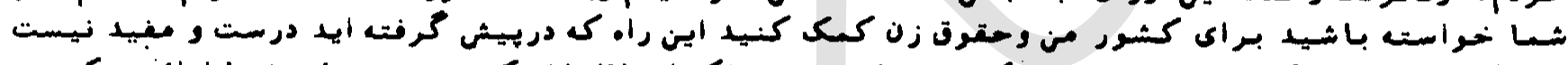

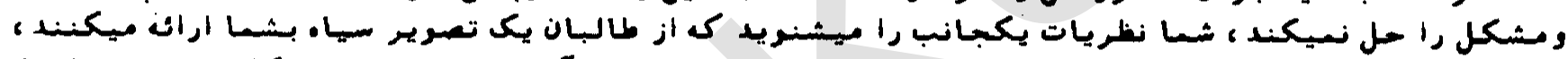

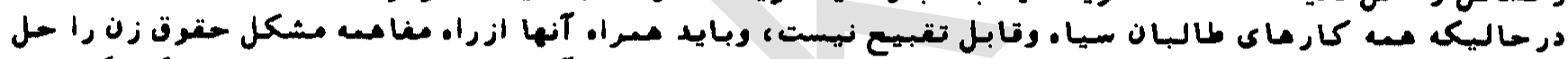

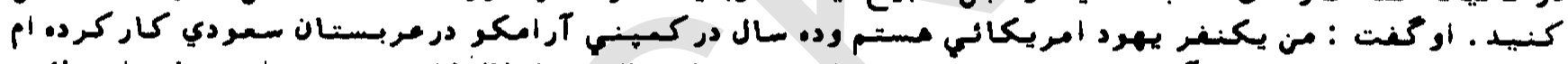

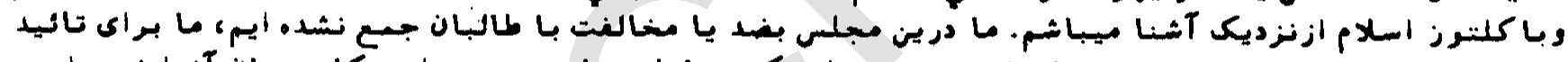

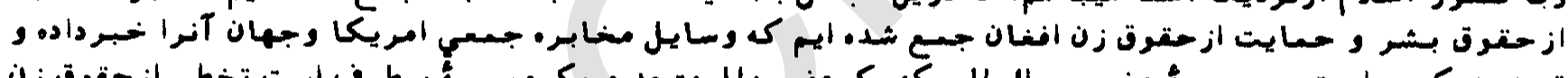

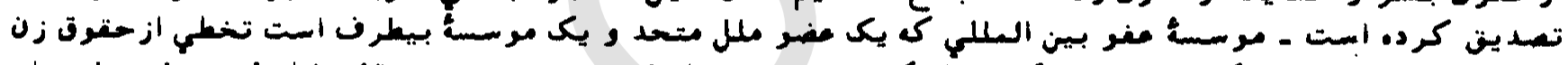

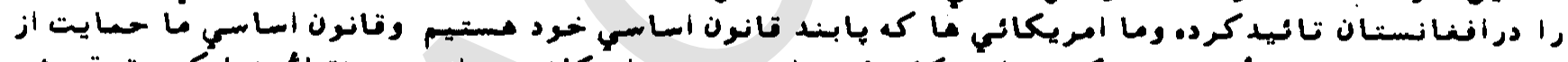

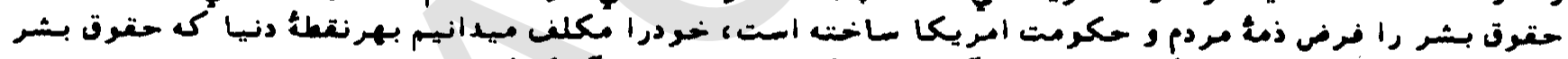

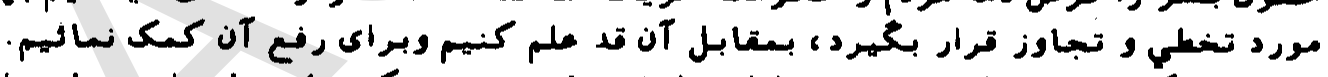

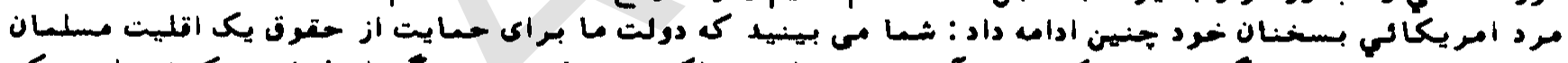

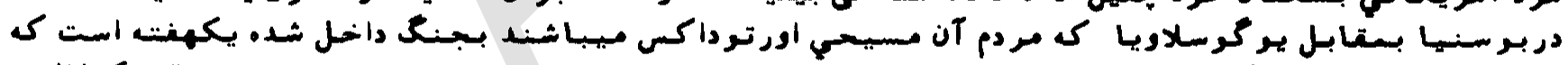

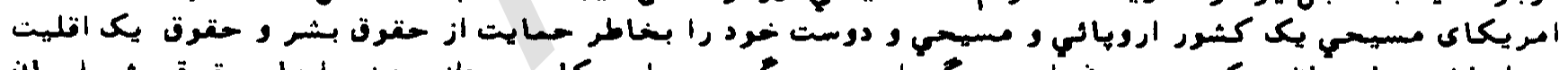

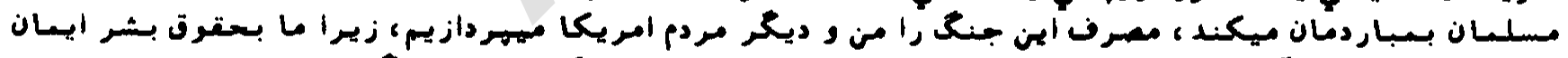

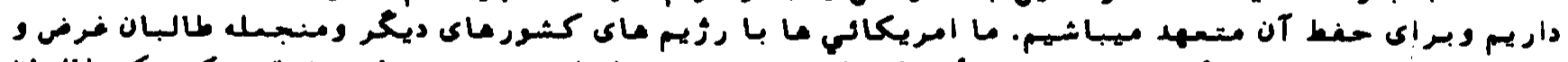

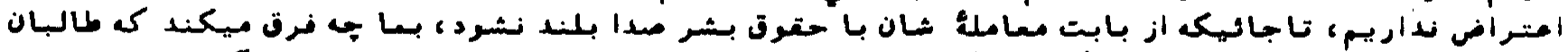

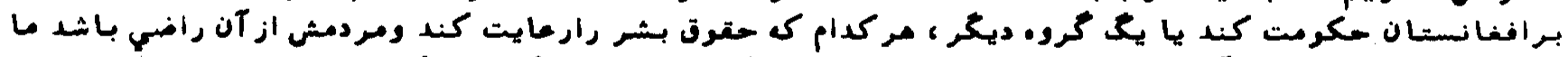

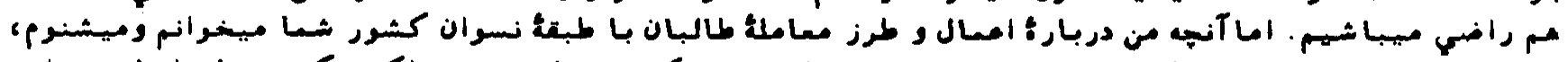

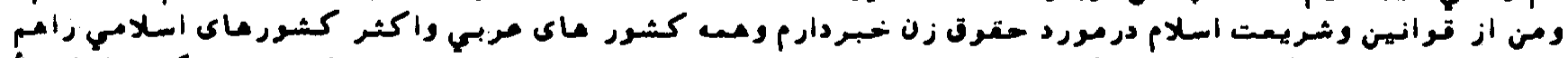

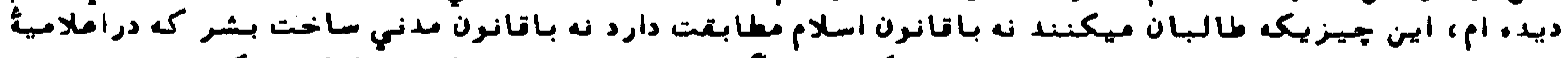

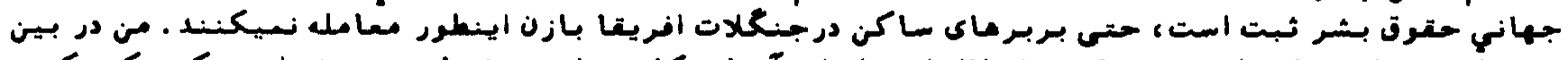

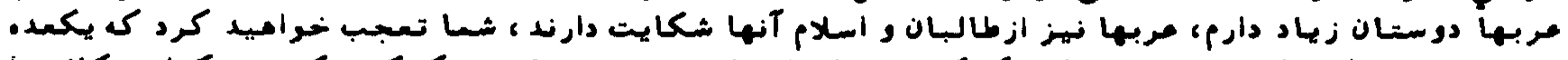

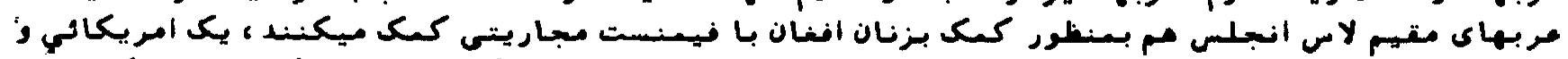

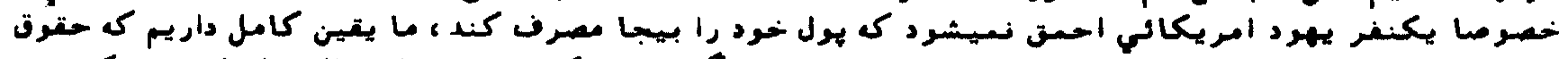

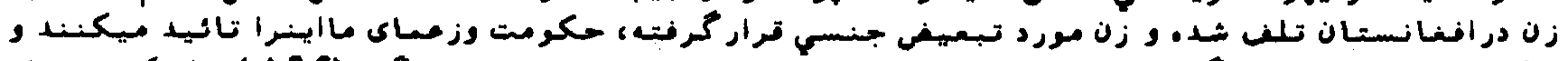

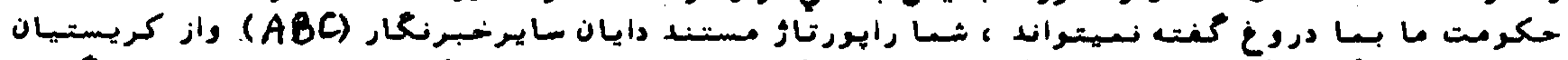

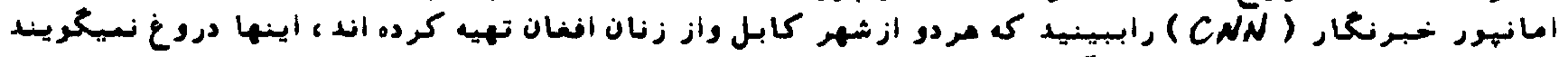

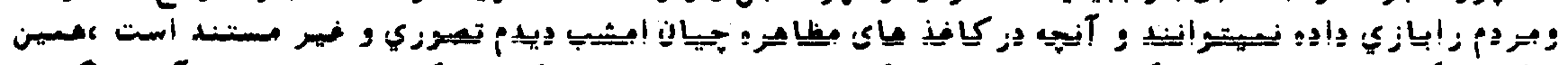

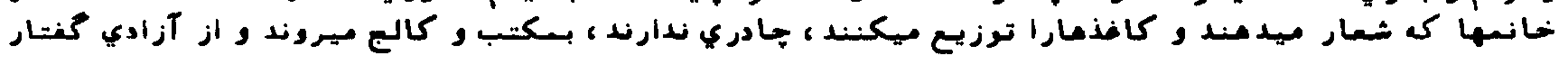




\section{4}

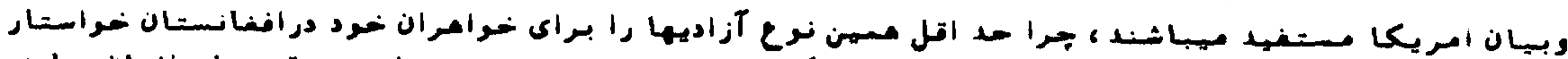

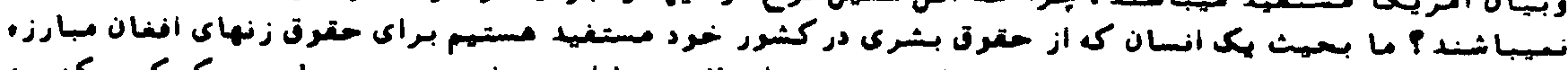

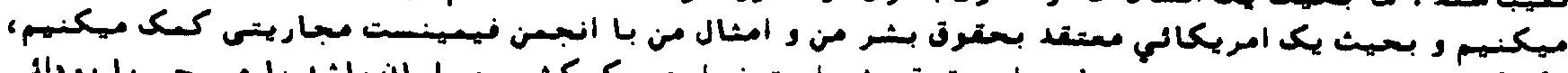

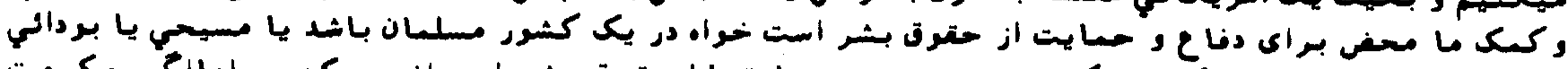

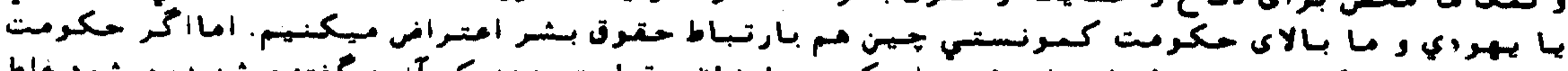

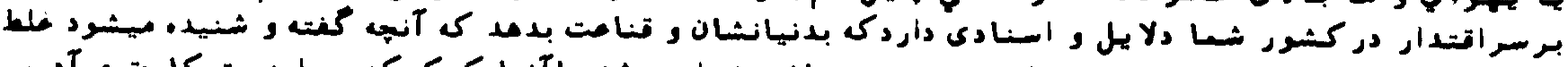

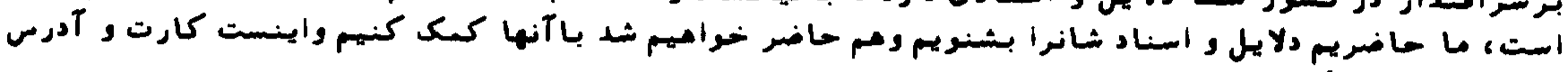

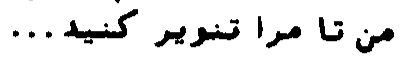

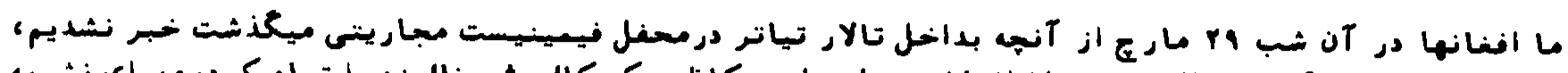

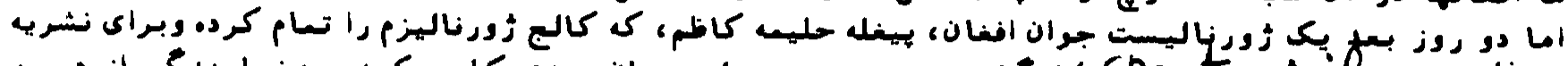

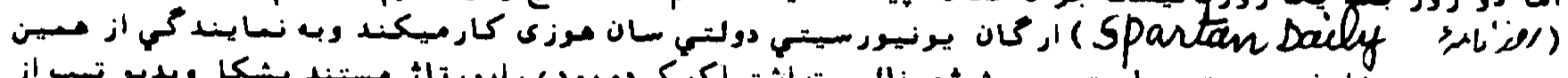

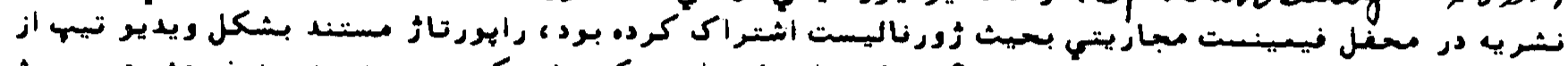

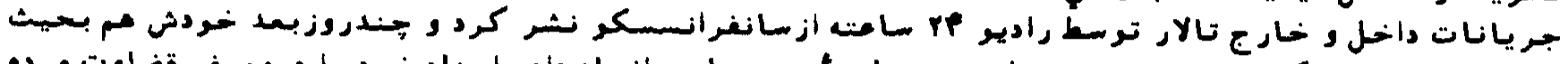

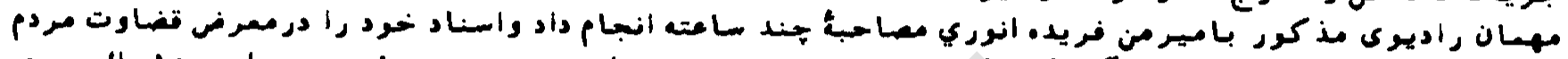

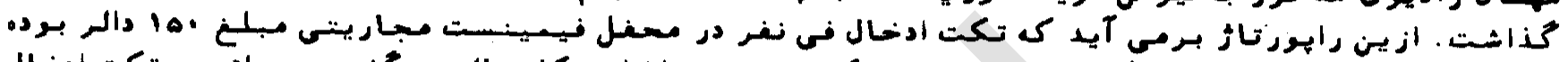

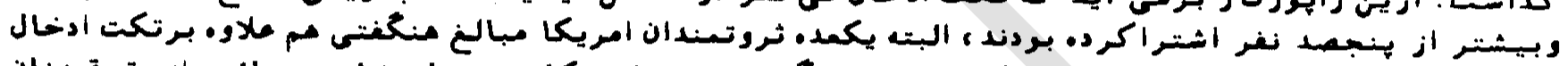

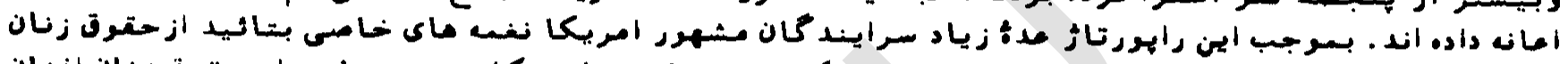

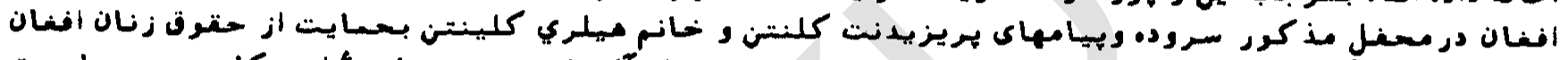

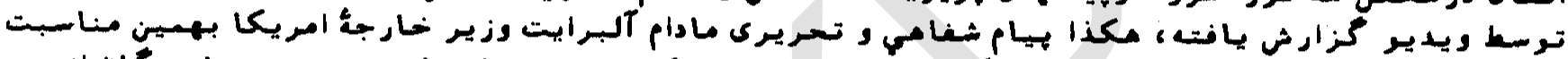

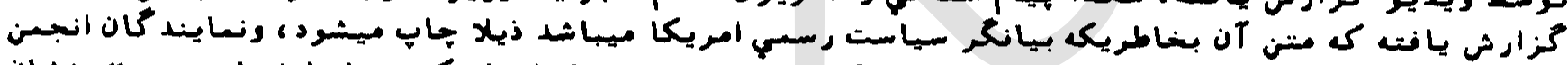

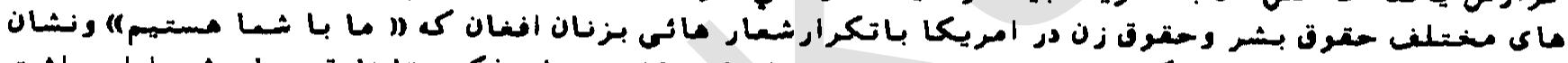

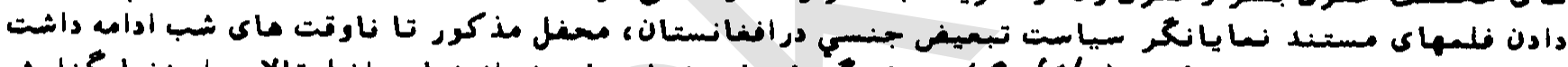

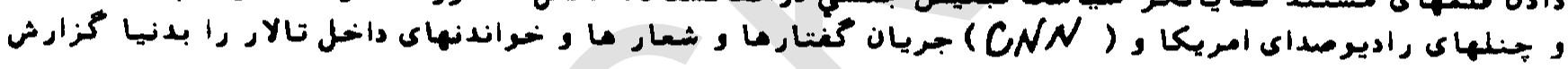

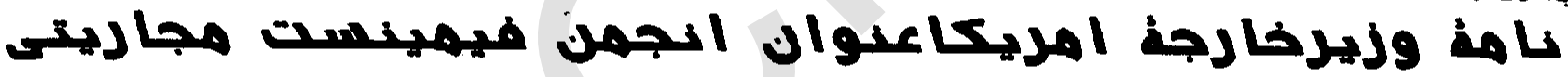

مبرد

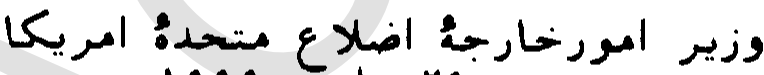 \\ 1999 مورد}

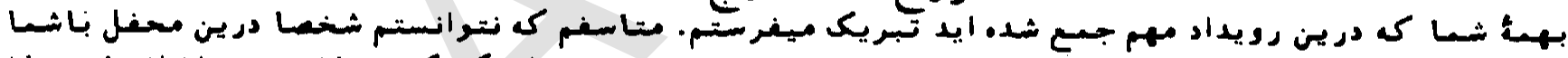

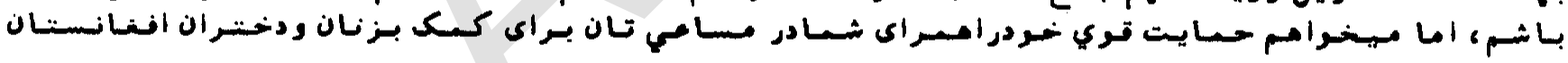

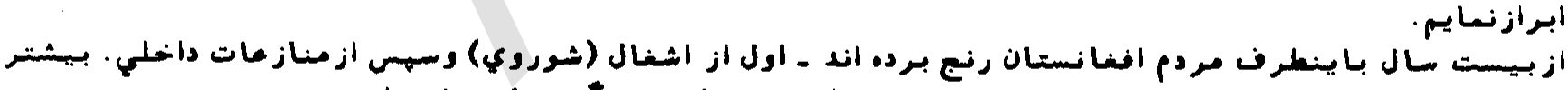

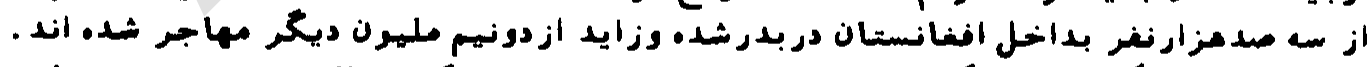

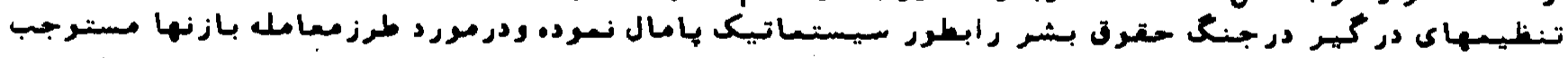

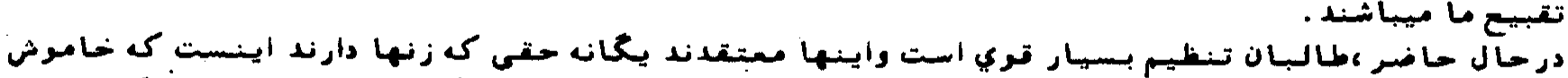

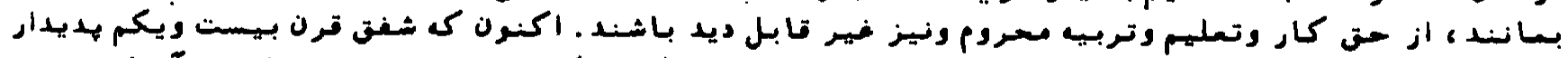

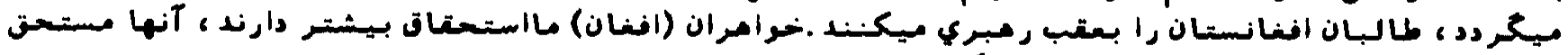

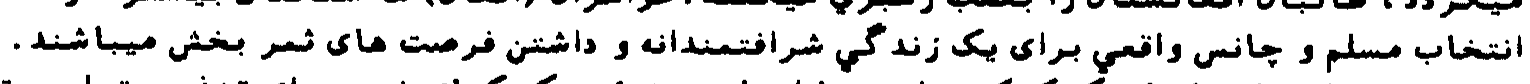

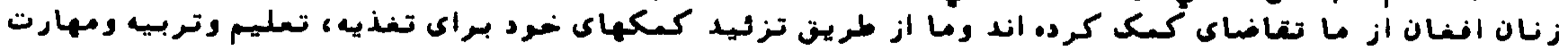

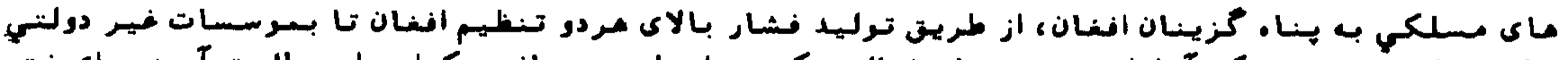

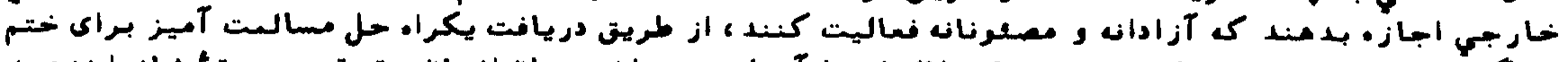

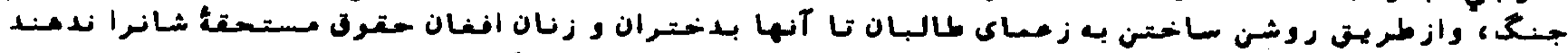

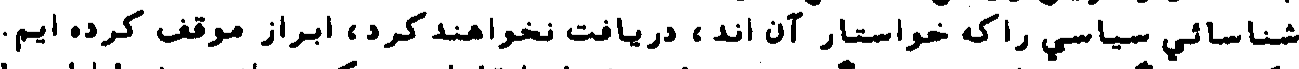

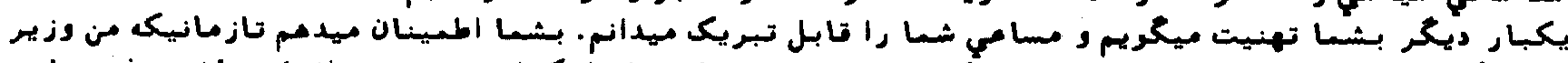

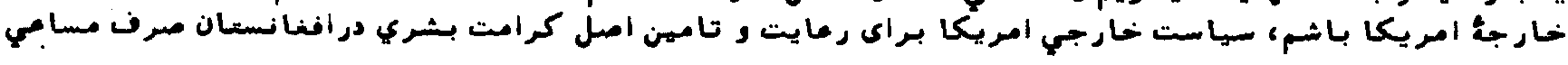

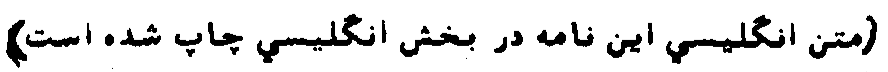
خواهد كرد. (مادلين ك. بانها آلبرايت) 


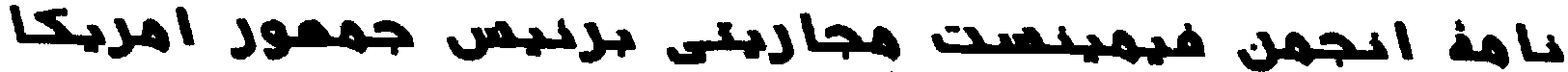

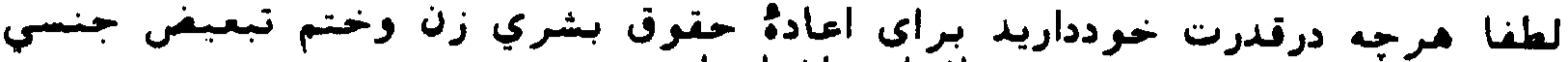

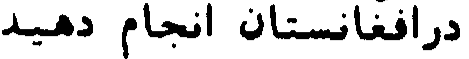

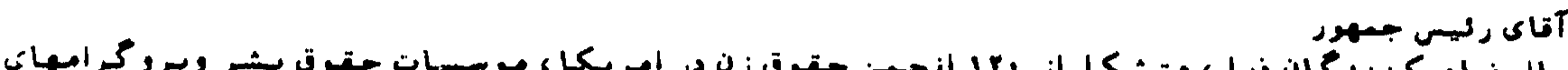

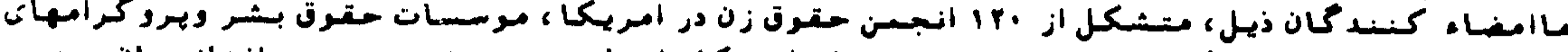

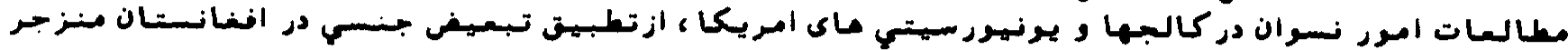

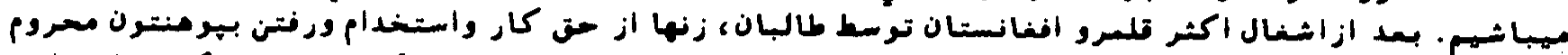

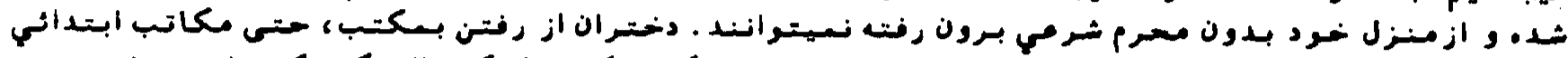

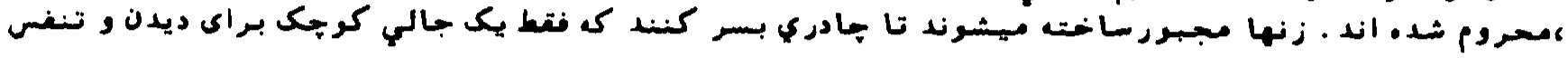

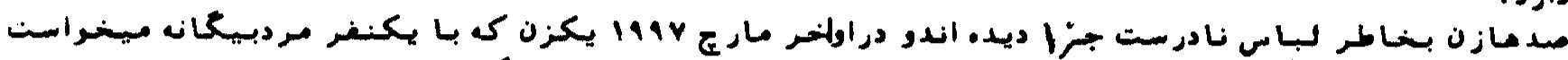

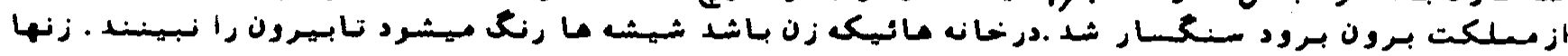

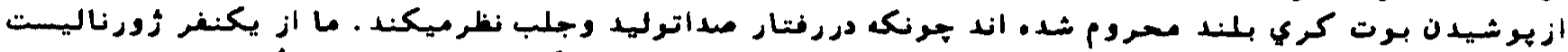

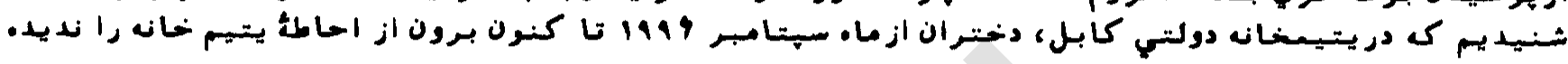

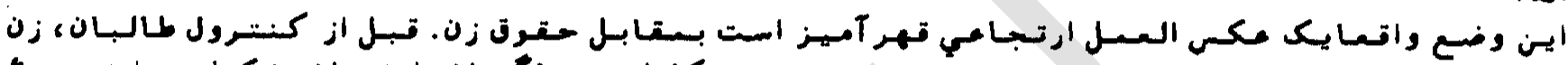

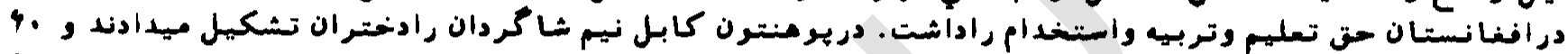

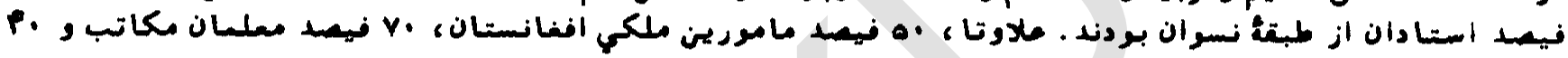

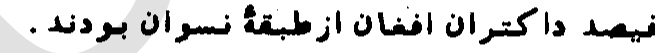

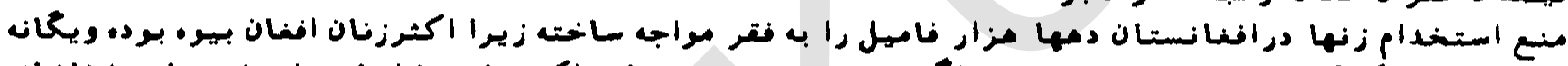

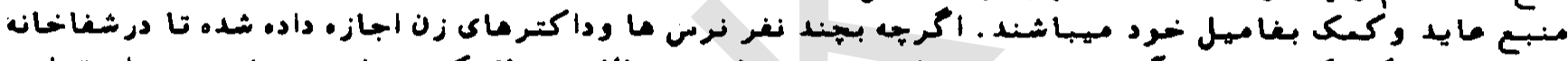

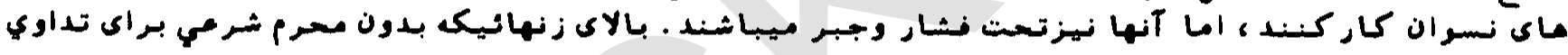

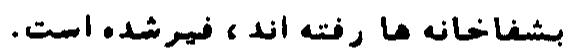

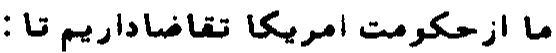

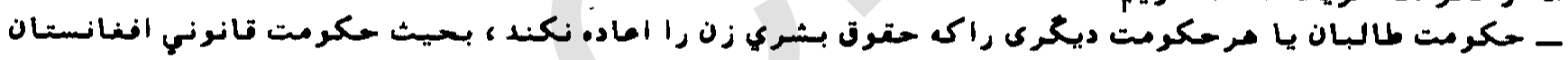

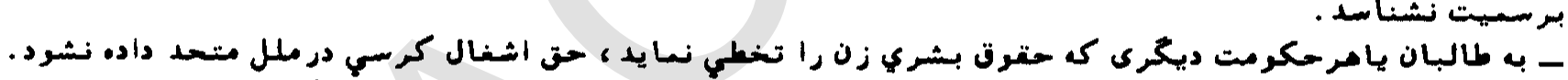

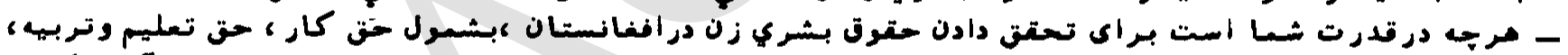

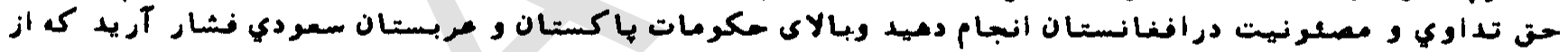

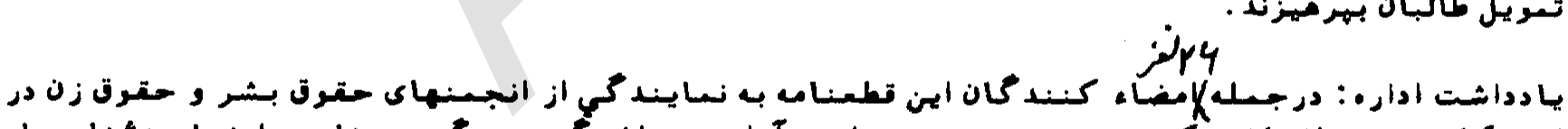

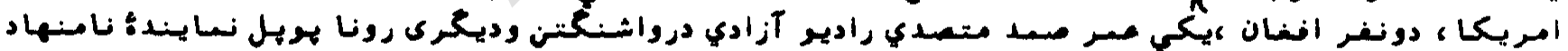

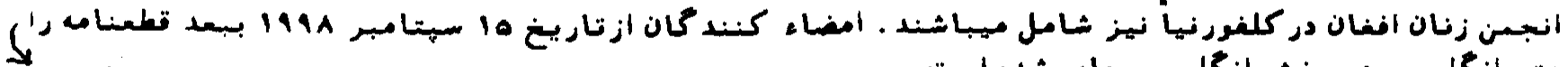

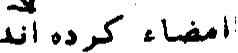

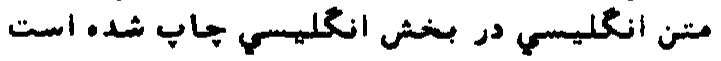

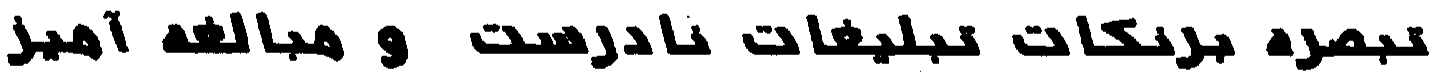

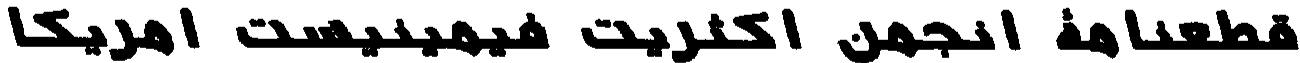

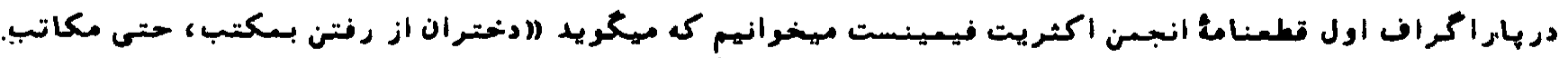

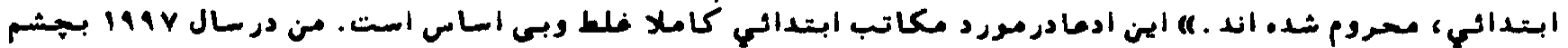

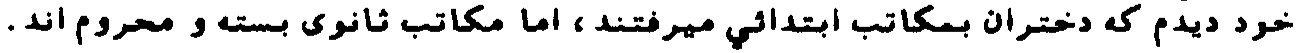

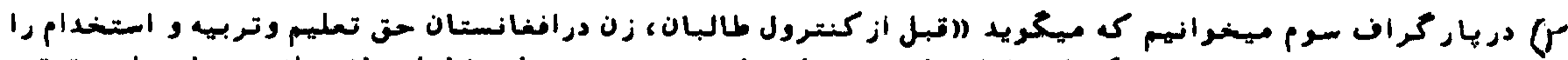

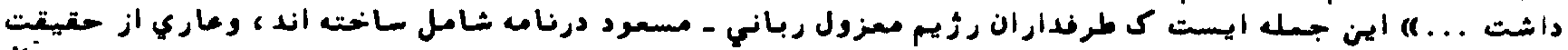

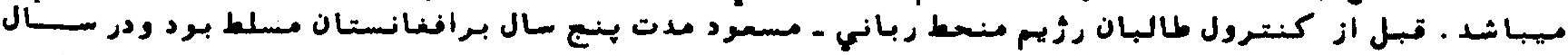

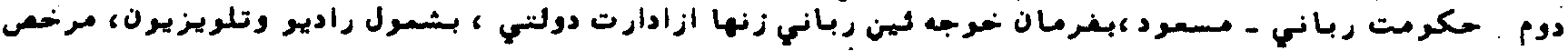




\section{6}

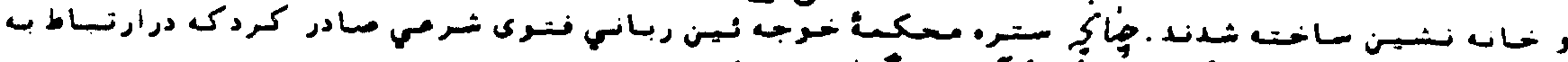

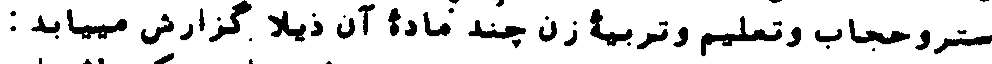

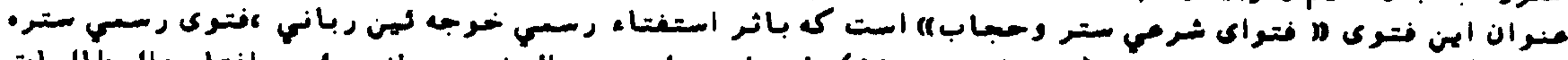

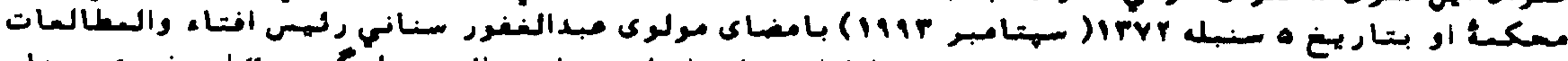

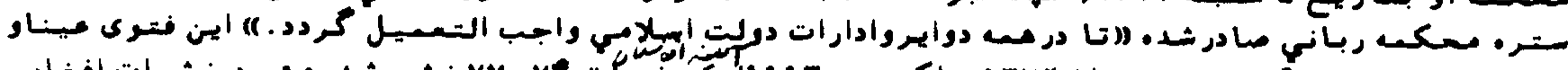

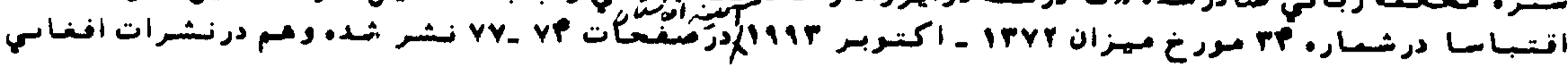

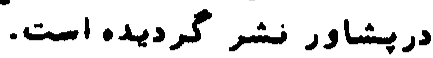

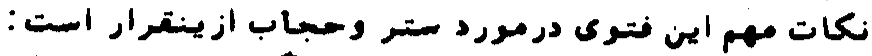

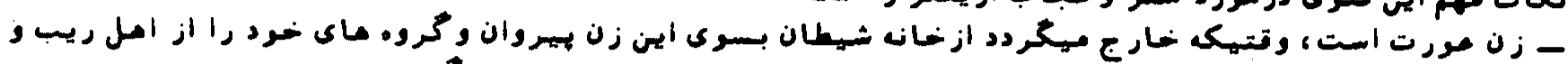

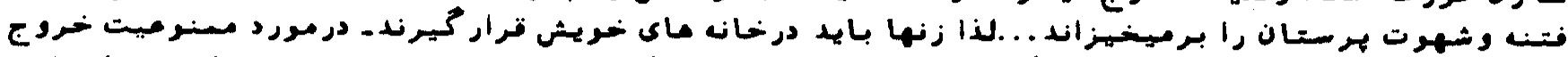

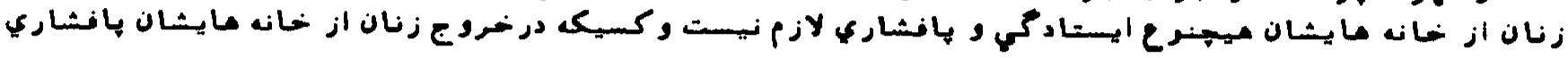

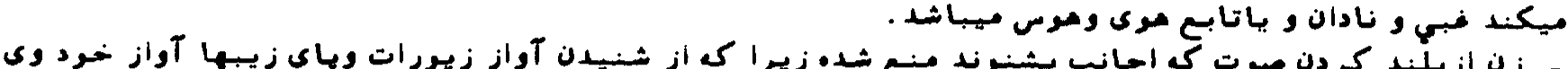

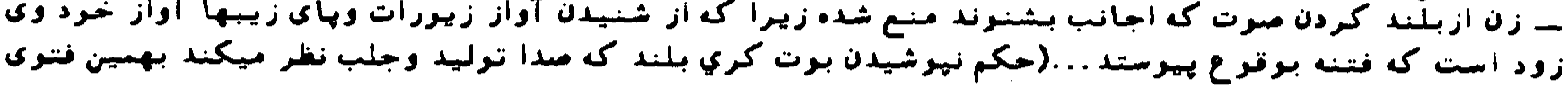

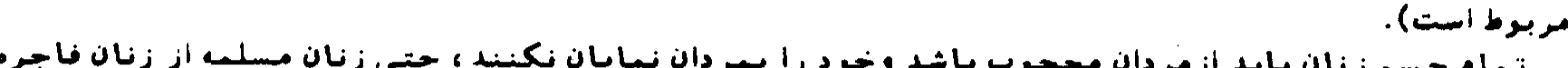

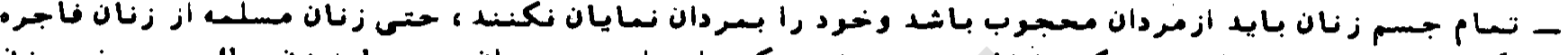

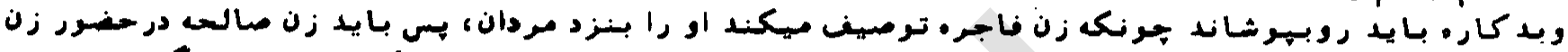

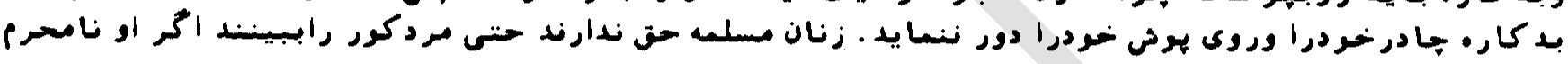

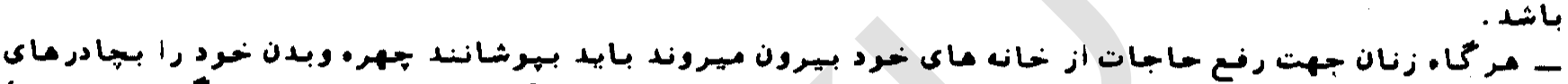

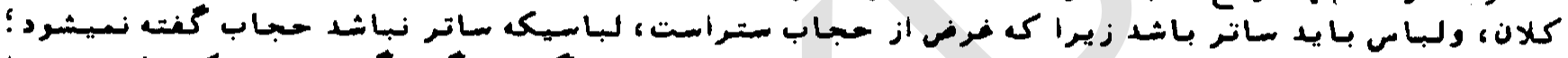

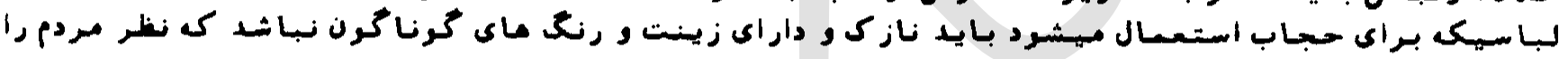

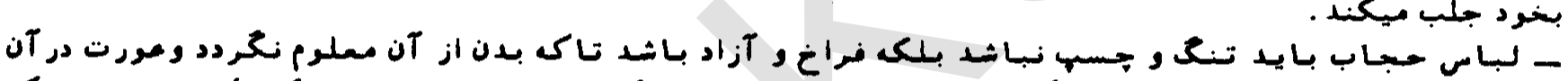

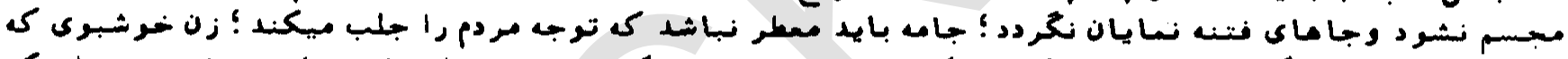

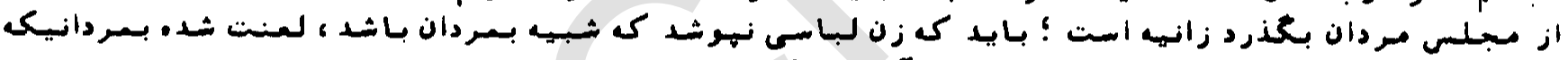

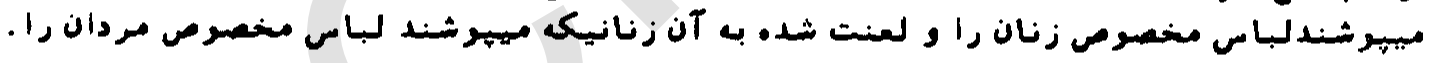

دربار \& تعليسم وتربيت ويا طلب ملم زنان:

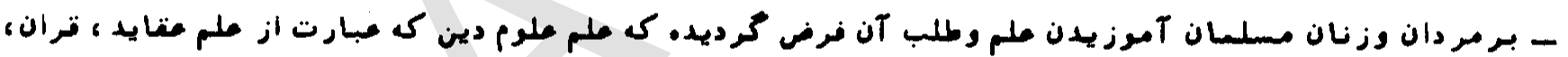

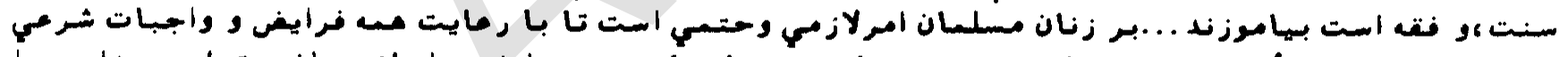

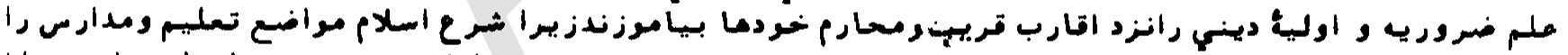

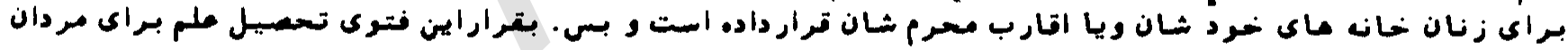

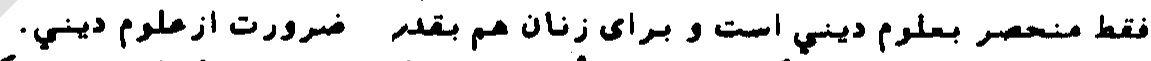

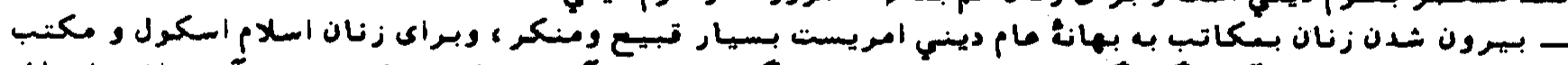

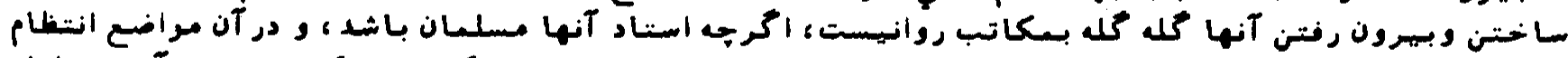

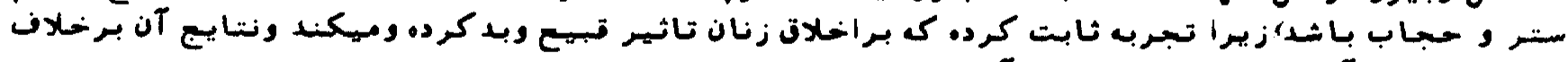

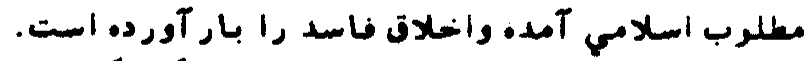

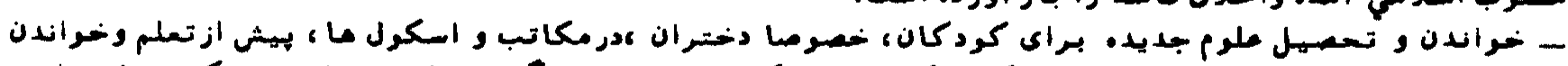

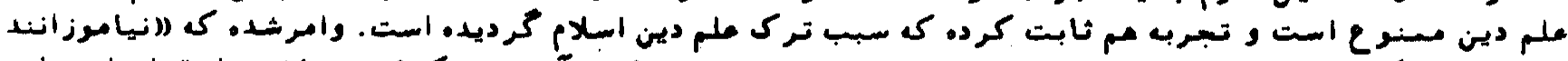

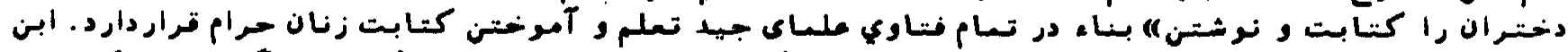

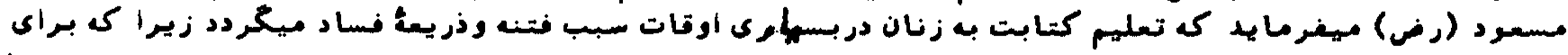

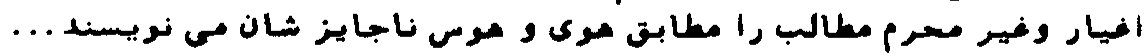

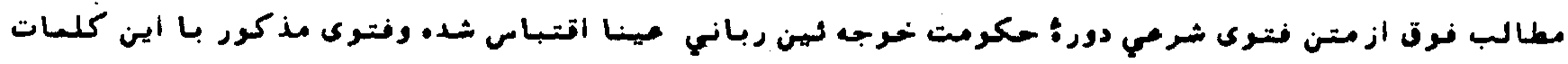

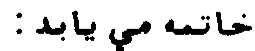

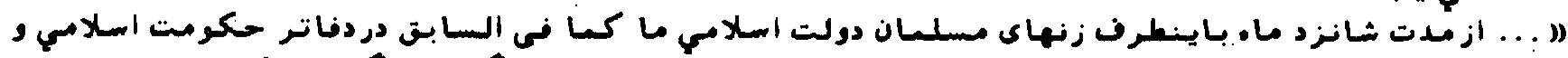

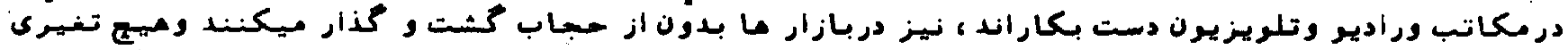

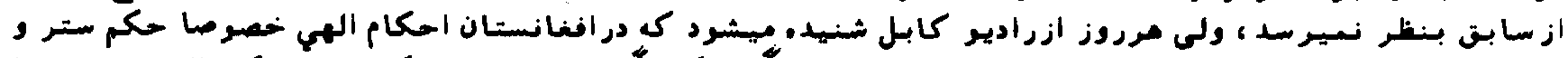

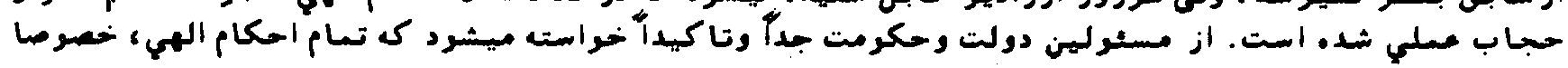




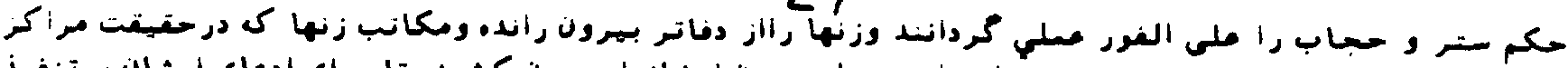

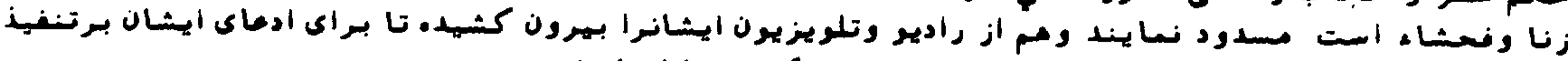

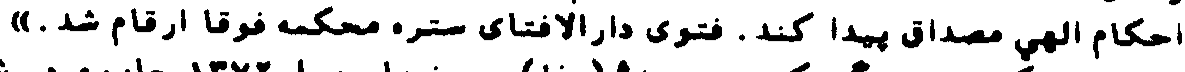

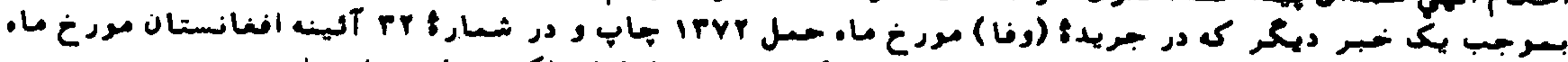

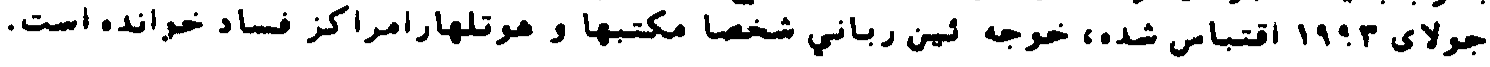

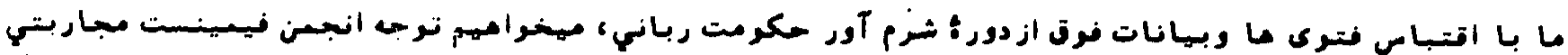

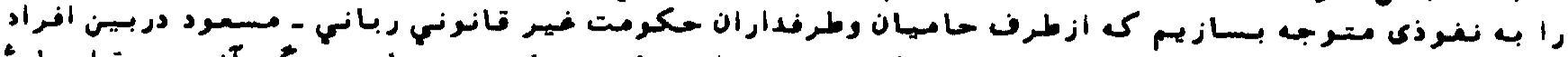

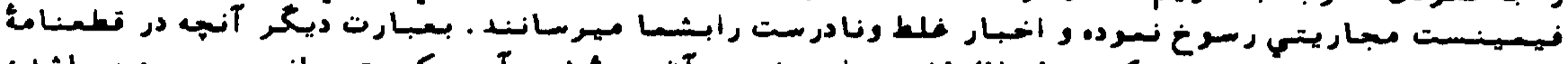

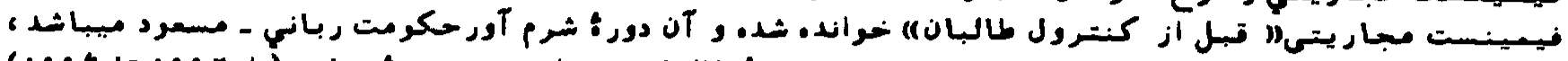

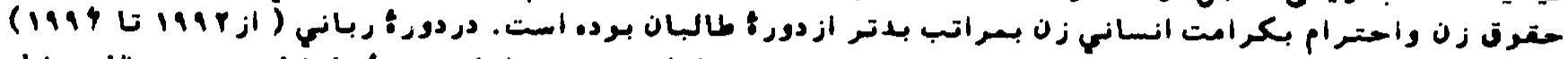

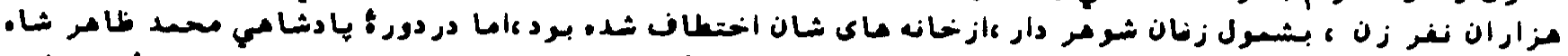

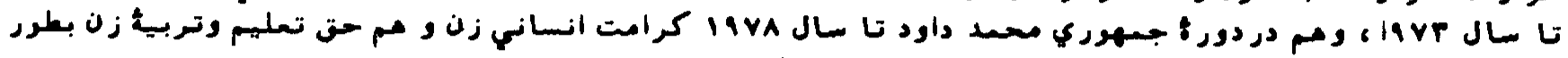

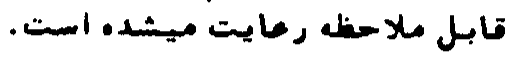

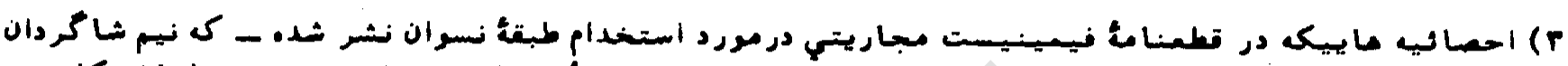

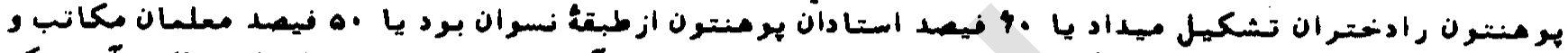

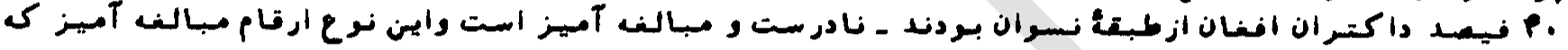

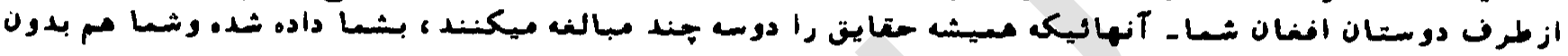

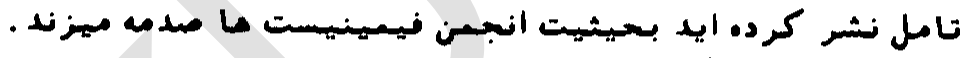

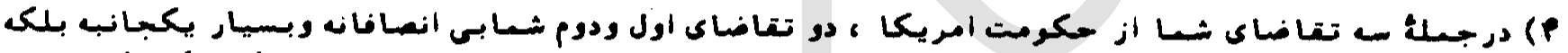

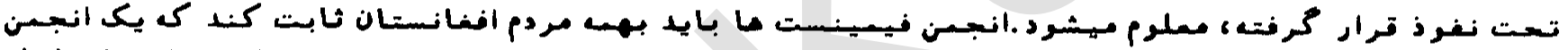

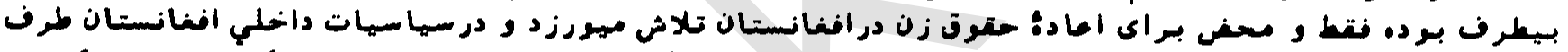

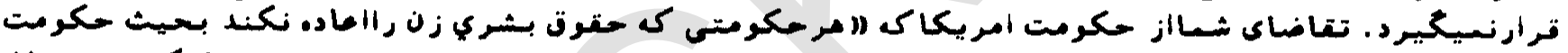

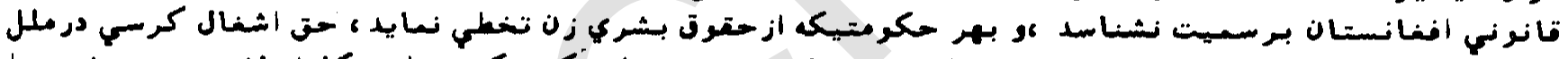

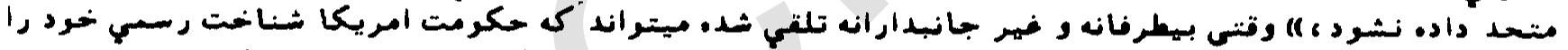

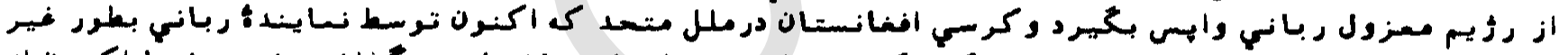

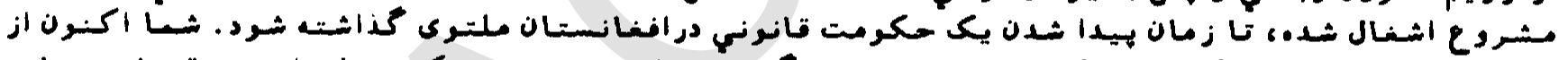

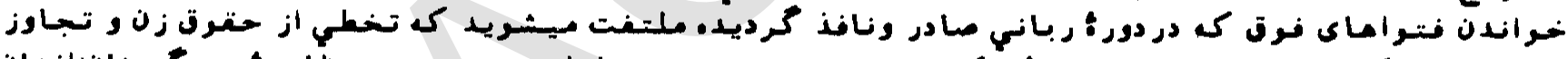

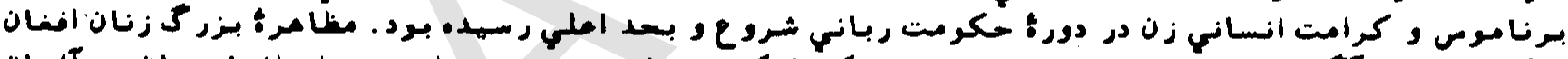

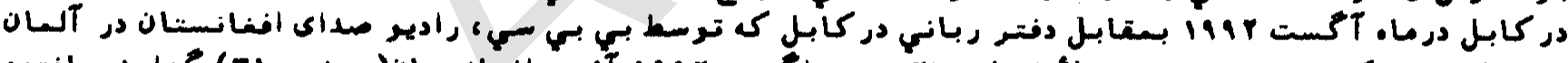

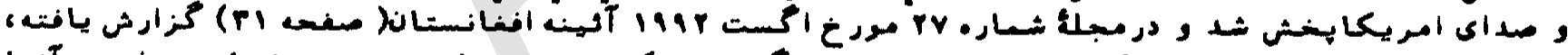

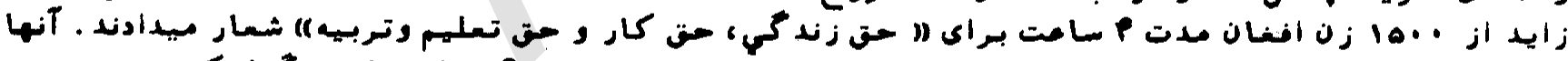

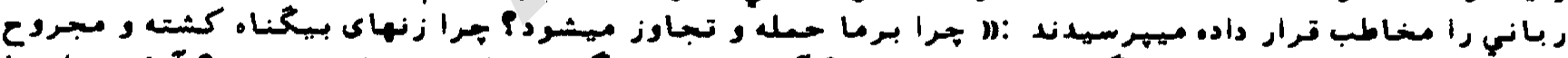

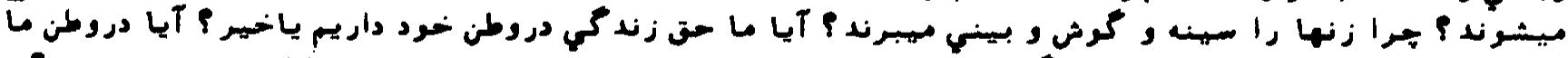

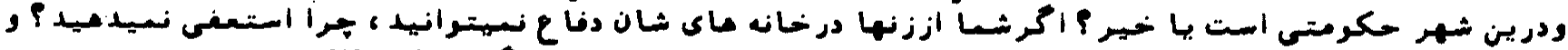

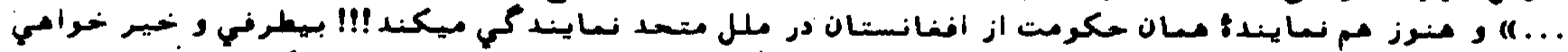

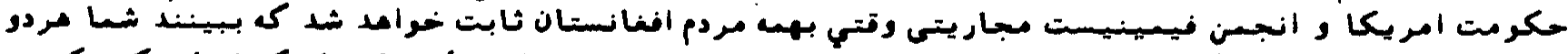

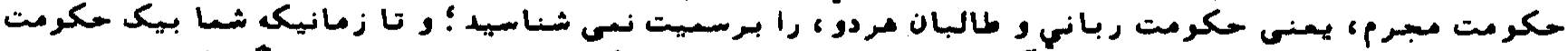

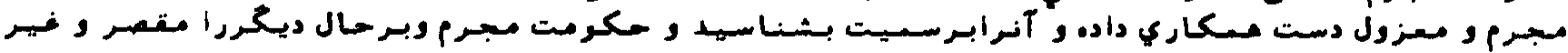

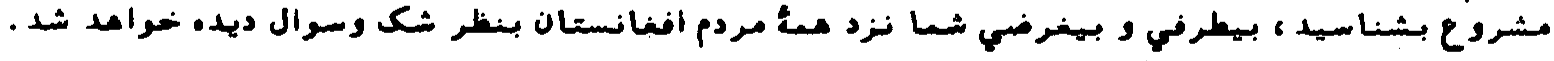

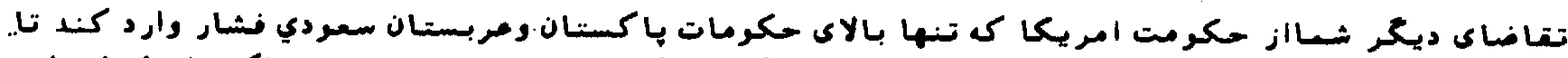

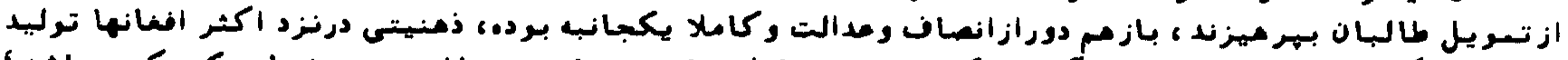

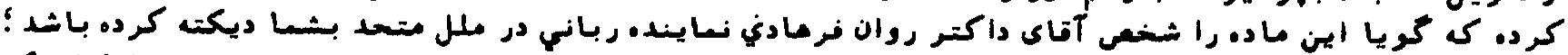

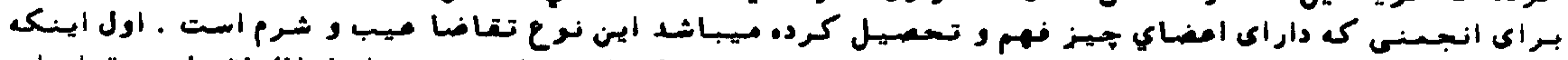

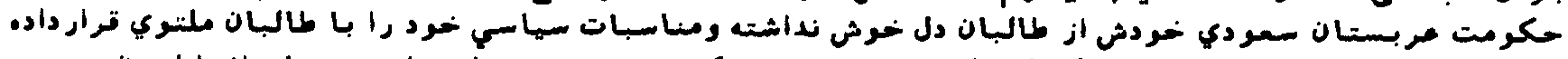

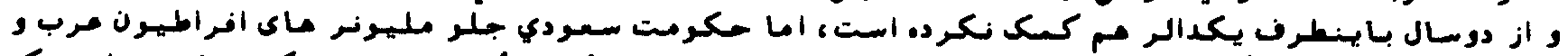

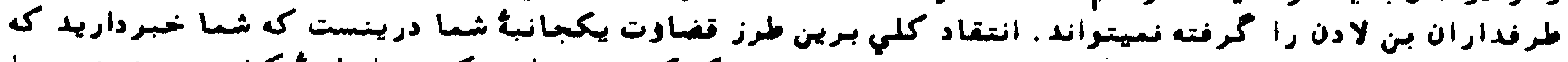

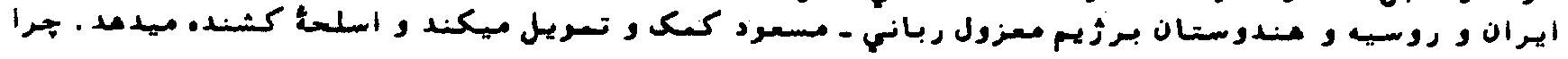


بحيث يك انبسين بـيطرف از مـكرمت امريكا تقاضا نكرديد تا بالاى حكرمات روسيه و مند مم فشار مشابه وأرد

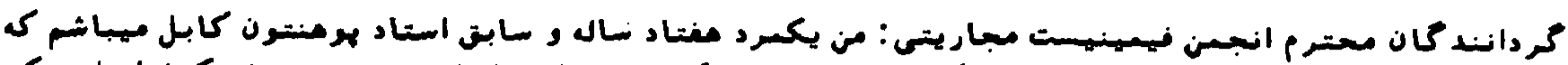

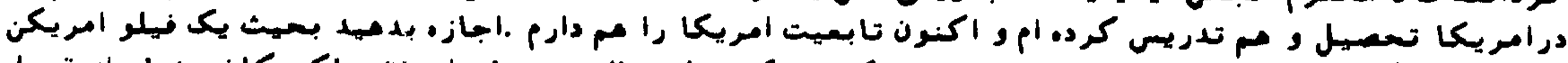

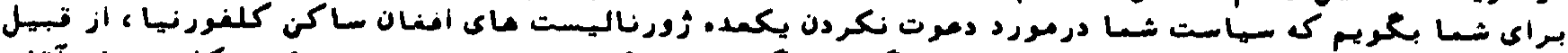

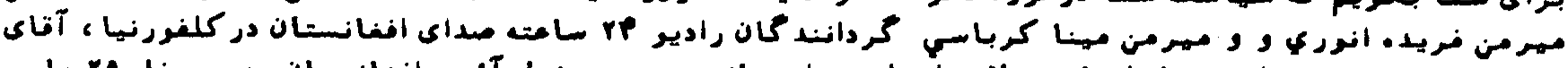

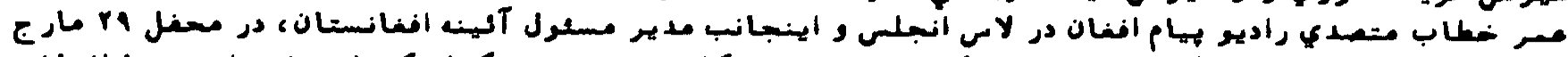

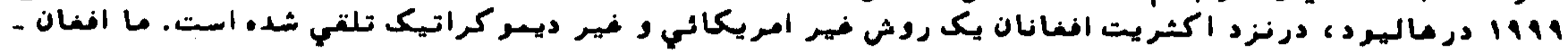

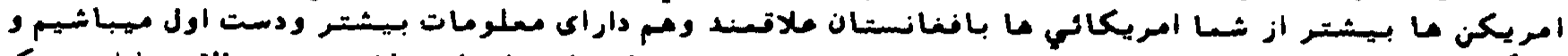

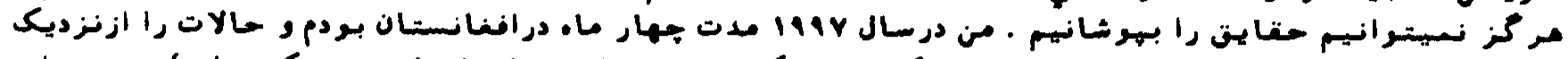

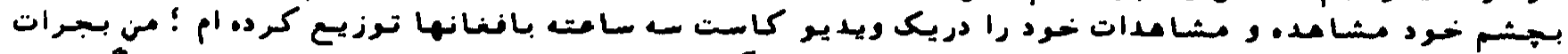

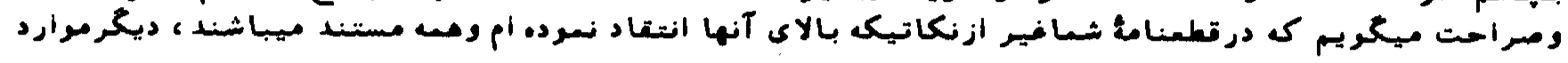

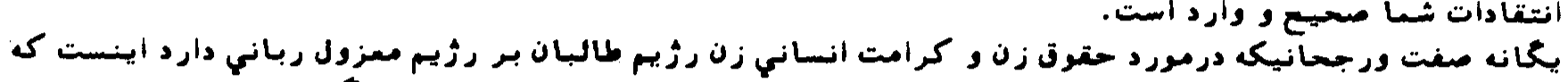

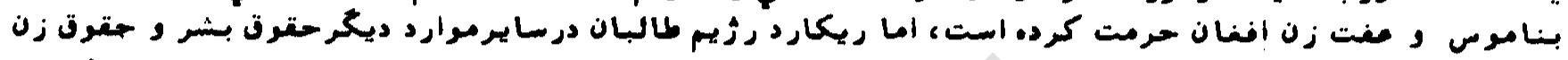

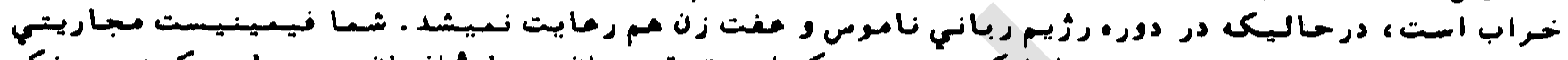

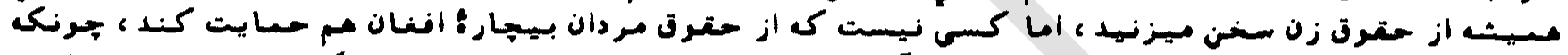

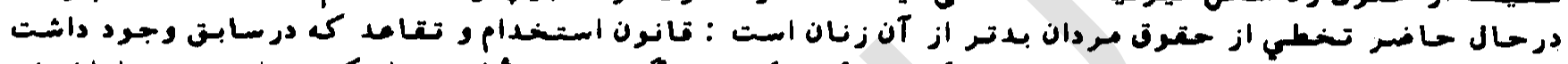

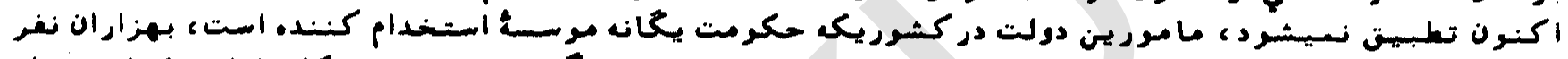

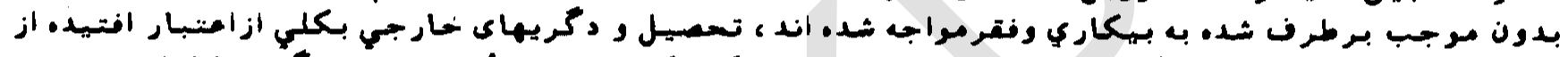

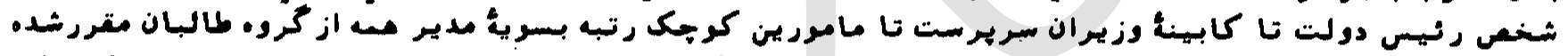

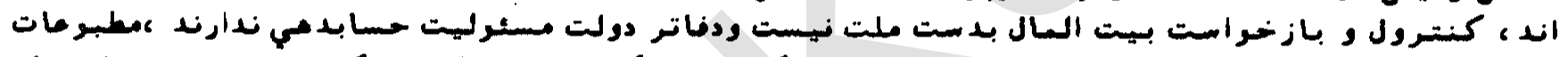

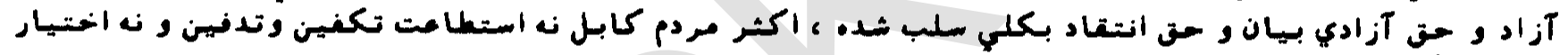

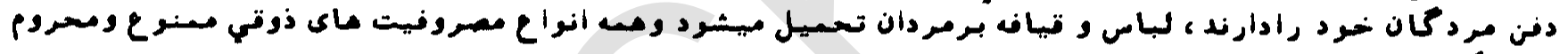

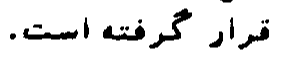

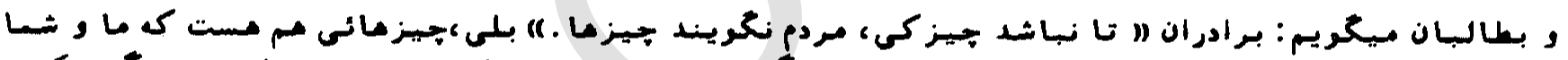

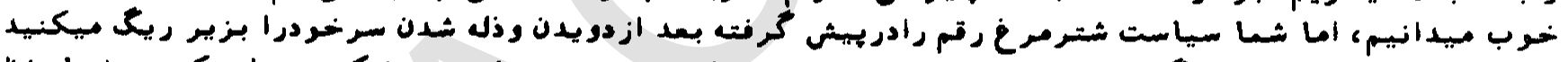

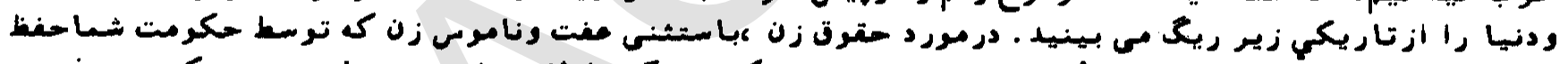

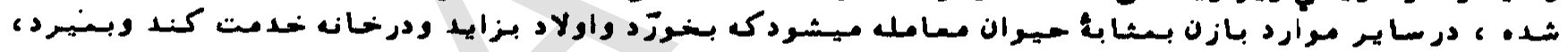

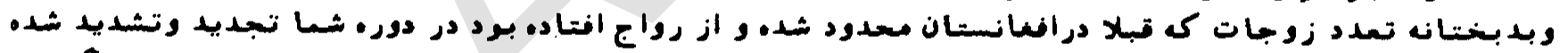

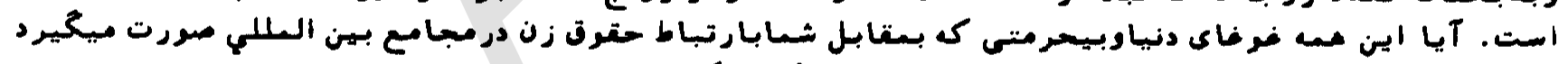

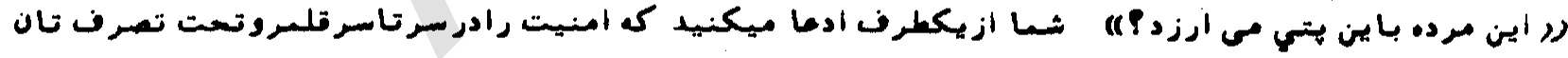

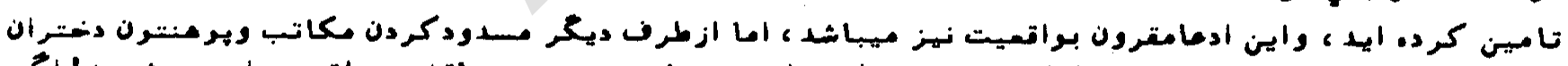

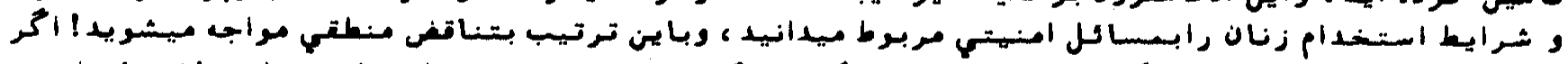

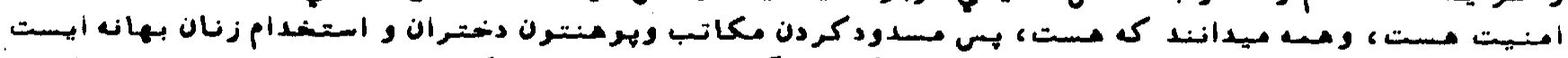

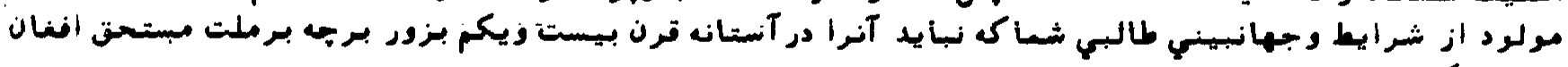

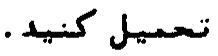

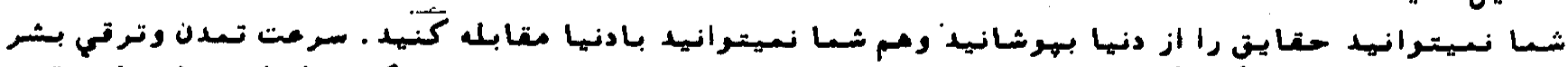

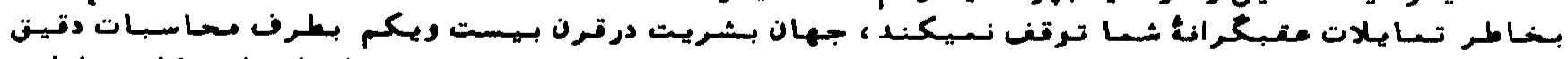

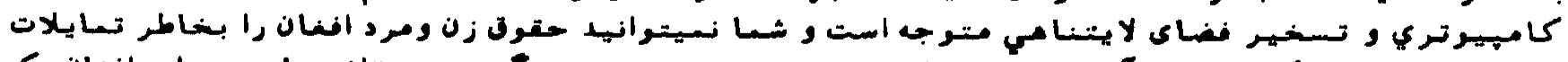

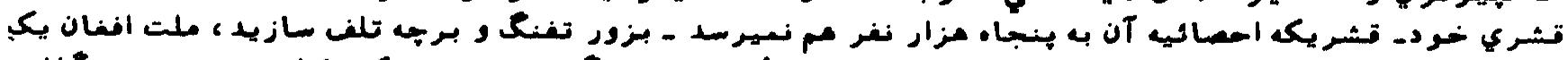

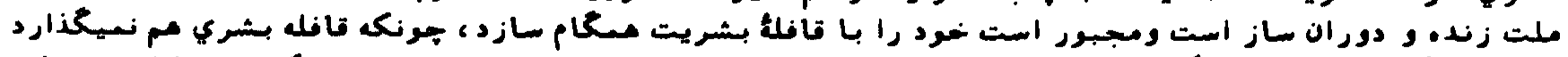

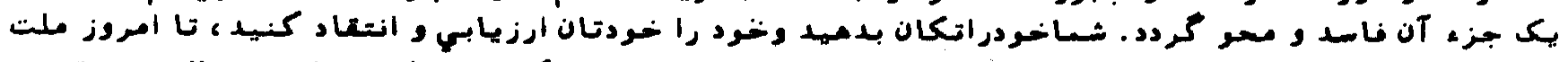

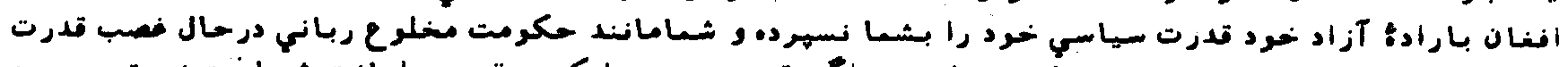

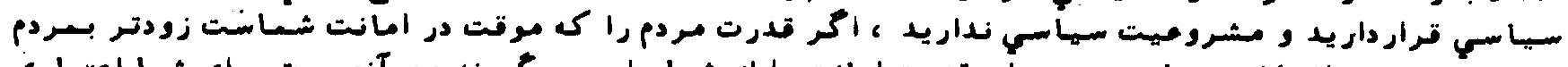

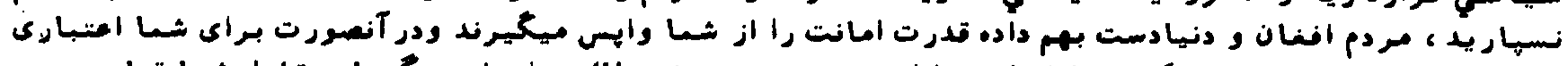

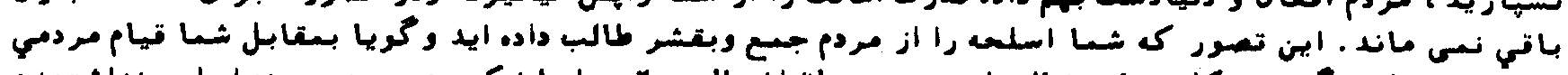

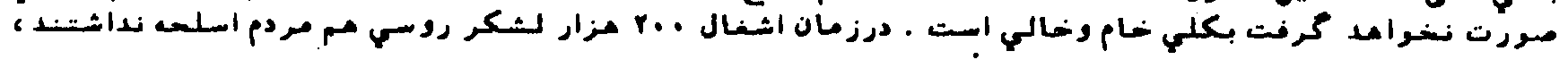




\section{9}

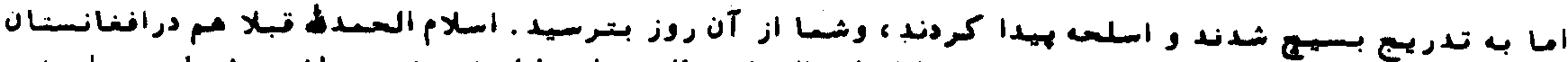

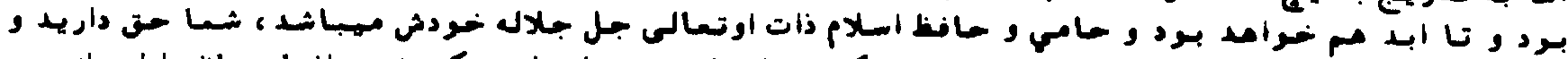

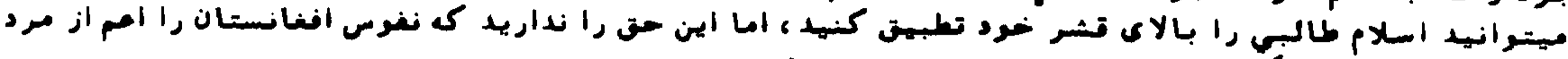

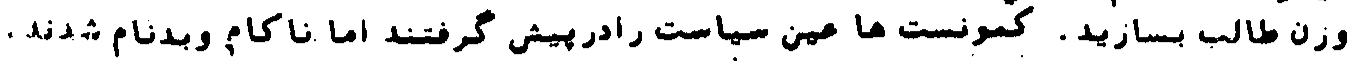

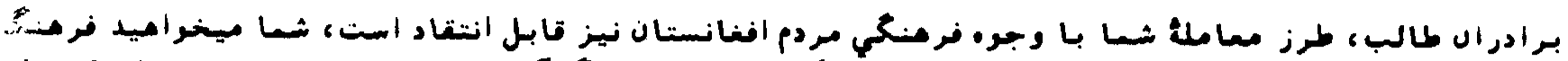

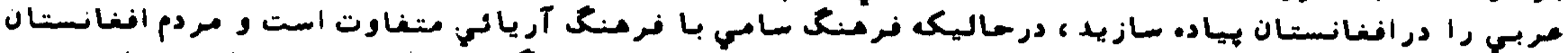

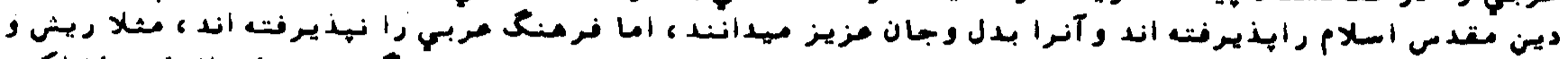

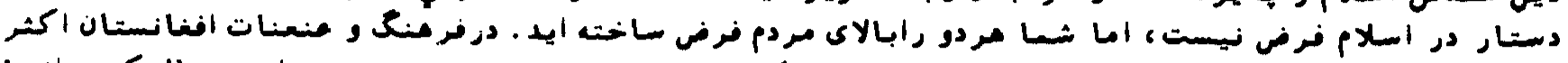

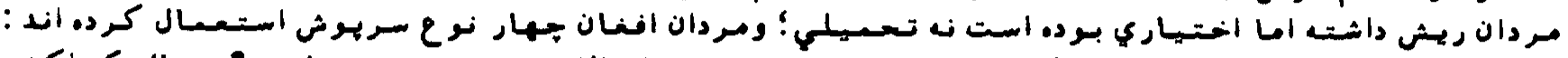

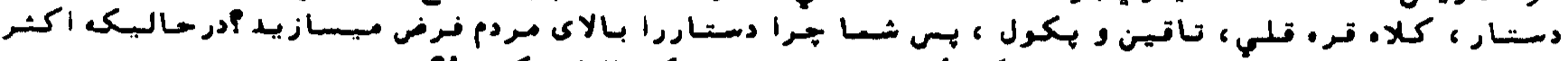

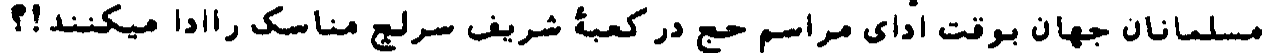

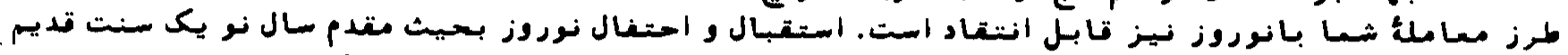

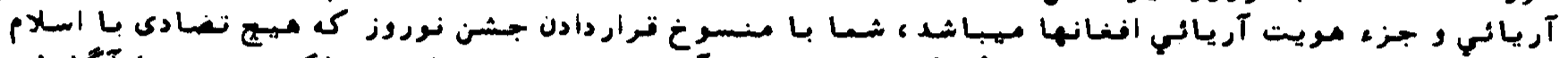

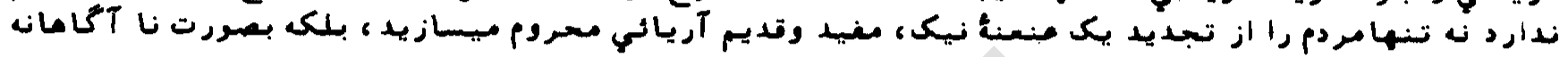

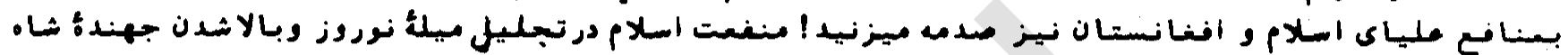

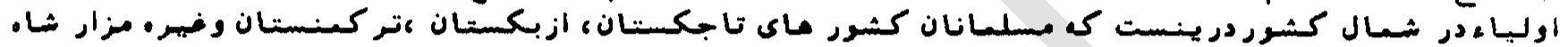

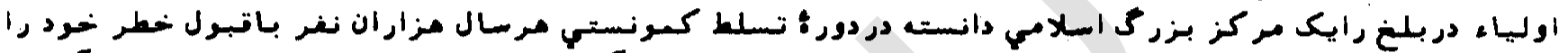

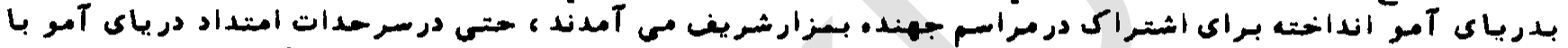

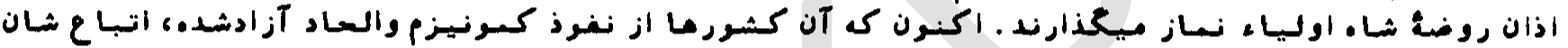

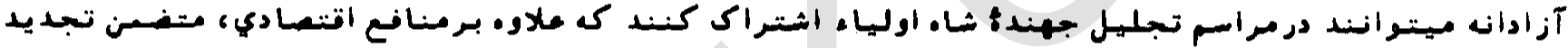

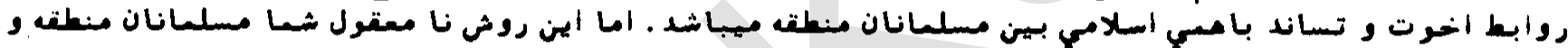

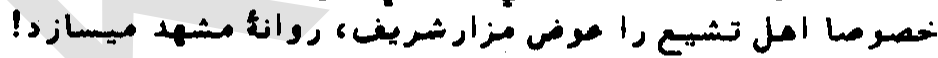

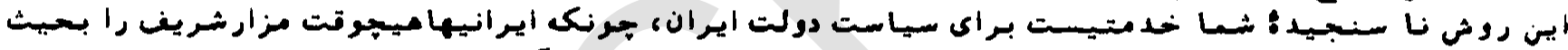

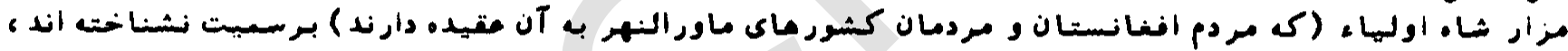

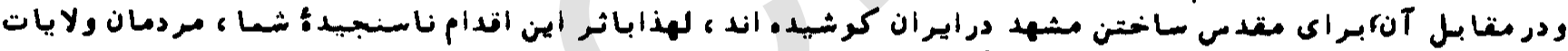

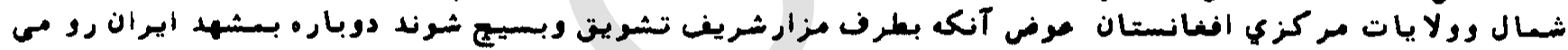

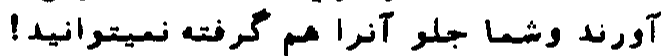

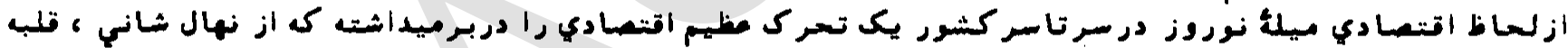

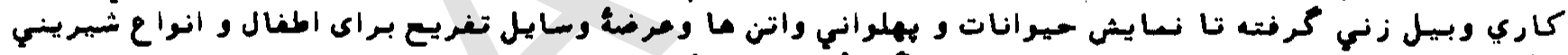

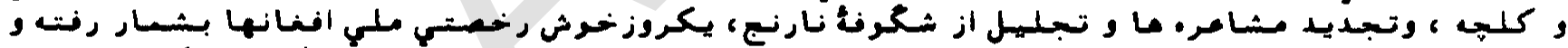

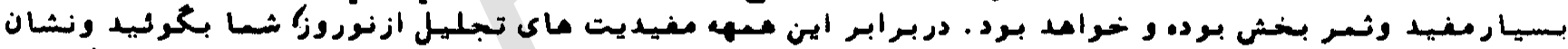

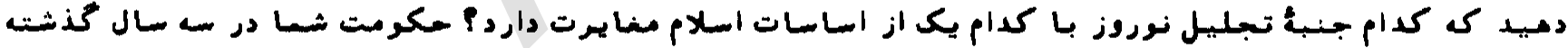

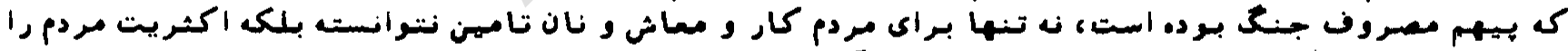

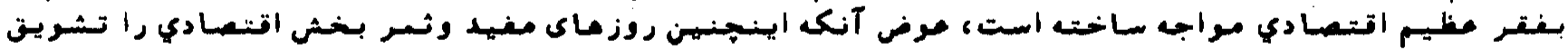

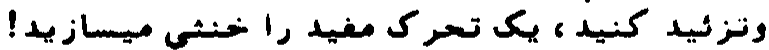

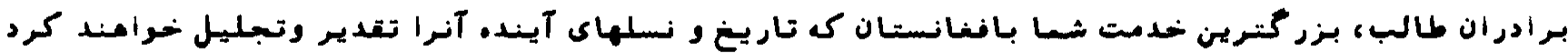

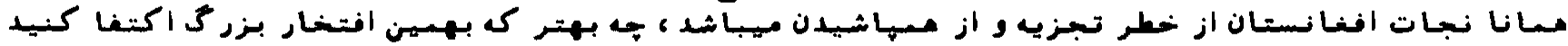

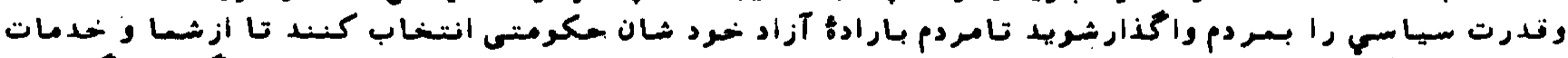

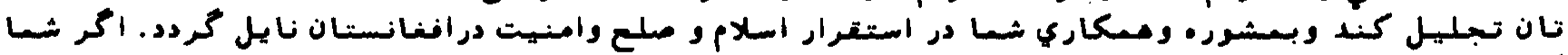

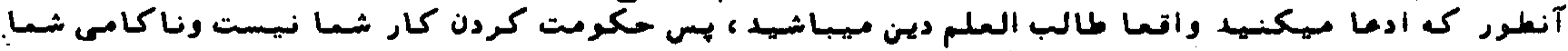

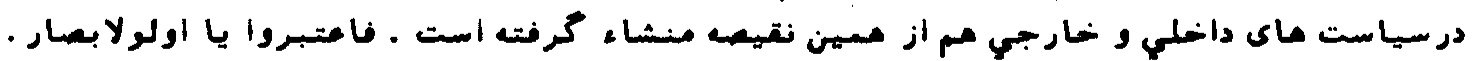

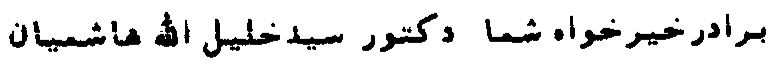

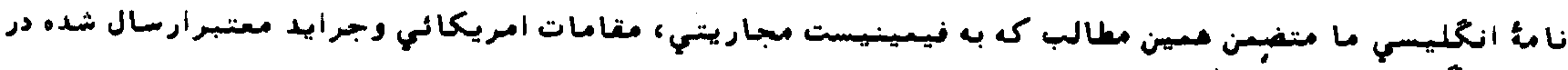

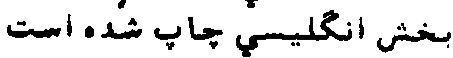



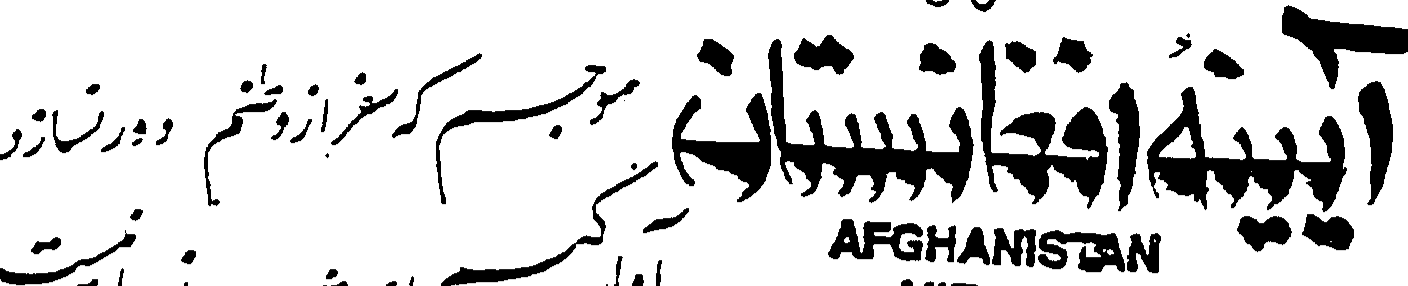

AFGHANISTAN

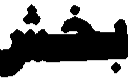

-

.

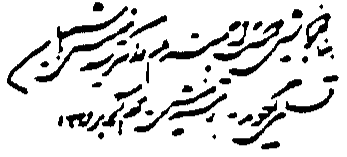

MIRROR

RO. BOX 408

MONTCLAIR, CA 91763

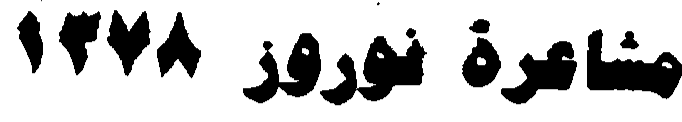

Fis (909) $625-6751$

(909) 626-8314

\section{جداد جاد}

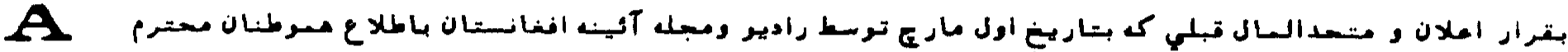

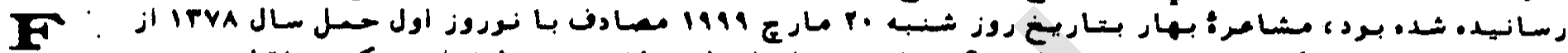

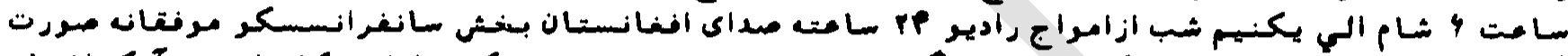

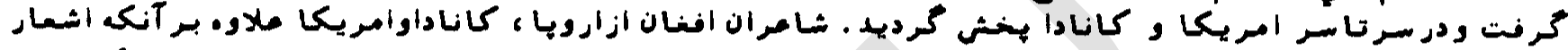

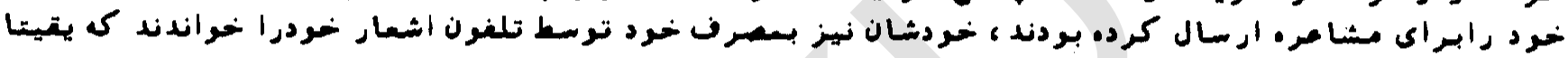

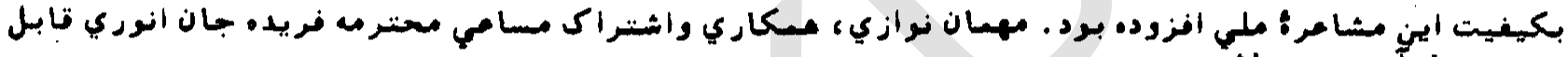

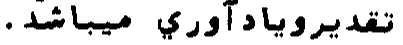

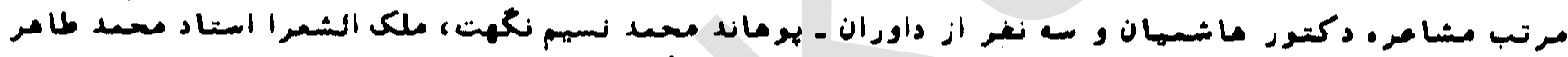

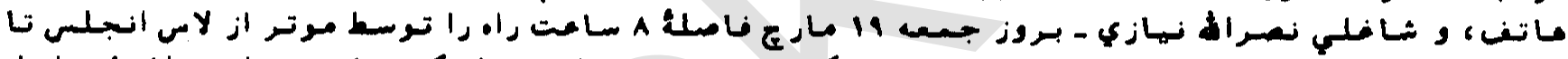

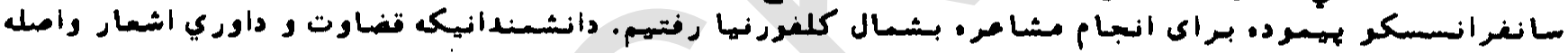

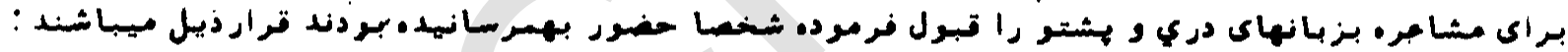

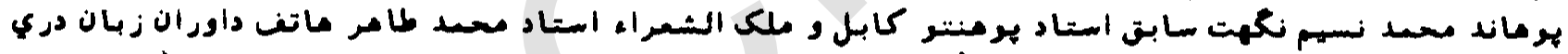

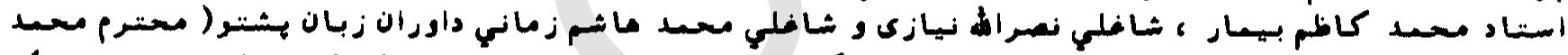

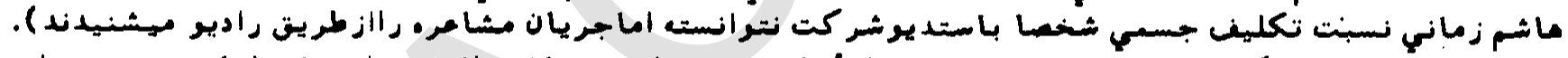

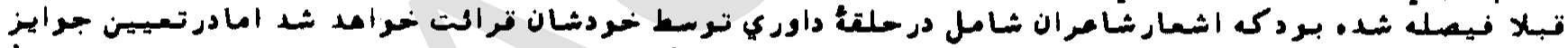

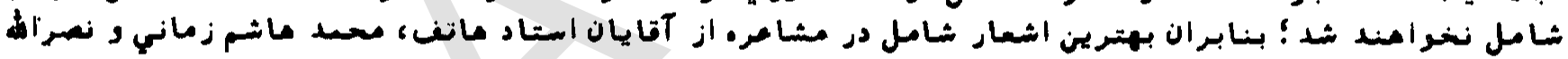

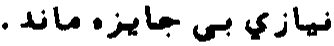

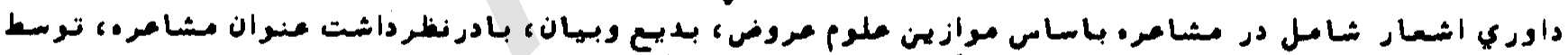

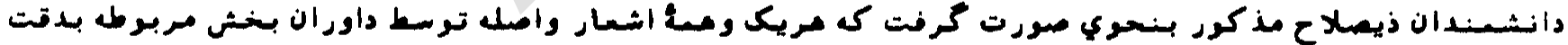

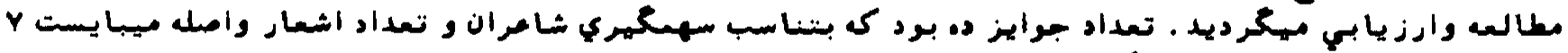

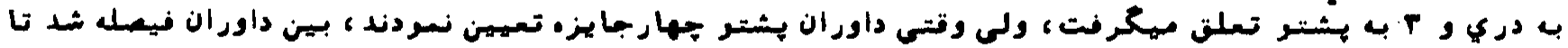

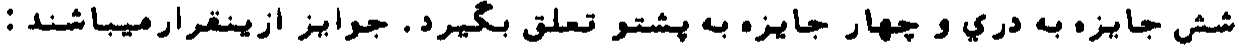

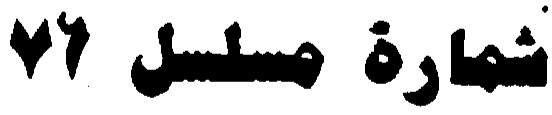

جروايز هشتر

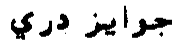

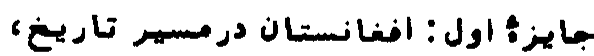

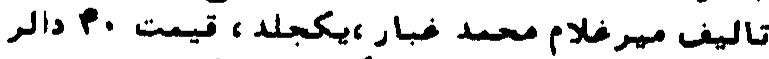

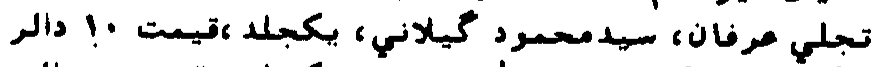

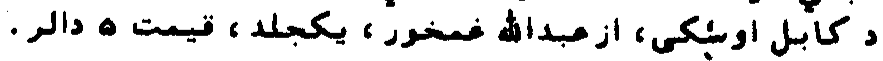

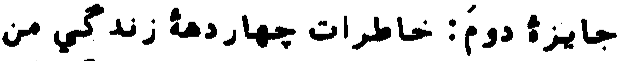

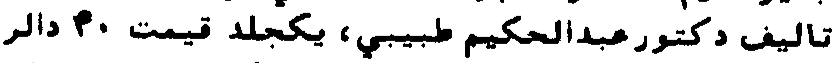

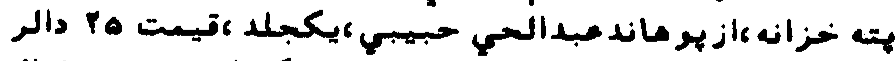

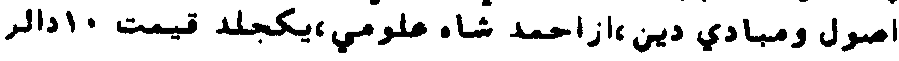

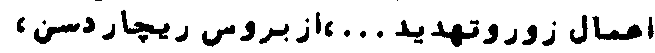

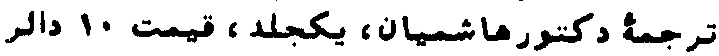

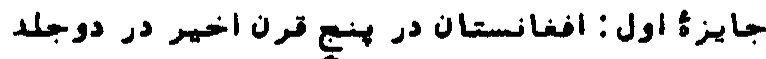

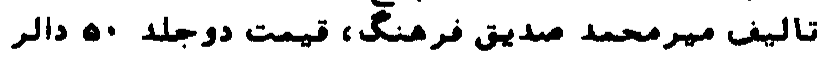

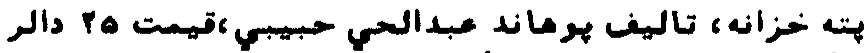

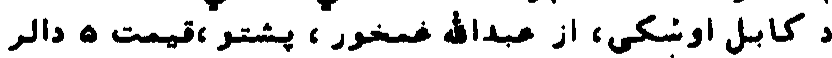

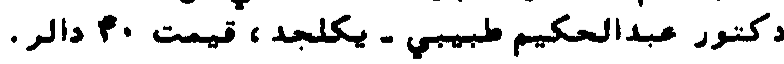

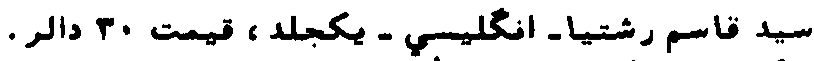

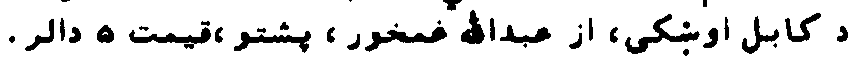




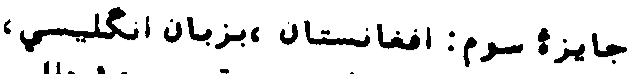

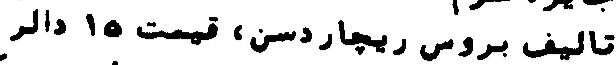

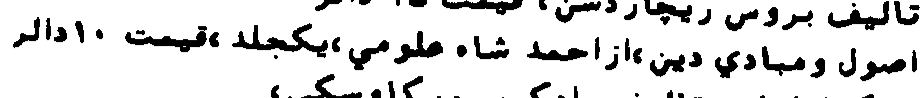

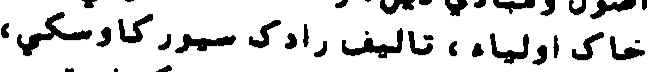

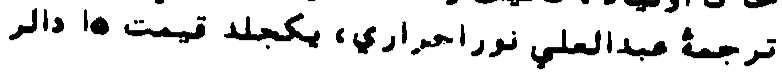

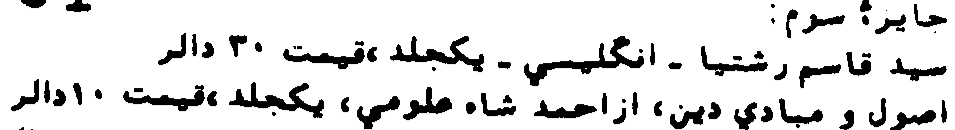

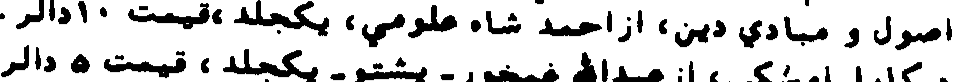

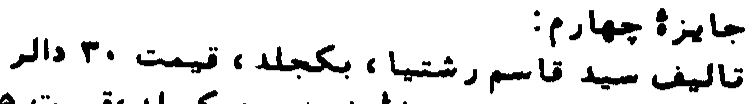

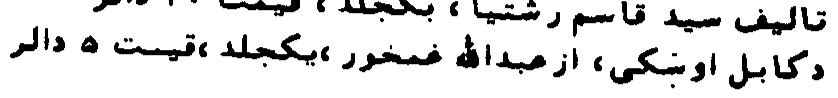

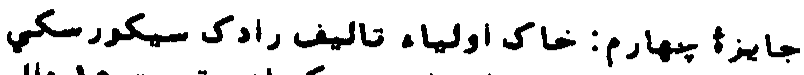

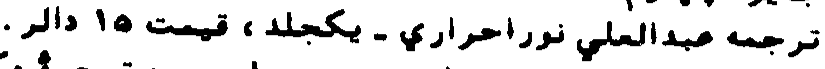

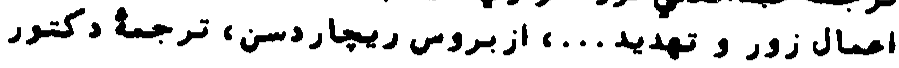

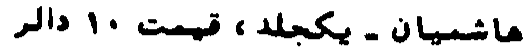

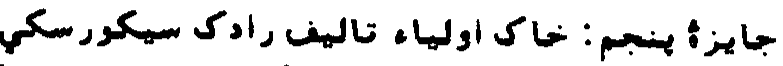

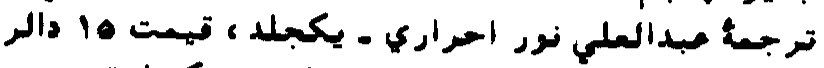

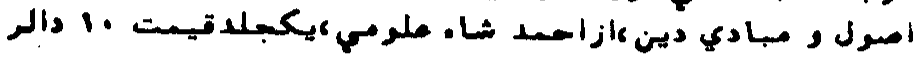

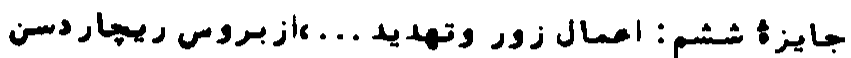

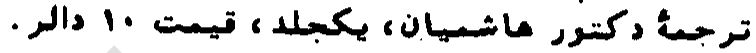

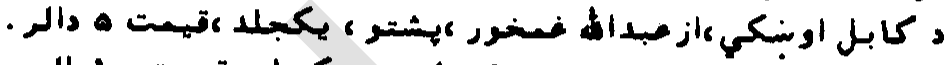

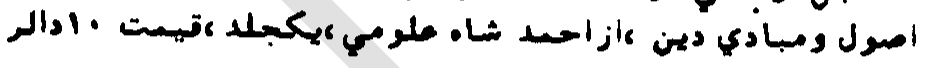

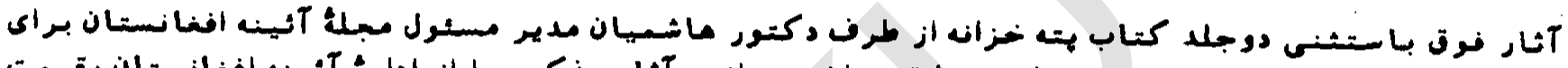

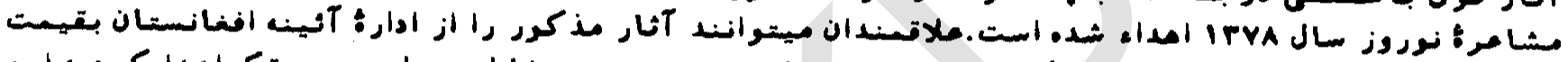

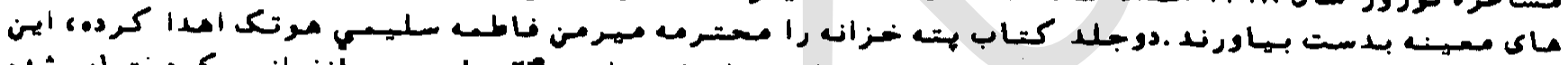

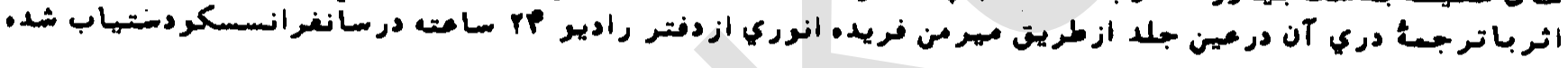
ميتراند.

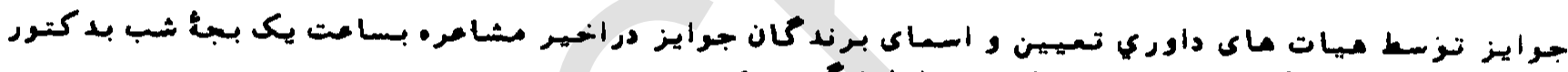

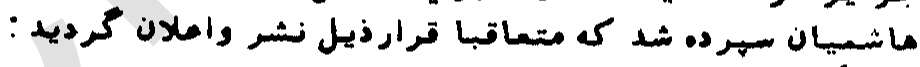

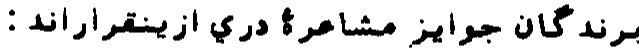

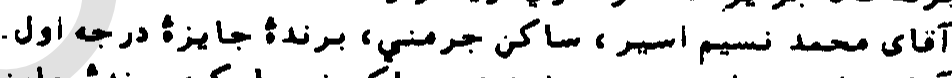

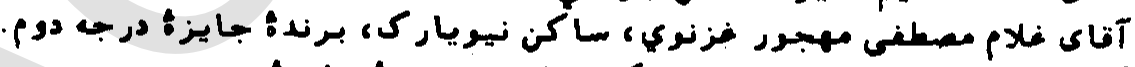

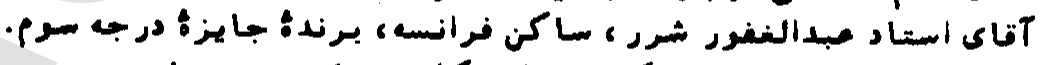

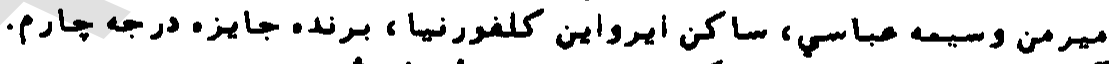

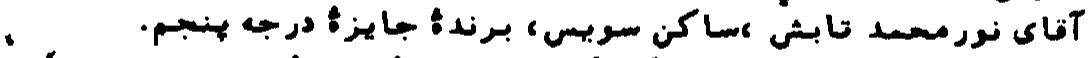

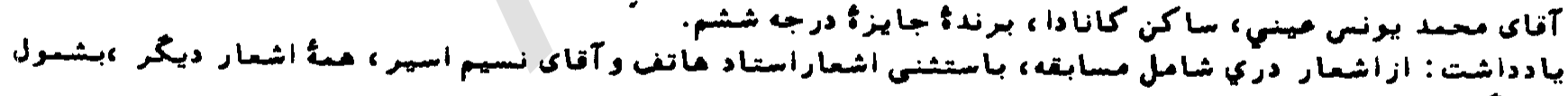

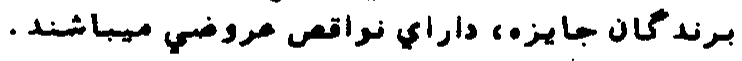

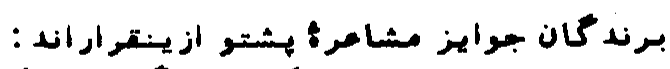

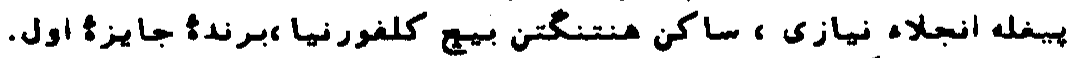

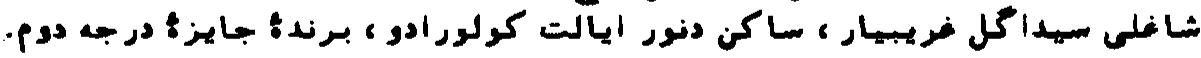

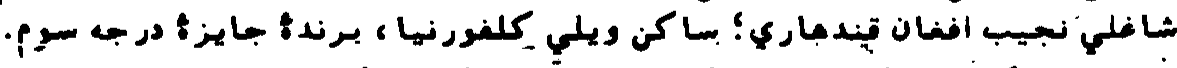

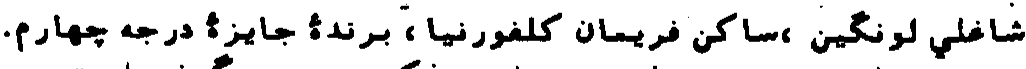

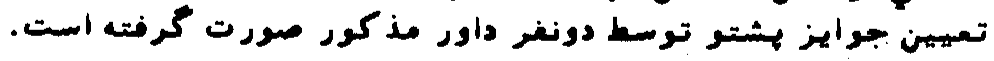

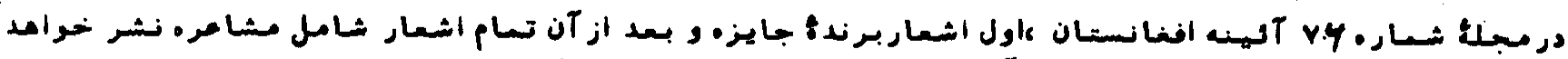

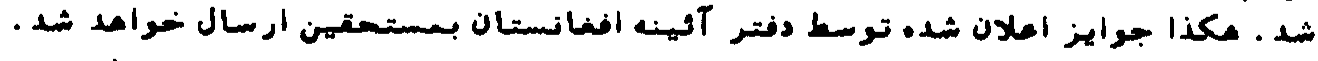

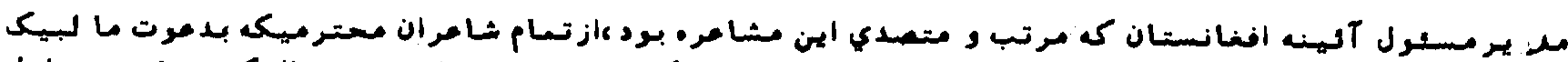

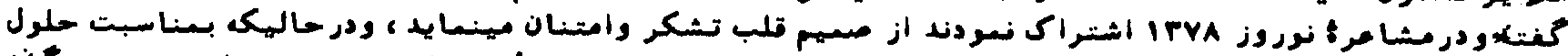

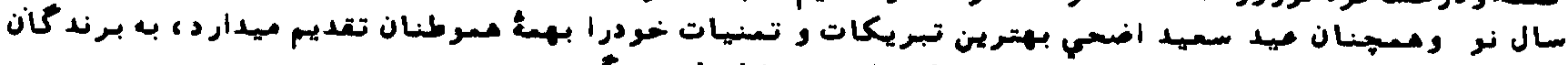

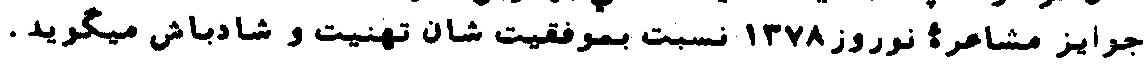

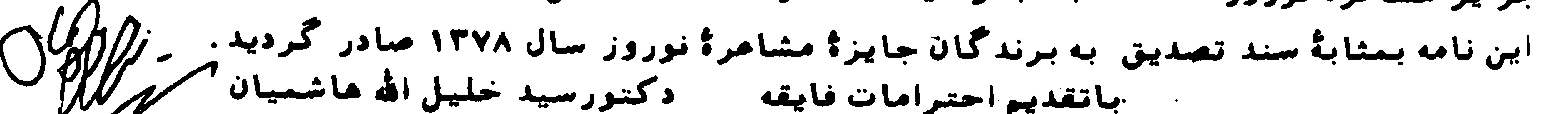




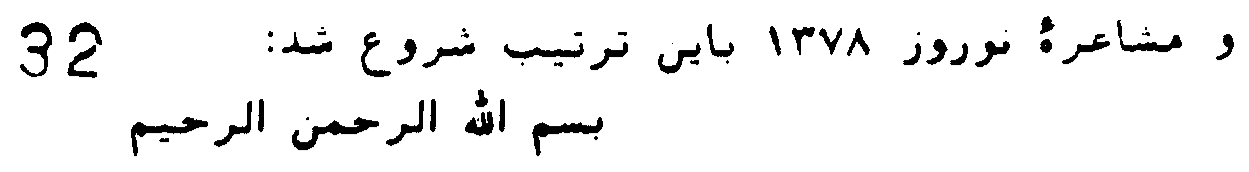

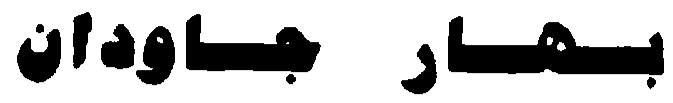

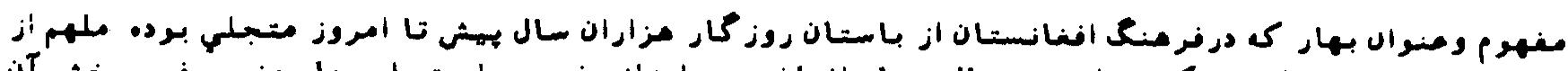

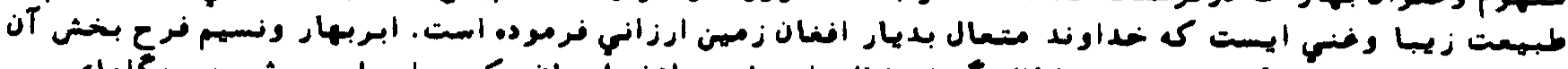

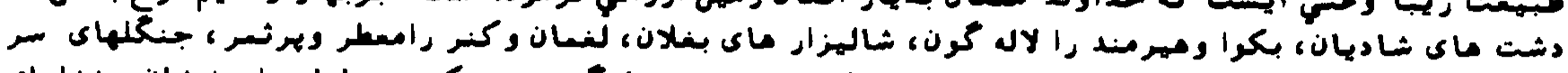

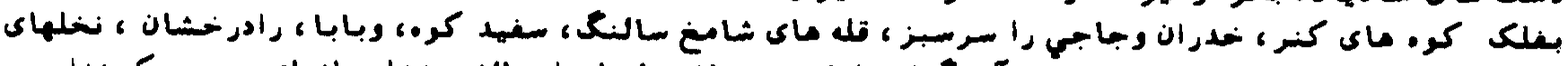

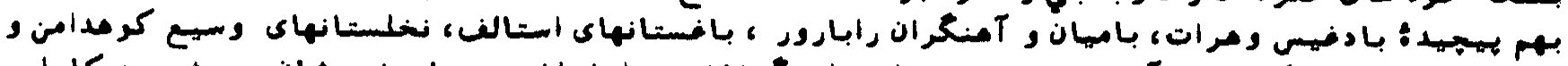

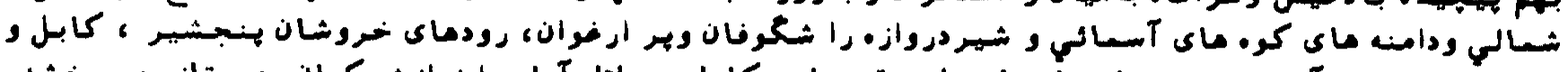

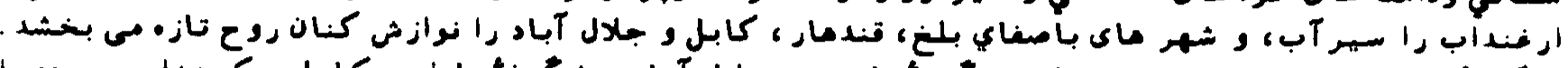

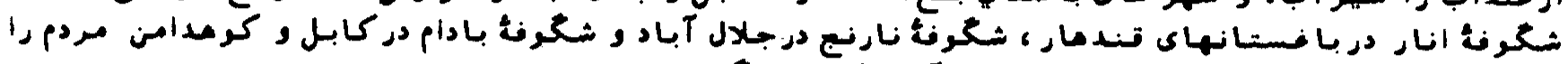

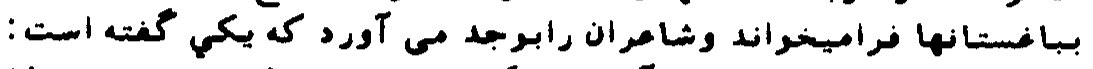

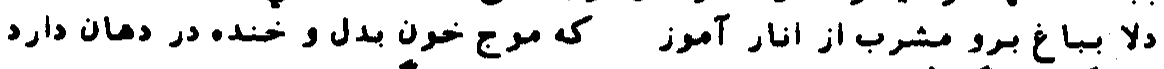

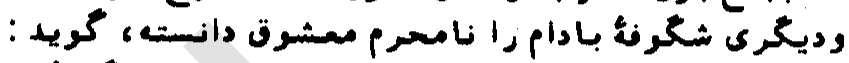

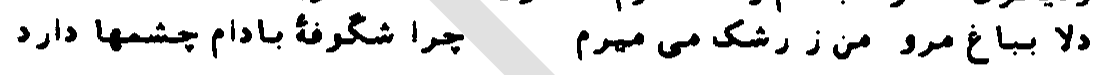

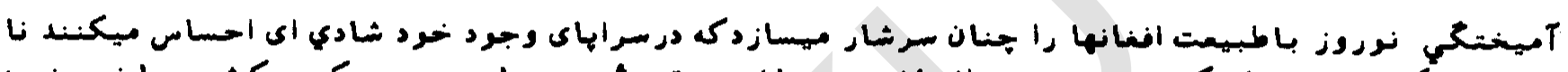

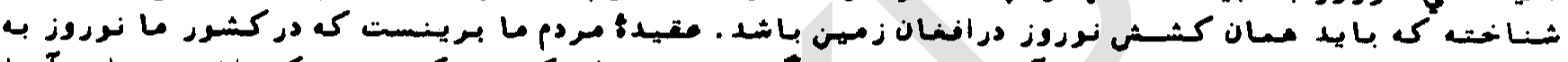

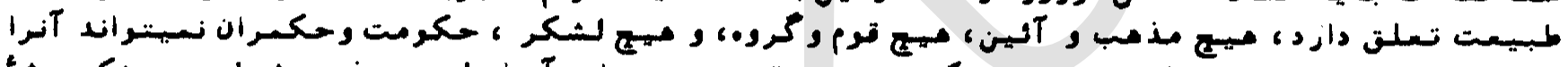

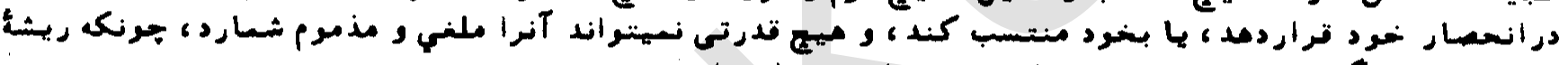

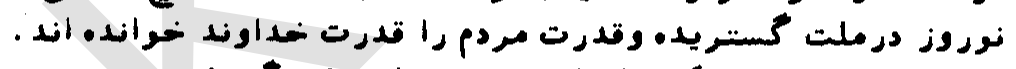

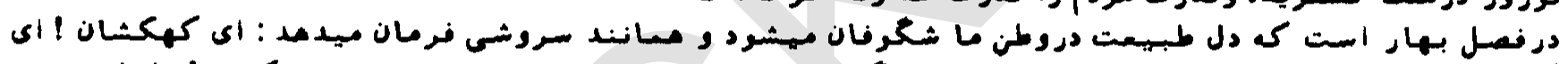

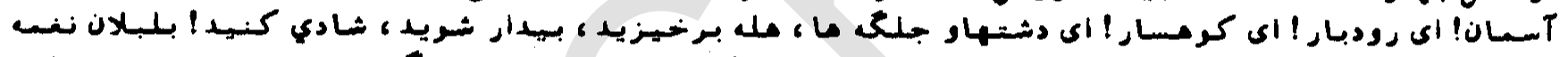

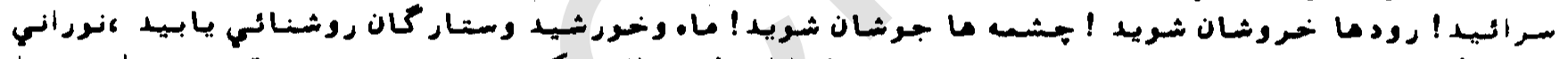

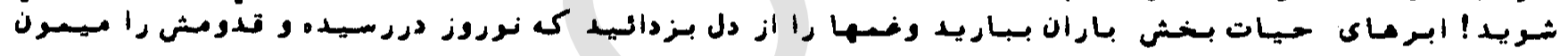
ماريد

\section{Jojgh Land ag}

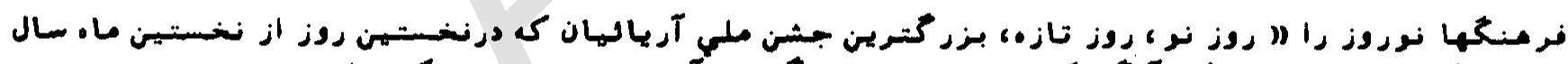

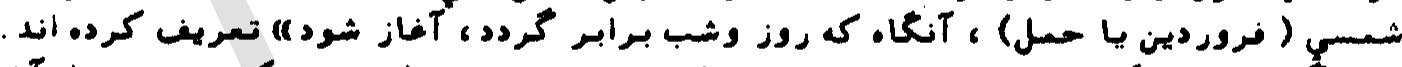

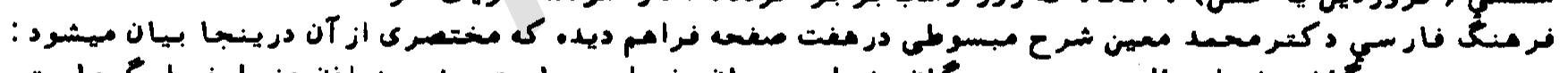

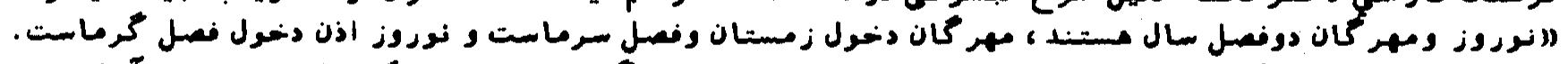

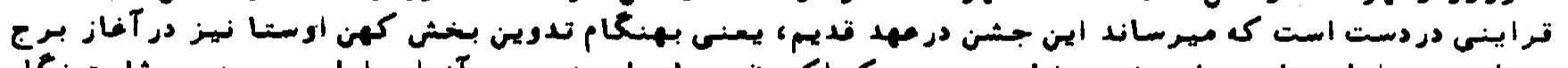

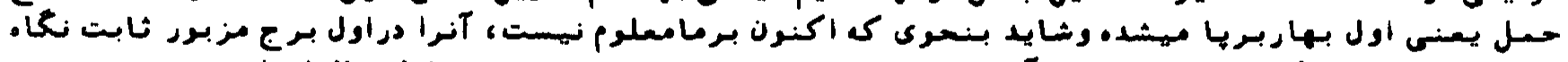

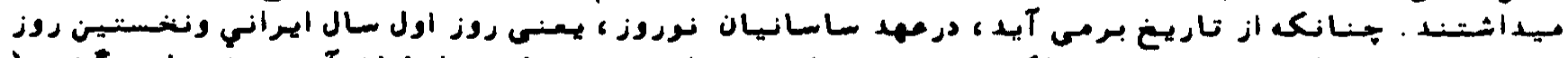

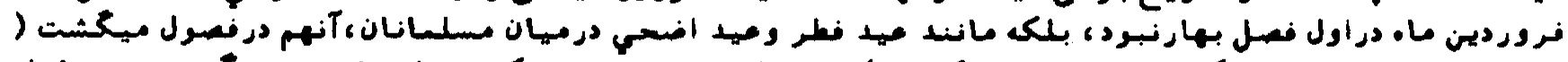

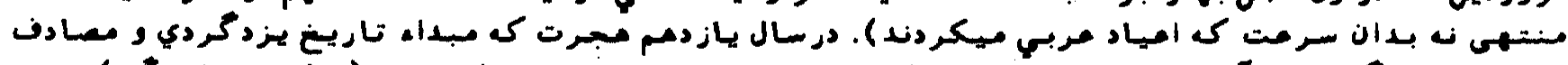

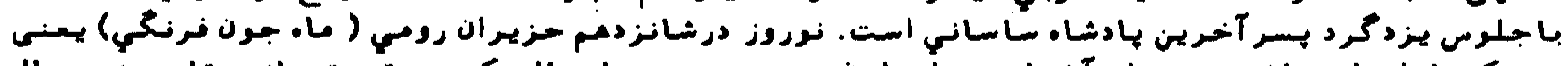

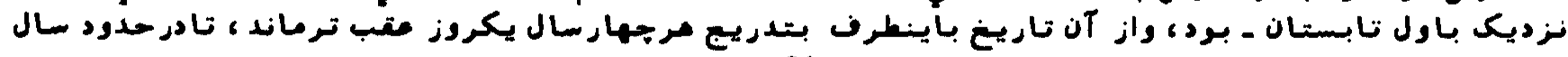

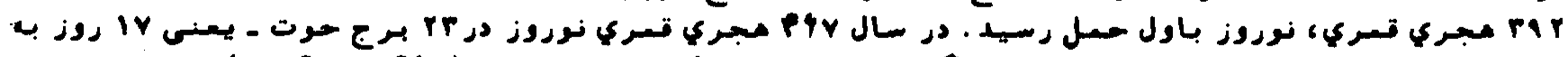

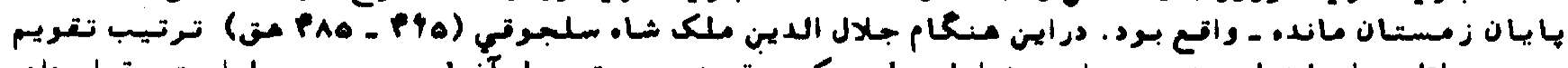

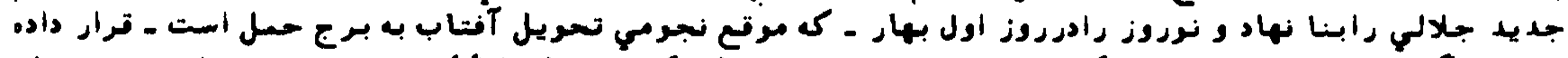

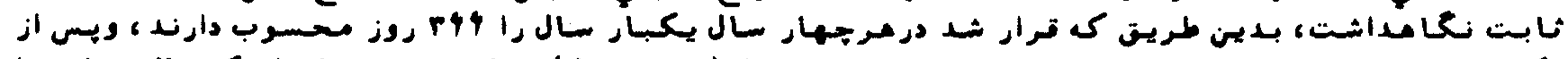

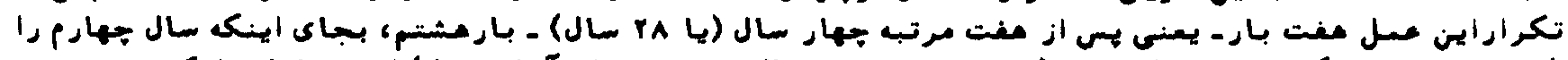

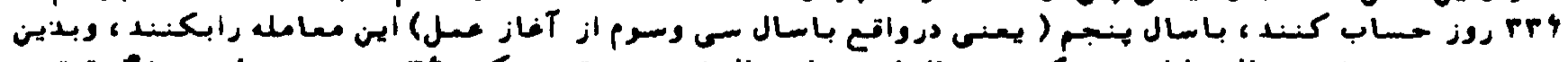

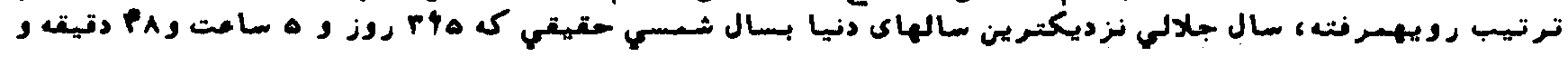

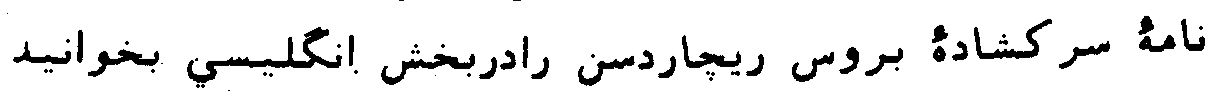




\section{3}

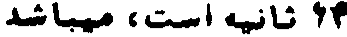

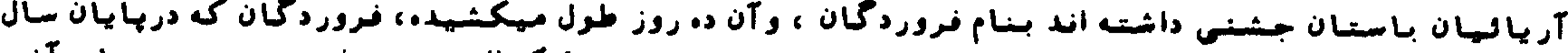

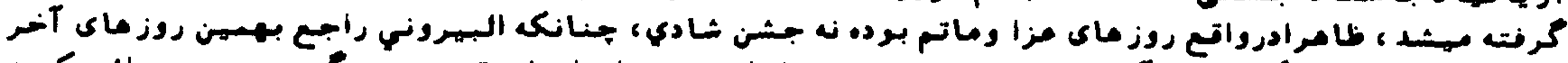

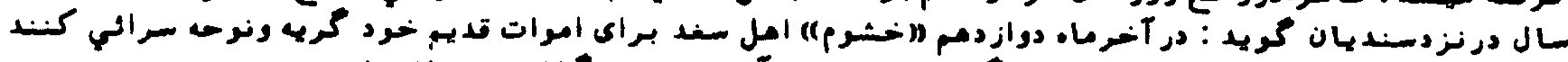

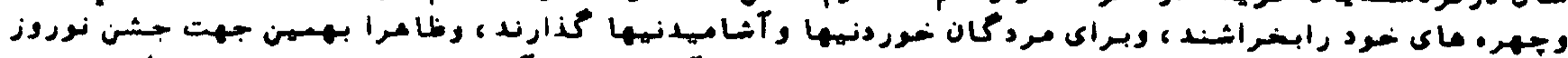

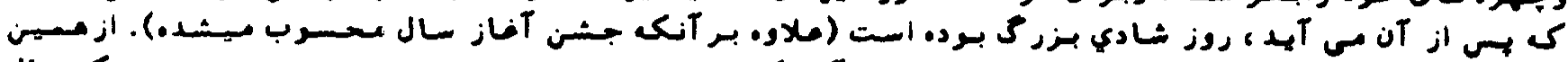

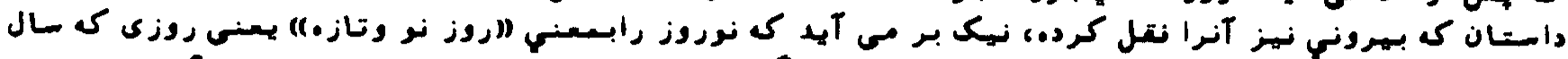

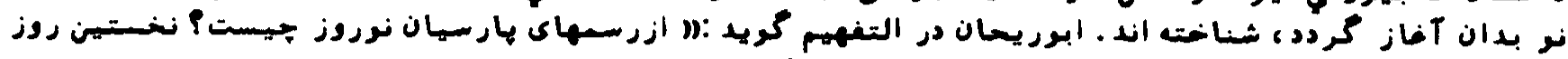

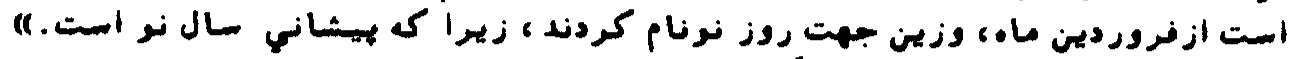

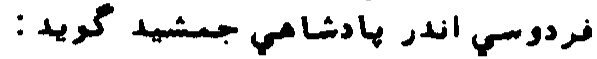
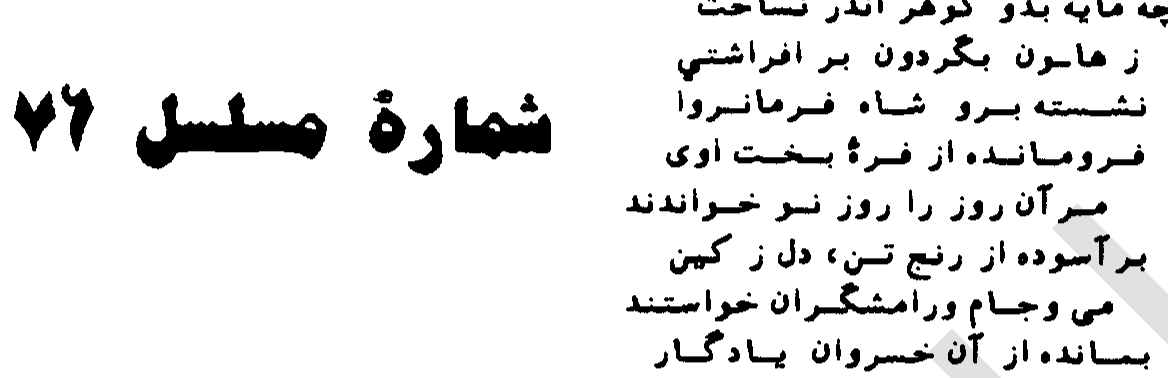

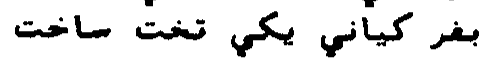

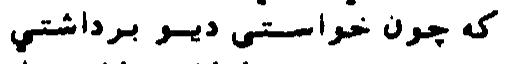

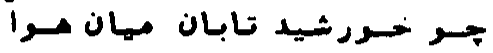

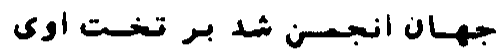

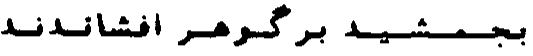

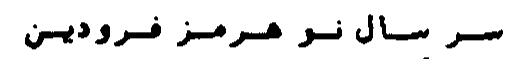

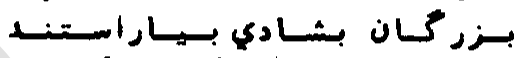

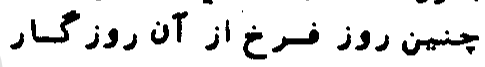

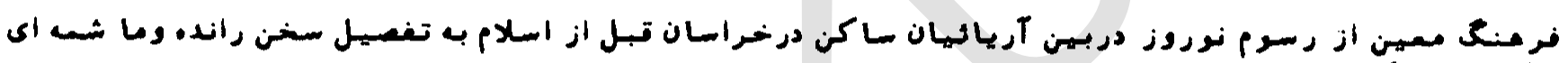

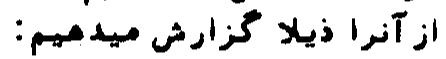

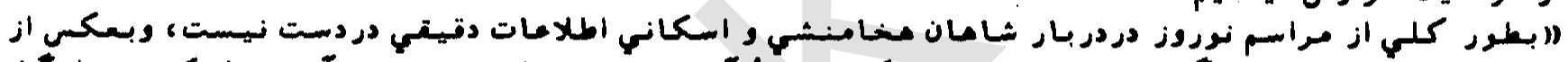

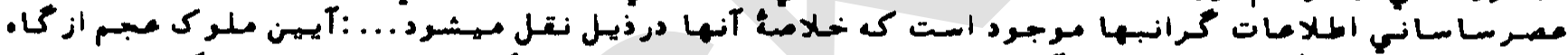

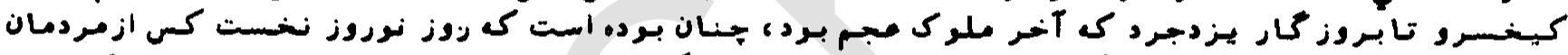

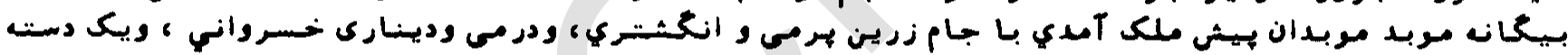

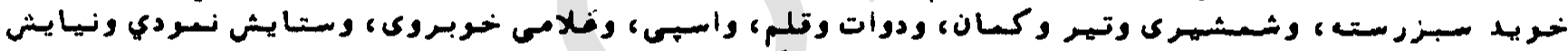

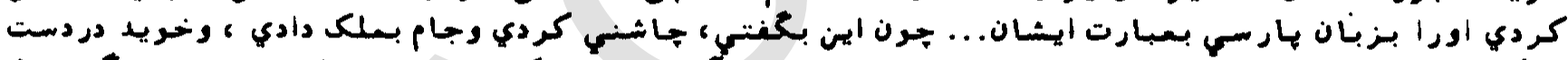

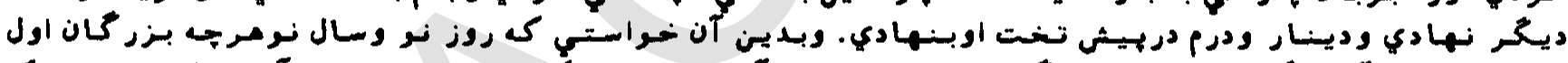

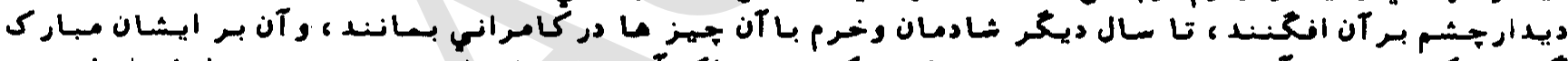

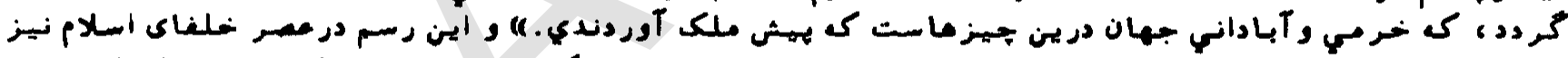

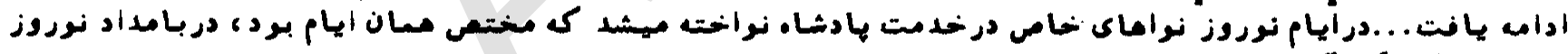

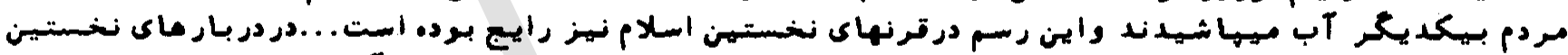

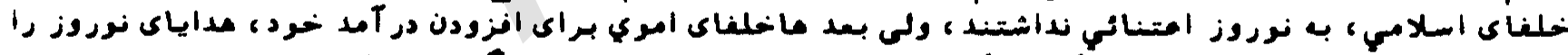

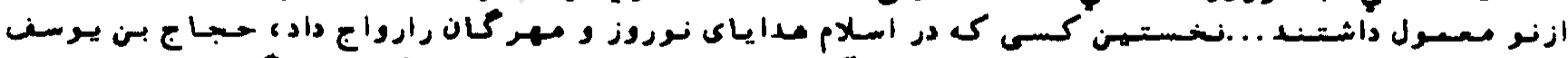

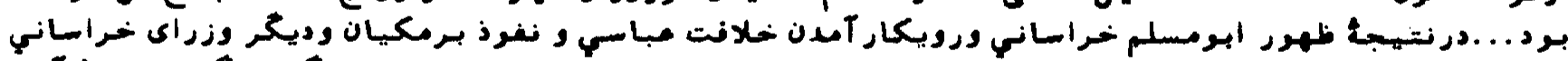

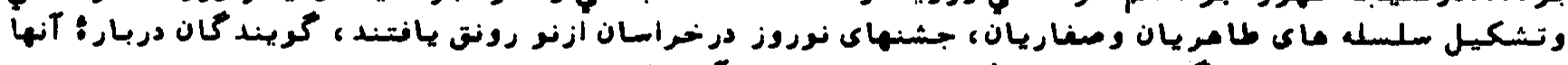

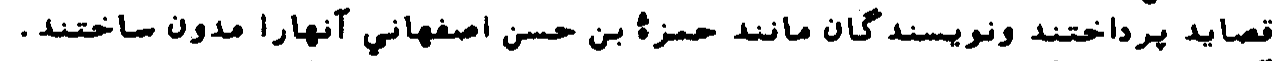

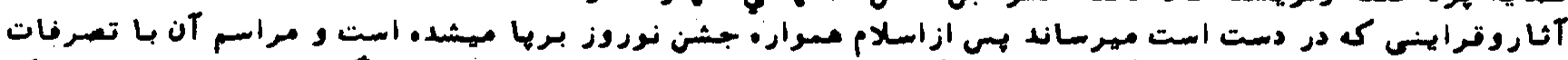

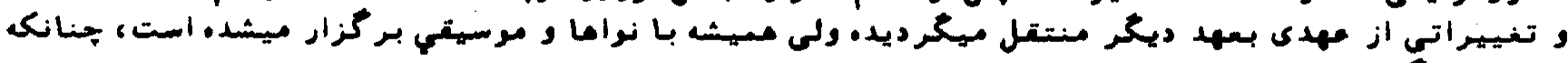

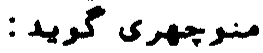

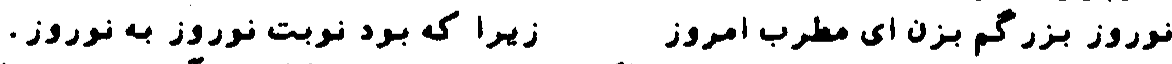

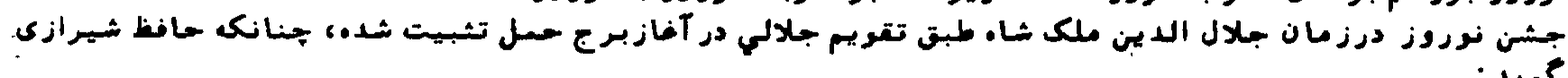

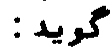

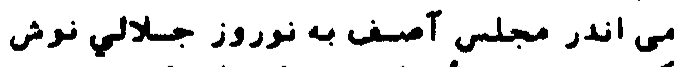

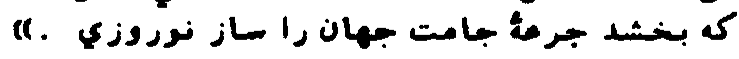

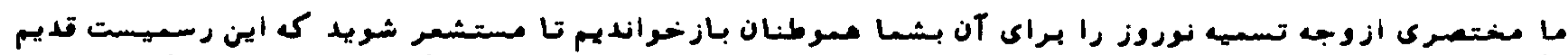

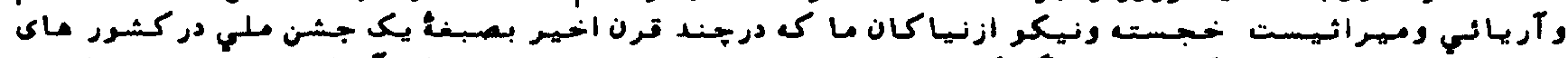

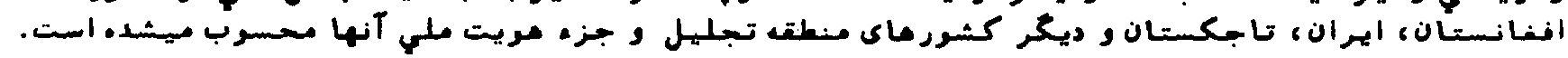




\section{4}

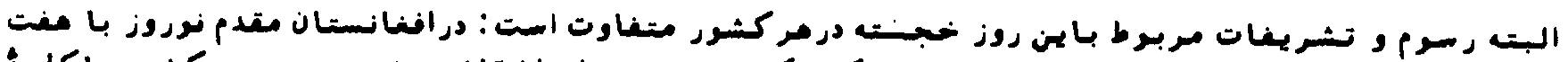

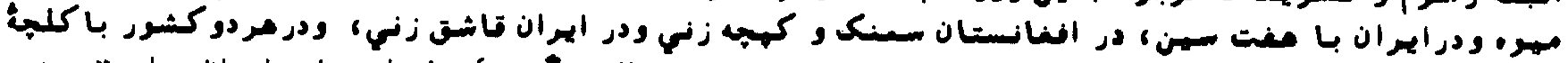

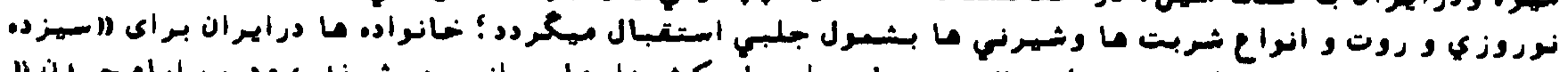

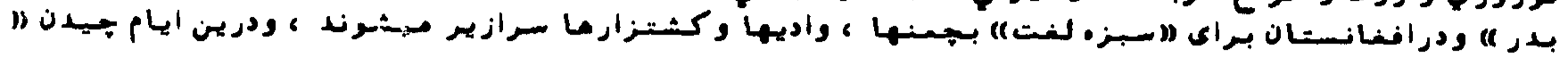

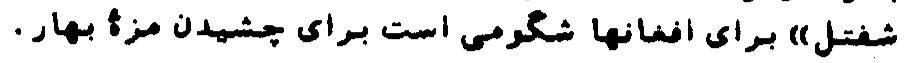

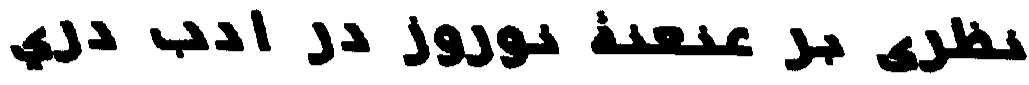

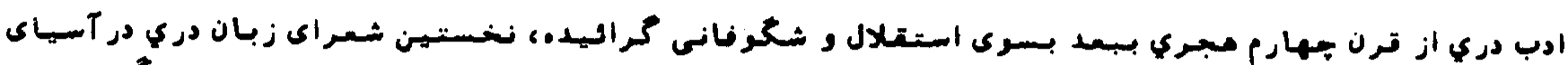

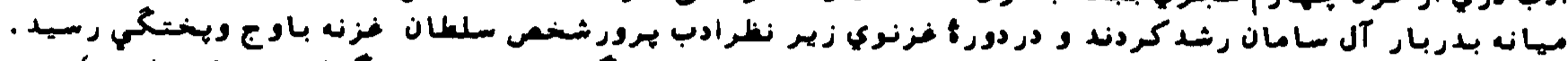

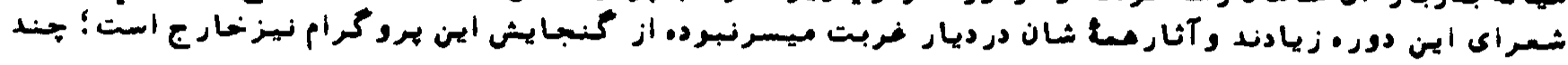

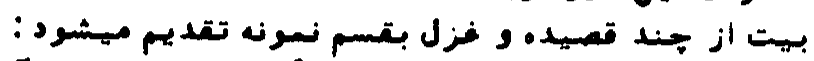

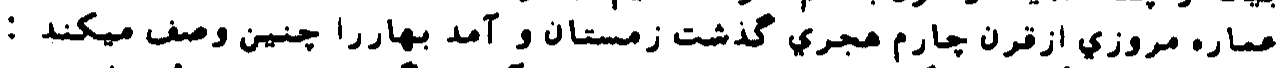

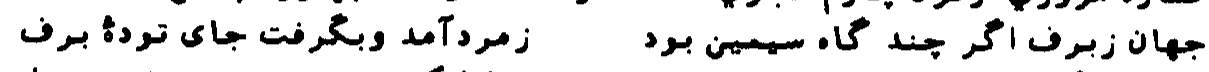

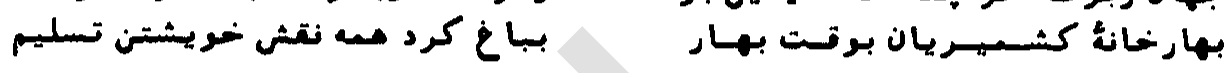

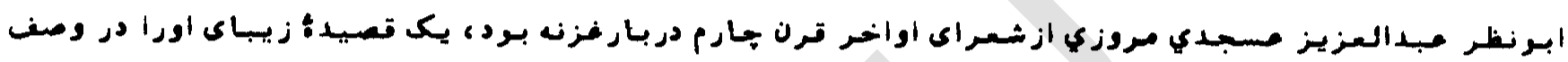

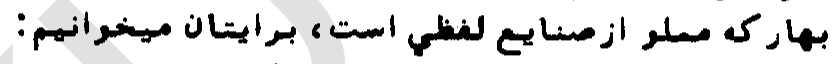

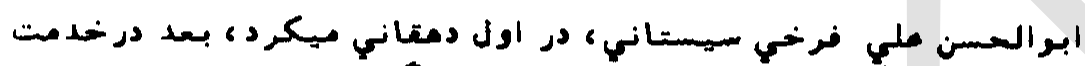

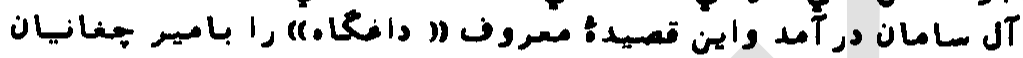

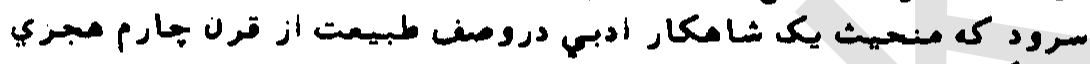

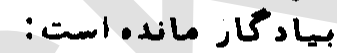

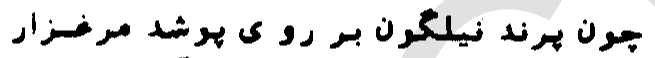

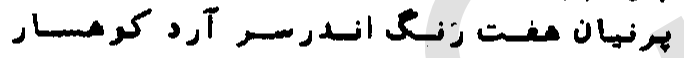

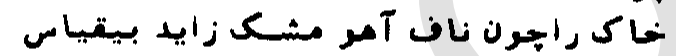

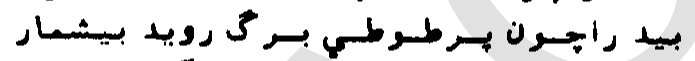

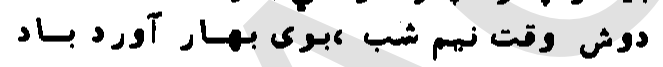

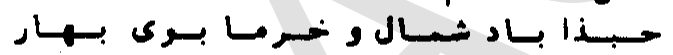

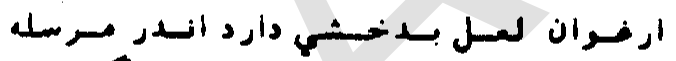

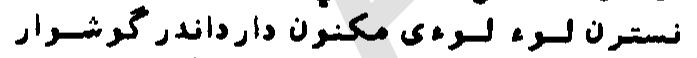

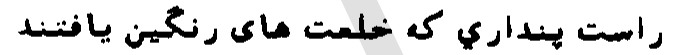

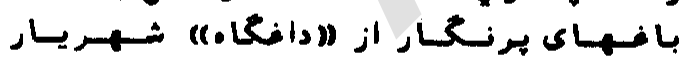

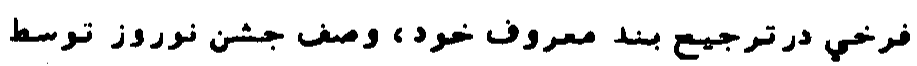

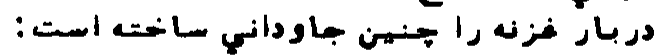

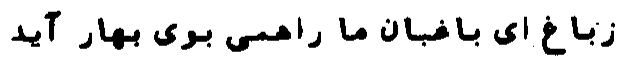

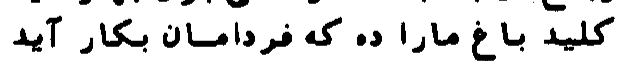

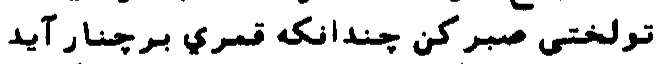

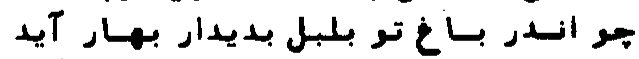

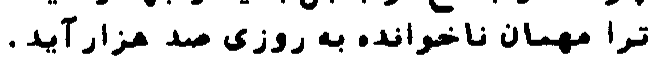

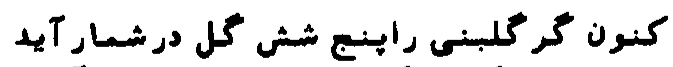

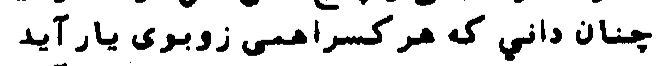

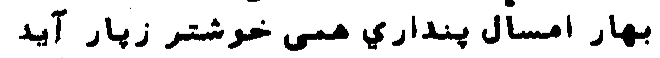

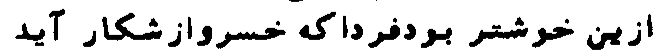

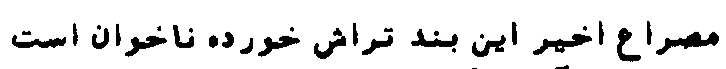

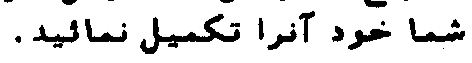

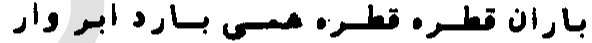

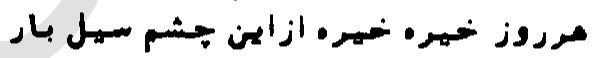

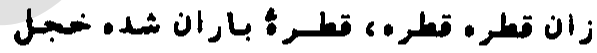

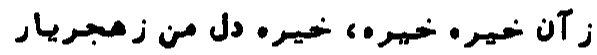

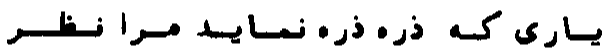

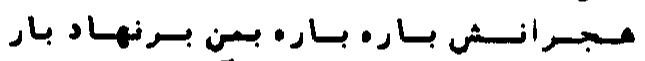

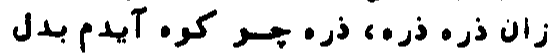

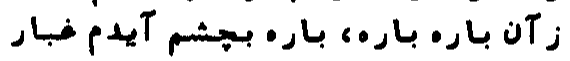

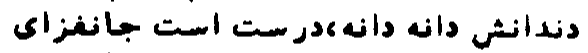

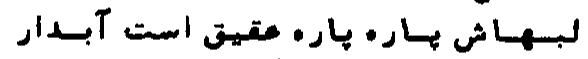

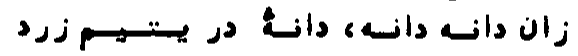

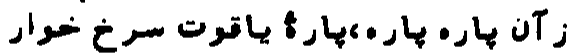

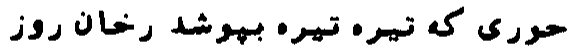

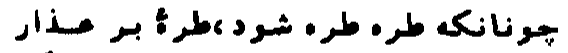

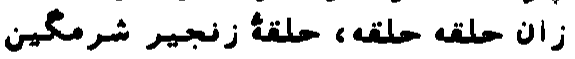

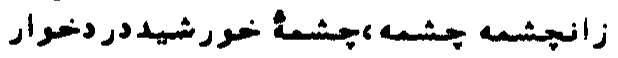

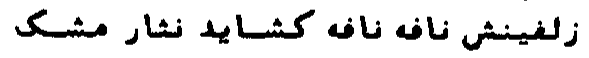

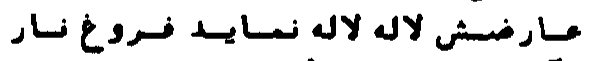

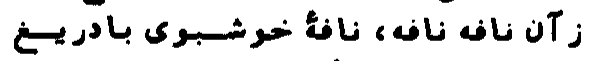

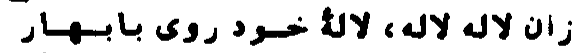

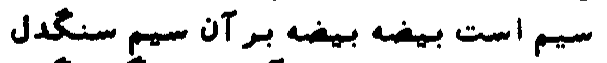

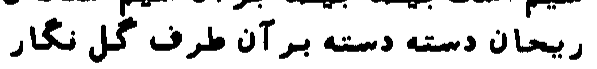

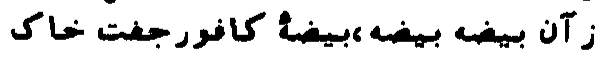

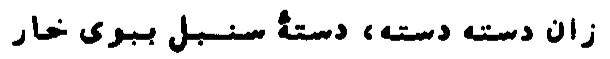

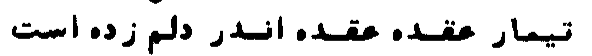

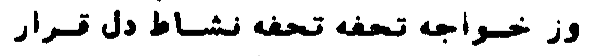

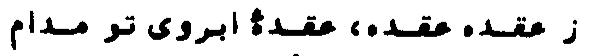

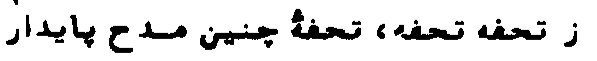

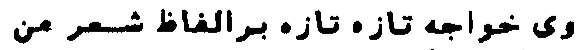

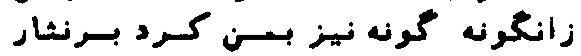

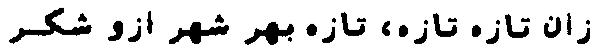

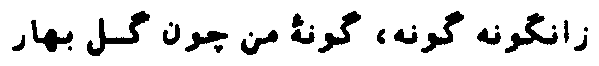




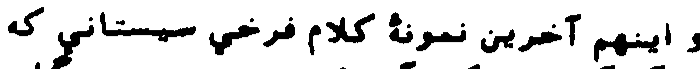

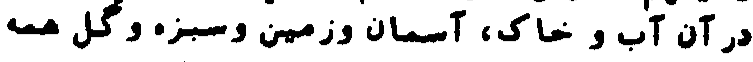
رادرتابلوى بهار مقبرل تعسو ميرنمر ده است:

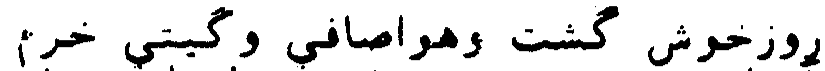

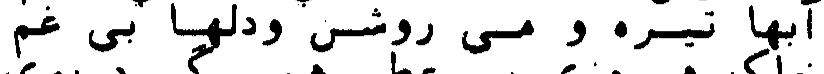

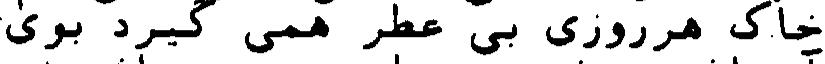

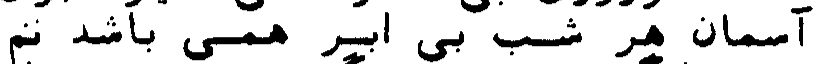

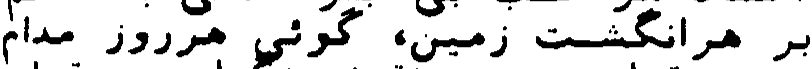

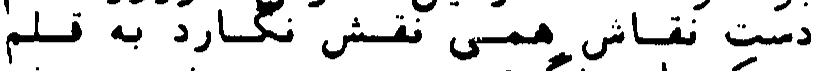

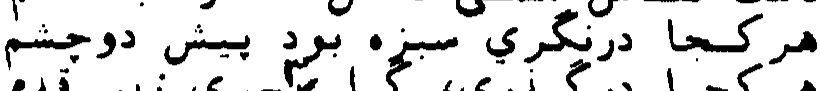

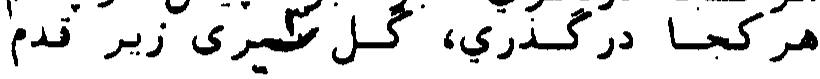

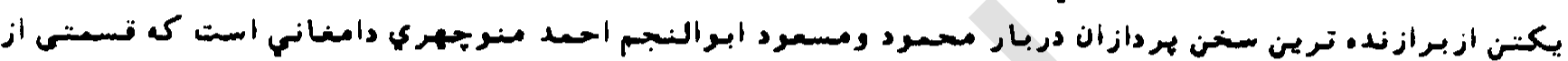

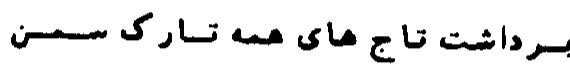

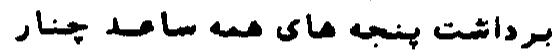

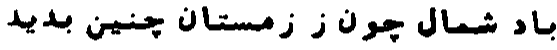

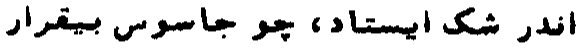

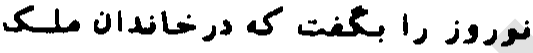

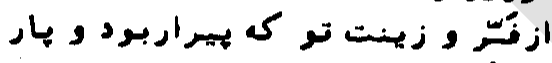

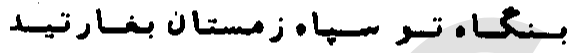

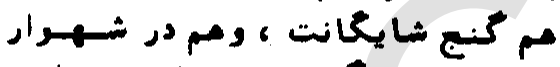

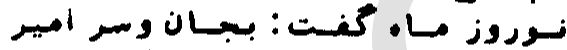

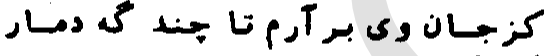

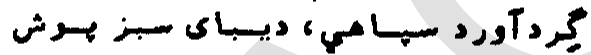

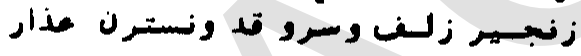

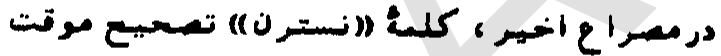

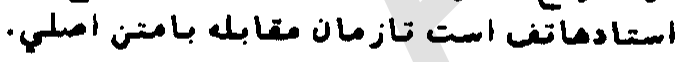

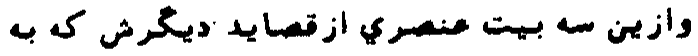

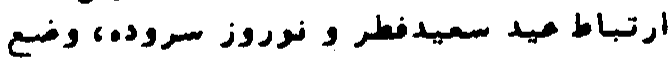

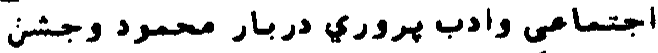

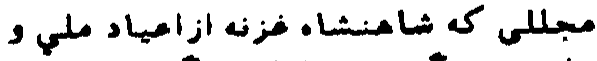

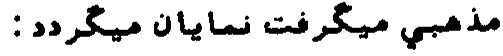
به از مسيد نشسناسم از روز كار به از مدع خسرو ندر آمسرز كار

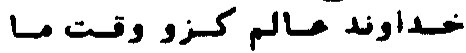
معه سـال ميداست وليل ونهار

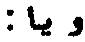

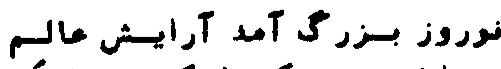

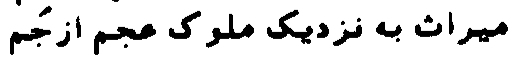

فرخه معر درازى سهري كرد ومرسال دربمدال .

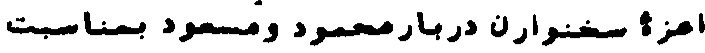

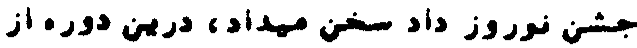

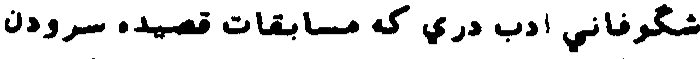

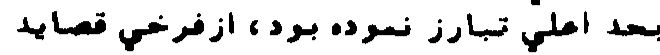

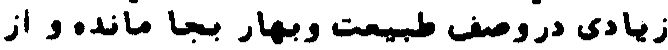

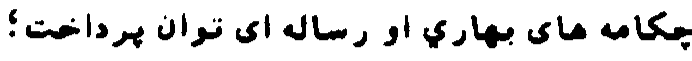

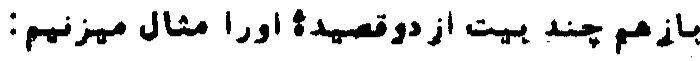

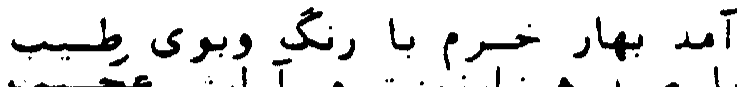

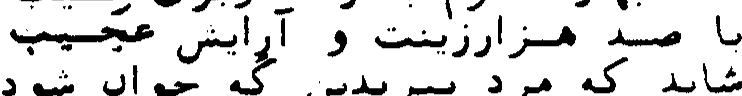

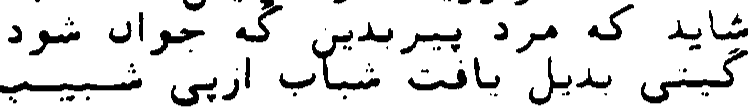

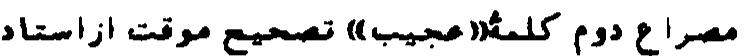

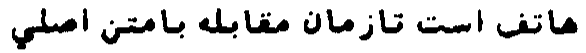

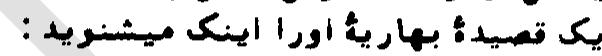

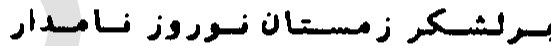

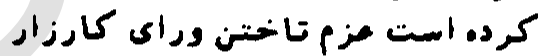

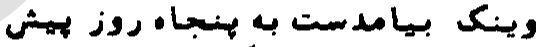

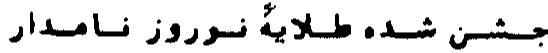

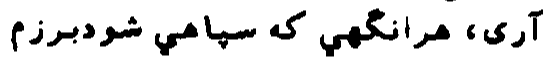

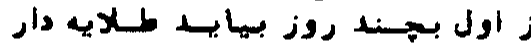

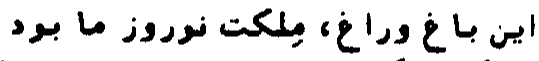

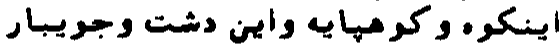

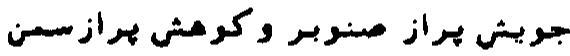

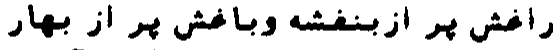

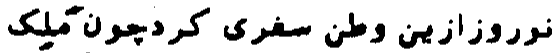

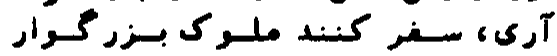

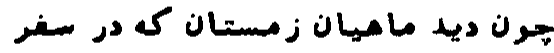
نـوروز مه بماند ، تسريب مهى خهمهار

قصيده سراك بلند مرتبت ديخر دربار خزنه

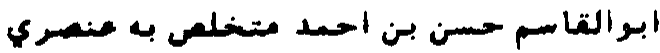

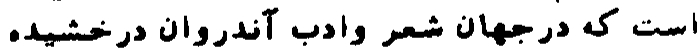

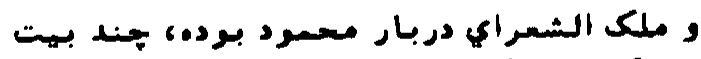

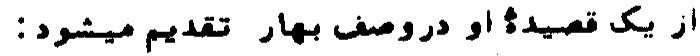

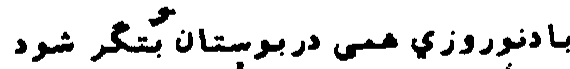

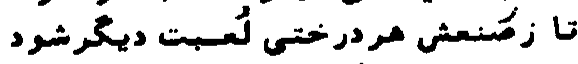

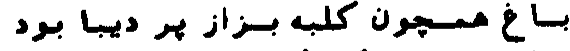

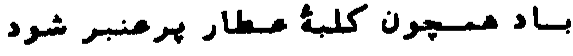

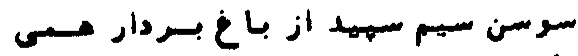

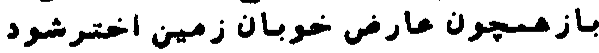

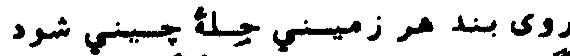

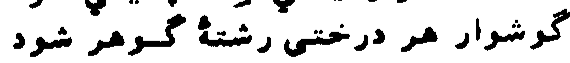




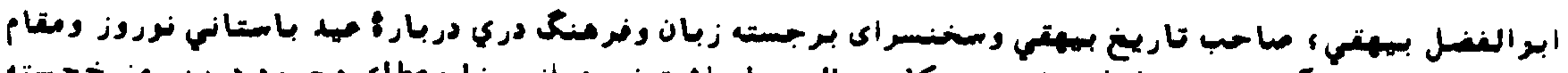

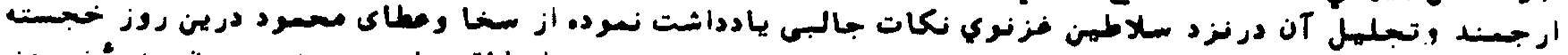

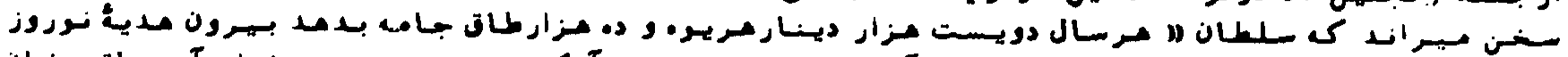

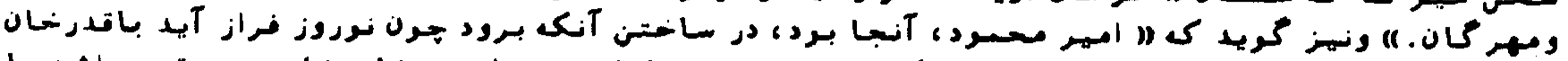

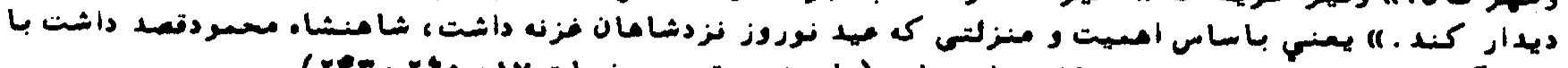

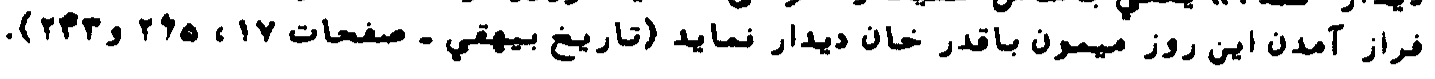

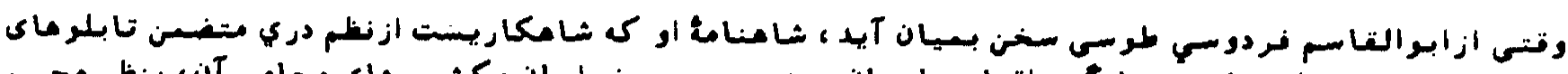

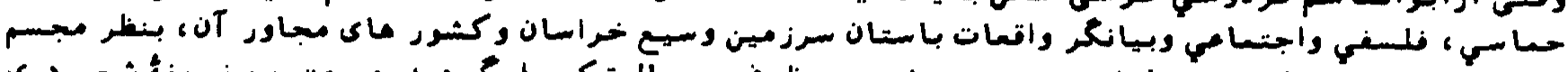

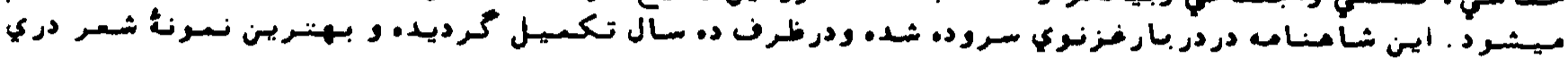

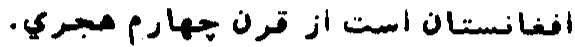

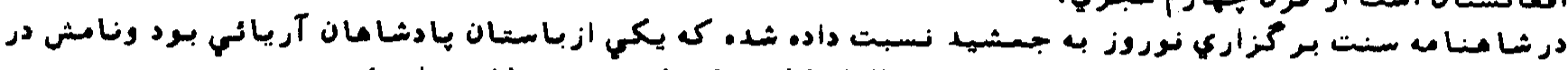

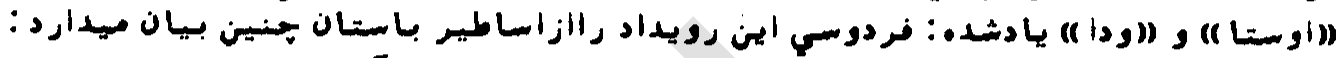

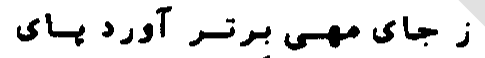

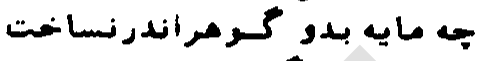

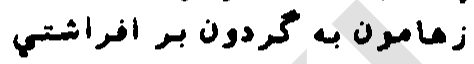

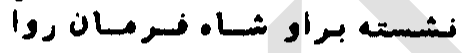

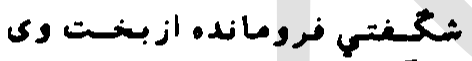

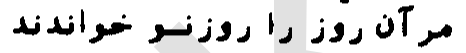

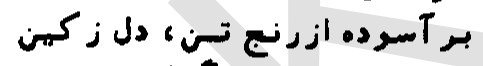

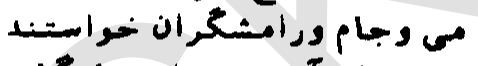

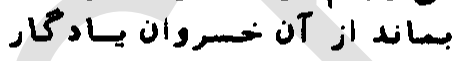

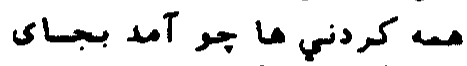

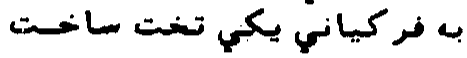

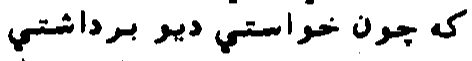

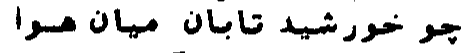

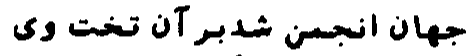

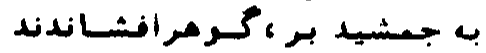

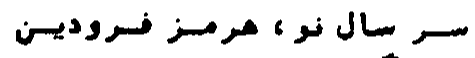

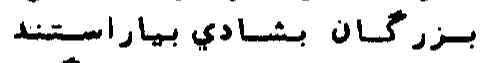

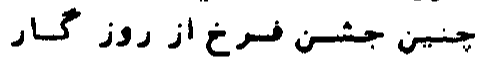

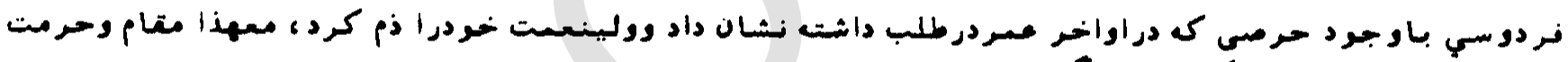

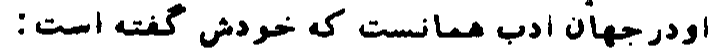

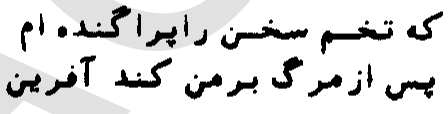

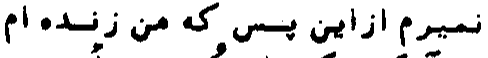

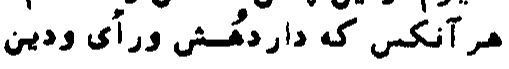

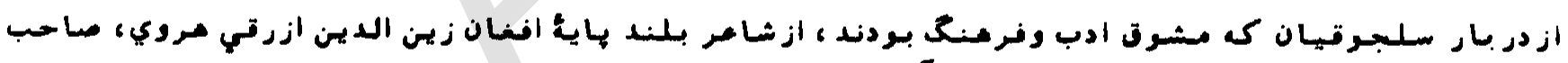

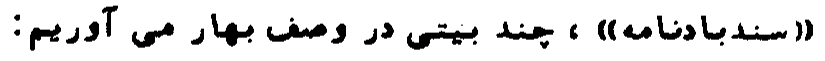

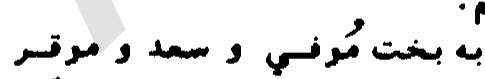

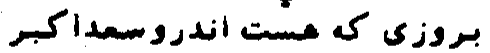

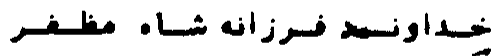

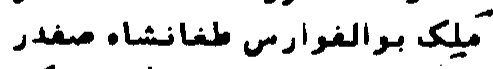

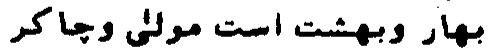

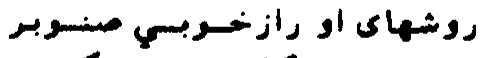

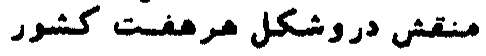

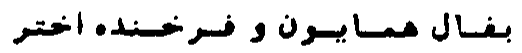

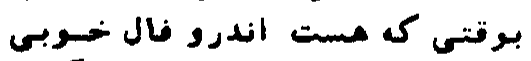

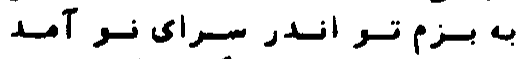

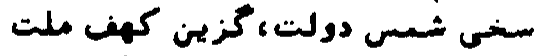

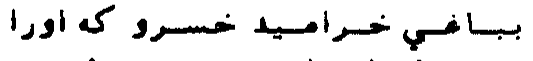

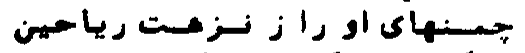

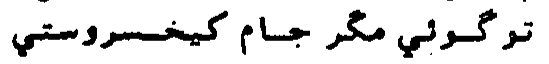

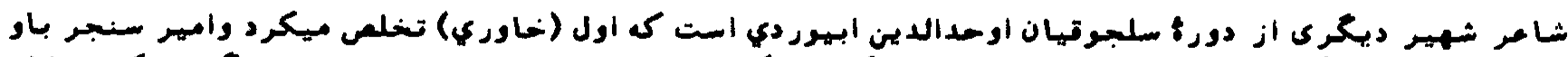

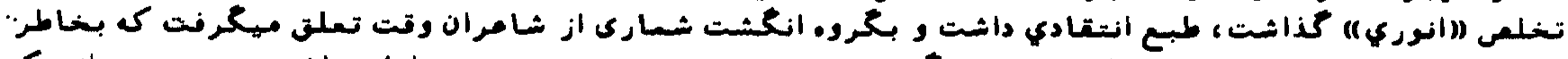

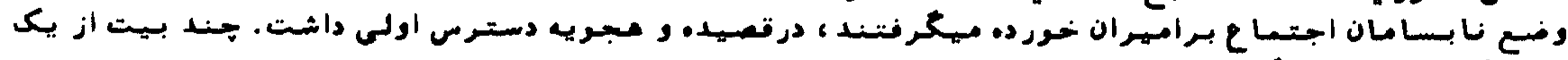

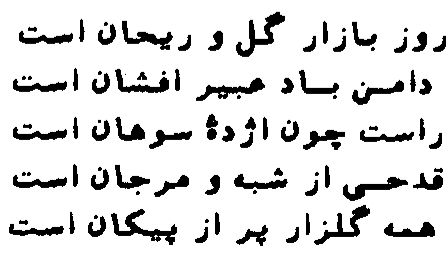

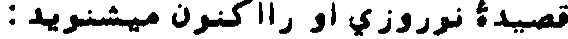

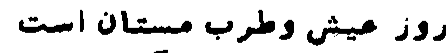

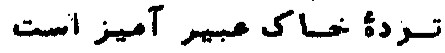

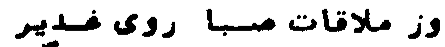

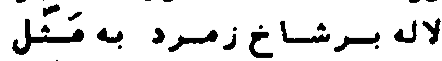

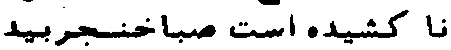




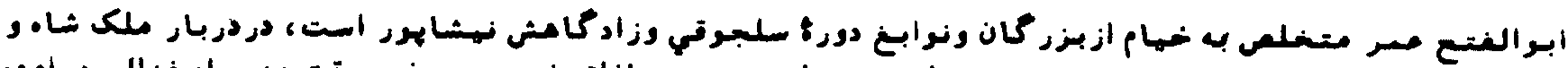

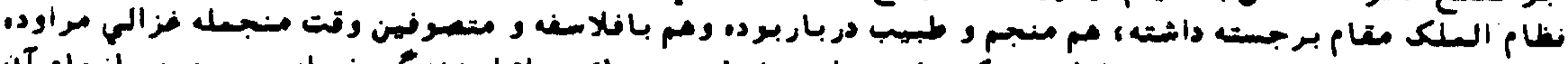

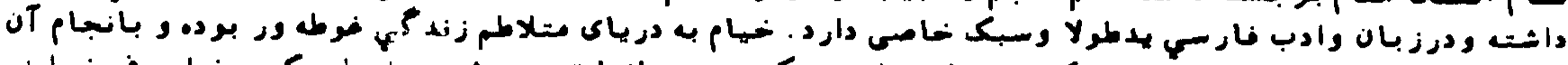

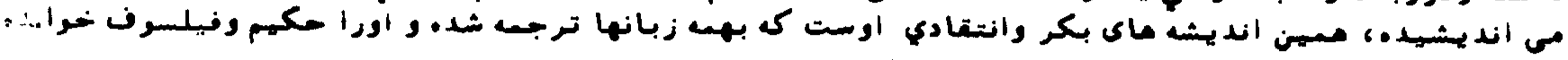

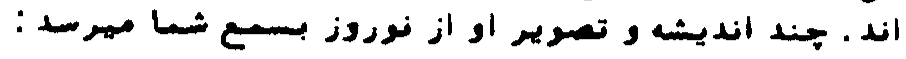

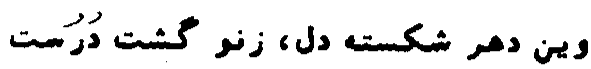

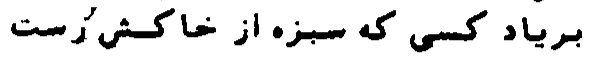

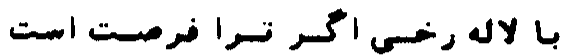

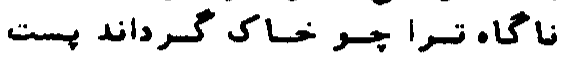

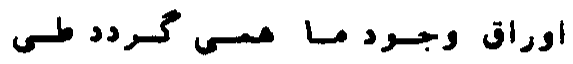

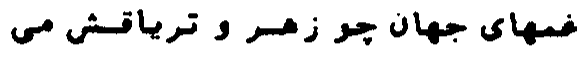

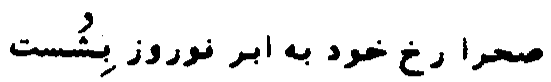

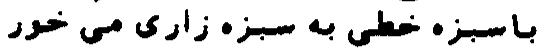

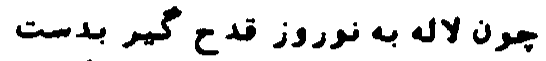

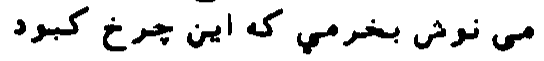

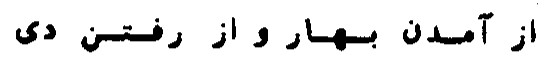

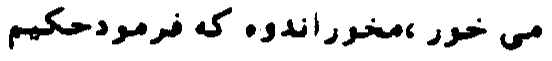

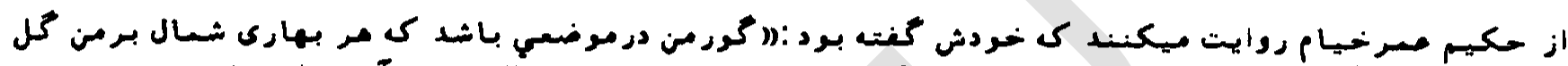

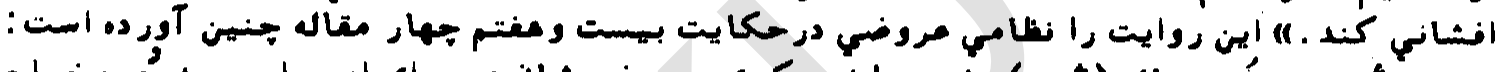

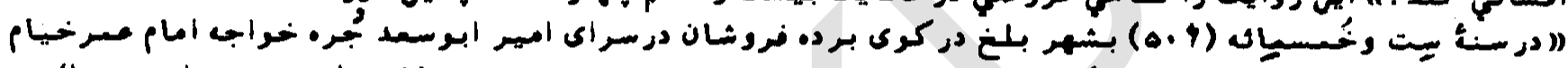

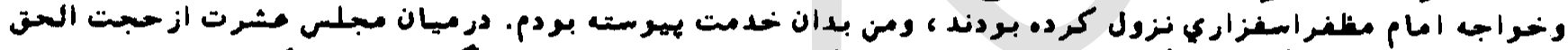

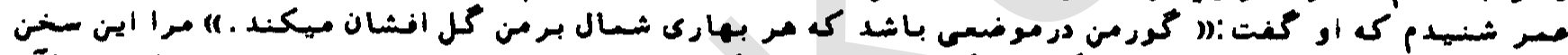

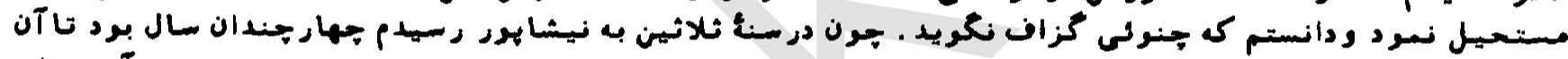

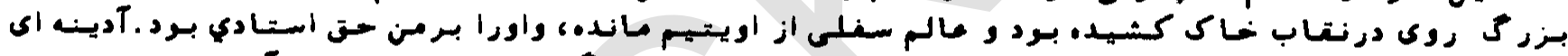

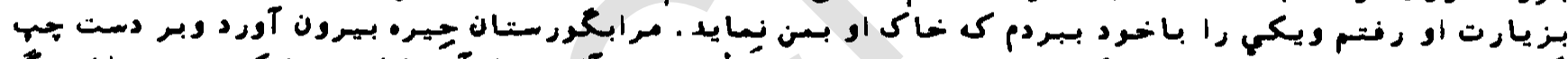

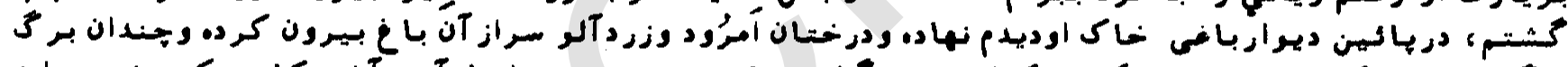

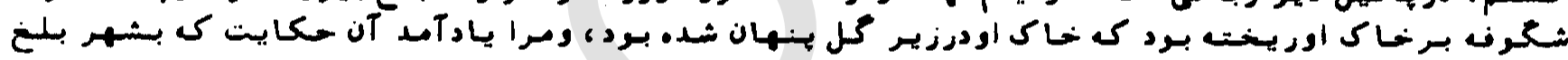

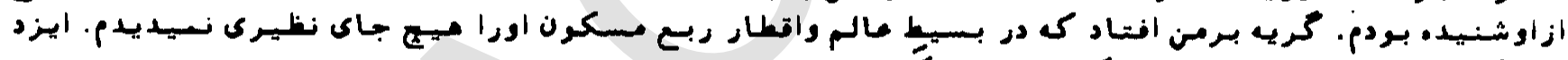

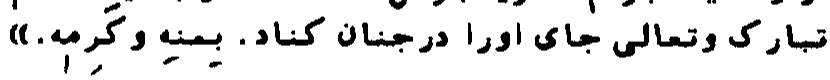

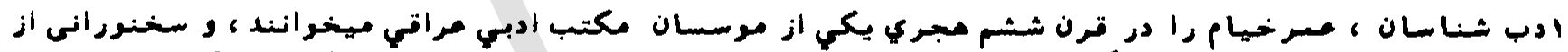

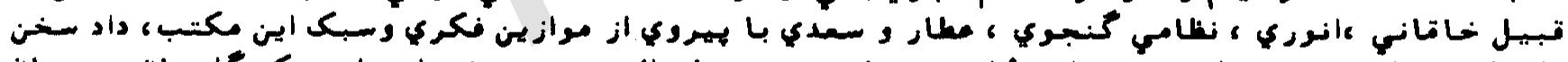

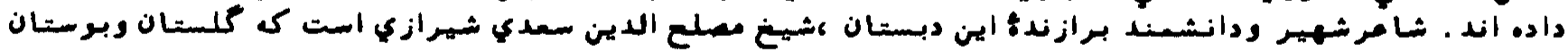

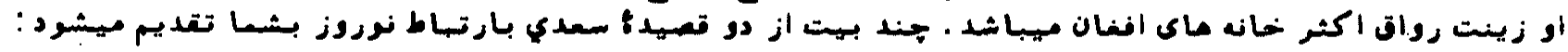

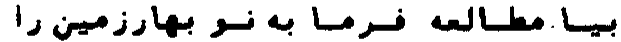

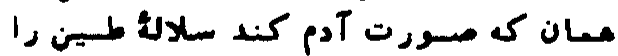

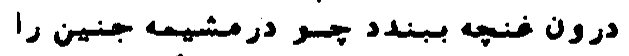

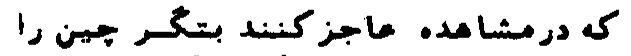

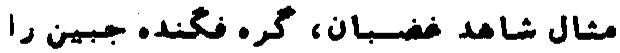

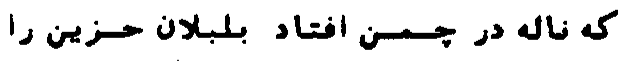

لهكر زحست سـرما ز سرما برغاست

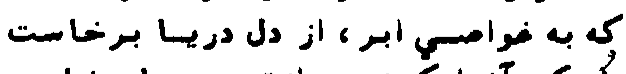

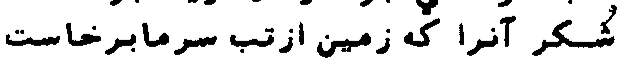

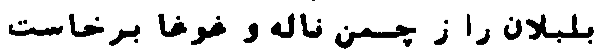

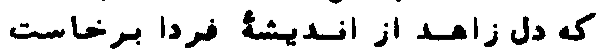

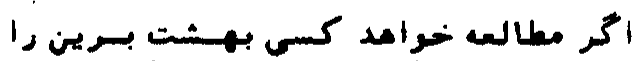

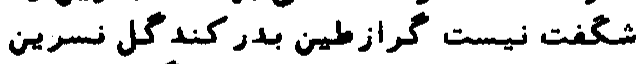

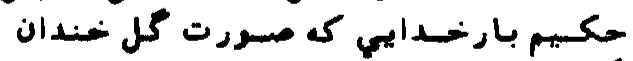

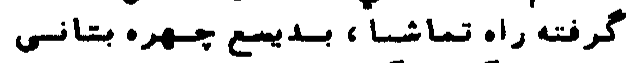

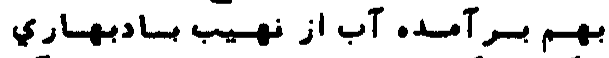

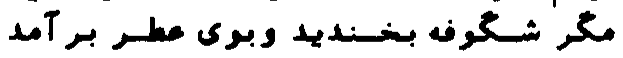

و ئا

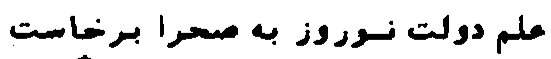

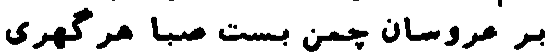

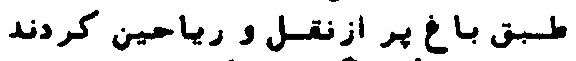

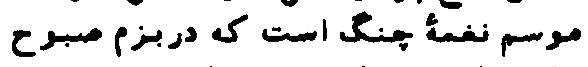

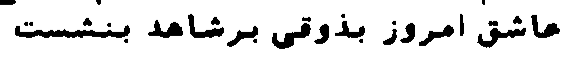




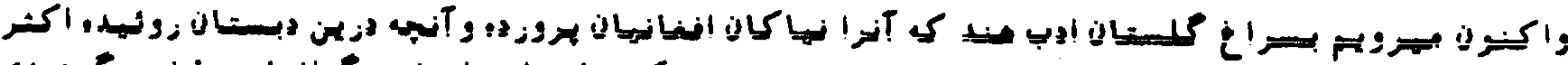

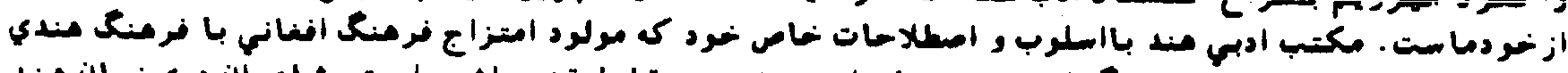

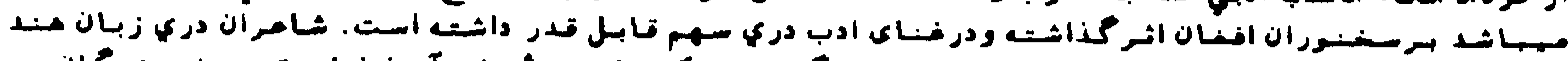

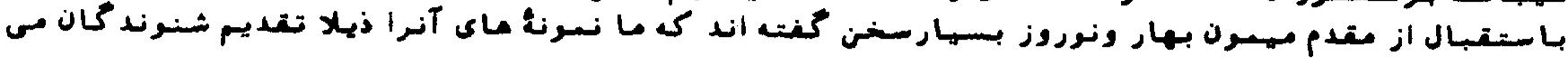

نمانميم:

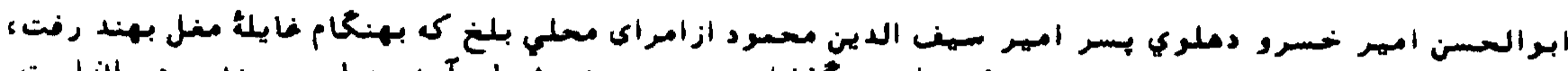

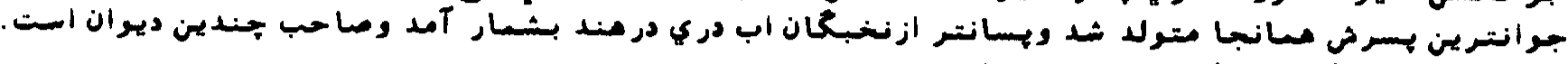

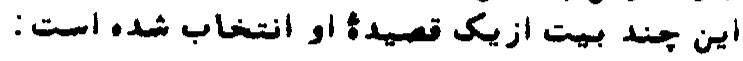

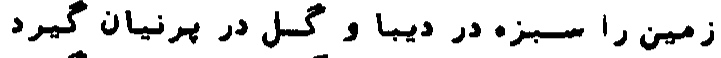

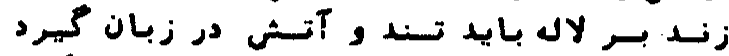

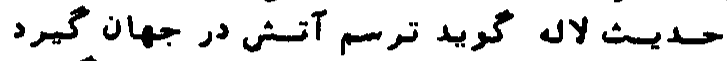

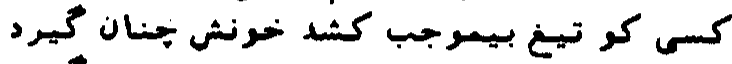

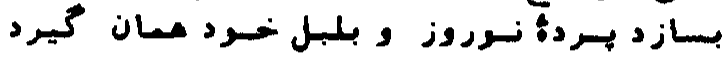

بـشم بــ روز مرا موسم باران آورد

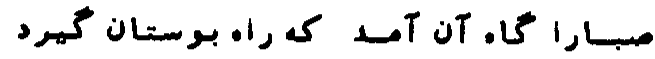

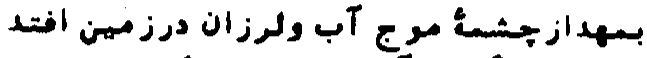

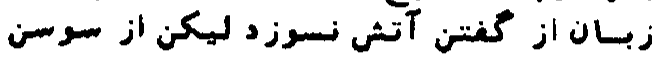

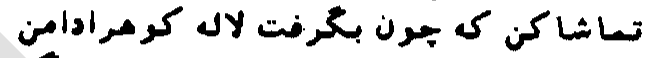

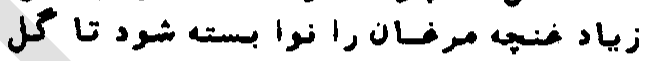

\section{actiojul}

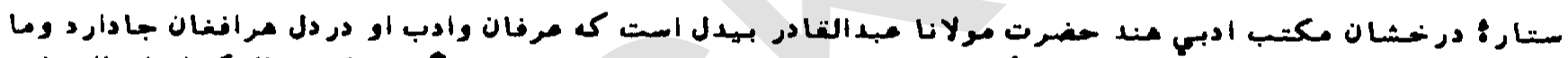

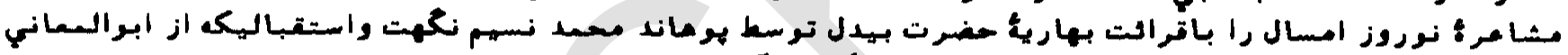

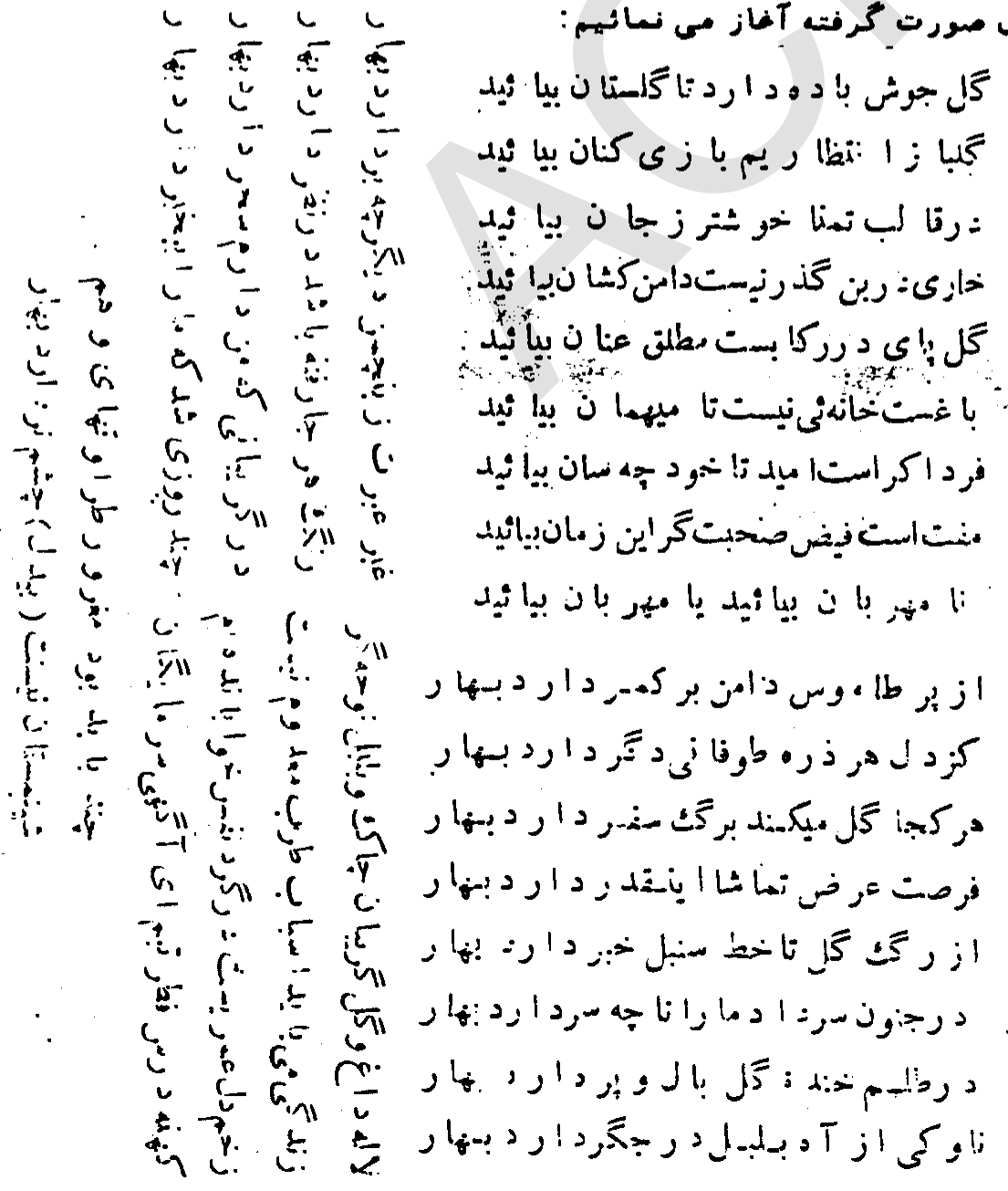

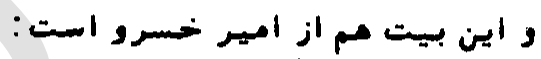

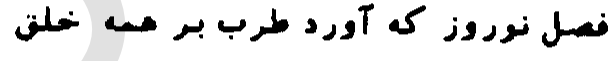

\section{.}


39

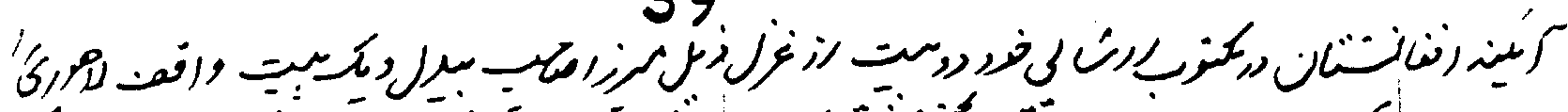

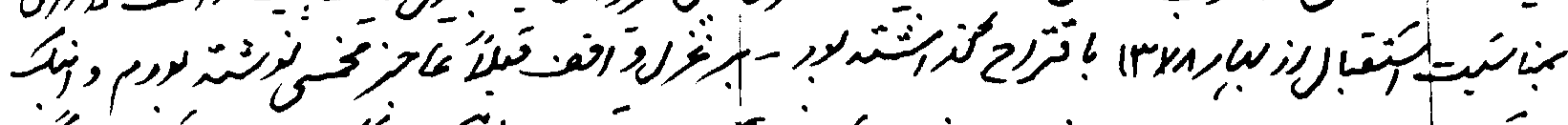

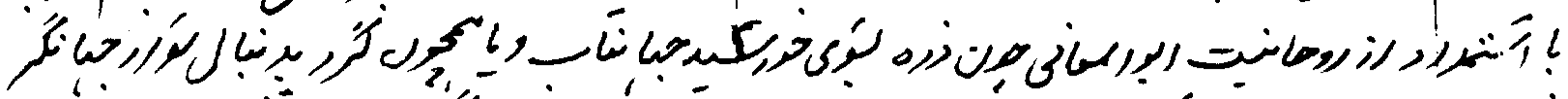

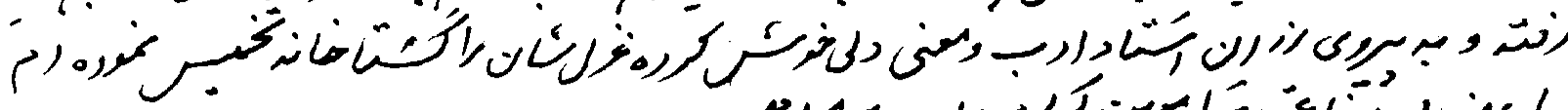
"

$$
\text { تخ }
$$

!

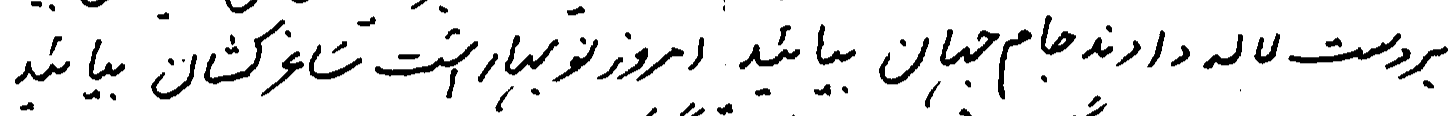

定

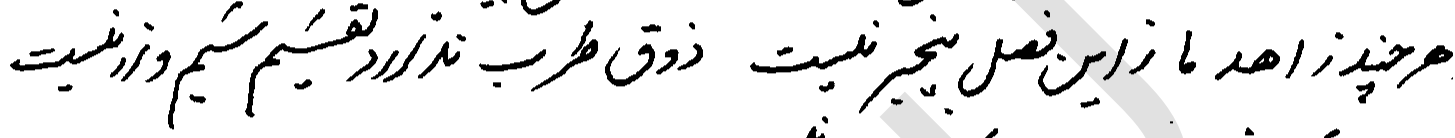

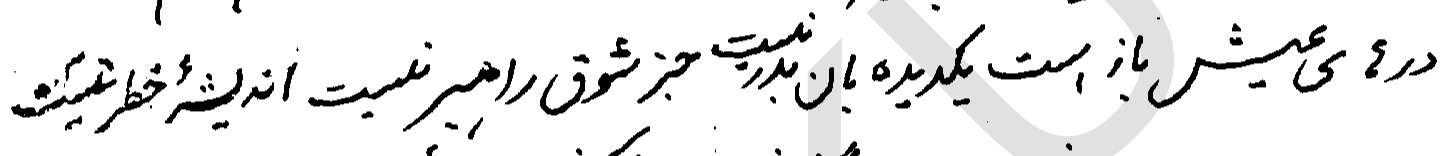

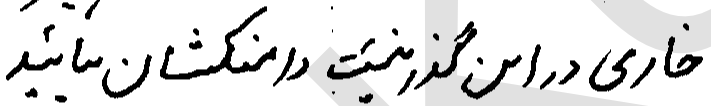

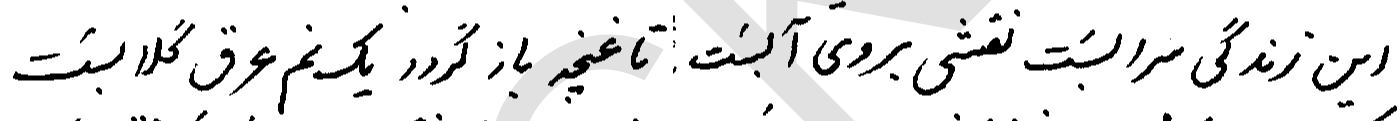

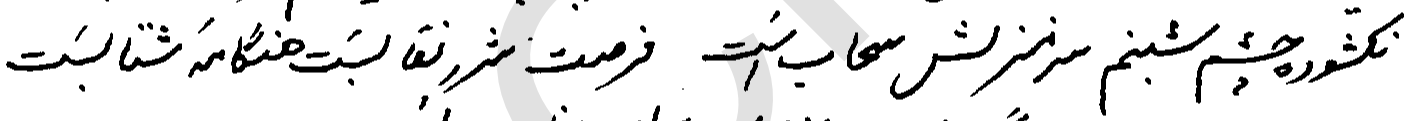
每

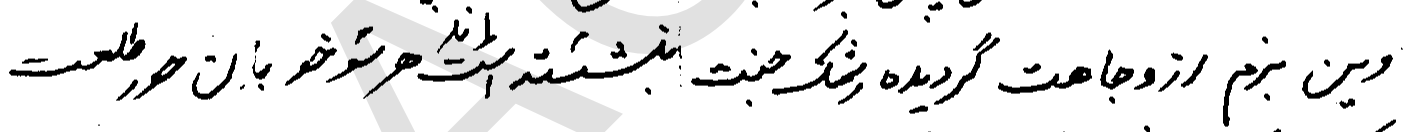

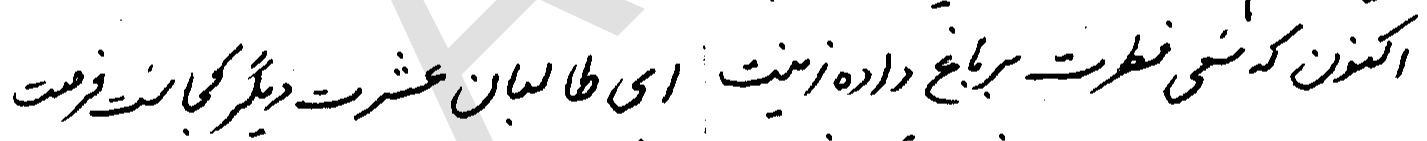

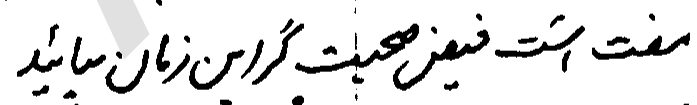

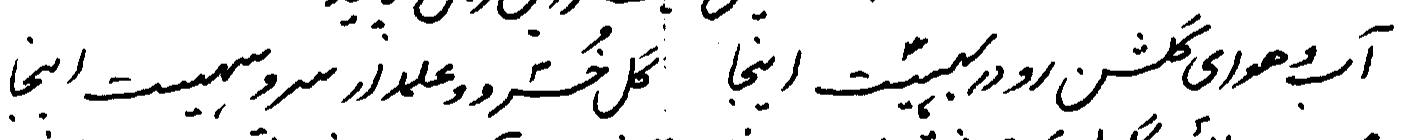

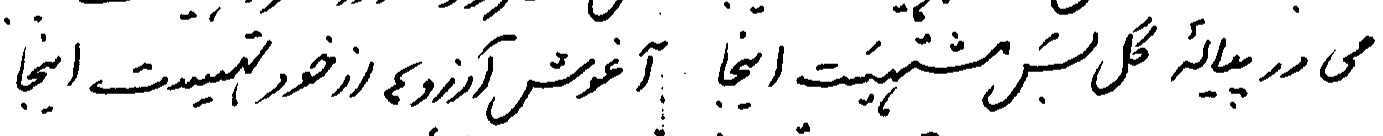
(ن)

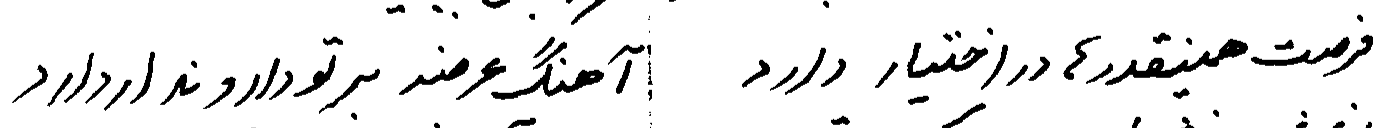

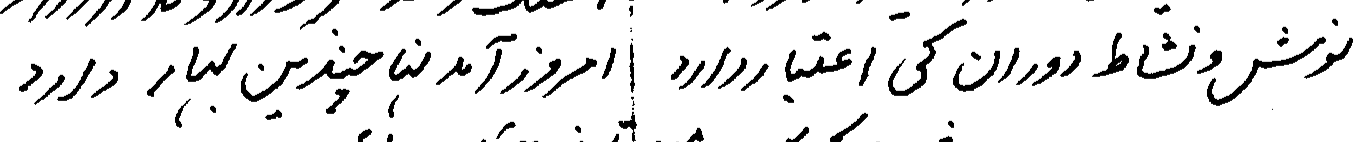

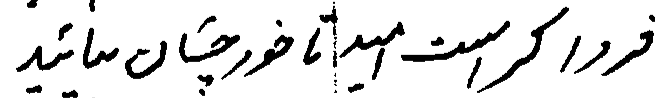

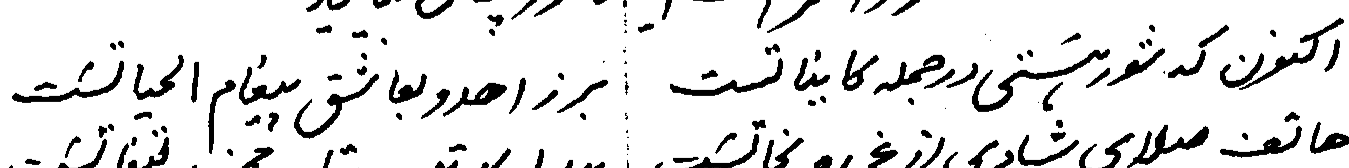

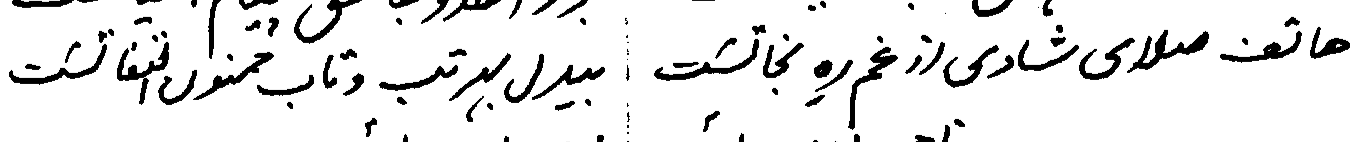

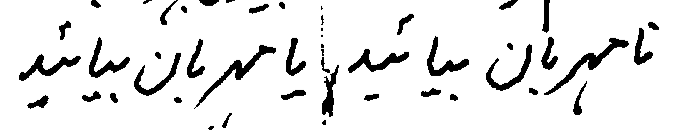


40

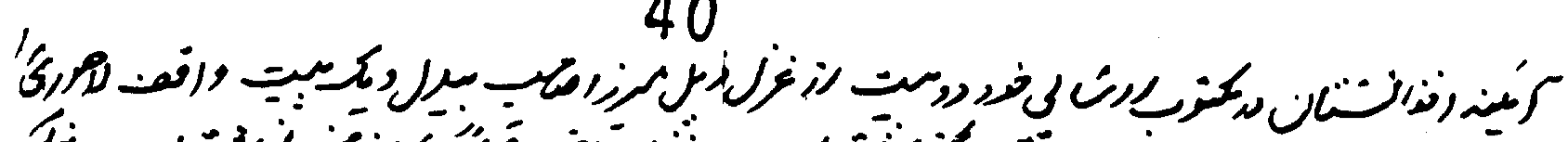

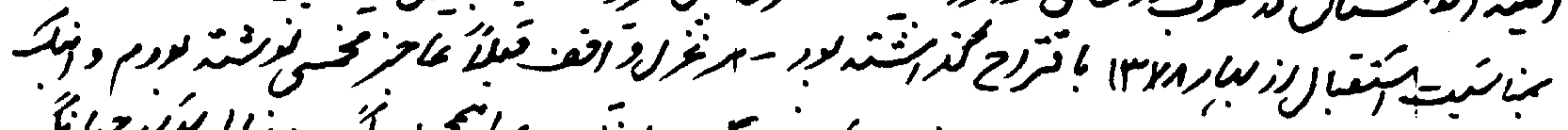

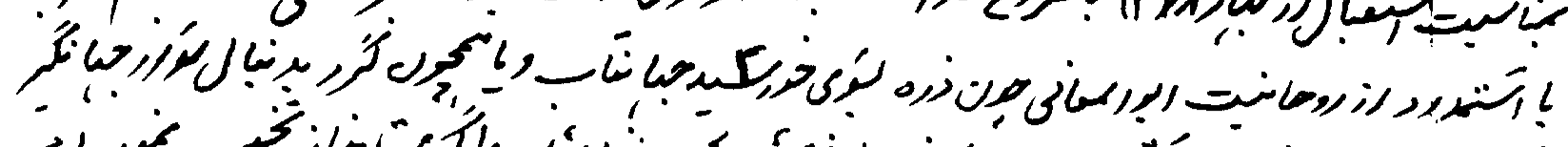

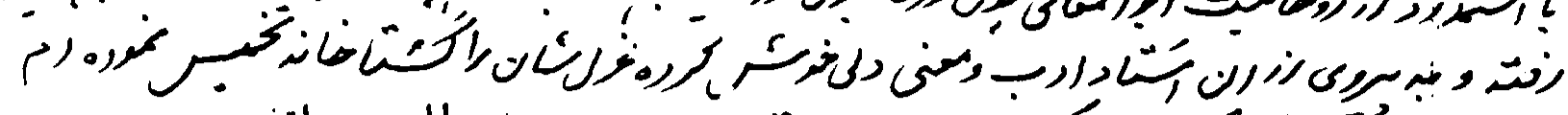

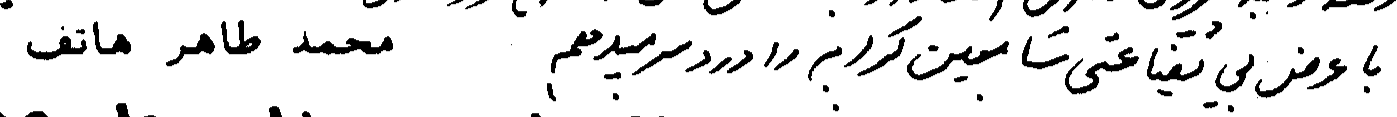

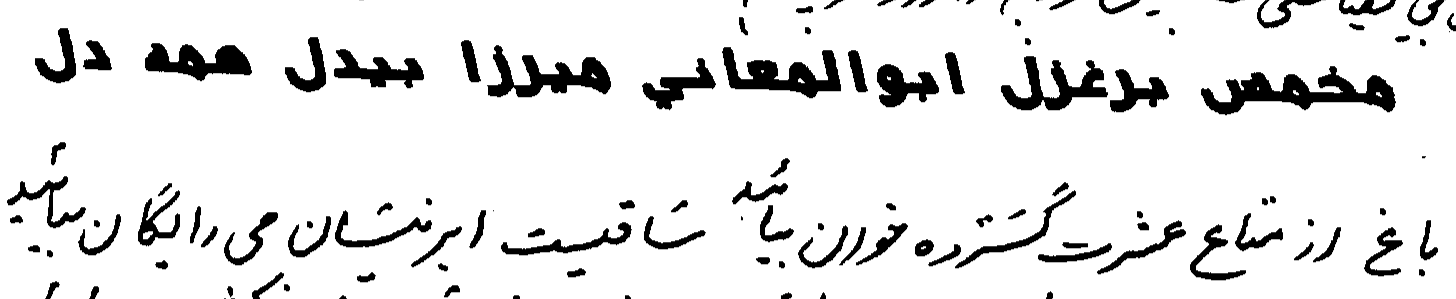

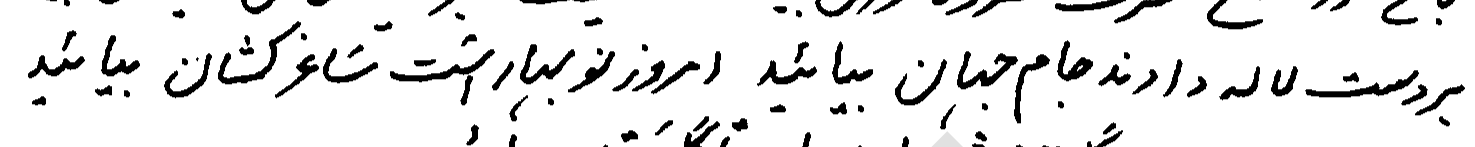

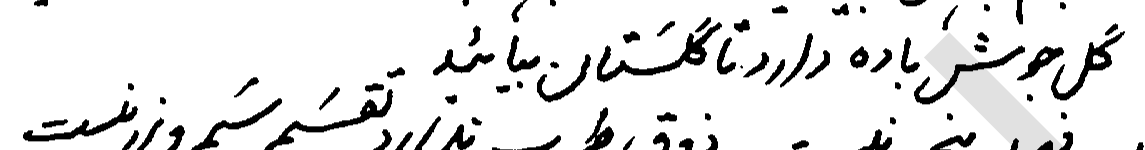

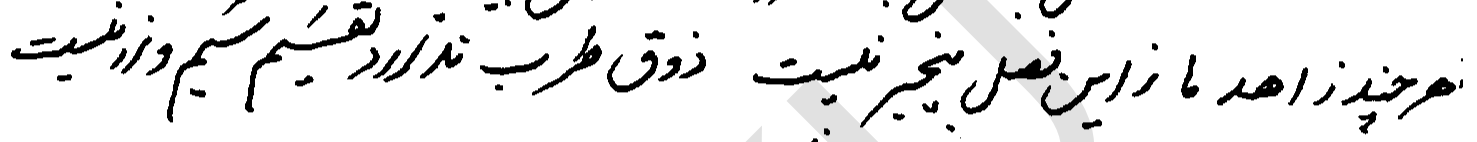

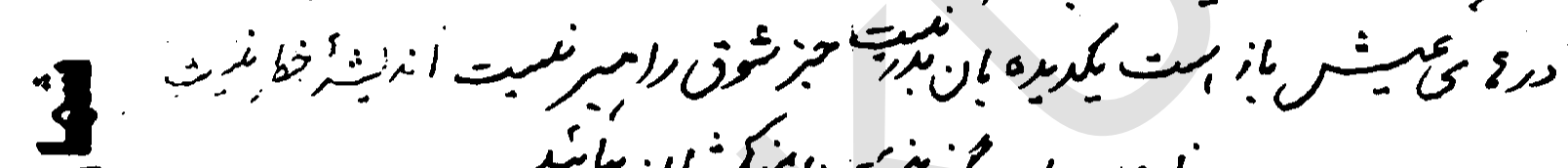

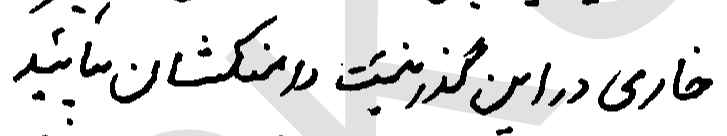

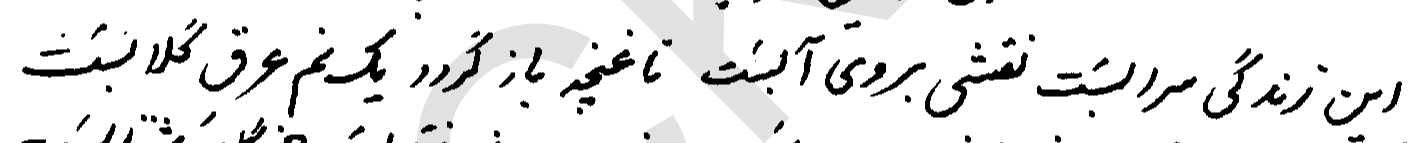

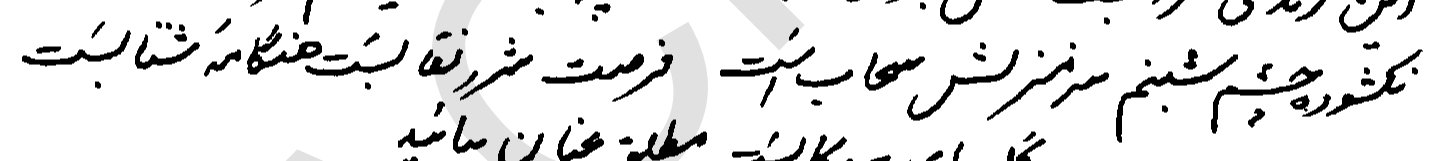

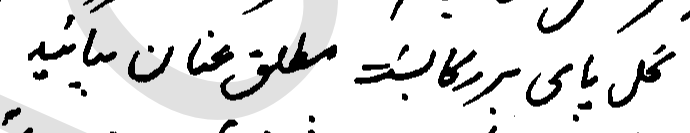

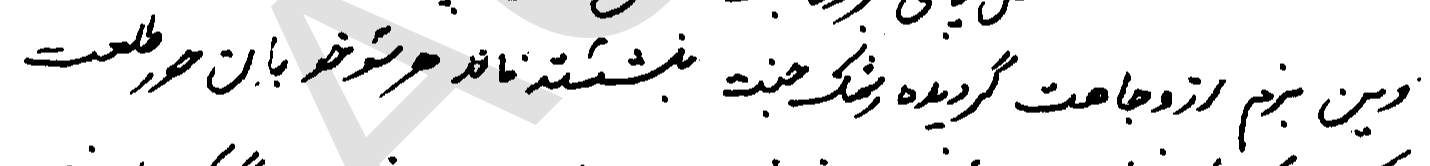

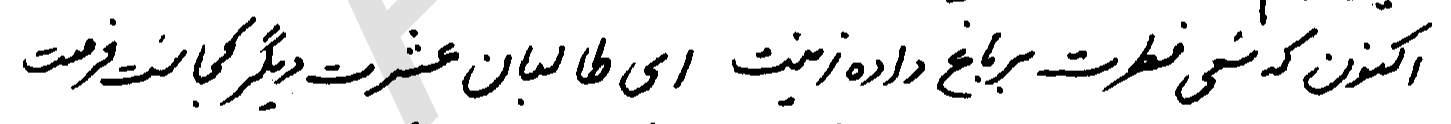

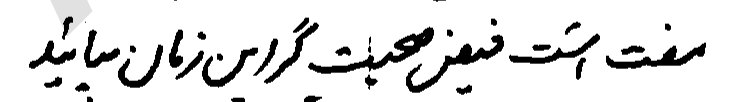

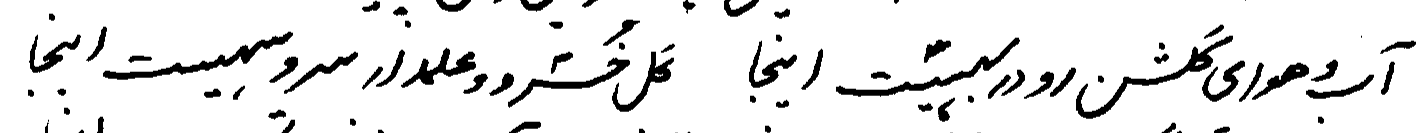

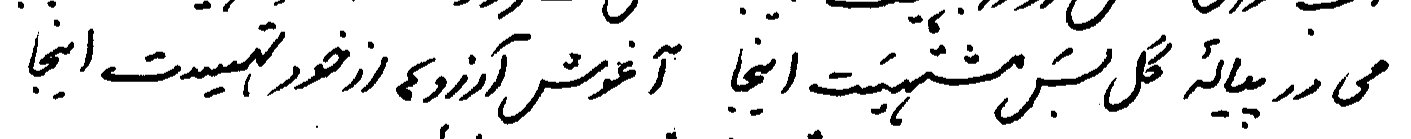
>

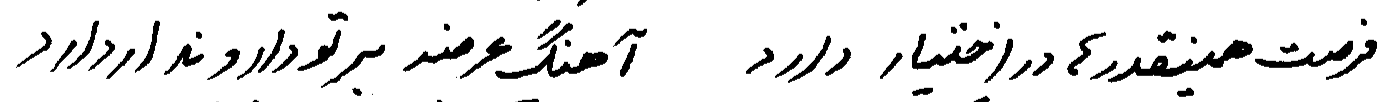

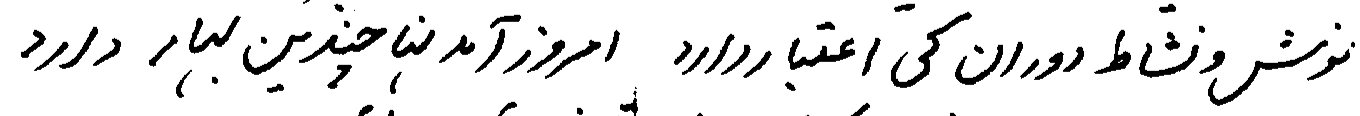

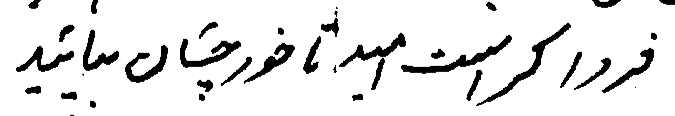

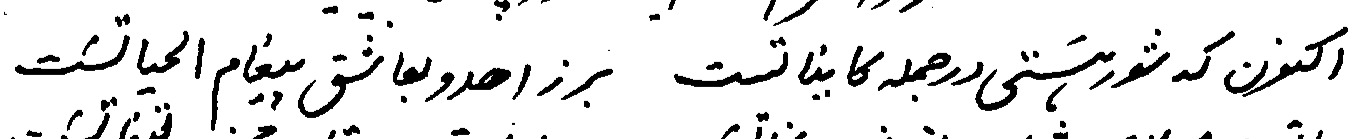
هاثت ملارى

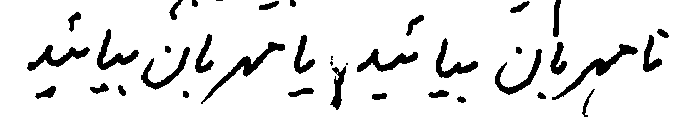


تطعة ديكر امتاد ماتف باستتبال از مجري

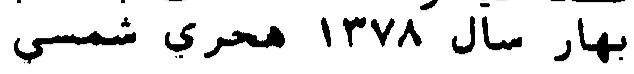

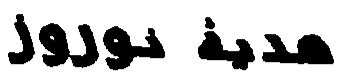

اين خضر بي خجسته بهاران سبزيوشي

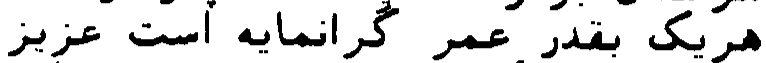

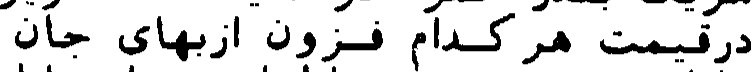

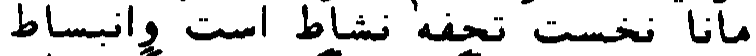

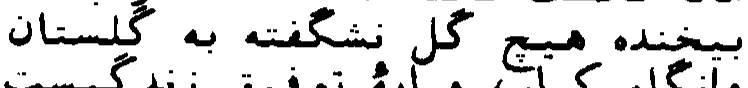

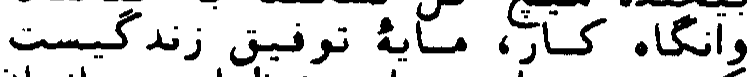

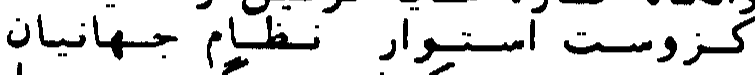

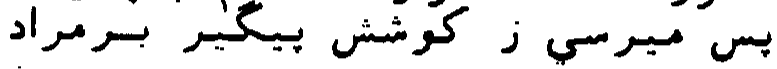

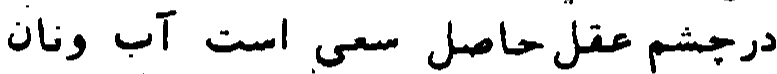

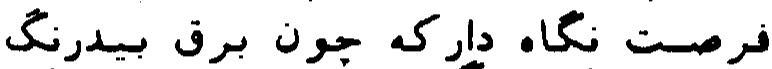

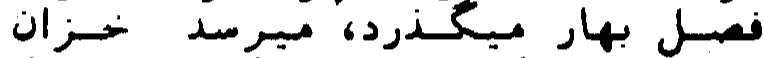

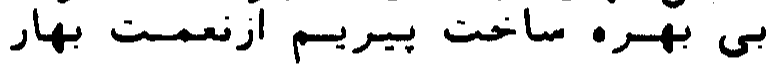

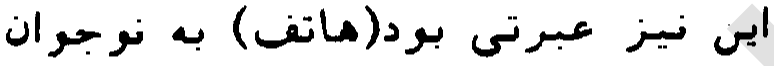

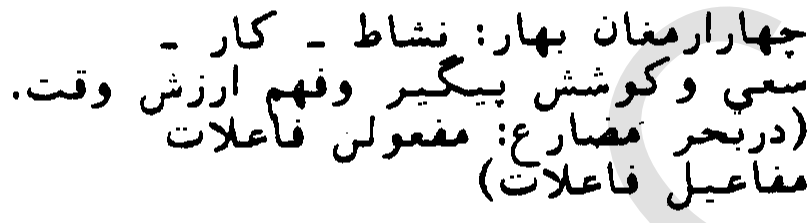

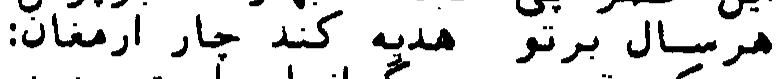

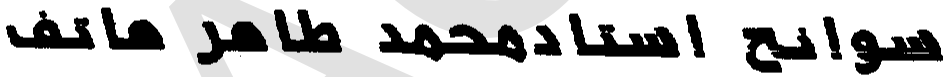

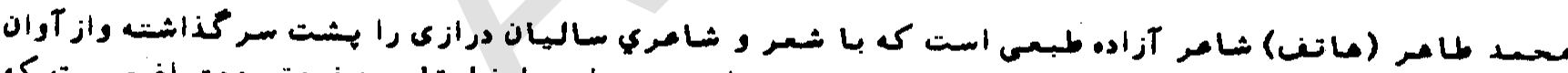

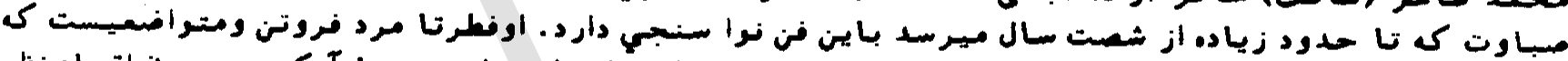

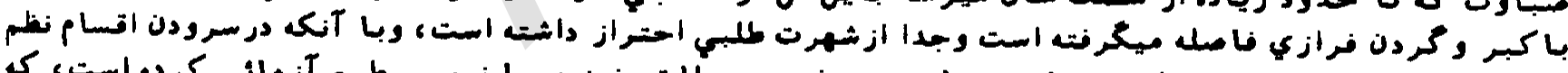

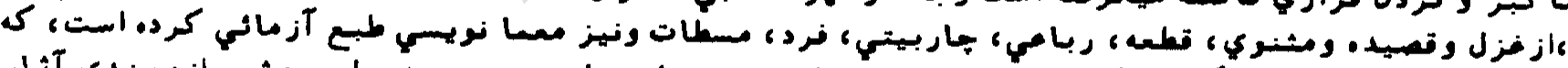

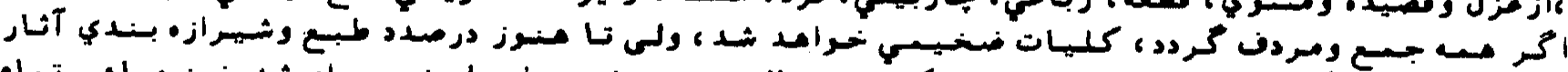

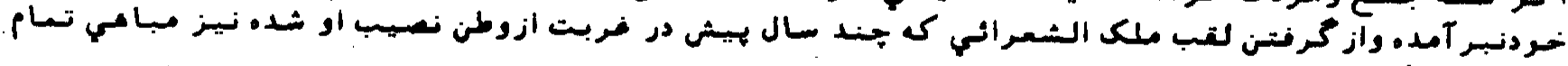

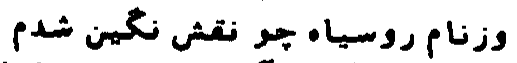

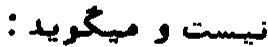

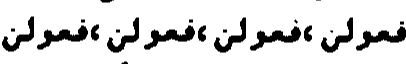

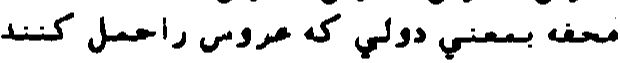

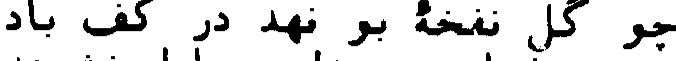

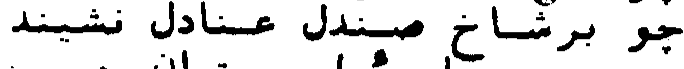

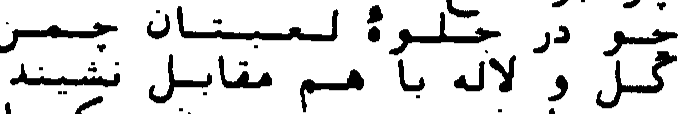

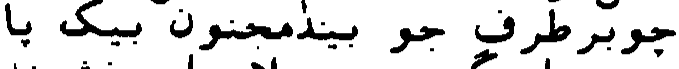

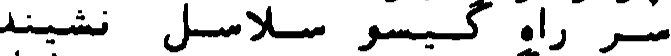

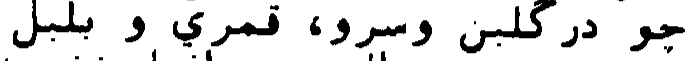

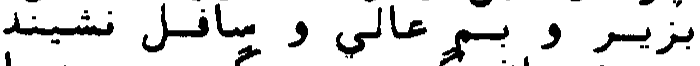

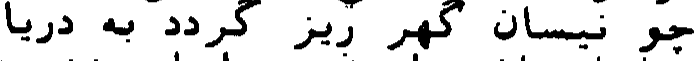

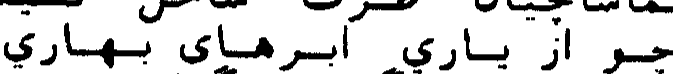

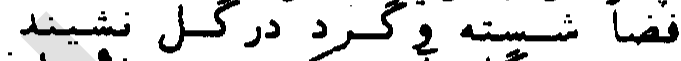

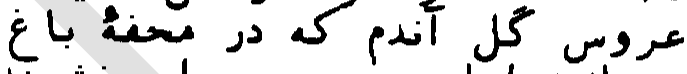

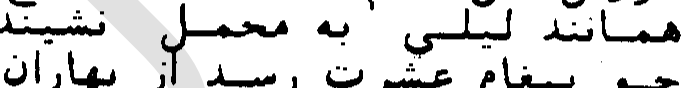

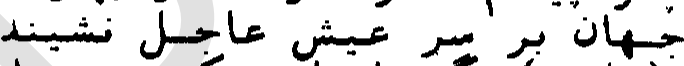

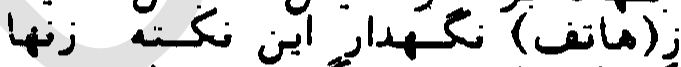

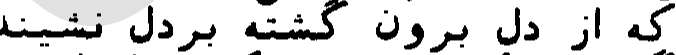

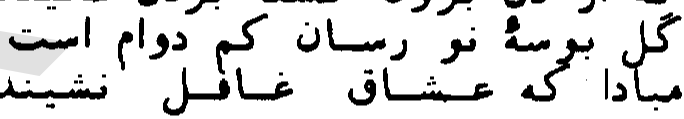
In:-

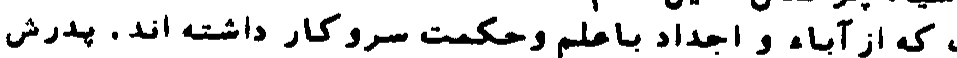

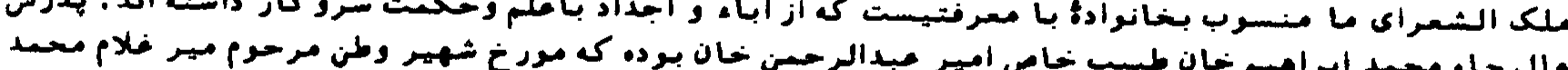

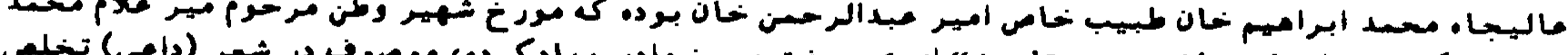

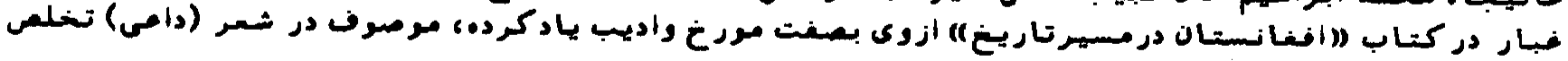

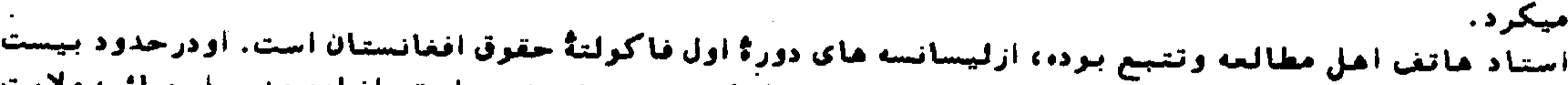

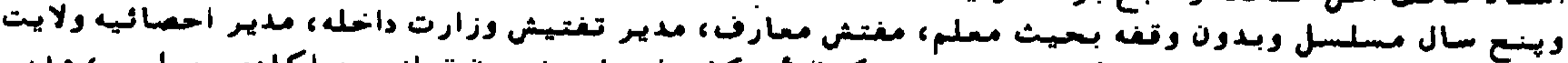

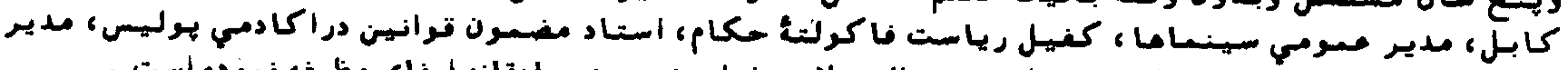

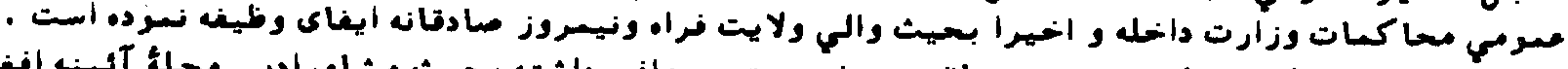

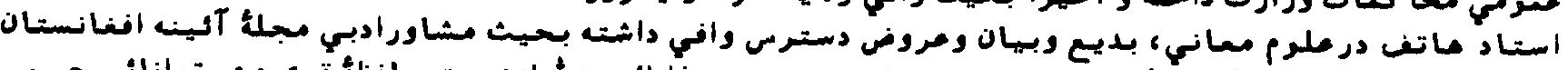

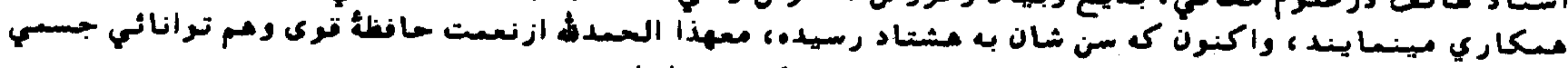

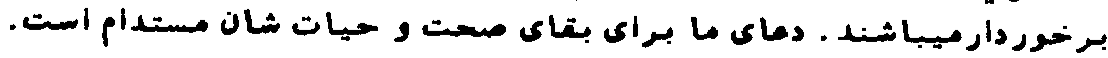




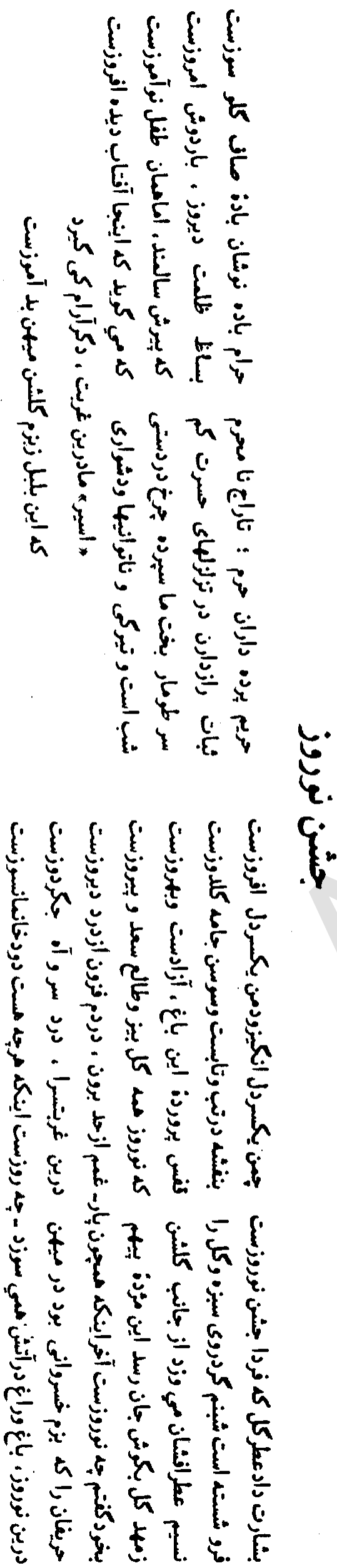

\section{داستان نوروز 42}

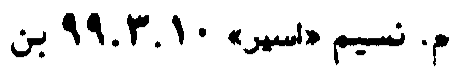

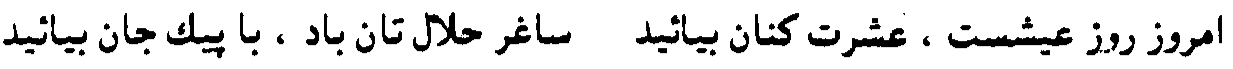

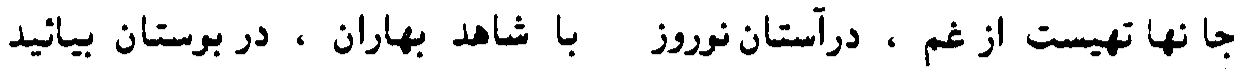

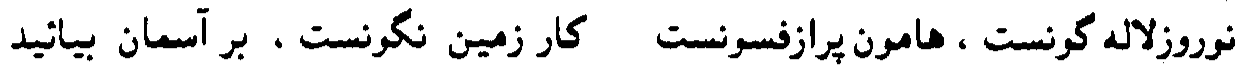

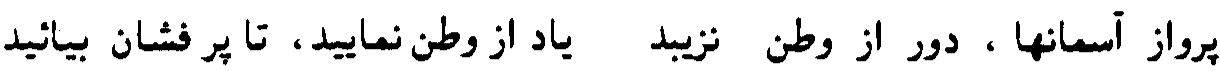

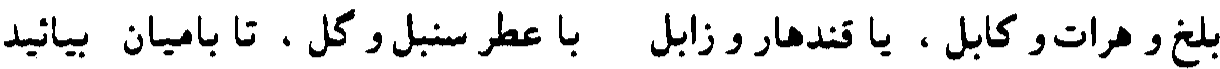

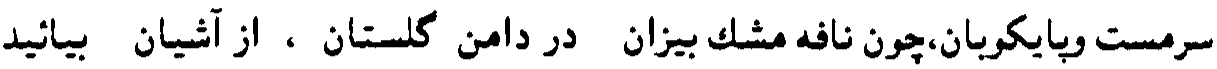

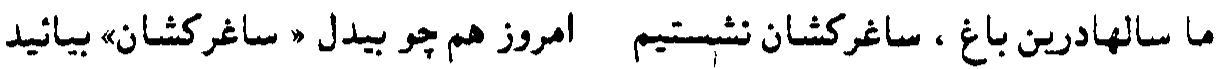

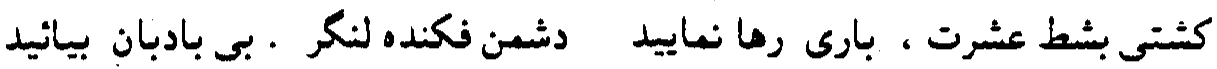

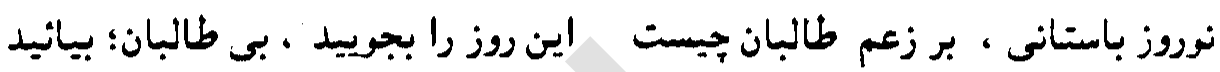

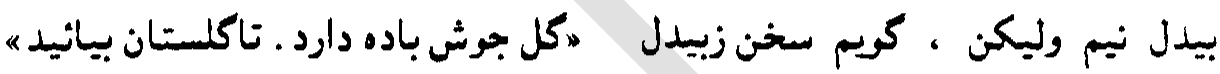

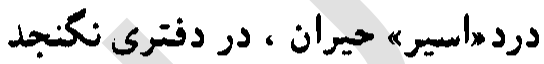
تا باشعابكريد ، اين داستان ؛ بياثيد ، درديد برندهُ جايزهُ درجه اول مشاعرةً دري

\section{بهار در غريث}

جه خرميست ، كه فصل بهار مى آيد به كرش ، زمزمه ماى مزار مى آيد

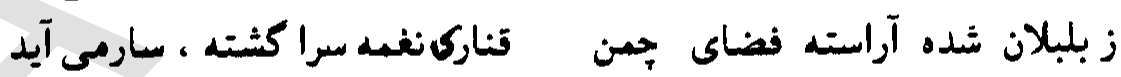

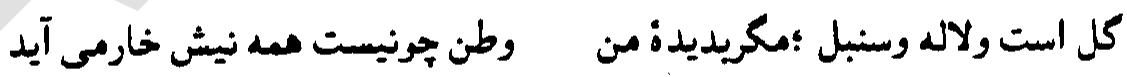

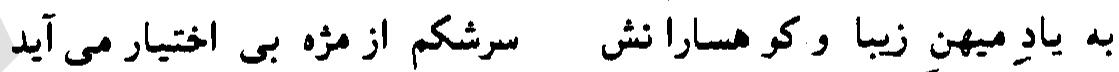

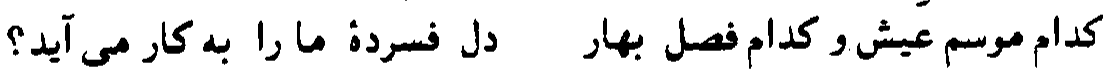

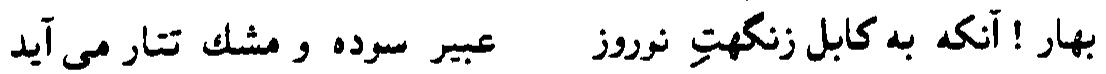

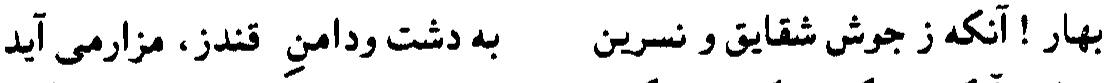

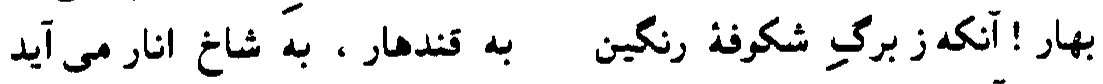

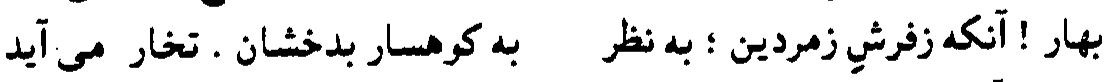

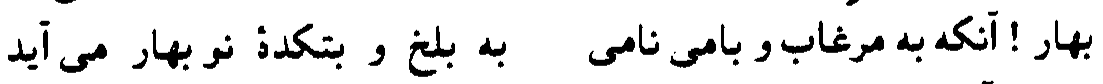

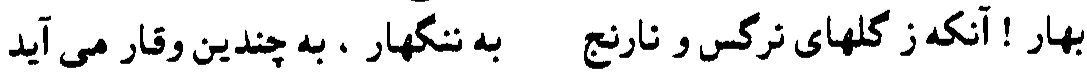

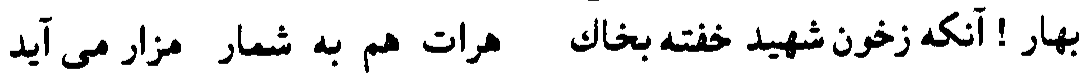

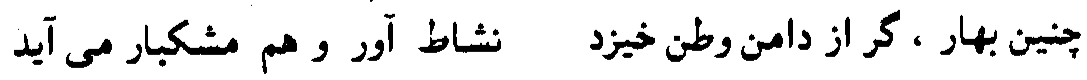

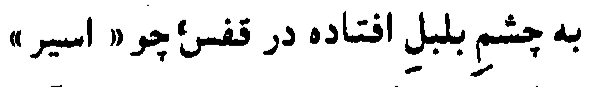

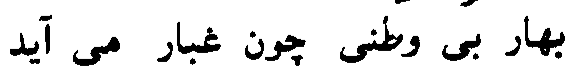

ناهُ سر كشادةُ بروس ريجاردسن زادربخش انظليسي بخوانيد 
sh

Sing hars.

axil

نغارش : :كتورسيد خليل اله ماثبـان

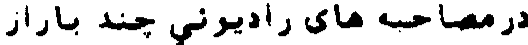

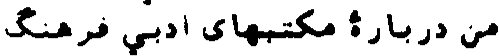

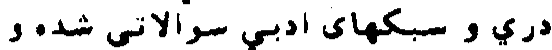

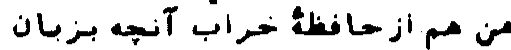

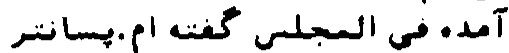

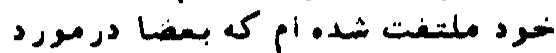

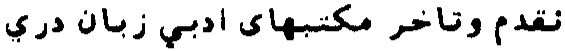

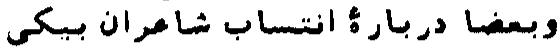

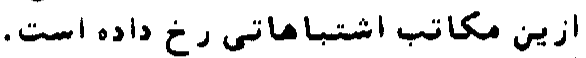

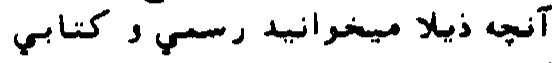

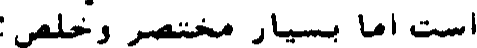

بطور مقدمه بايد مرض شعرد وك اصطللاجات

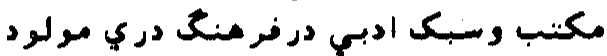

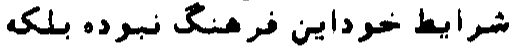

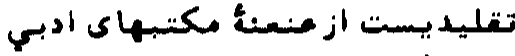

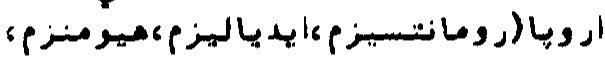

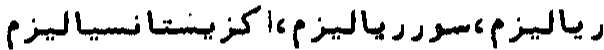

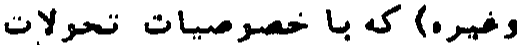

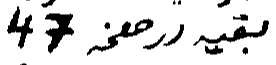

برندة جايز؛ُ درجه مسرم مشاعر مُ دري

\section{4}

از امتاد عبدالغفرر شرر ازفرانسه

كابل آن رثك كلستان ياد باد باد

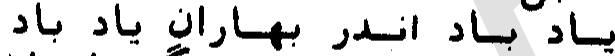

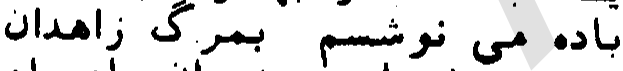

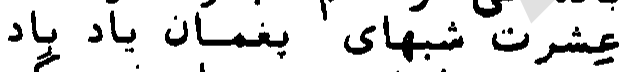

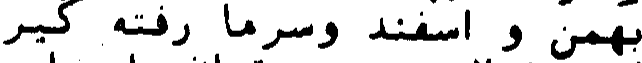

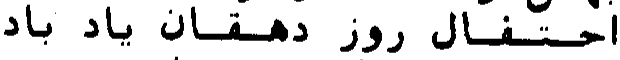

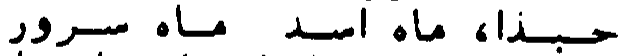

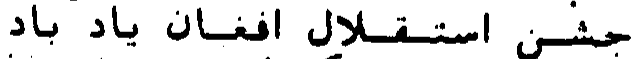

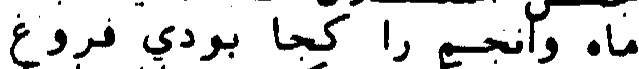

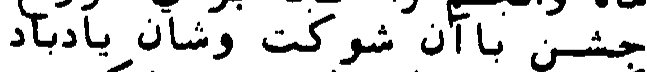

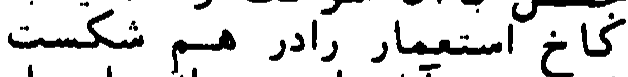

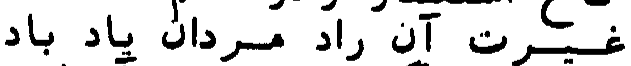

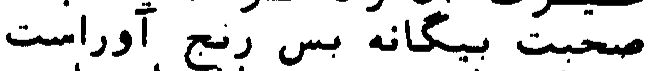

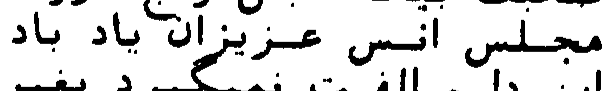

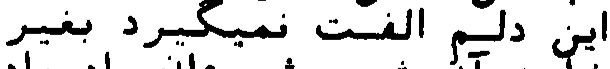

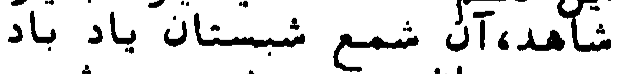

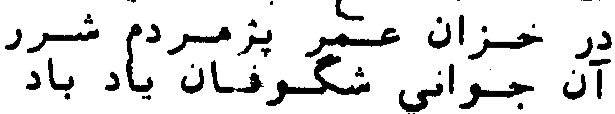

\section{3 نوروزدرون}

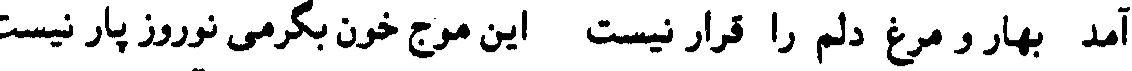

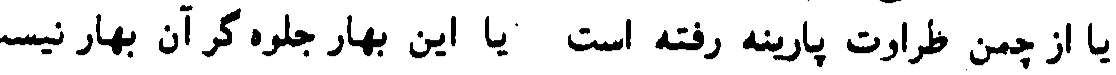

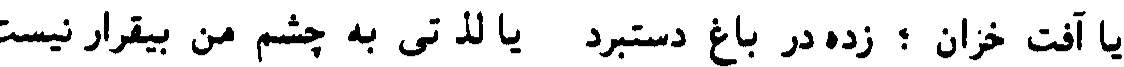

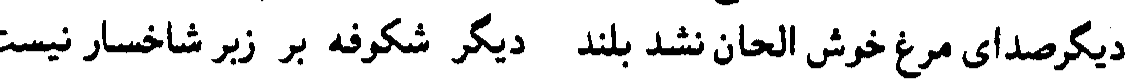

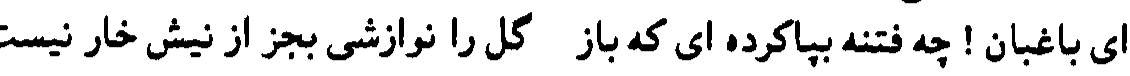

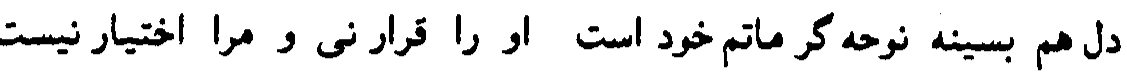

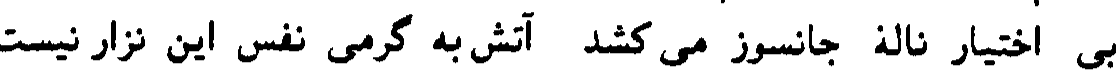

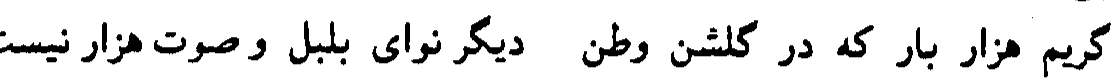

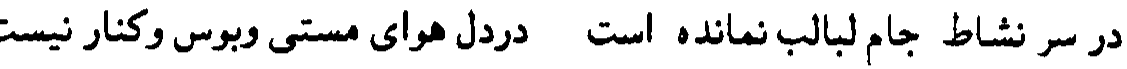

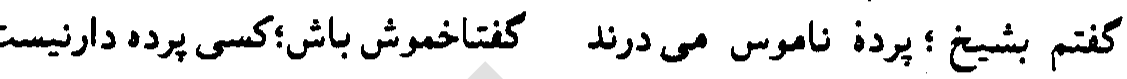

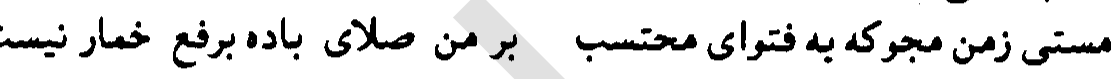

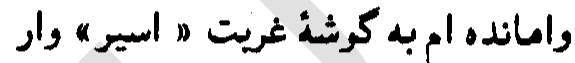
بر خود اميد و بر دكران اعتبار نيست عريت 
برندُ جايزٌُ دربه بنجم مشاعر؛ُ دري

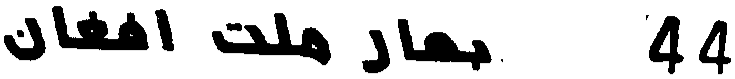

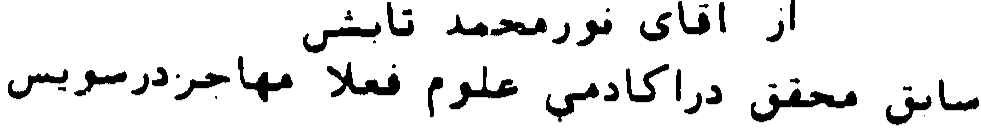

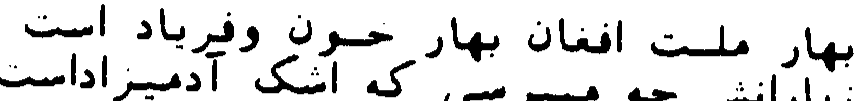

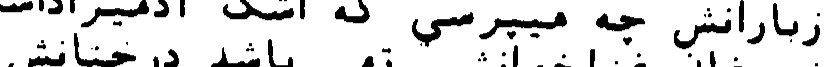

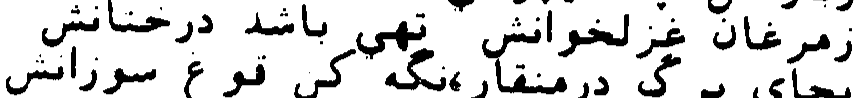

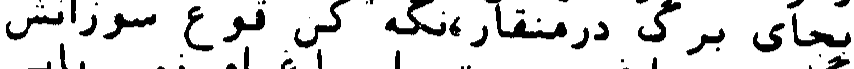

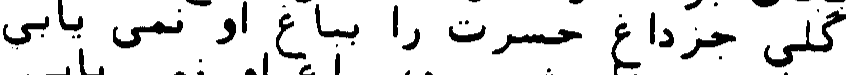

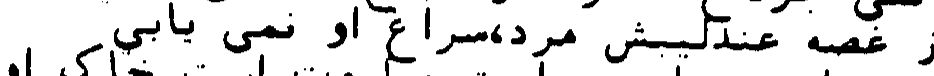

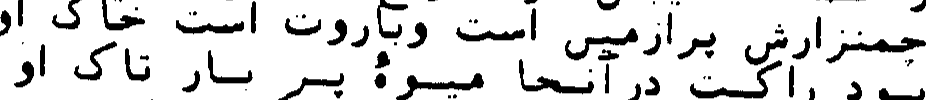

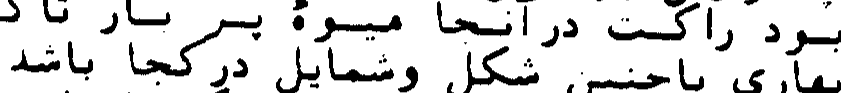

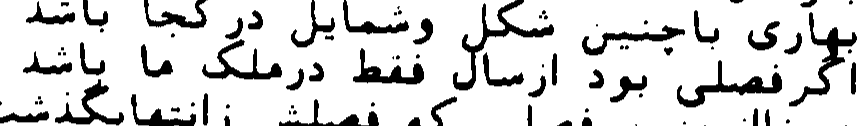

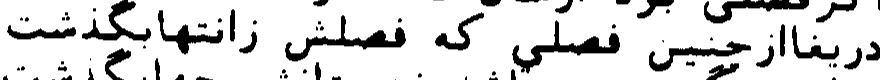

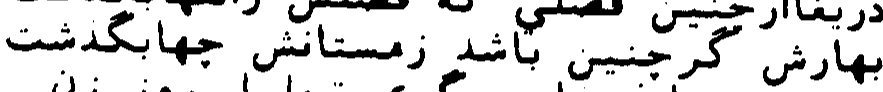

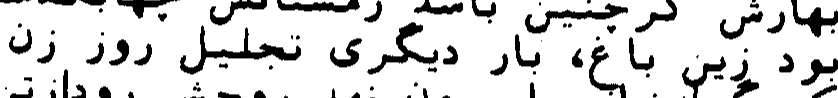

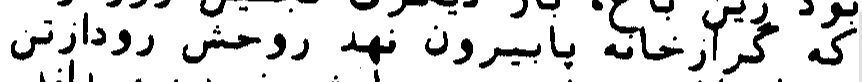

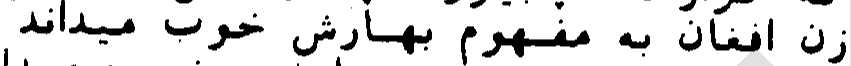

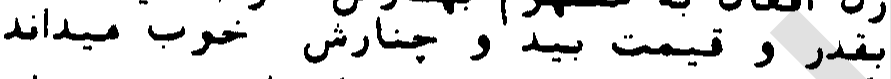

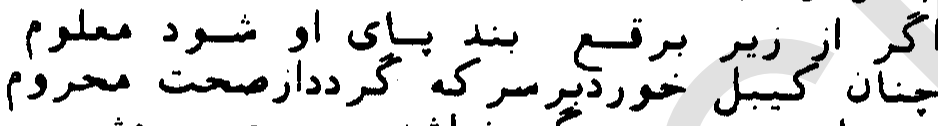

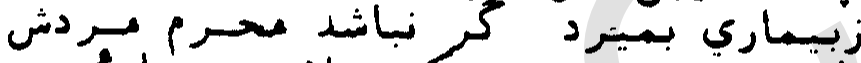

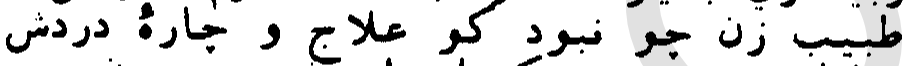

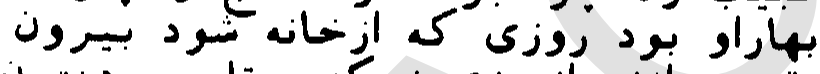

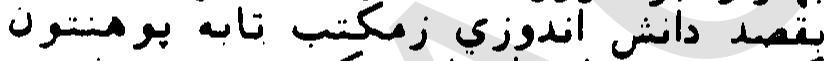

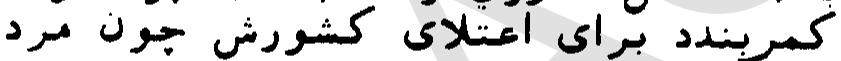

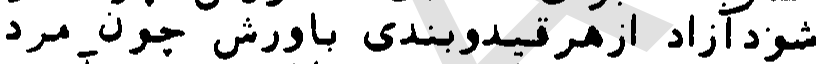

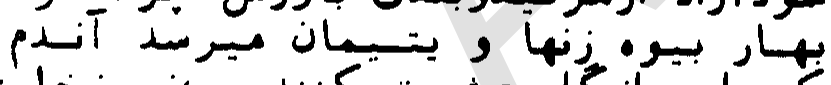

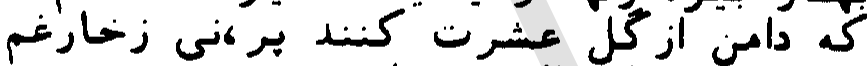

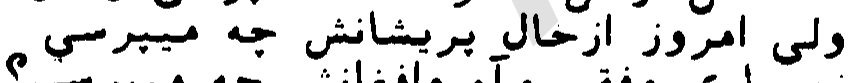

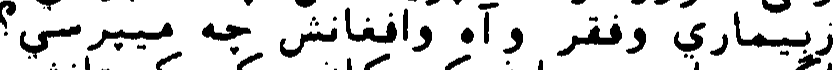

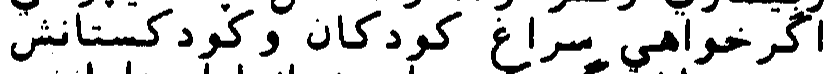

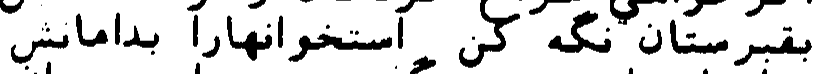

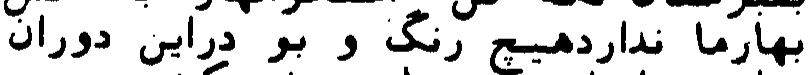

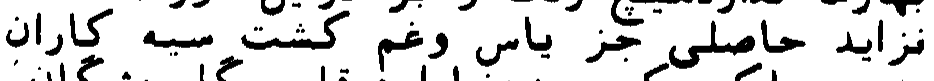

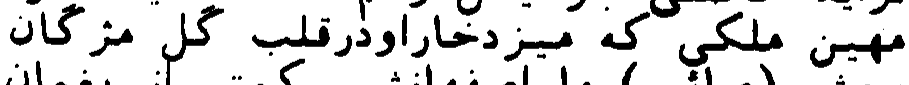

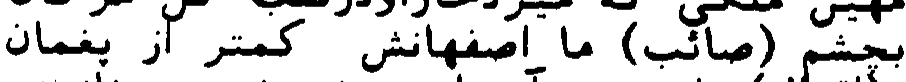

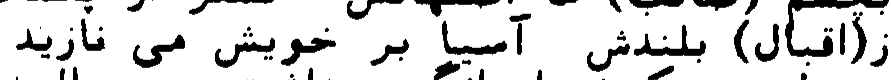

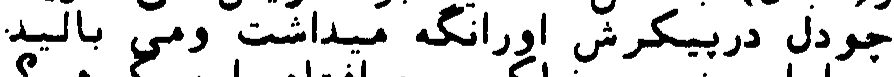

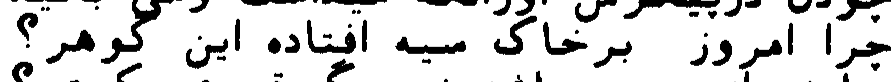

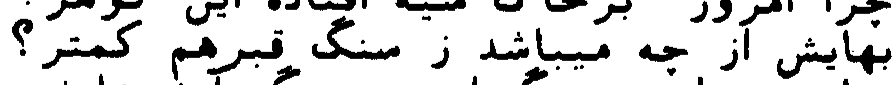

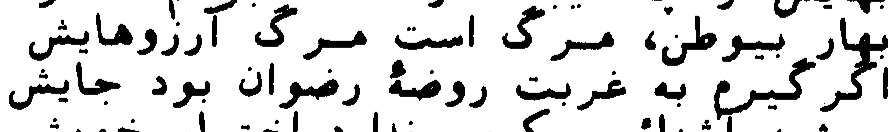

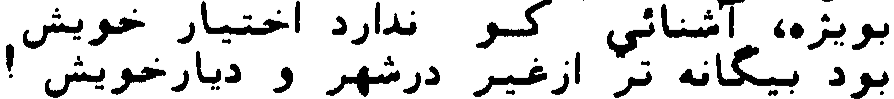

برندهُ جايز• درجه جارم مساعرُ دري

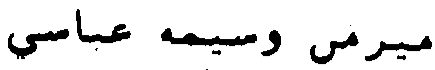

\section{بمار فئر}

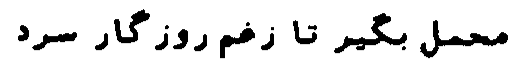

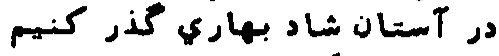

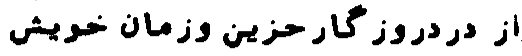

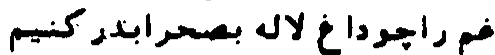

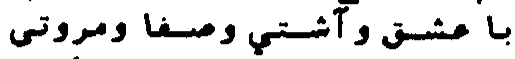

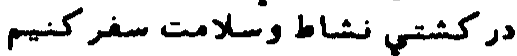

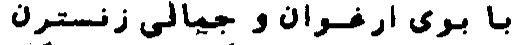

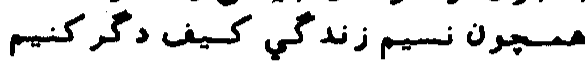

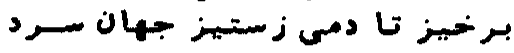

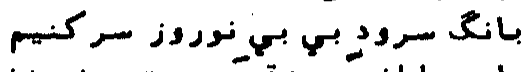

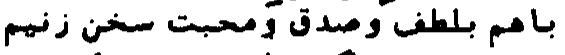

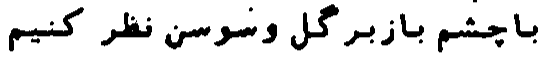

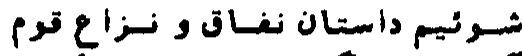

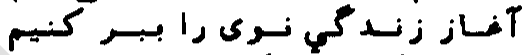

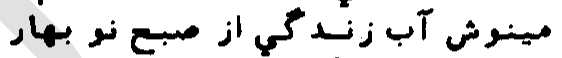

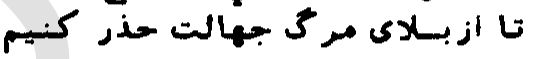

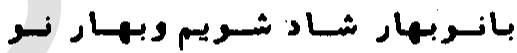

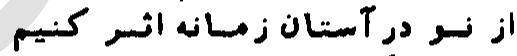

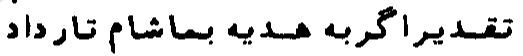

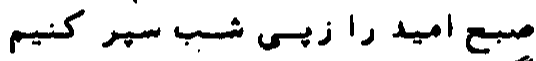

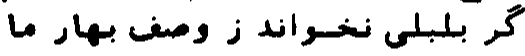

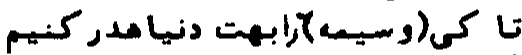

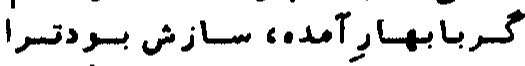
اى معرطن، بهاروطن راخبر كنيمر

بماركلمل وسيه مباسي

زبساد نسربهاران بـاز بـرى قلستان آيد

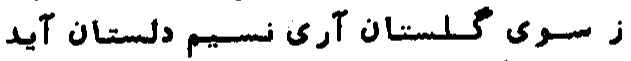

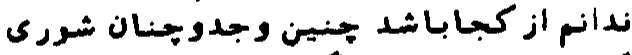

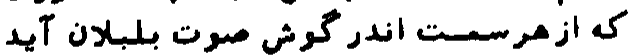

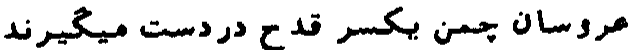

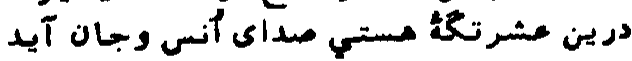

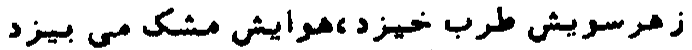

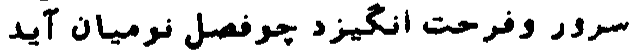
جمسن فرش است از الساس شبنم درسيحر كاهان

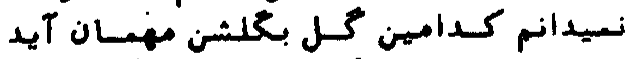

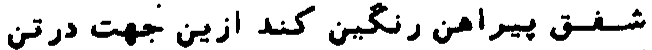

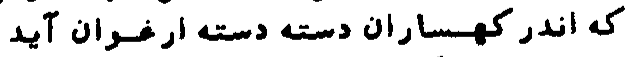

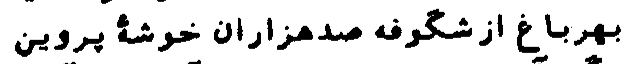

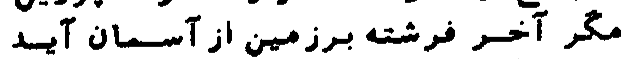

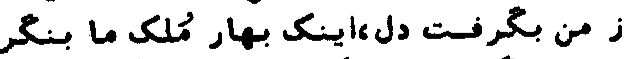

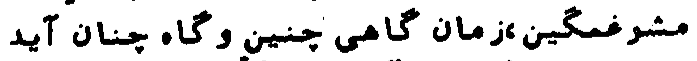

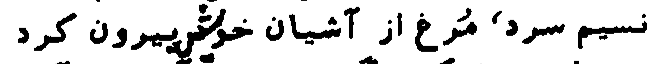

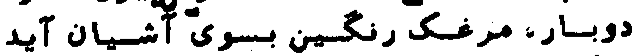

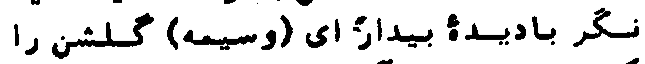

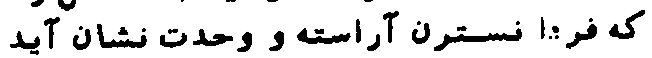




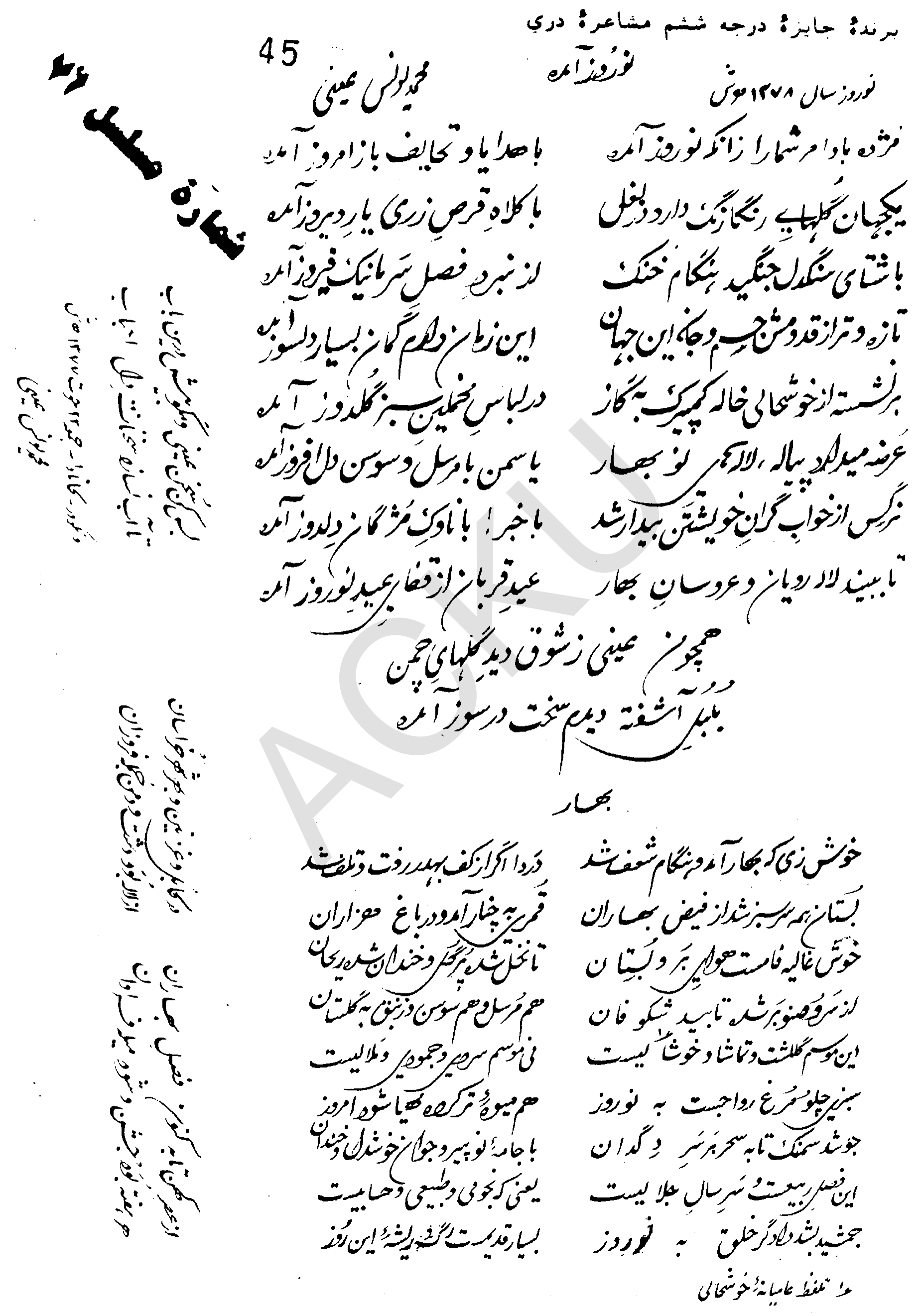




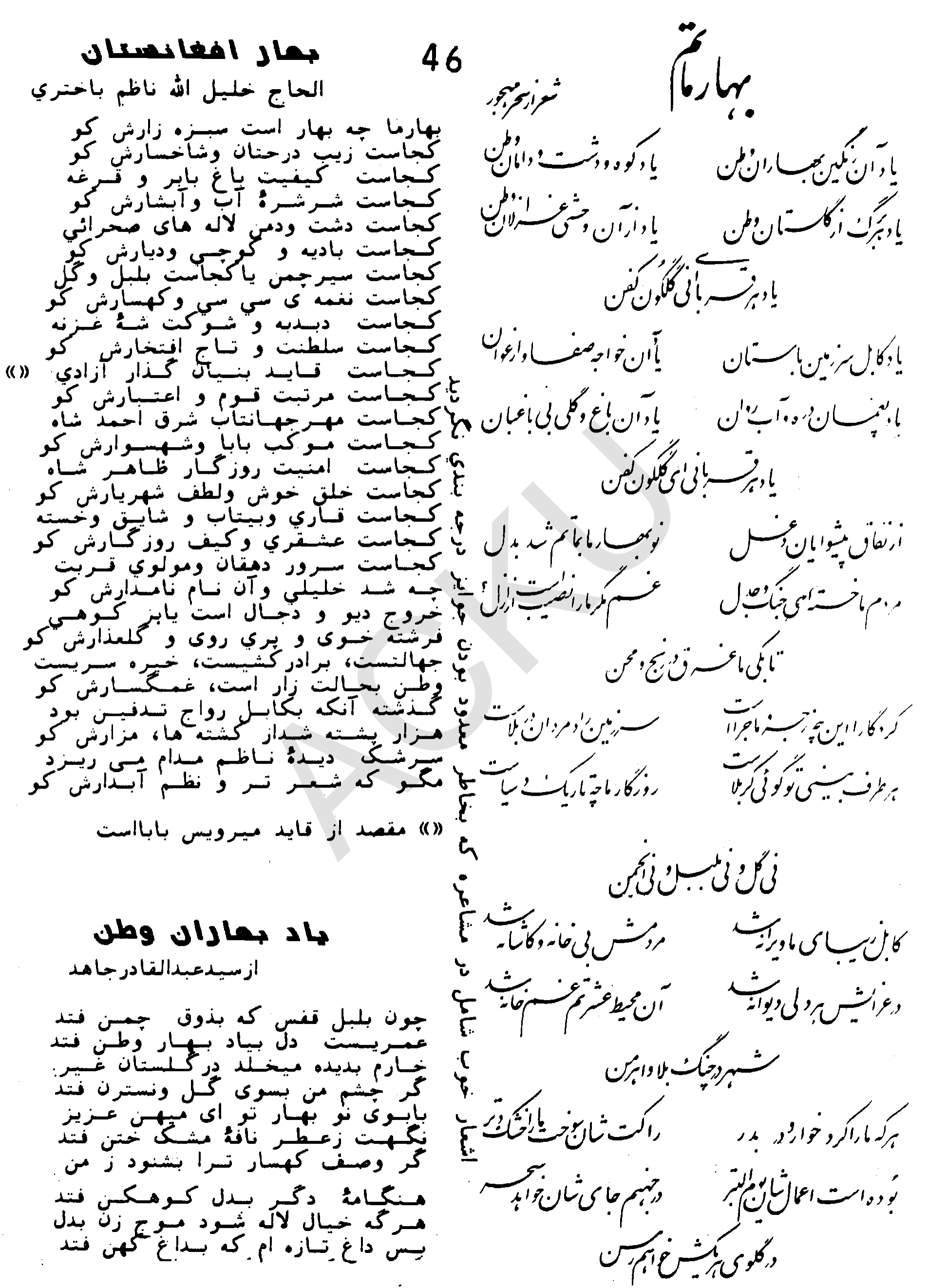




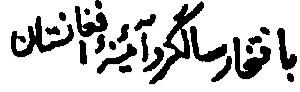

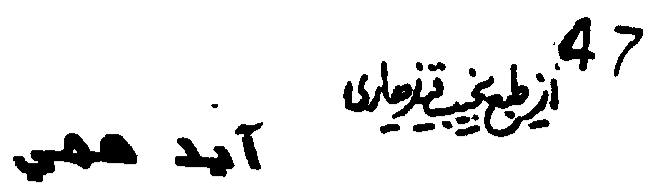

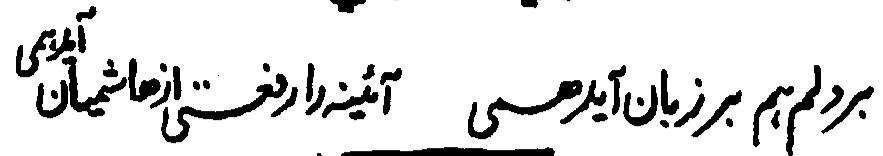

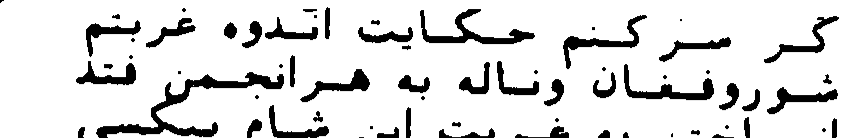

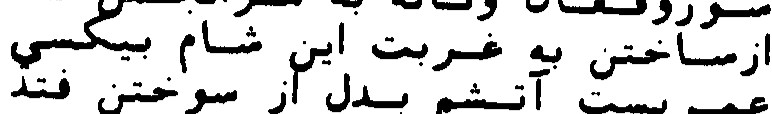

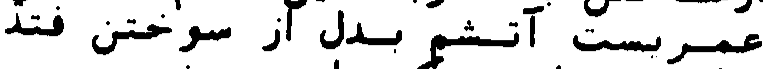

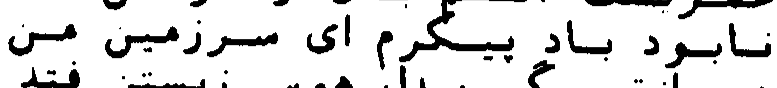
حس

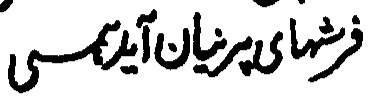

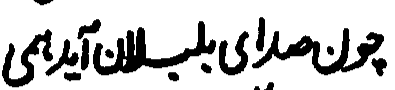

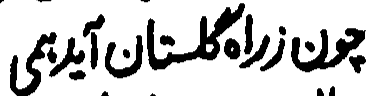

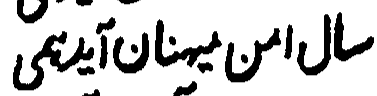

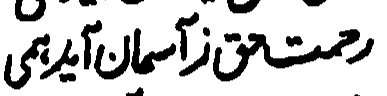

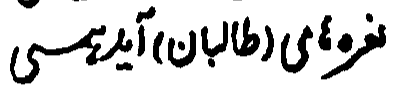

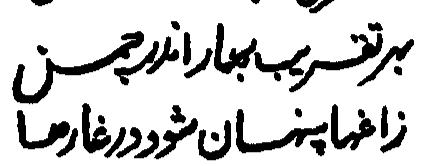

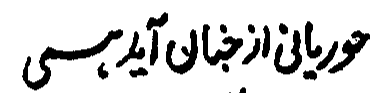

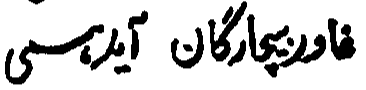

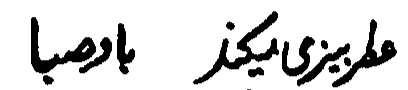

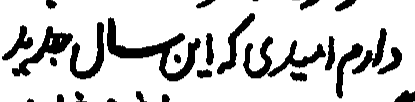

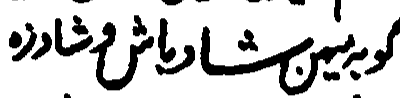

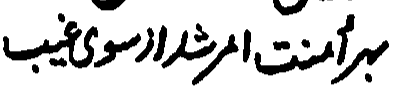

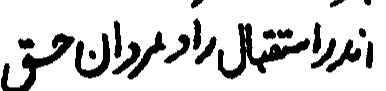

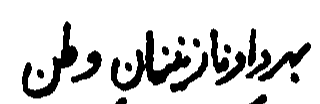

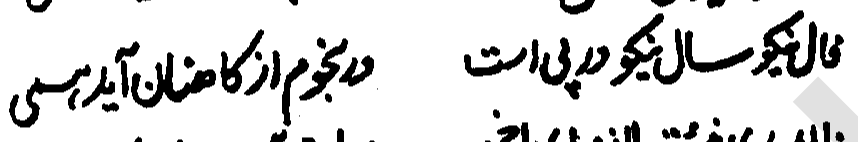

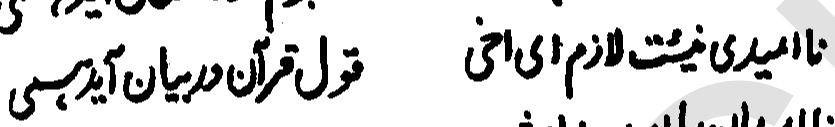

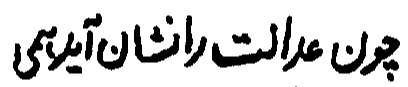

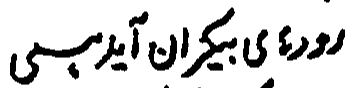

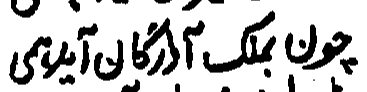

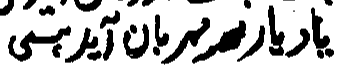
c6

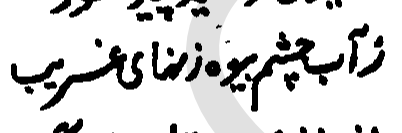

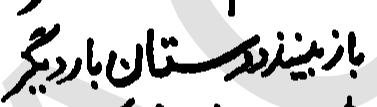

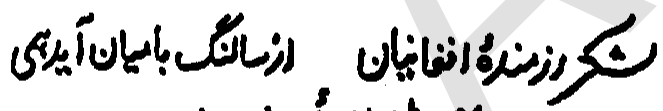
(1)

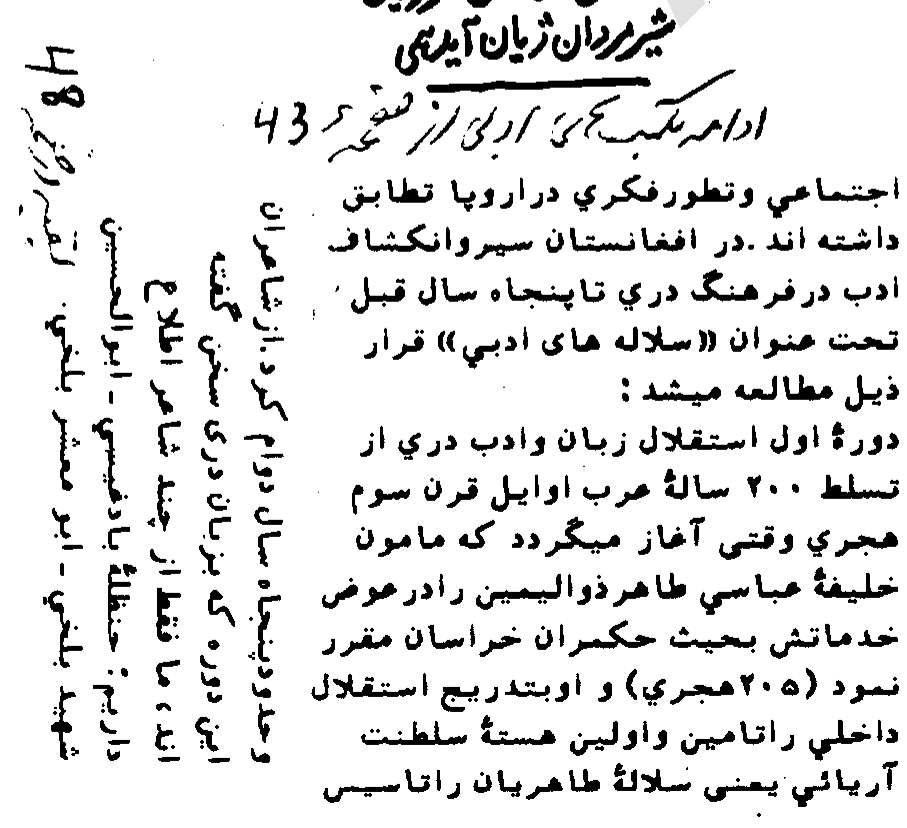

\section{بمار آرامي وطن}

$$
\text { از آقاى مسمد كريم شبون: }
$$

.

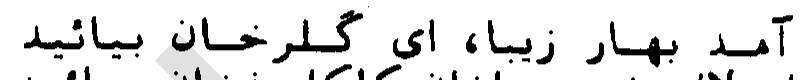

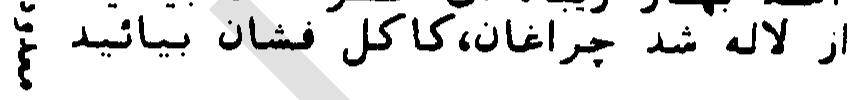

زين آمدآمدنها، خوش فرصنيست درراه

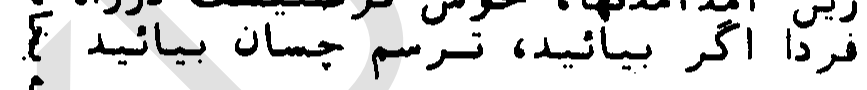

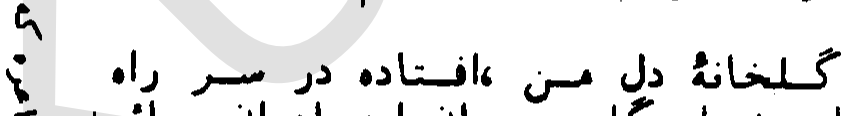

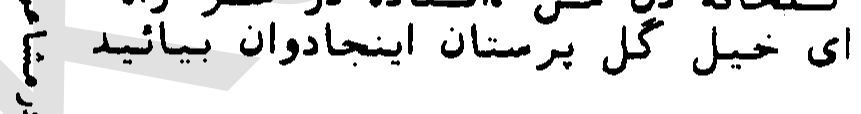

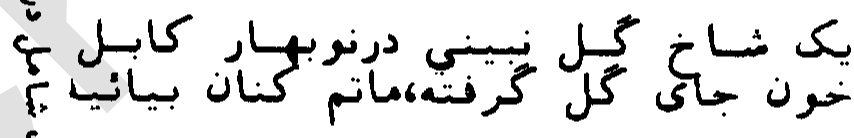

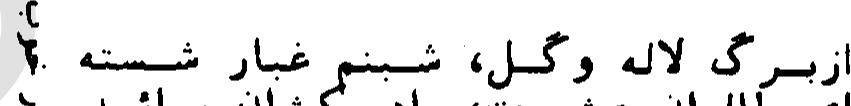

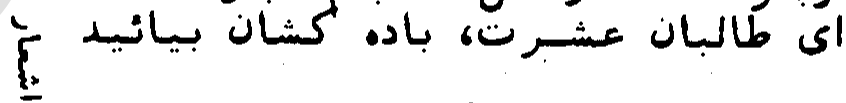

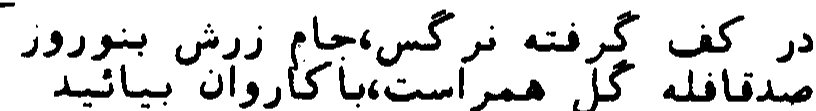

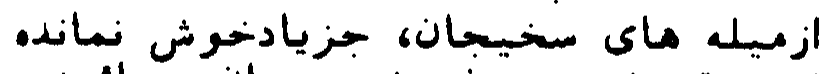

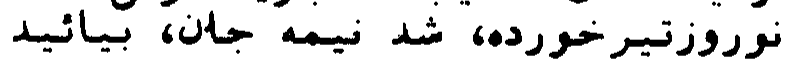

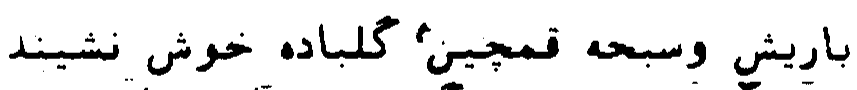

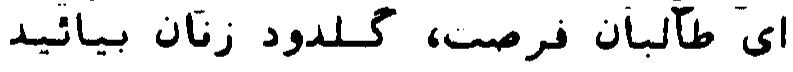

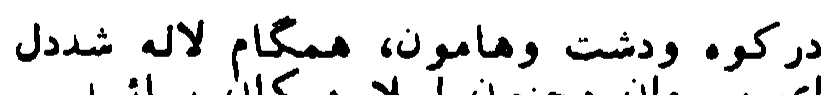

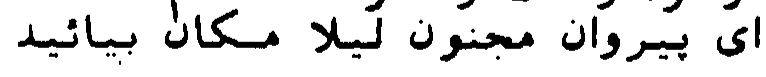

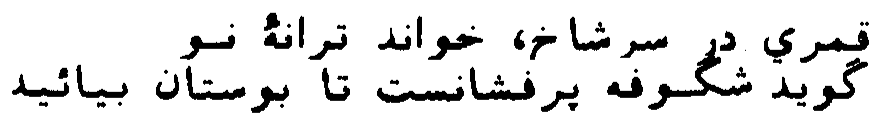

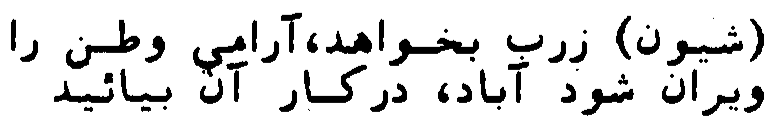

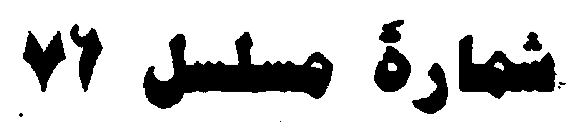




\section{viglay ow}

\section{ازحبيبي (سها)}

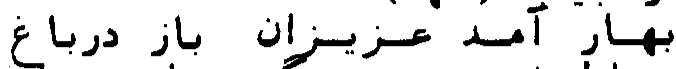

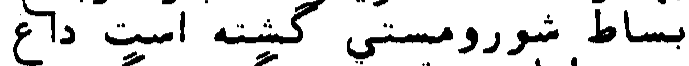

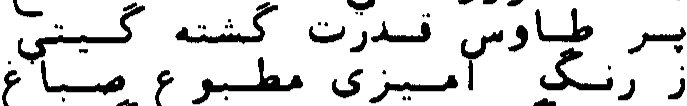

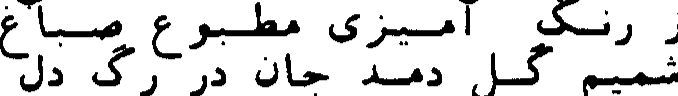

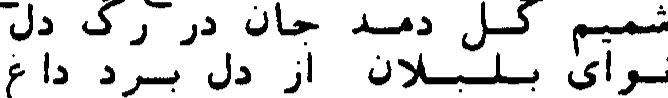

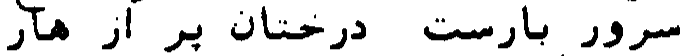

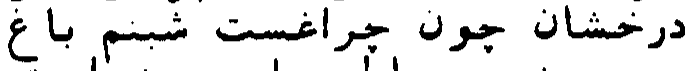

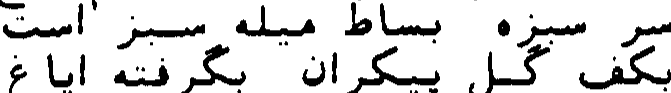

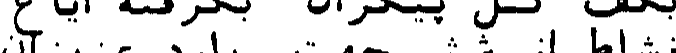

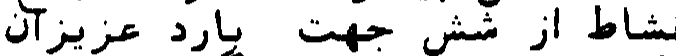

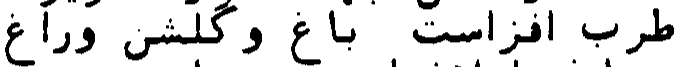

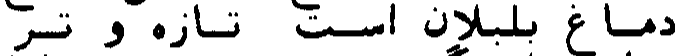

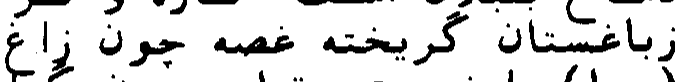
(مهاب) را نيستان

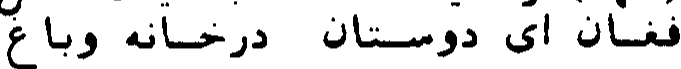

\section{جماد وقراد}

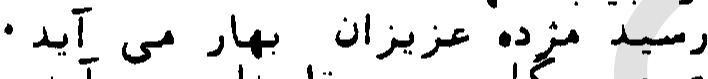

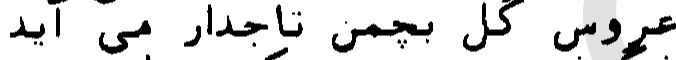

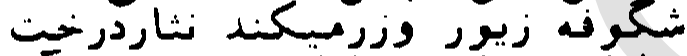

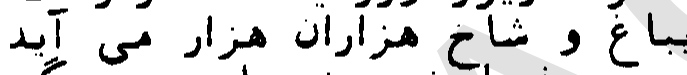

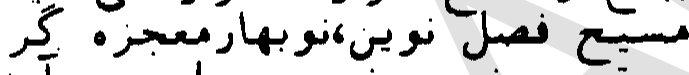

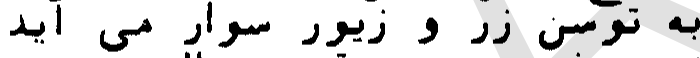

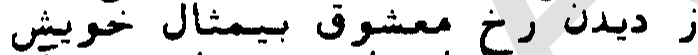

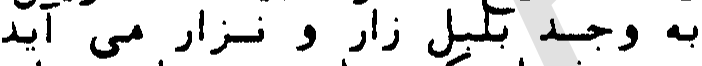

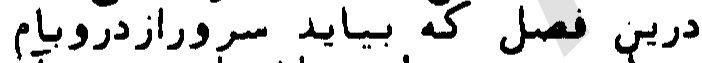

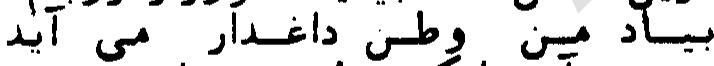

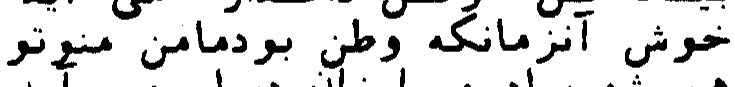

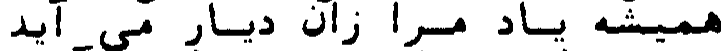

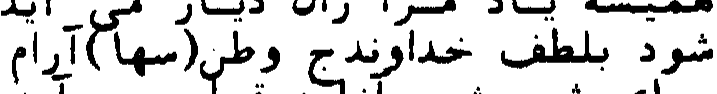

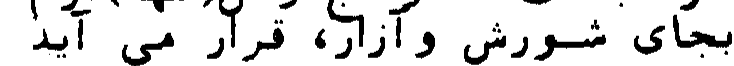

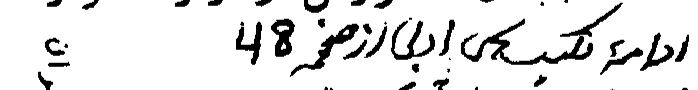

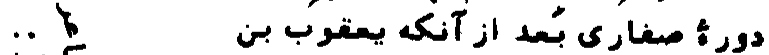

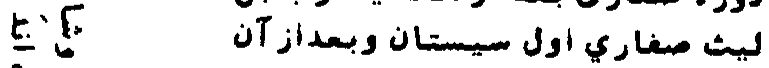

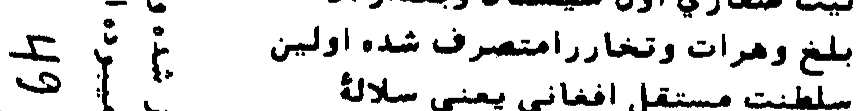

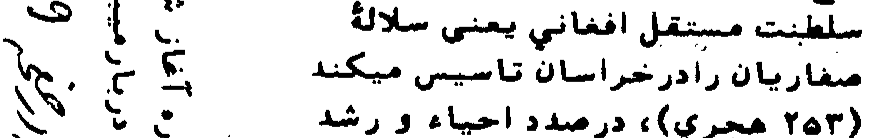

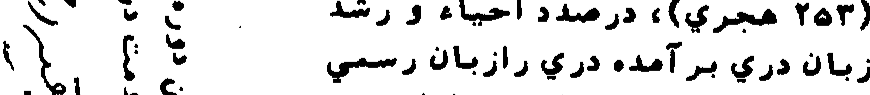

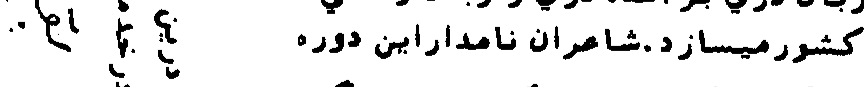

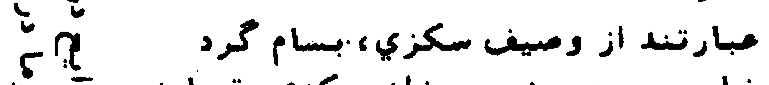

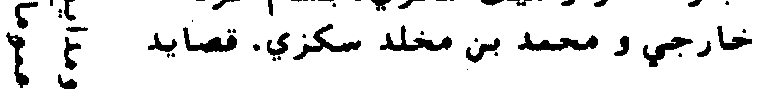

3

3

$\frac{1}{2}$

3

$\frac{5}{3}$

3

\section{همد كاهل • " سال ههل}

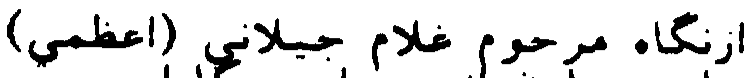

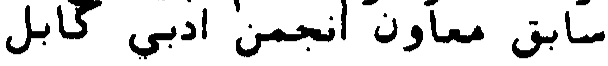

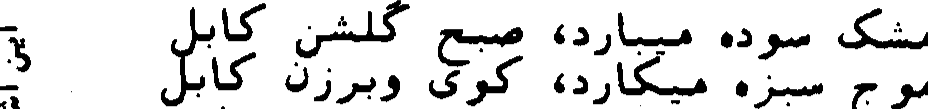

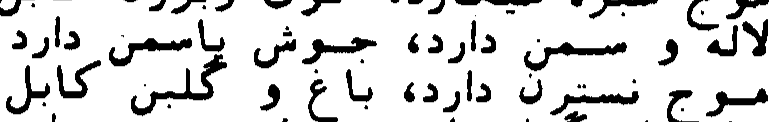

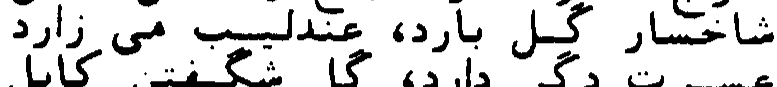

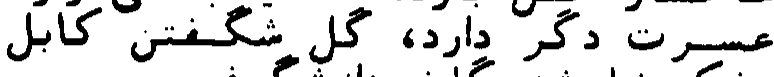

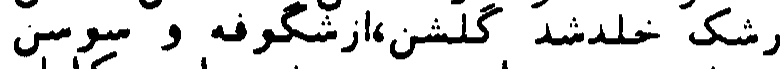

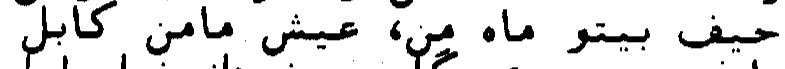

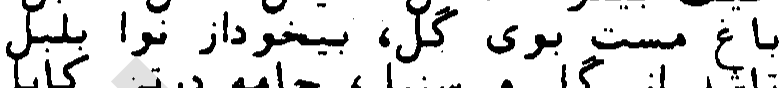

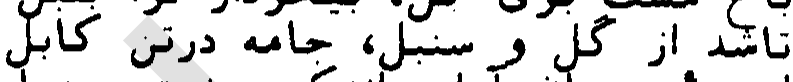

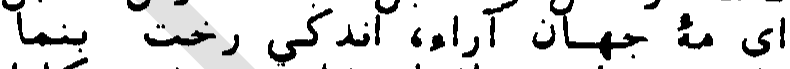

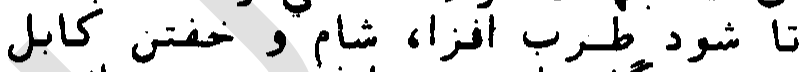

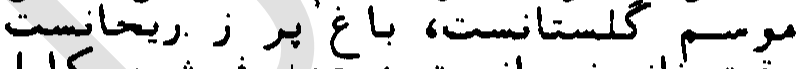

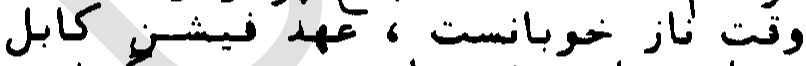

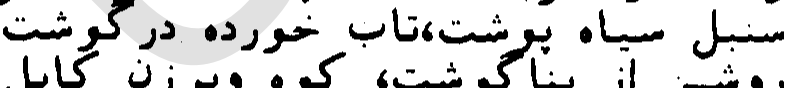

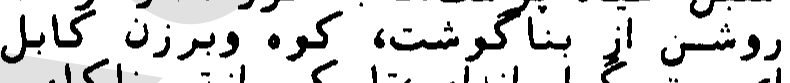

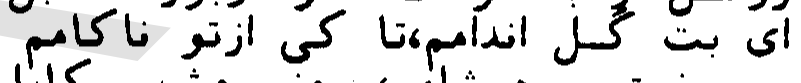

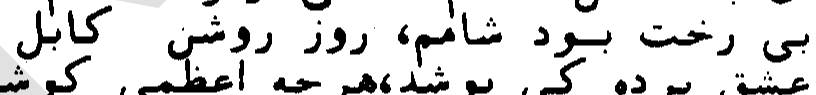

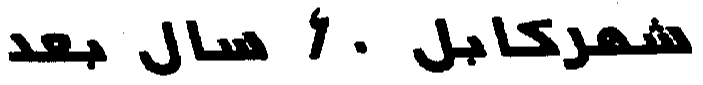

$$
\text { ازعبدالوهاب (غانل) }
$$

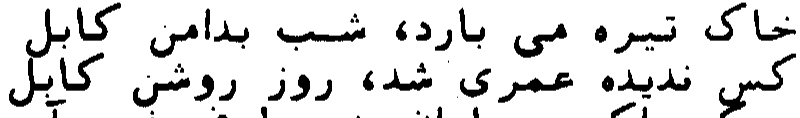

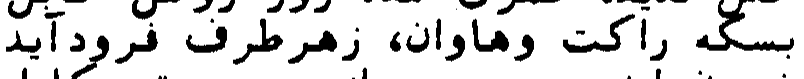

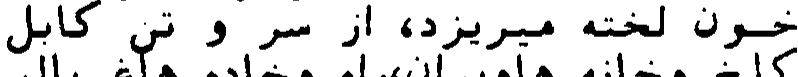

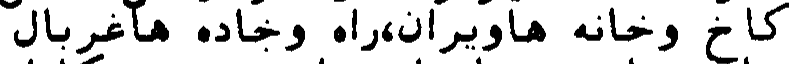

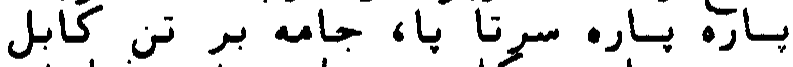

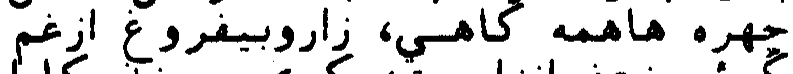

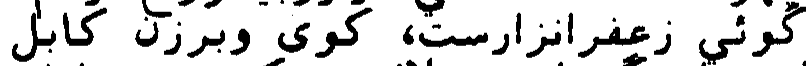

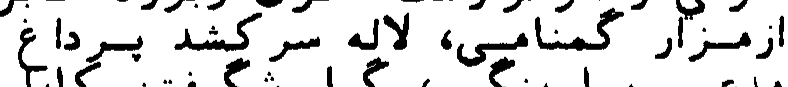

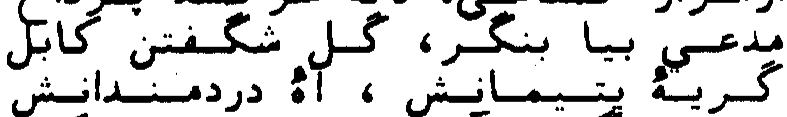

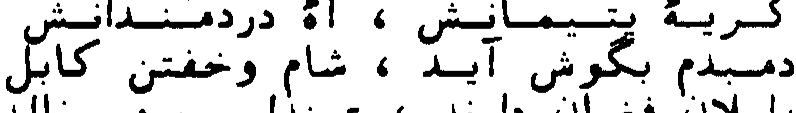

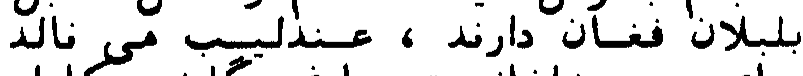

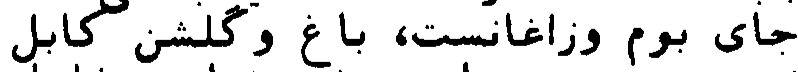

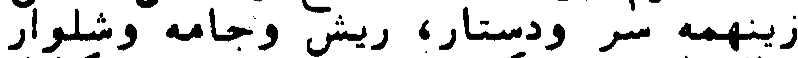

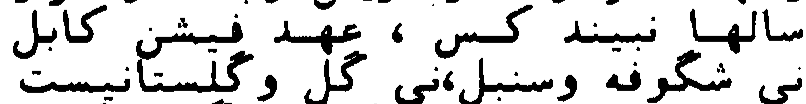

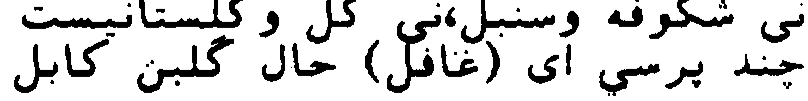




\section{همار ملع}

منظومة ديكر ارآتاى احمد صديق حياء

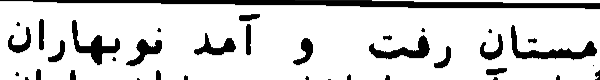

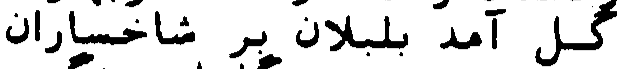

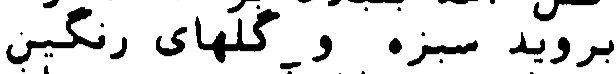

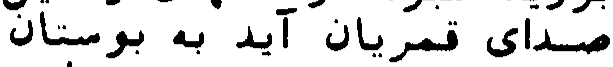

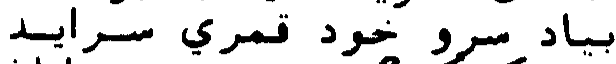

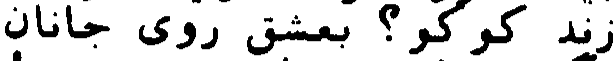

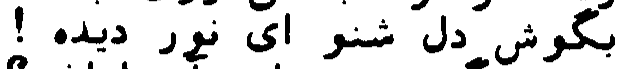

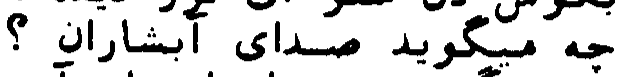

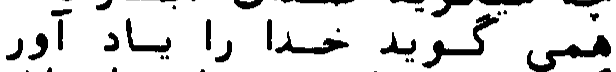

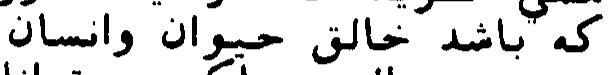

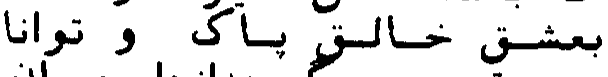

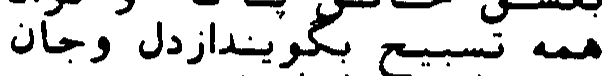

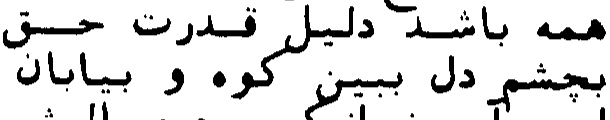

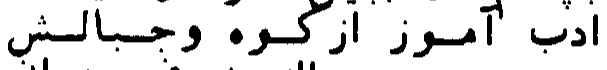

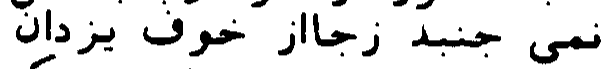

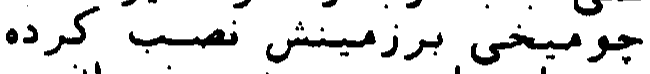

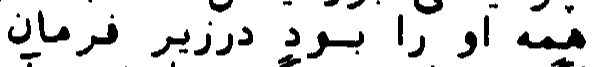

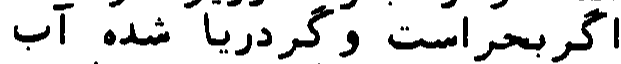

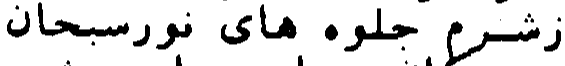

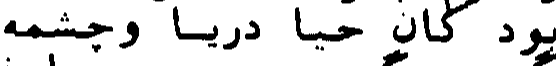

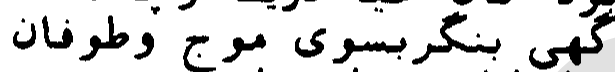

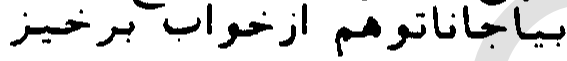

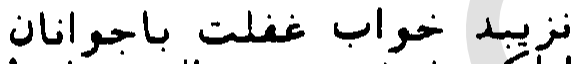

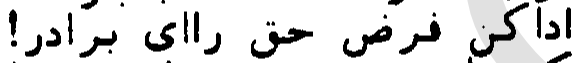
كم تاخنشي شودوسي

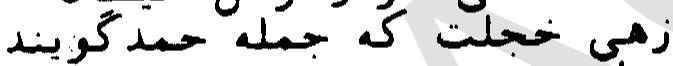

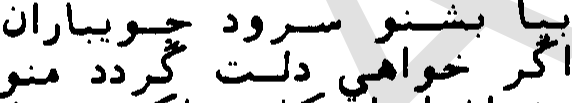

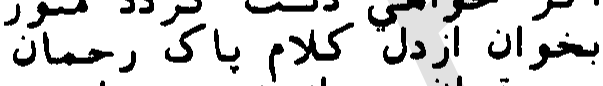

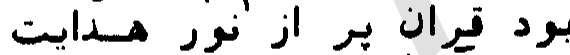

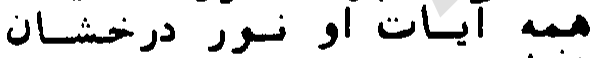
شفاء ورحمت وختير وسعادت

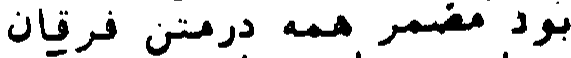

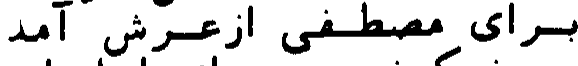

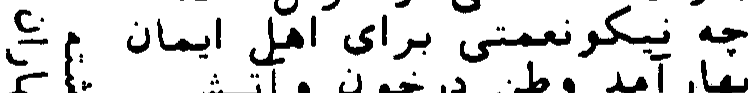

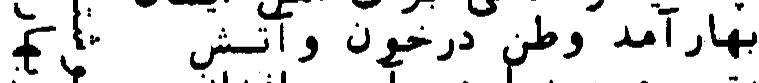
ك.

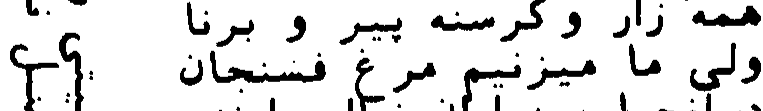

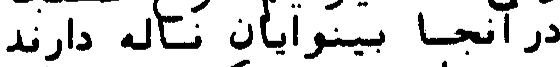

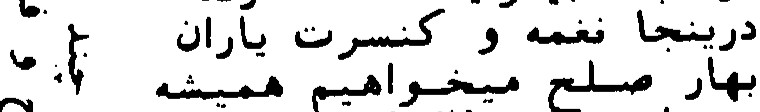

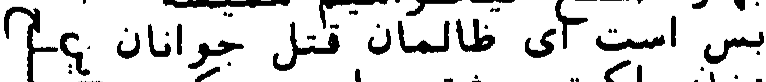

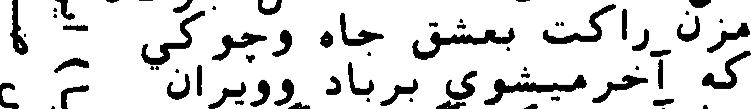

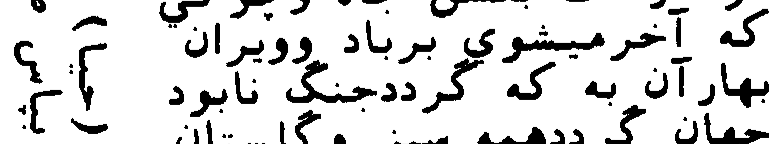

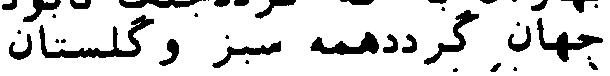

از آناى احمد صديق حياء ازآستراليا

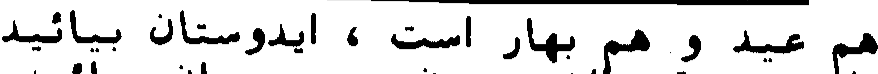

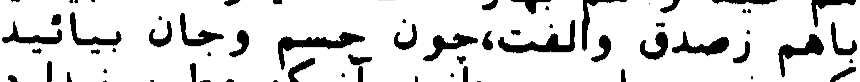

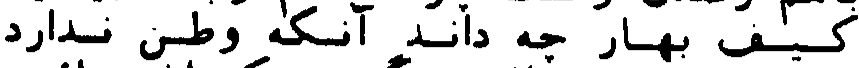

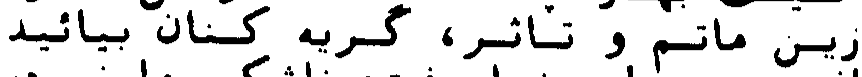

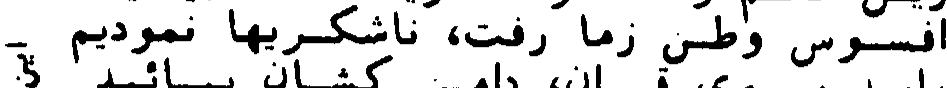

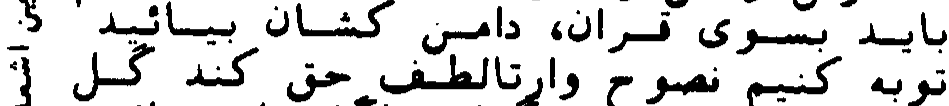

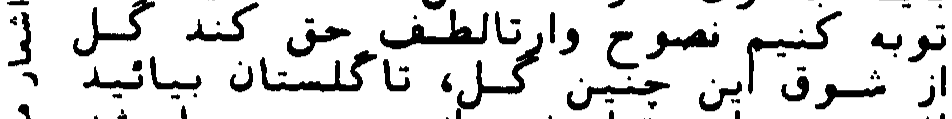

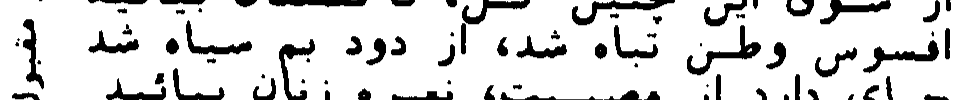

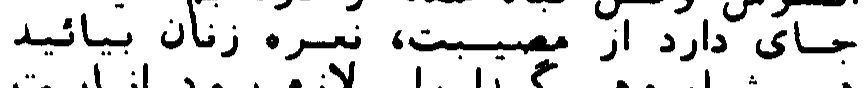

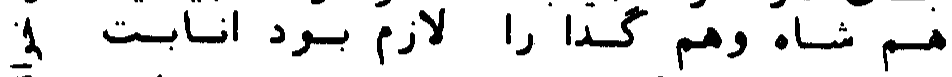

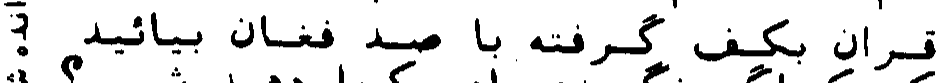

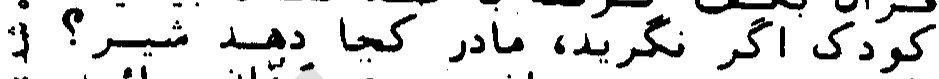

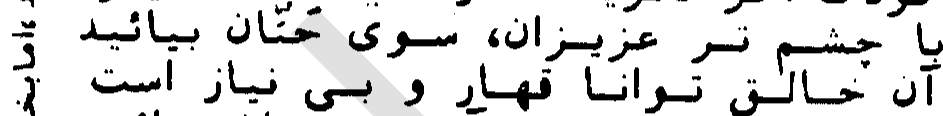

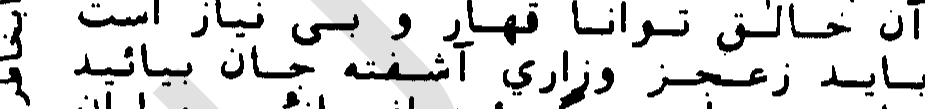

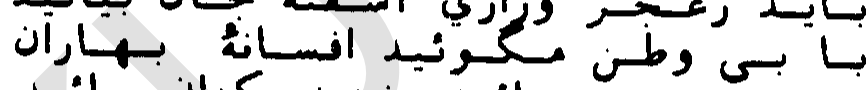

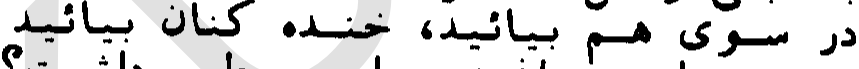

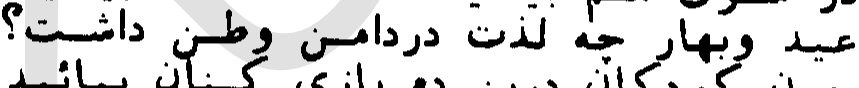

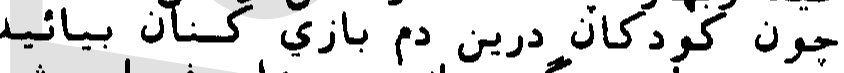

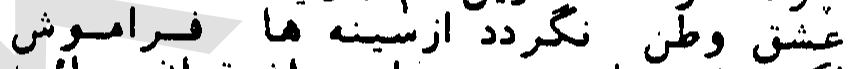

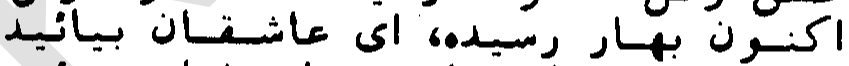

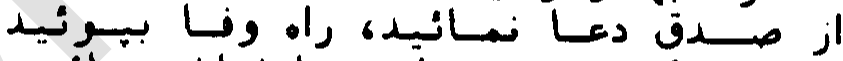

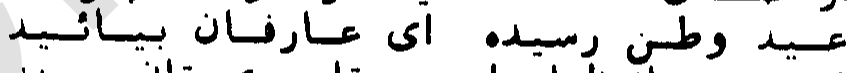

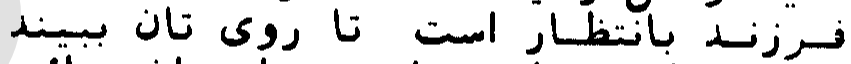

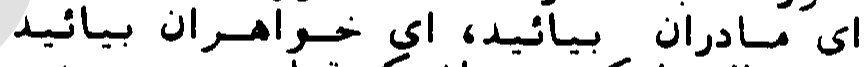

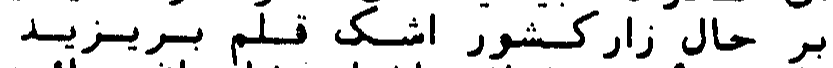

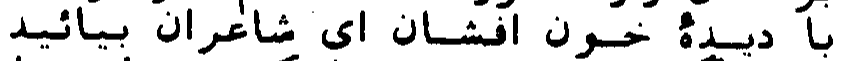

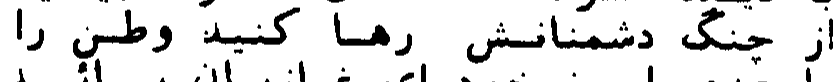

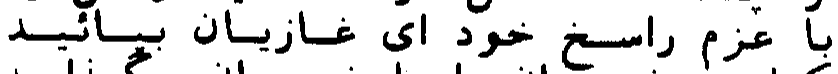

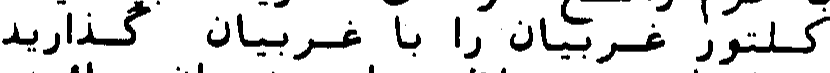

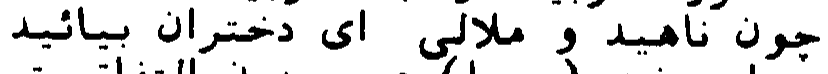

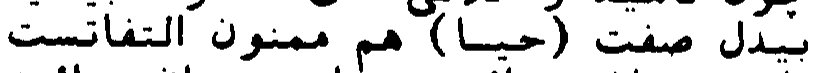
نأ مهـربان بيـاتيديد يـا مهربـان بيانيد

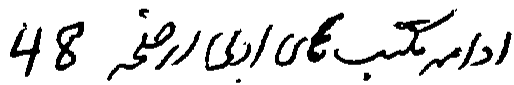

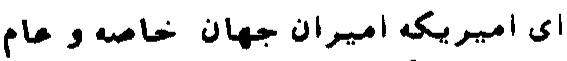

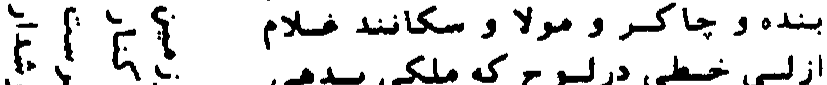

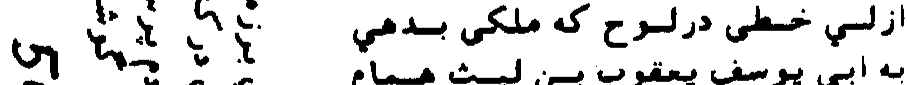

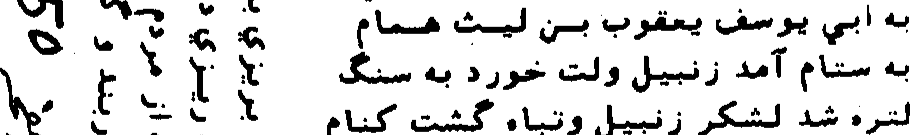

لتروشئ لشكر زنبيل وتبا.

(ازوميف سكزي)

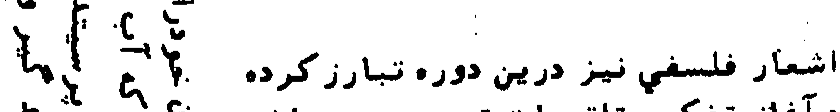

|l. درى راتشيل ميكند أين دوبيت ازمخلد 


\section{بمار الهغانسيان عزمل}

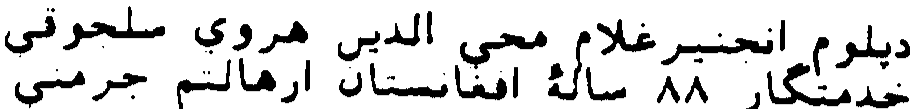

برشكو. است نعرونتينت وناج سلطان بهار

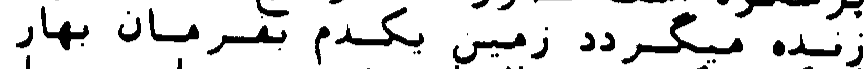

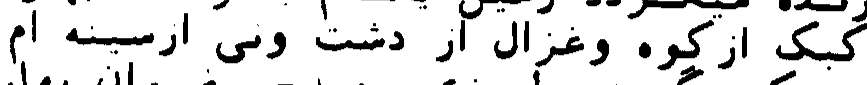

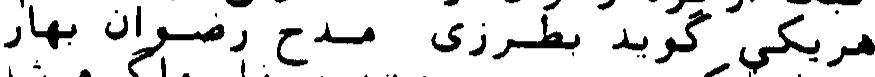

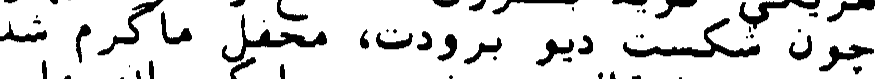

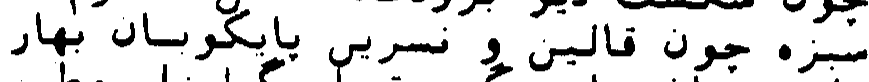

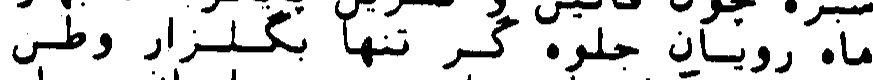

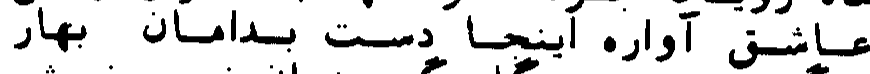

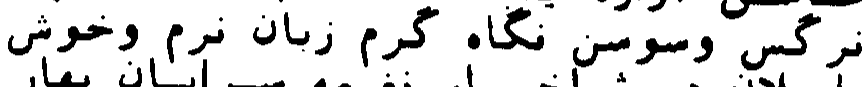

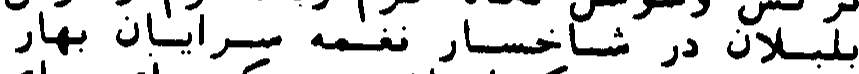

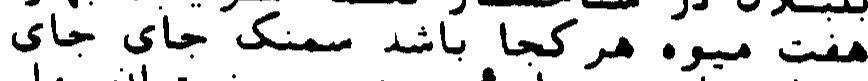

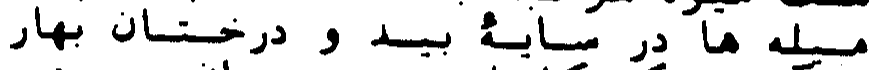

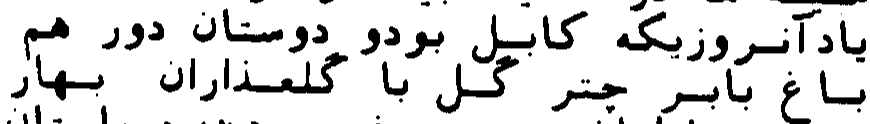

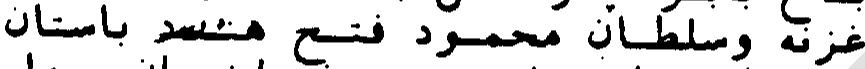

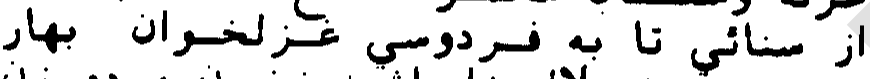

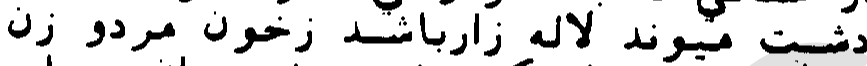

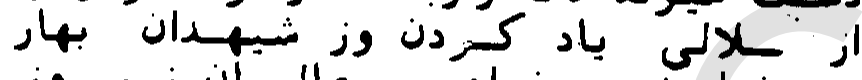

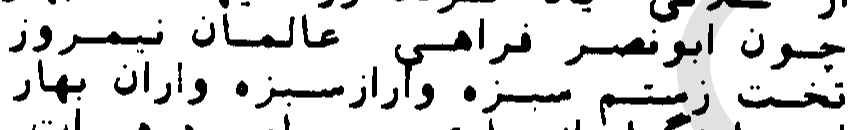

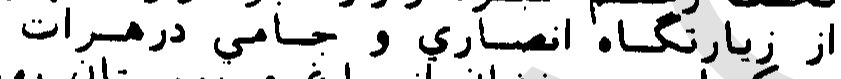

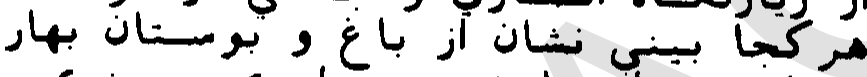

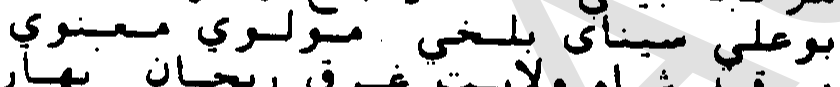

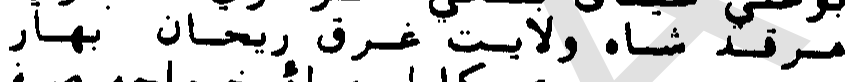

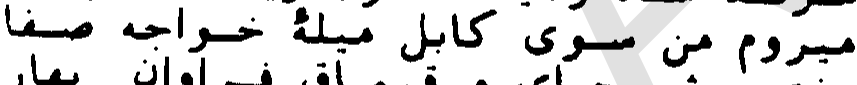

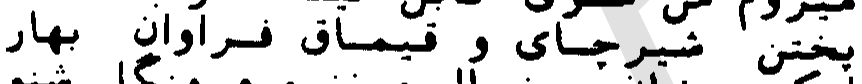

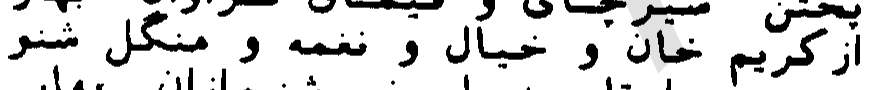

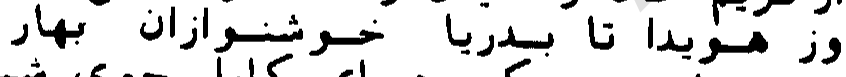

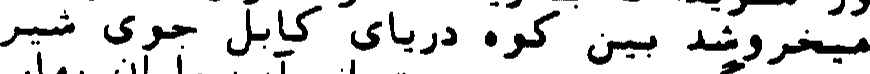

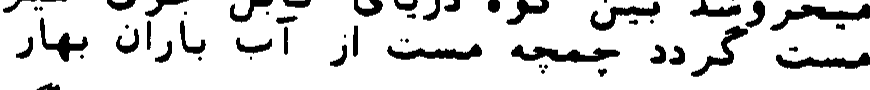

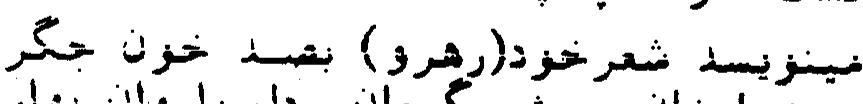

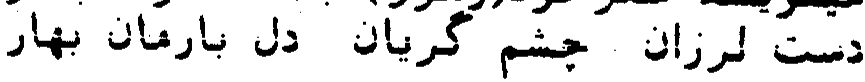

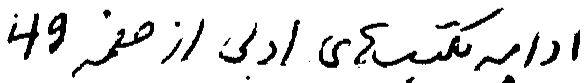

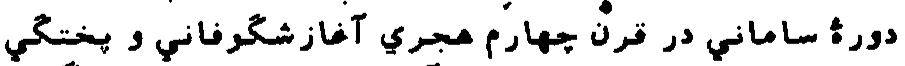

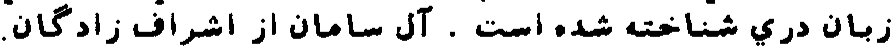

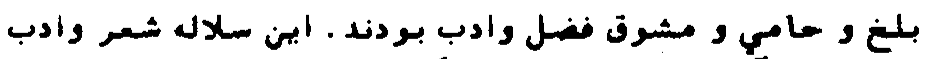

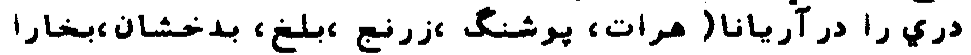

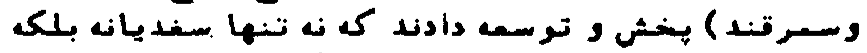

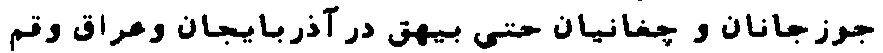

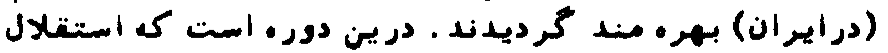

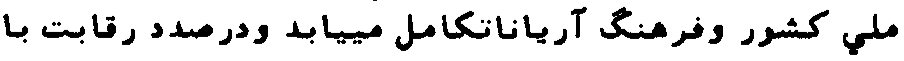

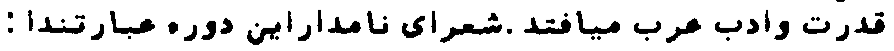

\section{$2+99$}

از عسدالهمد ومابزاده فرياراز آلمان

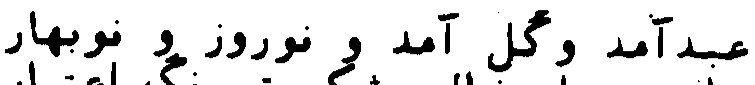

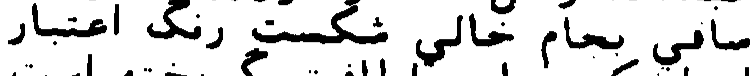

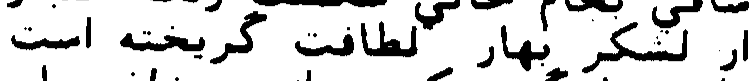

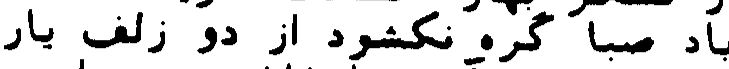

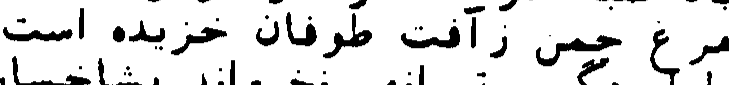

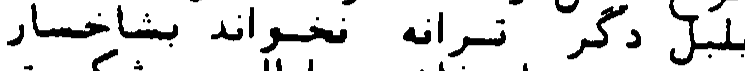

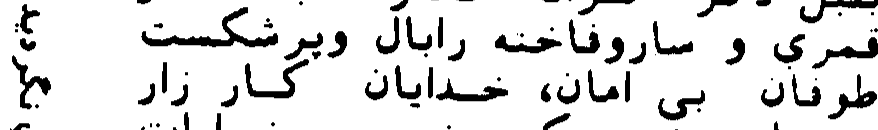

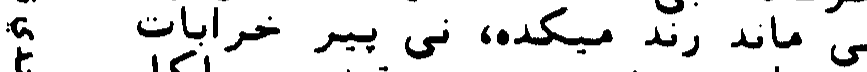

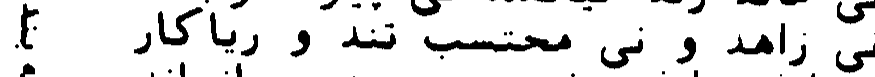

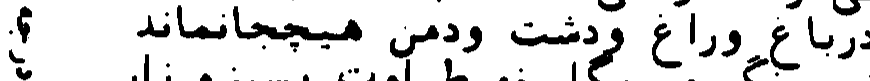

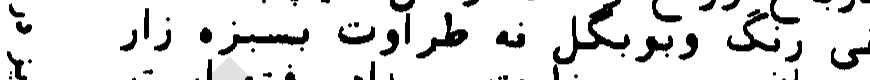

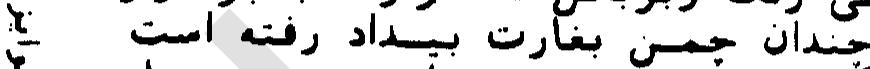

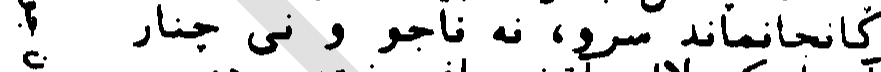

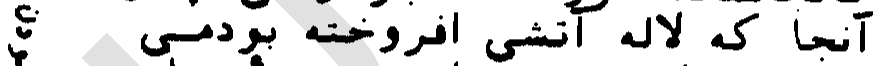

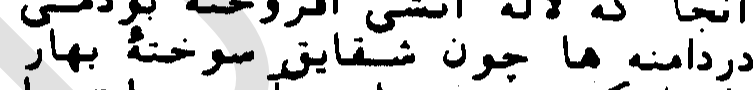

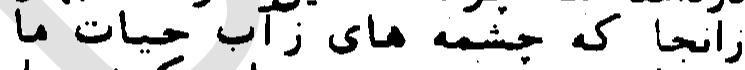

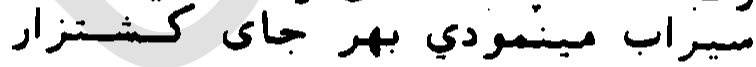

مام وطن بنخون وبه آتش نشسته امتح فوريت

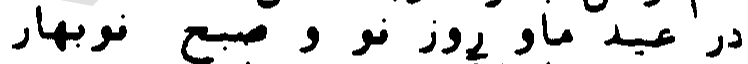

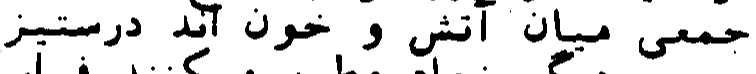

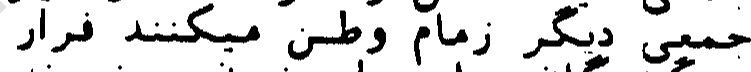

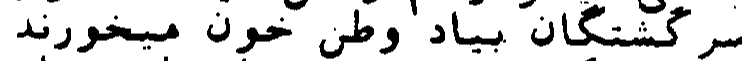

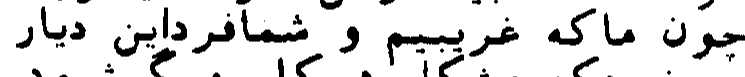

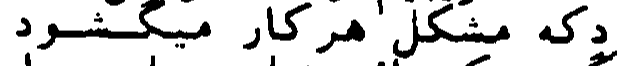

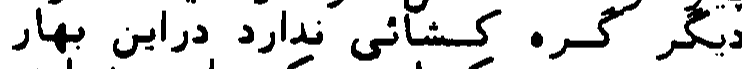

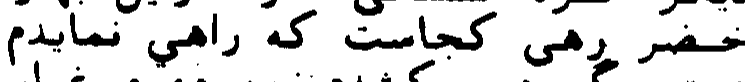

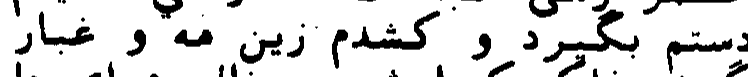

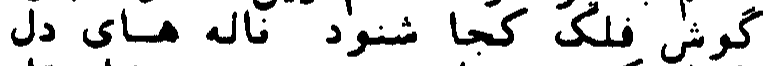

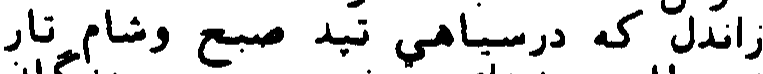

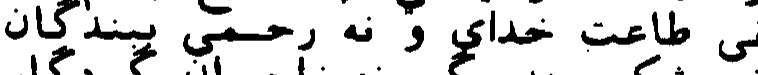

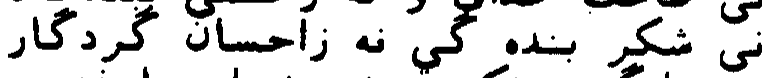

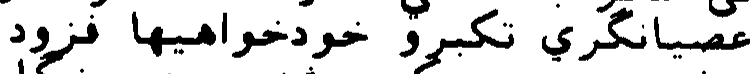

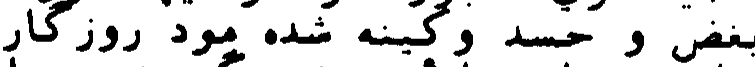

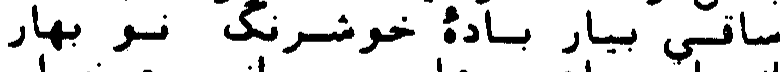

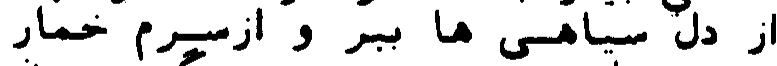

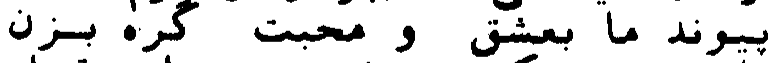

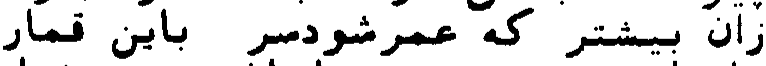

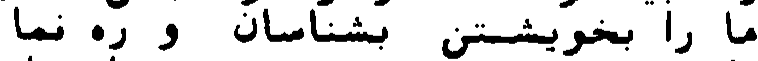

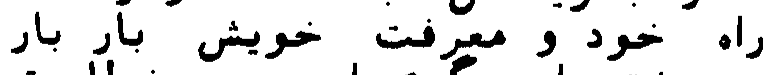

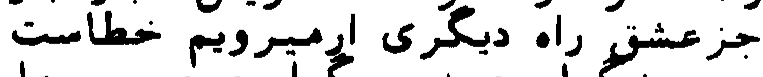

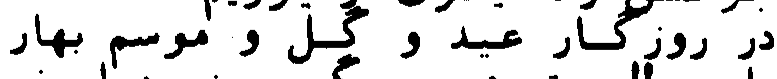

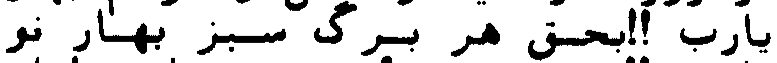

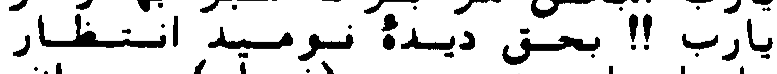

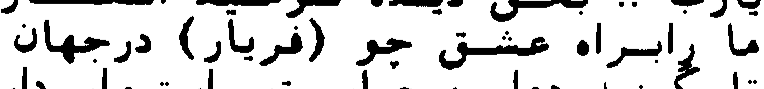

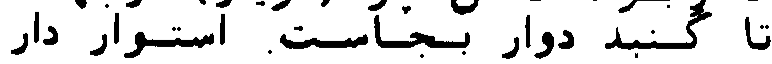




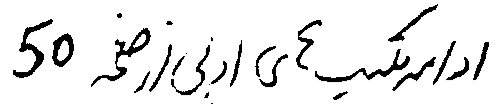

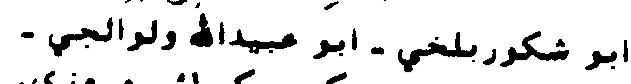

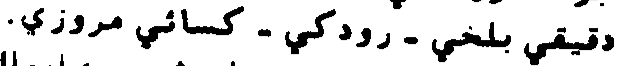

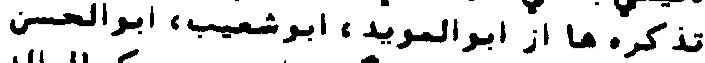

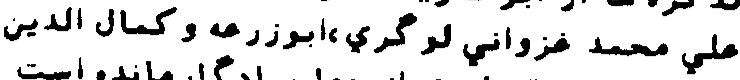

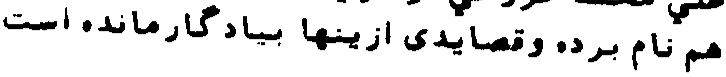

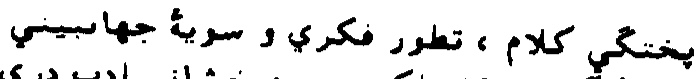

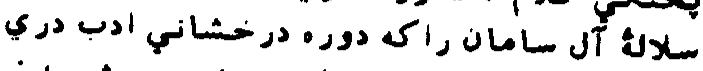

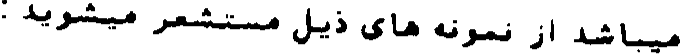

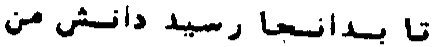
हूँ है।

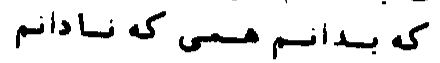

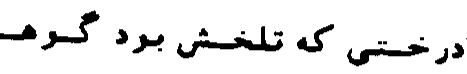

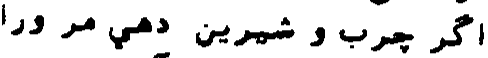

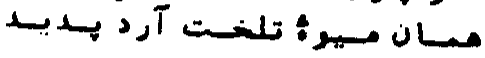

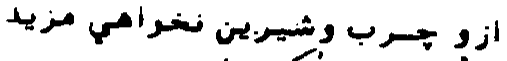

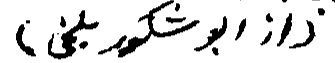

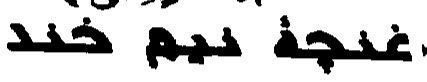
ازعبدالصمد ومابزاده فريار ازجرمني هـي

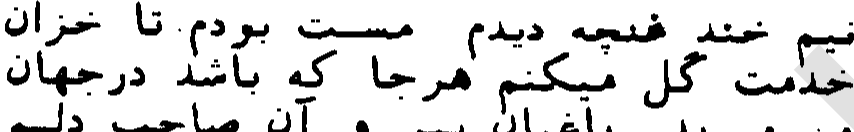

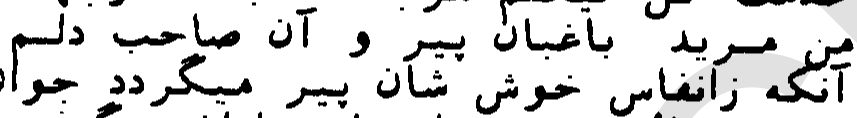

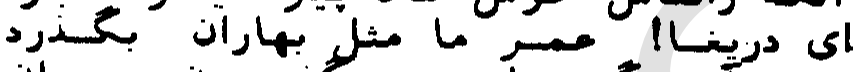

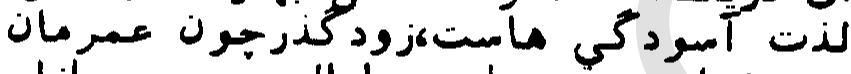

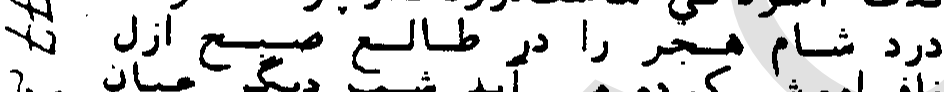

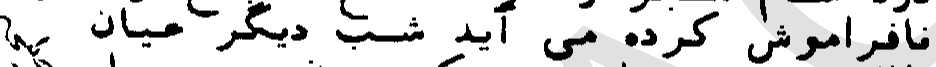

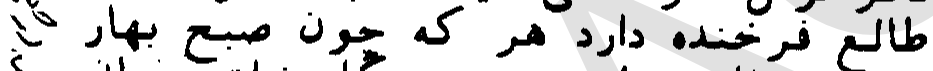

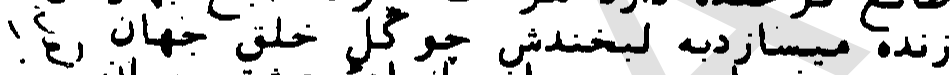

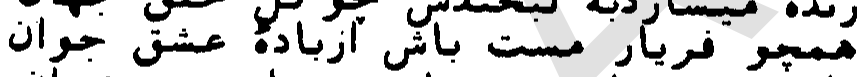

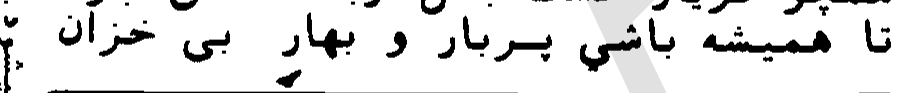

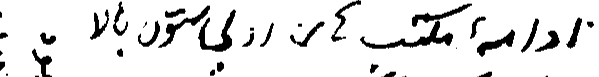

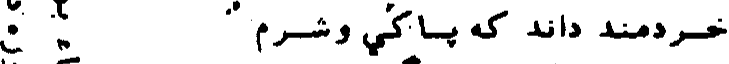

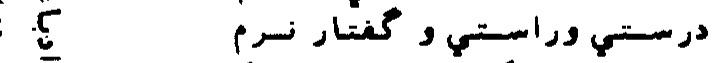

\&.

5

if $x$

$57 z^{w}$

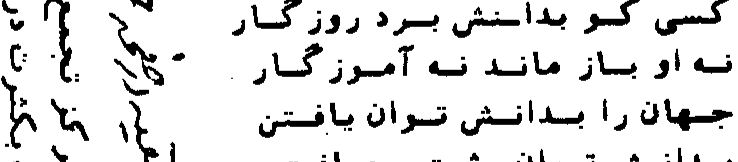

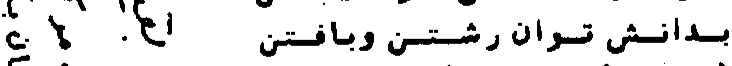

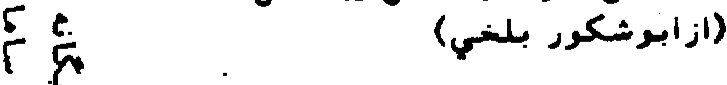

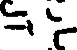

$r$

క

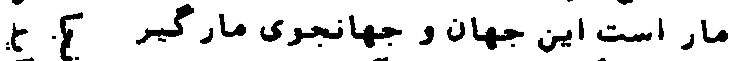

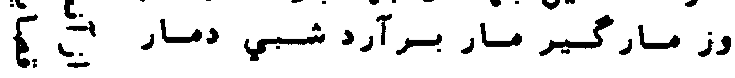

51

ازز نعراله نياوى

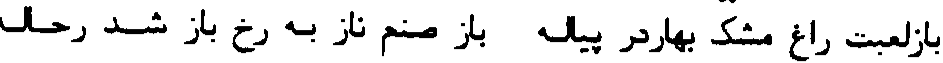

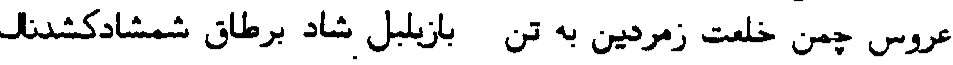

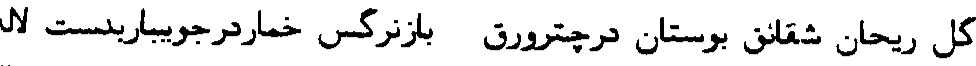

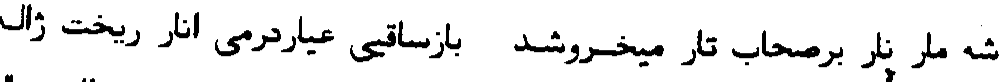

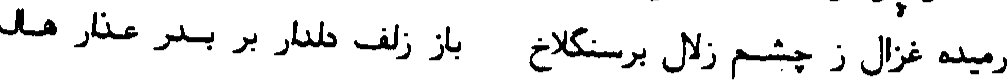

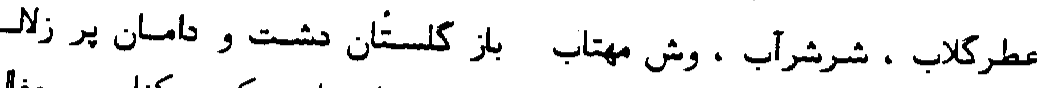

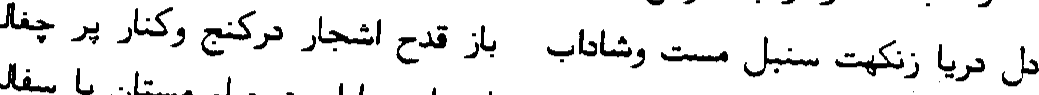

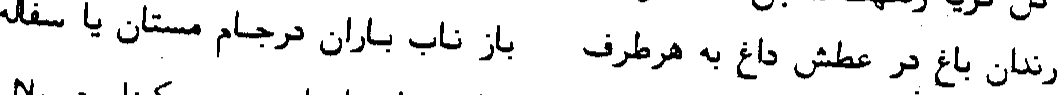

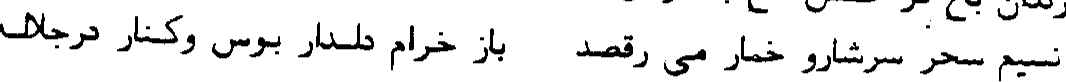

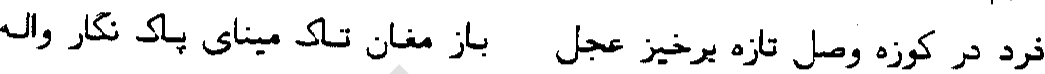

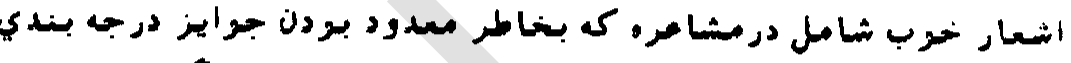
نجر ديد

\section{حلول جماد}

\section{ازعبدالصمد ومابزاده فريارازجرمني}

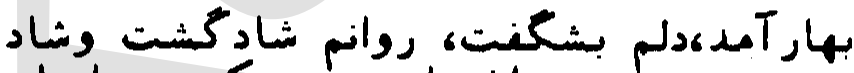

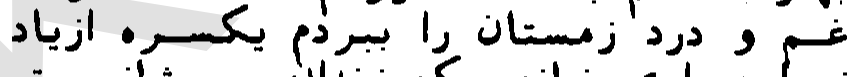

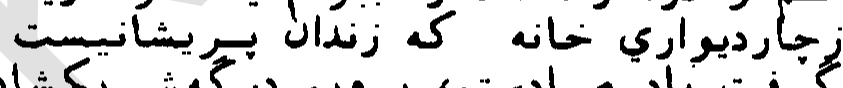

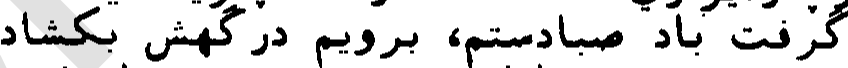

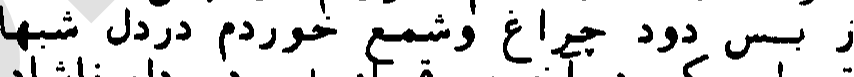

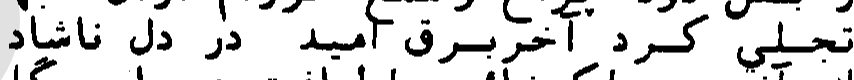

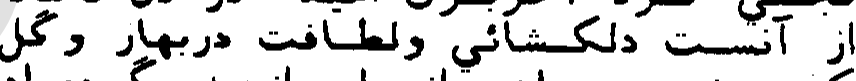

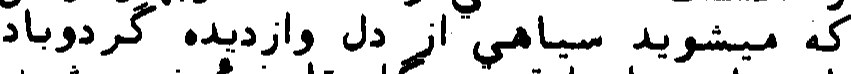

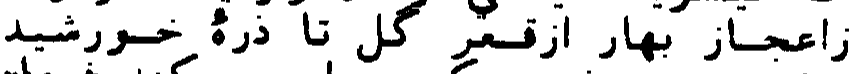

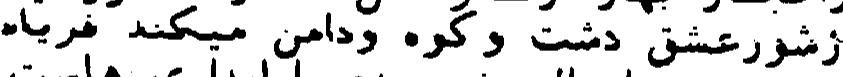

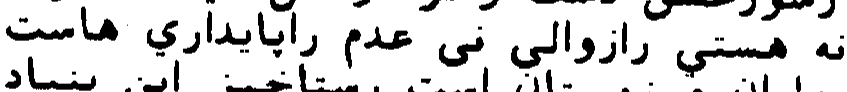

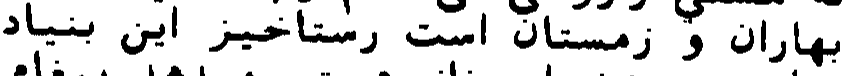

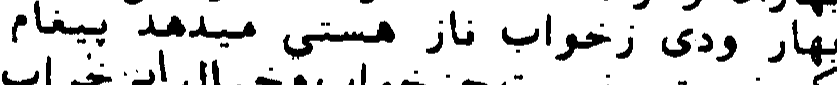

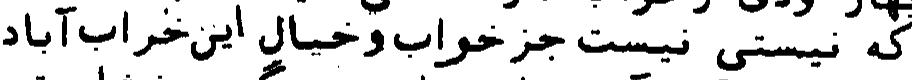

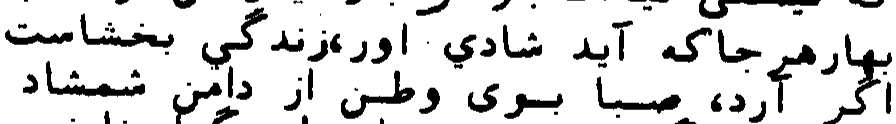

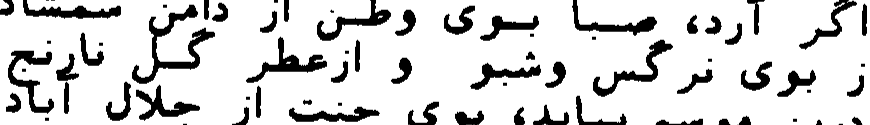

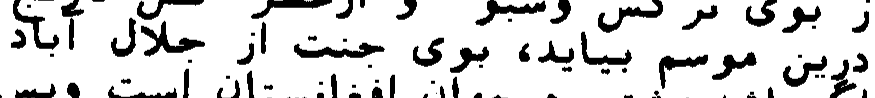

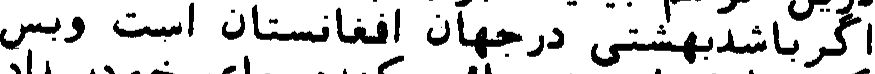

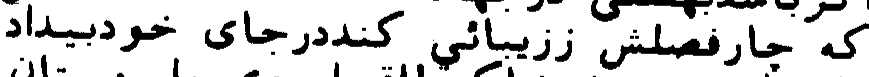

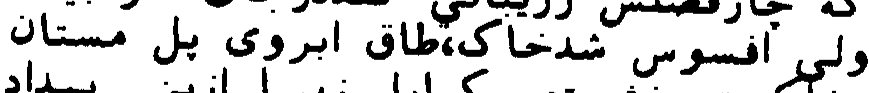

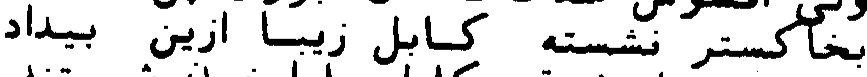

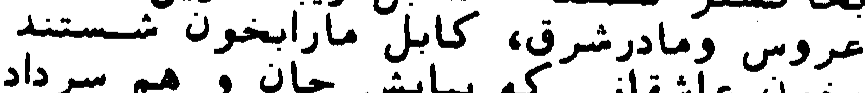

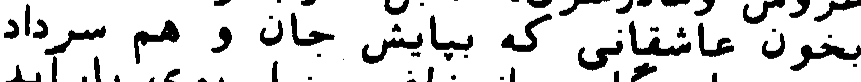

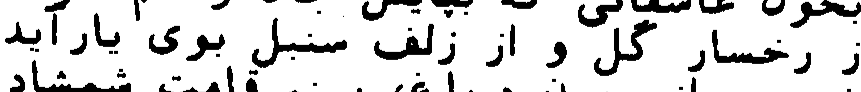

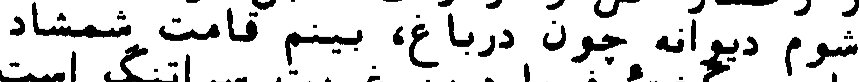

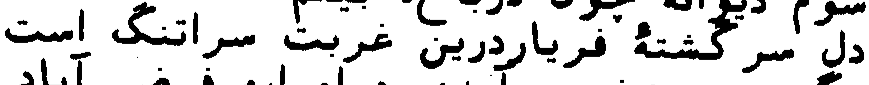

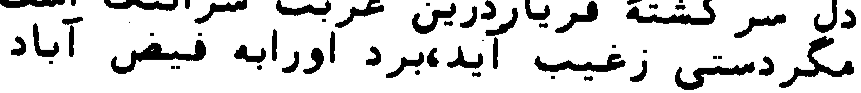




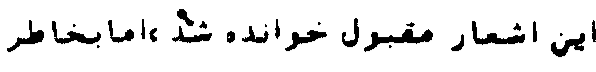
معدودبردون جوايز درجه بندي نغرديد

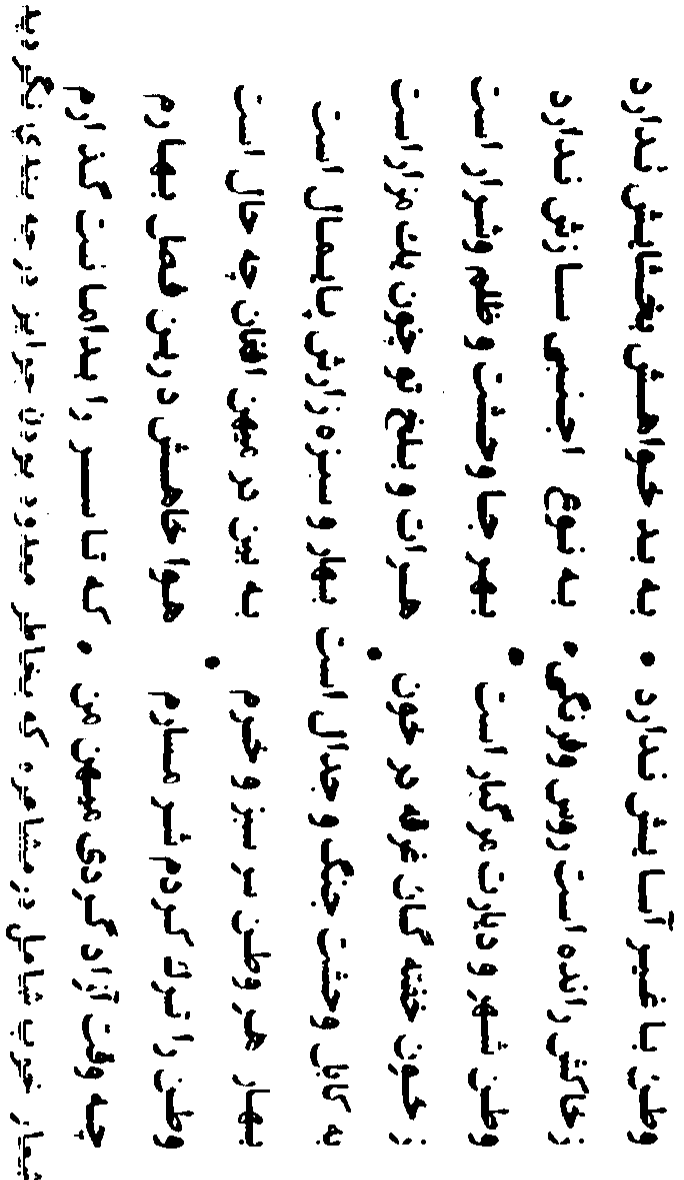

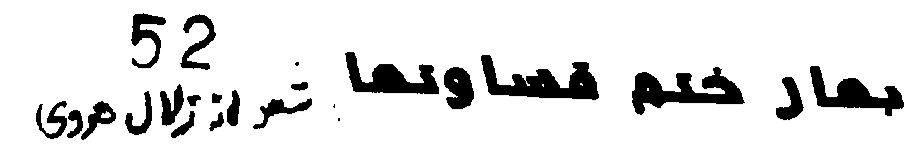

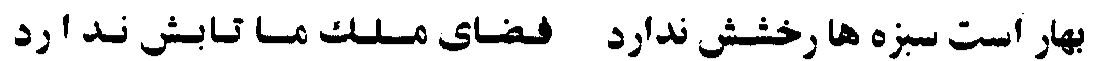

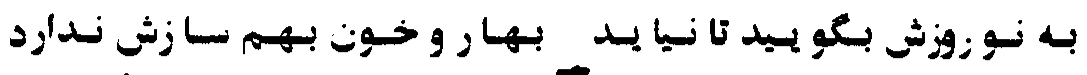

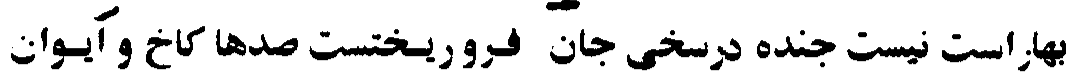

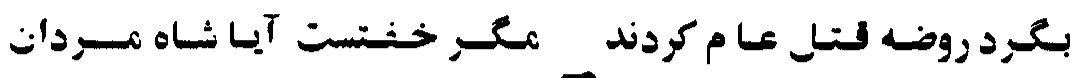

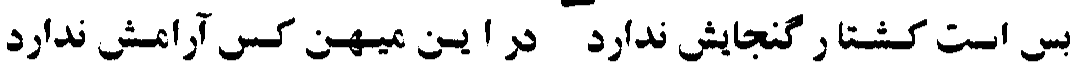

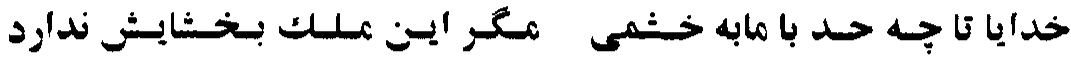

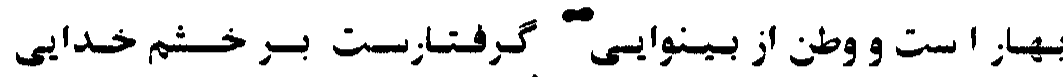

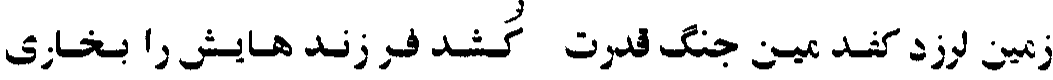

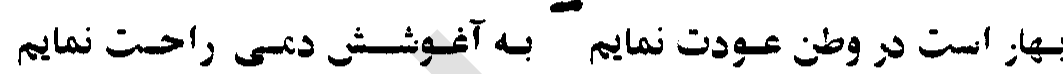

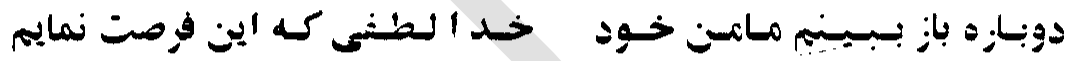

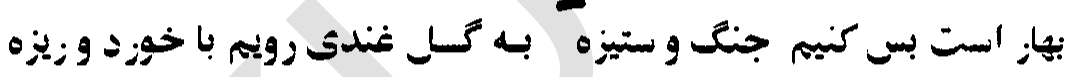

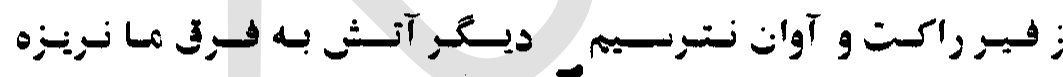

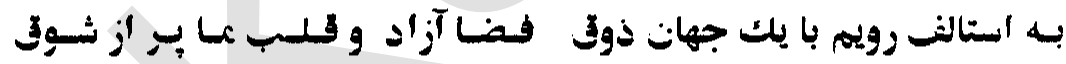

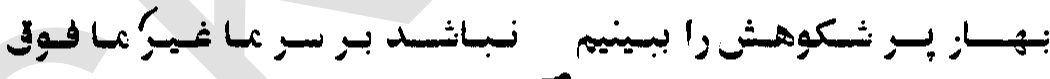

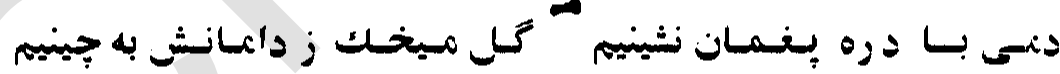

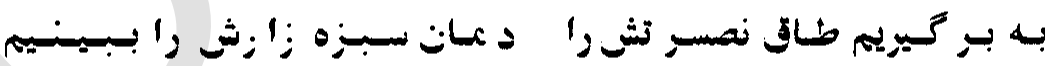

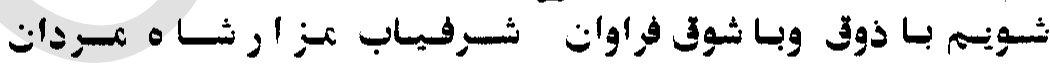

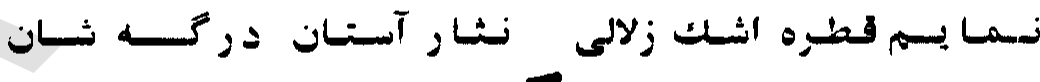

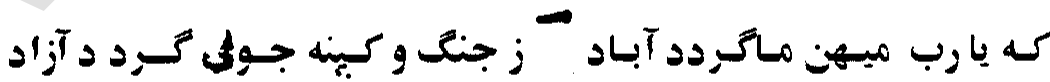

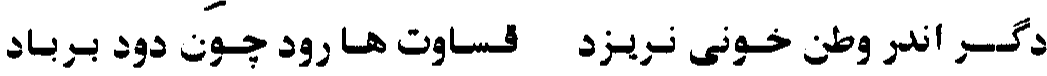

\section{Fige whas yha}

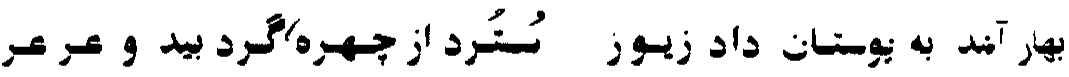

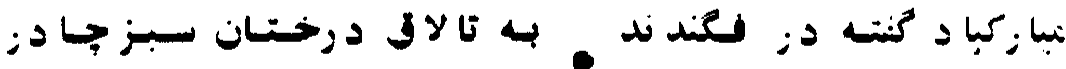

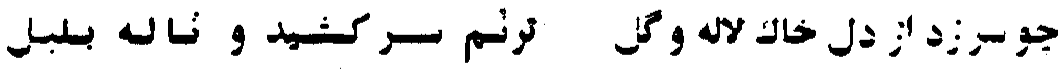

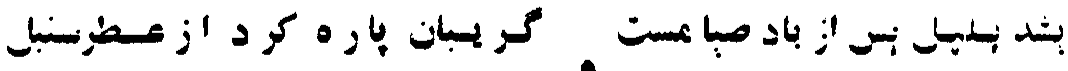

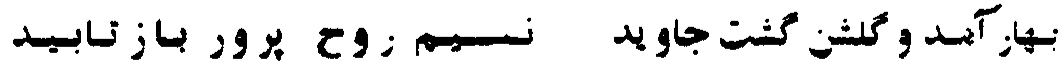

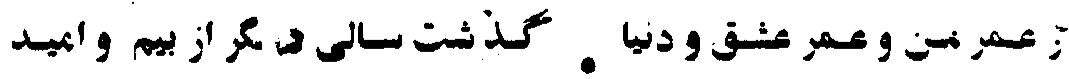

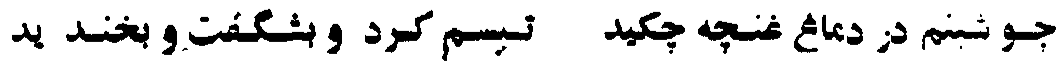

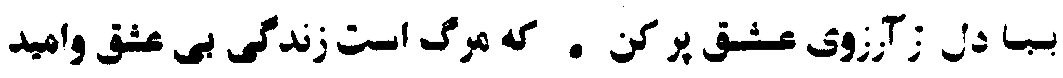

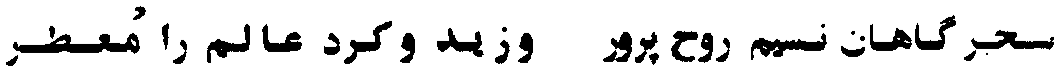




\section{بهاد بمار كامل}

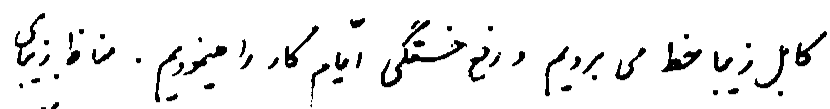

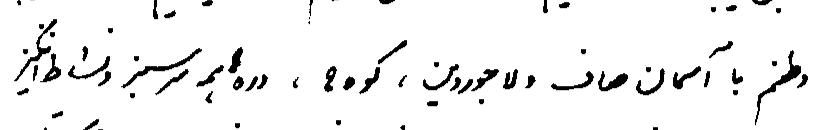

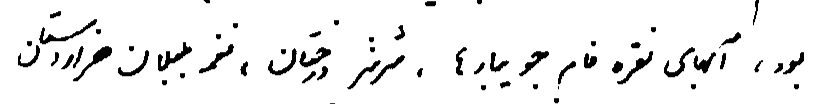

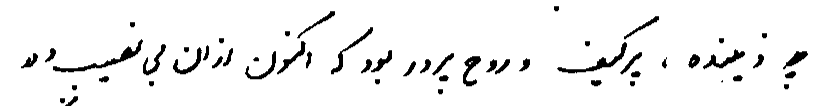

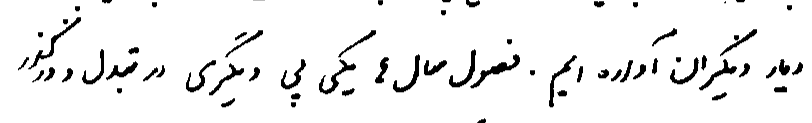

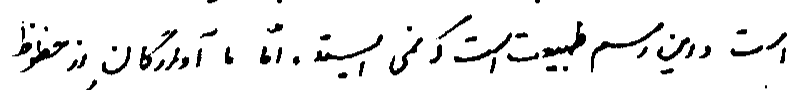

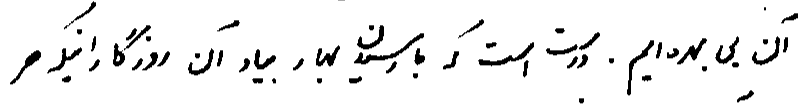

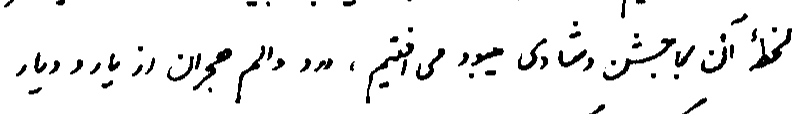

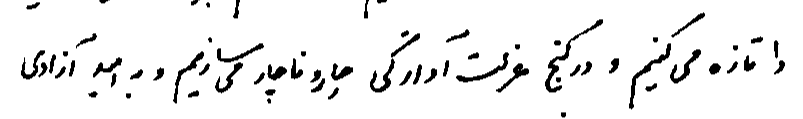

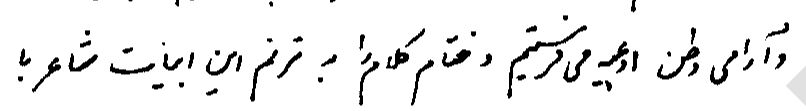

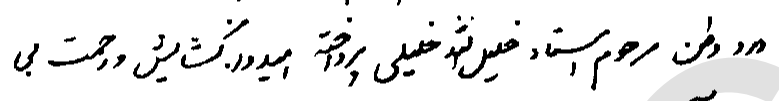
•

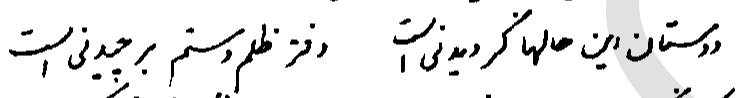

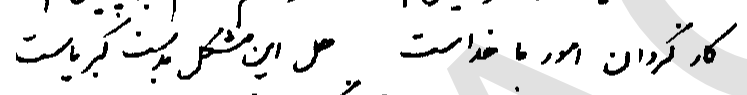

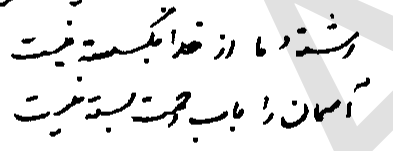

sing

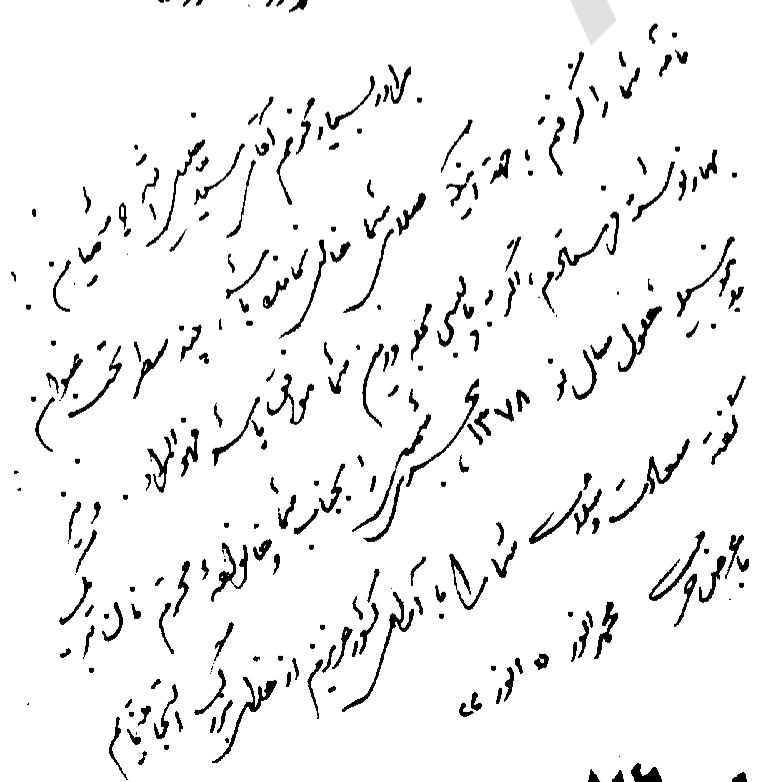

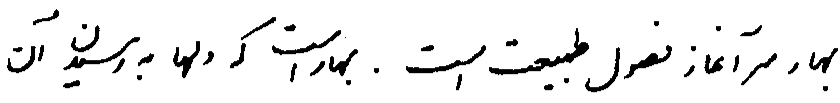

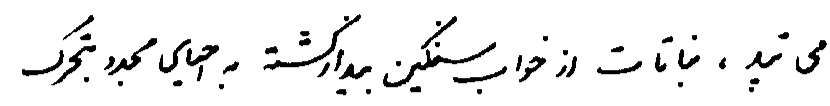

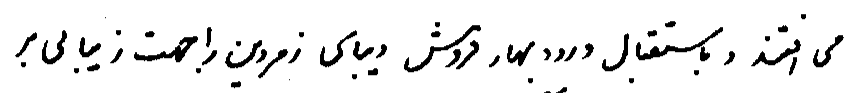

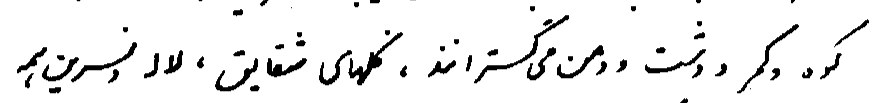

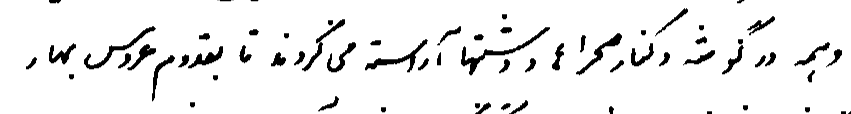

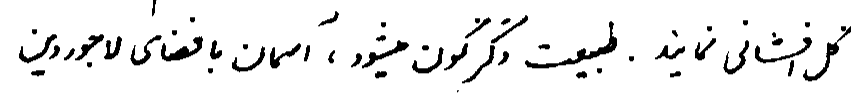

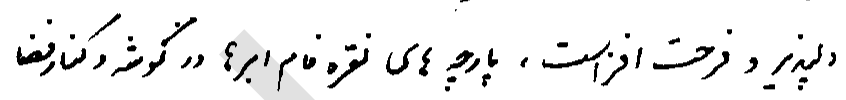

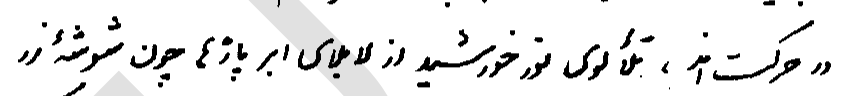

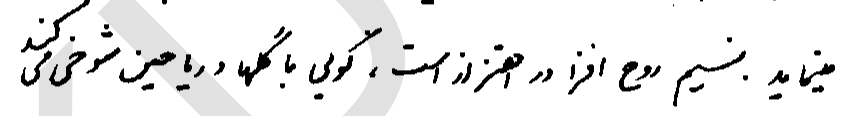

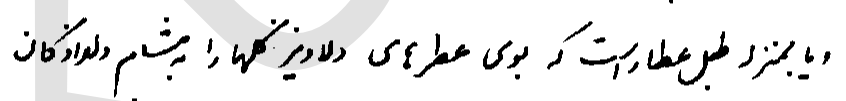

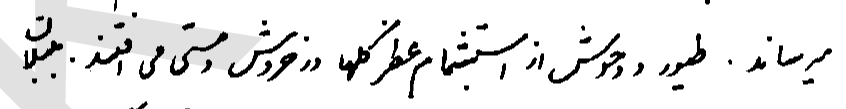

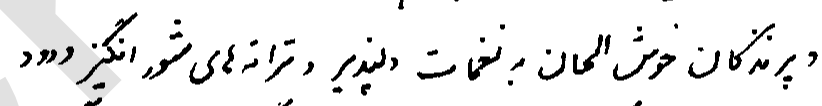
.

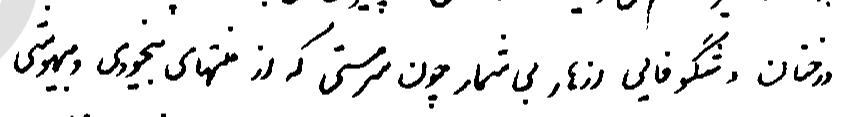

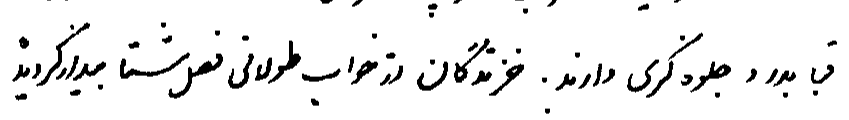

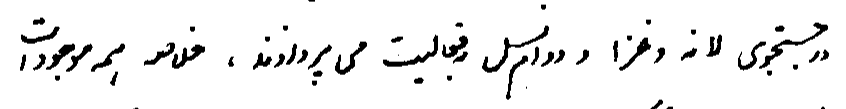

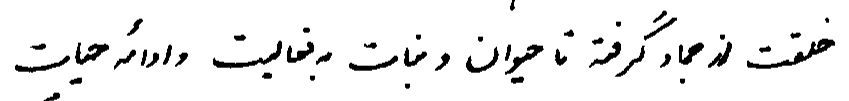

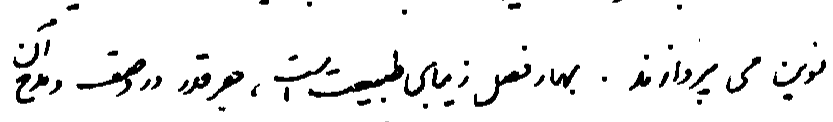

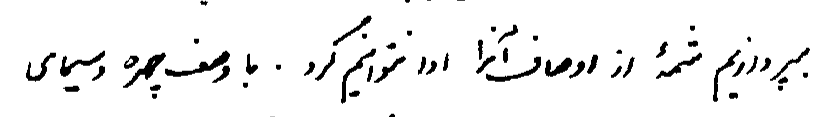

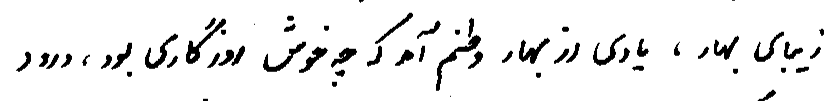

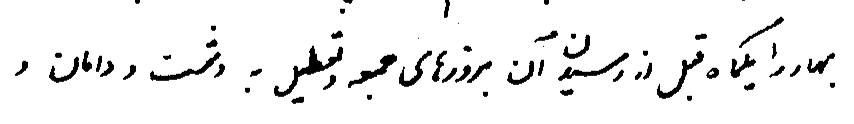

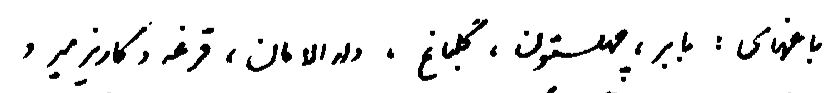

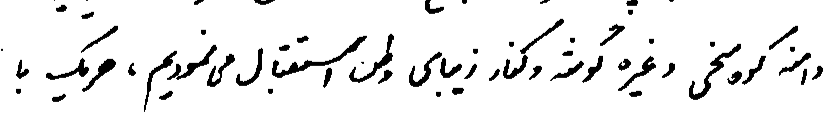

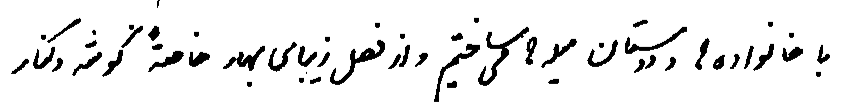

\section{شمارة مساتسل}




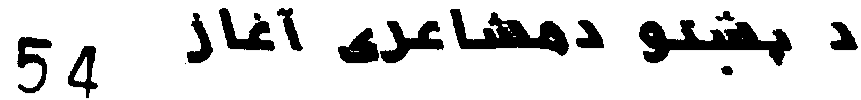

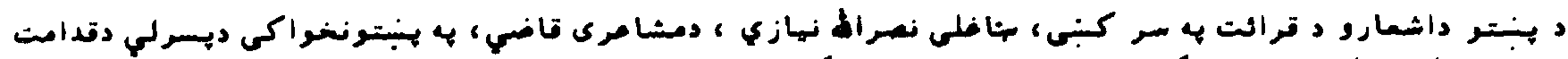

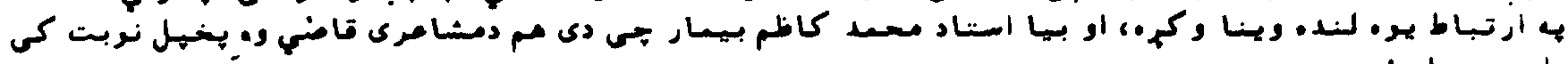
إسى وروبلى

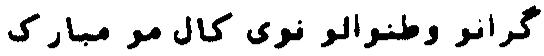

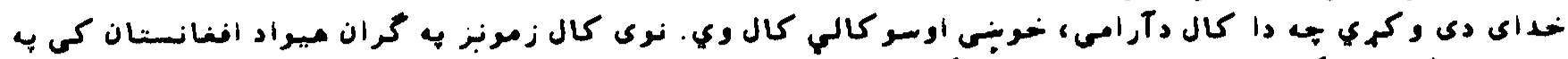

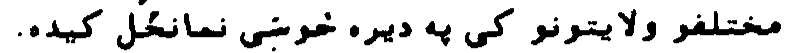

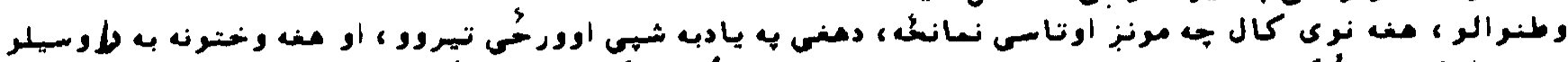

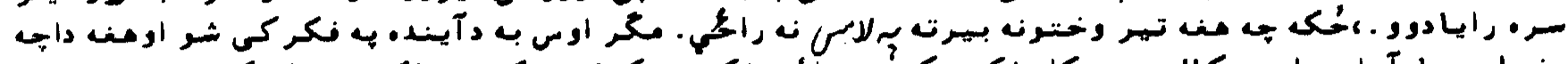

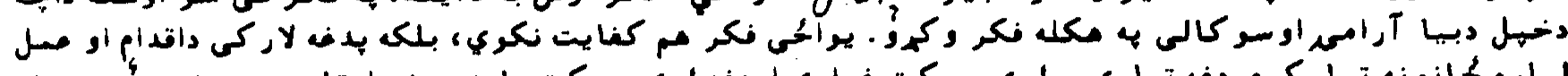

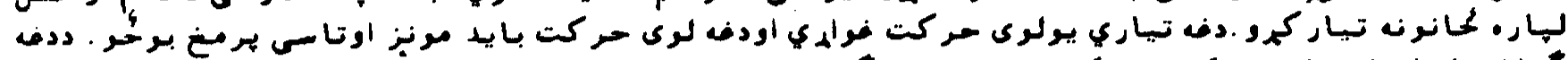

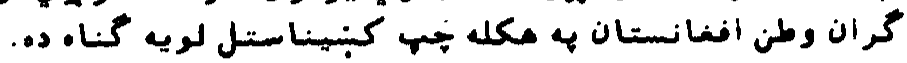

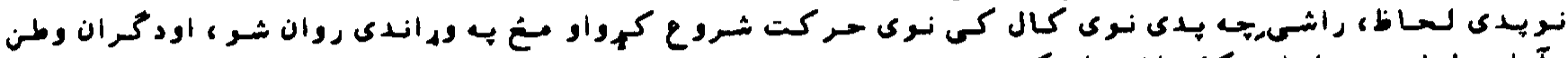

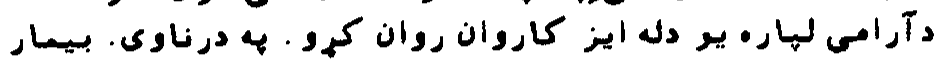

\section{دغ}

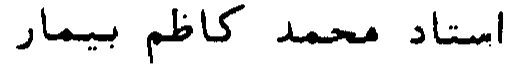

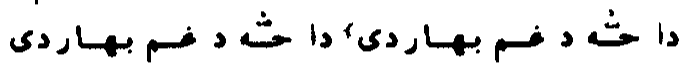

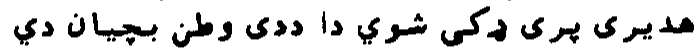

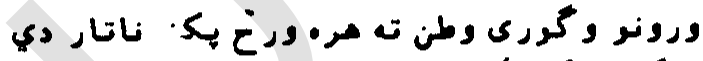

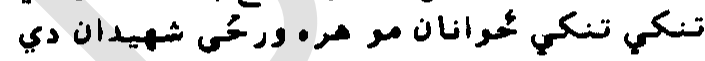

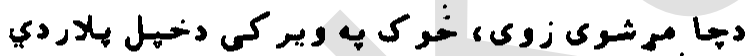

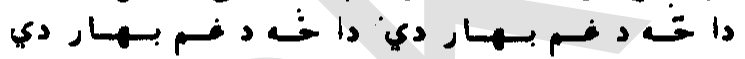

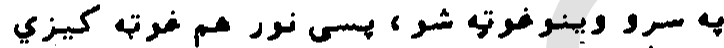

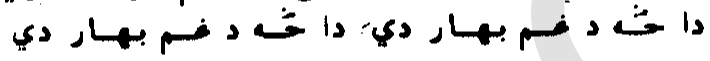

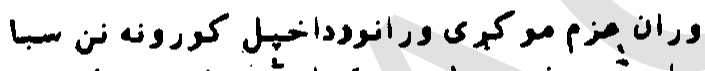

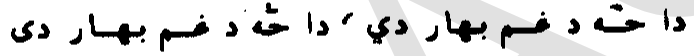

بى بلاره دربه در دى أوس د خماكر به كورونر

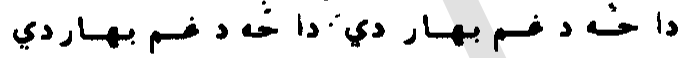

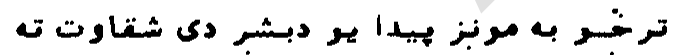

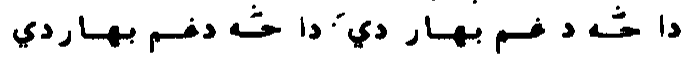

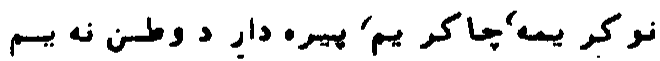

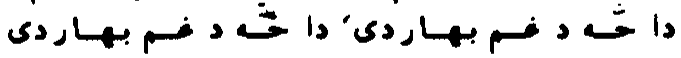

فيرت اوس به دوى كى نشتــ دتيرشور افنانانر

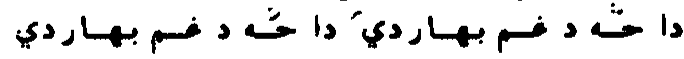

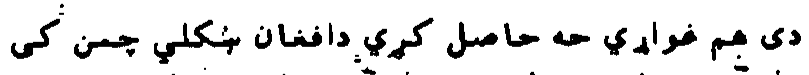

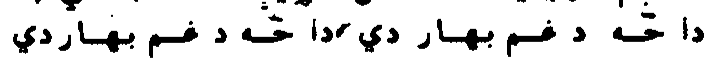

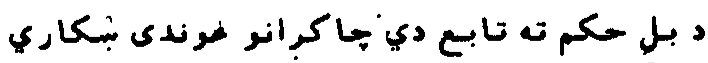

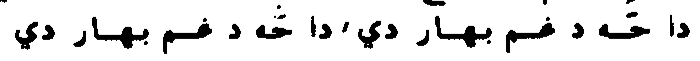

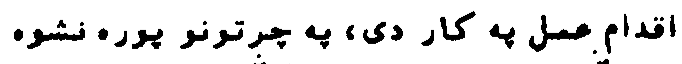

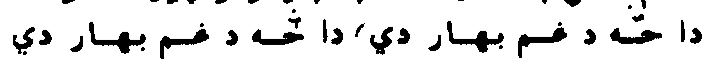

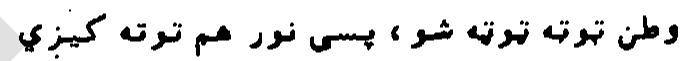

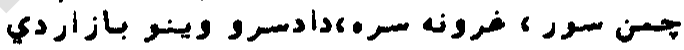

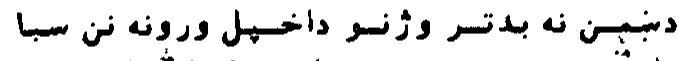

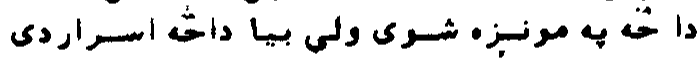

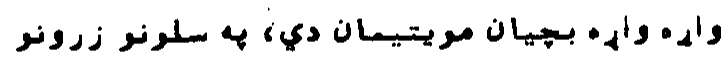

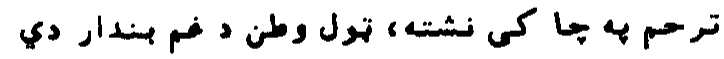

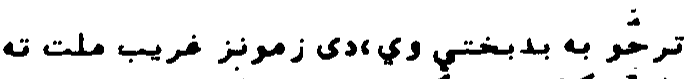

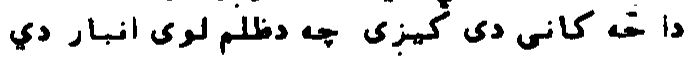

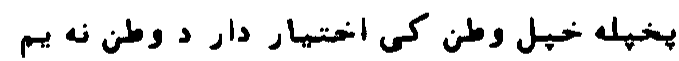

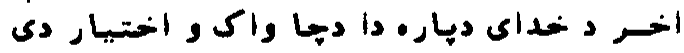

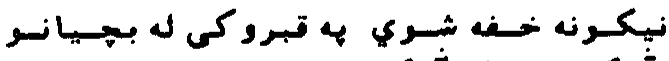

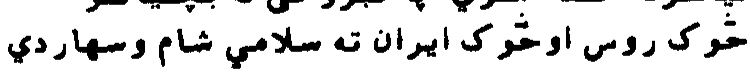

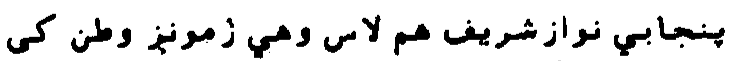

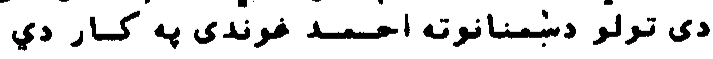

توماندان او رمبران مر نركرانو خوندى مئكاري

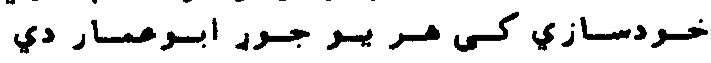

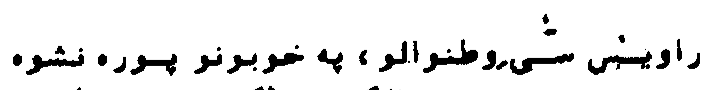

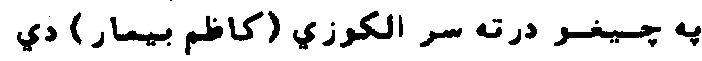




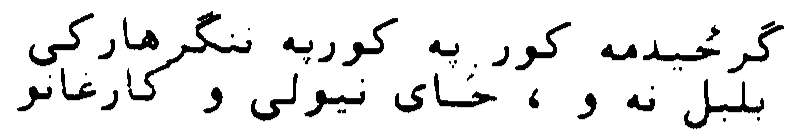

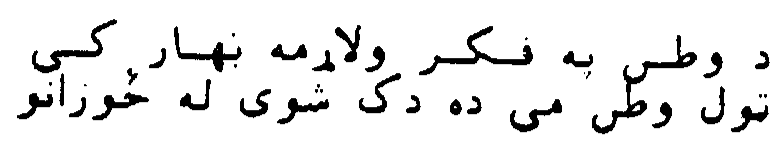

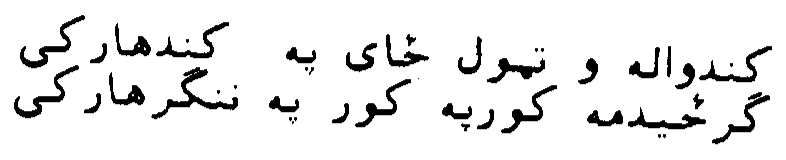

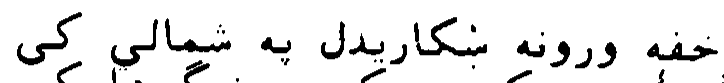

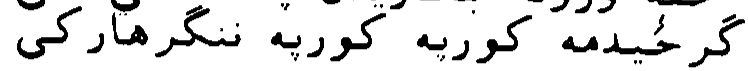

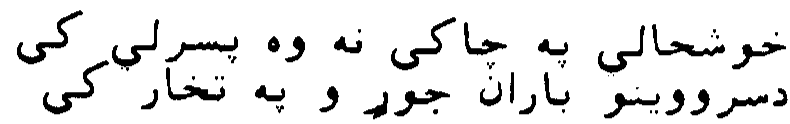

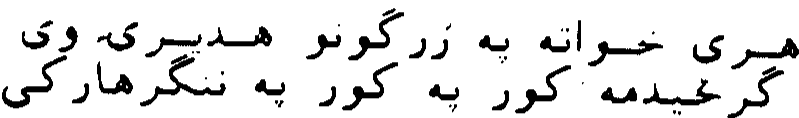

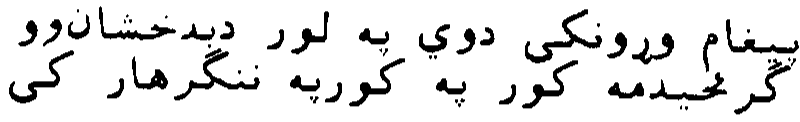

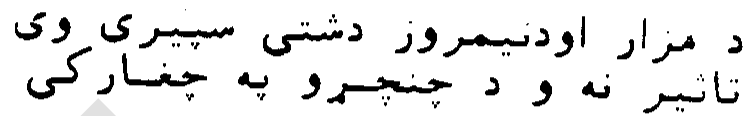

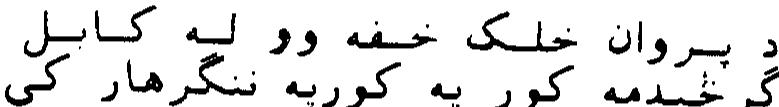

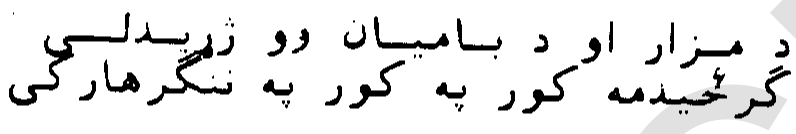

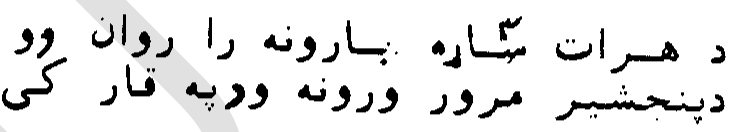

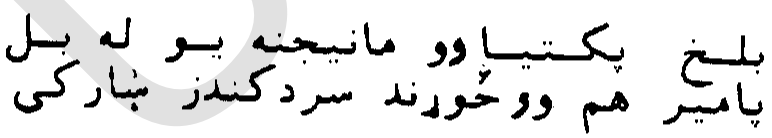

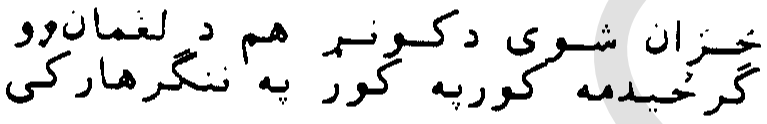

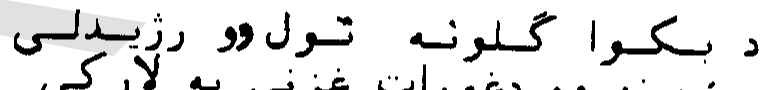

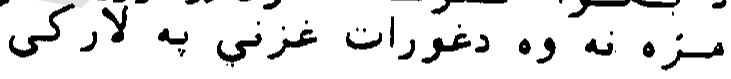

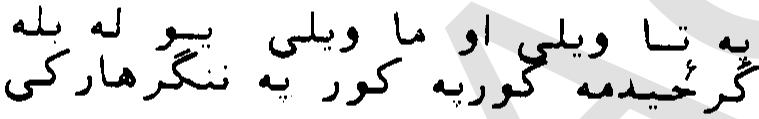

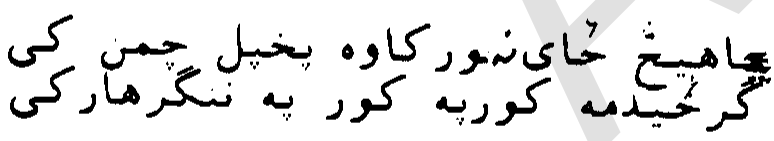

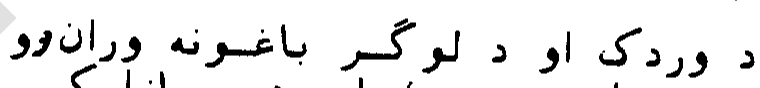

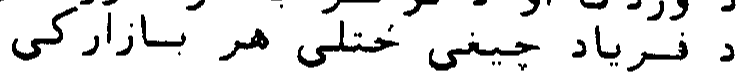

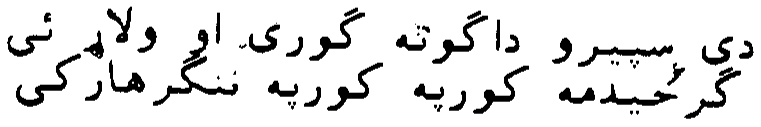

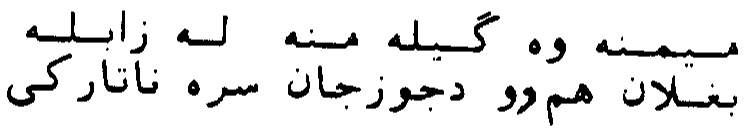

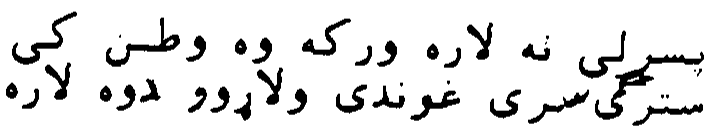

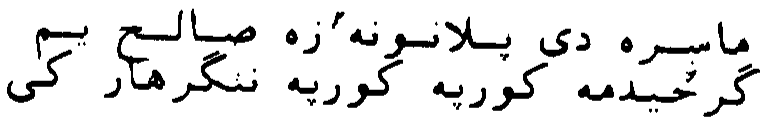

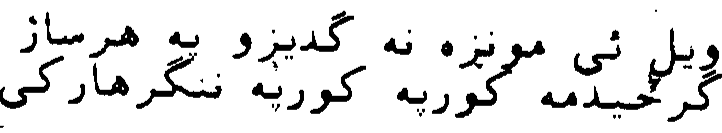

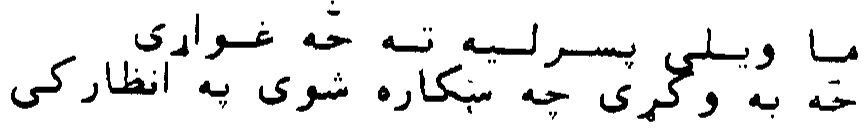

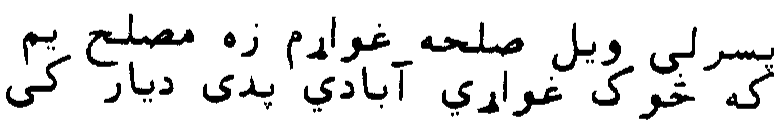

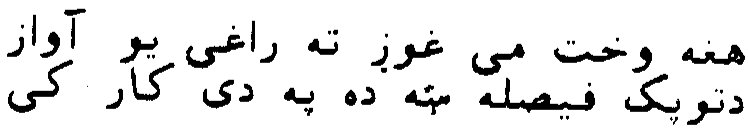

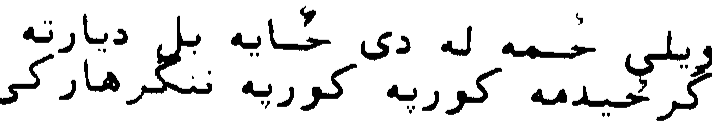

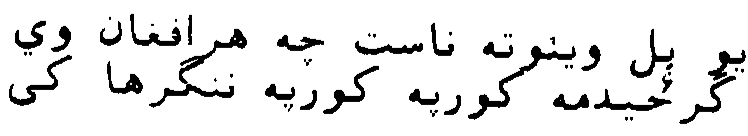

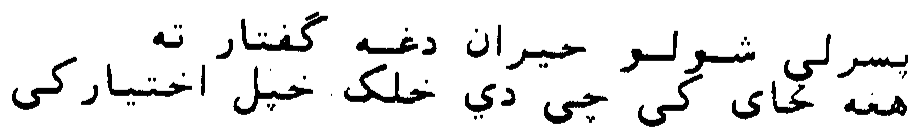

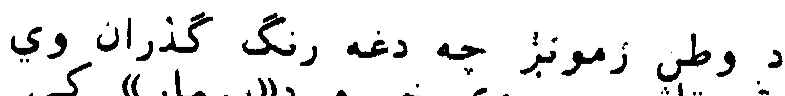




\section{6 د محمد ماشم زماني شعر}

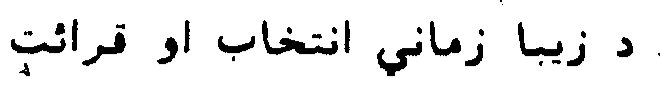

\section{دودנ عנפה}

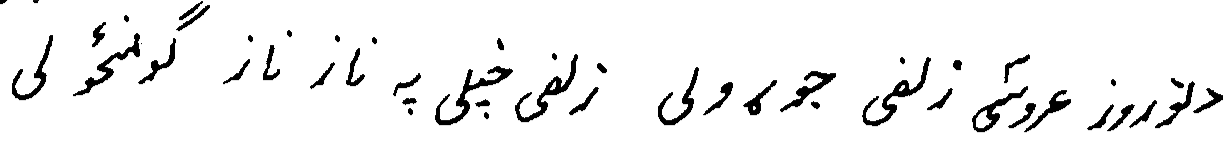

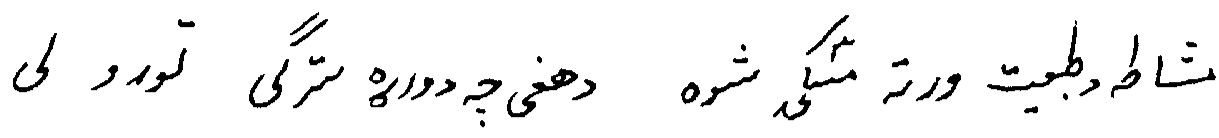

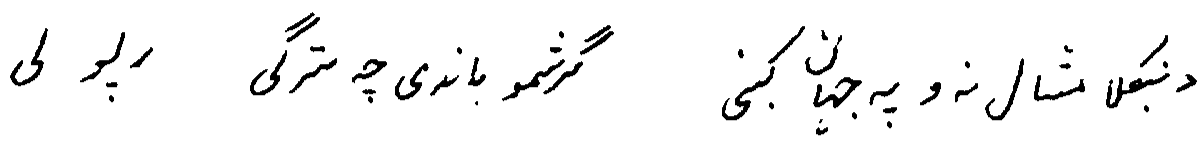

ti, $i$ is $q$ :

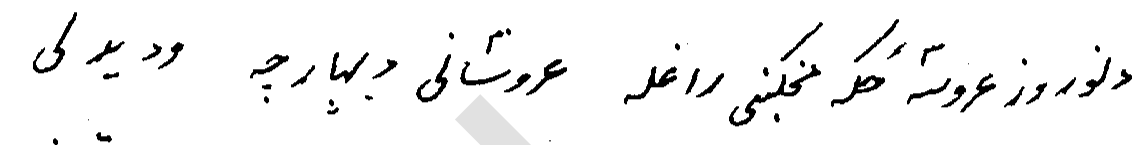

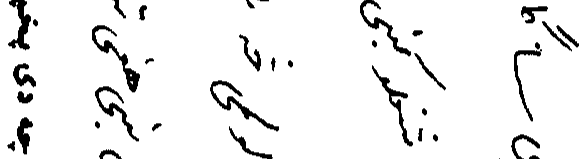

F a

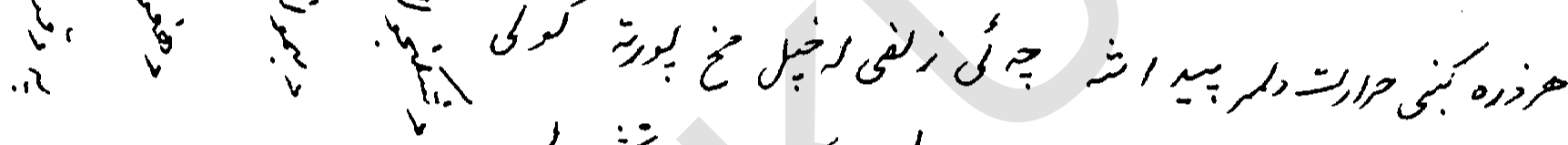

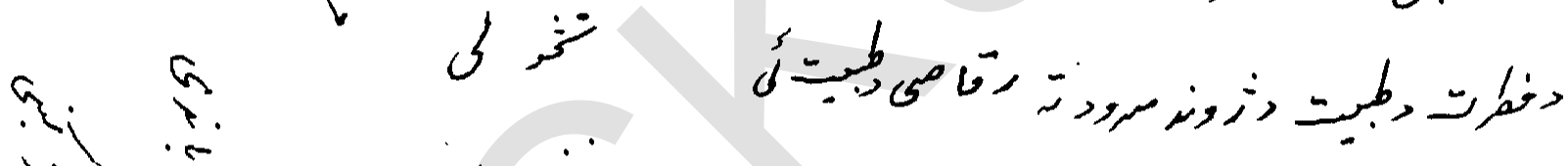

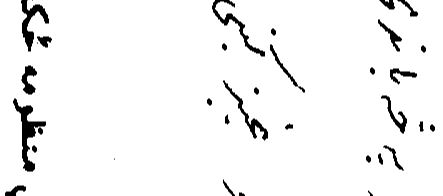

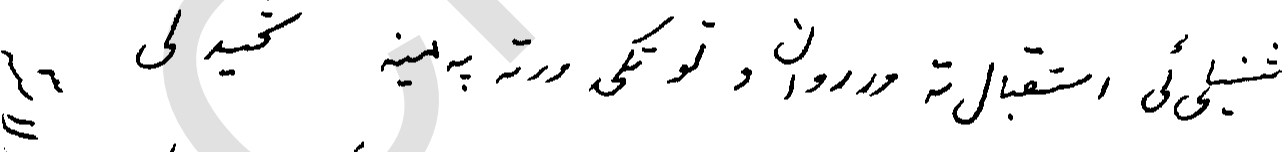

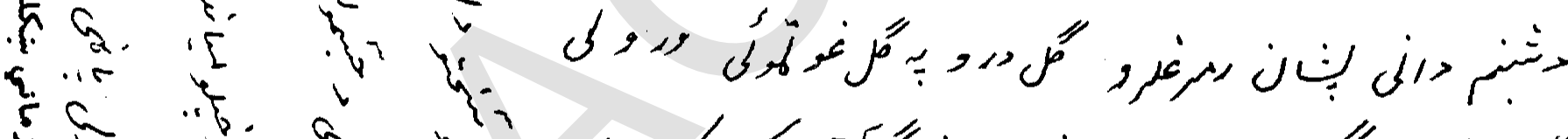

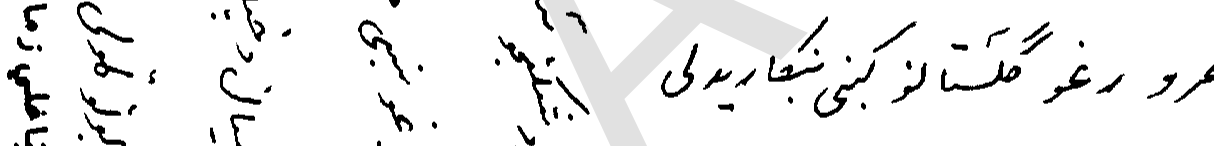

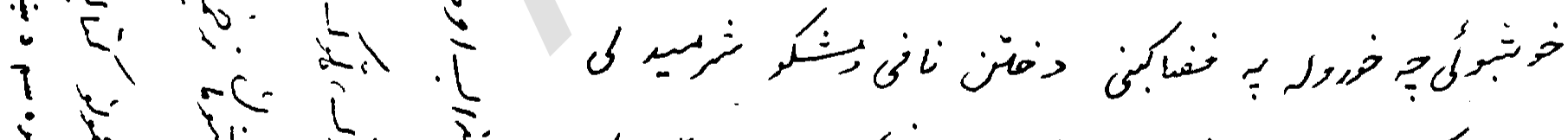
خ

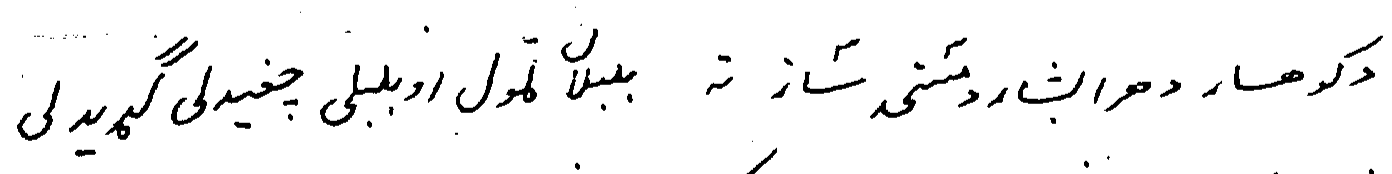

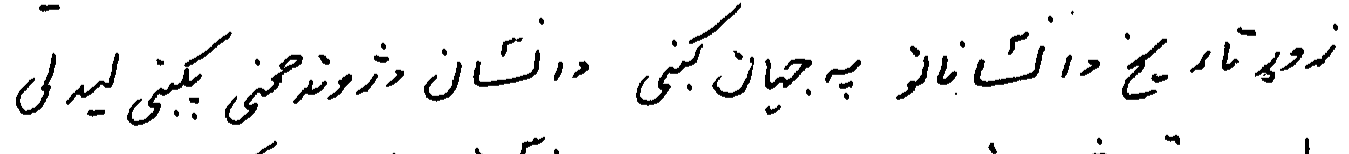

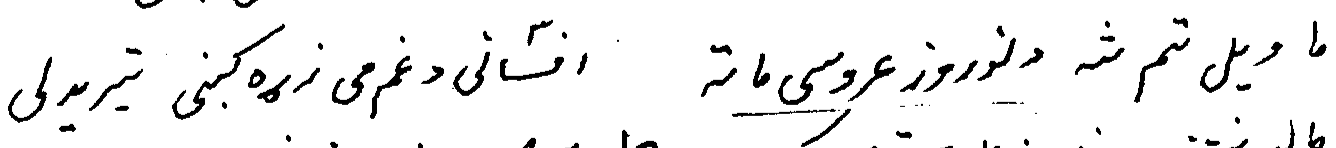

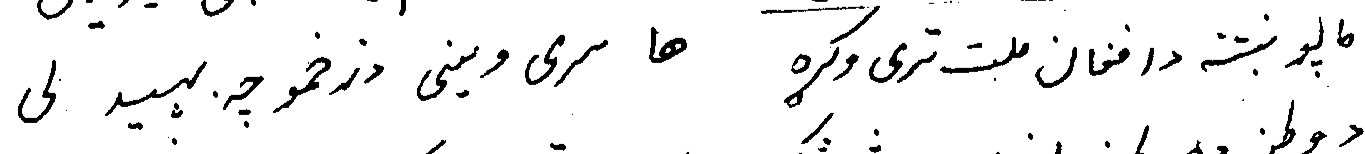

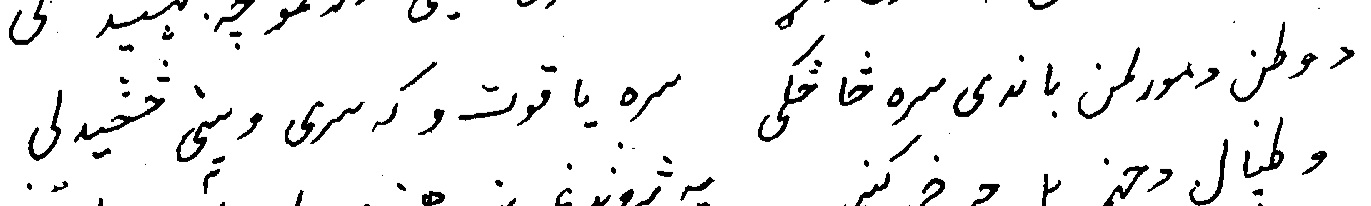

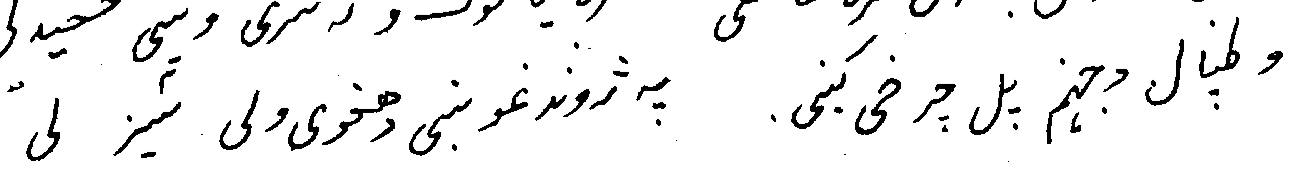


א.

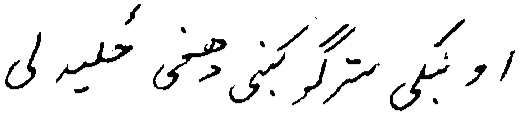

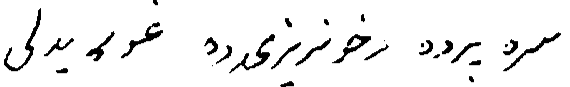

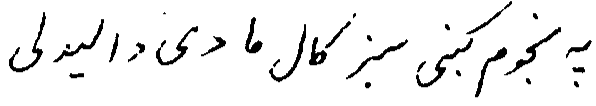

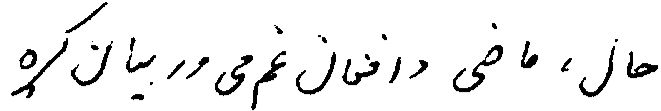

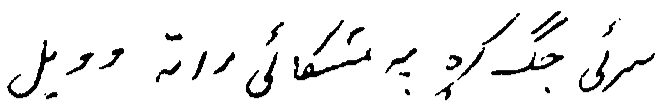

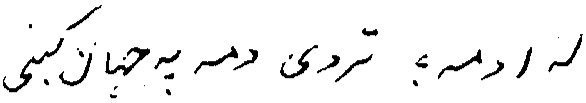

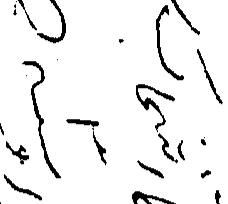

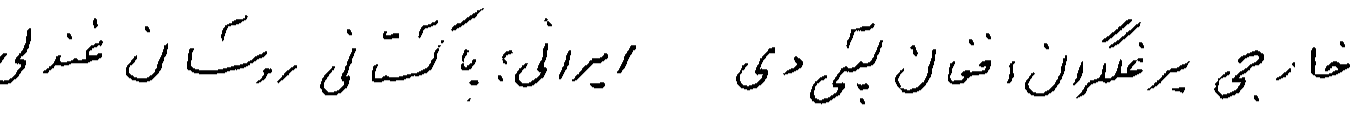
$\sum^{2}, \frac{1}{2}, \frac{1}{2}$

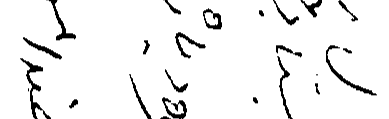

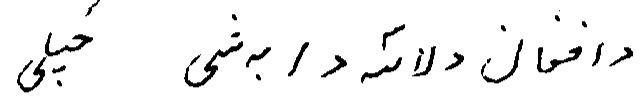

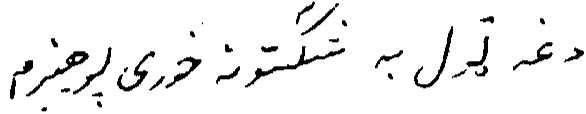

$v^{\circ}, h^{\circ}$

S $i \frac{i}{5}$.

b.

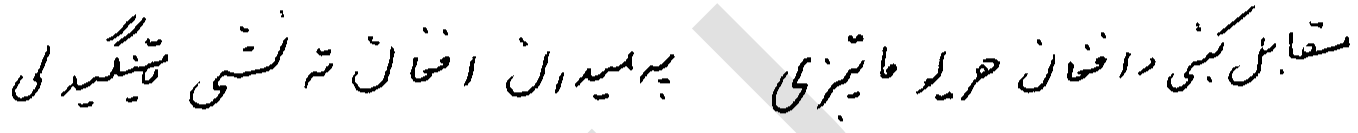

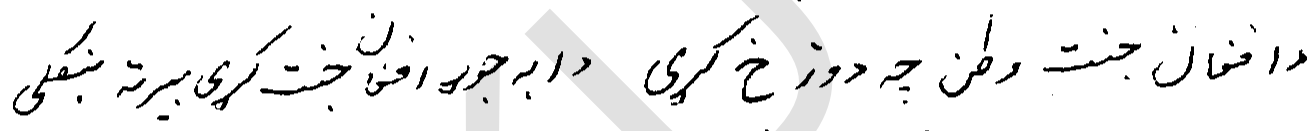

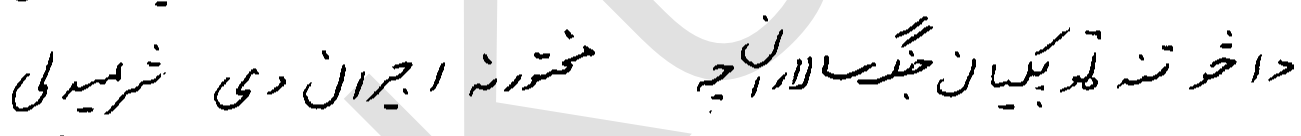

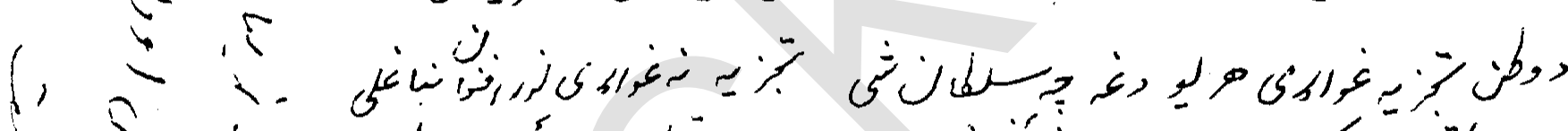
$\therefore$ : : ते हो

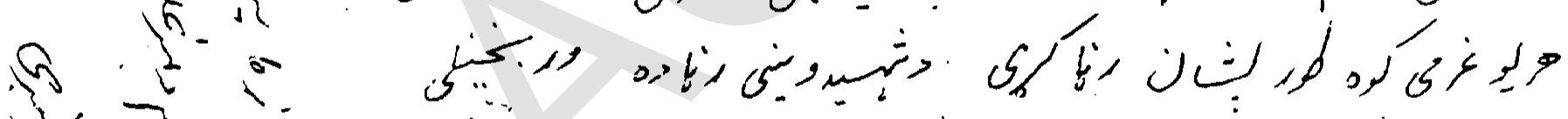

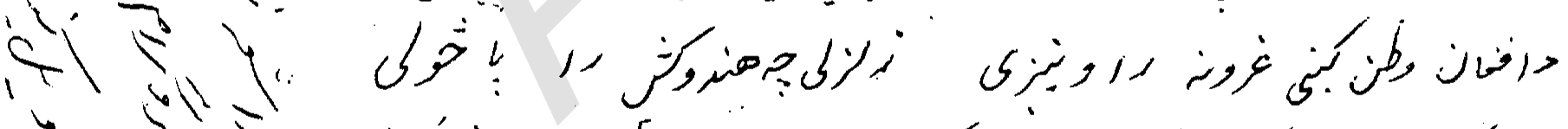

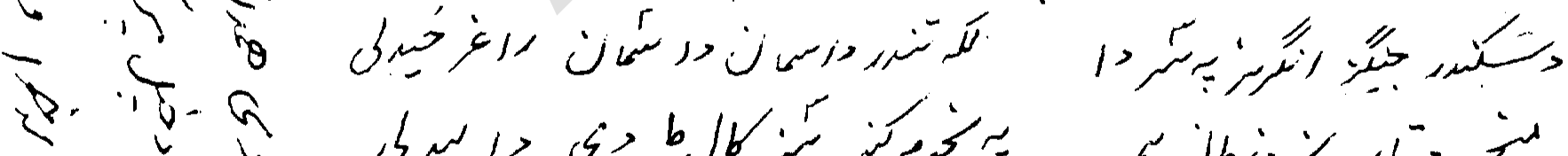

बे

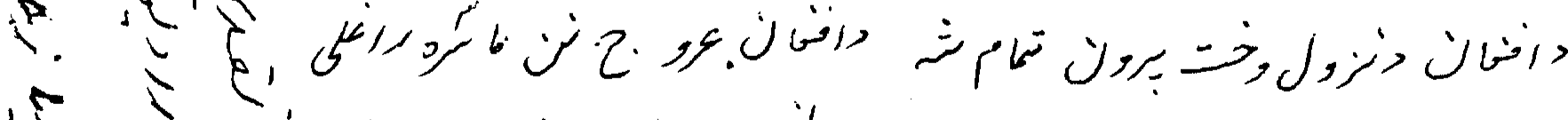

v.

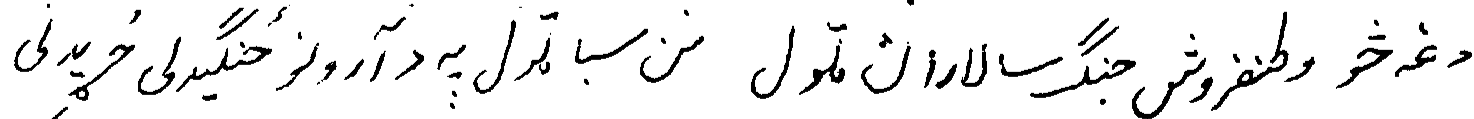

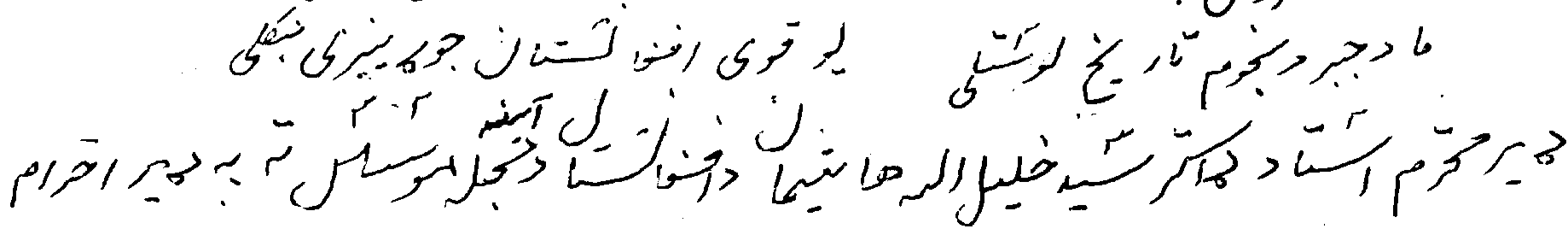




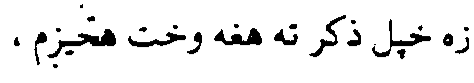

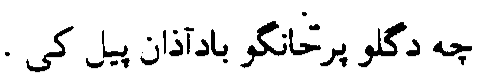

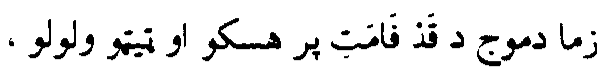
اود ليوال بلبلـ د حال بر الفلاح اتدا ده .

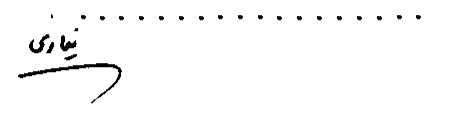

58

\section{|}

أز نصسرال نيازي

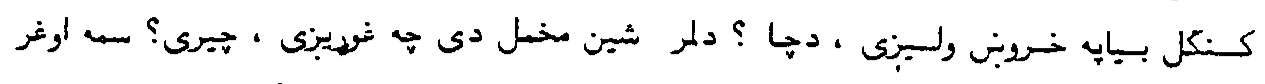

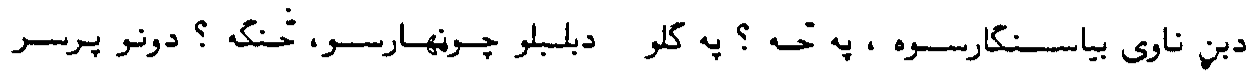

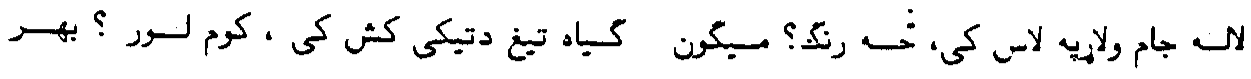

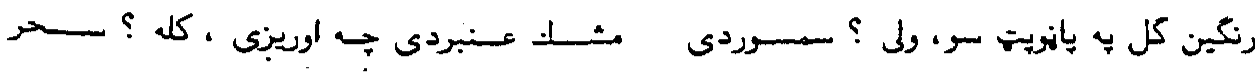

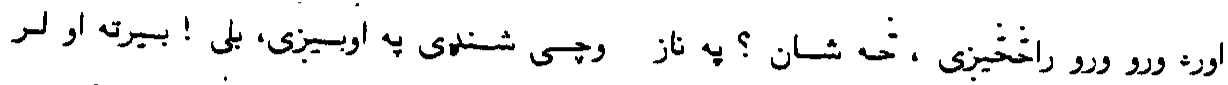

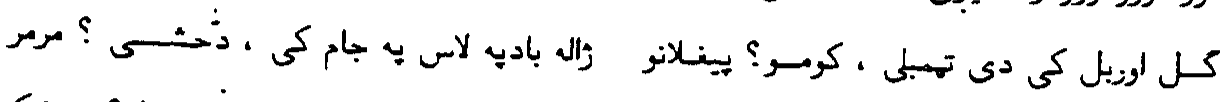

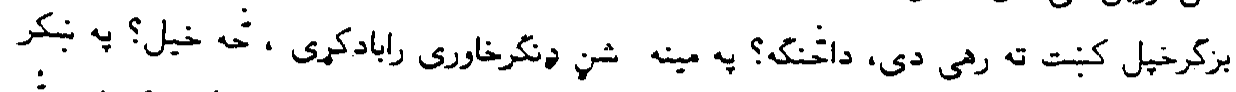

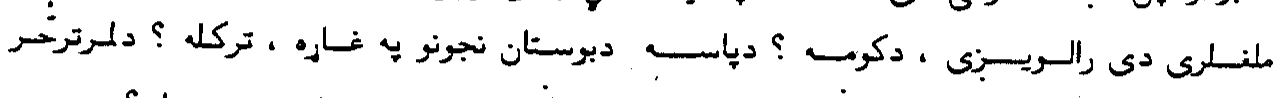

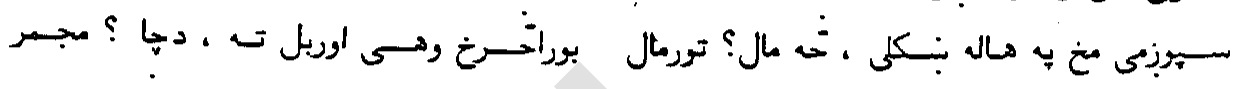

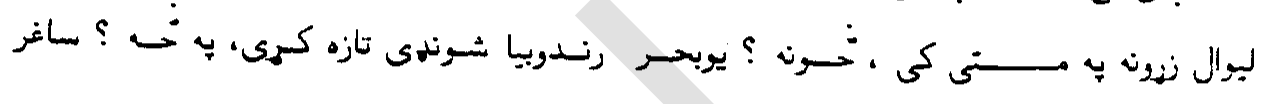

دباد نيلى خين · اويه بيره ثى ينده كى، ديسرلى بان؟

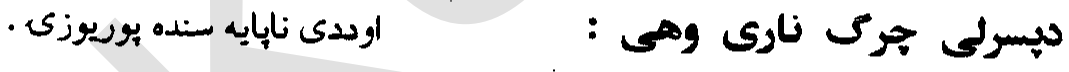

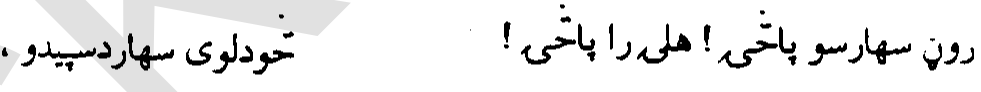

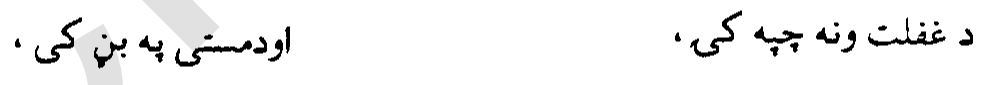

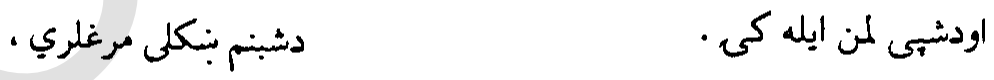

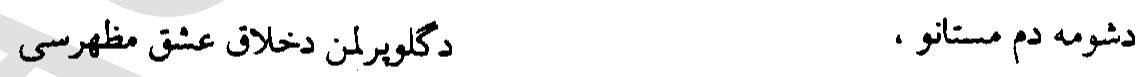

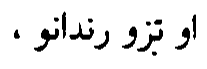

a

t.

$q$

5

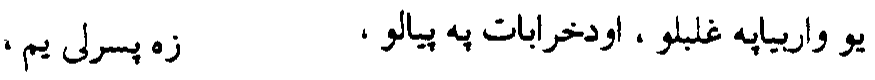 سره غنجه مى قبله ده. به كلواوبانهوكى غلغلى كدى كى.}

ايى دزمى خوبه ! اى دهيلودنرى رنكين خوبه ! ! محراب مى رونه جينه ، اود لمر رونبانه بلوشه ده . تمبى دجبغويه سنكرودى ورى كه.

غراوبيديا مى تسبيح او مصله دهـ ، دسهايه برخه مى طهارت اودسيل به خهو ابو تن اوجامى ولم. زماد ثنا جزيان دسيوزمى تجله ، اودخالإنده لمرسره اوزرغونه ده . دعبادت توله زرى مى دغمى به خير، جلى اوبل كيزى .

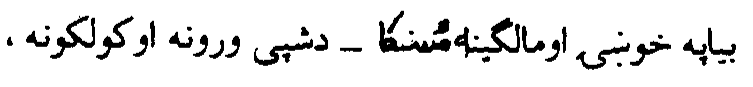
درنا برمخ ويرانبته . ديسرلى جرك ننكار كوى : زرسئ دمينى ملهلى وياروى ، اودباران به اوبككو دتكلف بارى ونروى . دخوزمنومينوكوكارى ؛ دغرو او خنكلو آزانكه كى. 


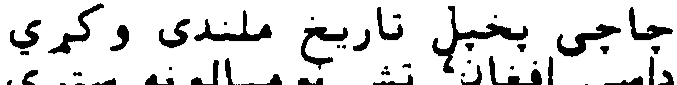

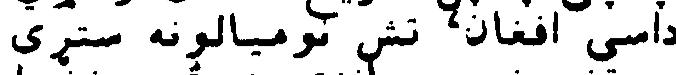

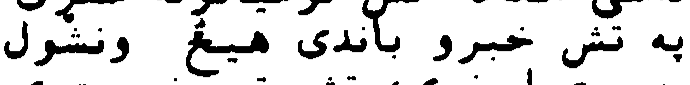

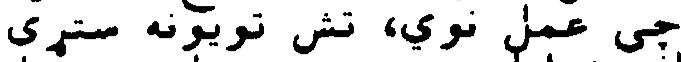

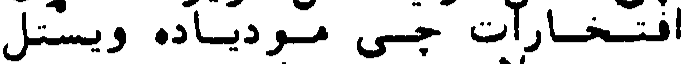

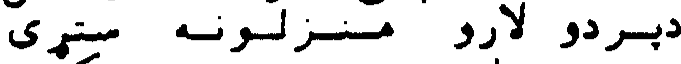

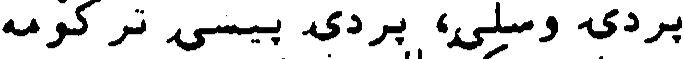

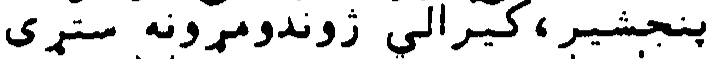

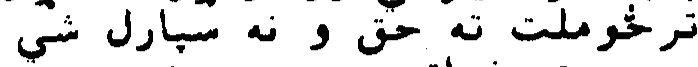

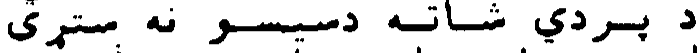

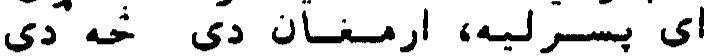

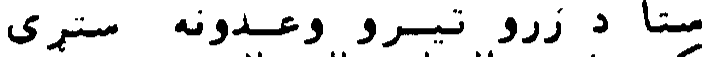

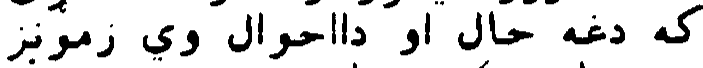

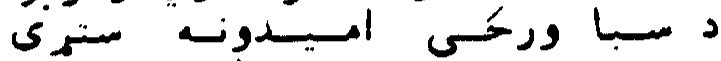

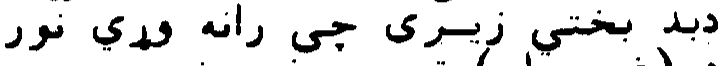

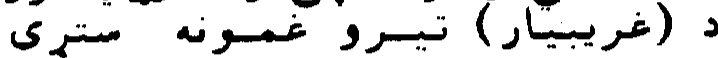

\section{9}

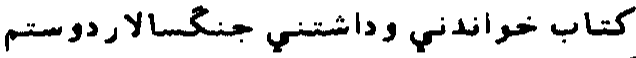

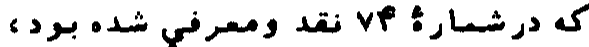

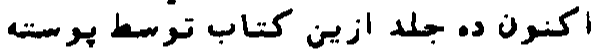

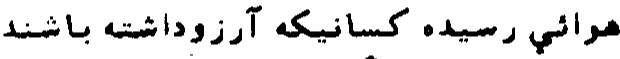

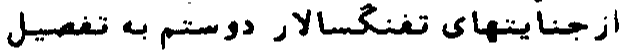

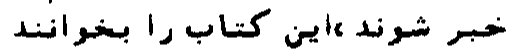
قيمت V دالر ـ مرجي كئ آئينه أفغانستان
59

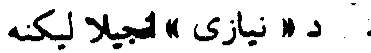

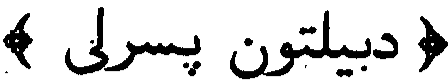

خليُّ وائى بسرلى بيابه زاتلون دى زمابه زوندكى غم هموار دتوريلتون دى بسرلى مى بيله تا بسرلى نه دى له تاليرى زما بهارد زهه به خون دى

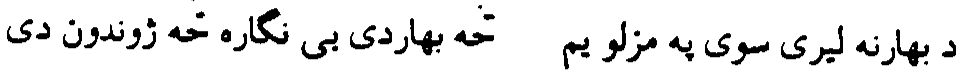

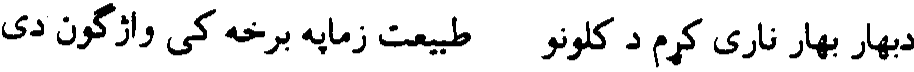

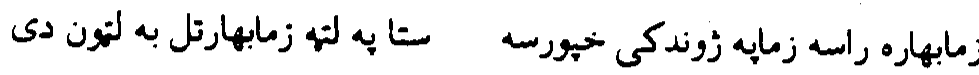

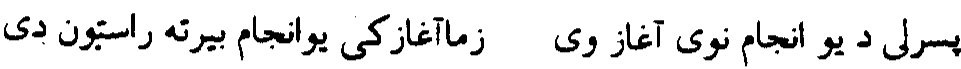

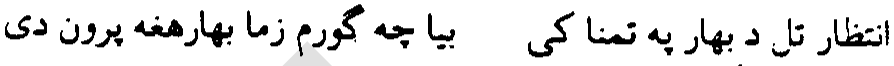

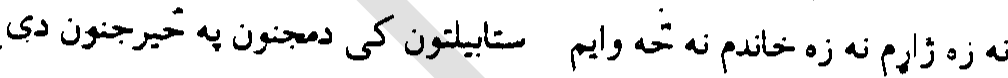

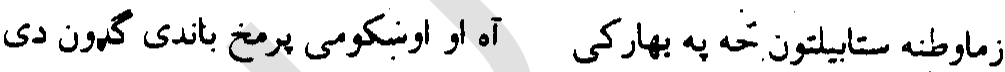

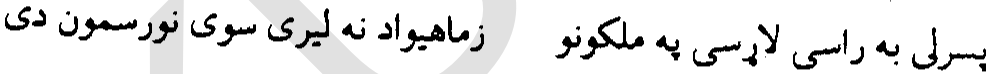

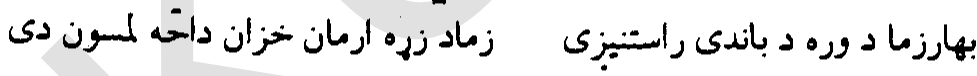

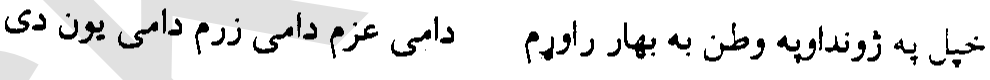

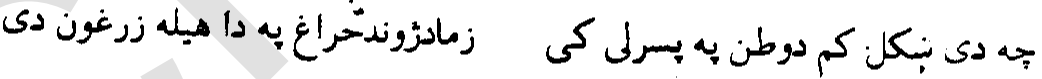

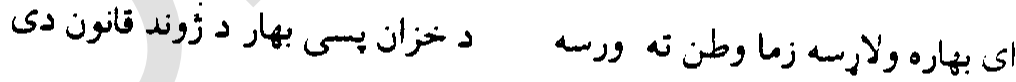

دبنبنتو دمشاعري خحلورمه درجه ورونكى

\section{alj alj assta.}

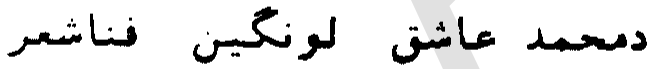

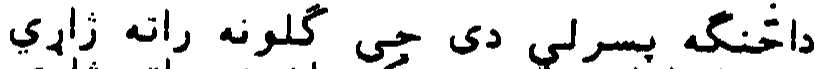

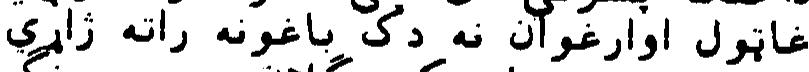

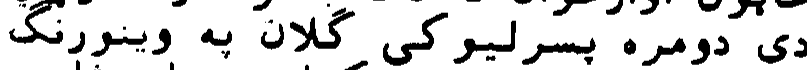

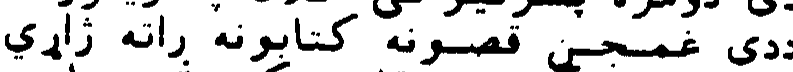

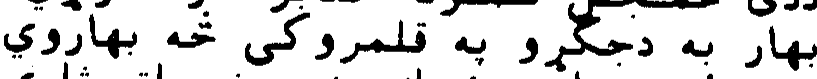

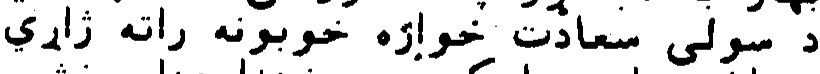

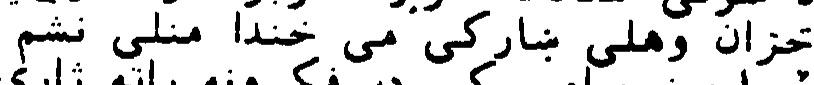

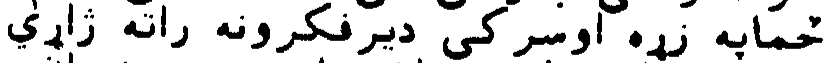

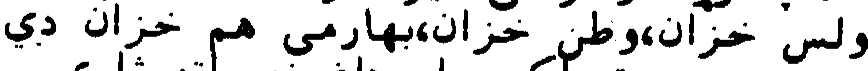

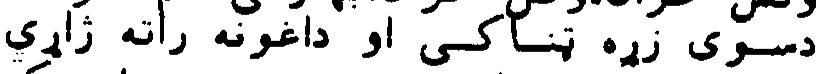

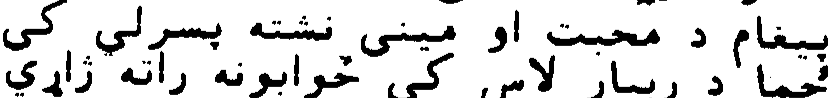

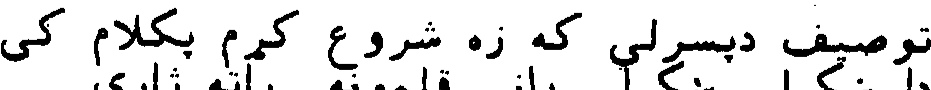

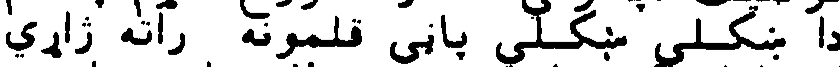

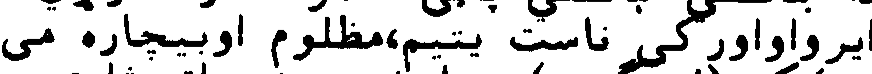

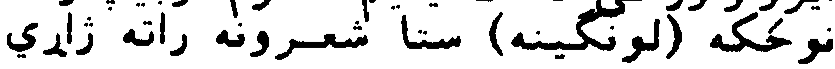

ديسبتو دمشاعرى دومهم دربه ودونكى

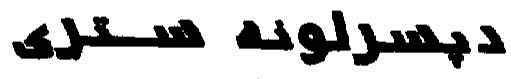

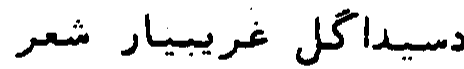

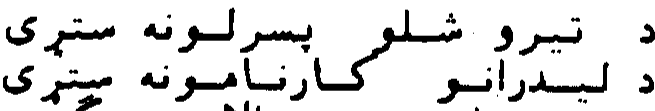

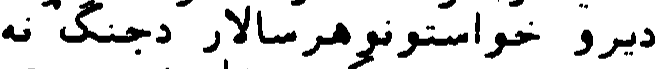

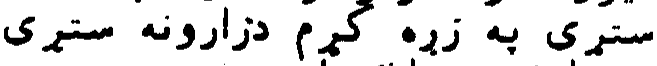

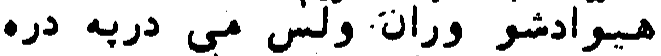

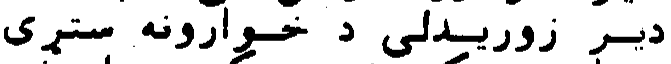

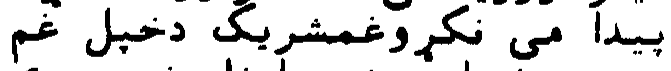

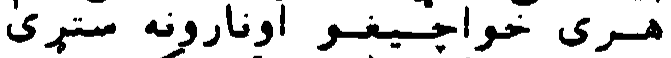

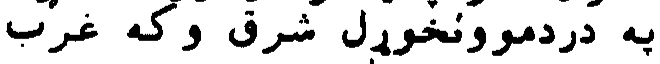

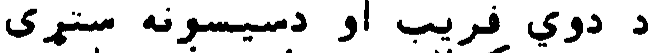

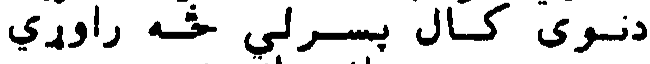

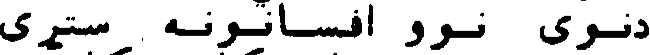

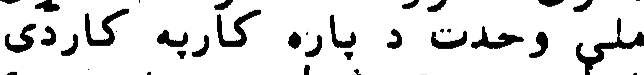

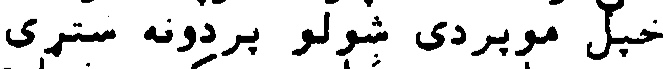

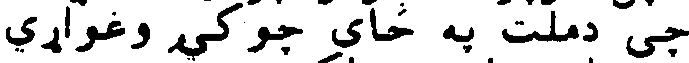

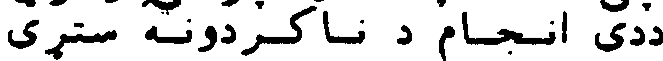




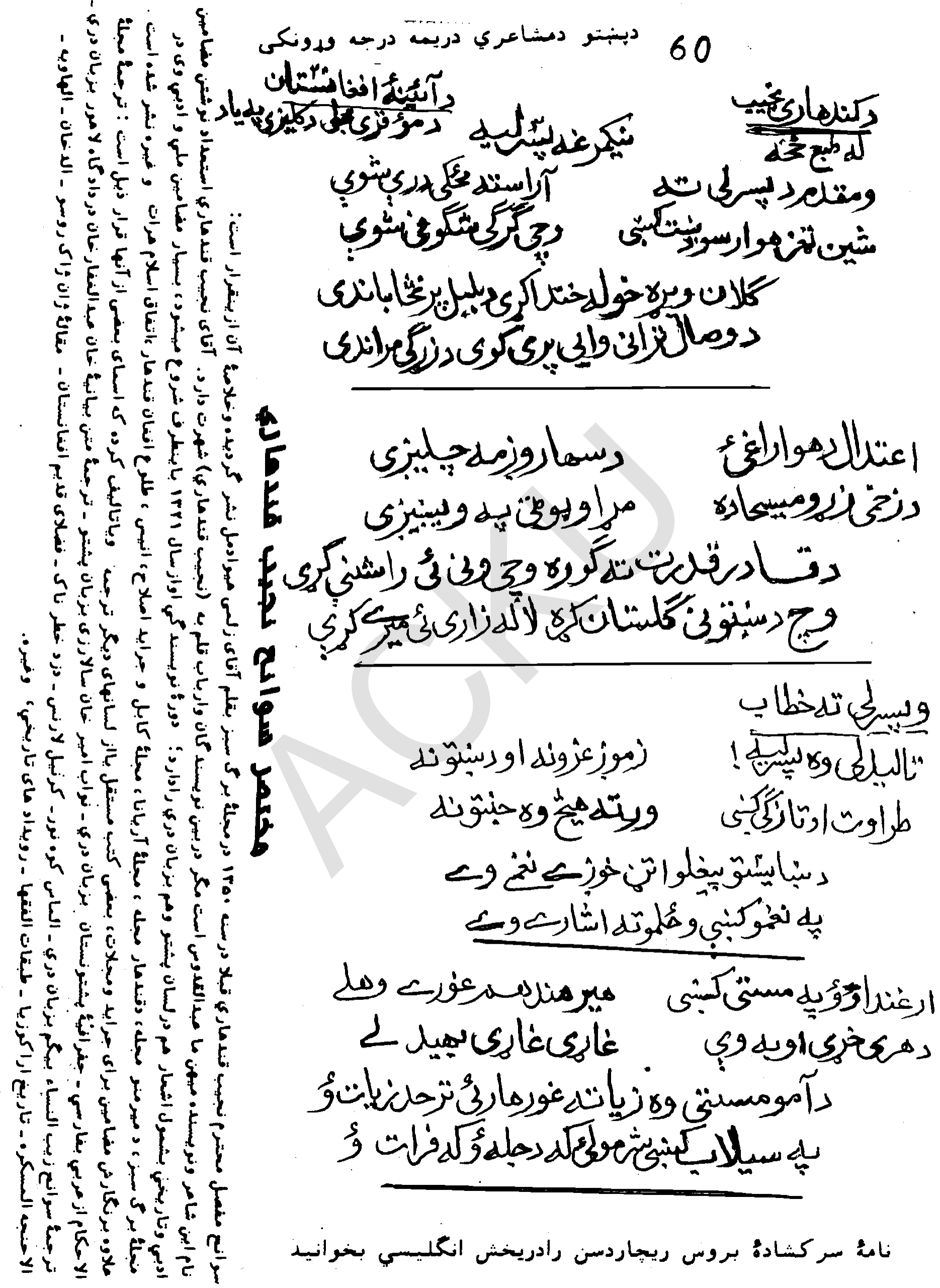




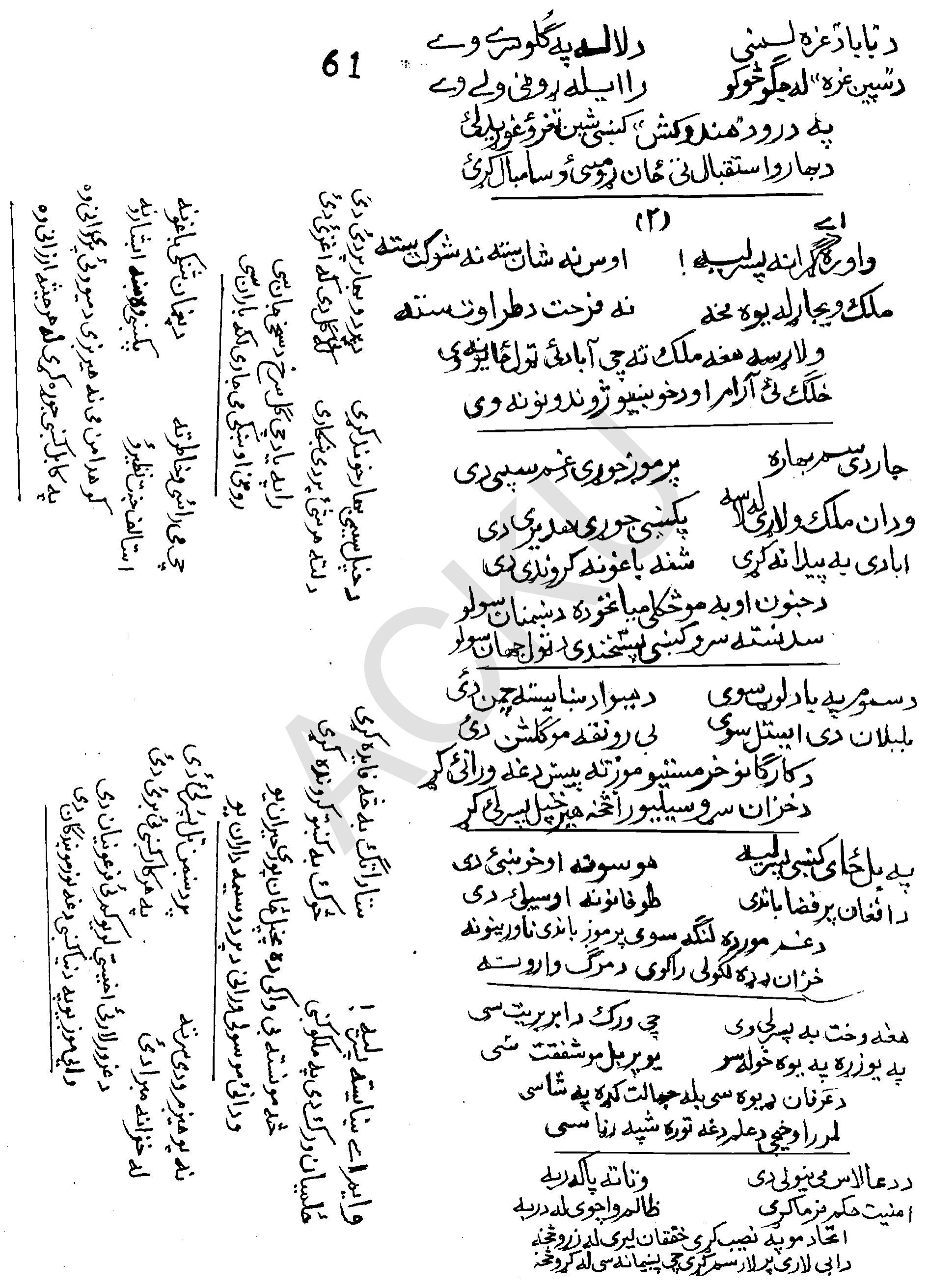


62

انجنير ملطانجان كليوال

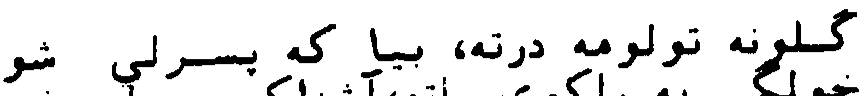

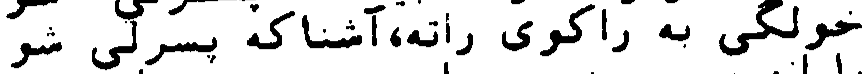

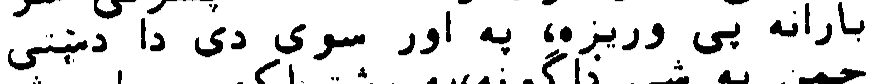
•

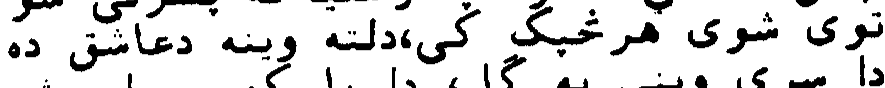

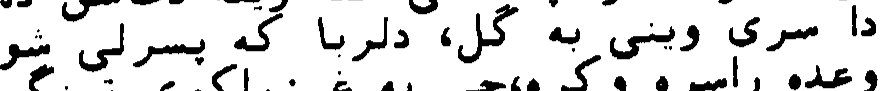

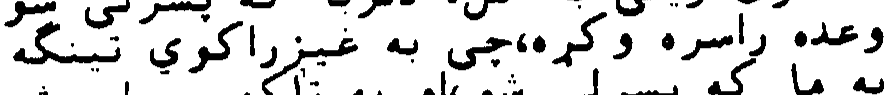

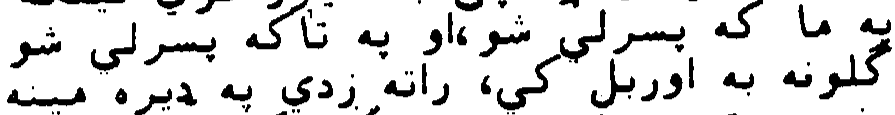

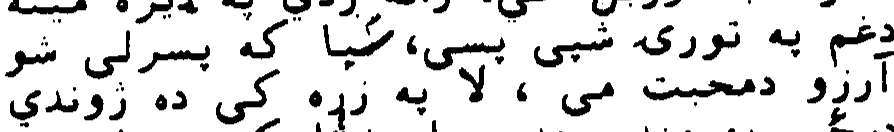

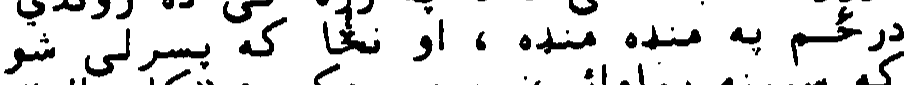

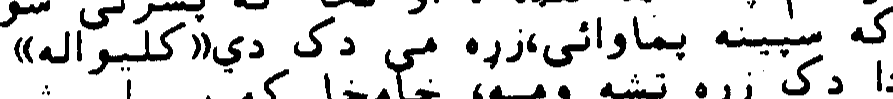

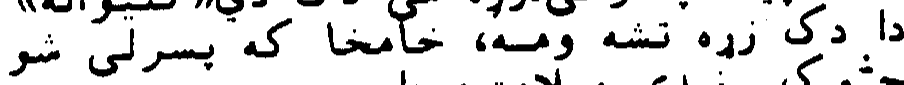

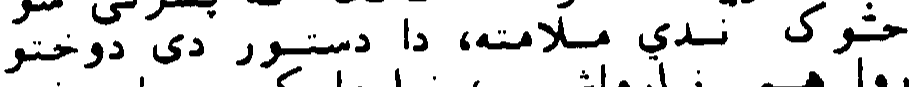

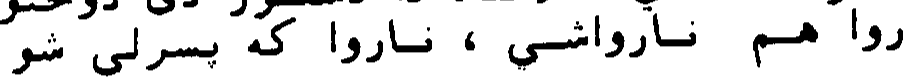

\section{الما دكلي بسرلي \\ سلطانجان كليوال}

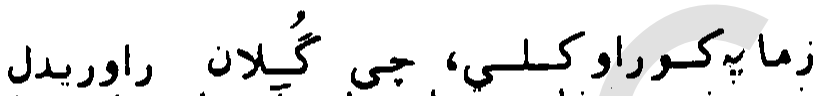

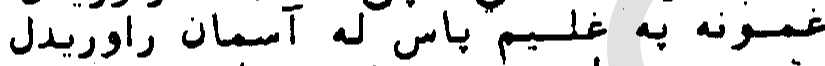

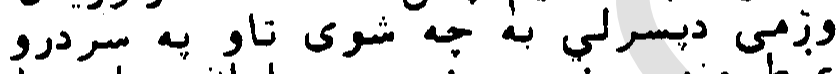

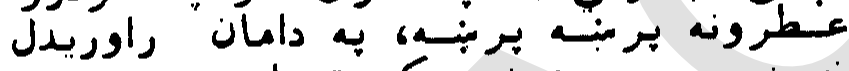

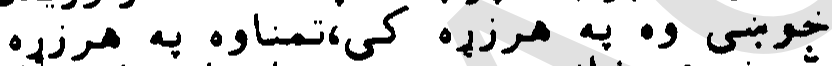

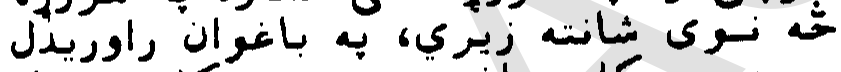

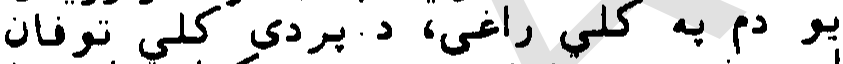

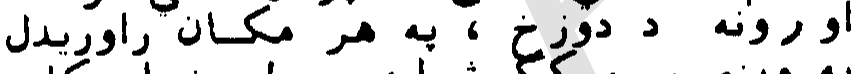

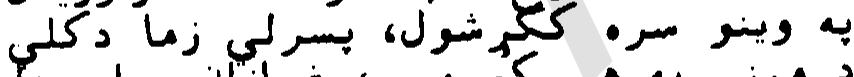

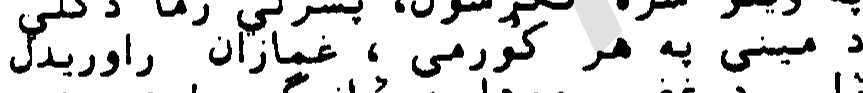

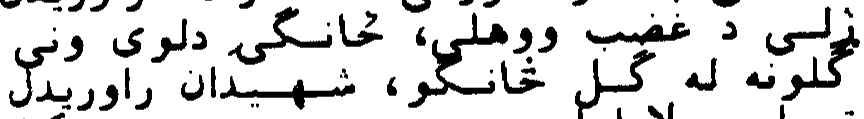

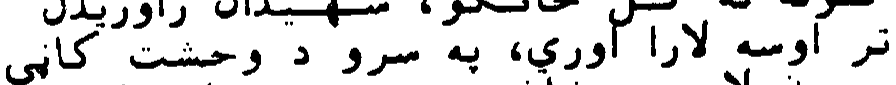

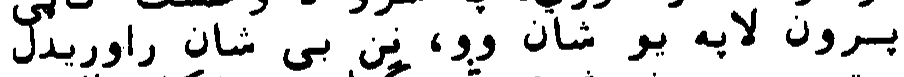

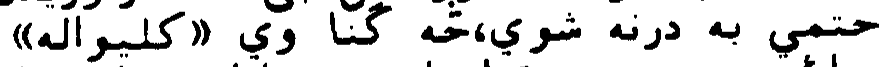

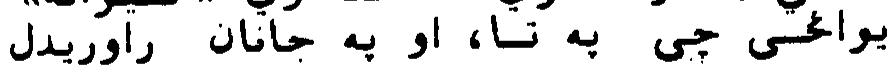

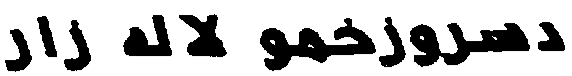

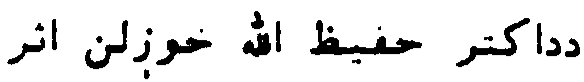

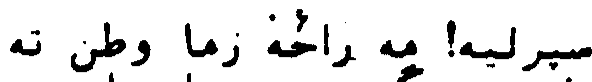

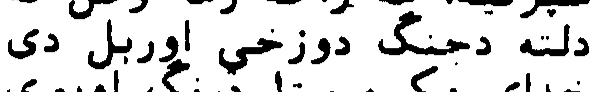

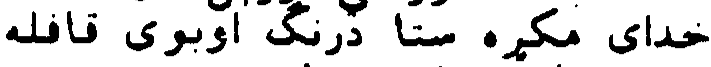

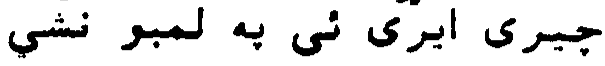

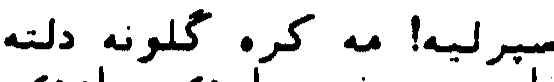

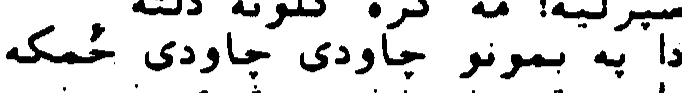

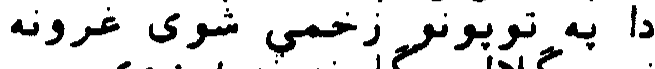

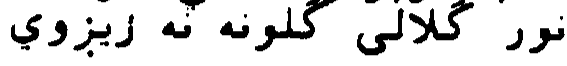

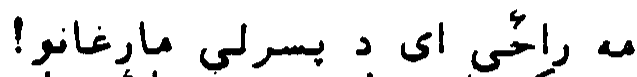

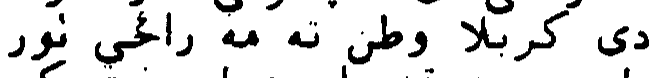

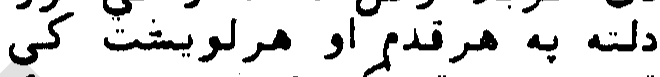

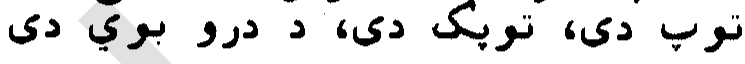

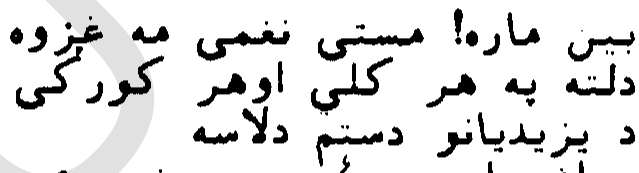

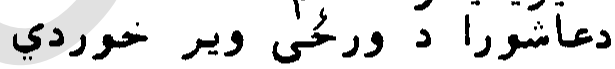

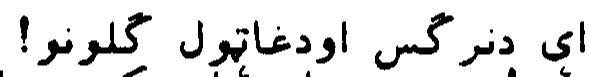

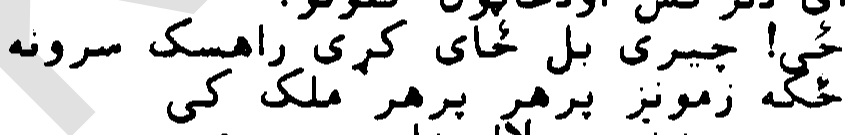

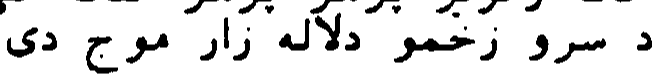
خر اي سبرليها كَ راحسى، واورها

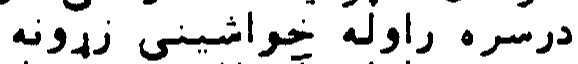

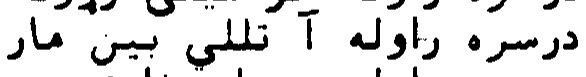
درسره راوله دسولى ناوى

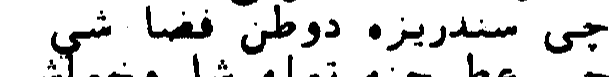

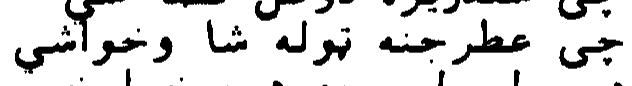

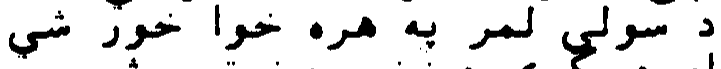
او دجيكرى دوزخي ميخ تور شئي

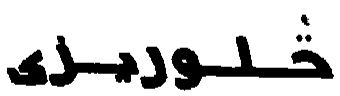

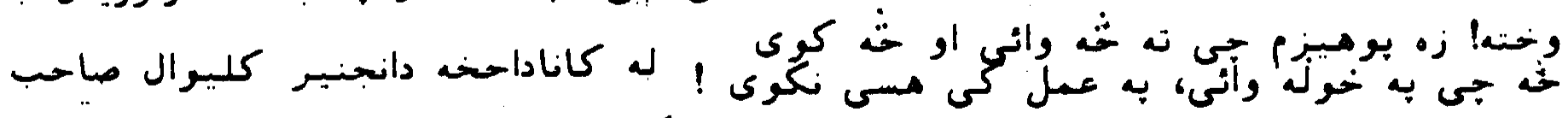
سكلى اشعار بس له مشاعرى نه ورميـده خر , به مشأعر• كى شامل كنل كيز

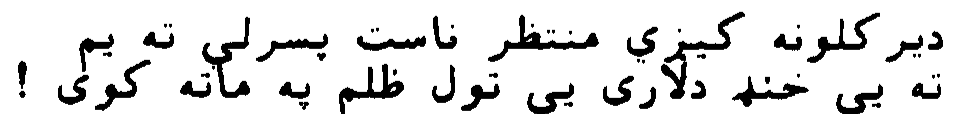

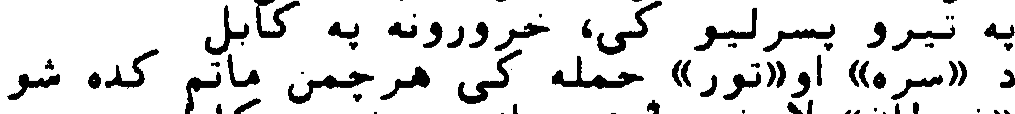

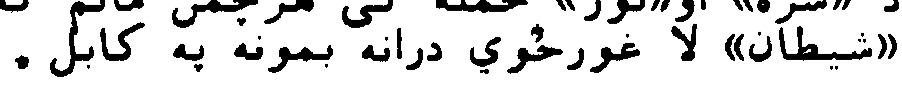


هوكي اولى راخي

$$
\text { ملطانجان كليوال }
$$

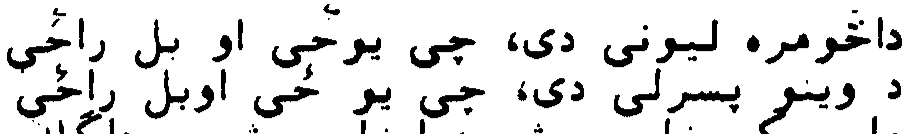

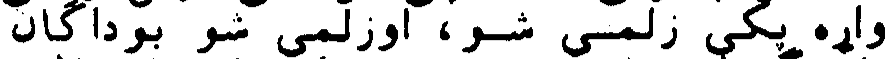

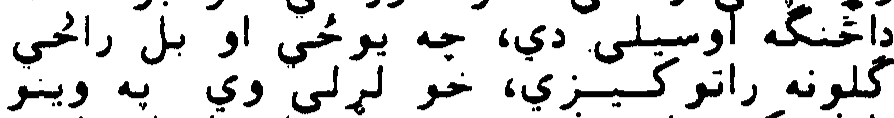

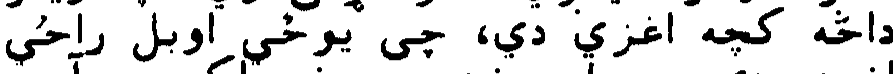

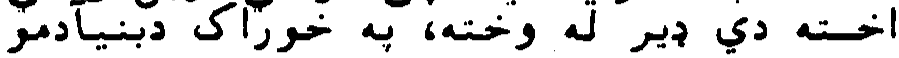

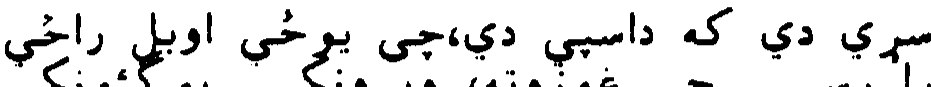

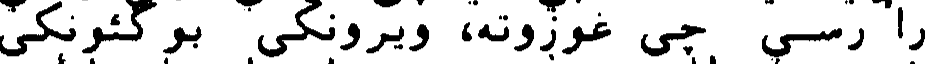

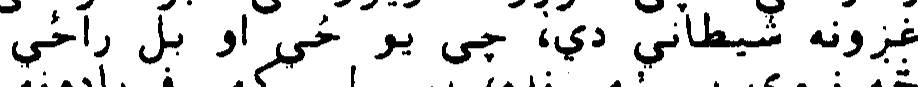

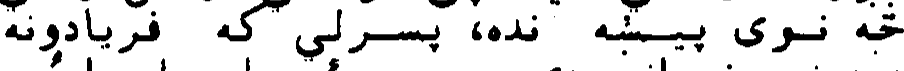

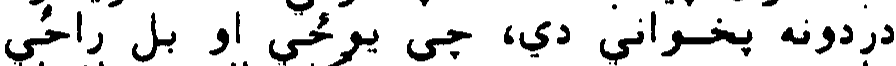

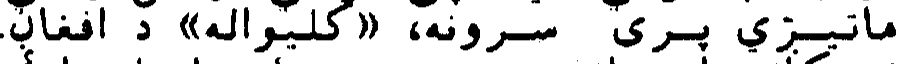

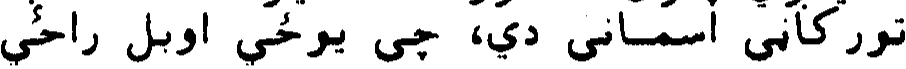

تورنتو - كانادا، دمارج لسم، 1999

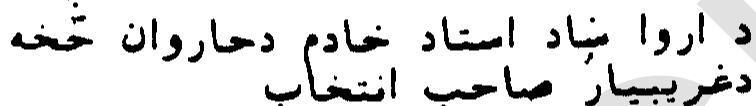

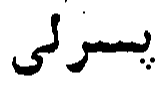

دنسا. مذر مبا بهلو دى دجمن را غلى دئ

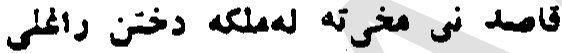

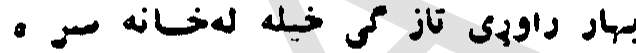

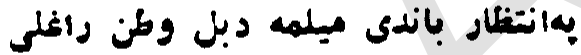

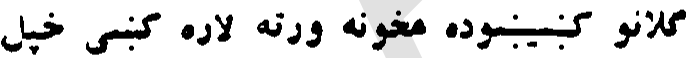

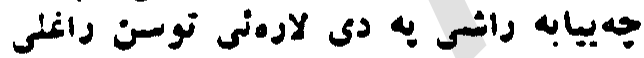

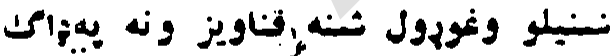

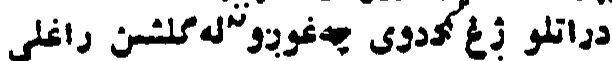

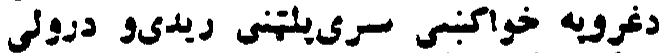

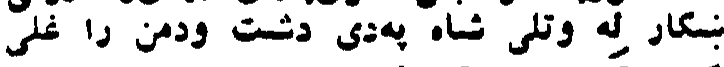

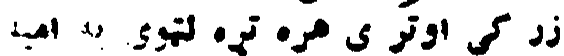

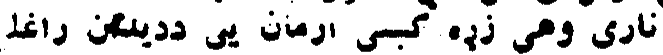

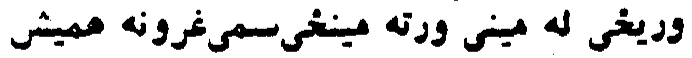

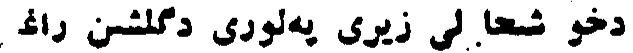

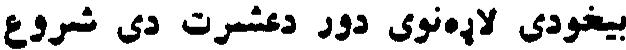

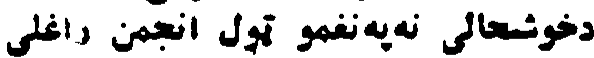

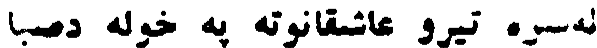

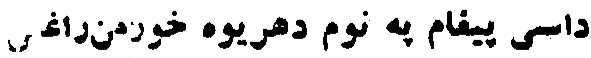

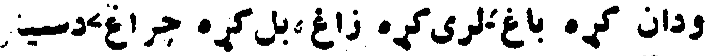

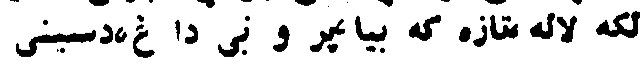

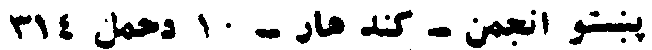

محمدمعصوم هوتلك

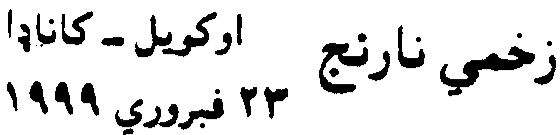

يارانو! مأمحفل ته تش لاسونه دي رالاي

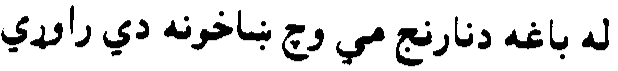
كرم نه يم ملامت نه يم قلم مي ويني رُّائي بيتو كي مي زخمونه و دردونه دي رالصي

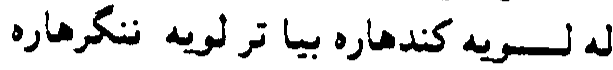
تاريخ د دوونارنجو داستانونه دي رالصي

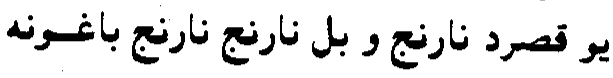

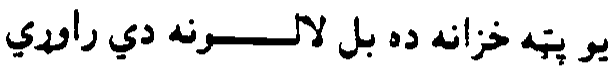

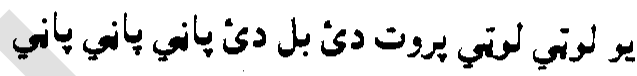

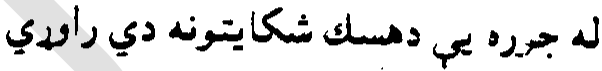
نارنج دئ دري لدي و مهده ده ورانه ورانه له خبلو له يردو يعي فـــيادونه دي رالضي له بوتو و له كانو جيغي خبزي اسمانسوته

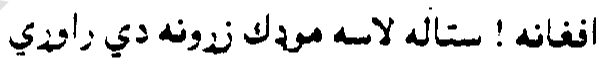

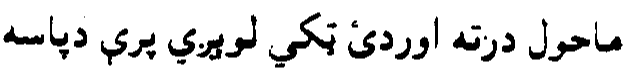

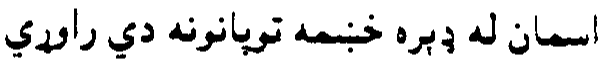
بربنبنا اوروندلنسوني و تناوي زهر بِاشي

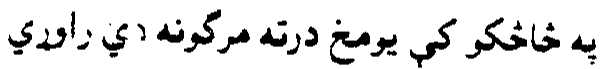
وطن دي كريلا دئ عاثشوري دي اخترونه

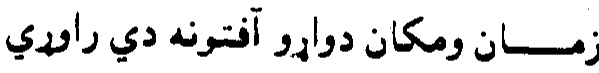

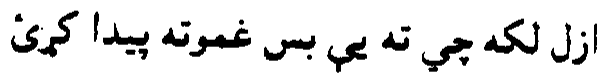
تقدير مكر ستا برخه اجلـرنه دي رالضاي. درجار سم در واري سم ستركي لجربشاني كره نخلاصي جي با درته اغزي و با كلونه دي رالدي

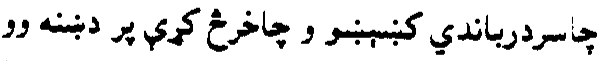

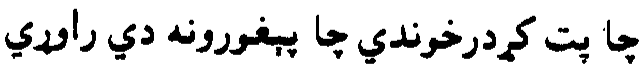

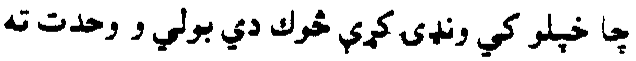

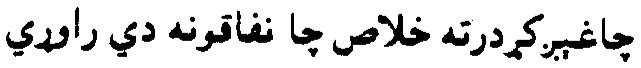

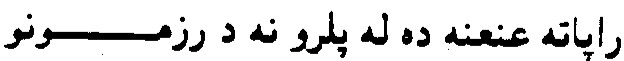

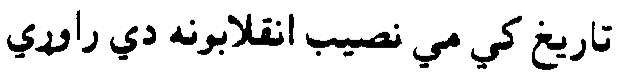
يقين مي دئ بر دغه ازموينبت بيا بريالى يجي

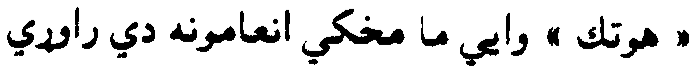




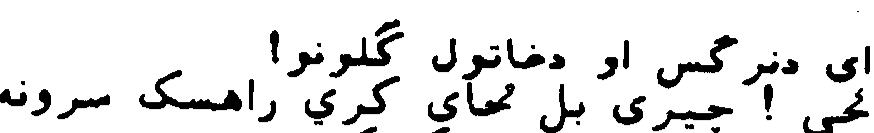

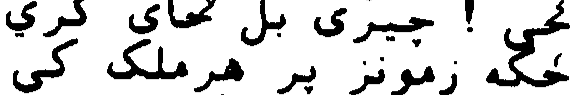

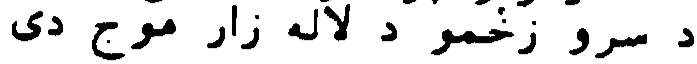

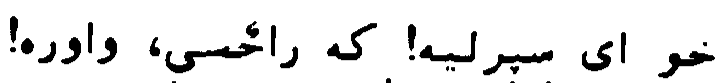

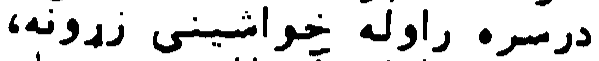
درسره راوله دآ تللى بين زئن مار. درسر• راوله دسولى نيلى ناوى

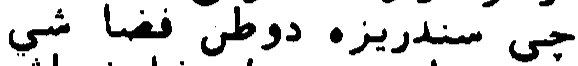

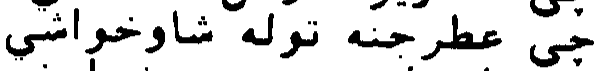

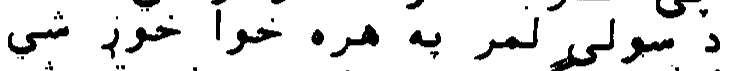

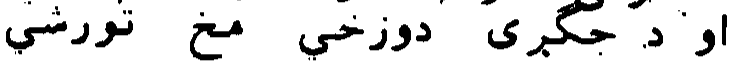

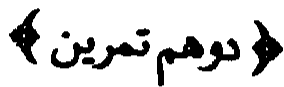

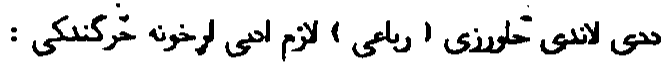

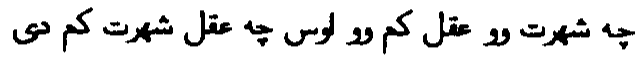

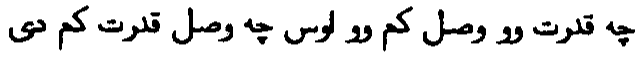

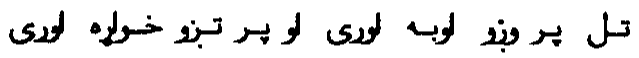

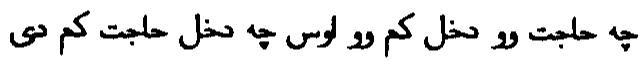

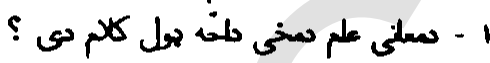

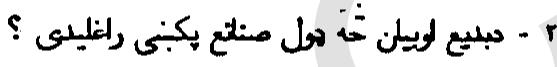

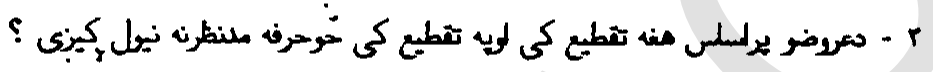

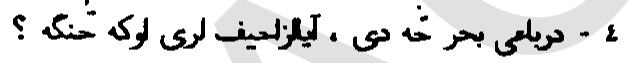

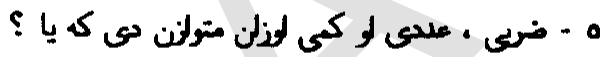

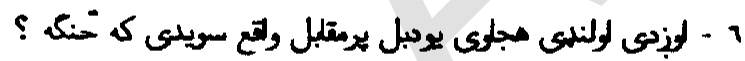

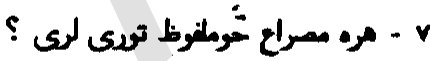

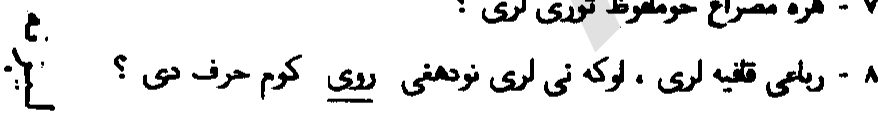

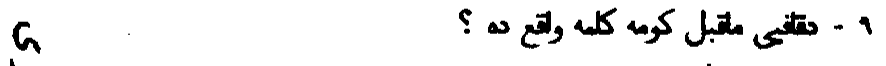

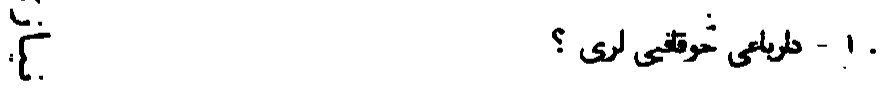

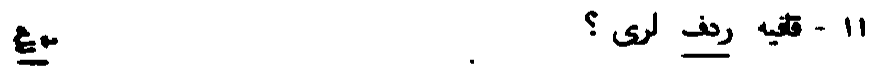

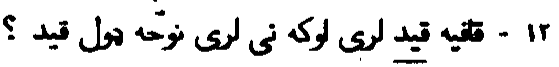

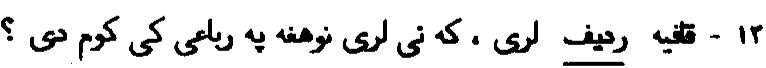

$$
\begin{aligned}
& 18 \\
& 10 \text { - مليه د تلِيس حرف لرى ؟ }
\end{aligned}
$$

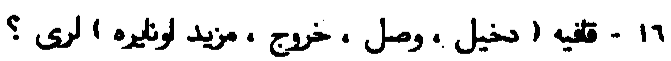

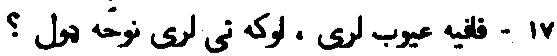

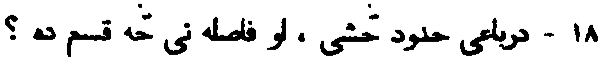

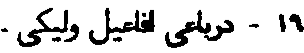

34

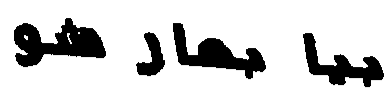

عبدالمسد ومابزاد نريار

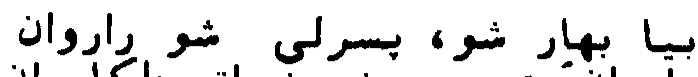

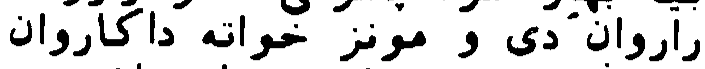

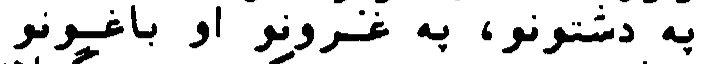

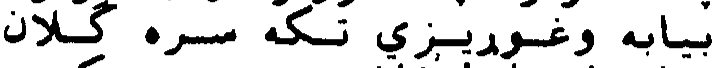

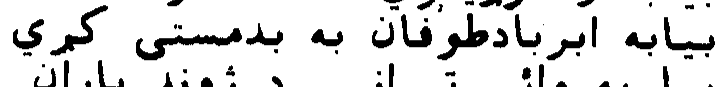

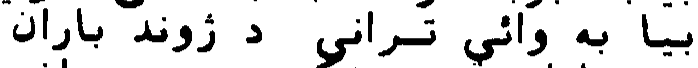

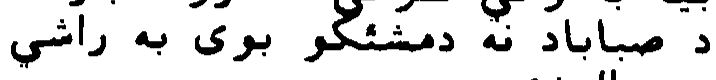

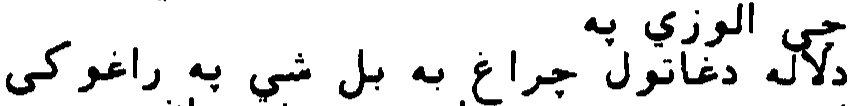

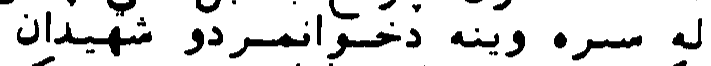

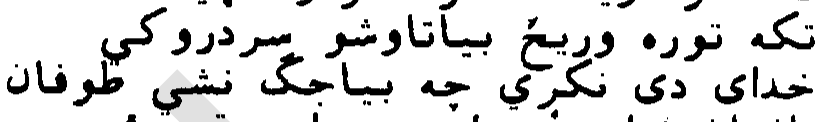

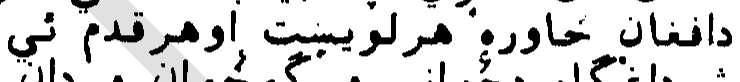

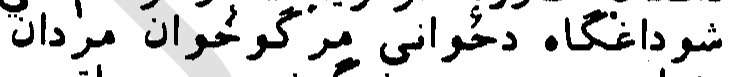

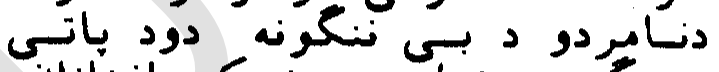

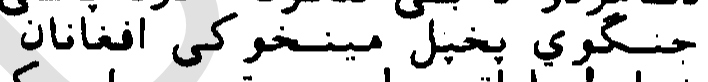

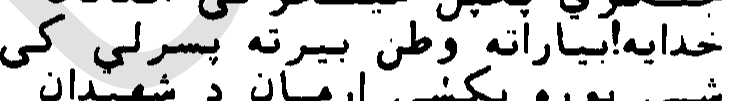

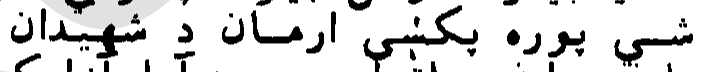

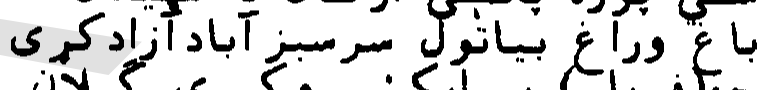

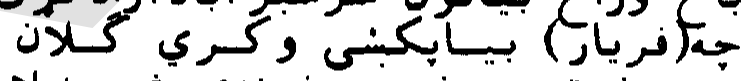

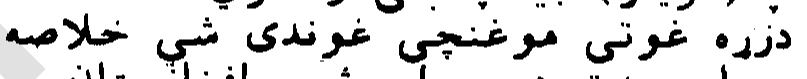

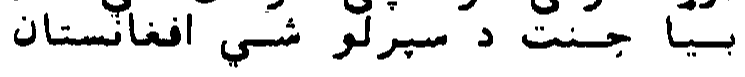

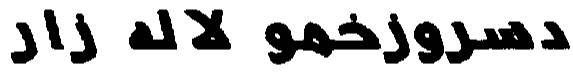

داكترحفيظ الله خوزلن دتورنتو كاناداحخه

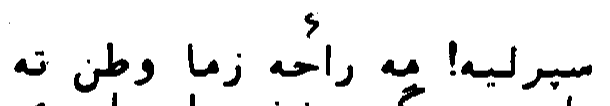

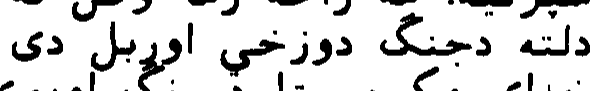

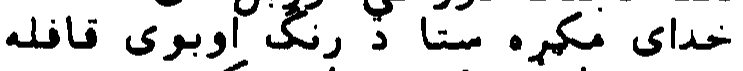
جيرى ايرى نى به لمبركي ني نشي

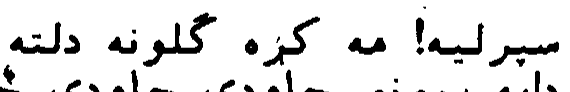

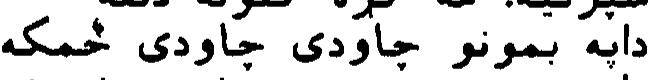

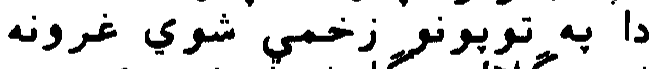

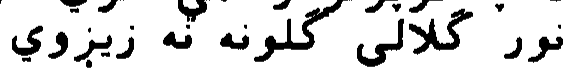

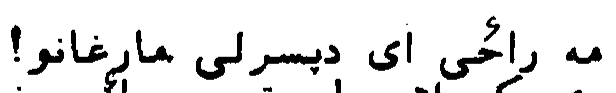

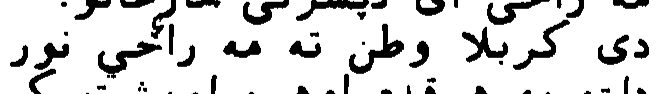

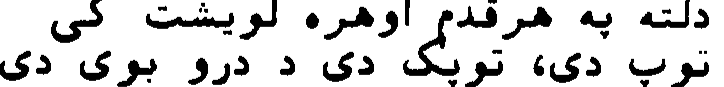

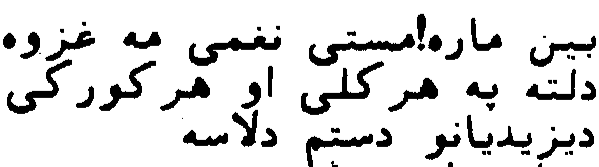

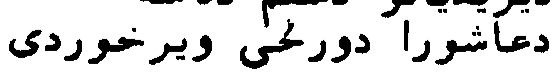




\section{د منلى او مهربان خدأى ج بنوم}

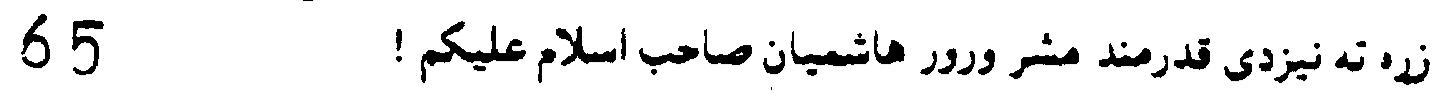

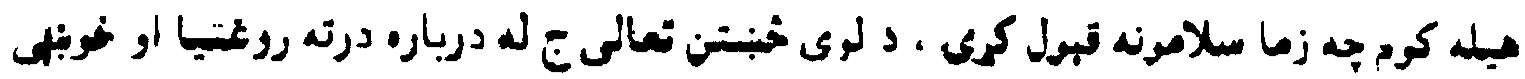

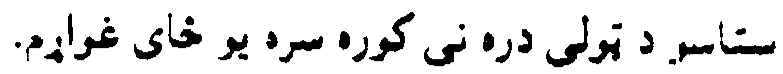

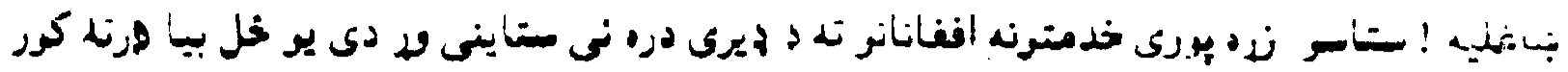

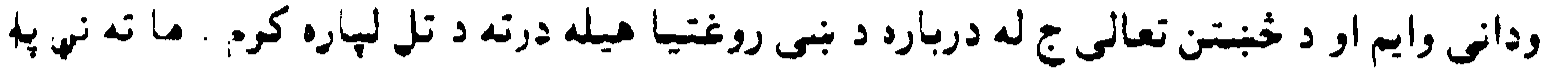

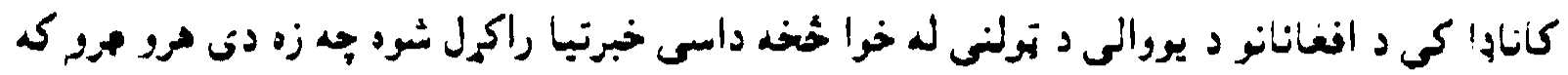

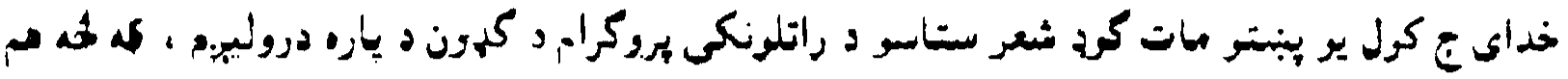

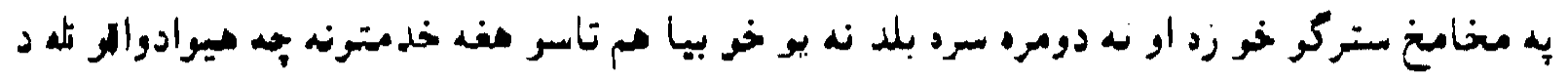

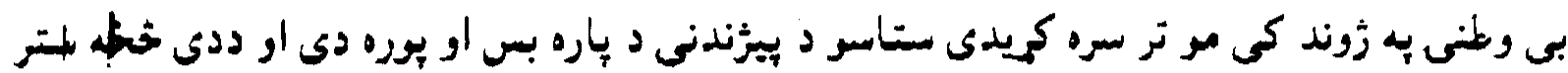

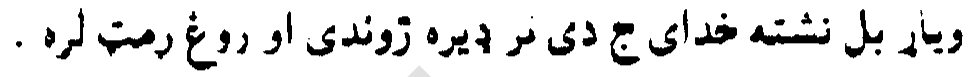

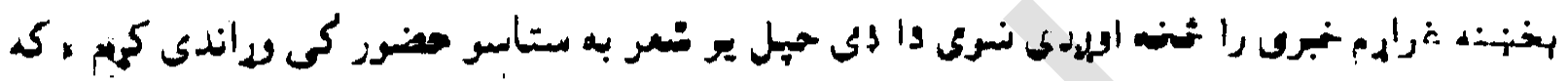

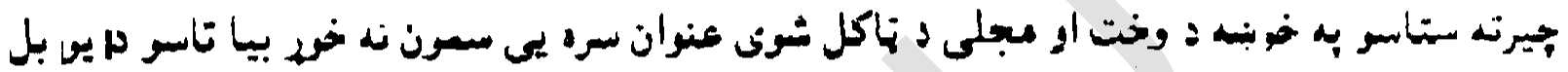

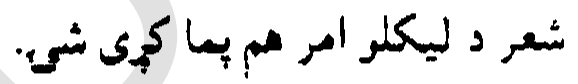

$$
\text { به درناوى }
$$

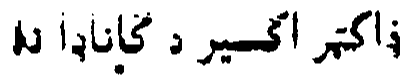

$$
\text { د نارنج ميله }
$$

د نارنج ميله نتكرهار كى كيده كرانو

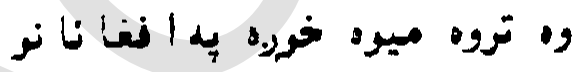

$$
\text { ترل بـ راغلل د كخابل مرأت بخكيا نه }
$$

بلغ ننكرماركند مار وه خاك دشاعرانو

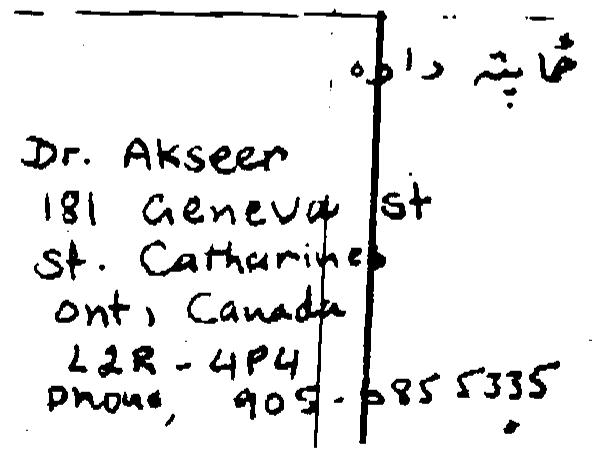

$$
\text { د سيين غر اومندوكث باميردنخوكر }
$$

وه بغار به به شين بن كى د توتيانر

$$
\text { ارغنداب به ياديده تخت سفر كى }
$$

د دوضى شاه اوليا • سيل د سخيانو

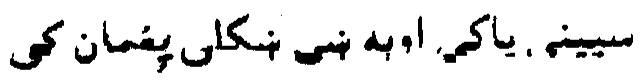

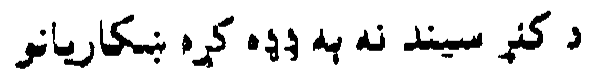

د باميانو بوتان ياد د بلغ تاريخ كى دئ

د مهى بـ لار كاروان د رونبانيانر

احمدشاه بابا ميرويس كه شاه محمودوه لاس يع لند كي د انكريزمم دمندوانو 


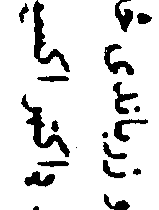

5

666

د درات باسسون كمى بريد وكم نويجيانر

$\sum_{5}$

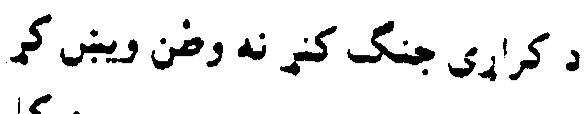

دوبل بالاحصار غبر د زمريا نو

\&

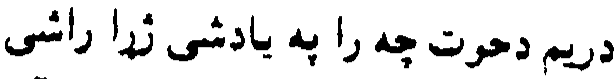

كوم أراز جه اوجت نميندوكيبيبانو

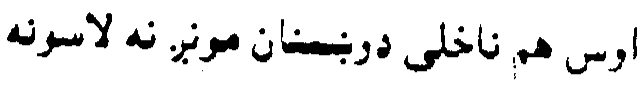

نن يع ثحه حالت راودى دى دوستانو

$$
\text { وروردوروربه مرك خوشنال لاسونه سره كره }
$$

د سرو وينر ججد بهير دى د خوارانو

وطن وران كيميه دوستى كى كارنيانو

هر سيى بعربر. مطلب كيى بدمرغى درد

خدائ ديار، خبلد خوبنه خبل اختياركيى

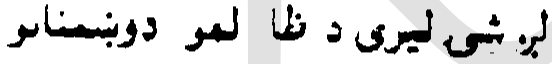

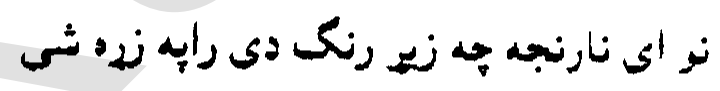

خداى خد, كم وكرو سبل ستادكلنو

ל.

سبين كلان دى مياوى بنكارى بـ باروتر

جلال كر بن كي ديرى ورانى د بنرانو

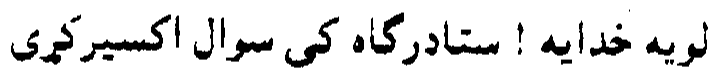

وطن خلاص كى زمونب ز; دمطلبيانو

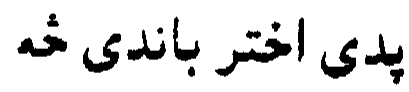

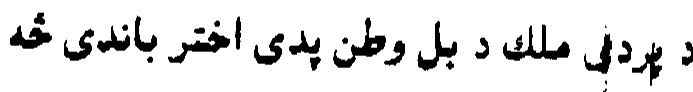

نور جا مو بنه نعتر بنكلى بستر باندى ثد

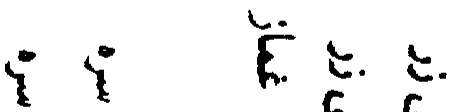

\& 9.9

ह.

\&. है:

c. क

है

E.

द.

है.

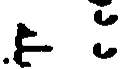

t.

$t$

د زوند ناخوالم بى خهلوانو بردى درباندى ثي

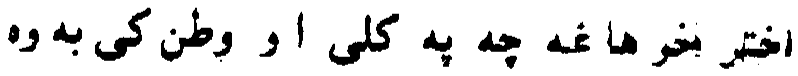

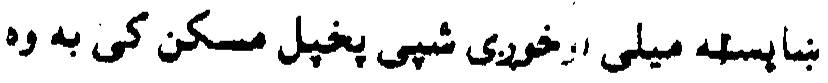

ريبار خنداكى ما ليدلى بل خبر باندى ثه

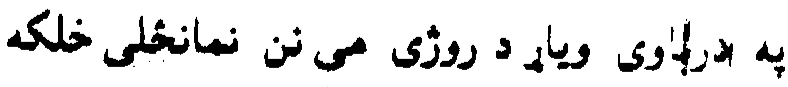
تروئمس بسى د سيين صبا خرب مى لبدلى خلكه 


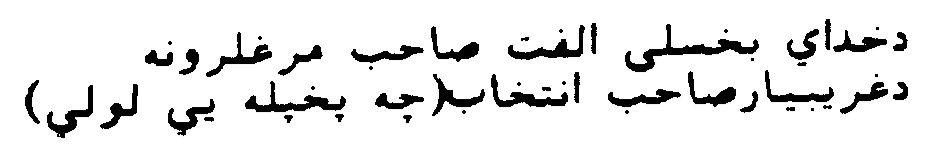

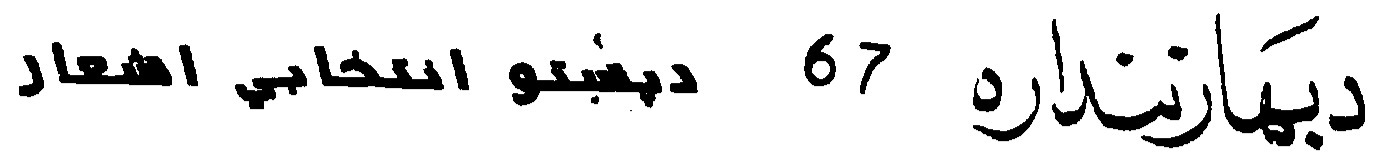

Ang J X L

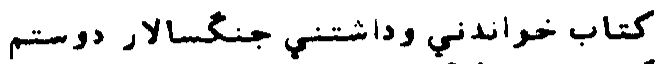

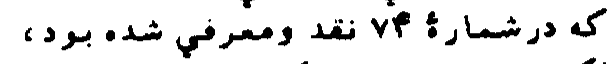

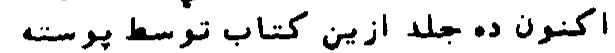

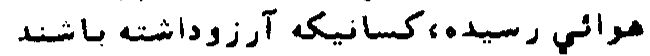

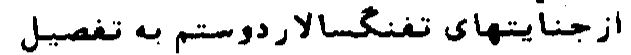

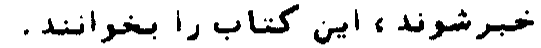

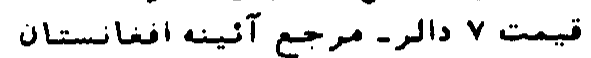

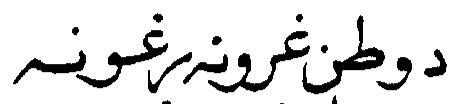

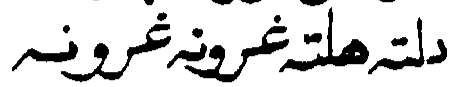

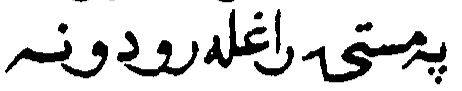

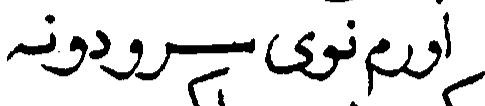

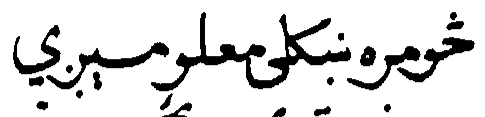

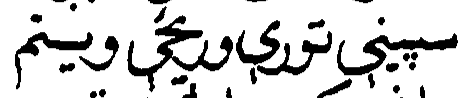

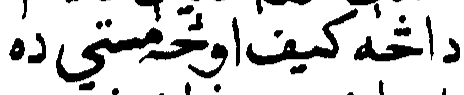

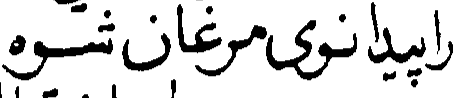

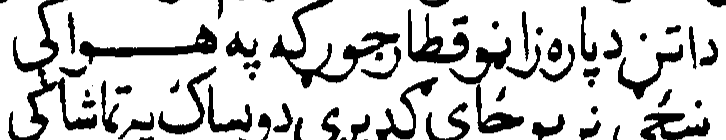

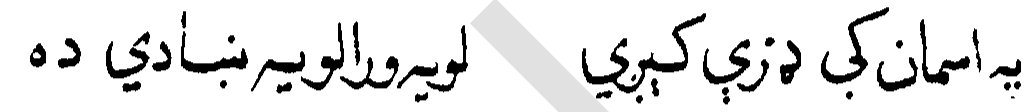

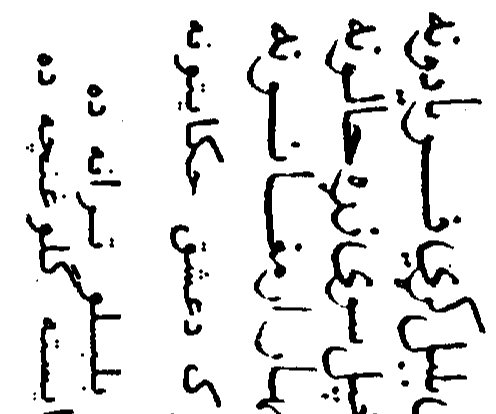

Fo

के $\bar{G}$

然, $q, a$

r.e. $\varepsilon=\xi$

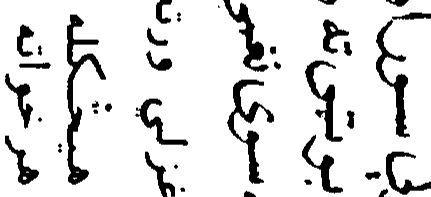

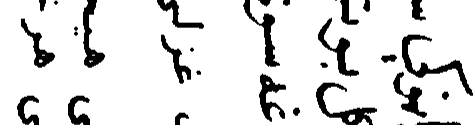

द. द. दे. हैं हैं

to

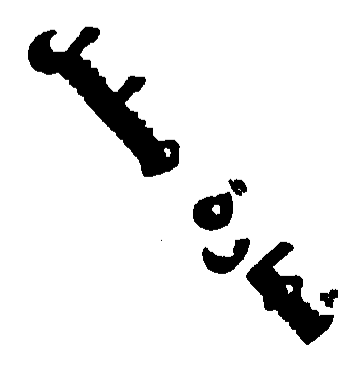

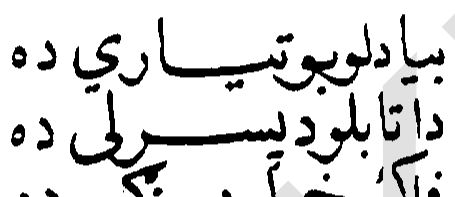

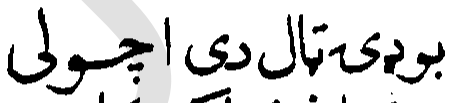

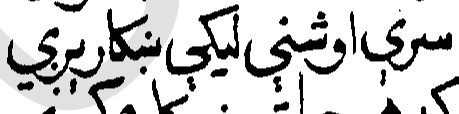

كونجاتربن

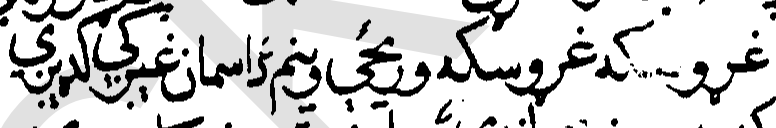

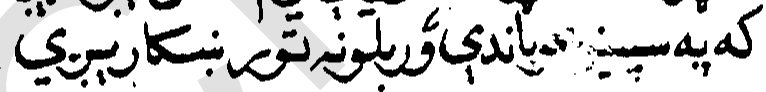

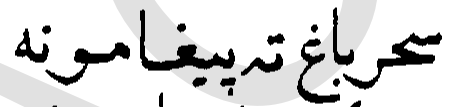

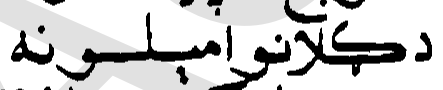

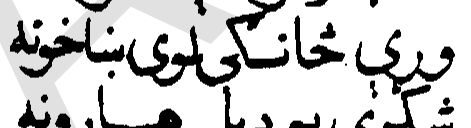

dis 1.20

•

oL

$\int_{1} l_{L}$

-

(

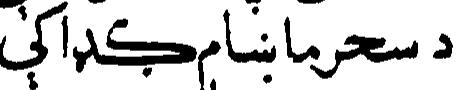$$
\text { (1) }
$$

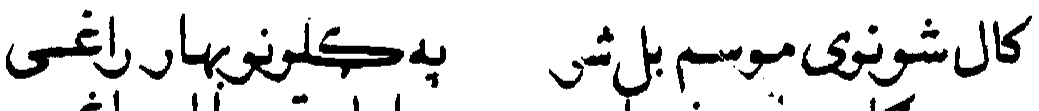

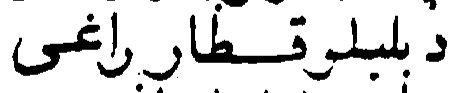
S

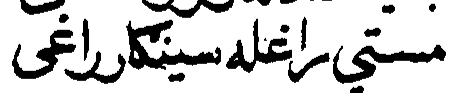
ن

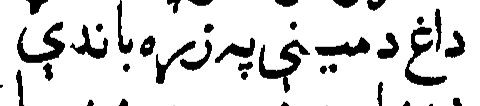
Livis

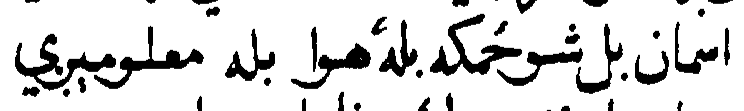

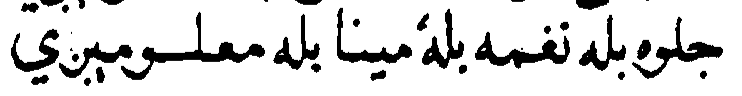




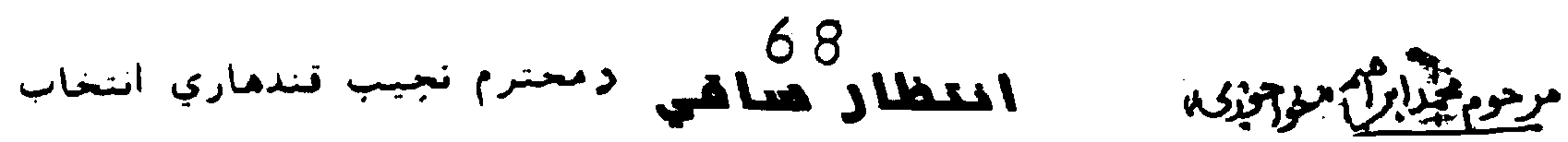

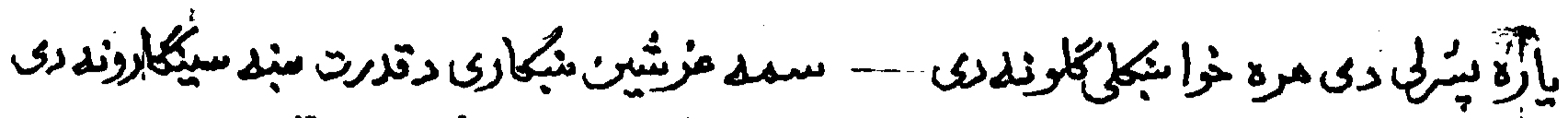

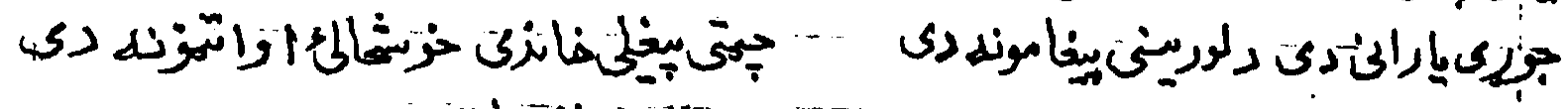

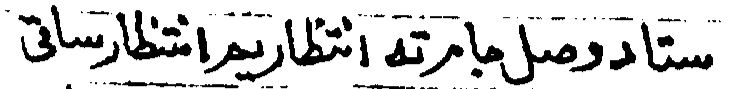

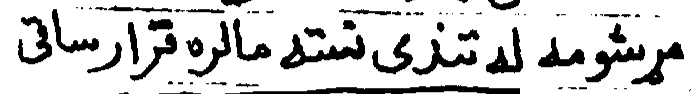

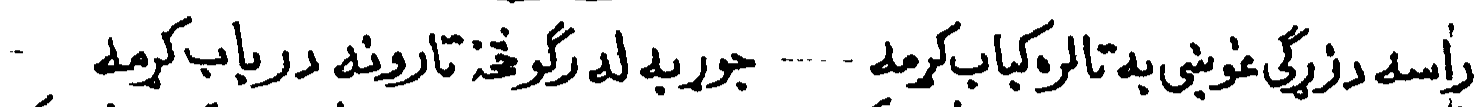

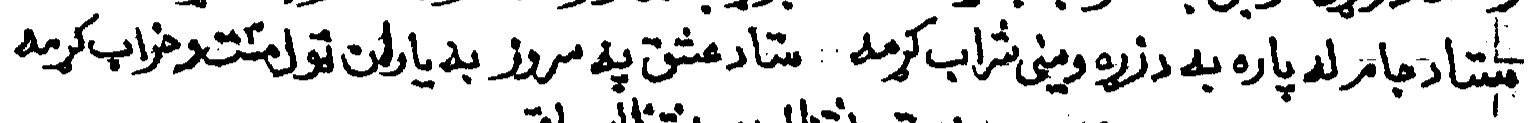

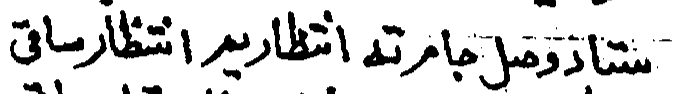

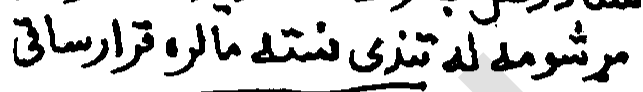

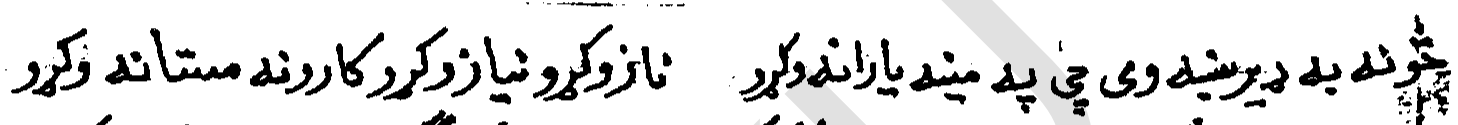

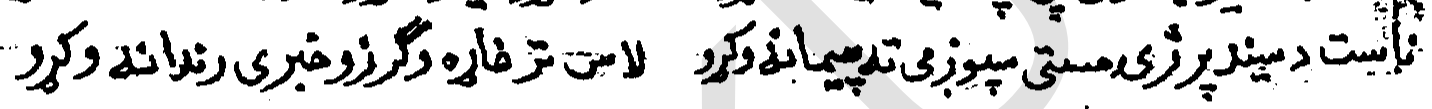

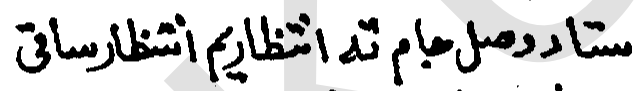

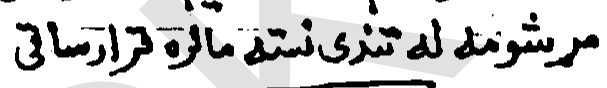

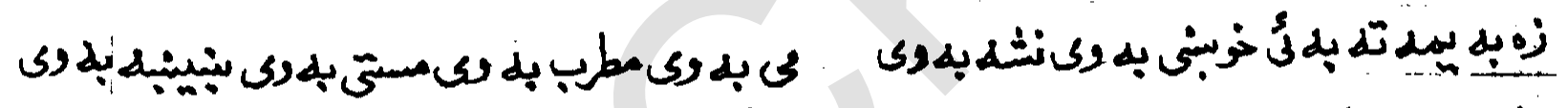

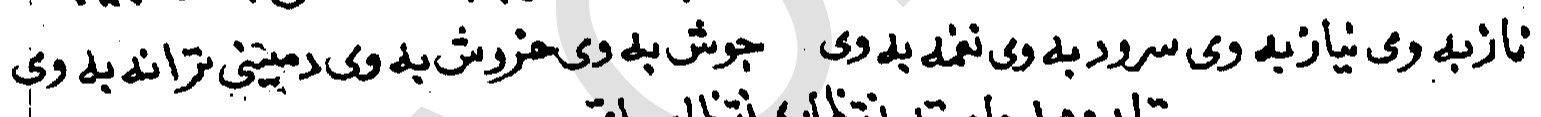

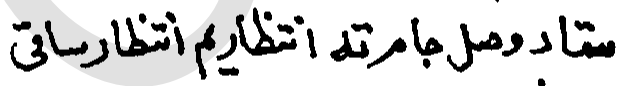

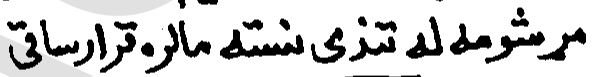

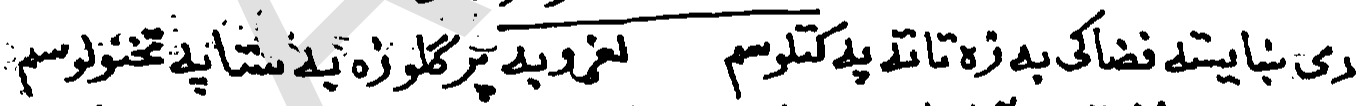

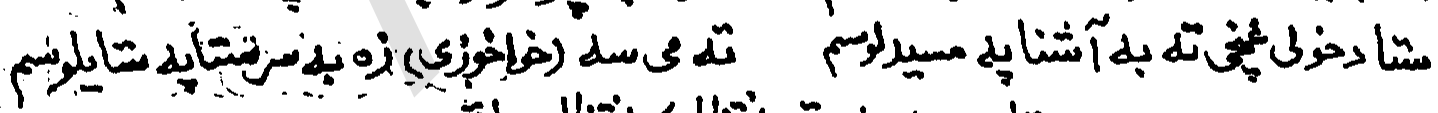

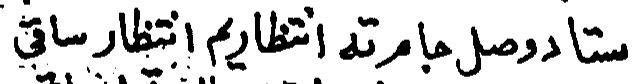

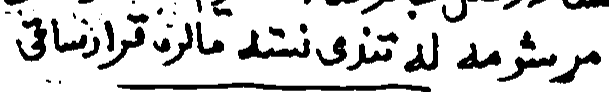

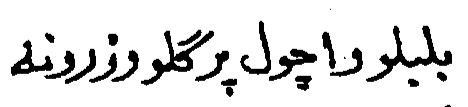

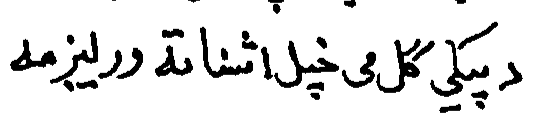

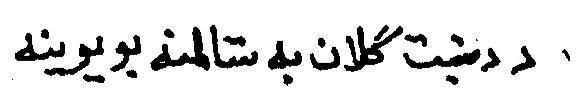

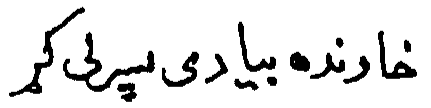

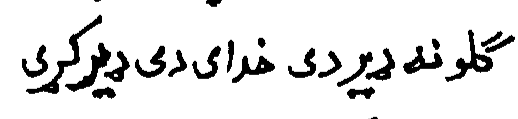

سابه بياكرى بإيزي

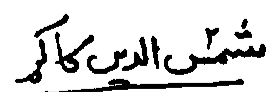

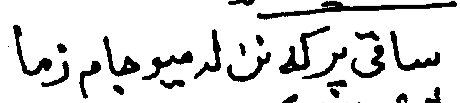

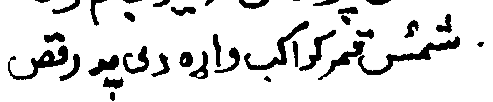




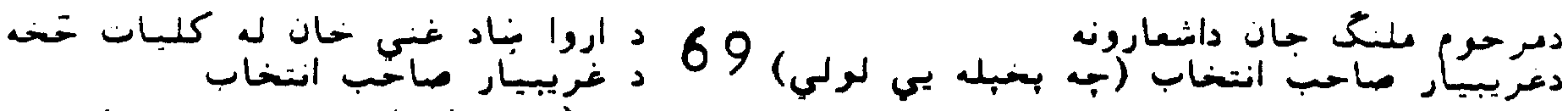
(جى استاد بيماريي لولى)

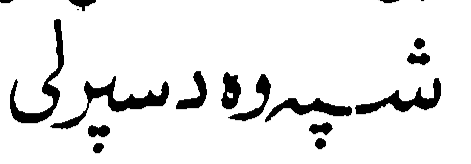

مو سم دإسمر لى دى

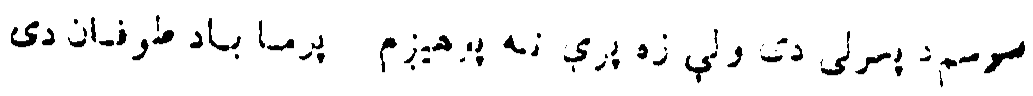

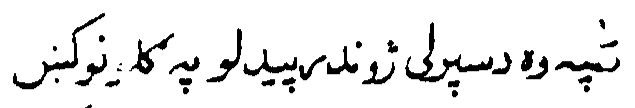

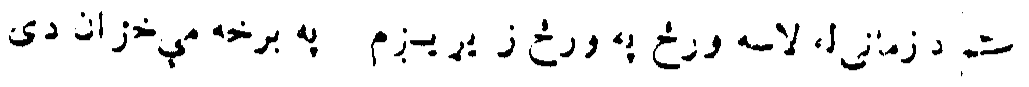

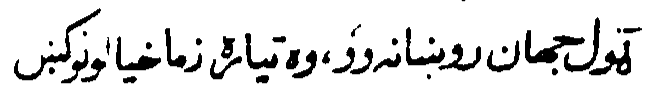

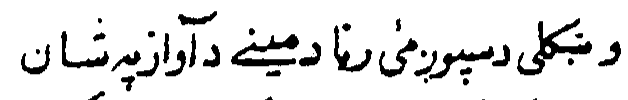

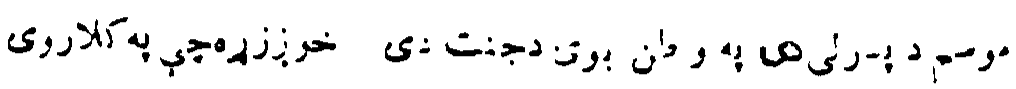

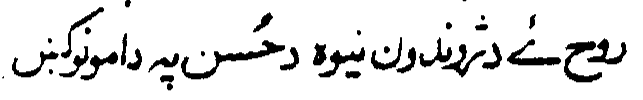

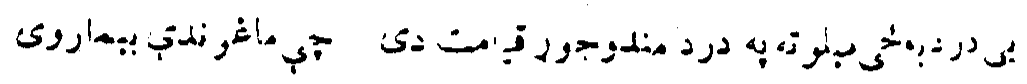

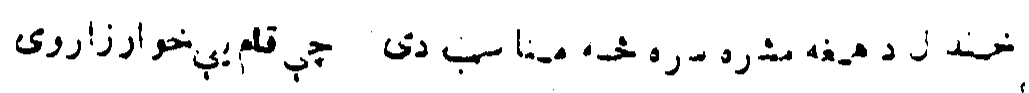

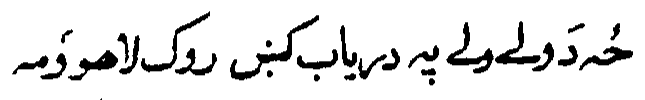

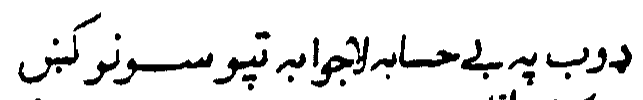

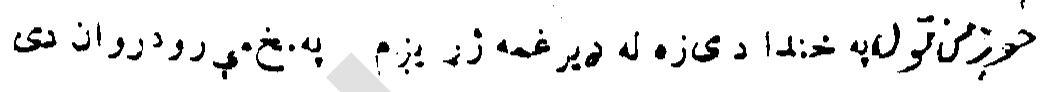

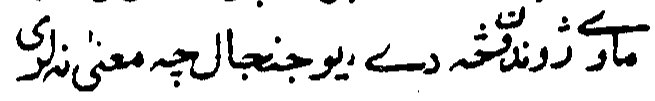

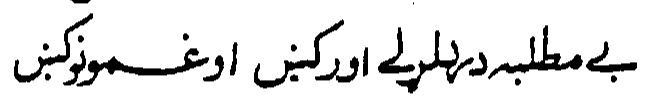

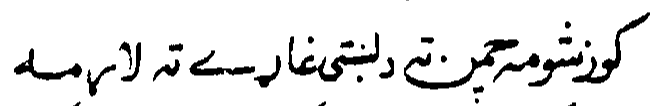

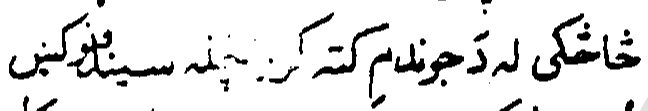
l...

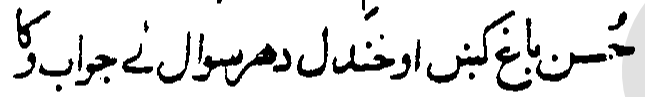

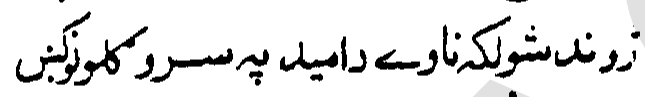

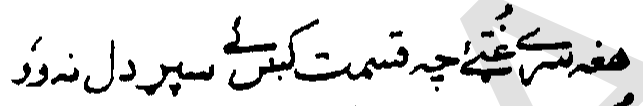

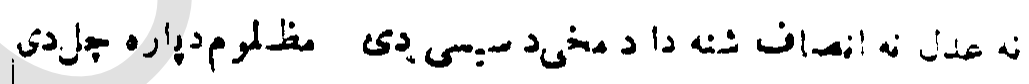

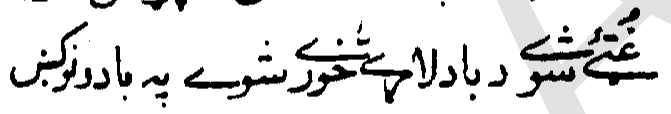

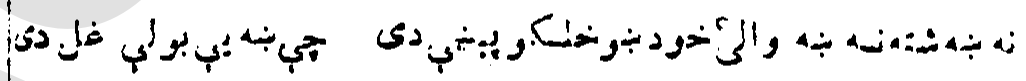

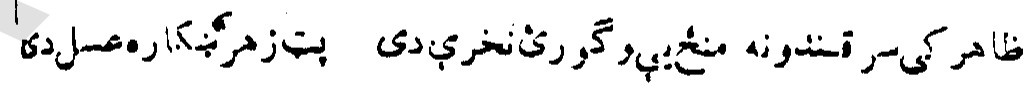

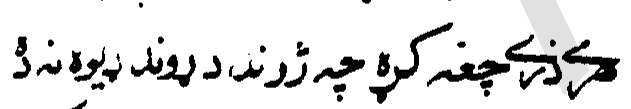

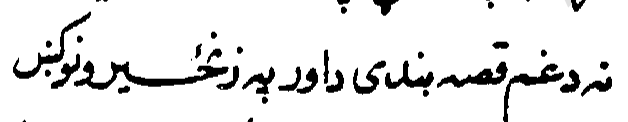

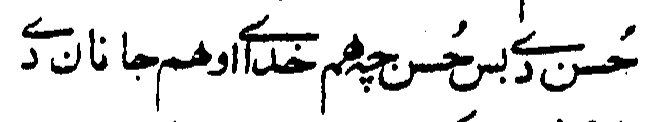

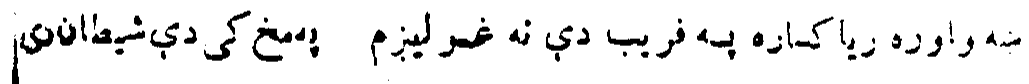

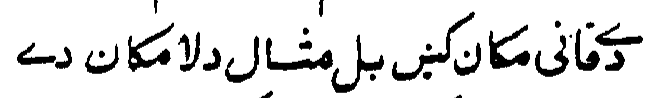

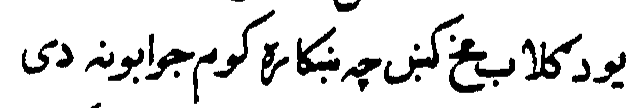

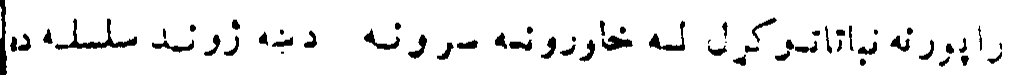

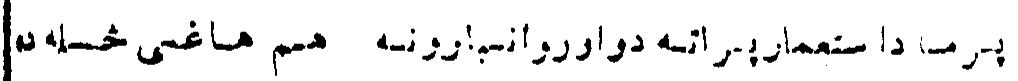

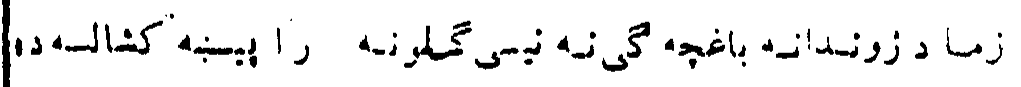

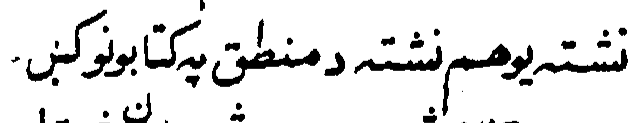

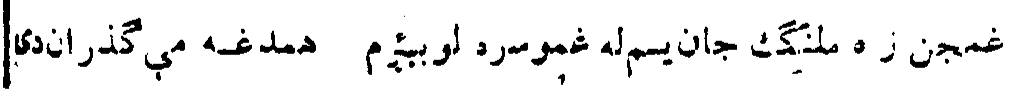

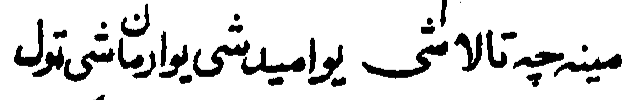

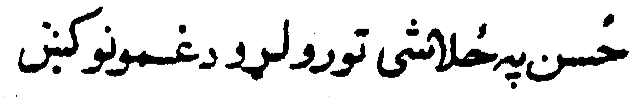

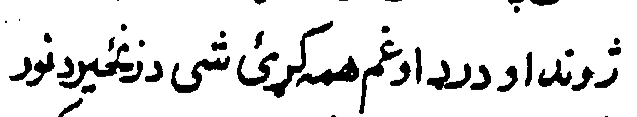

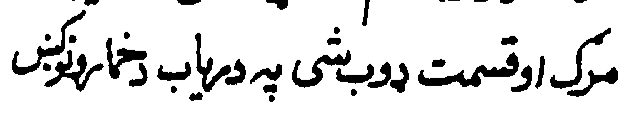

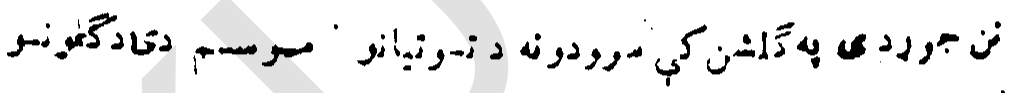

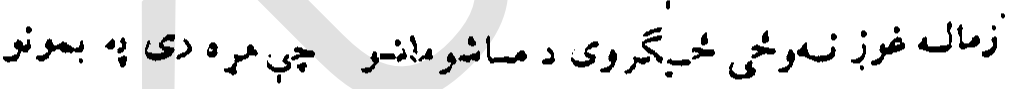

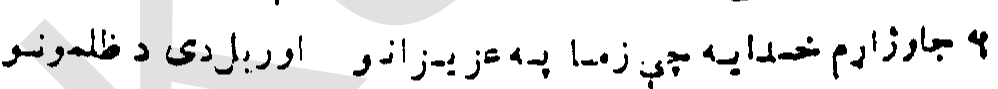

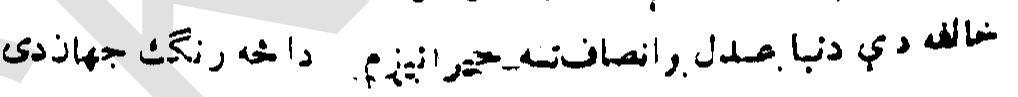




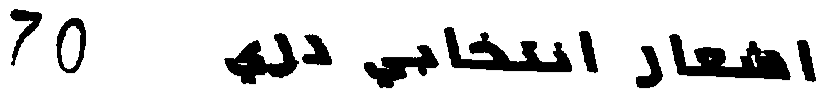

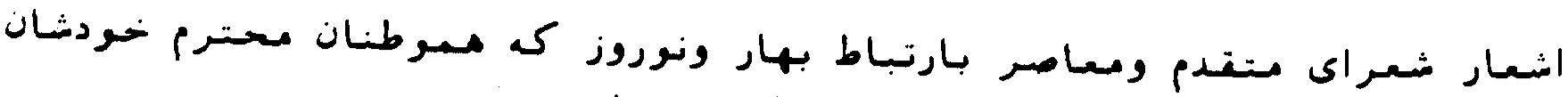

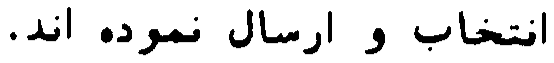

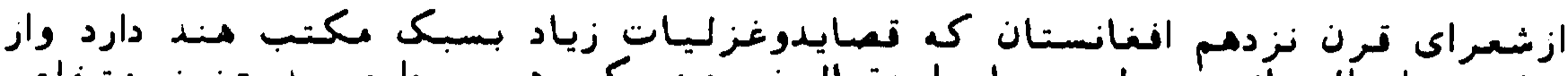

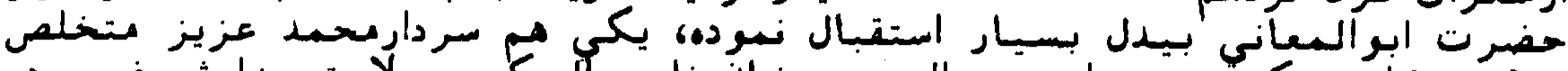

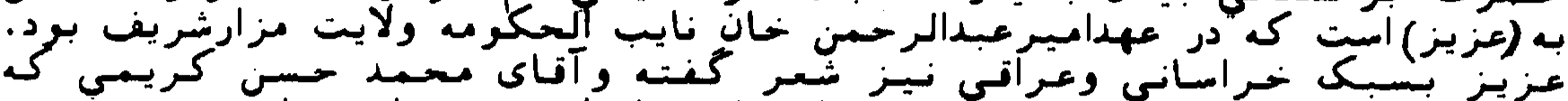

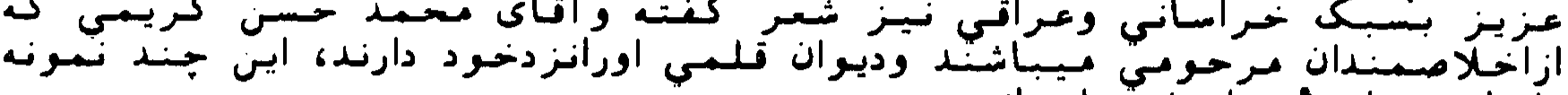

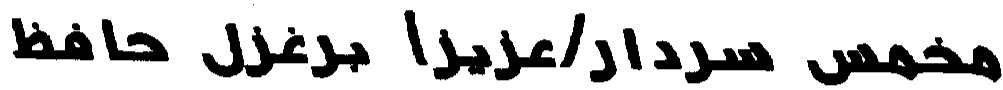

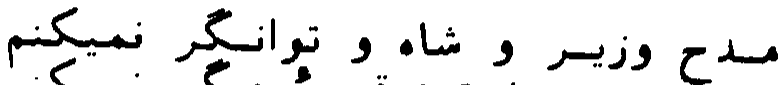

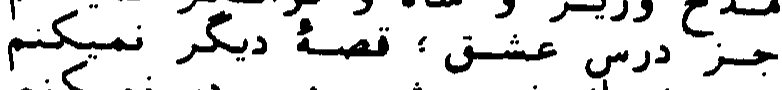

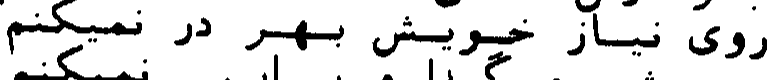

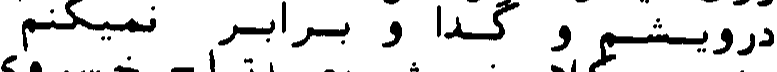

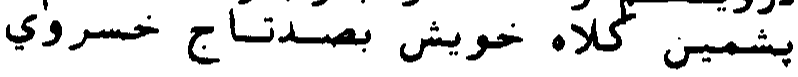

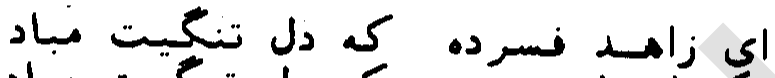

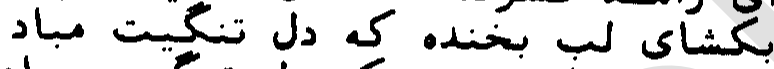

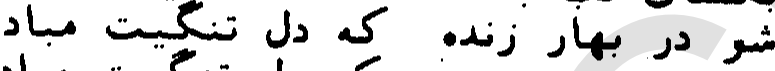

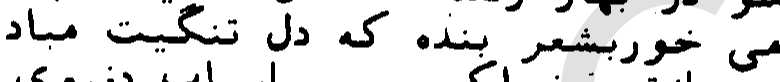

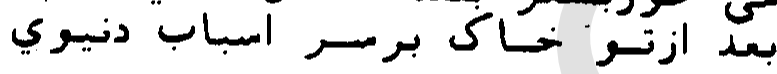
كـرديم جون مطالعه يكسر كتاب امن

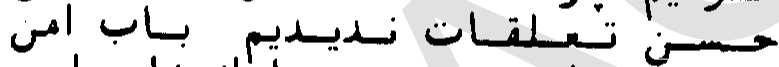

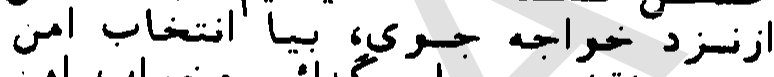

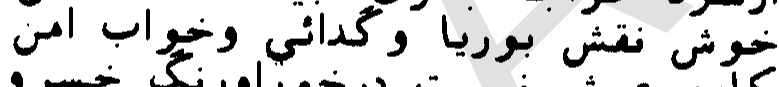

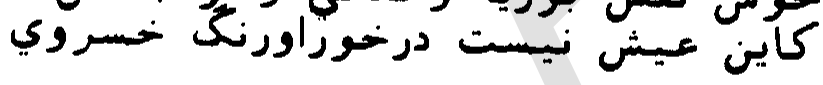

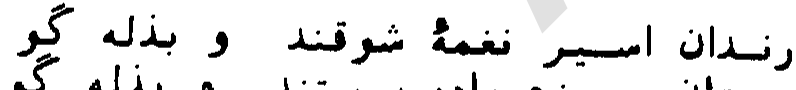

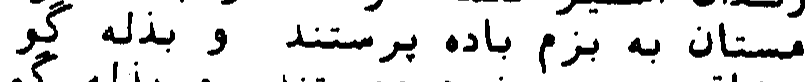

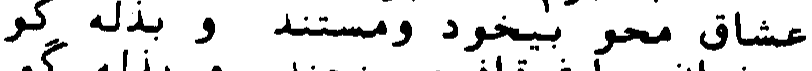

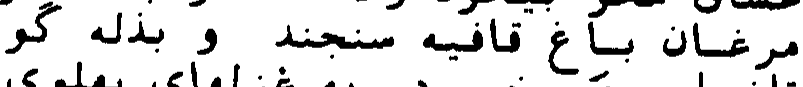

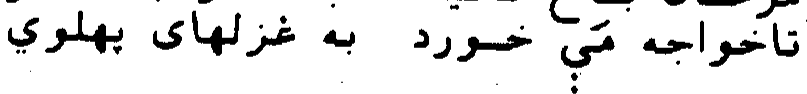

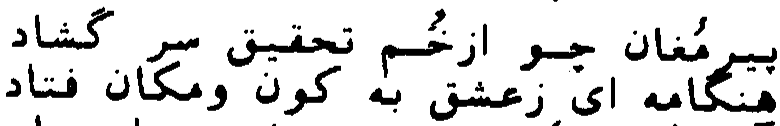

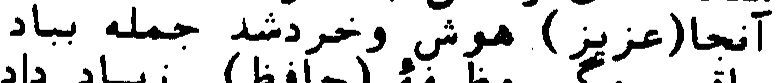

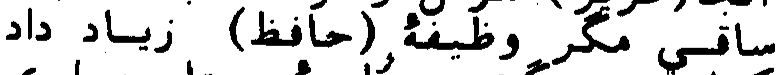

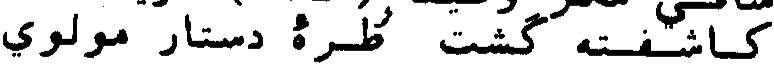

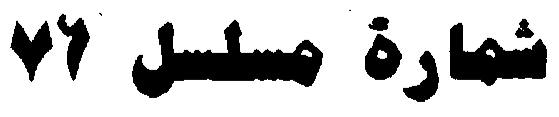

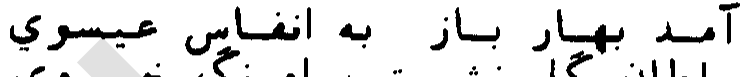

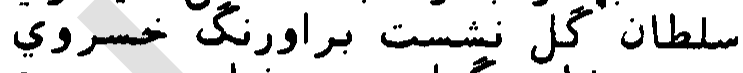

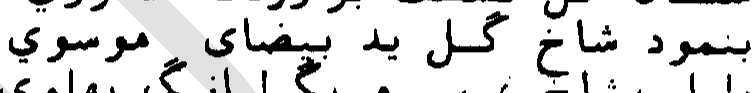

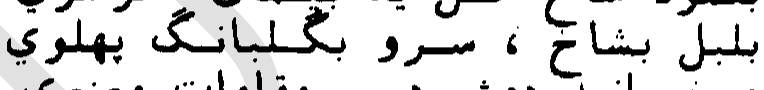

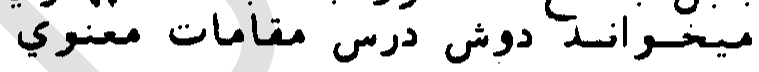

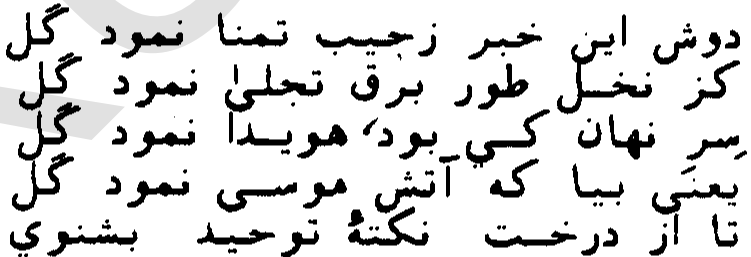

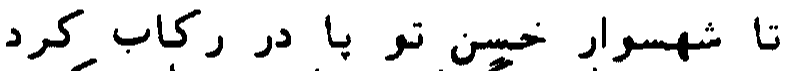

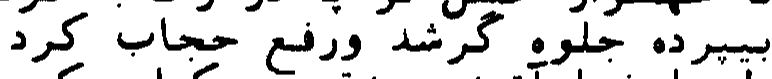

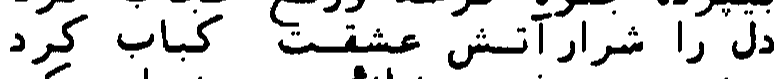

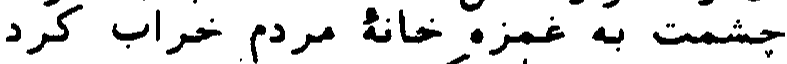

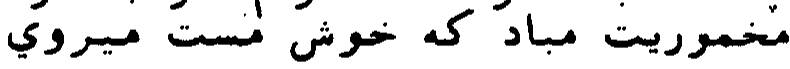

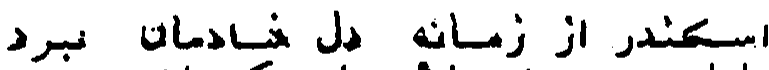

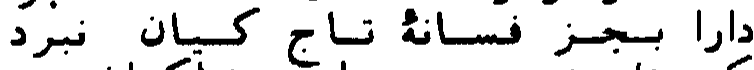

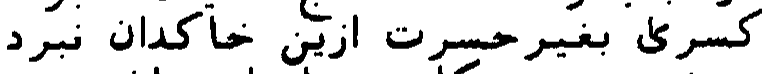

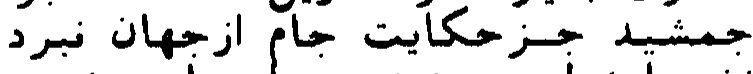

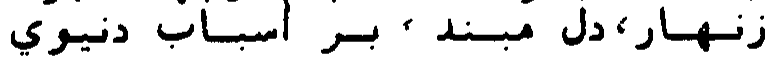
ظالم جفـا و جسور وستم تاكي اينقدر

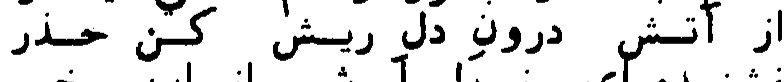

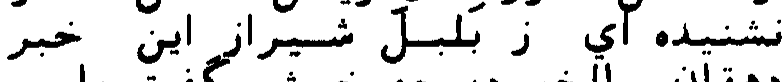

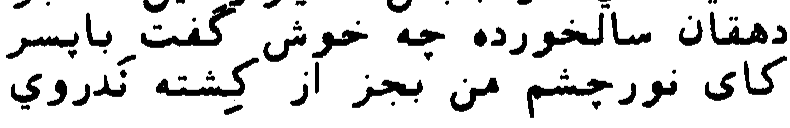

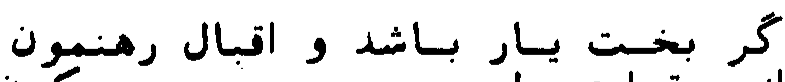

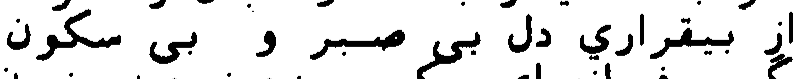

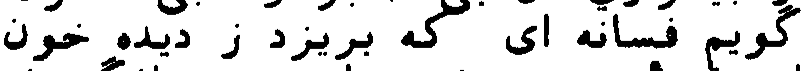

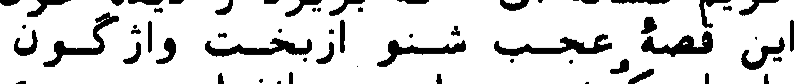

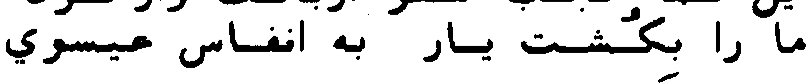




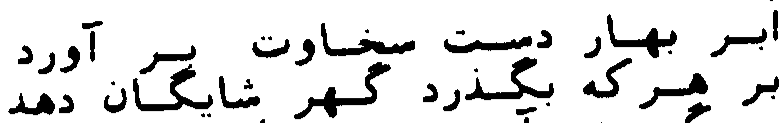

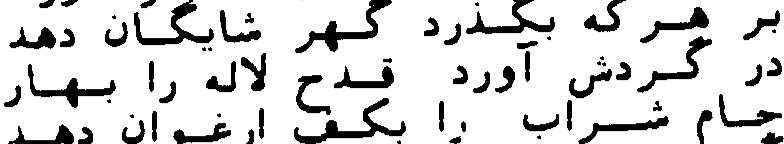

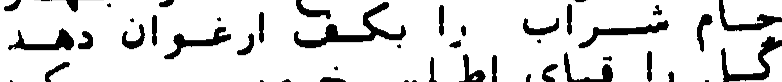

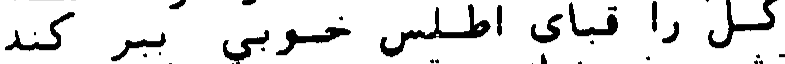

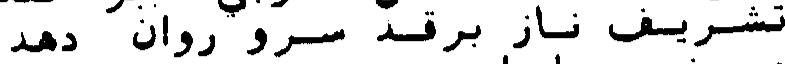

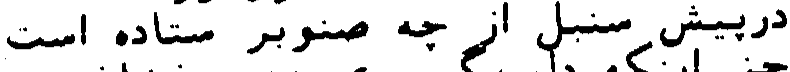

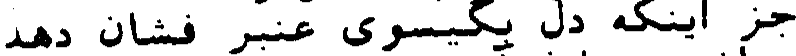

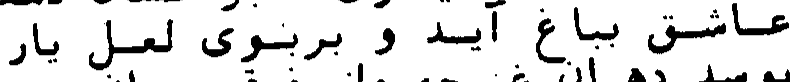

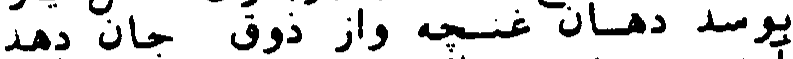

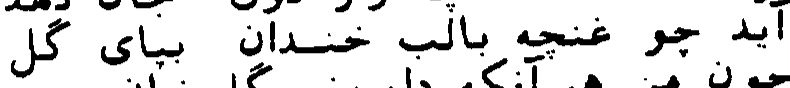

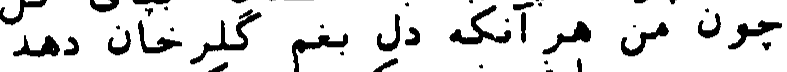

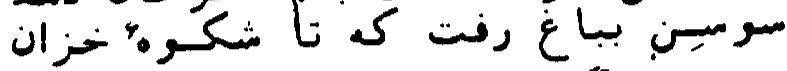

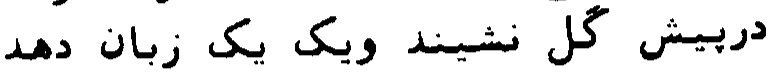

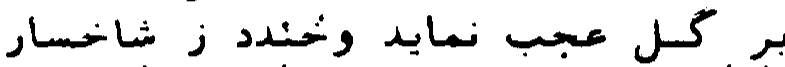

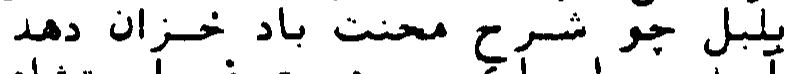

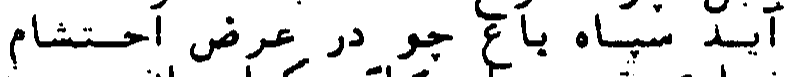

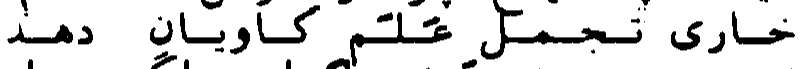

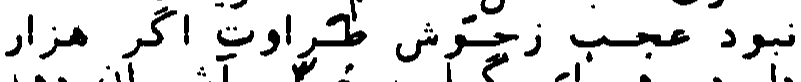

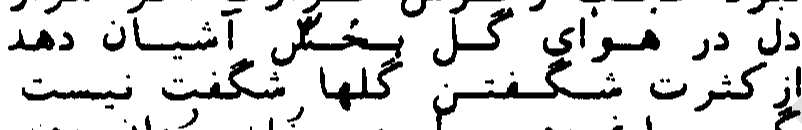

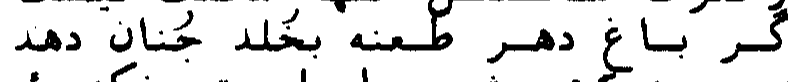

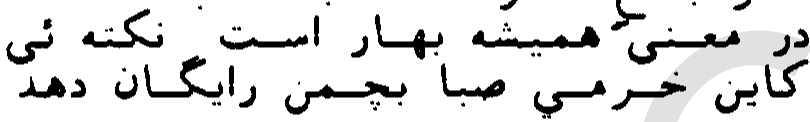

دربحرمغارع مثمن اخرب مكفرف محذوف

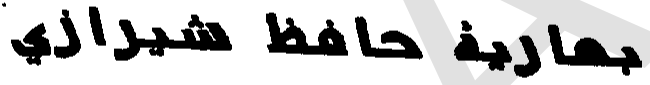

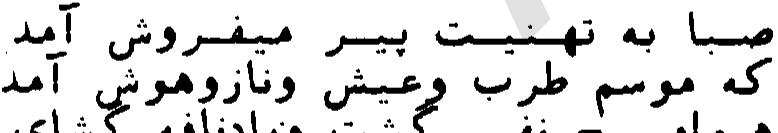

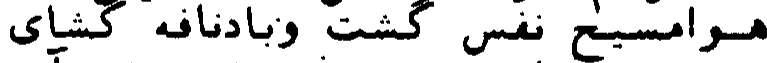

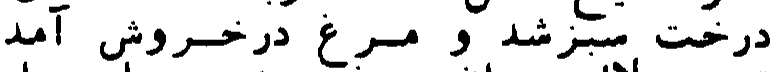

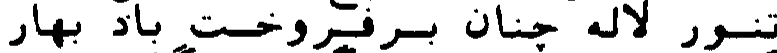

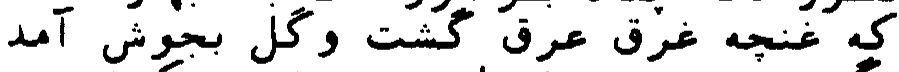

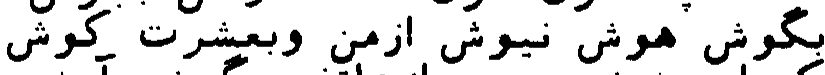

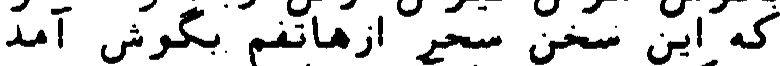

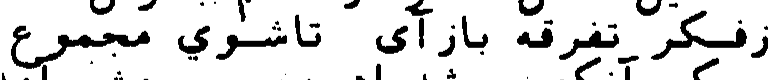

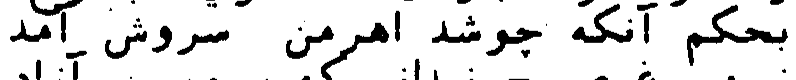

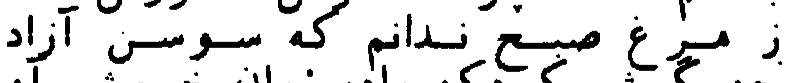

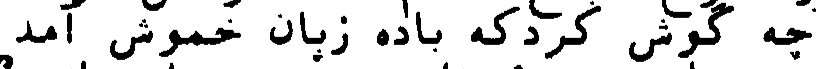

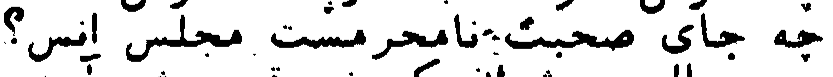

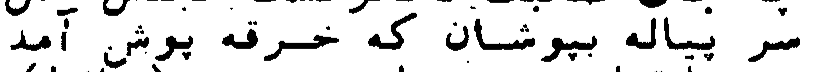

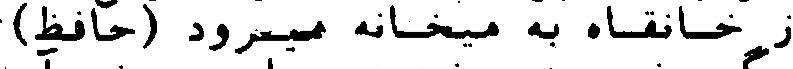

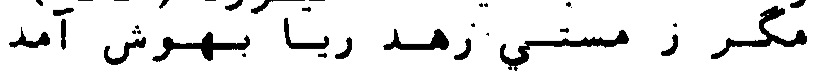

دوغرل مردار (عزيز) بسبك مكتب مندي:

\section{ع.4}

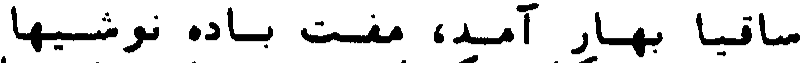

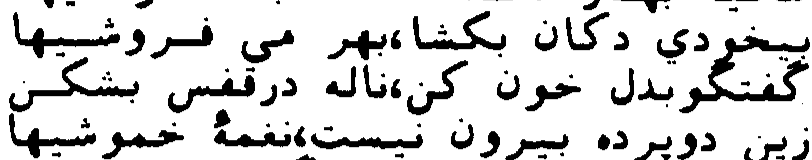

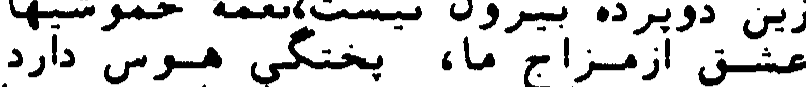

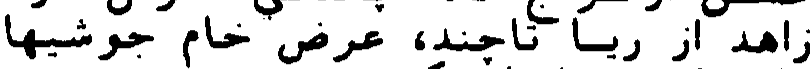

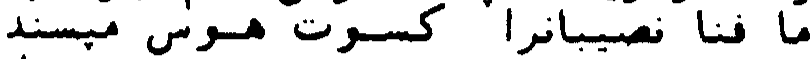

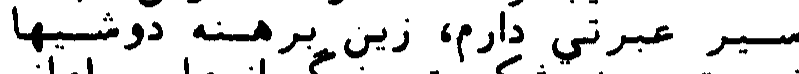

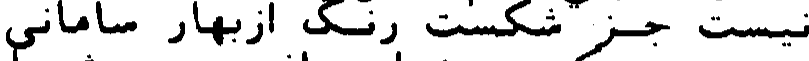

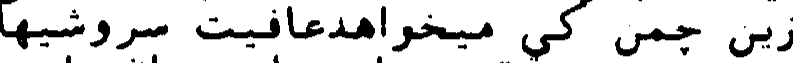

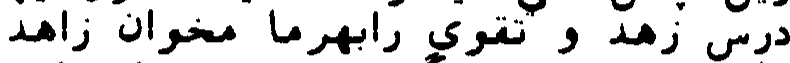

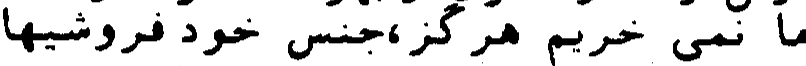

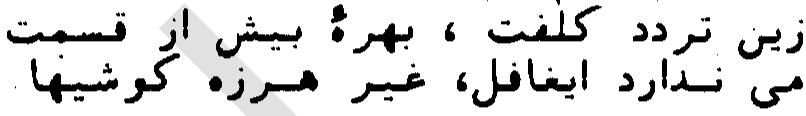

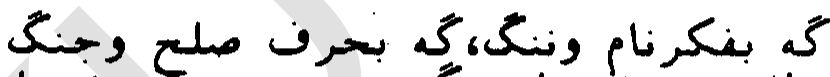

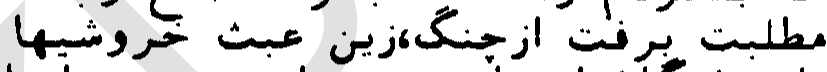

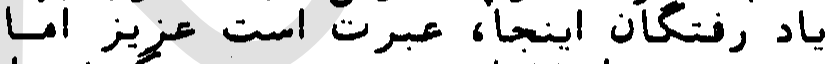

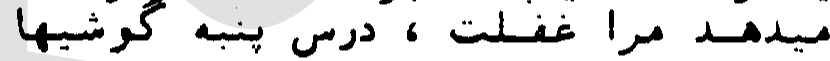

\section{1م:}

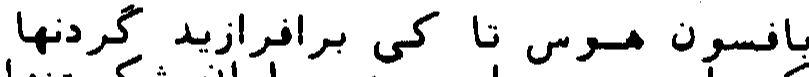

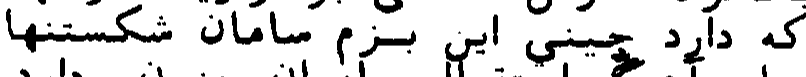

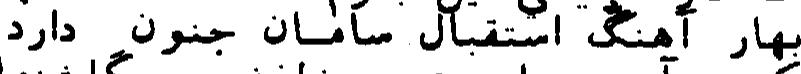

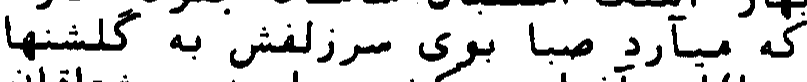

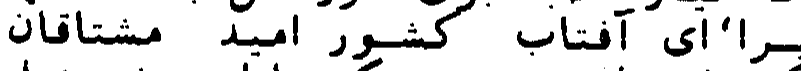

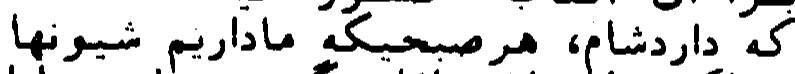

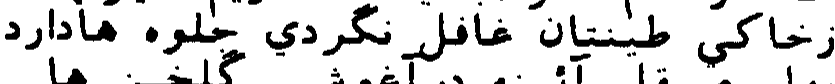

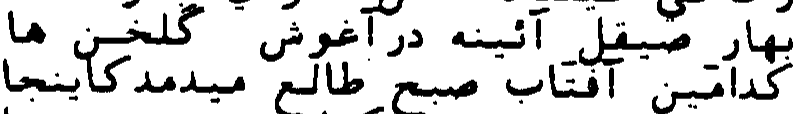

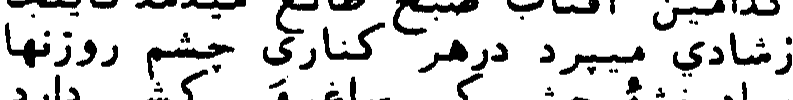

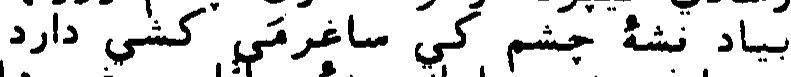

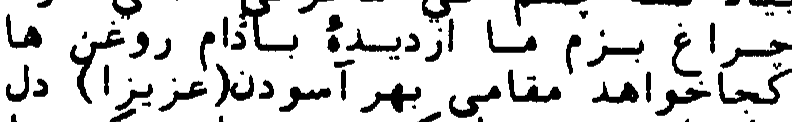

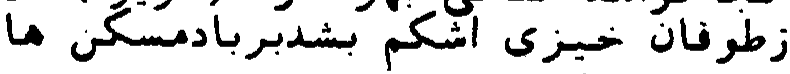

\section{بالب}

وقت امت آنكه باغ غر مراد جهـان دمهد

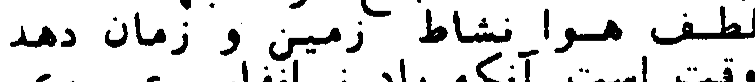

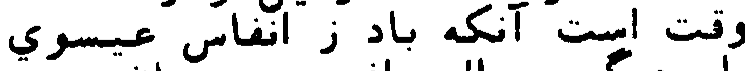

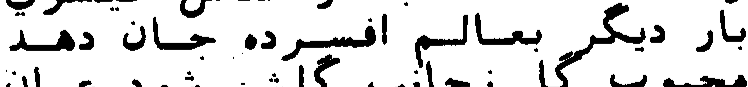

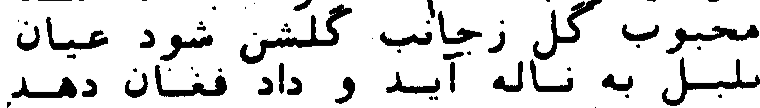




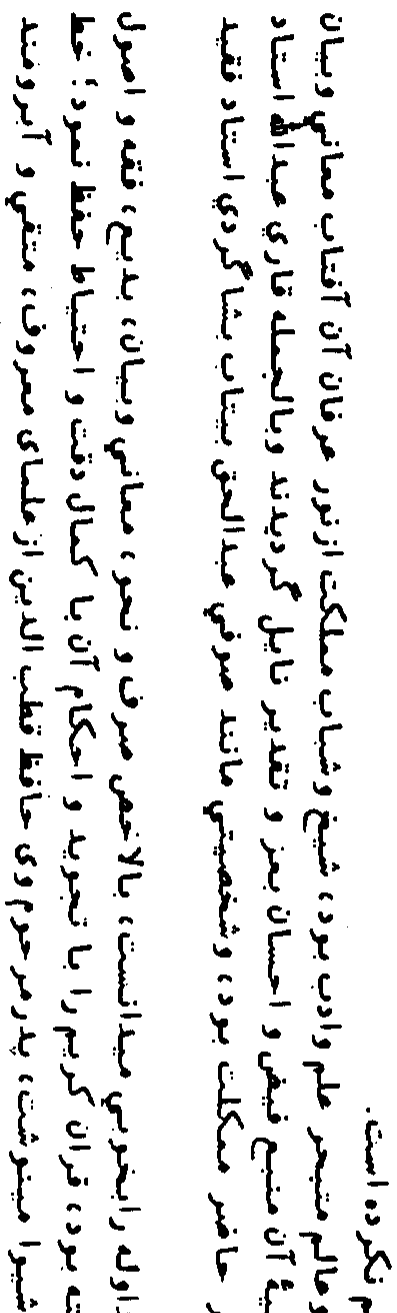

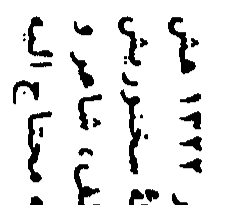

कह हैं

字定

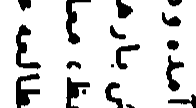

5

c.

$5 \leq\{$

$x \leq\{\leq$

a दूर है

t.

si

$\int_{0}^{\infty} \int_{0}$

\{. $\{:=$

$\overline{6}$

$5 \div 5$

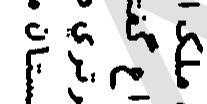
$t_{\pi}=\frac{1}{5}=5$

एद $\xi_{0}=\xi_{1}^{c} c^{-q}$

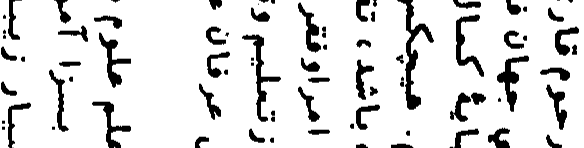

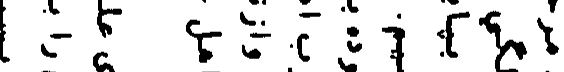

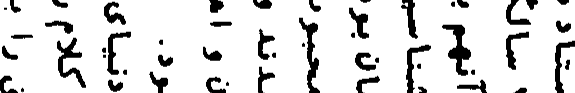

c:

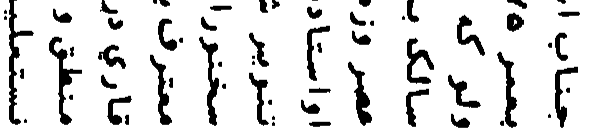

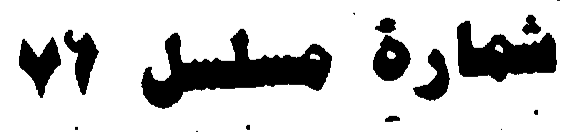

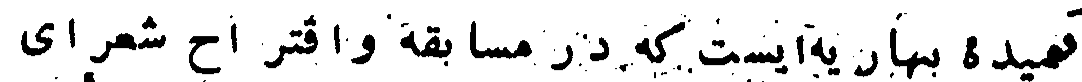

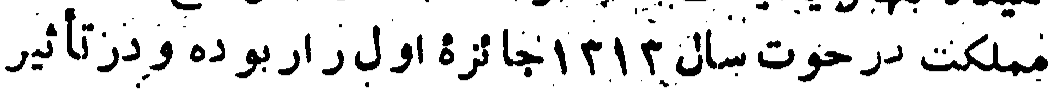

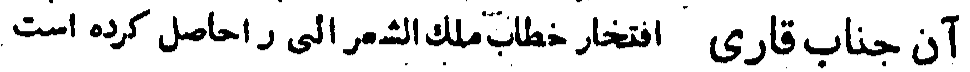

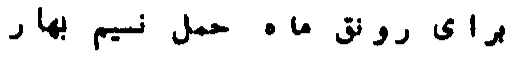

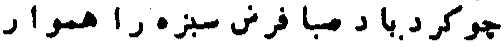

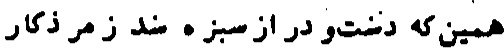

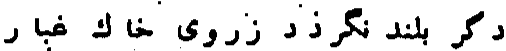

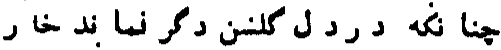

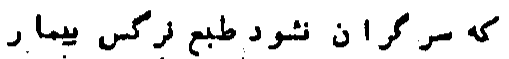

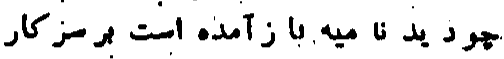

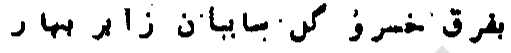

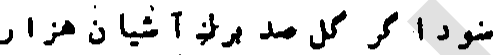

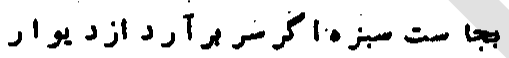

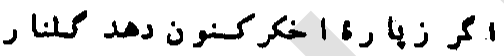

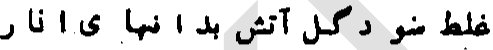

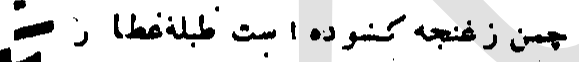

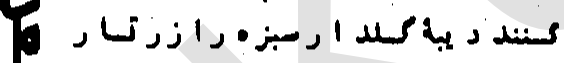

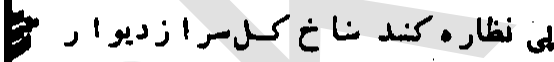

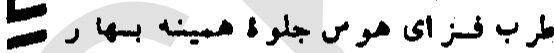

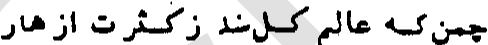

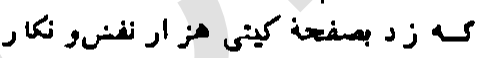

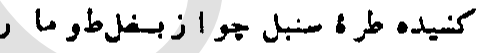

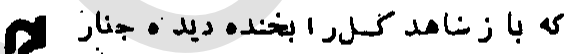

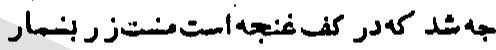

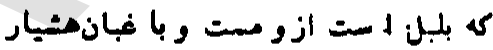

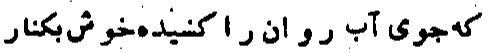

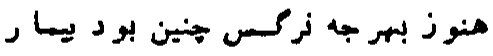

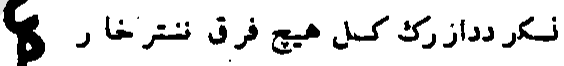

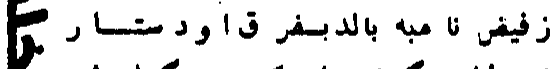

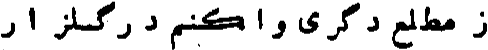

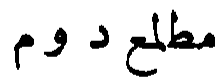

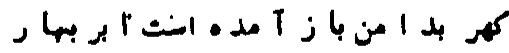

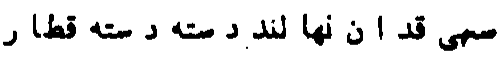

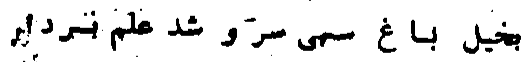

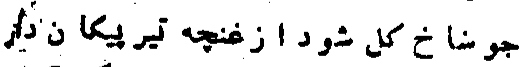

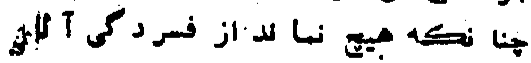

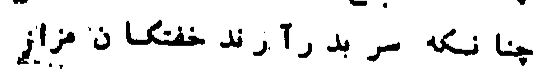

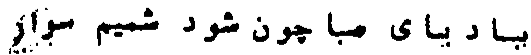

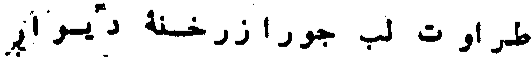

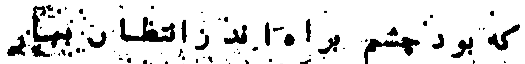
كن

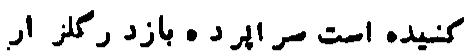

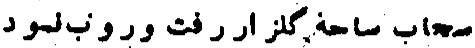

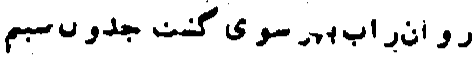

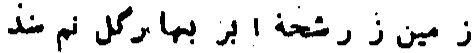

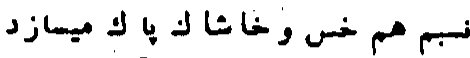

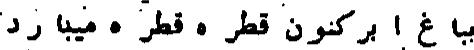

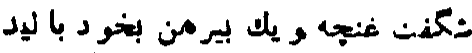

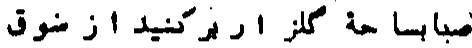

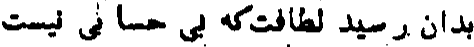

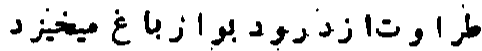

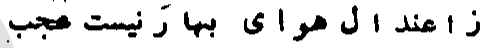

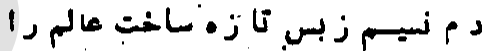

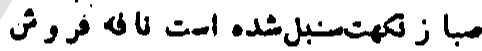

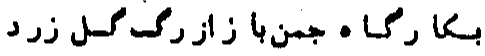

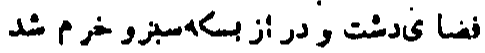

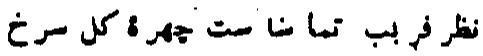

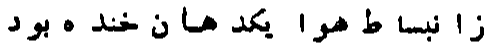

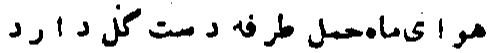

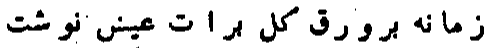

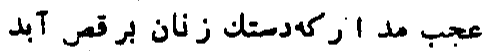

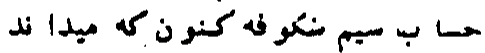

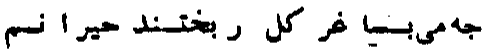

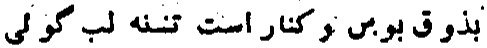

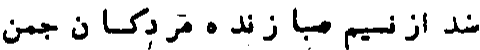

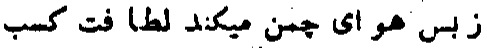

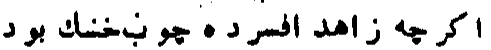

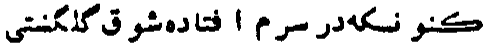

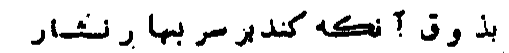

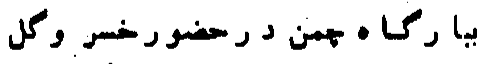

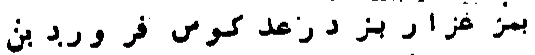

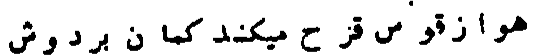

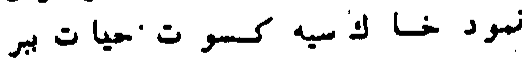

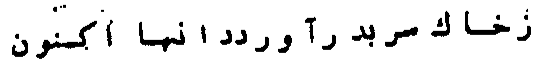

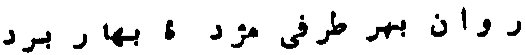

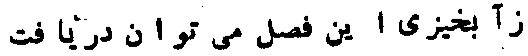

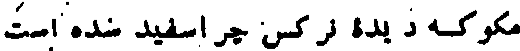

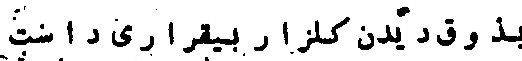




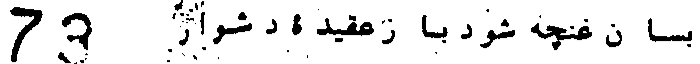

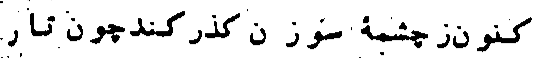

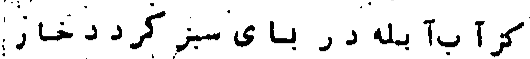

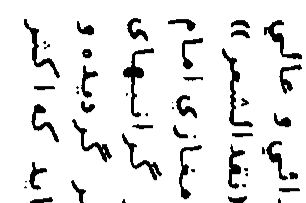
$\begin{cases}t \\ c\end{cases}$ $5, E\left\{\frac{5}{5}\right\}$ \& $\{? 5$

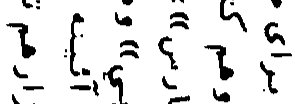

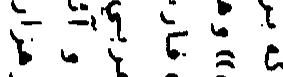
$\therefore$ ac $c$ द 5

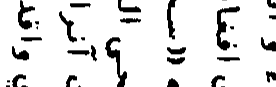

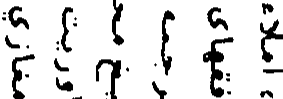

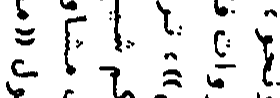

\}$_{0}^{c} \sum_{0}=0$

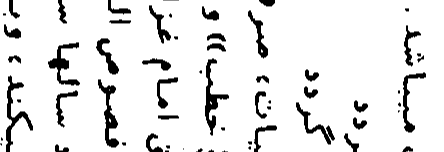

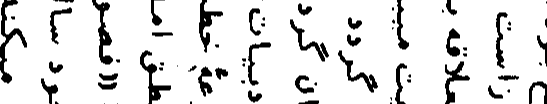
c. $\left.\}^{\infty}\right\}$

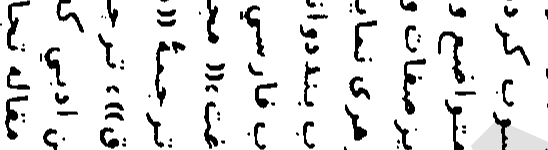

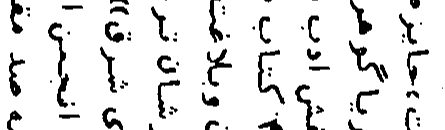

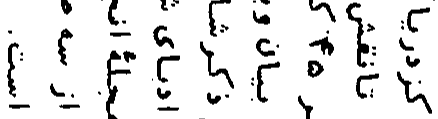

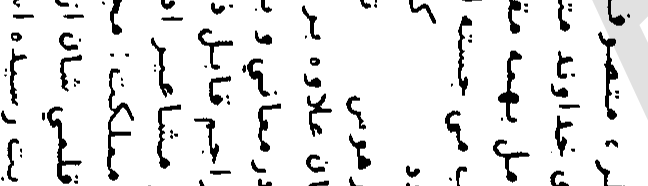

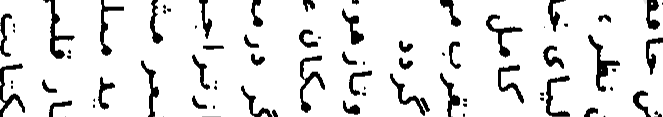

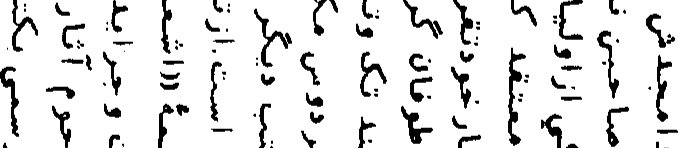

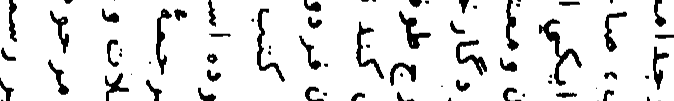

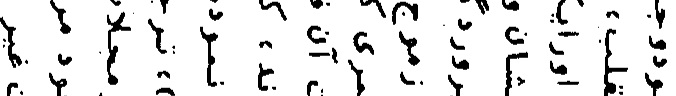
c)

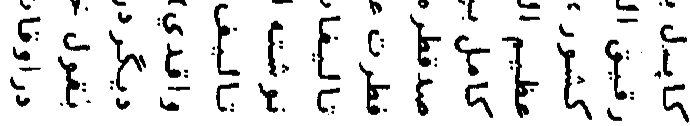

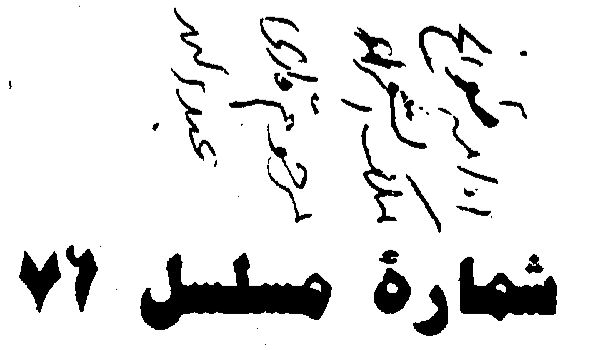

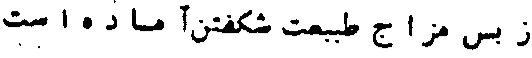

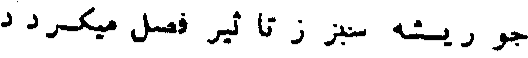

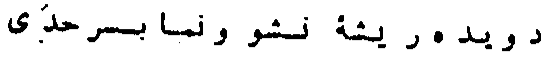

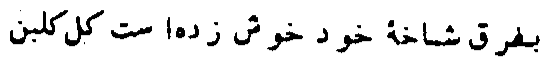

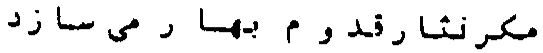

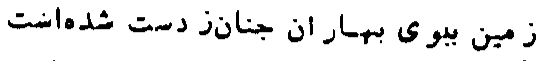

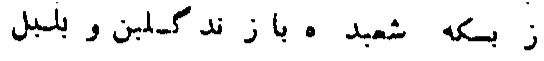

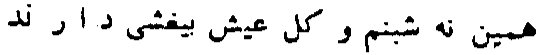

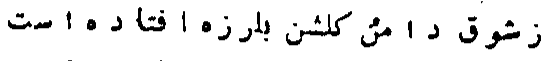

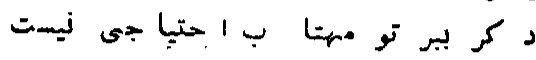

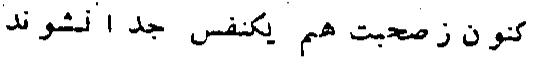

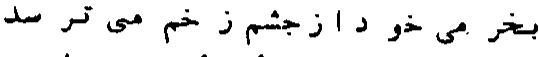

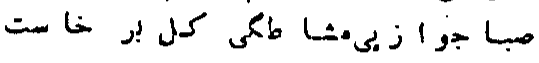

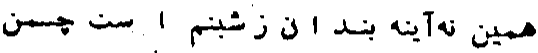

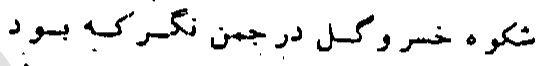

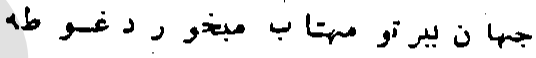

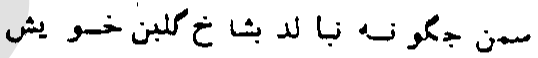

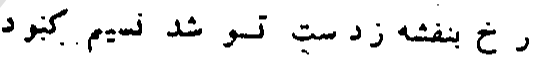

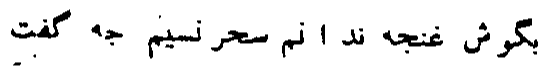

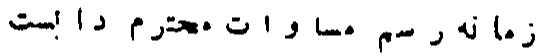

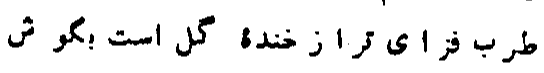

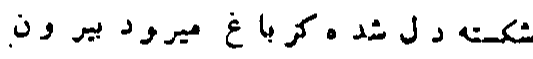

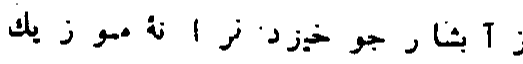

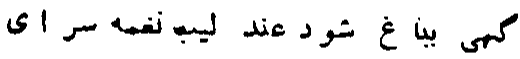

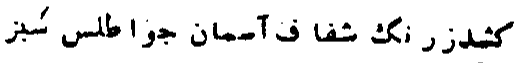

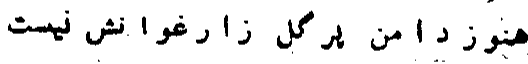

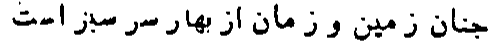

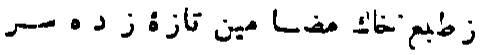

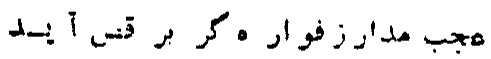

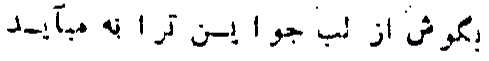

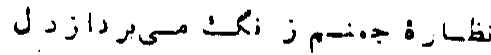

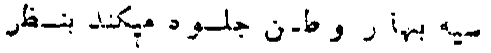

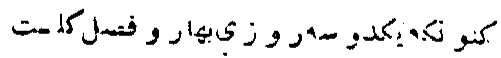

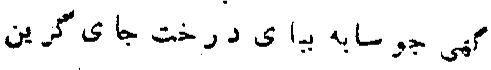

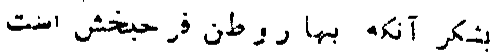

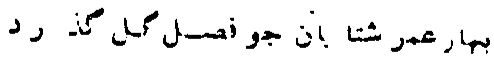

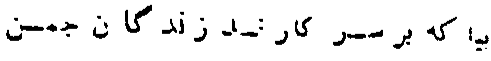

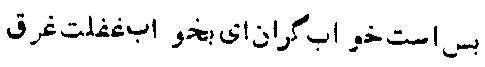

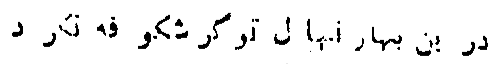

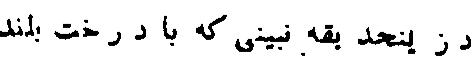

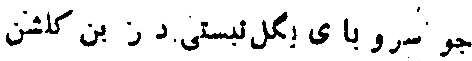

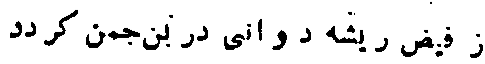




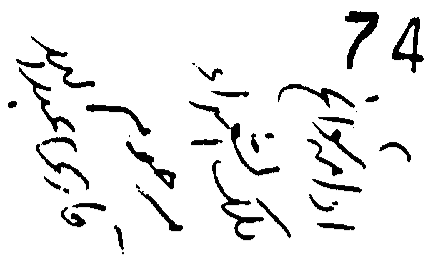

क.

ᄃ.

$\rightarrow=5 \%$

F $\{$ \&

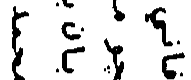

द. द

$\{i$

\& $2 \mathrm{c} \cdot \mathrm{q}$

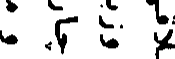

t.

$\rightarrow r \frac{c}{a} \cdot s$

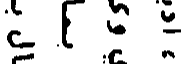

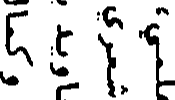

c. 5 .

$\bar{y}=6 ?$

c

दे

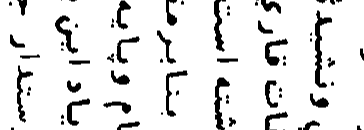

\& 5 :

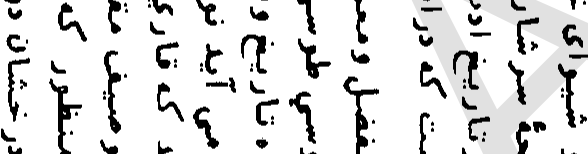

$\therefore t r q \dot{t}\{t 56$

Fs?

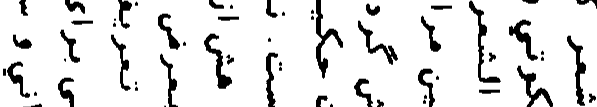

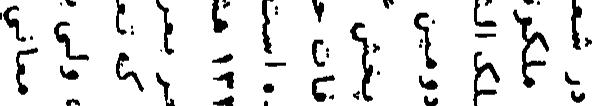

c)

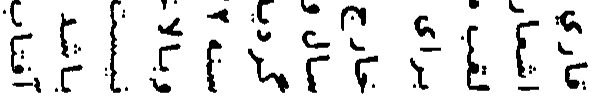

\section{?} iditg $\rightarrow$ ils

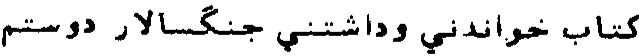

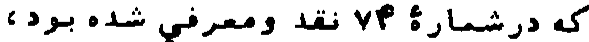

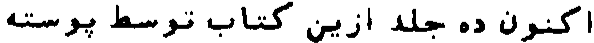

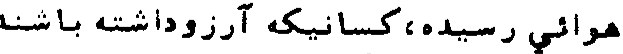

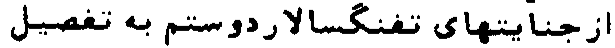

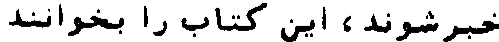

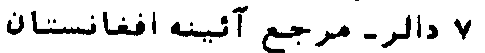

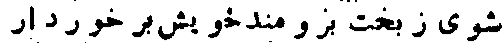

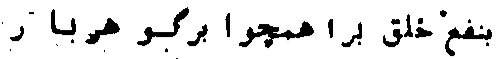

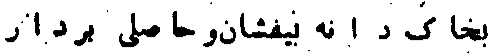

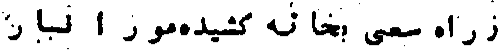

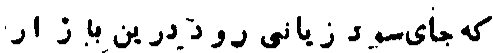

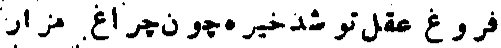

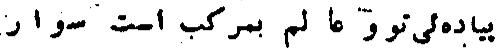

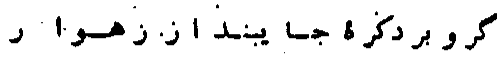

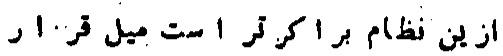

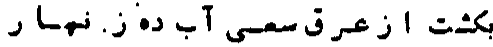

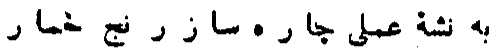

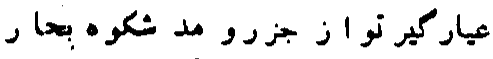

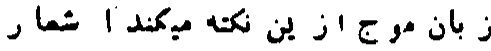

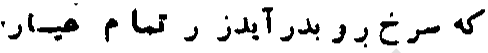

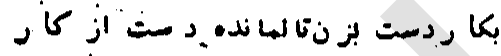

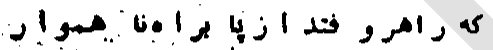

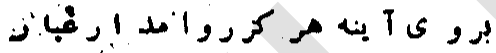

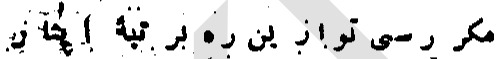

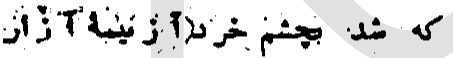

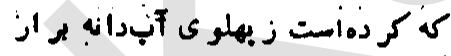

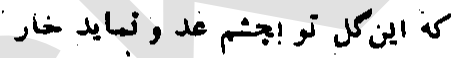

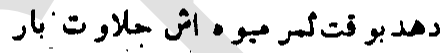

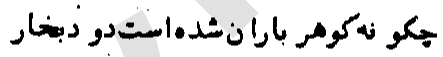
كمجر خ ر انبو دجز بجاملا ن نيكار نيكار وكرنهاز رم فر مشتر و دز دستيثنار

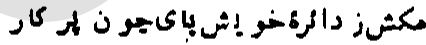

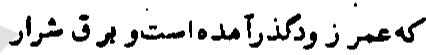

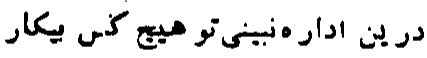

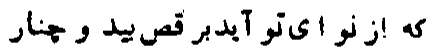

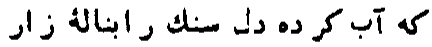
بكو كوعظجو ز اهدفتادت ازجي كذار

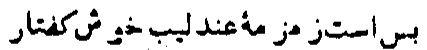

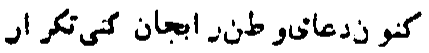

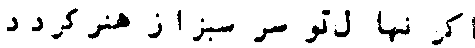

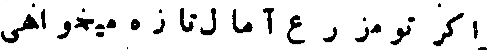

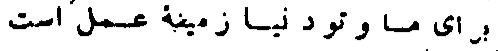

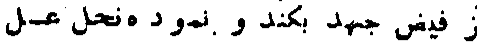

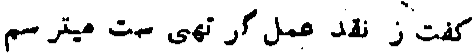

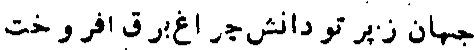

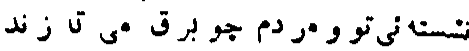

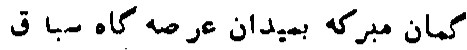

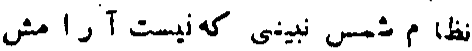

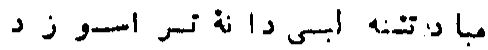

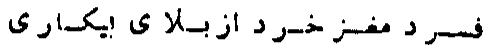

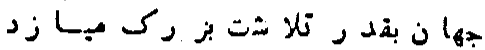

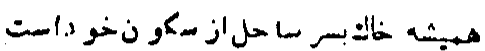

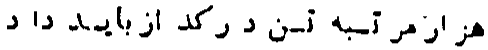

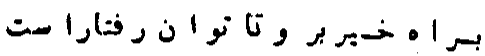

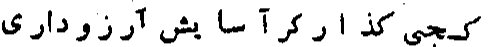

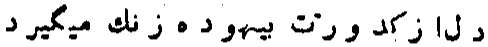

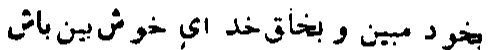

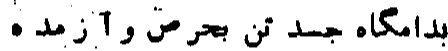

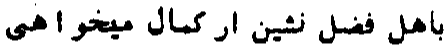

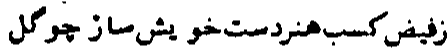

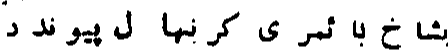

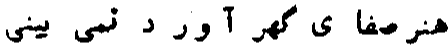

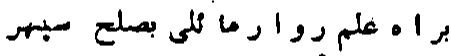

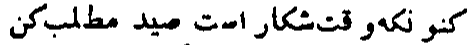

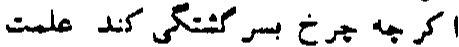

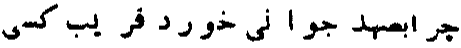

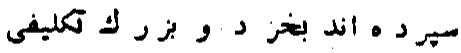
بس استخامهمر بر تضمفير بلبل نيست

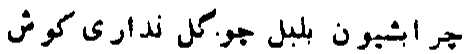

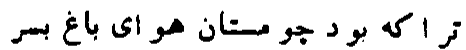

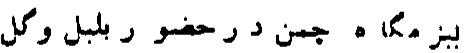

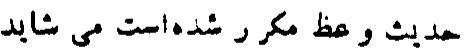

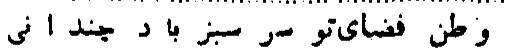

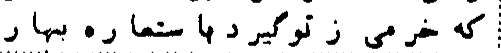

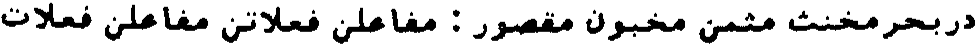

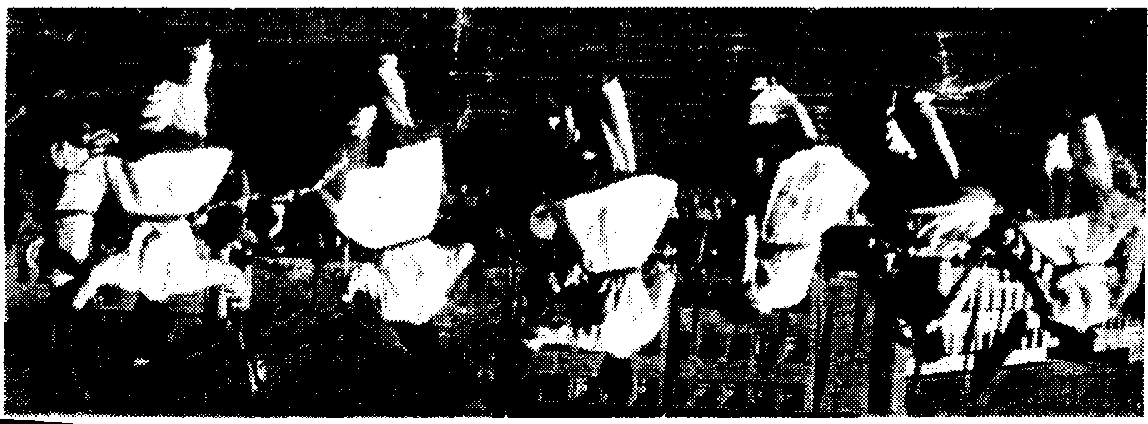




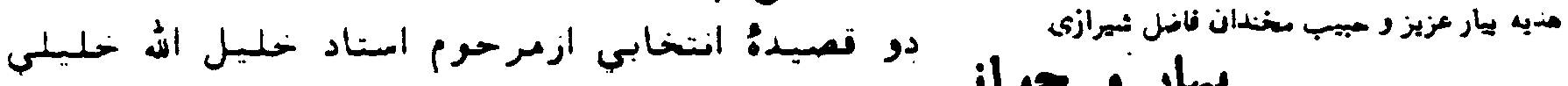

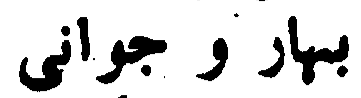

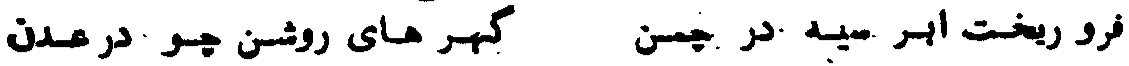
د

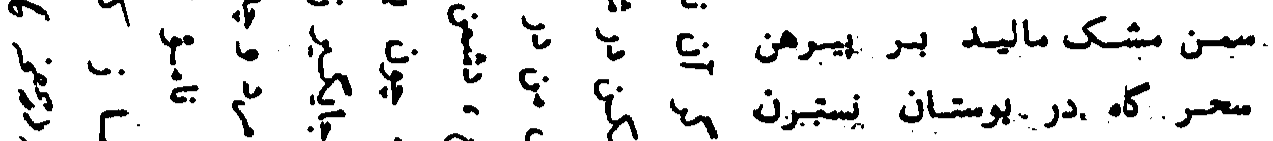

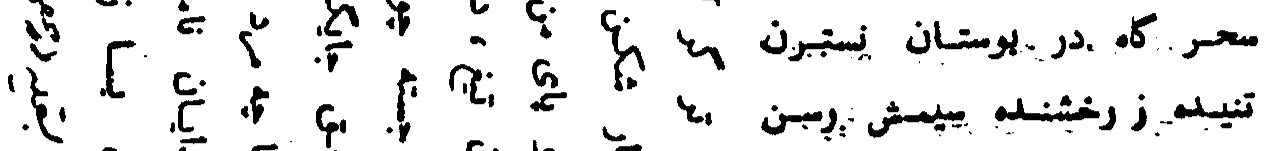
G

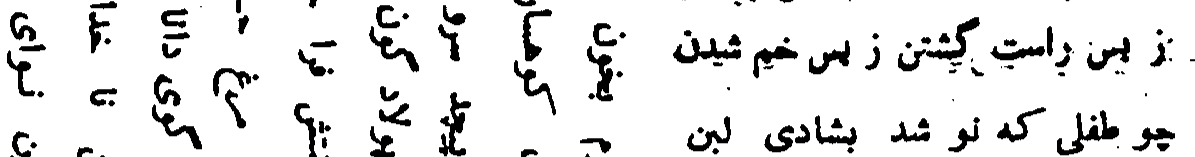

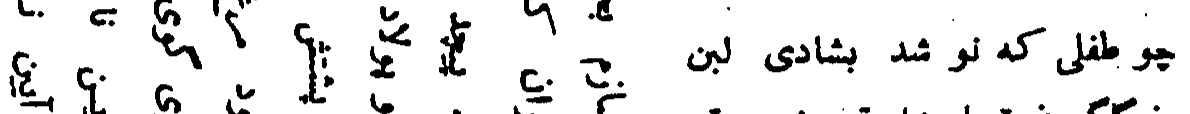

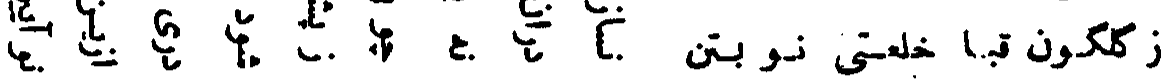

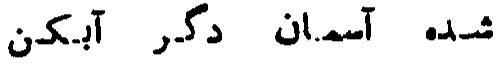

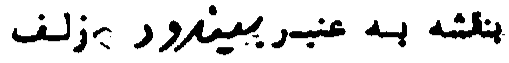

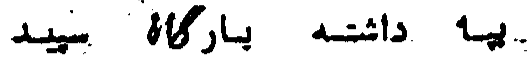

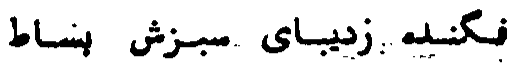

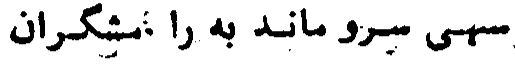

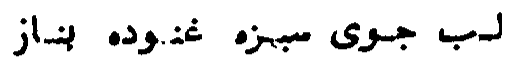
عروس همن : ارغوان كمرده باز

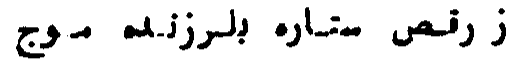

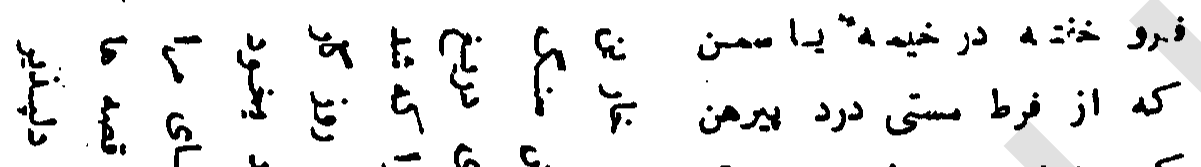
بكوث كل آمسته كويد سخغ ci. दे है

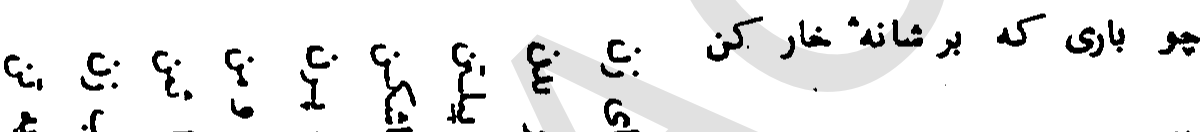
c.

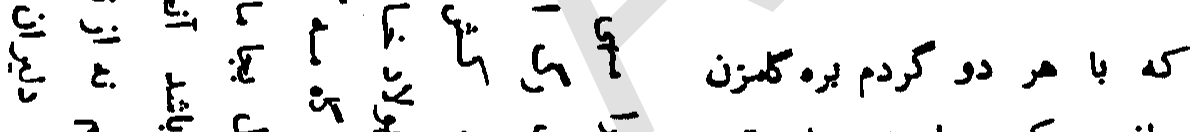

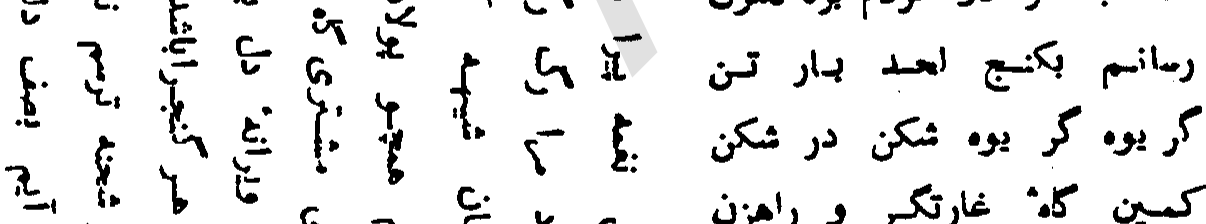

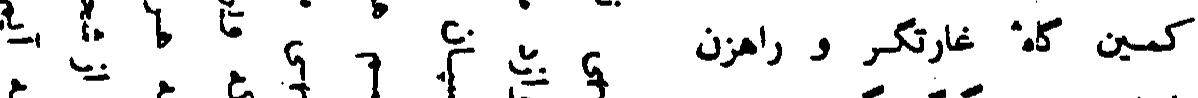

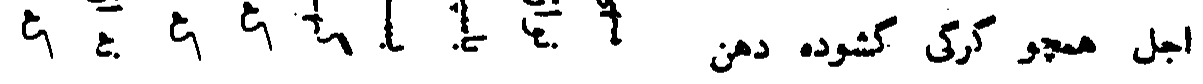

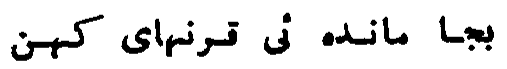

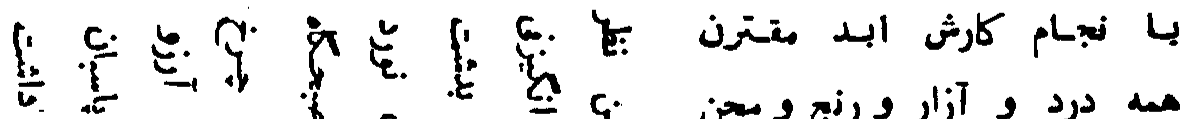
5 \&

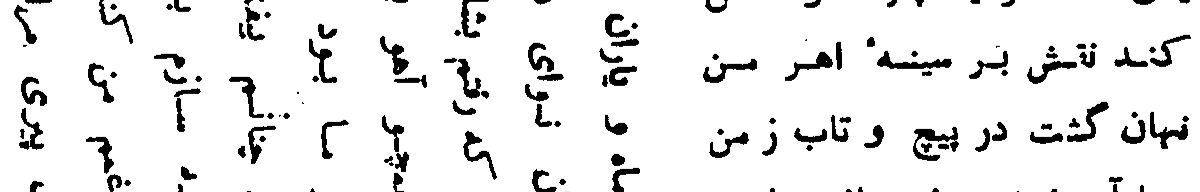

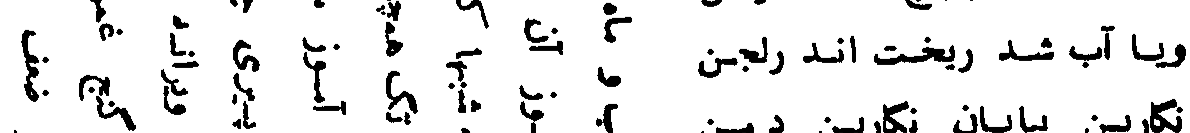

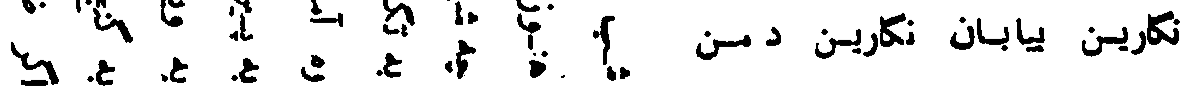

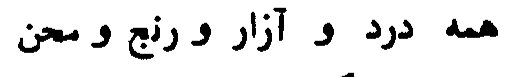

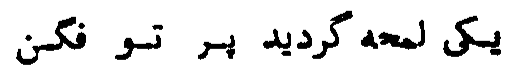

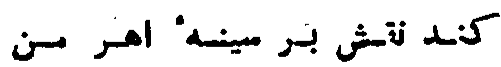
درنثيسد و خنديدو لسرزيدو رنت

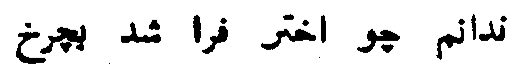
درينس جبوانى و شبهياى آن
جه در جام لاله فرو ريختند

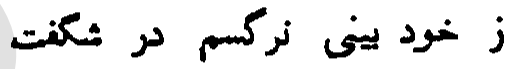
كهي نيم شب نور هـ نه در نهان

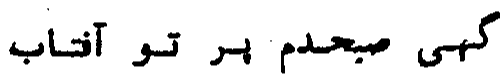
سر خلار را تيـز كرده نسيسم

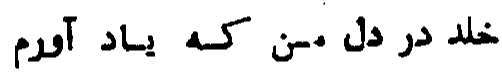
دربن يـام تساريك آوارك ما نسار انبار كثتهـ بدل

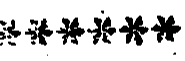




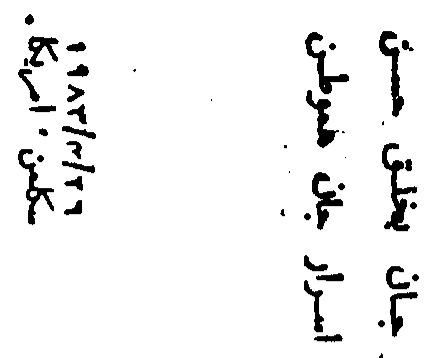

c. 5 .

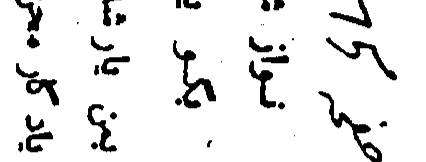

占点起

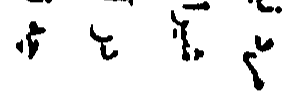

i.

की

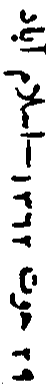

$\frac{1}{3}$ 告

?

$\dot{2}$

c. cis

t.

$\because$.

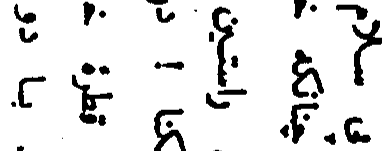

$\xi$

q $c_{1}\{:\{\}$

$\frac{G}{5} \quad \frac{G}{b} \quad l$

ह.

范

t.

ह
از استادخليل اله خليلي

\section{بمناسبت نوروز rarar}

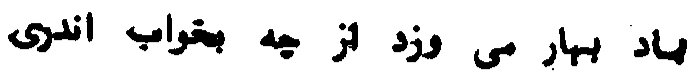

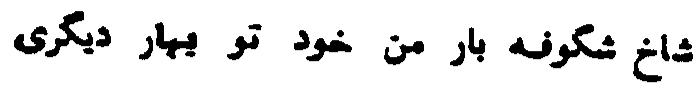

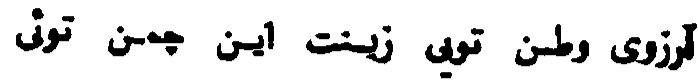

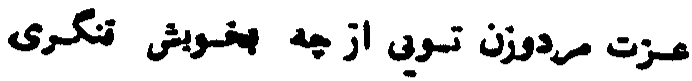

اهر من زمانه مكم مدملت كد در معان تهري

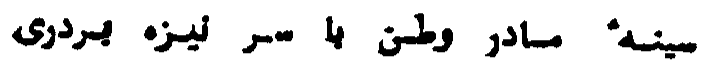

دثمن بـ خدا ترا جلو. دهد هزار رنعك

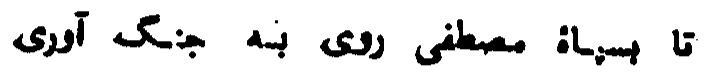

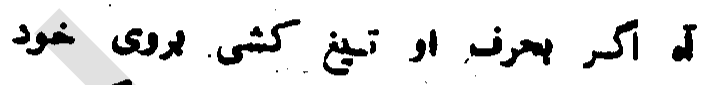

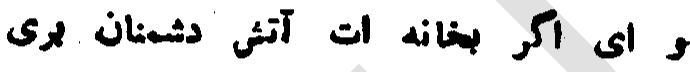

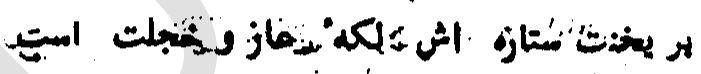

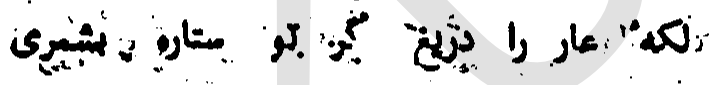

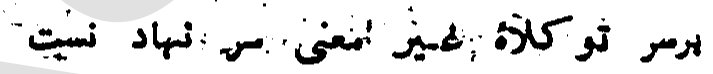

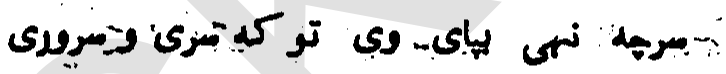

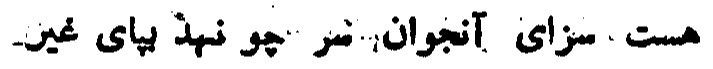

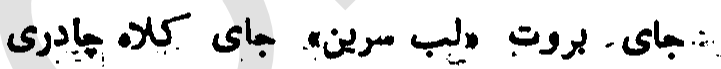

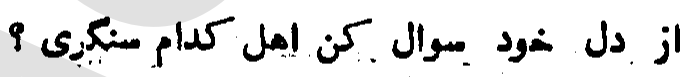

سنكر دوست زويترو سنكر دشمن از تنبا.

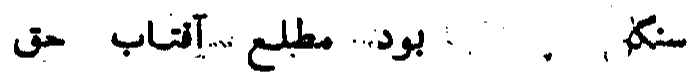

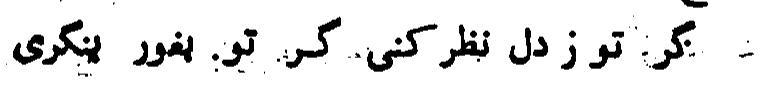

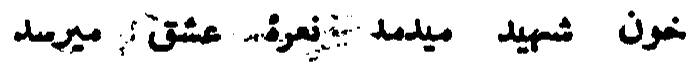

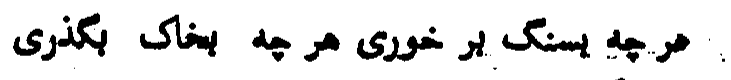

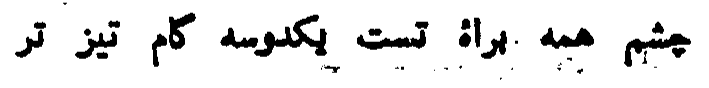

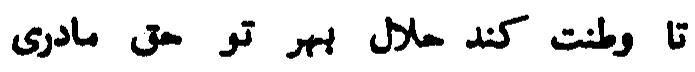

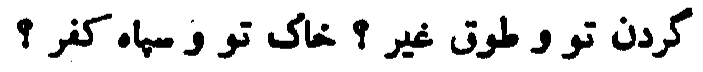

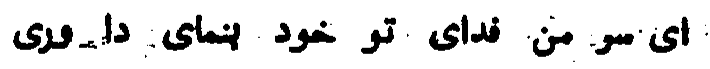

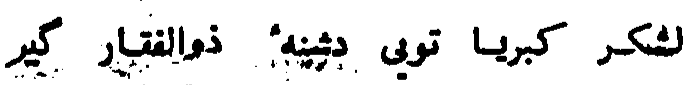

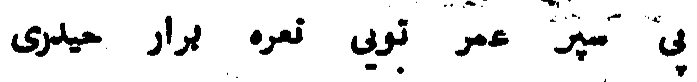

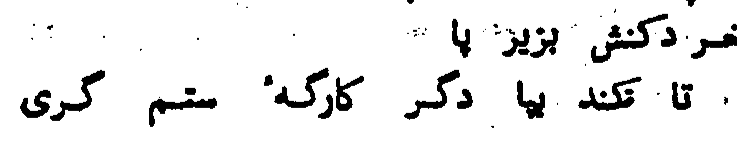

از بخت ستاره اش دور نكن كي. بعد ازيني.

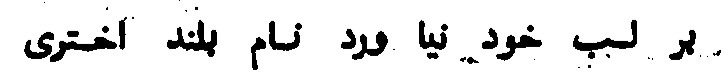




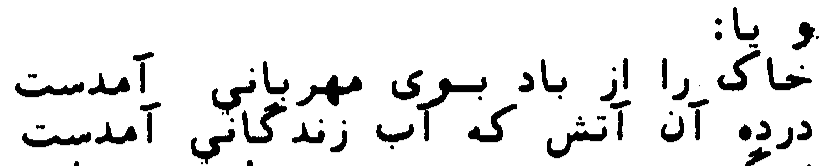

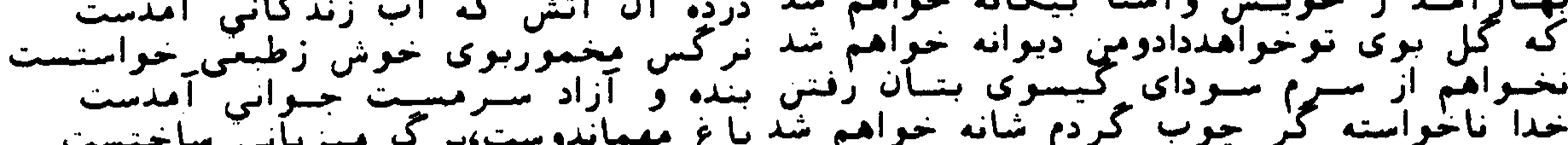

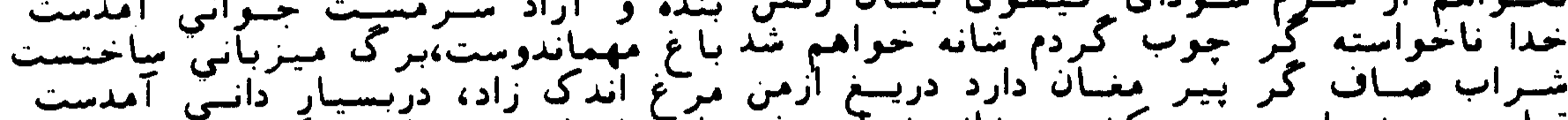

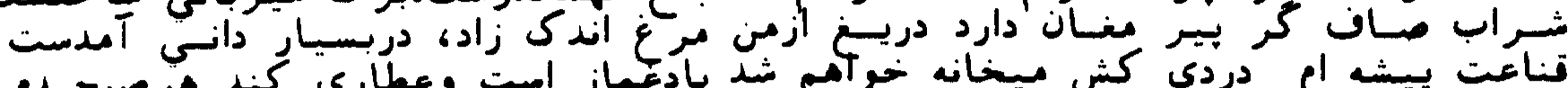

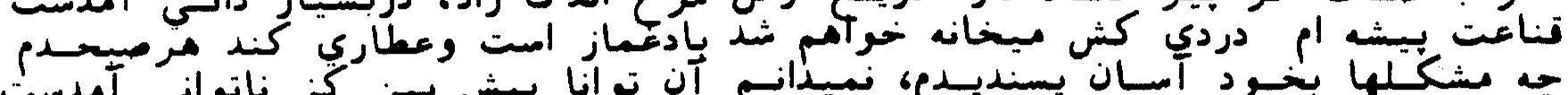

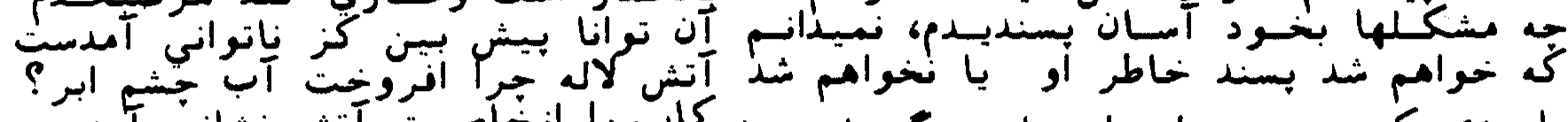

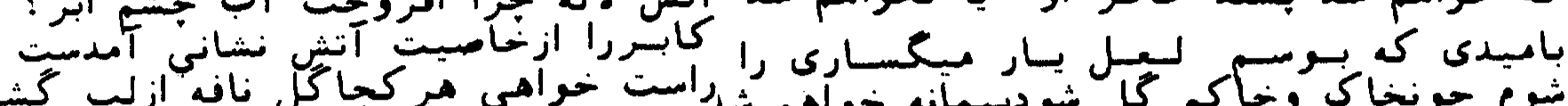

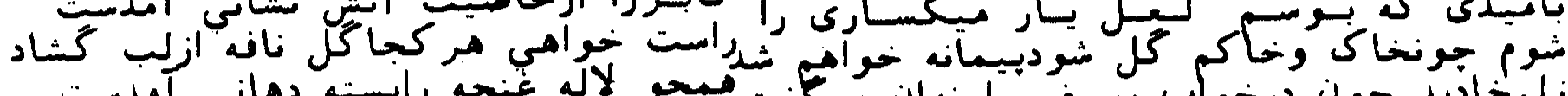

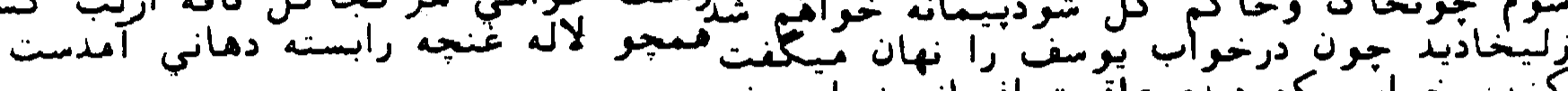

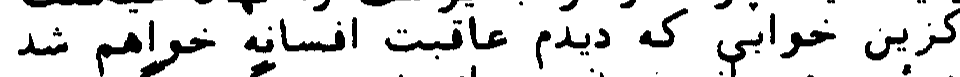

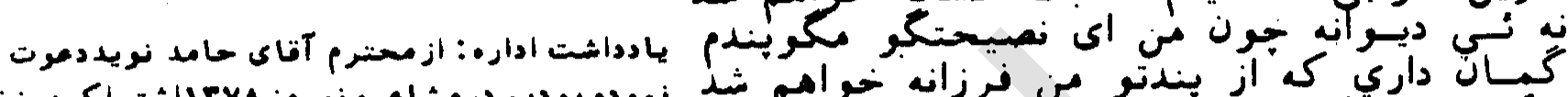

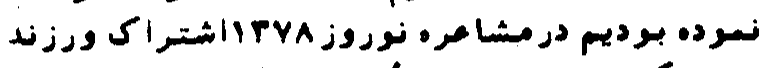

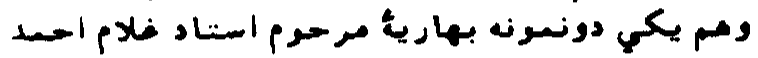

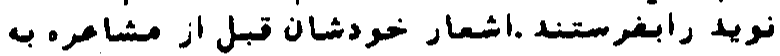

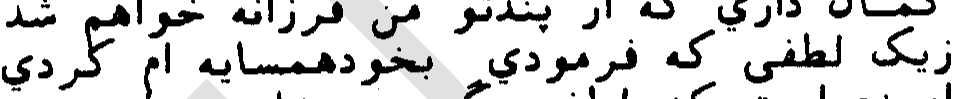

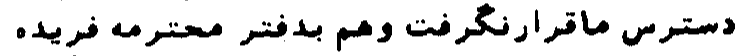

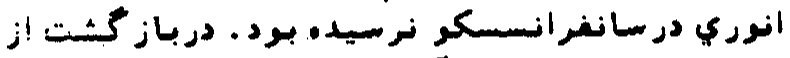

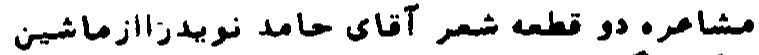

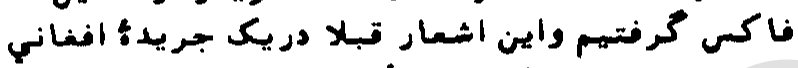

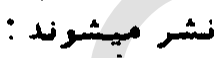

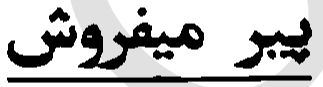

از طبـع حامد نويد

اكر شراب دد مد يبر مى فروش مرا!

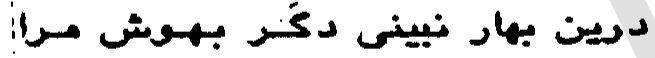

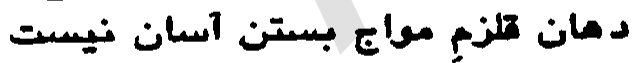

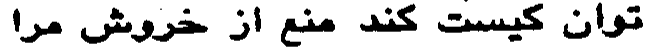

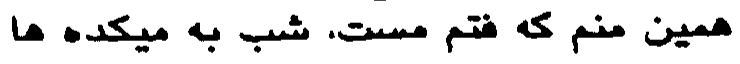
سحر برند خراباتيان بدوش مئ مرا

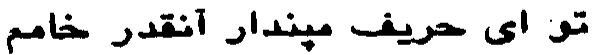

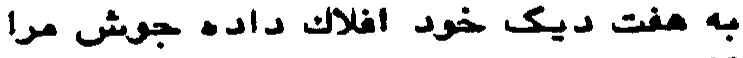

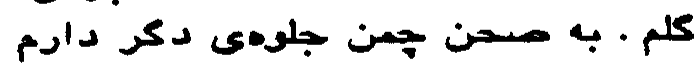

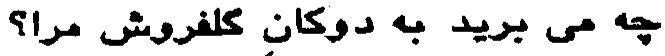

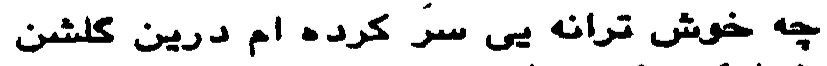

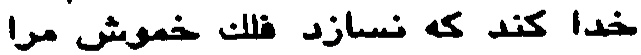

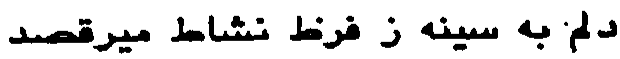

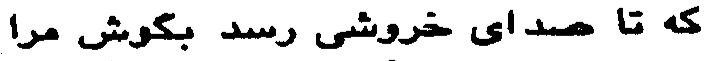

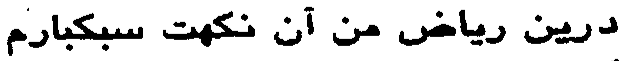

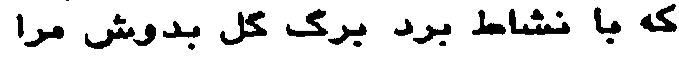

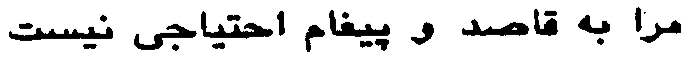

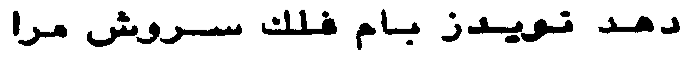

\section{جند رمونة كعام هجدودساد}

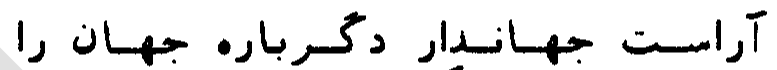

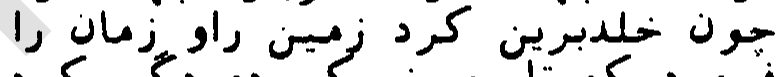

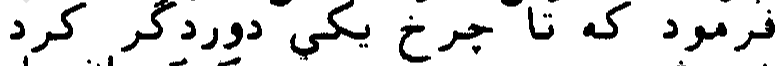

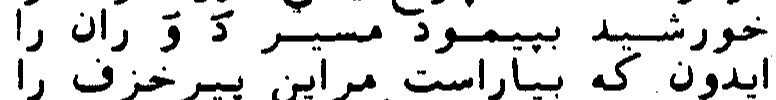

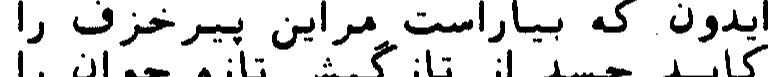

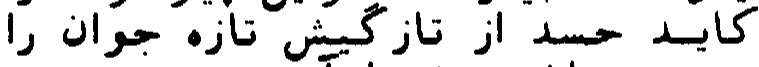

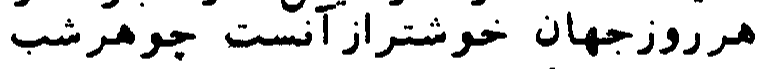

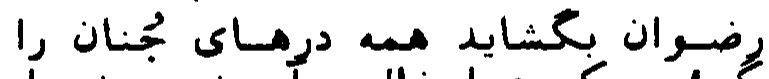

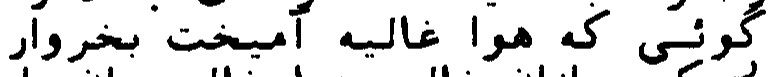

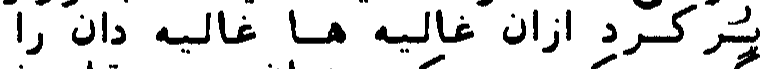

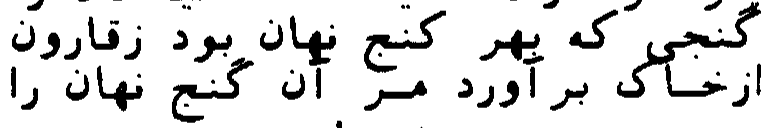

$$
\text { إبزون = }
$$

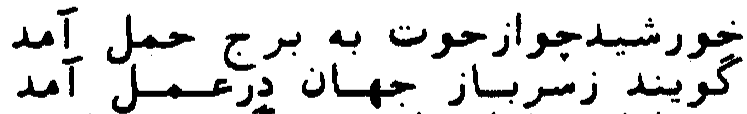

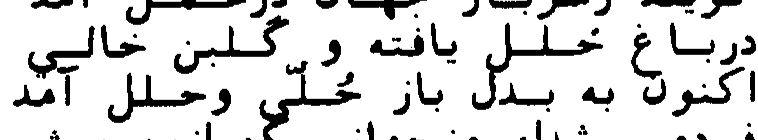

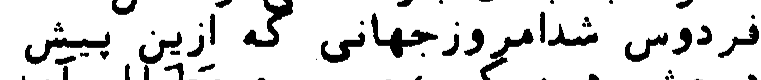

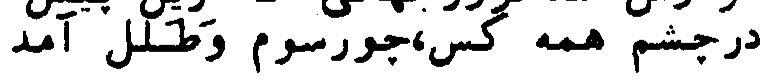

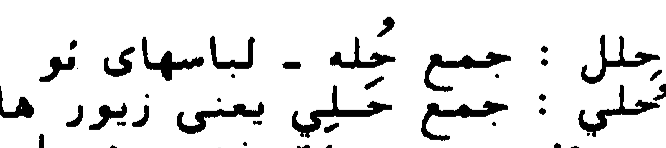

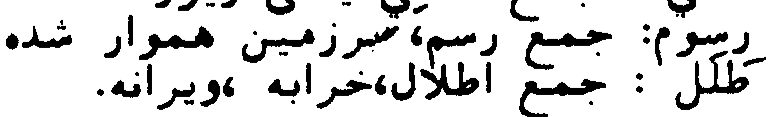




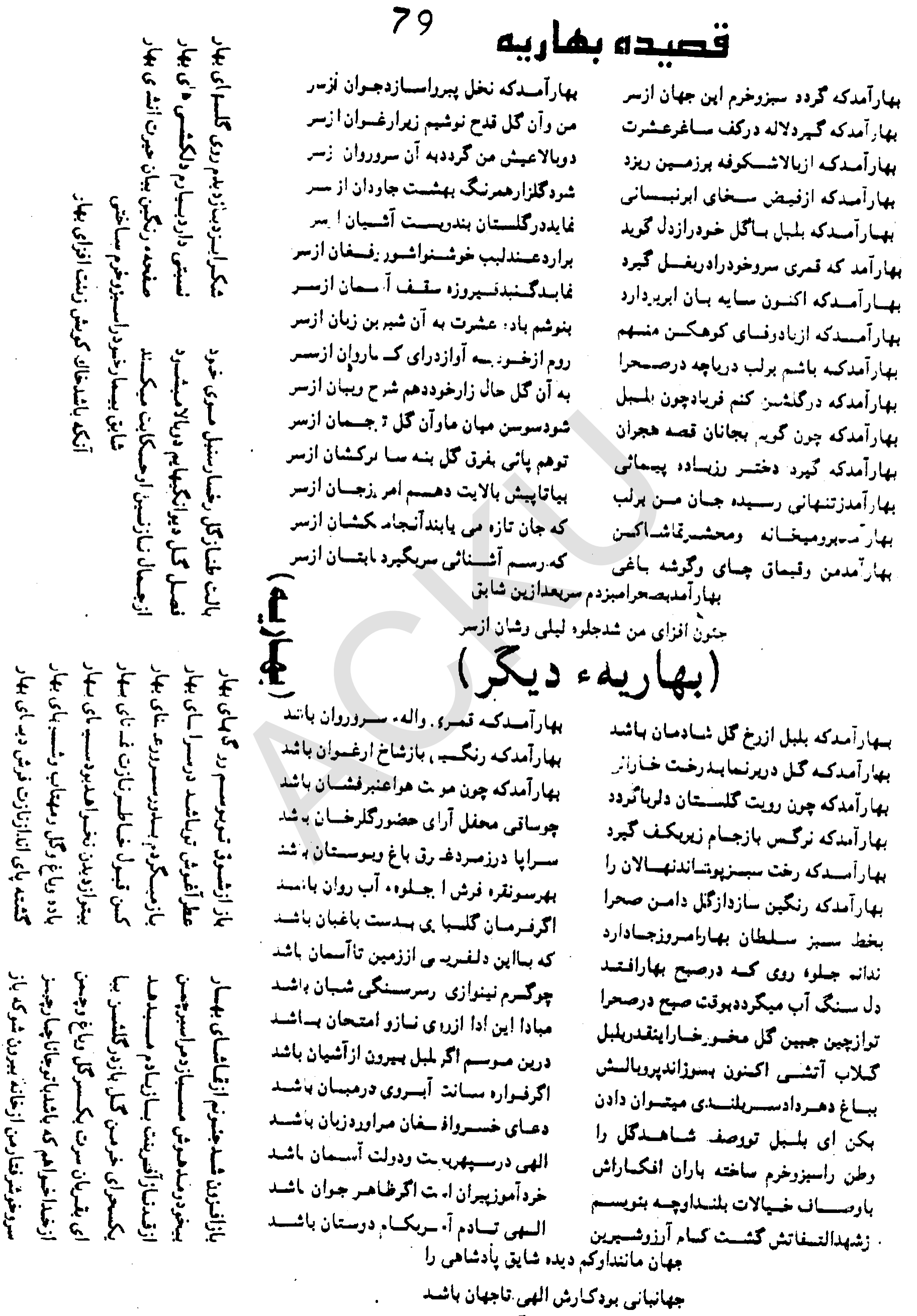




\section{بر كو به آفناب}

از طبع حامد نويد

نبود درين بهام دماغ شكنتخ

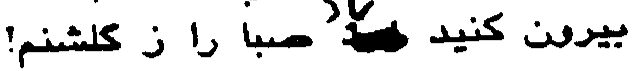

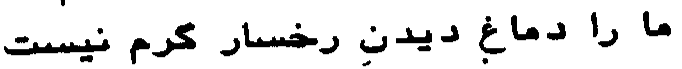

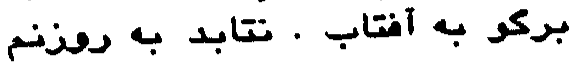

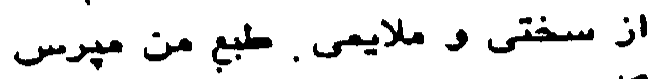
كامى ذ موم نرمتر و مات كاه امنم

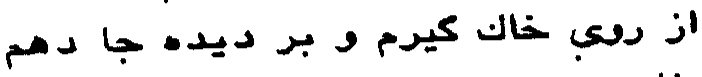

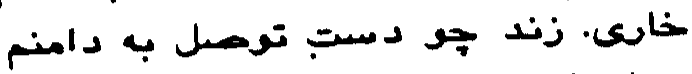

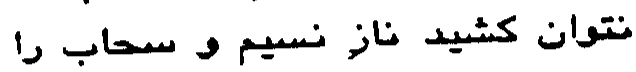

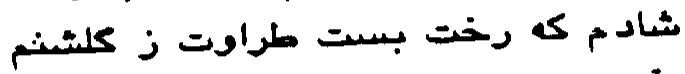
آن لاله ام كه فارغم از ناز باز باغبان

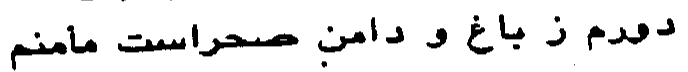

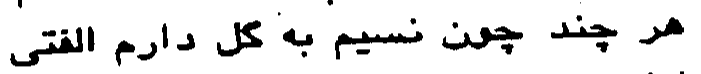

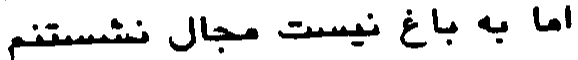

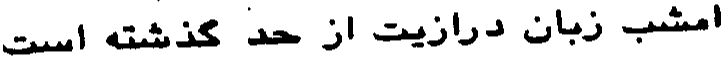

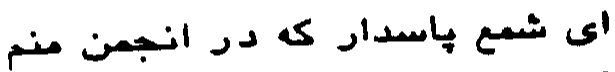

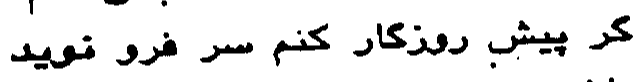
باشد بـ تيخ تيز سزئ سناوار كردنم

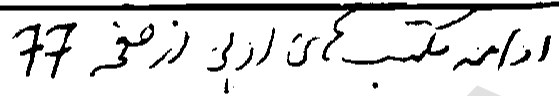

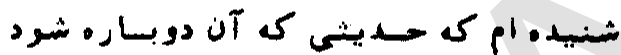

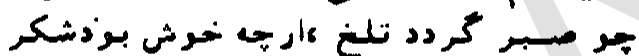
(ازفرخي سيستاني) عردي)

من نه مرد زن و زمر وجرامبم

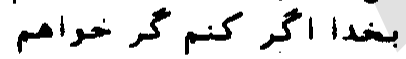

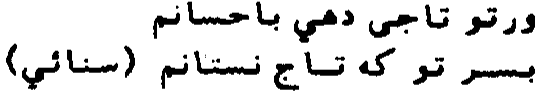

حكيم كفت كسى را كه بـتت والا نيست

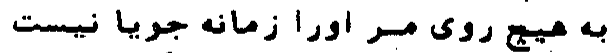

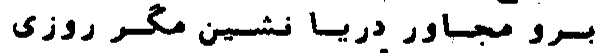

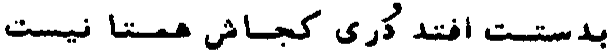

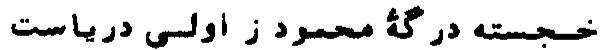

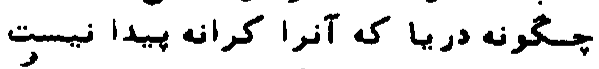

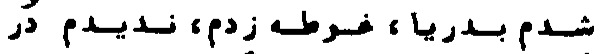

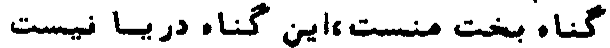
(مكيس فردوسي طوسية)

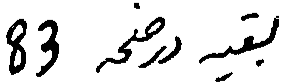

\section{0 (أيضا بهاريه}

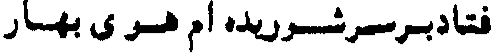

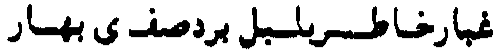

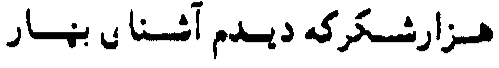
بغط سرغ نوشته است داجه إن بهـار

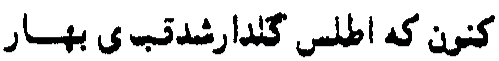

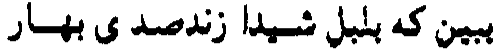

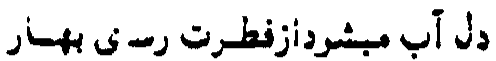

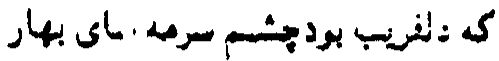

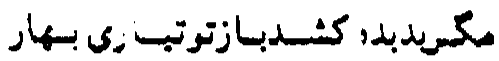

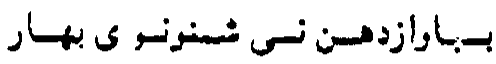
كه كثته عرمله انزاى أرن نسأى بهار كد مبرو مأى اجابت دمددء ماك بهار

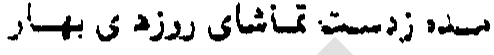

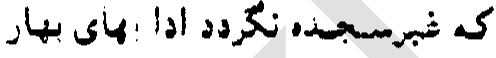

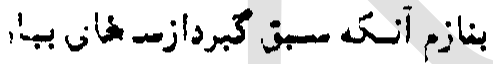

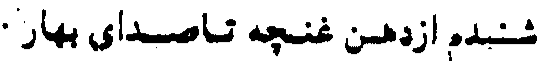

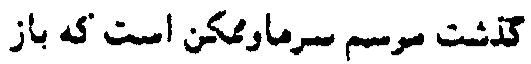

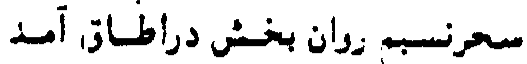

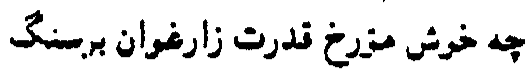

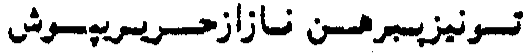

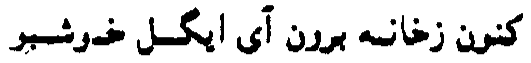

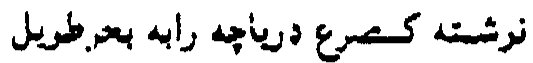

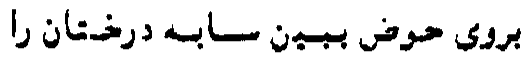

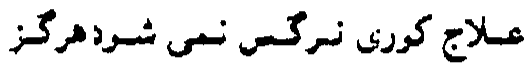

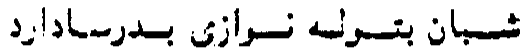

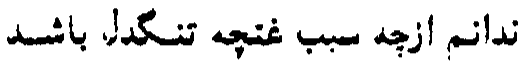

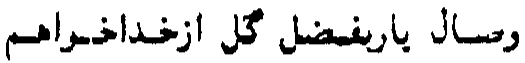

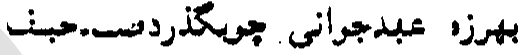

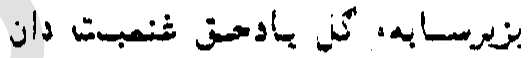

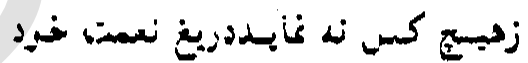
مكن مرامد جرشايت بشسوت نوش زنبشي

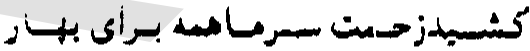

\section{}

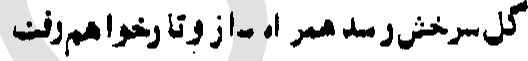

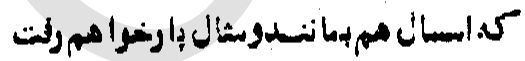

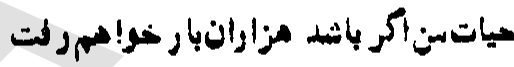
برايى هلى هرستكل دران دربهارغوا هم رنت

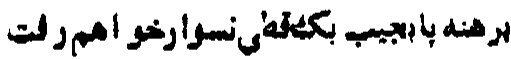

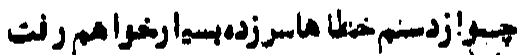

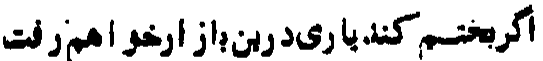
خدارايادكردنزدآنشستو ارخو اممر رنت

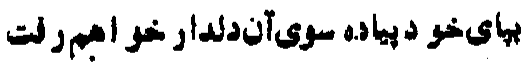

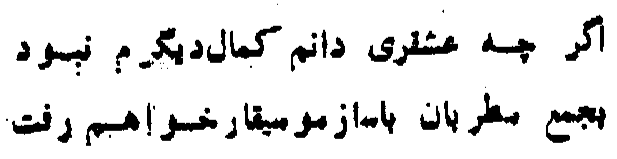

سرا مر لبلته و نغل بكر دكار. الست دل مسن عهازم ثـهر مسز ار. لمست

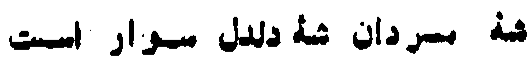

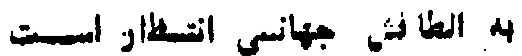
زهارت بهـن هـزاران درمهزاراهت

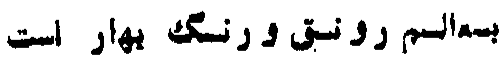

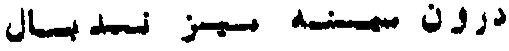

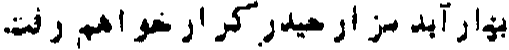

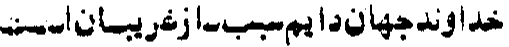

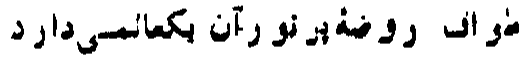

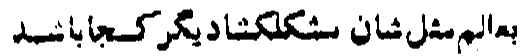

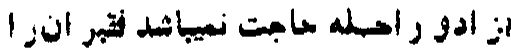
ميخى بانباكثهكا رانتطر بسيار تسر دارد خرابواخسنكان روكيهالمر انريداز ابيت يُجبه داو مدكر با هار هذ در دمنسيكر دد

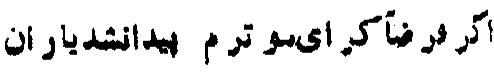
يسز ار است و مسز ار شسله بـسر دان

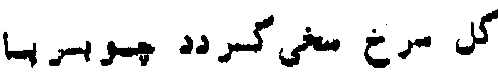

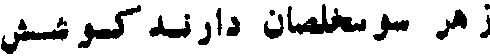




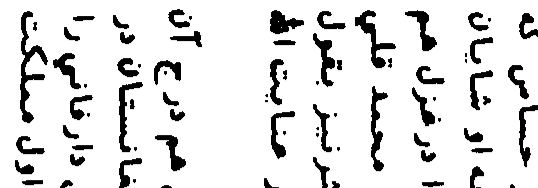

(E)

列

So

che

(a)

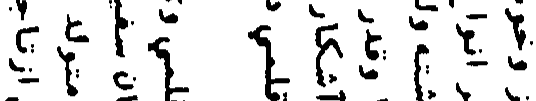

है

S.

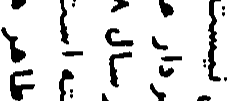

Sᄃc

te.te

$=0$

to

$q \cos t g=$ to r Fis a

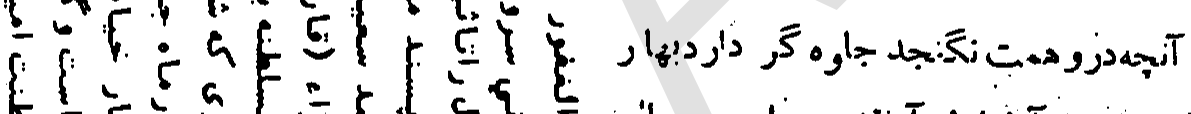

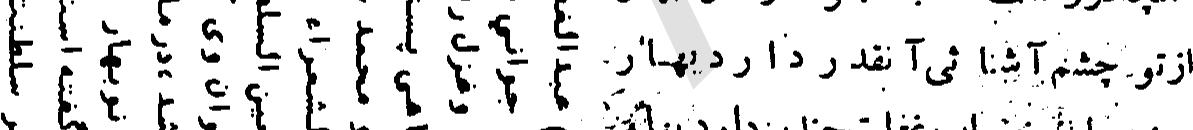

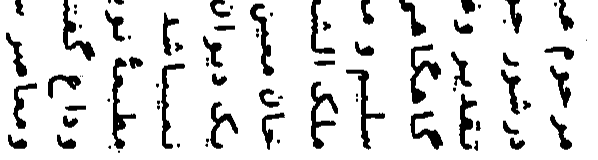

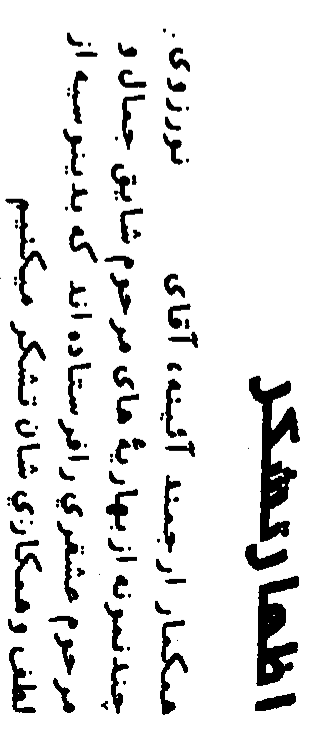

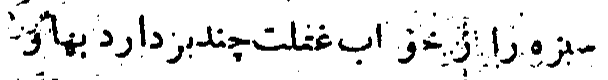

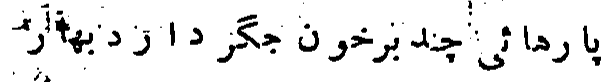

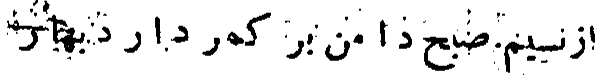

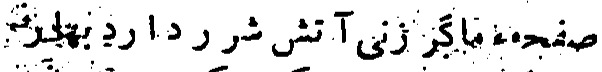
جز

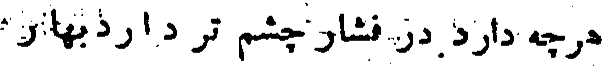

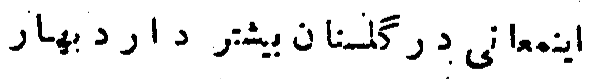

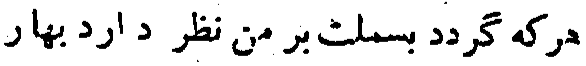

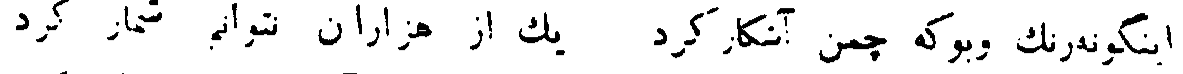

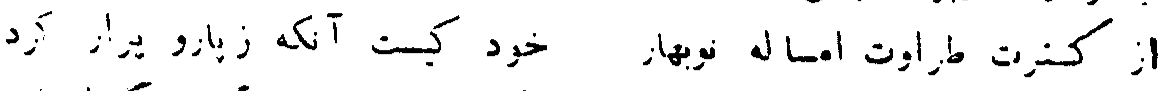

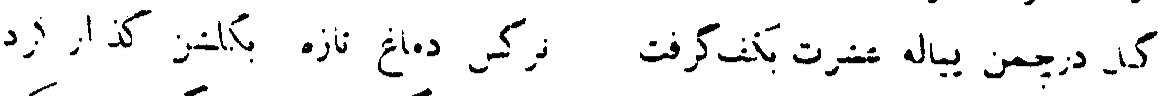

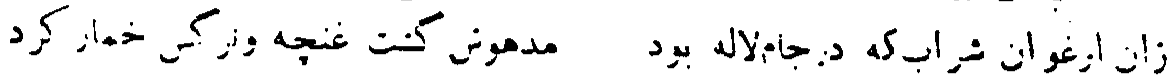

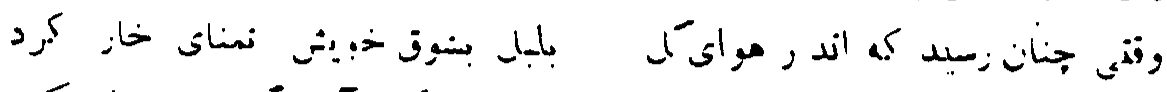

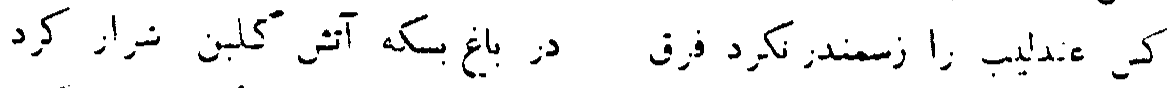

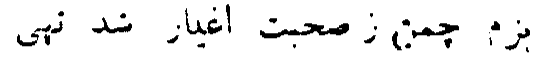

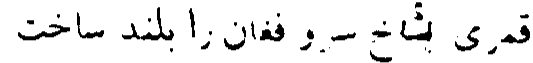

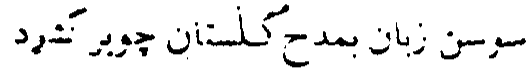

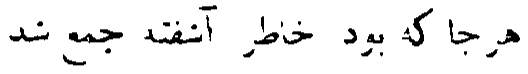

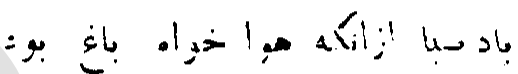

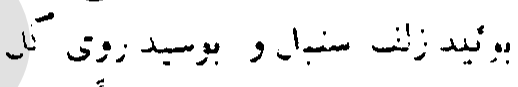

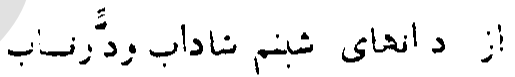

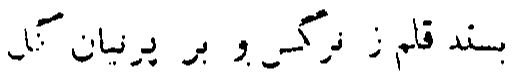

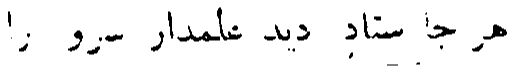

در بحر مضار ع مشمن اخرب مكغوف مسذوف

\section{يك بماربه ديكرهولا نا بهدل مهم دل}

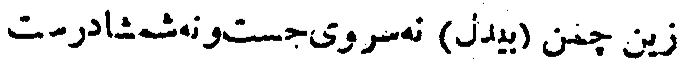

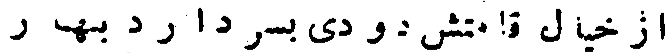



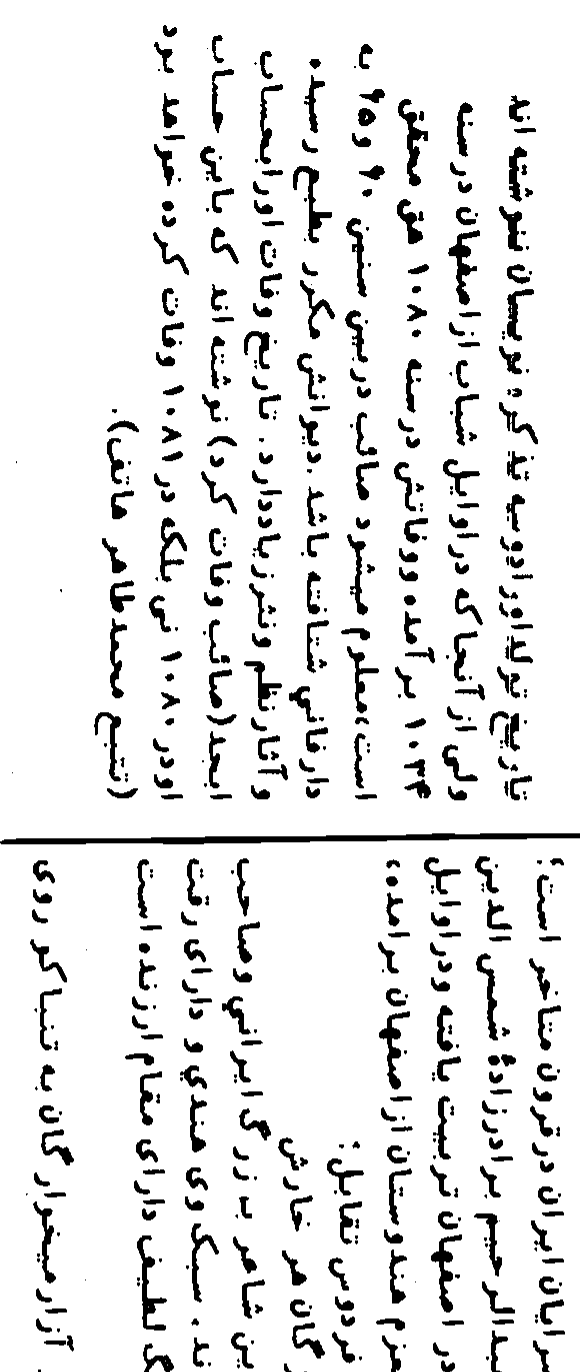

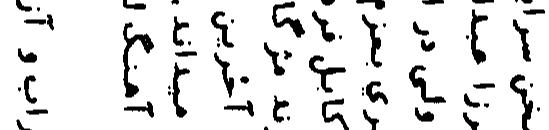

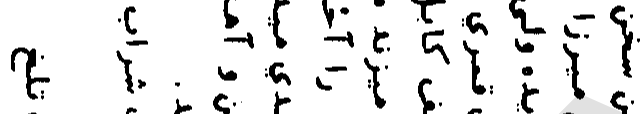

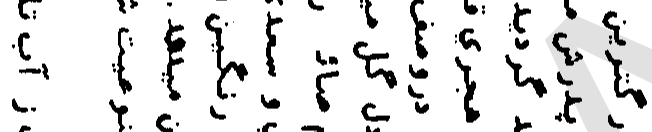

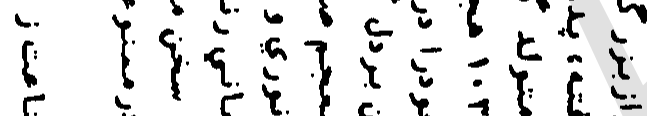

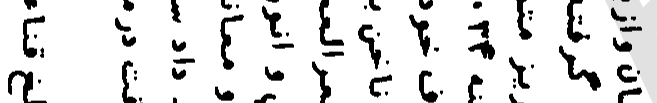

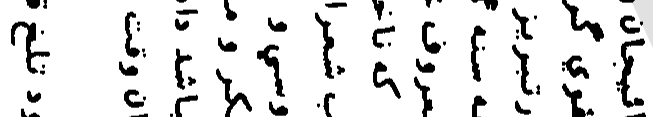

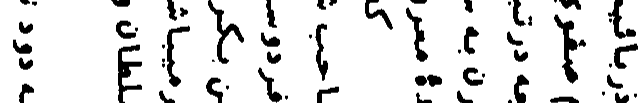

$\because F\}\{5$

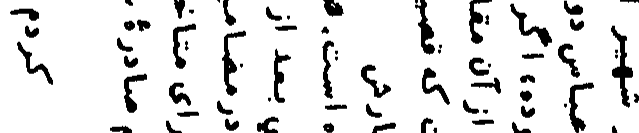

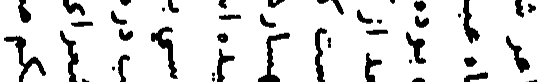

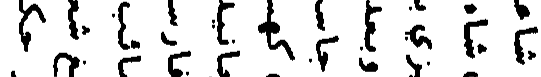

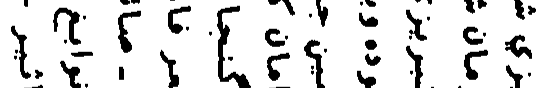

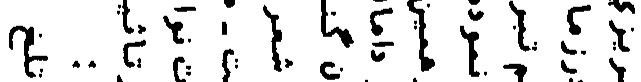

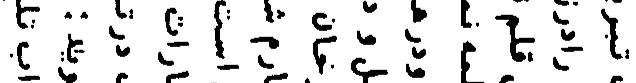

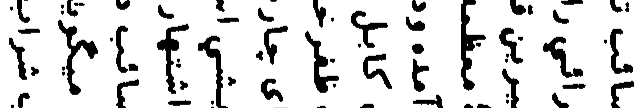

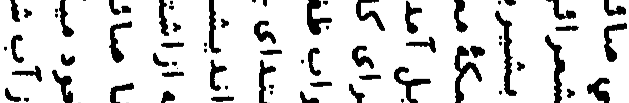

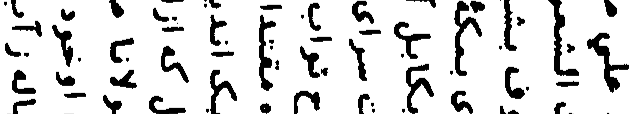

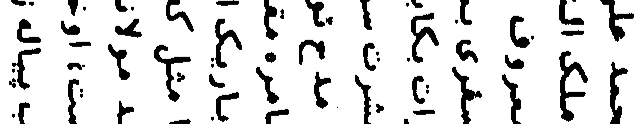
the lo
زمين را كند بحر كومر :مكوفه

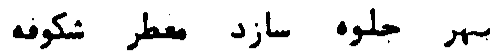

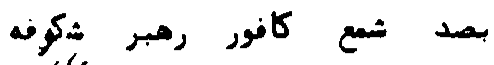

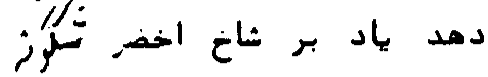

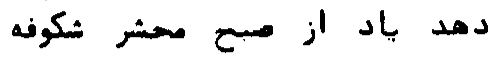

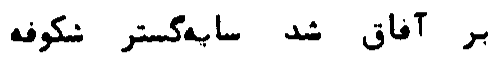

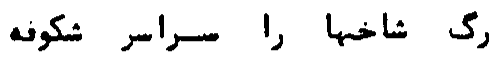

نهان شد جنان شاخهها در شكوف

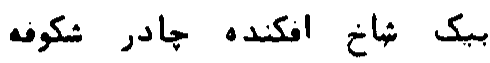

كه در مرده خورد ست ساغر شكوفه

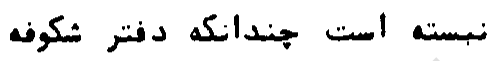

شود جهره با روى دلبر شكوفه

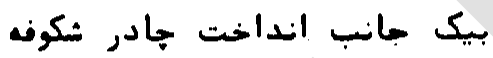

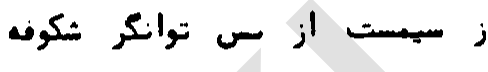

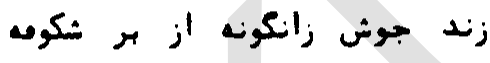

زند اينجنين غوطه كر در شكوف زئن

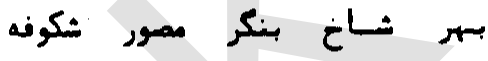

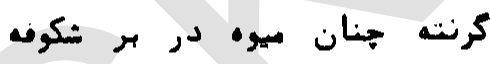

برآرد ز شاخ آنجنان صر شكوف هرد

زهر غنجه تنك شكر مر شكوف

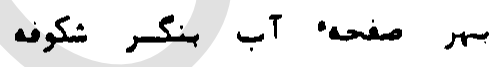
ز بس كرده شب را منور شكوف زمين را جو مهناب انور شكوفه كذر كن بستـان و هنكر شكومه

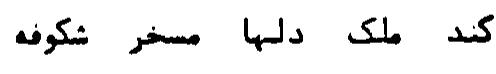
ز بس ربخت بر خاك اختر شكوفه

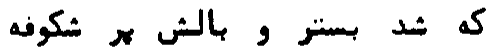

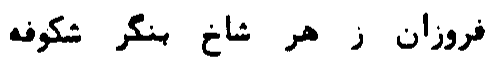
جو ريزان شد از شاخ اخضر شكوفه كم افكند دستار از صر شكوفه

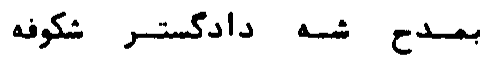
كه بر نام او ميزند زر لثكوفه كه بر خود نلرزد ز صرصر شكوفه

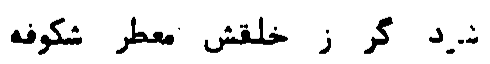

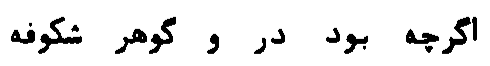

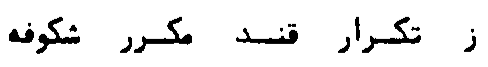

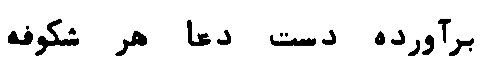

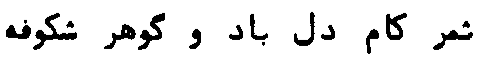

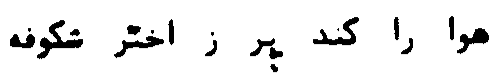

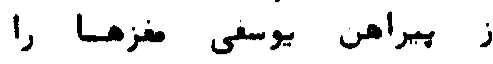

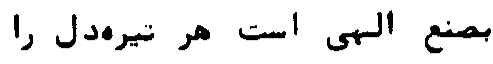

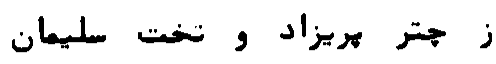

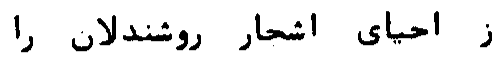
جو بال و ري برى بساط سليطان

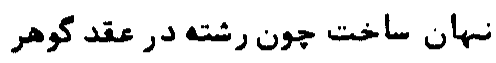
جو راهى كه از برف بوشيده كردد دئ

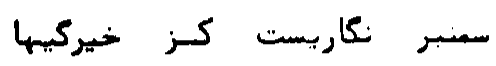
ز دئار آشغنهاش ميكند كل

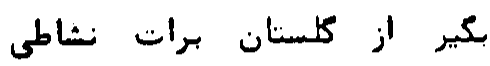
نقاب لطينى است كز خوشتطاشى لئى

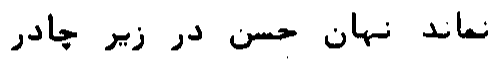

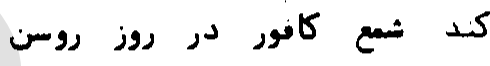
جو شيرى كه از مهر فرزند زايد

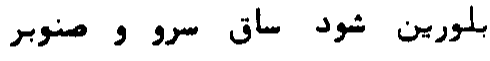
كه ديده قلم كاغذ از خودد هرآرد جو مريم كه عيسى بود در كنارش جو شيرى كز انكشت اطنال زايد بود درد از 'ان در نظرهاست شيرينك دارد

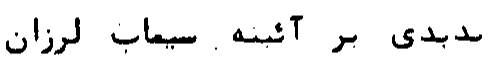
توان يافت فيض صبوحى دل شب

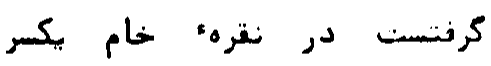

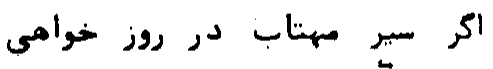

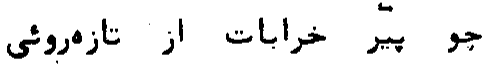
كم از كهكثان نيست هر كوجهباغى

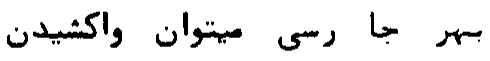

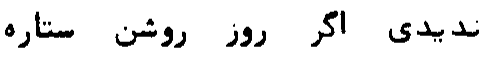
تو هم شيشه را بنبه بردار از سرون رون كرو كن بمى مر جه دارى ز بوشش

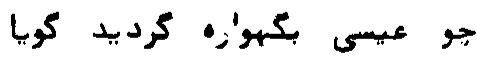

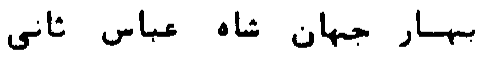

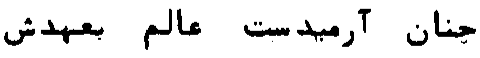
هرا مشكبو كردد از عطسه، كل

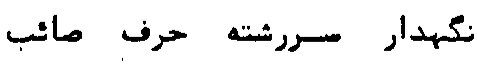

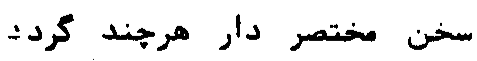
ثنا را دعا كن كه از شاخساران

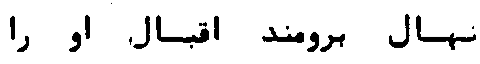




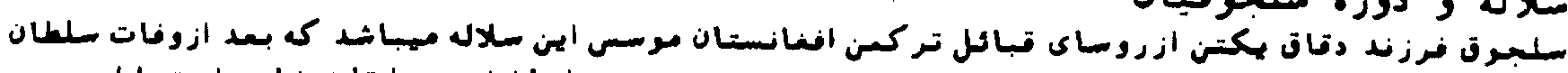

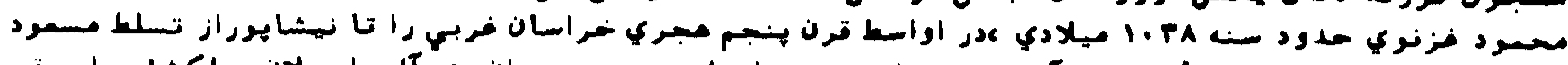

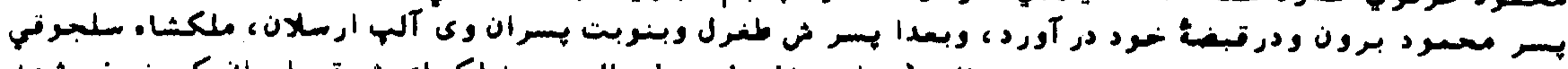

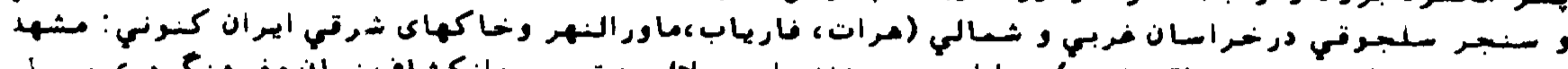

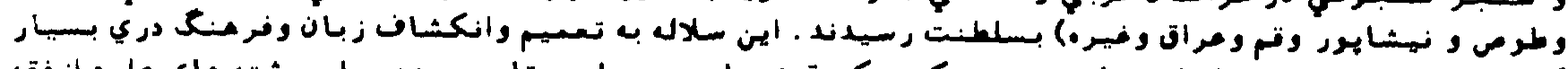

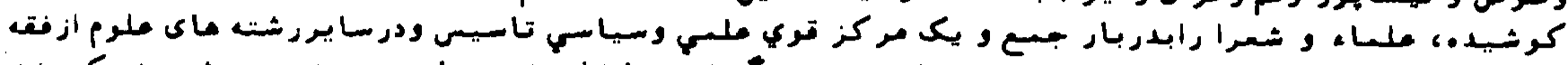

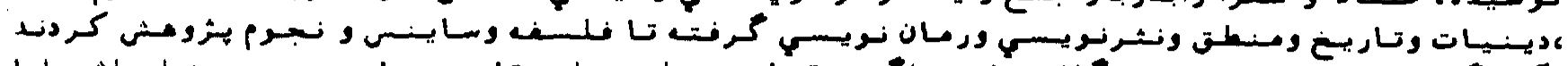

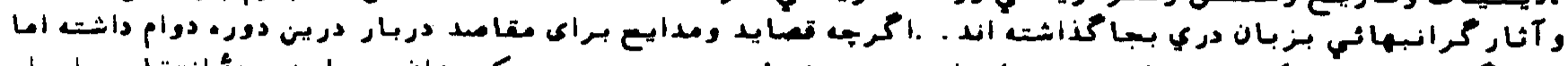

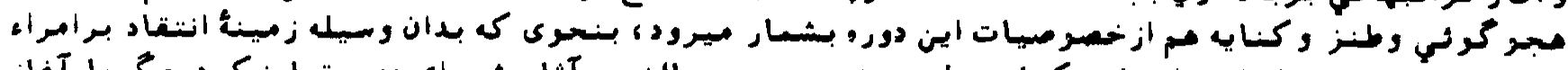

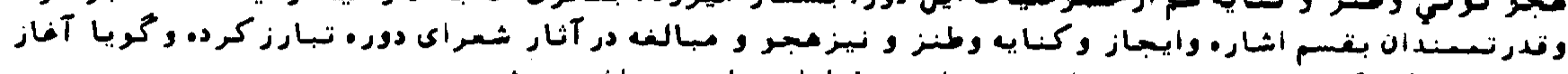

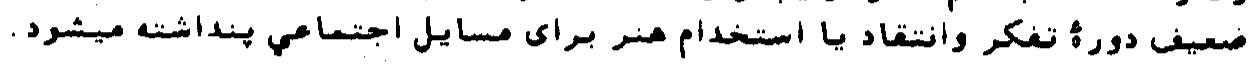

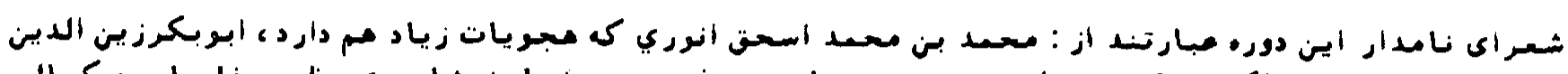

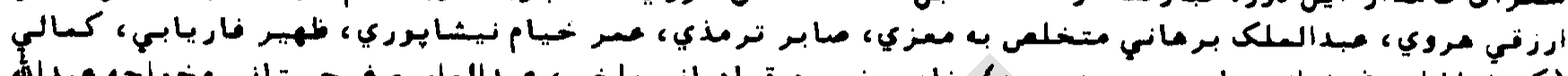

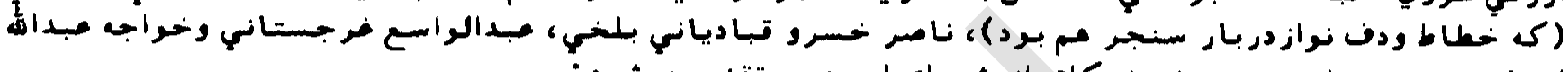

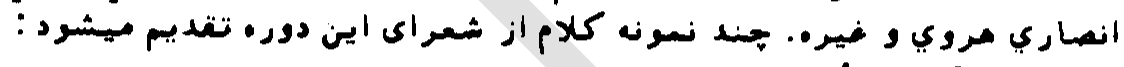

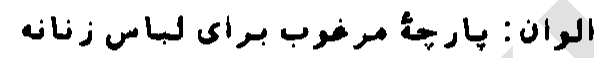

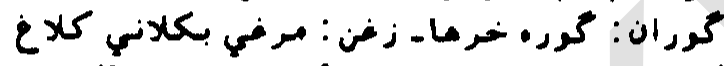

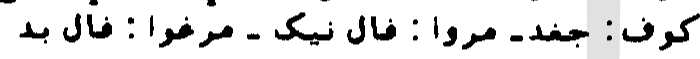

$\frac{a 2}{1}$

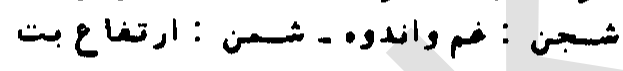

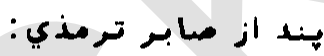

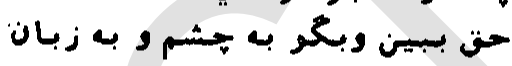

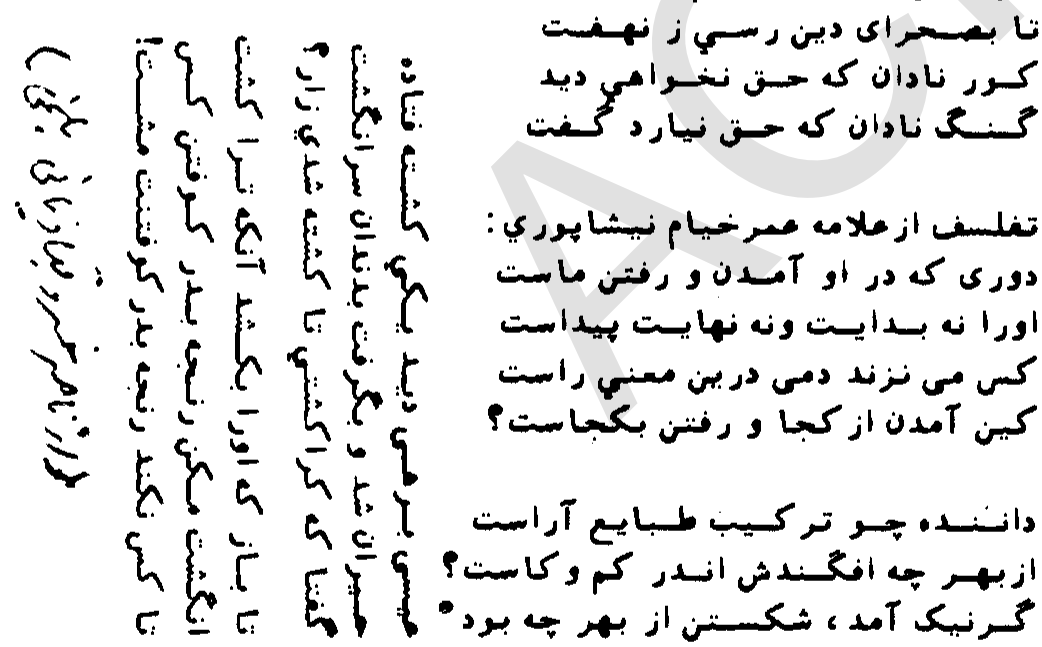

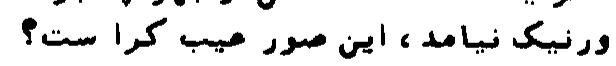

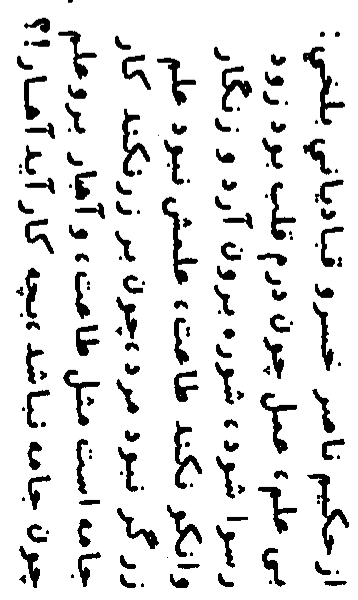

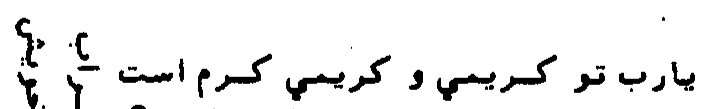

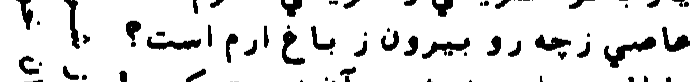

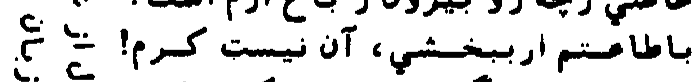

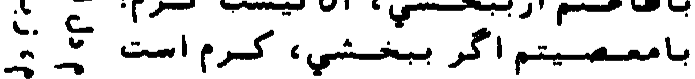

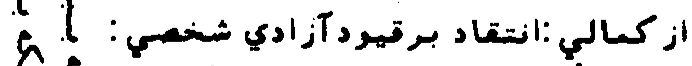

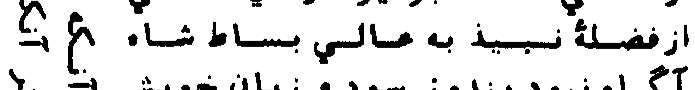

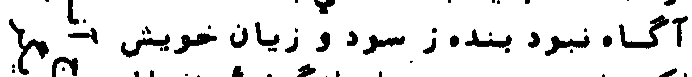

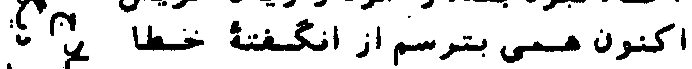

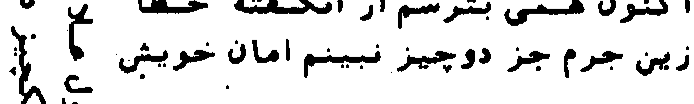
t.

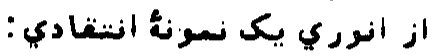

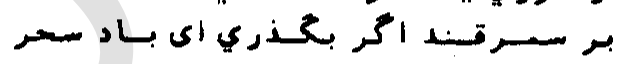

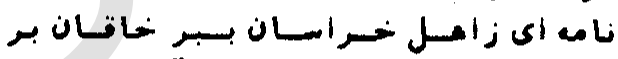

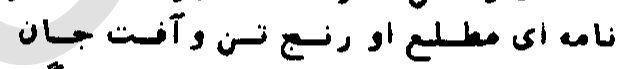

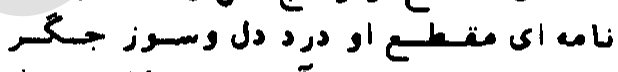

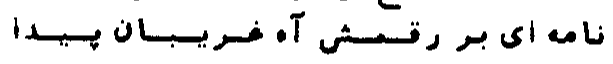

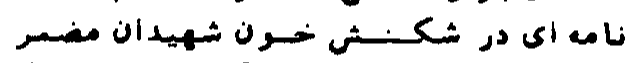

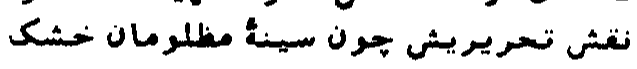

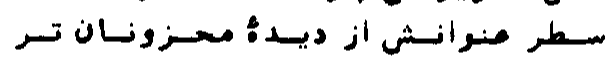
از ارزقي ، نسونة انتقادي برشاء :

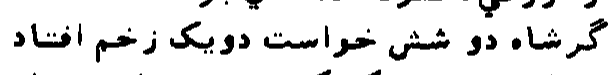

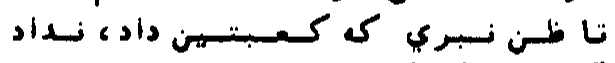

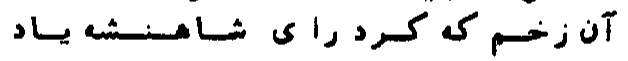

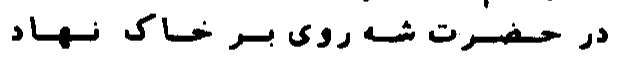

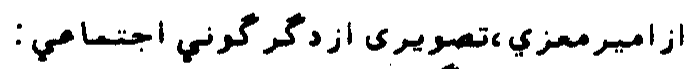

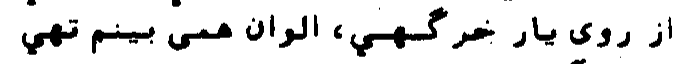

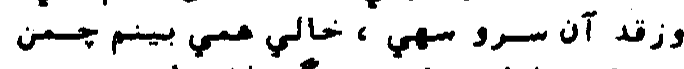

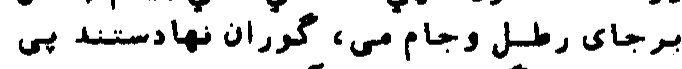

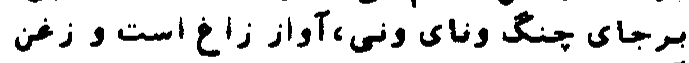

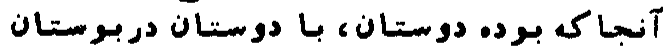

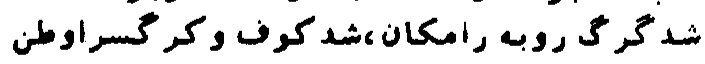

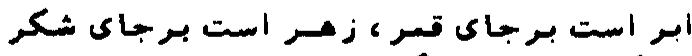

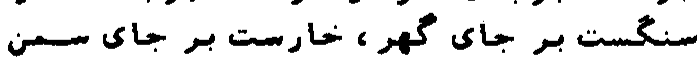

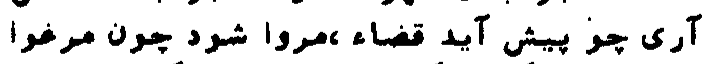

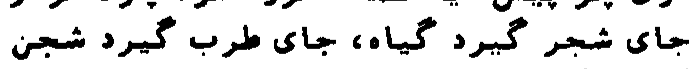

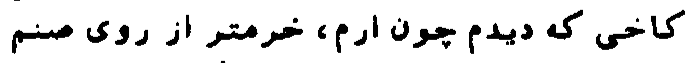

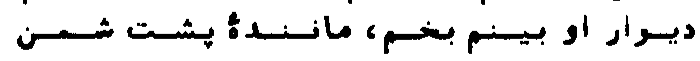

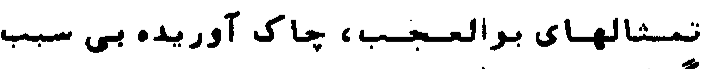

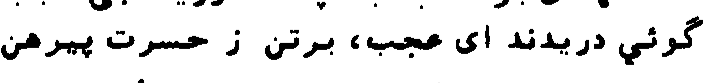
الثمارة هسلس 1 


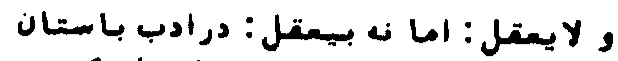

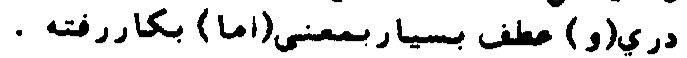

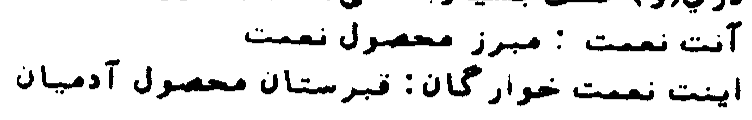

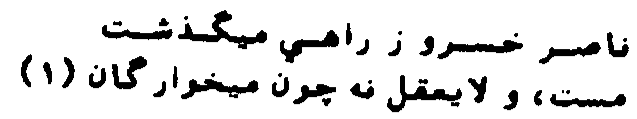

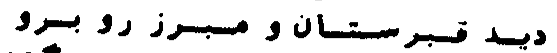

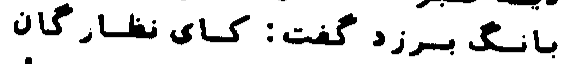

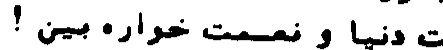

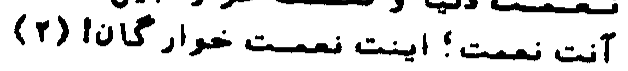

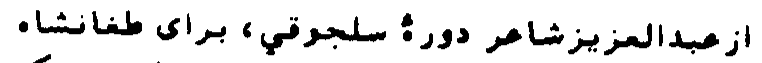

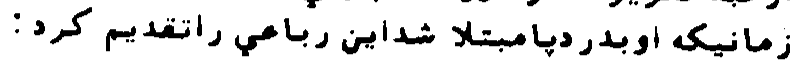

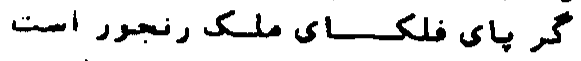

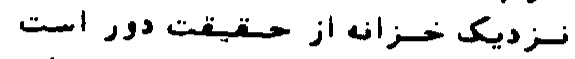

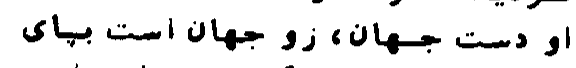

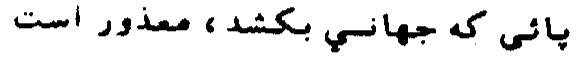

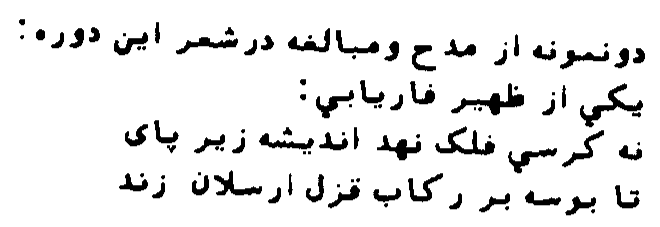

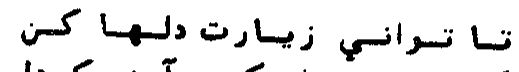

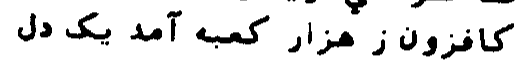

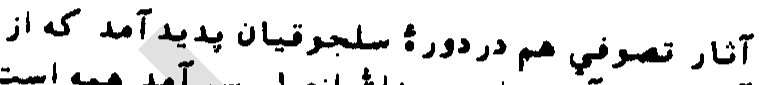

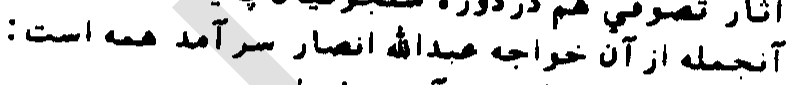

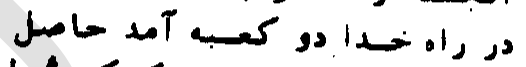

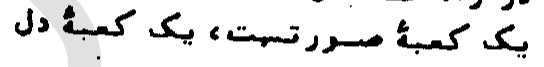

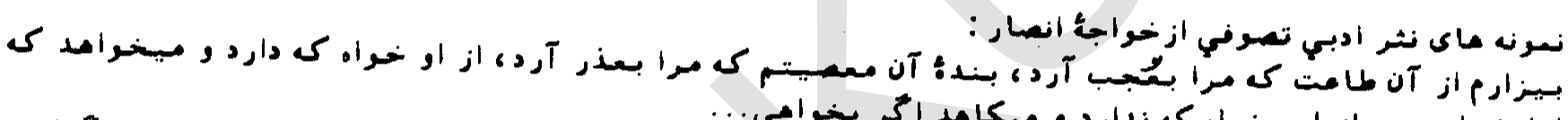

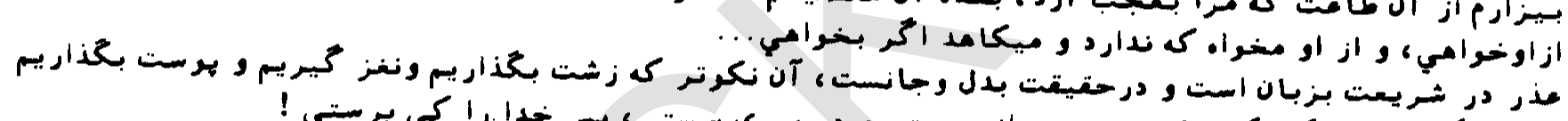

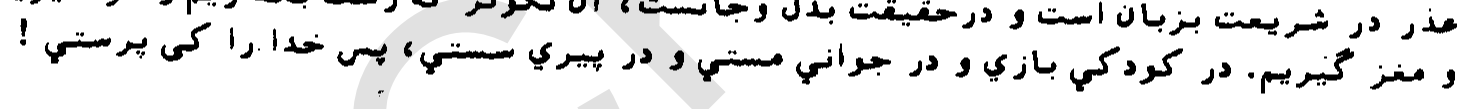

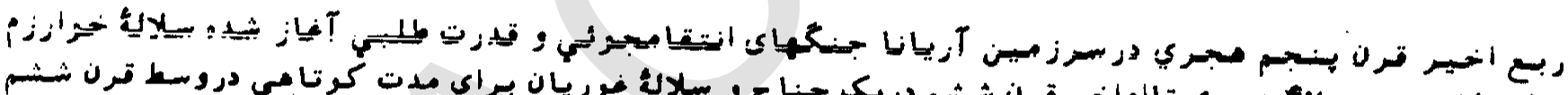

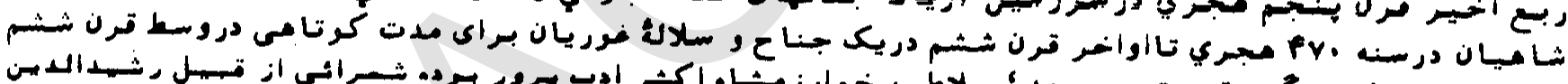

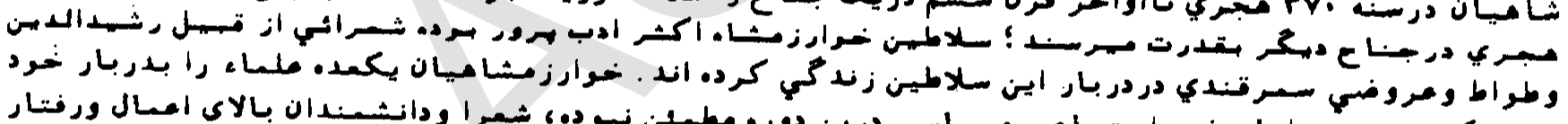

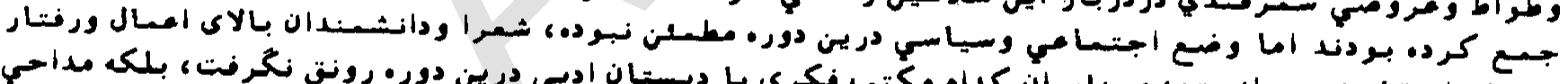

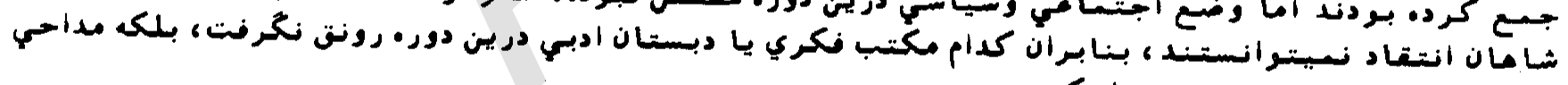

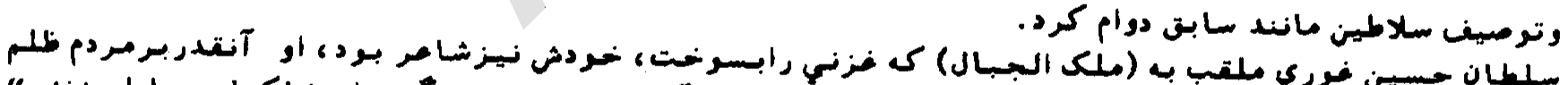

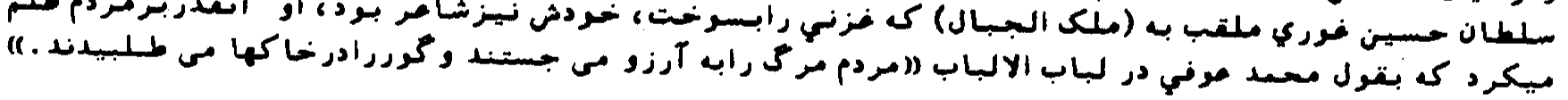

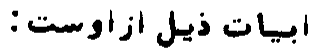

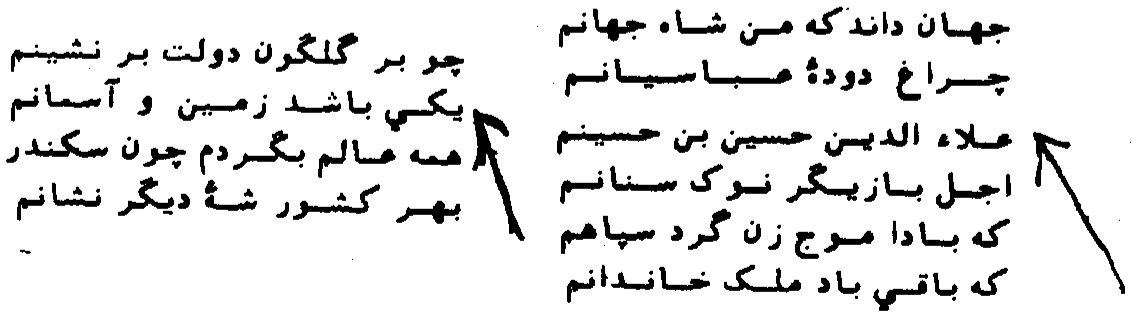

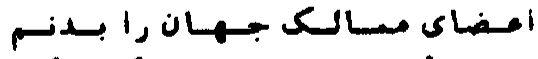

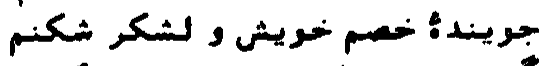

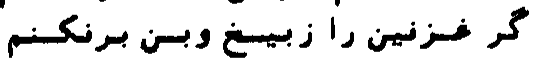

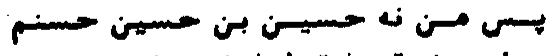

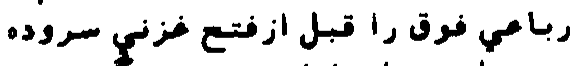

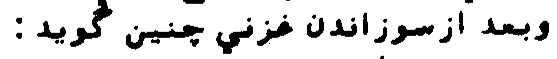

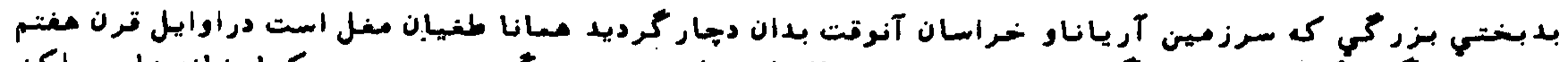

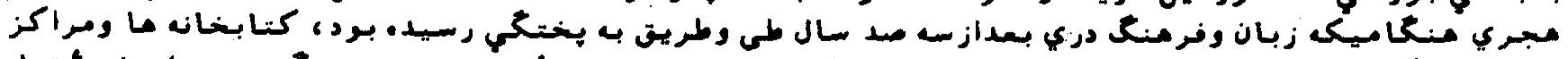

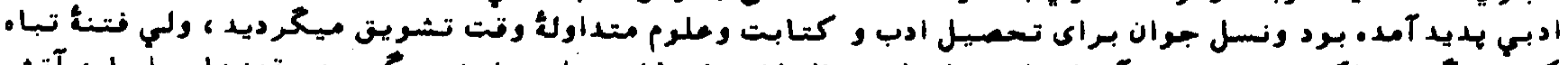

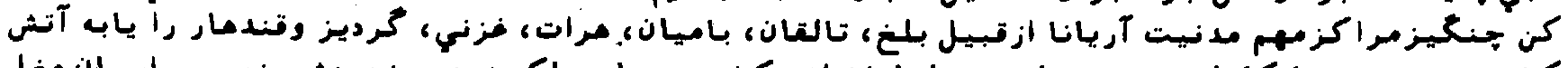

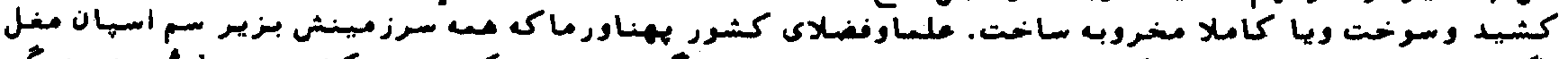

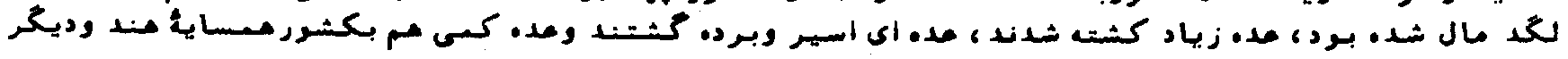




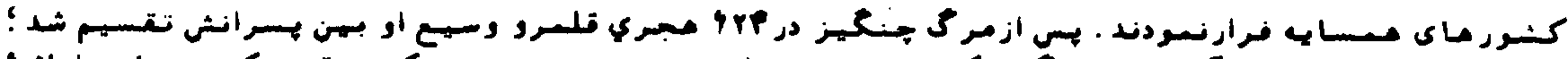

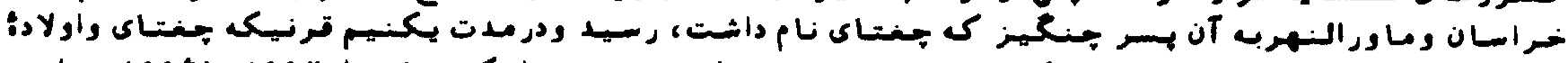

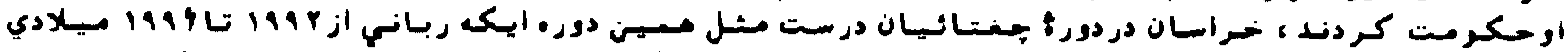

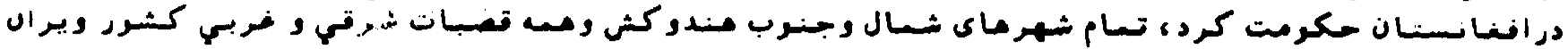

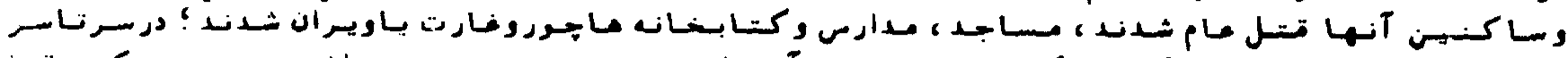

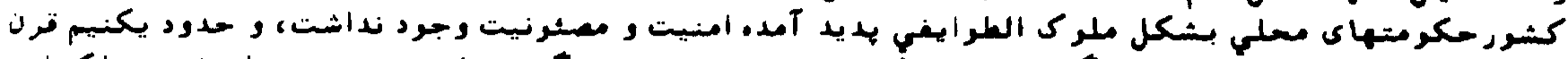

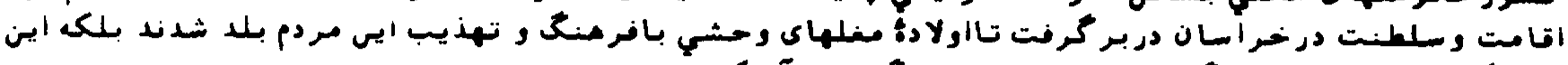

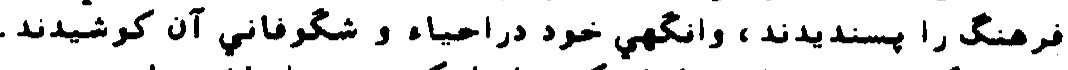

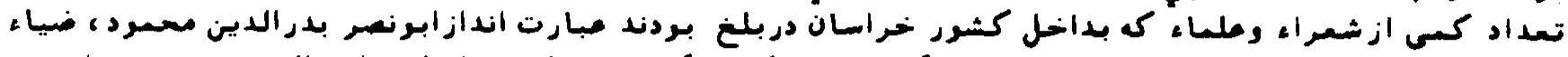

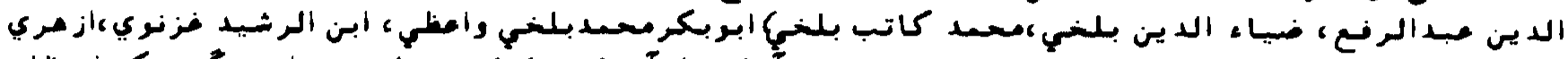

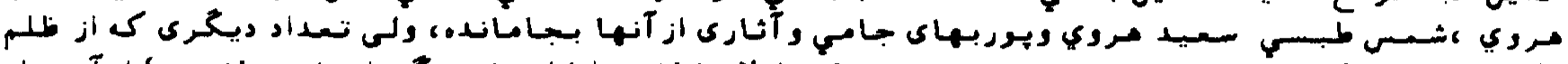

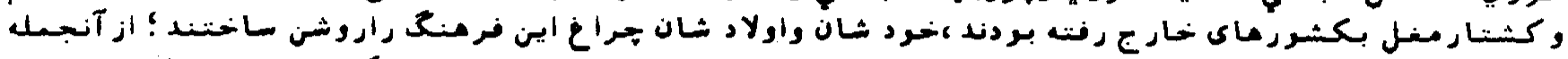

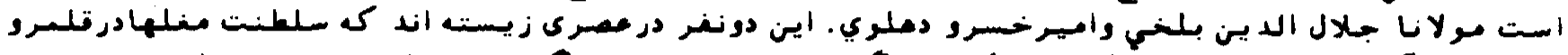

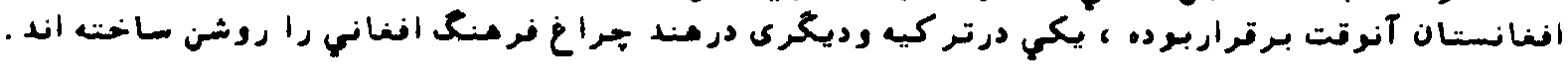

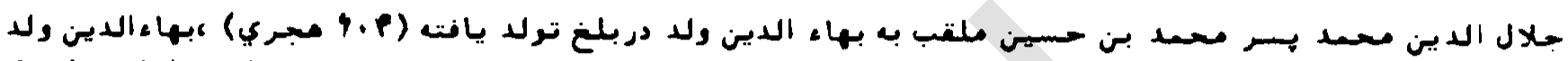

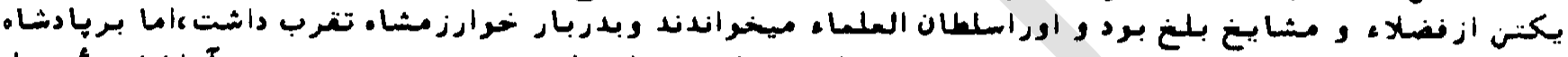

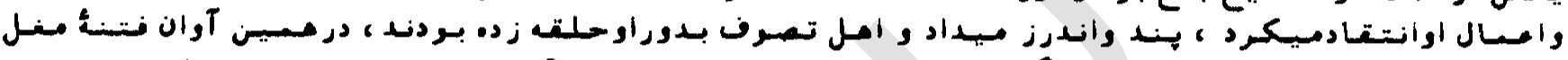

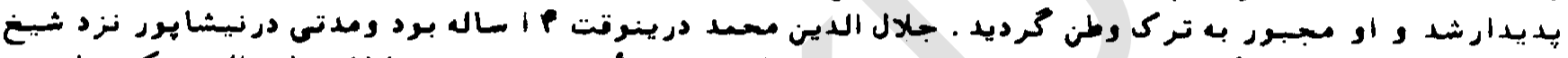

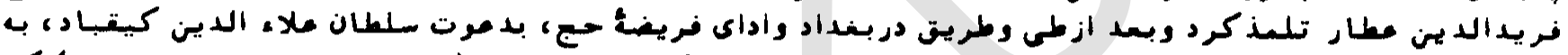

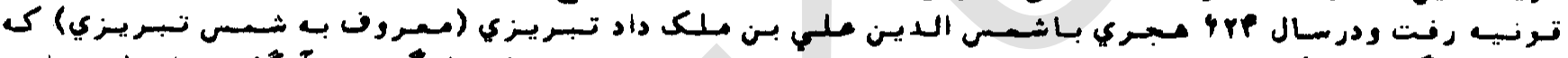

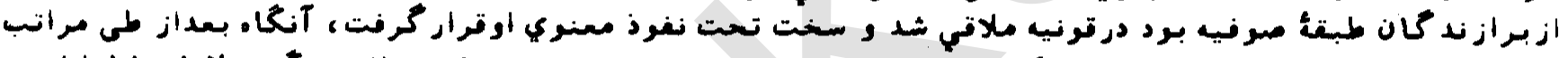

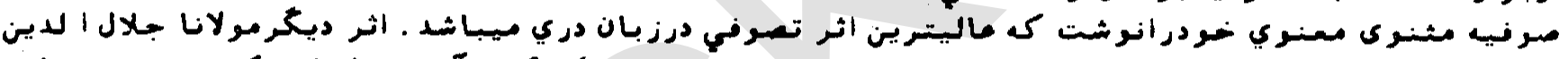

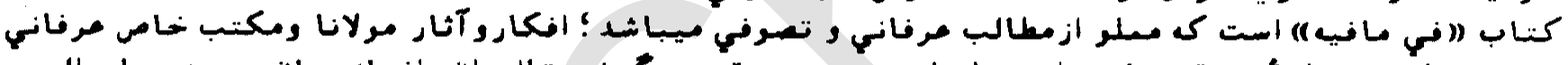

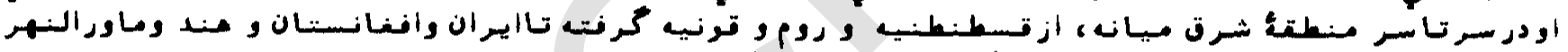

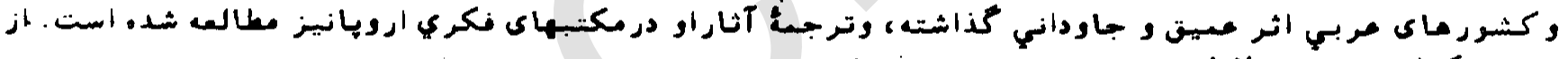

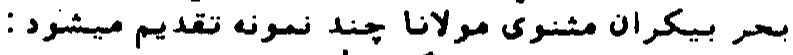

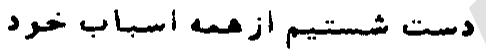

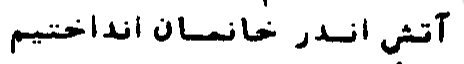

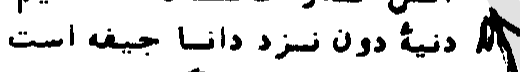

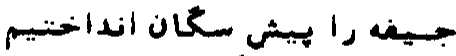

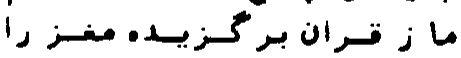

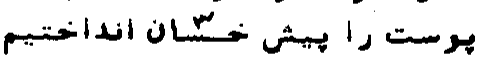

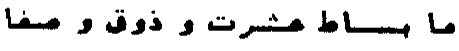

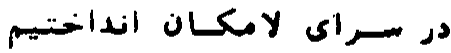

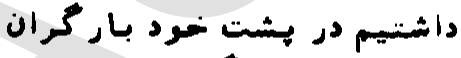

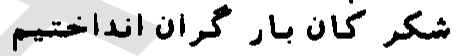

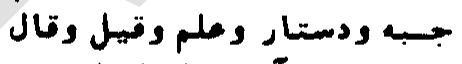

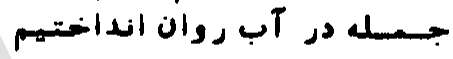

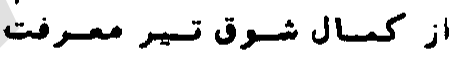

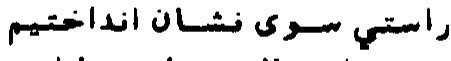

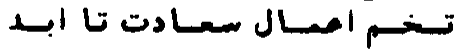

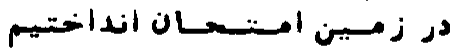

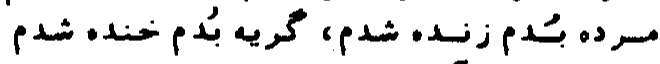

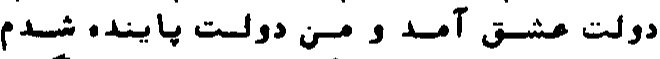

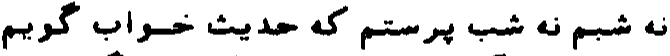

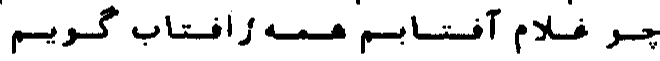

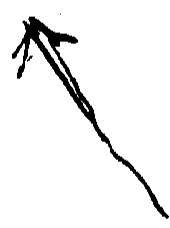

ما دل اندر رؤ مسر دان باخ اختيميم

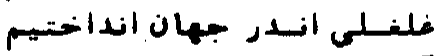

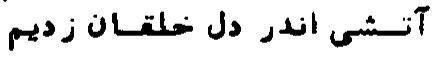

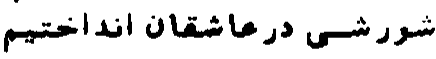

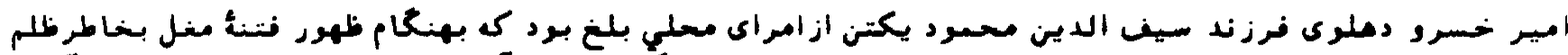

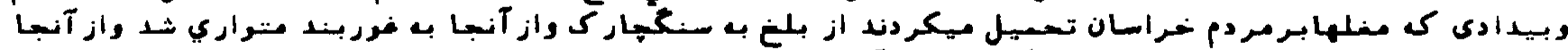

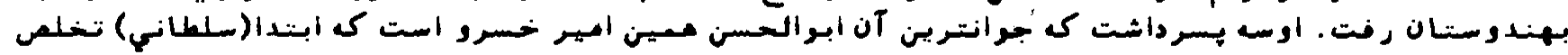

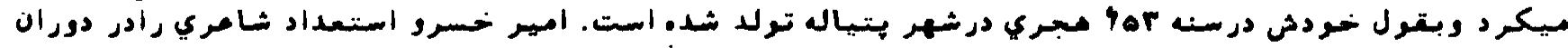

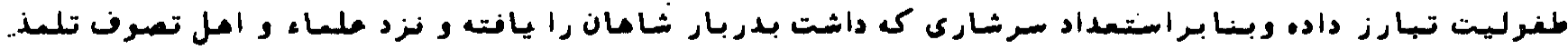

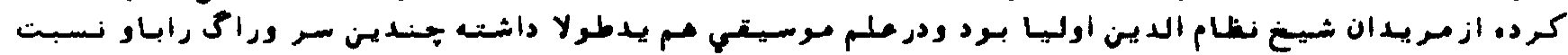

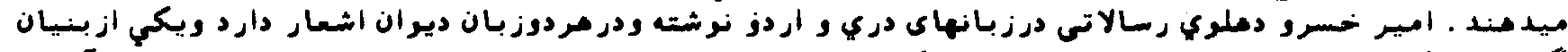

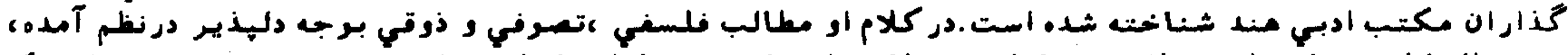

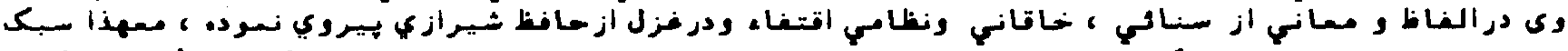

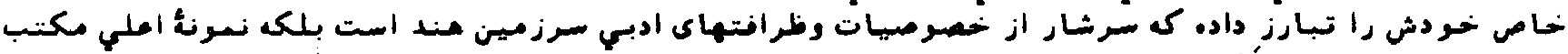

$$
\text { شمارة مسلسل Yنان }
$$

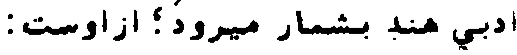




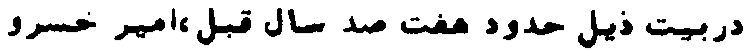

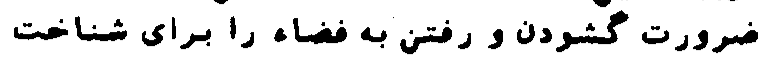

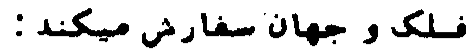

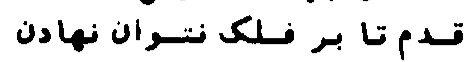

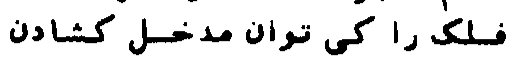

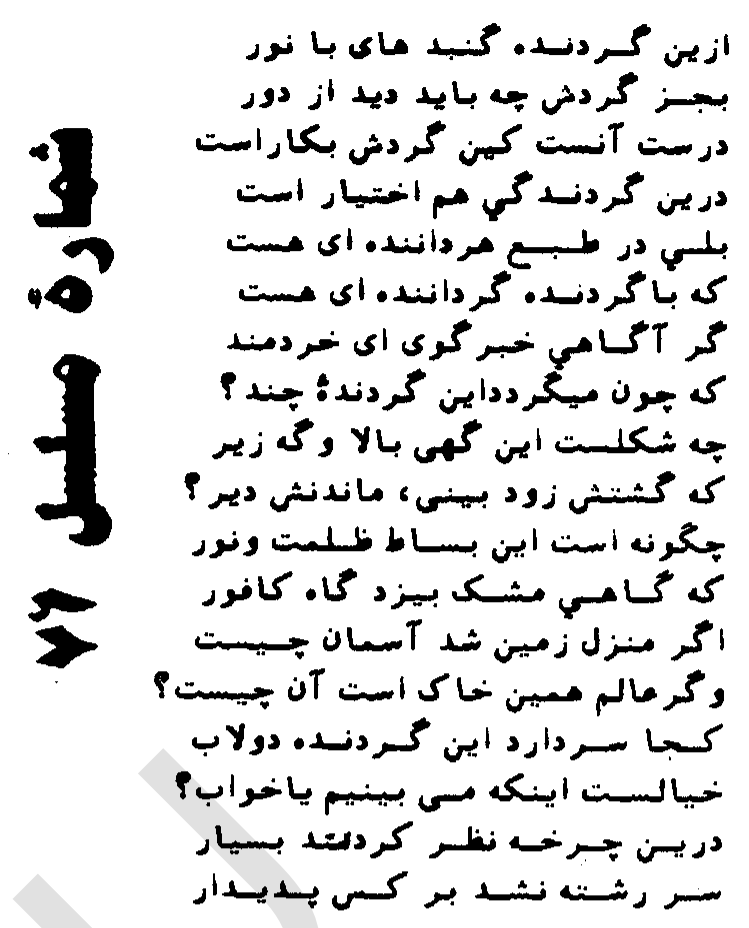

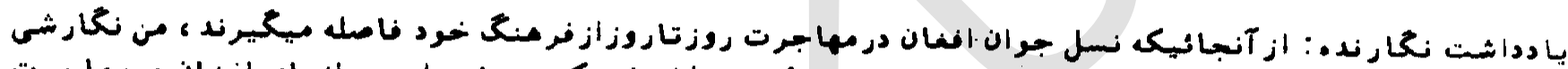

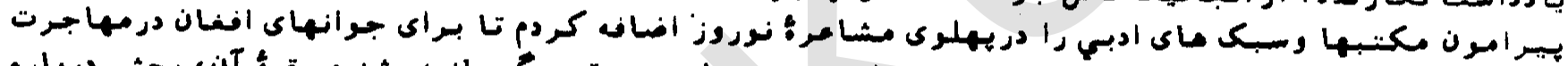

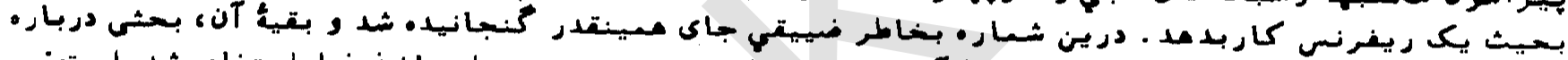

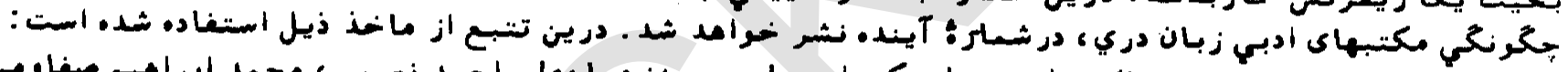

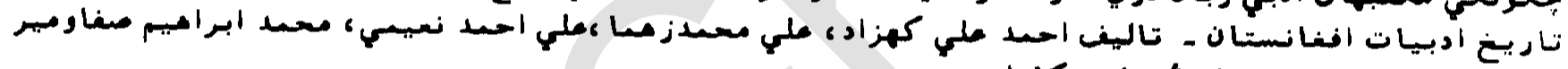

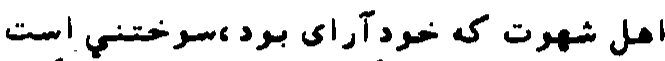

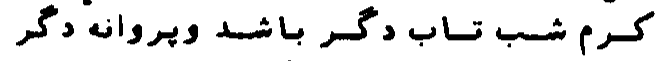

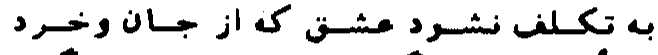

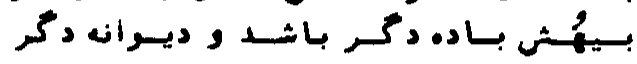

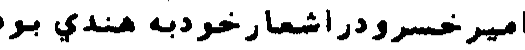

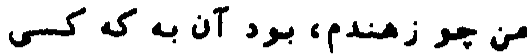

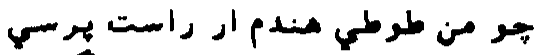

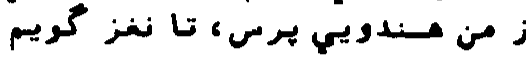

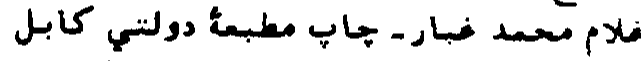

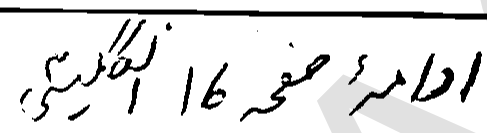

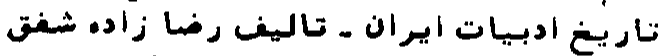

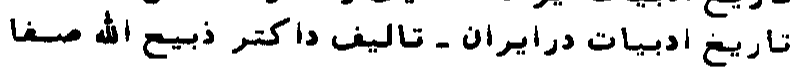

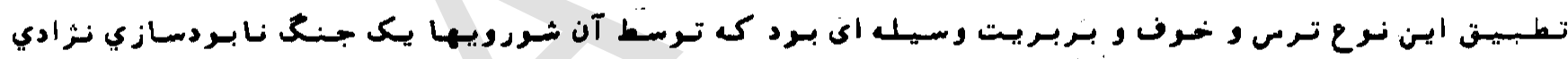

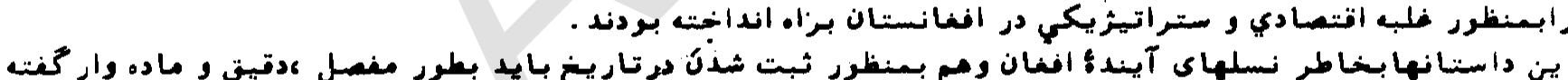

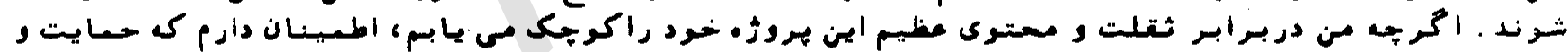

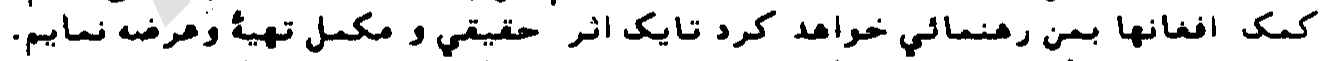

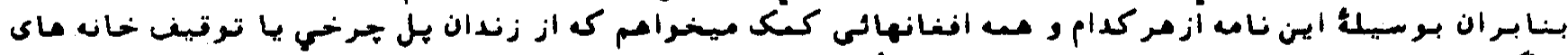

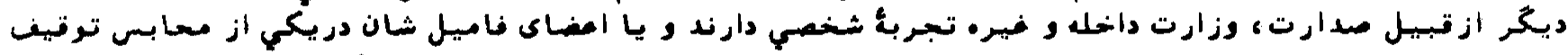

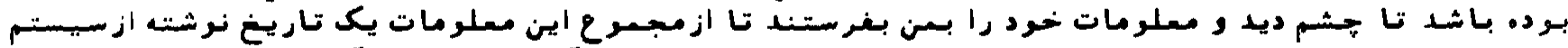

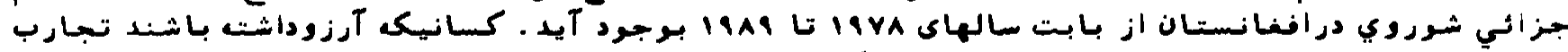

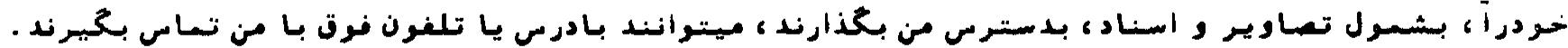

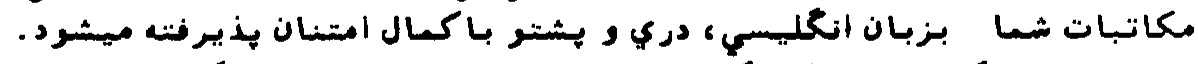

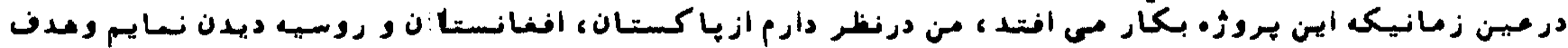

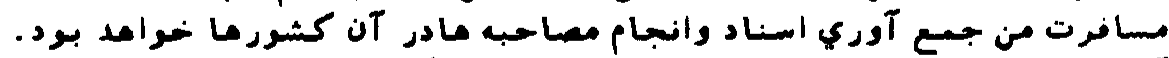

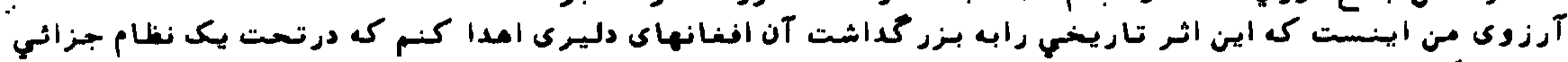

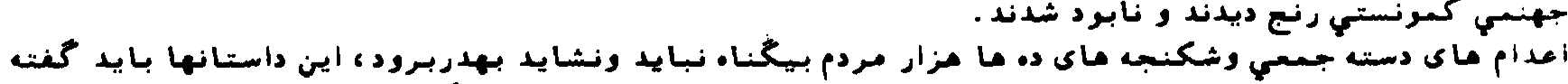

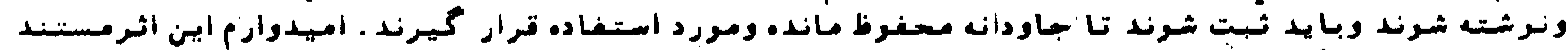

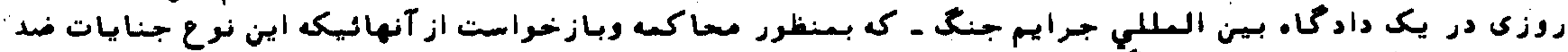

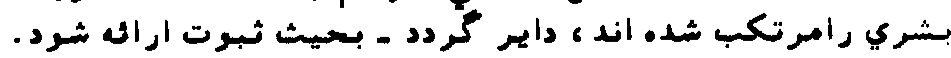

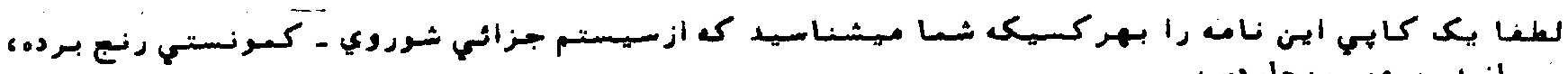

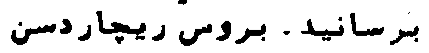


87

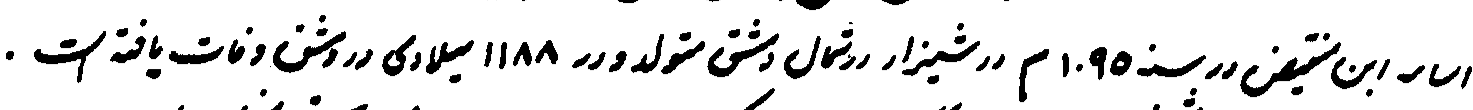

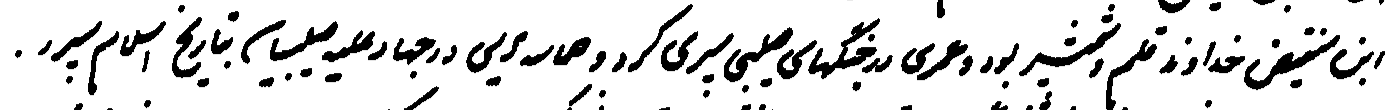

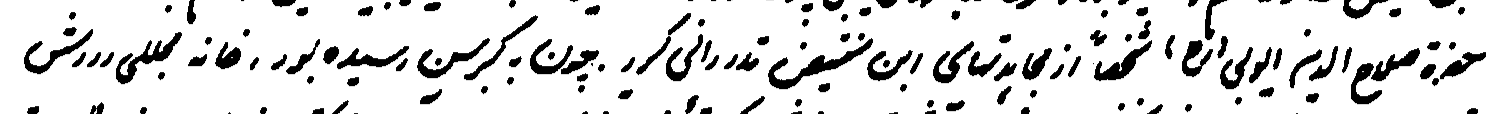

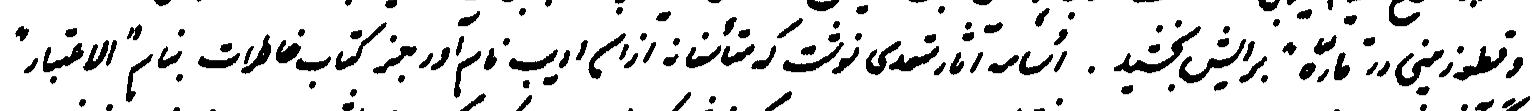

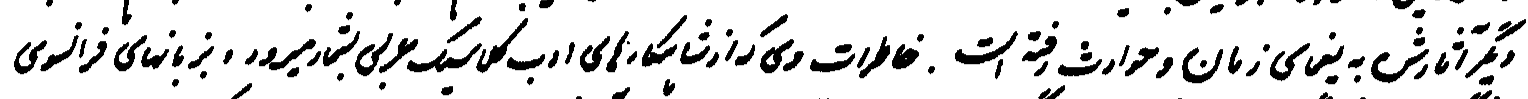

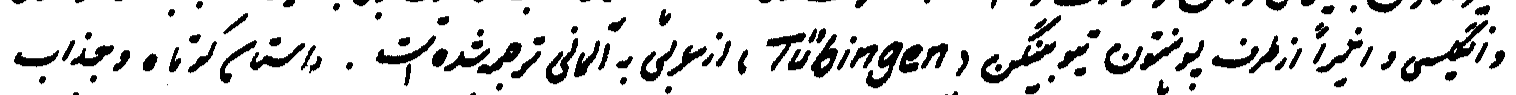

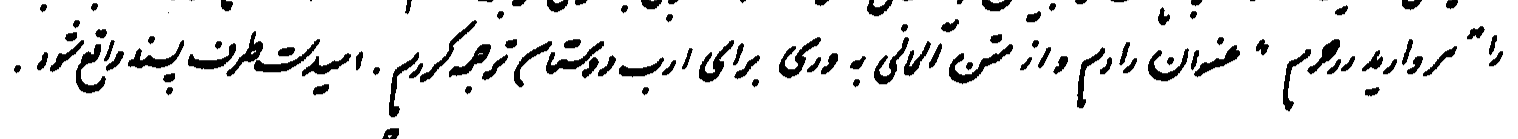
i

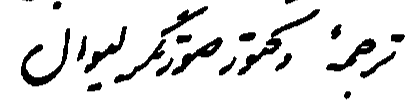

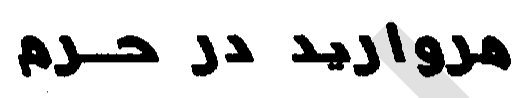

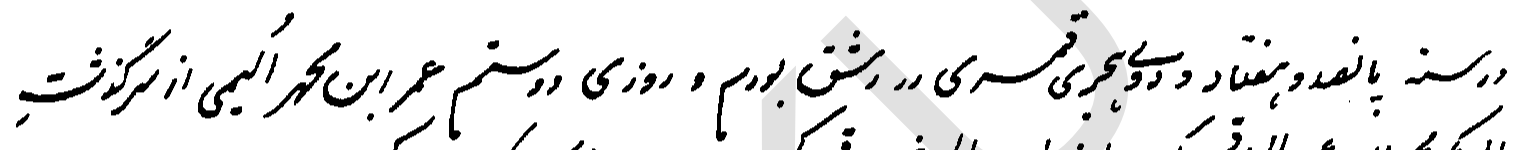

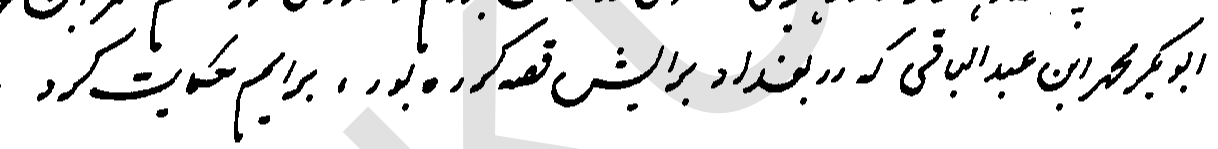

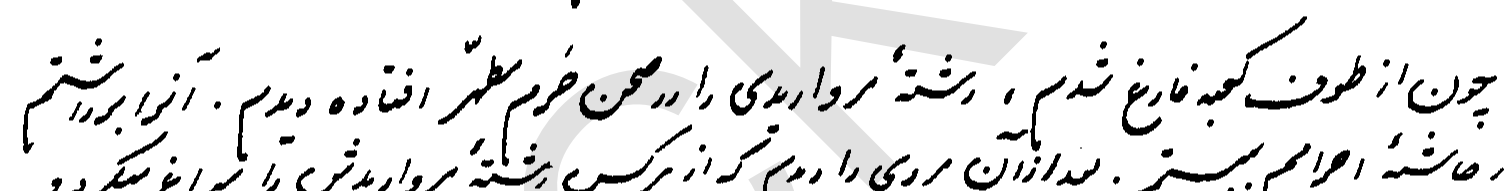

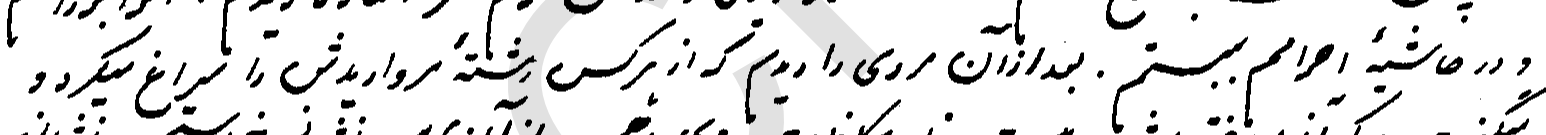

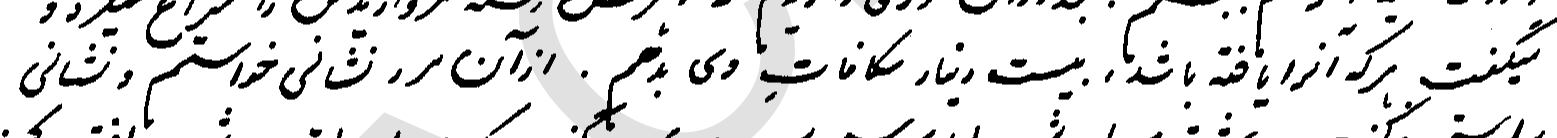

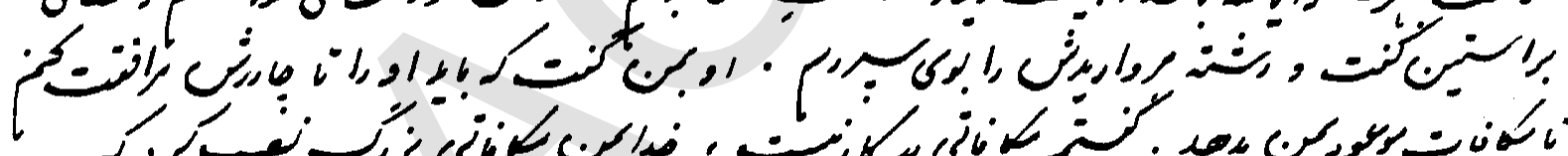

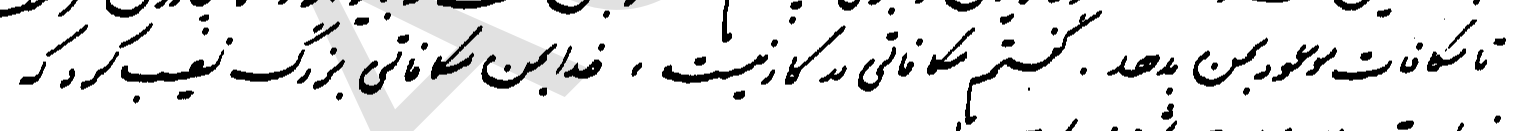
ب.

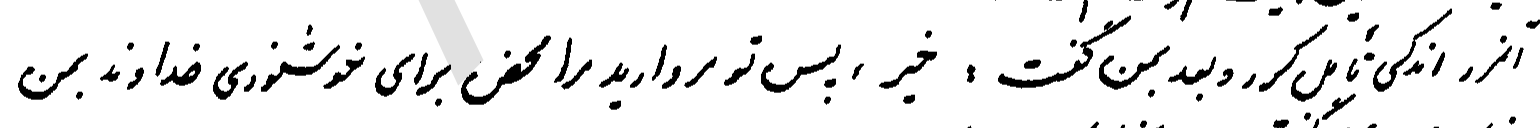

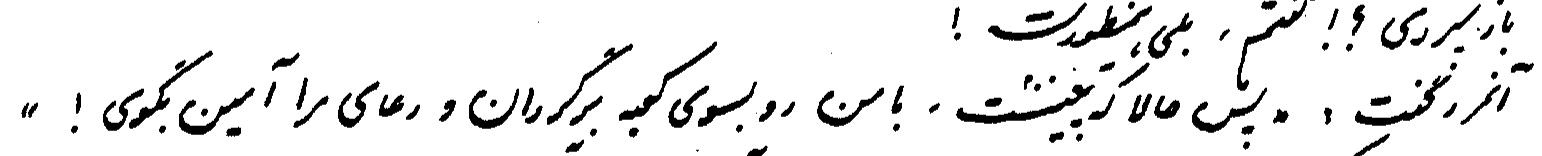

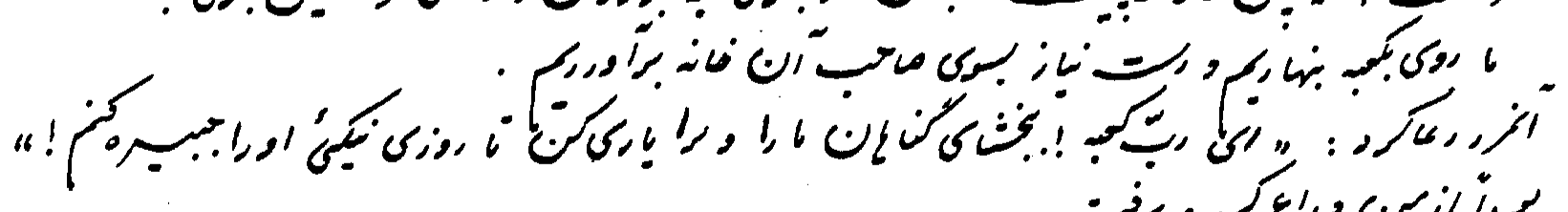
ب....

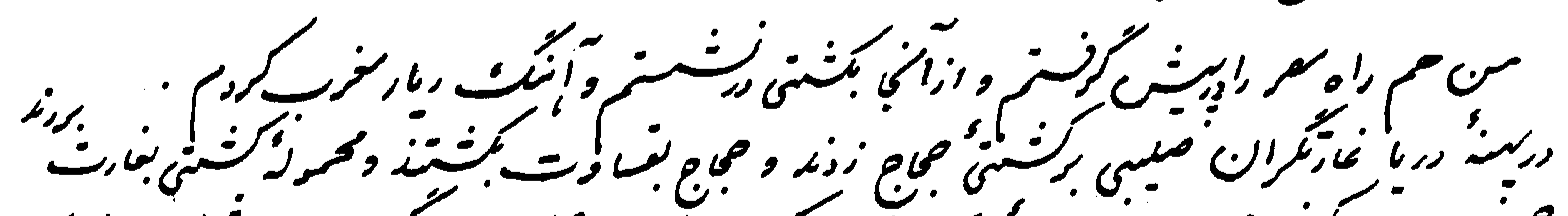

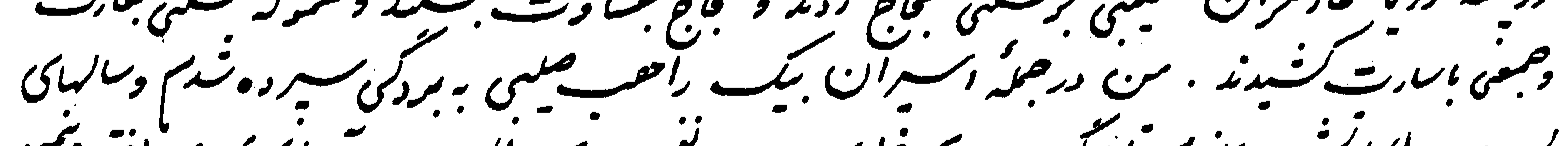

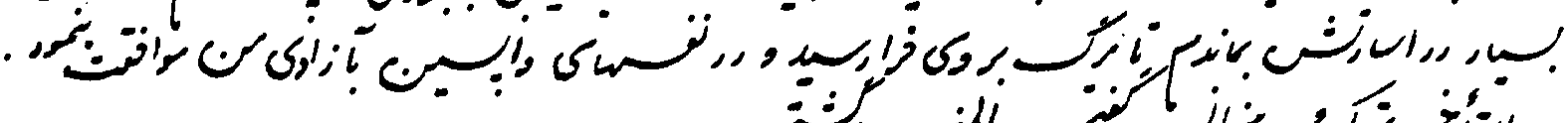

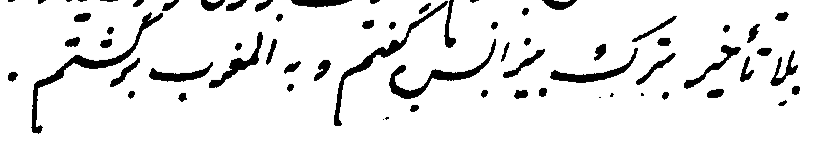




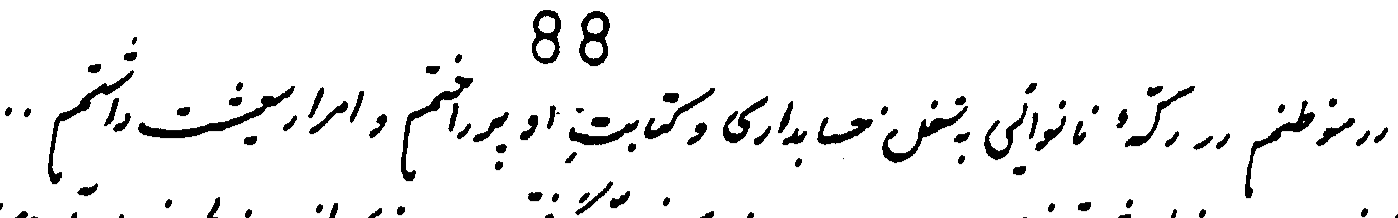

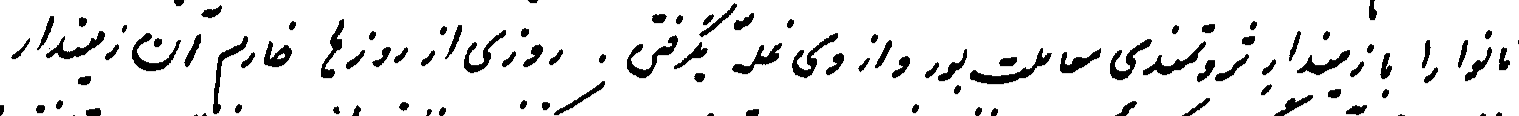

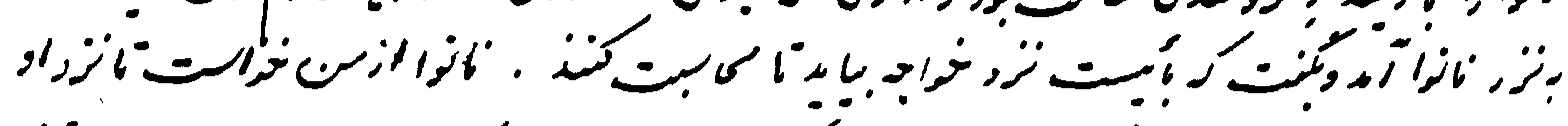

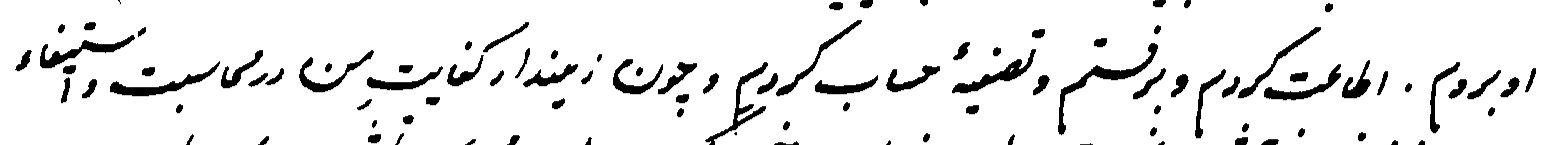

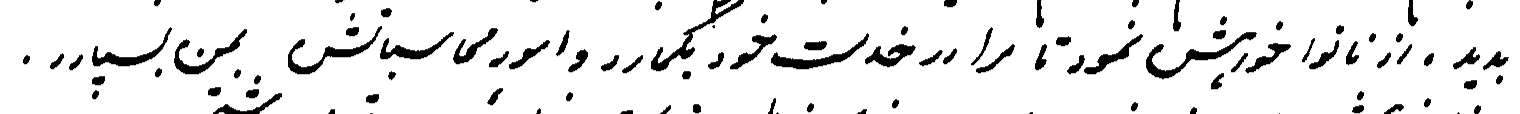

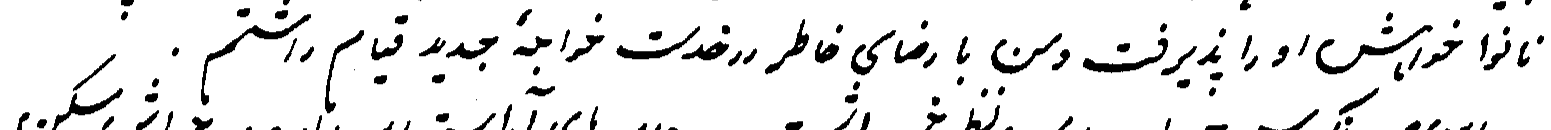

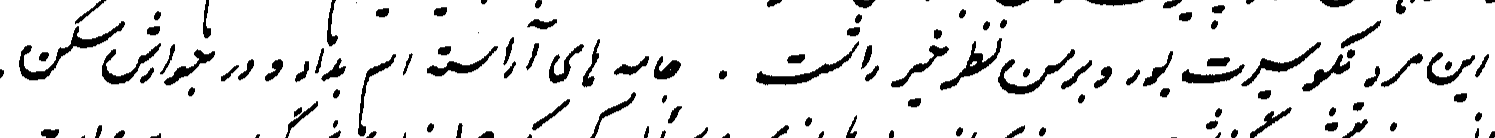

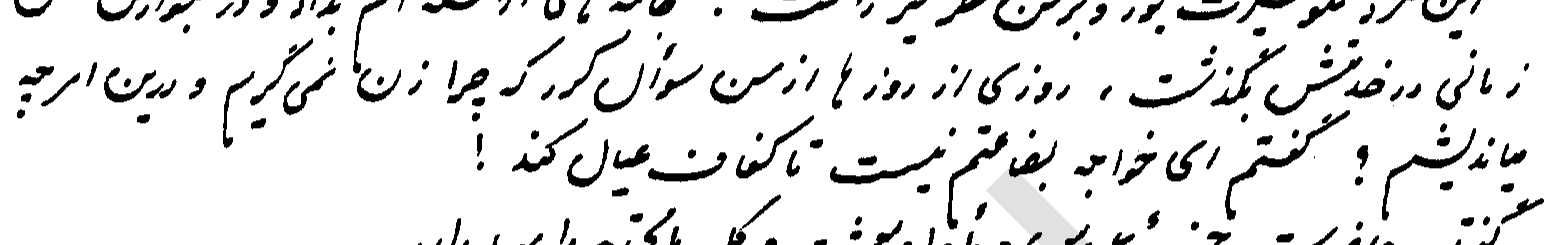
.

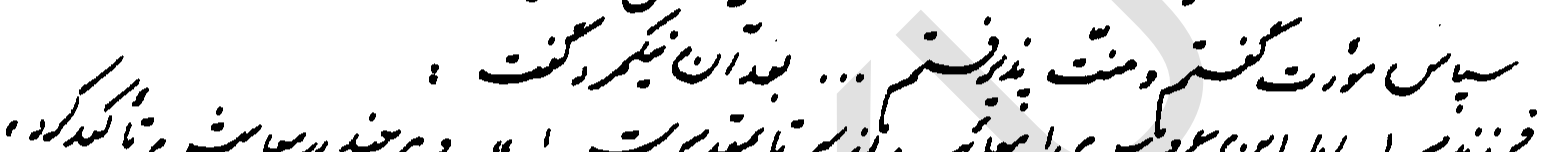

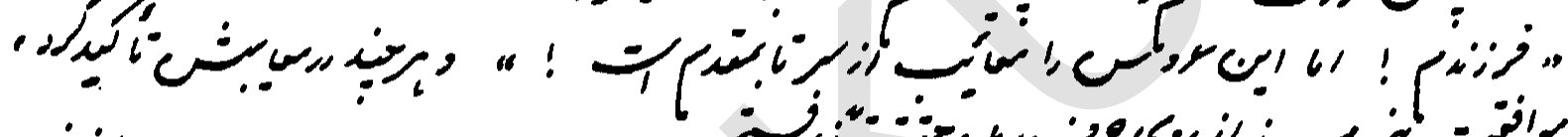

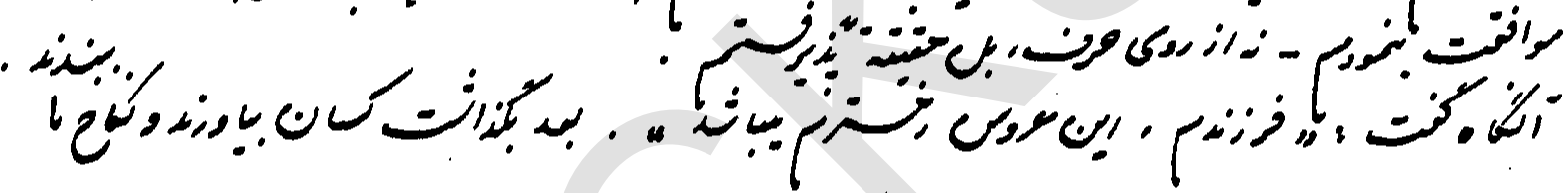

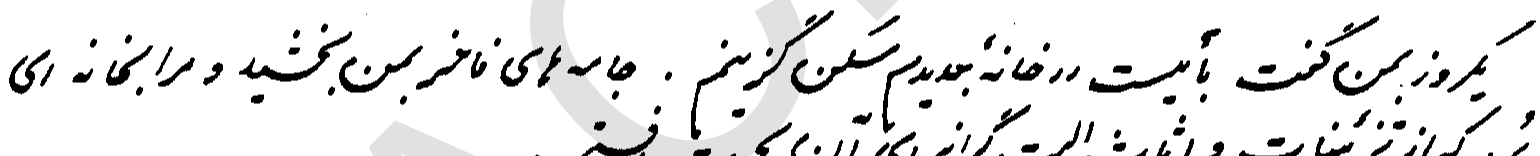

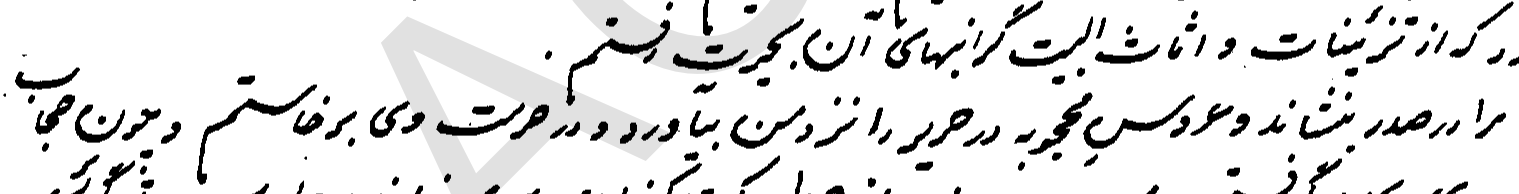

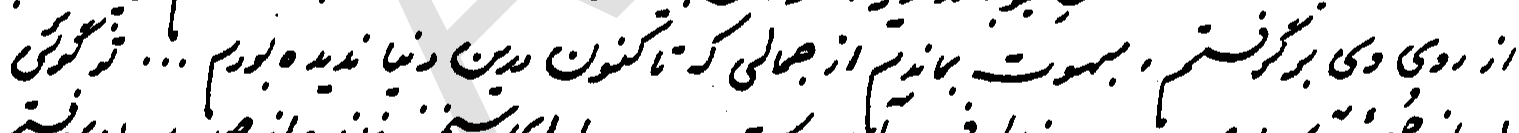

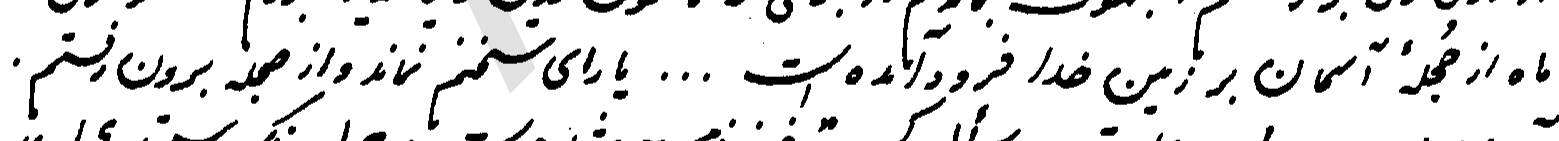

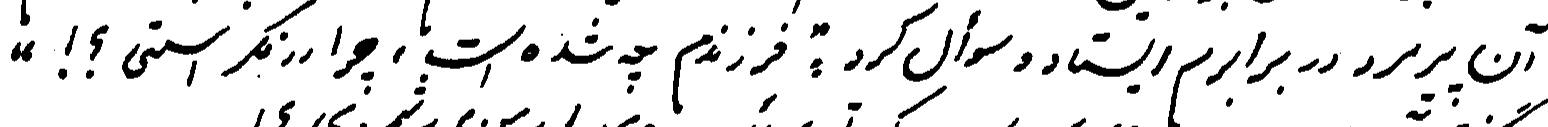
….

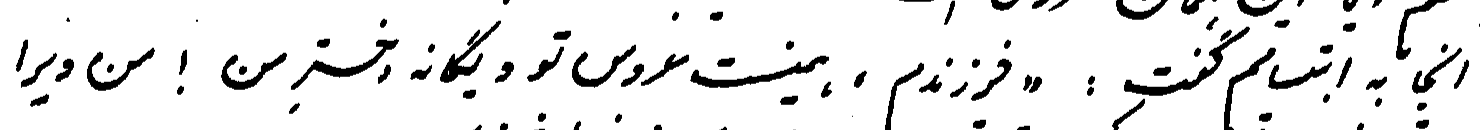

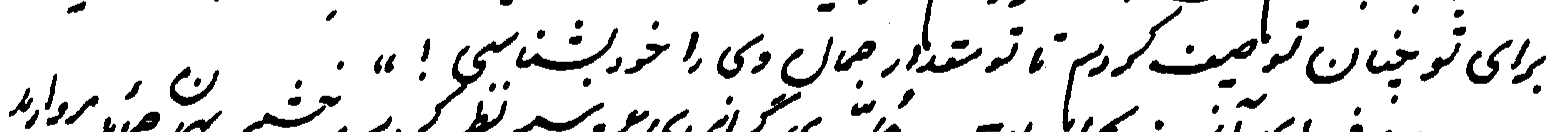

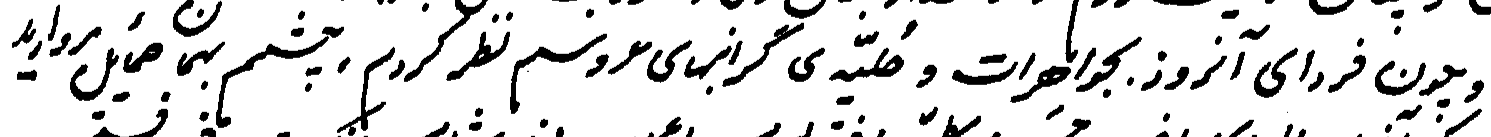

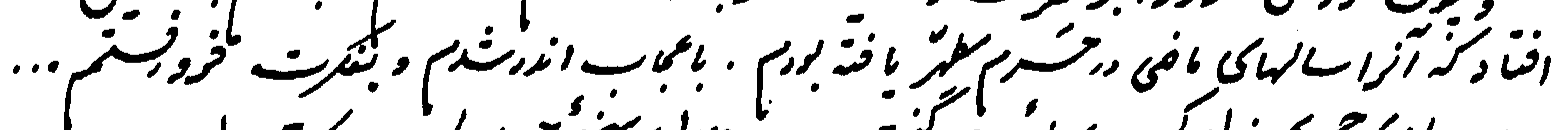

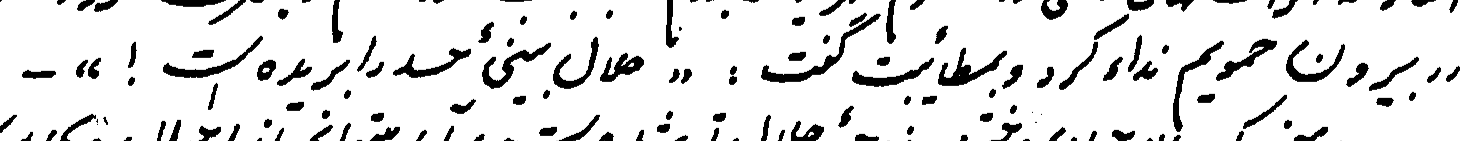

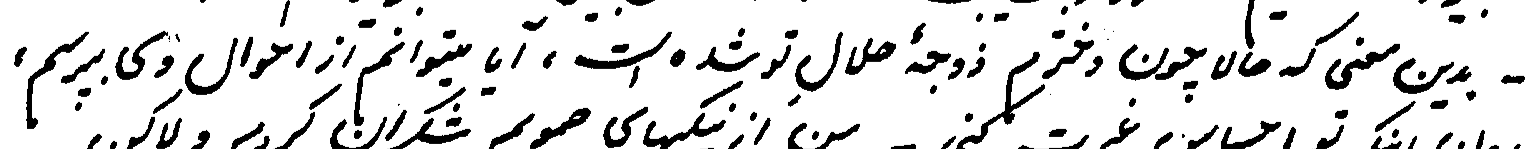

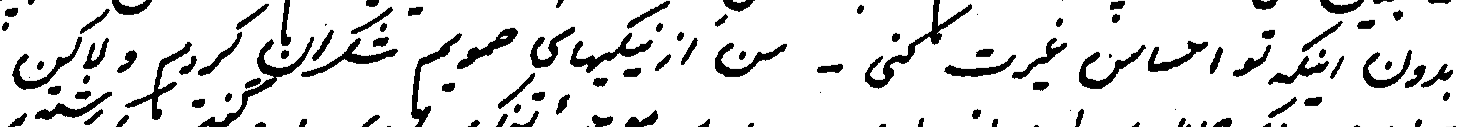

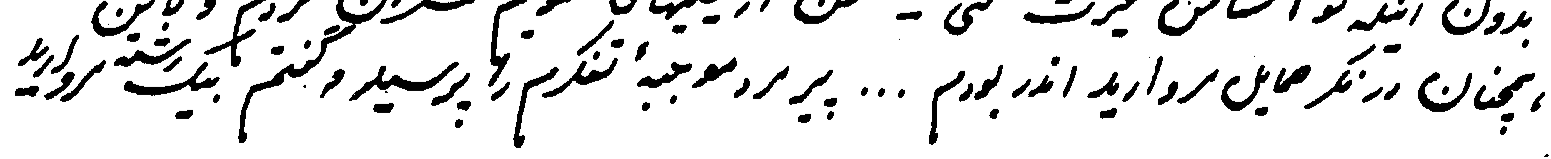


89

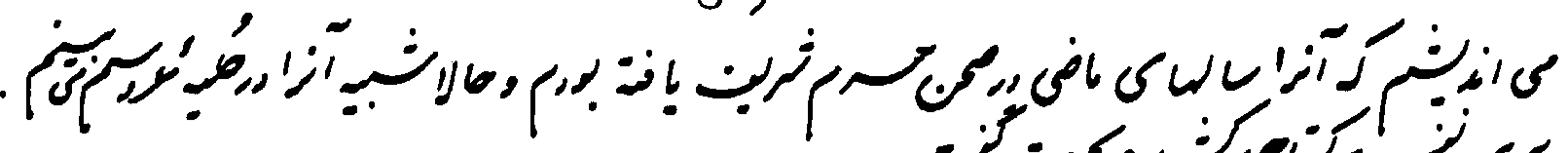

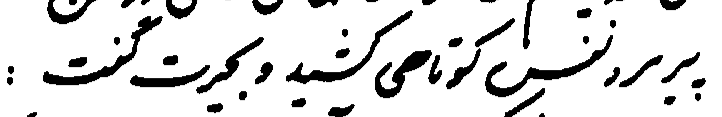

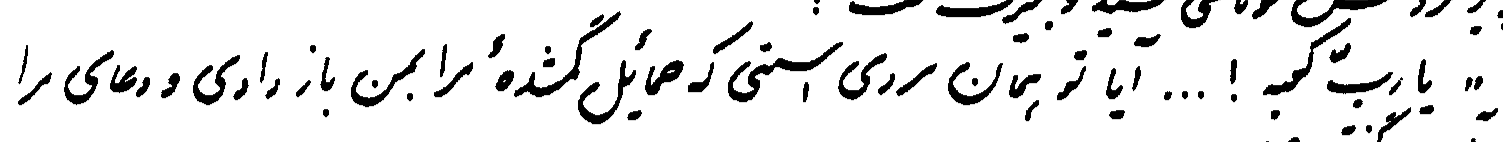

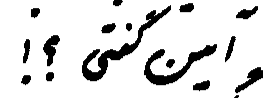

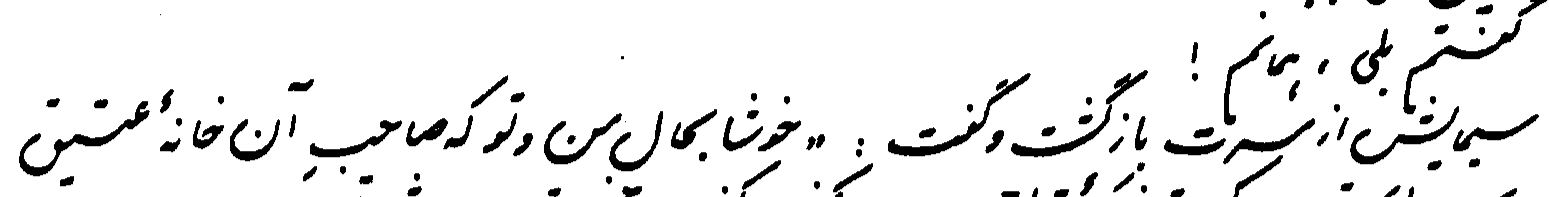
ت

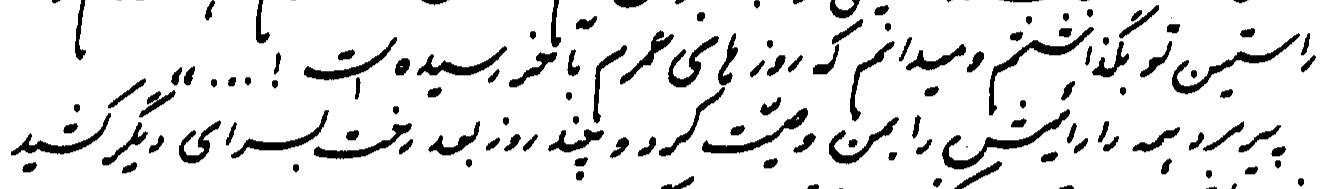

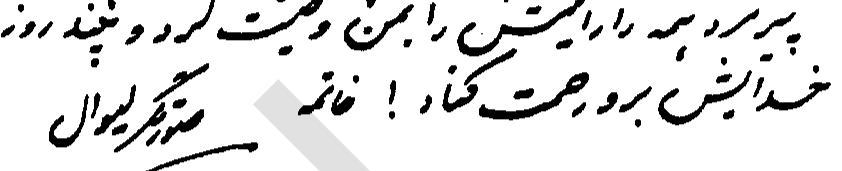
هوركي.

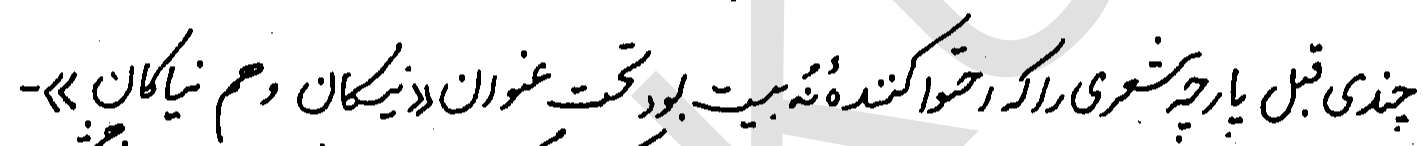

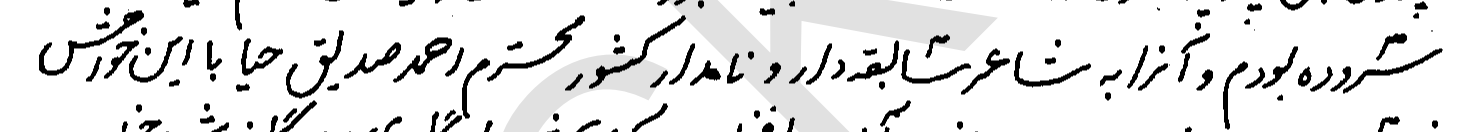
;

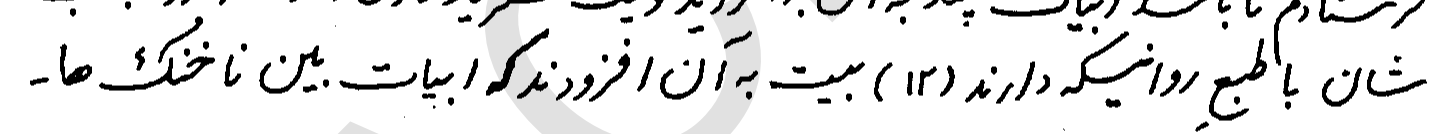
.

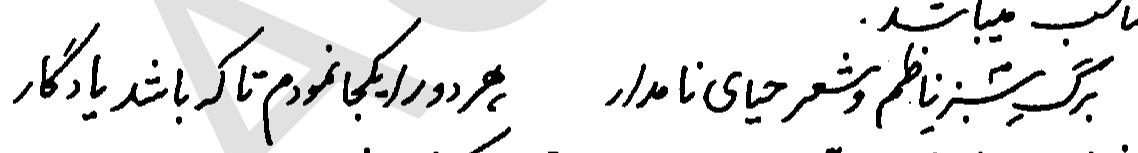

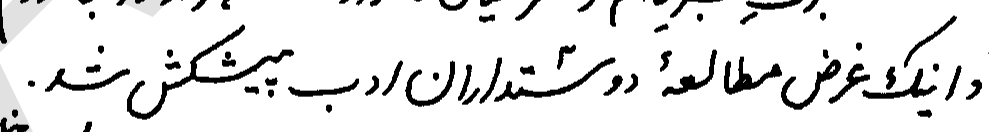

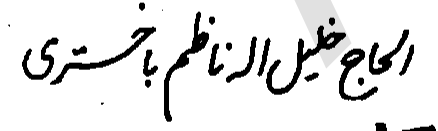
كبكان وصم نهاكاد

همروفيكر كاظم وحها.

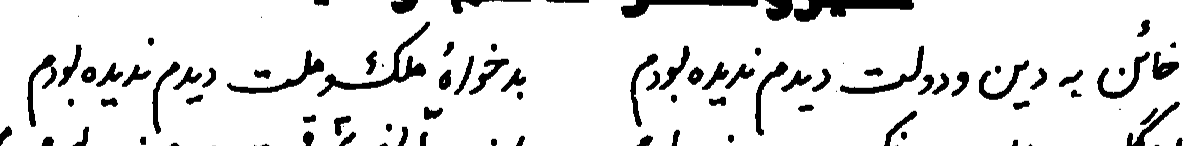

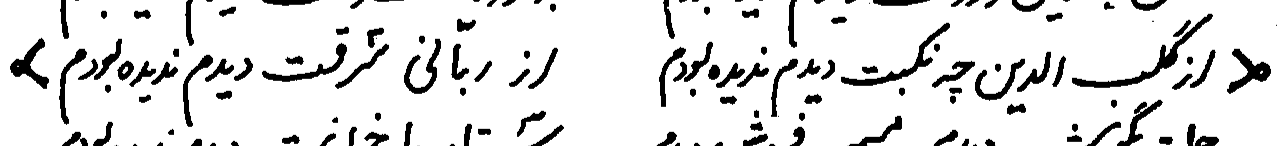

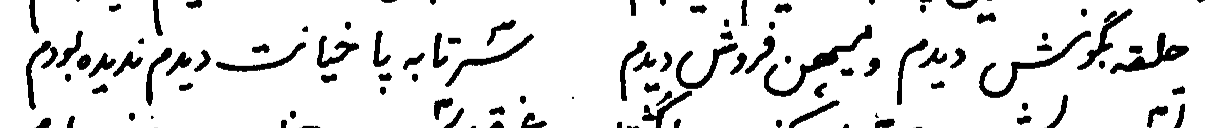

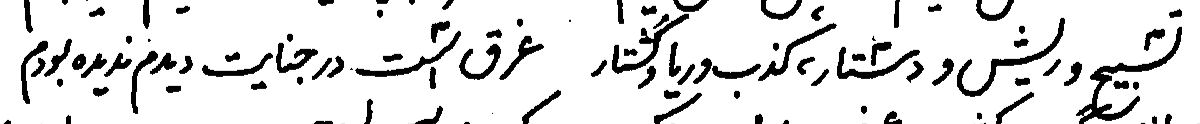

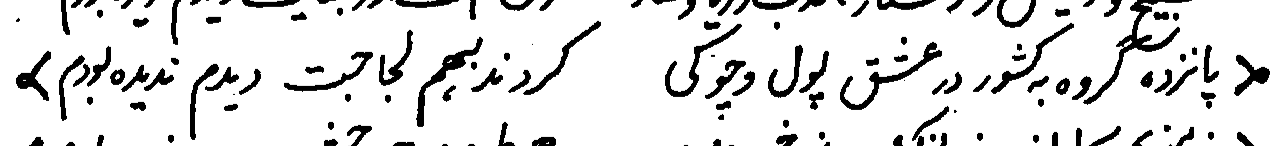
هק هان > 


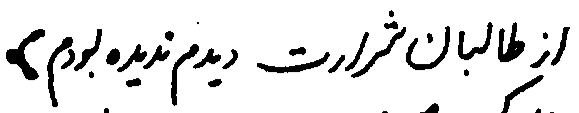

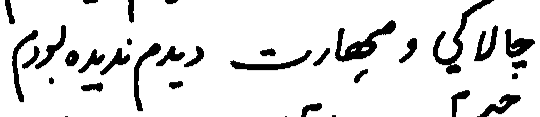

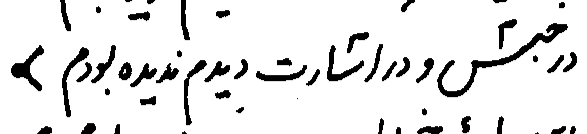
آن ت زتر

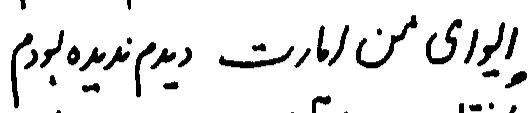

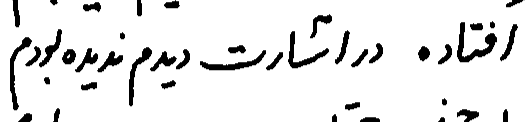

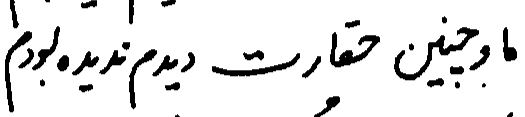

人) خر

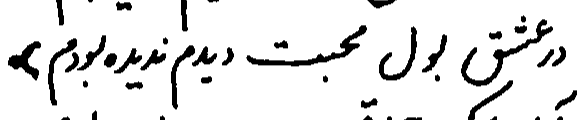

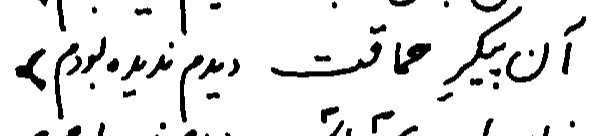
<

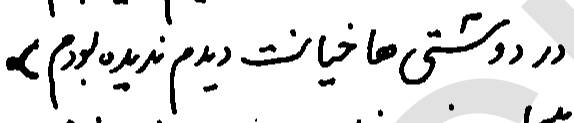

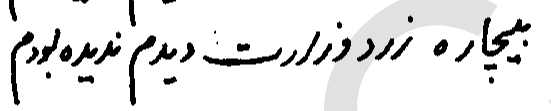

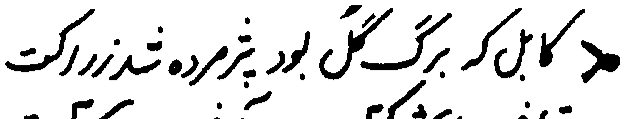

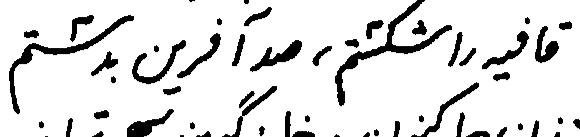
جا

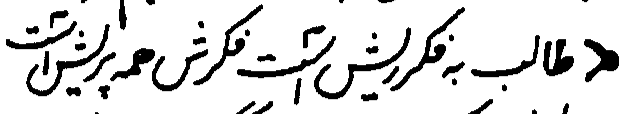

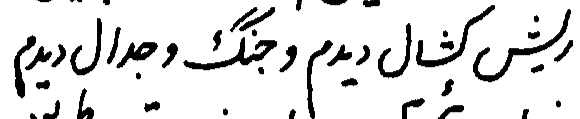

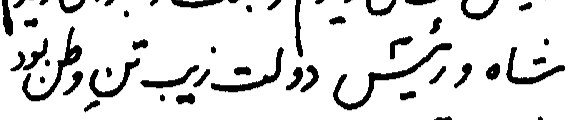

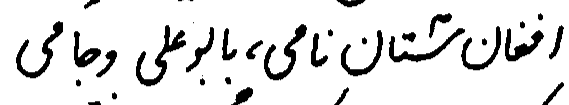
تيكان

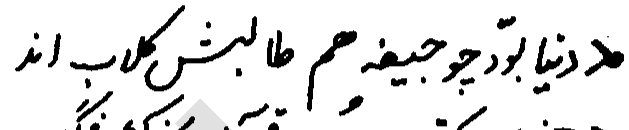

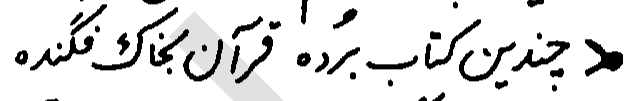

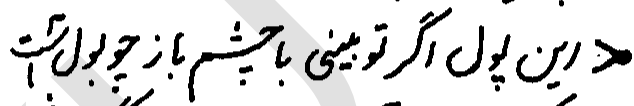

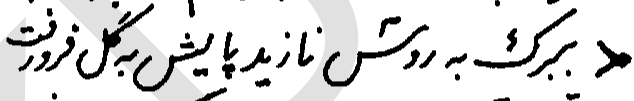
> > > >

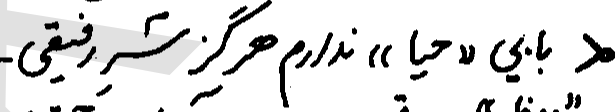

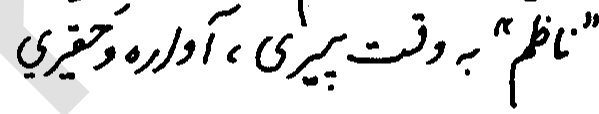

\section{جمار و دمقان هلمد الهغان}

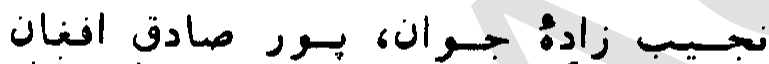

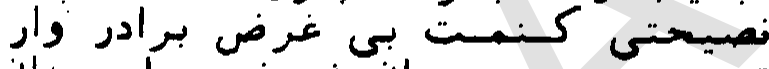

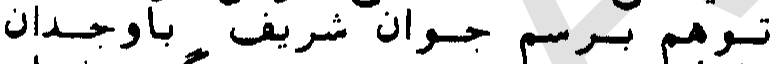

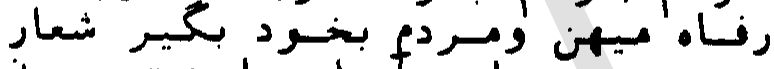

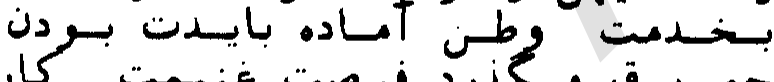

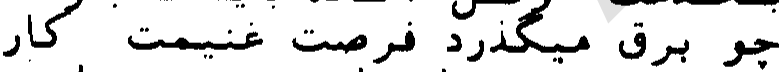

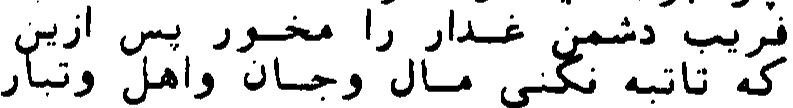

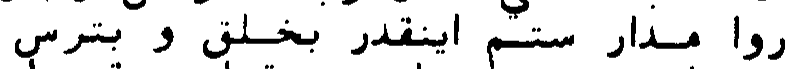

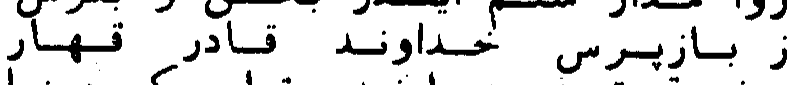

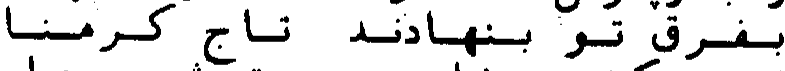

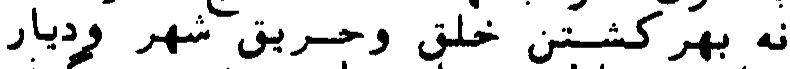

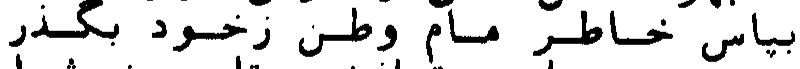

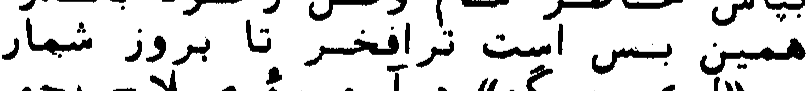

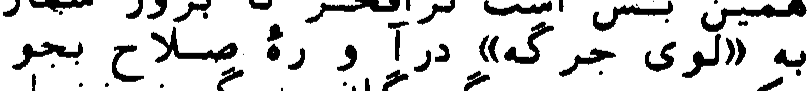

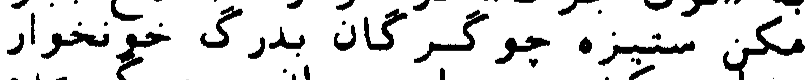

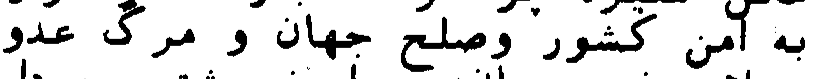

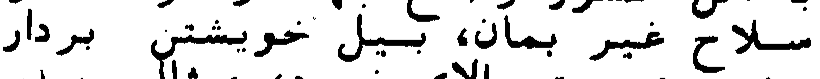

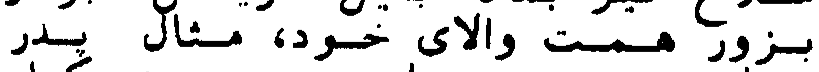

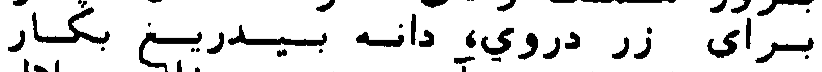

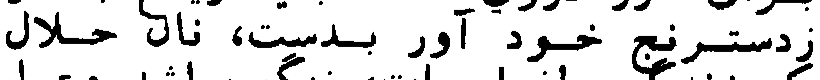

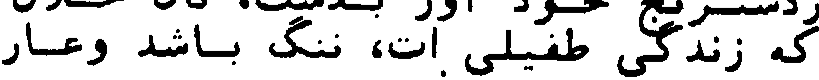

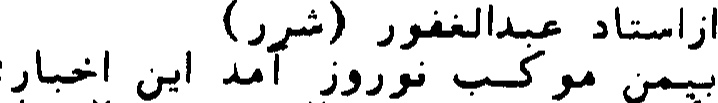

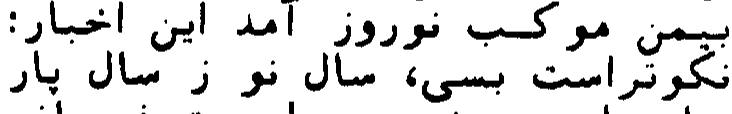

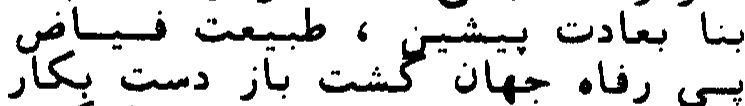

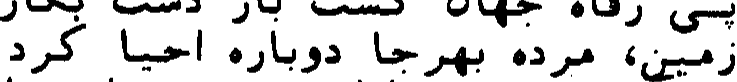

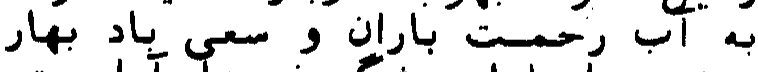

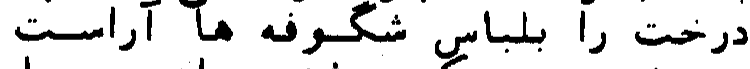

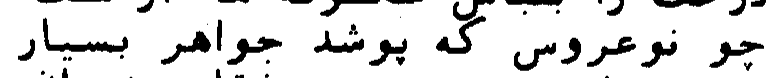

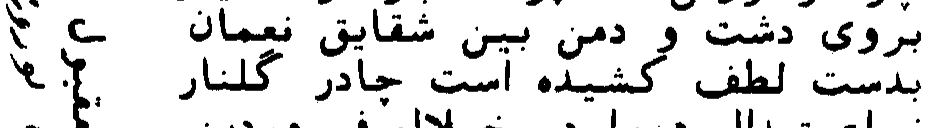

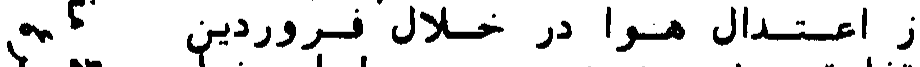

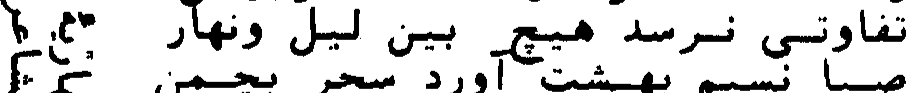

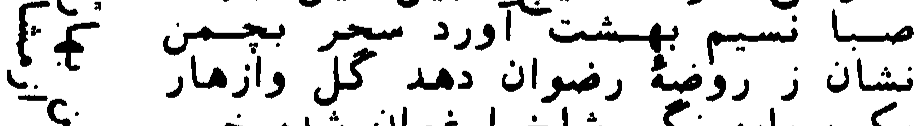

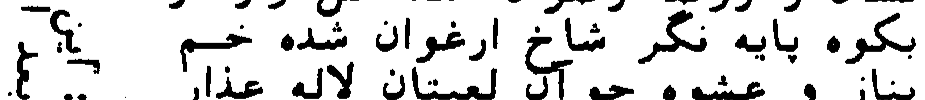

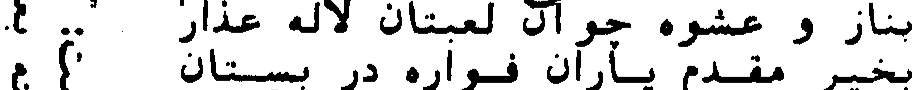

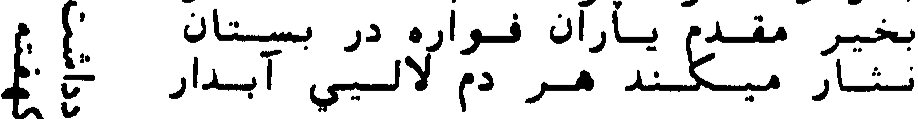
$\hbar$

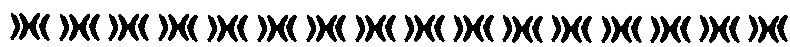




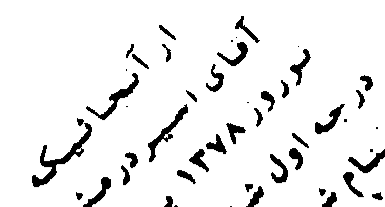

(4)

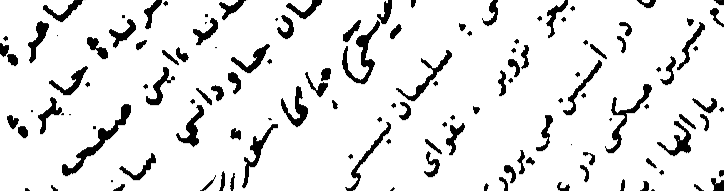

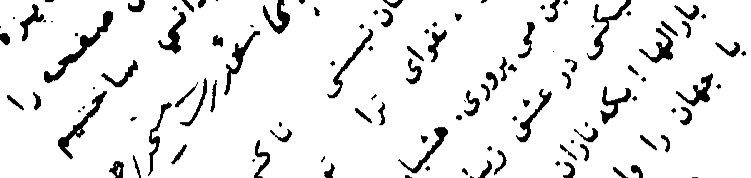

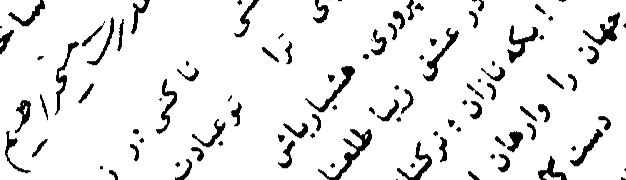

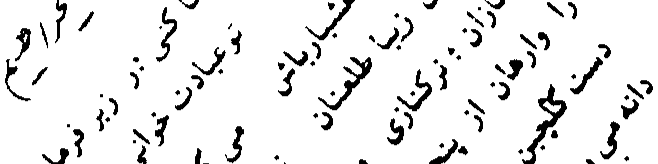

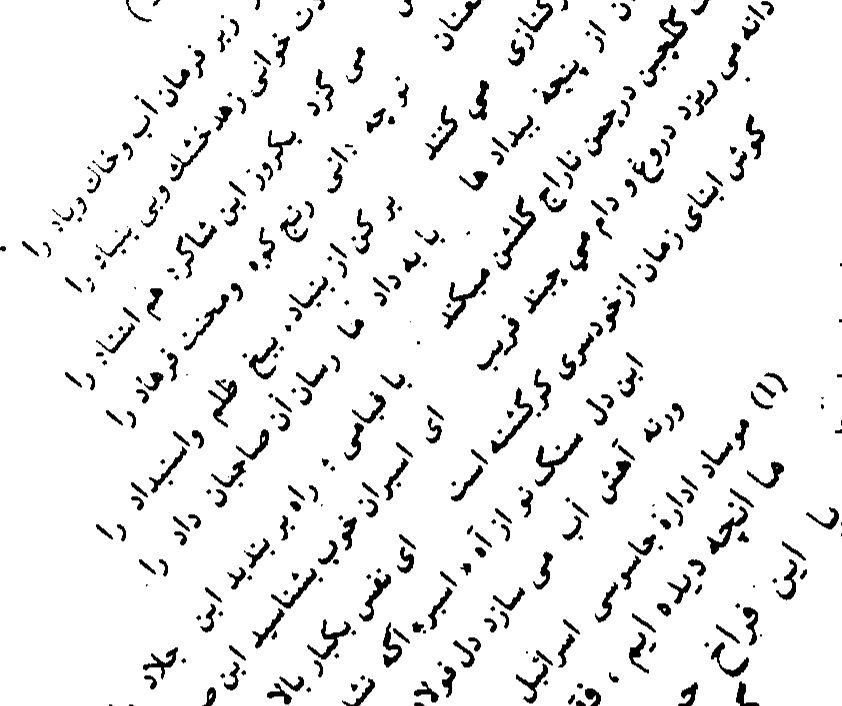

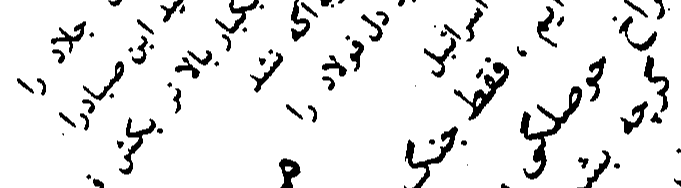

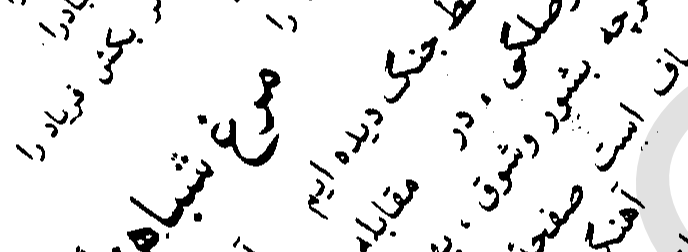

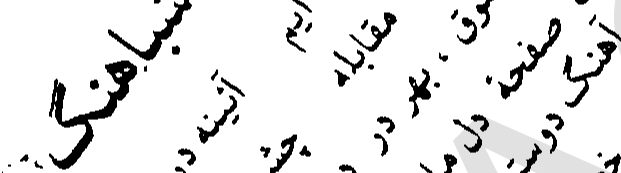

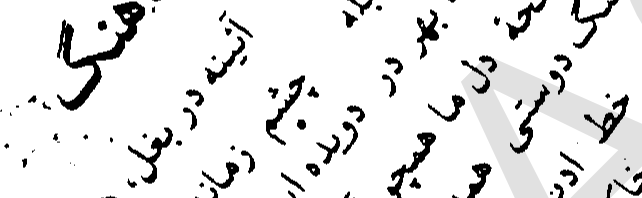

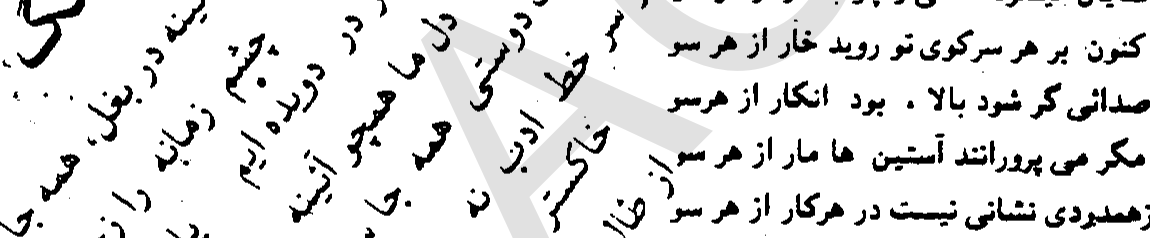
हो

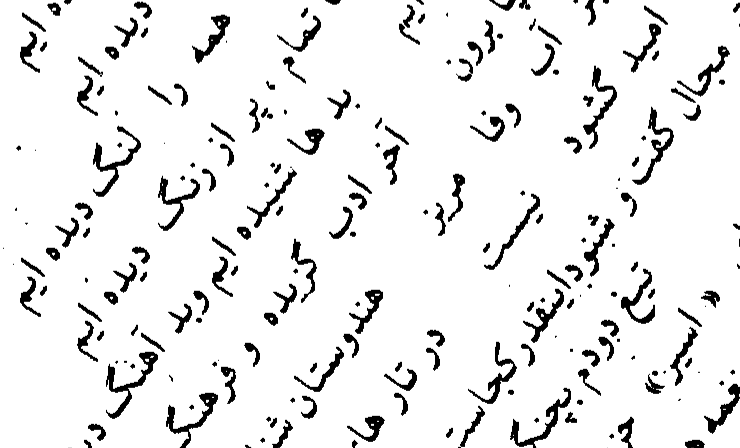
sen 2. हों है?
91 /ly

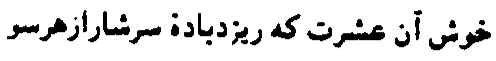

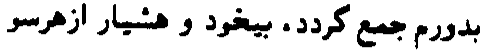

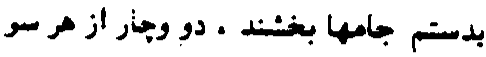

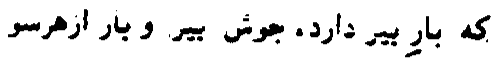

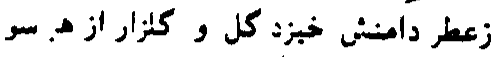

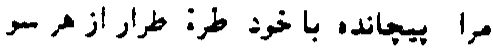

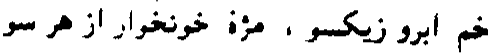

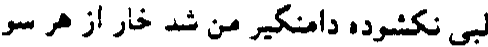

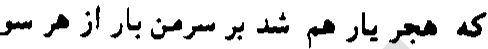

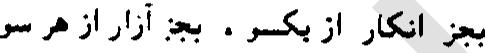

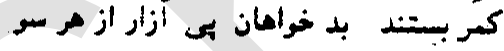

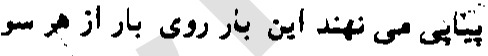

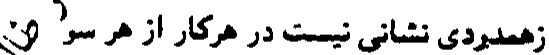

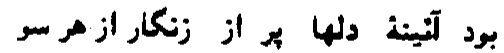

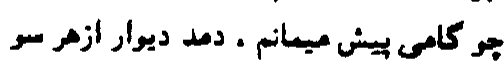

3 y
از جه در آتش كنيدى بصره وبغداد رأخُخ.

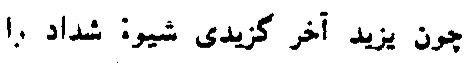

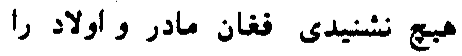

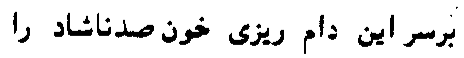

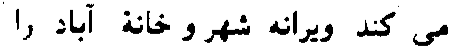

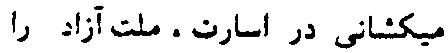
مكر CIA و كيد ونتنة ( موساد) رانان (1)

\section{o}

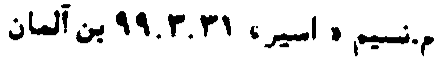

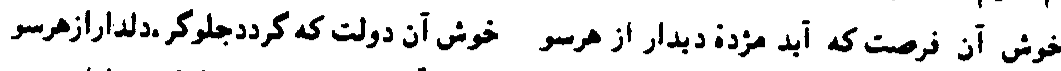

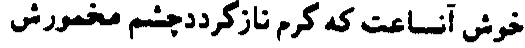

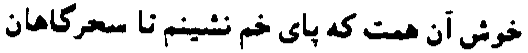

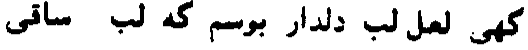

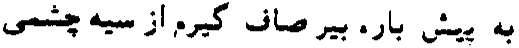

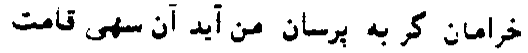

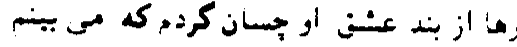

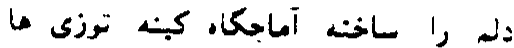

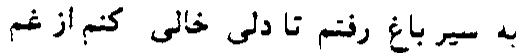
به نوشسم زندكانى بار سنكين أست. طالع بين

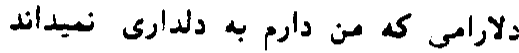

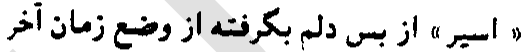

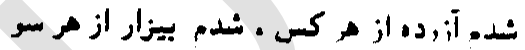

\section{مار آستصن}

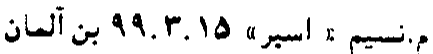

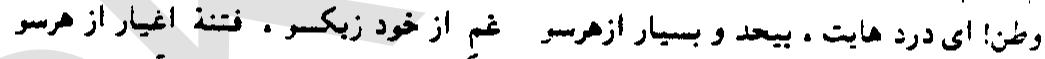

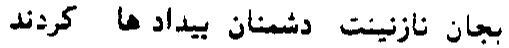

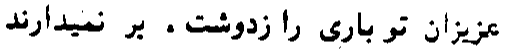

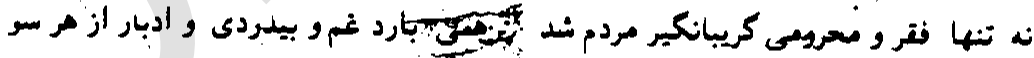

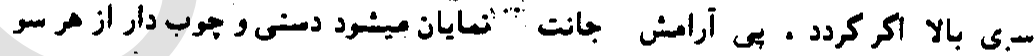

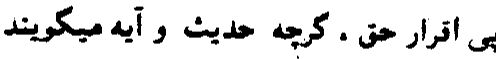

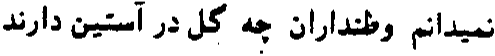

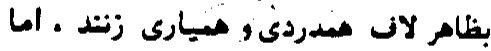

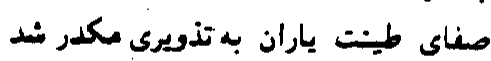

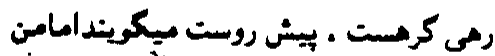

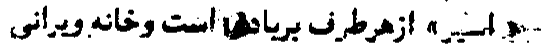

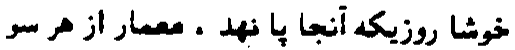

\section{تشُنه كام كربلا}

بمناسبت حملات مكرر هوائى بر عرات

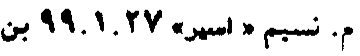

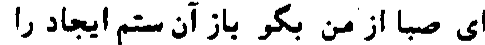

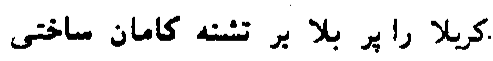

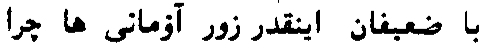

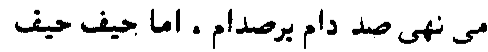
يو نفس سر كثت تا سر بطغيان مبكند

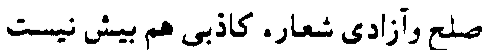

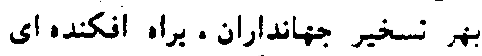




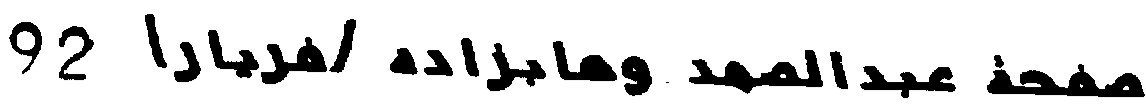

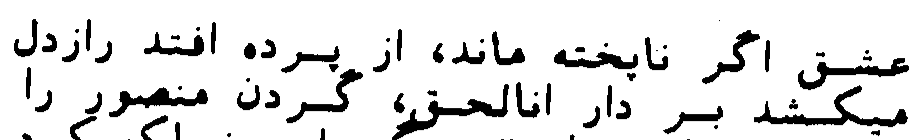

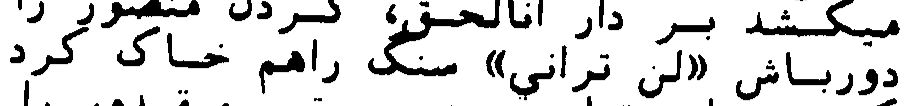

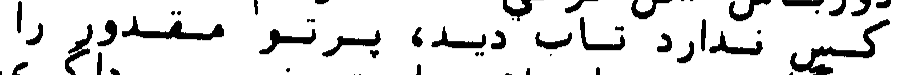

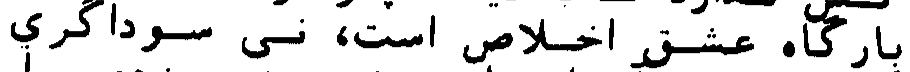

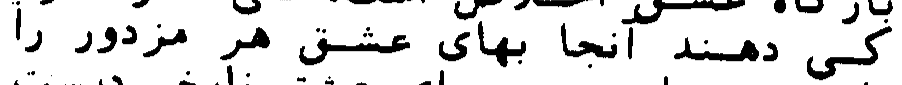

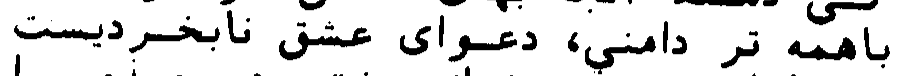

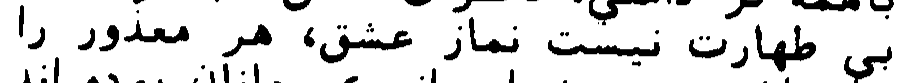

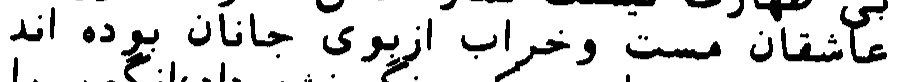

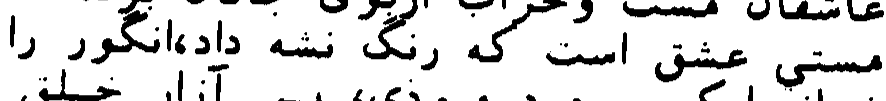

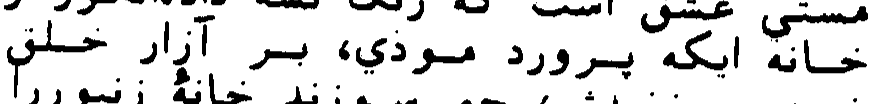

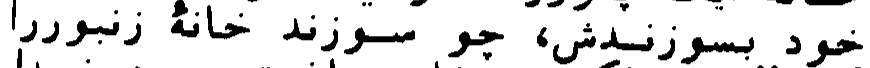

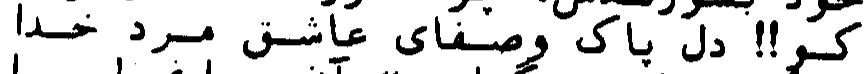

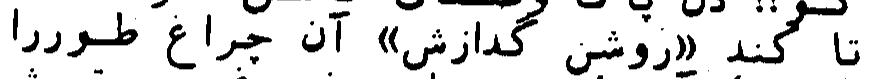

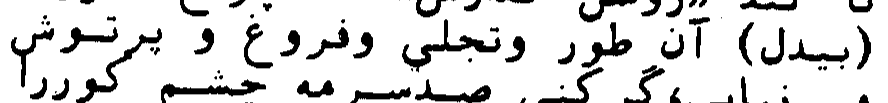

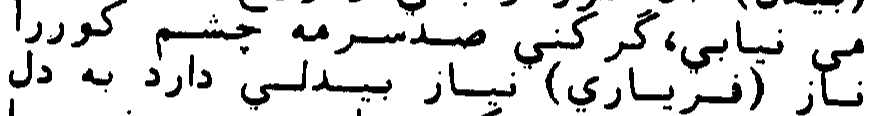

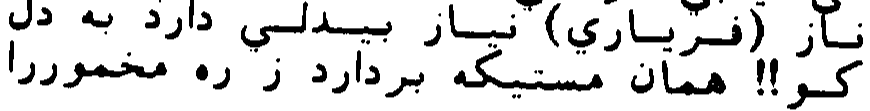

\section{شمارة مسلس}

\section{إمك هـ قدر}

ازآناي عبدالعمد ومابزاده (فريار)

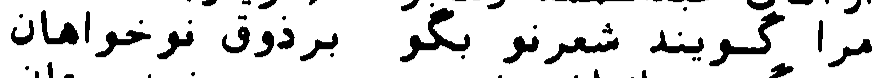

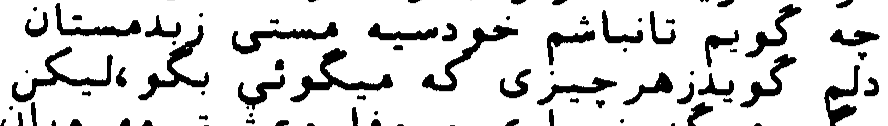

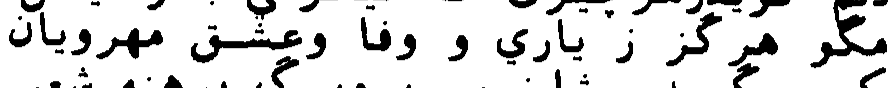

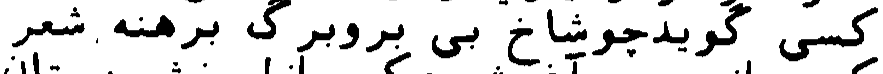

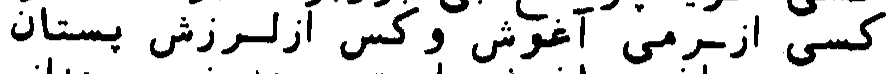

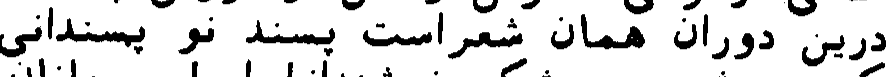

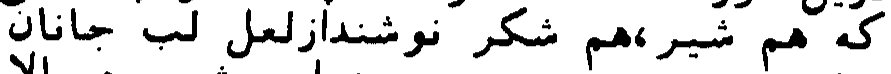

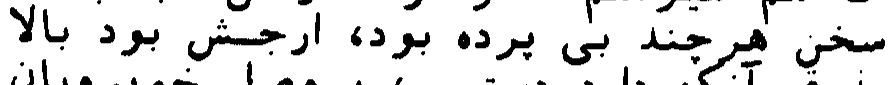

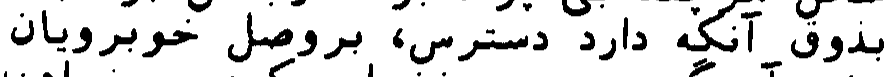

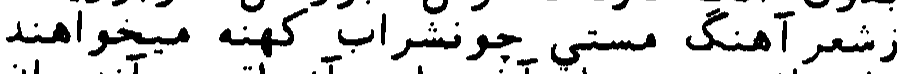

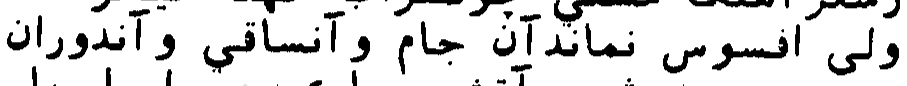

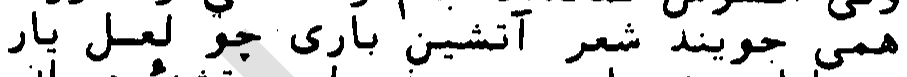

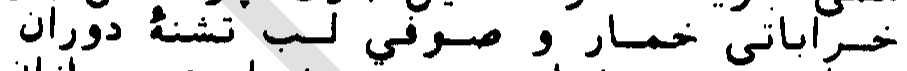

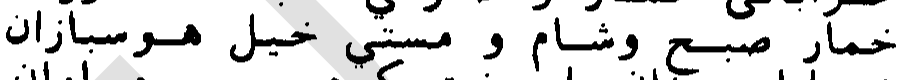

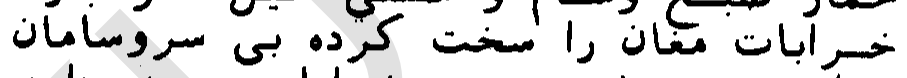

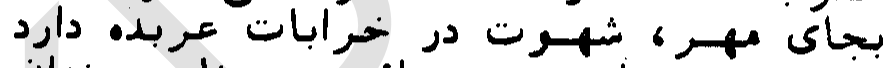

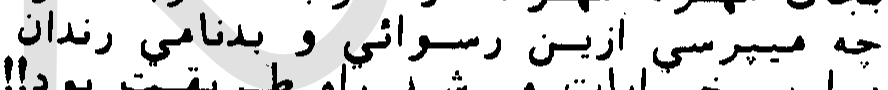

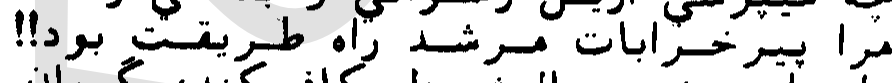

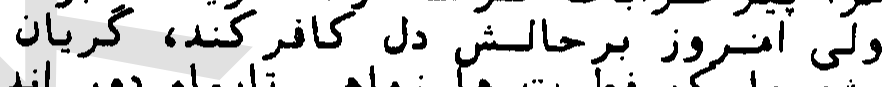

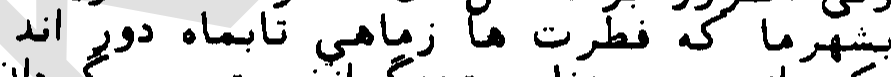

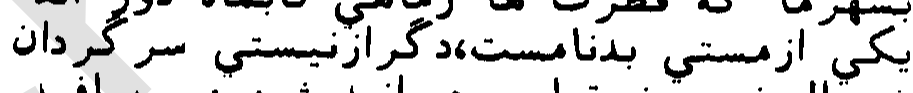

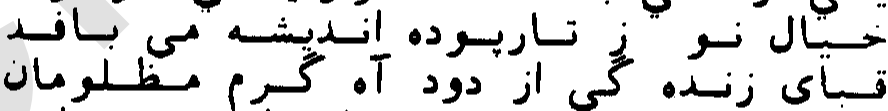

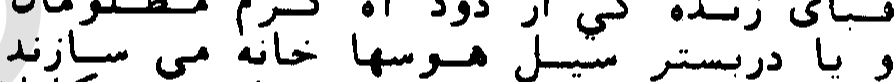

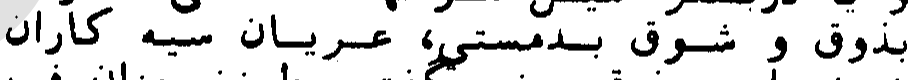

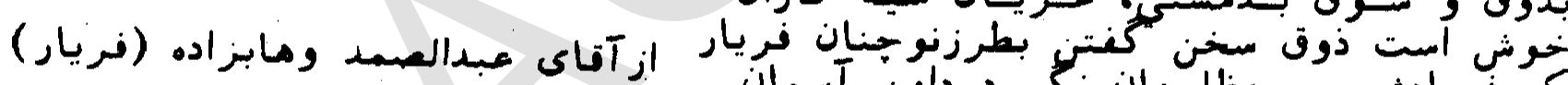

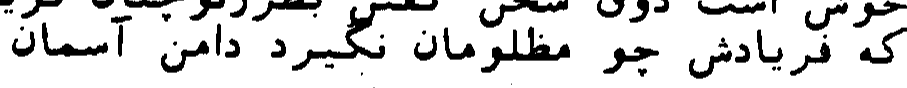

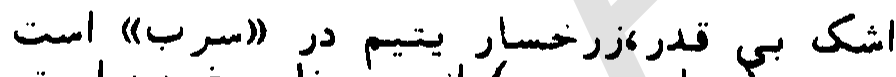

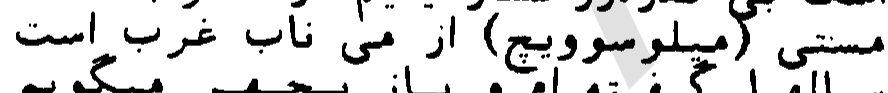

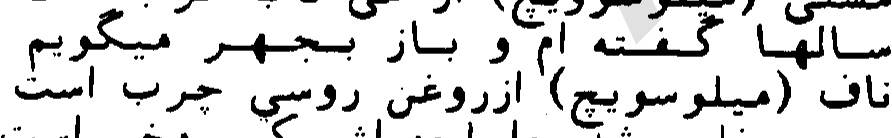

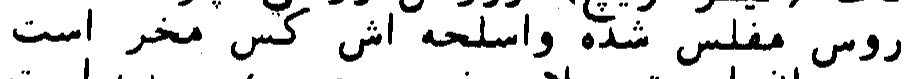

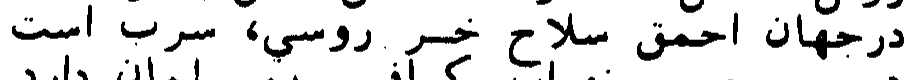

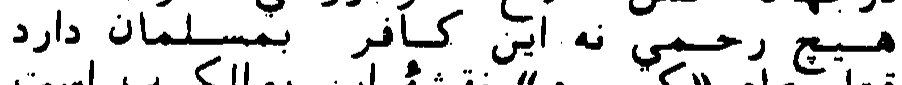

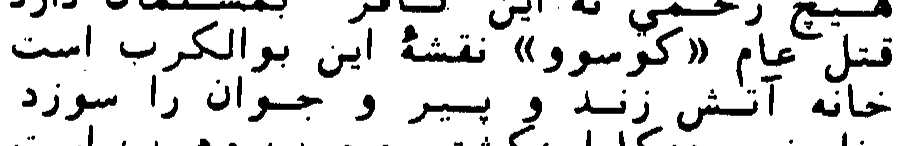

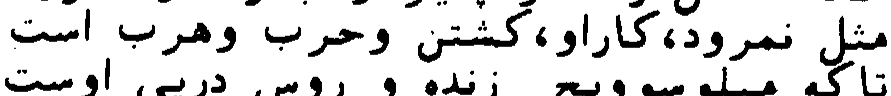

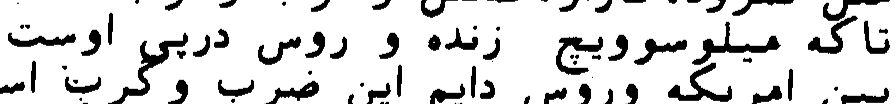

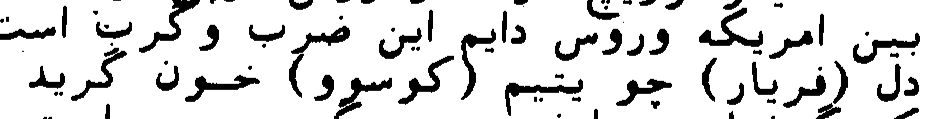

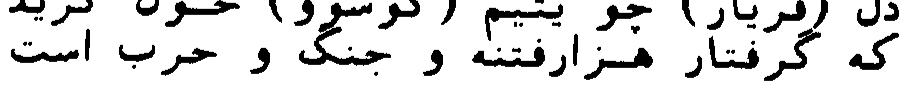

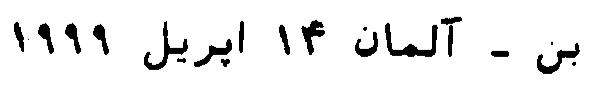

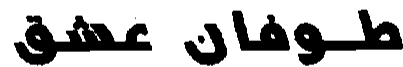

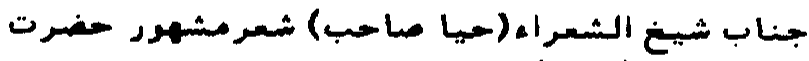

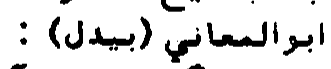

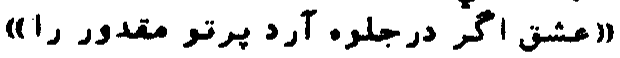

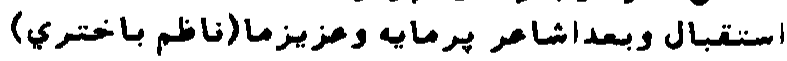

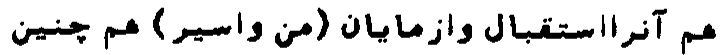

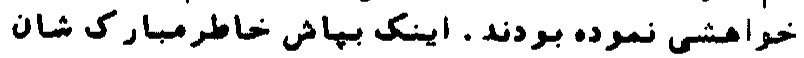

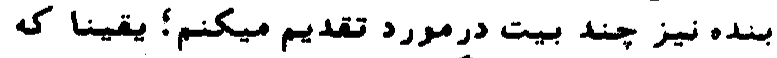

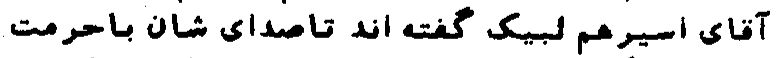

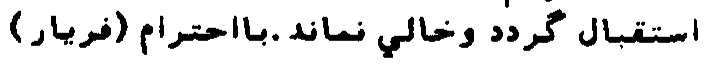

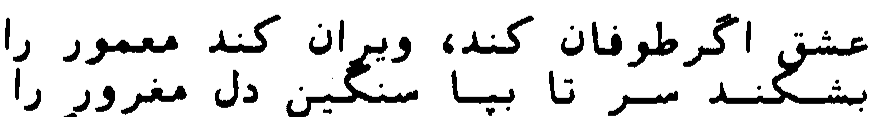

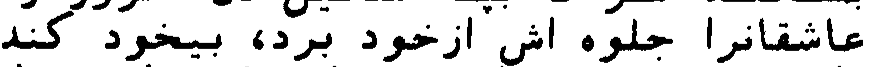

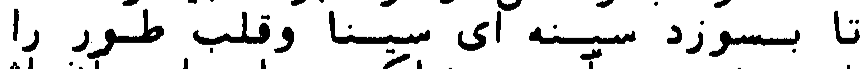

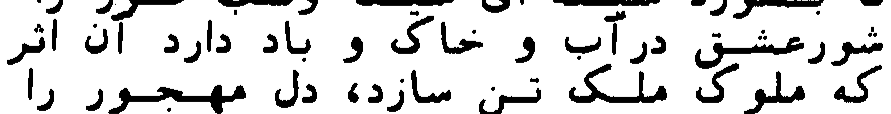


93

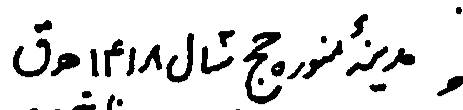

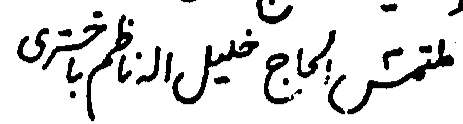

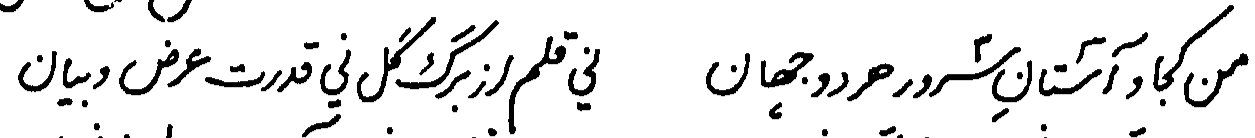

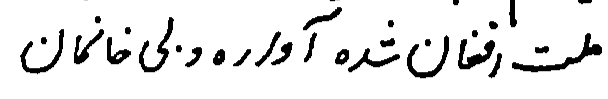

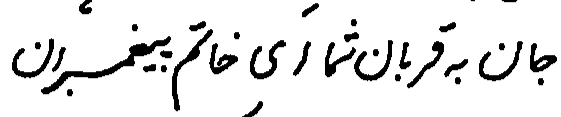

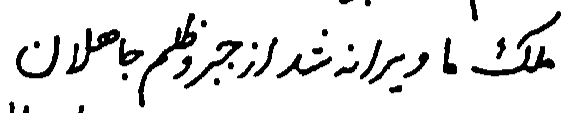

D
2

رزتفاك- ردرزن

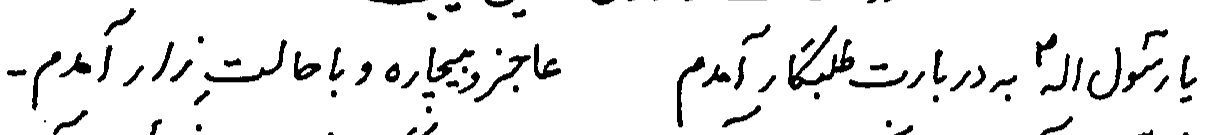

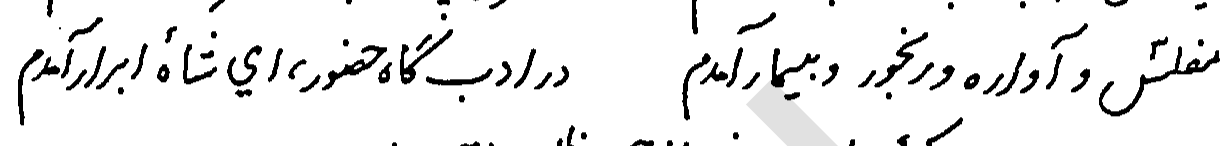

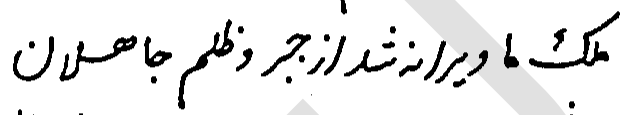

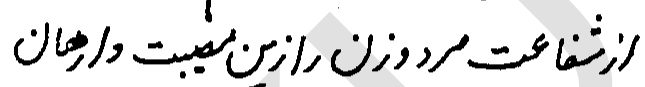

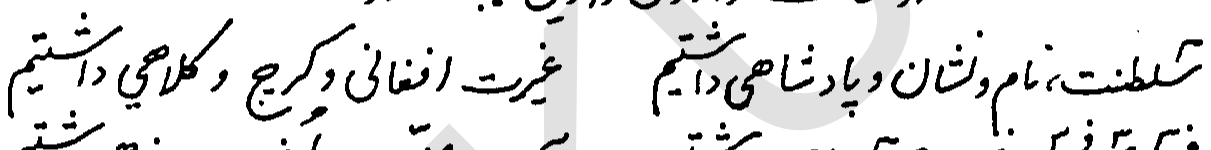

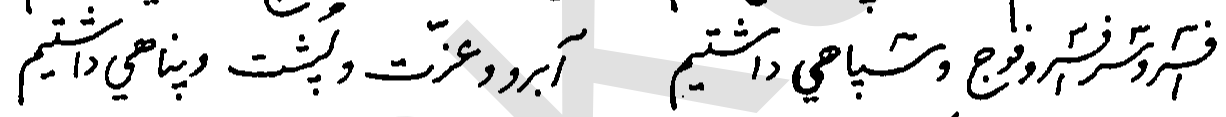

مكك إبران:

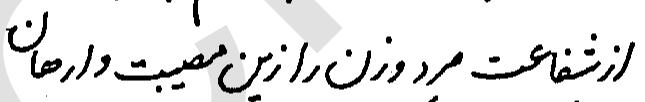

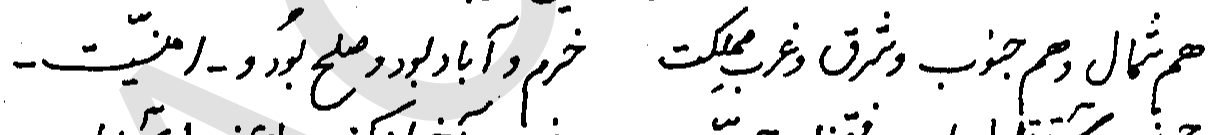

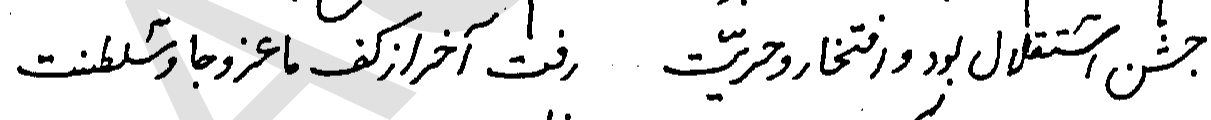

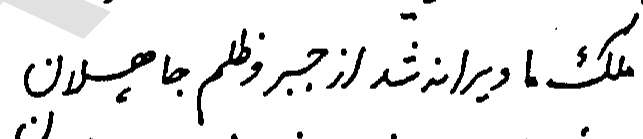

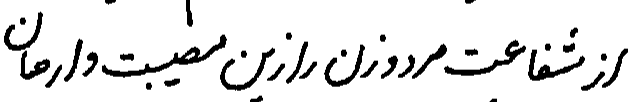

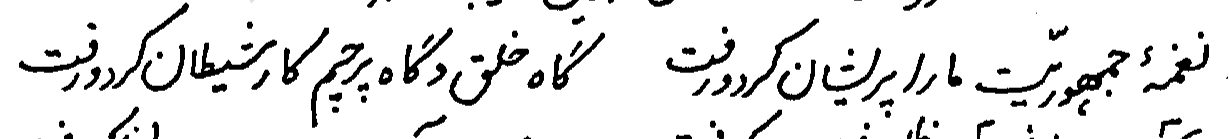

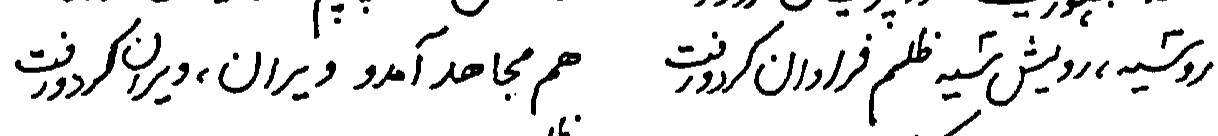

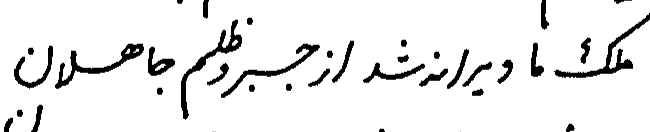

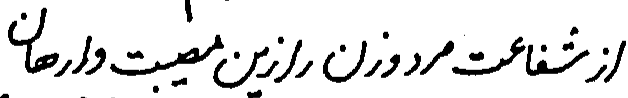

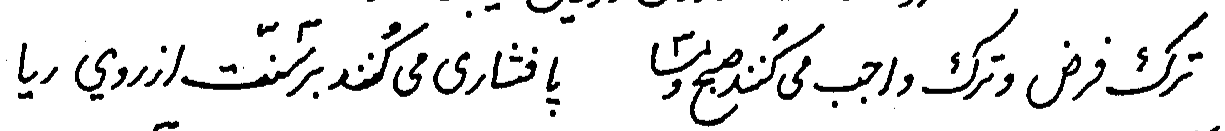

1 1

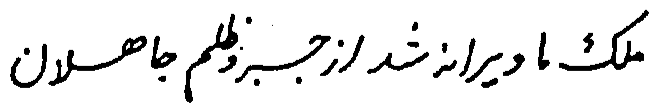

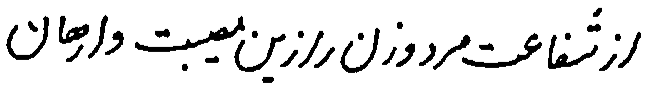


94

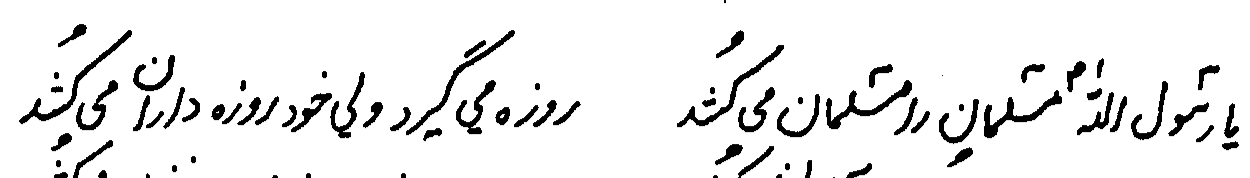

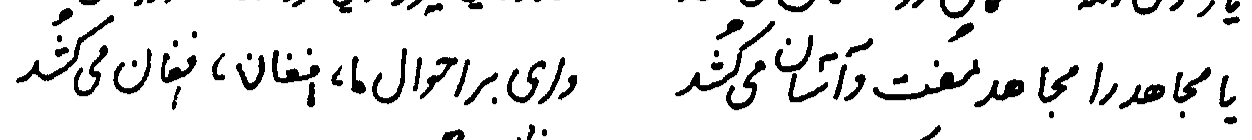

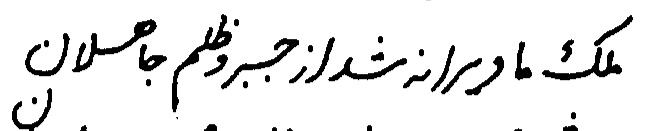

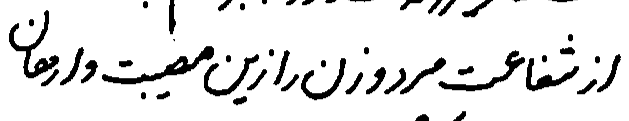

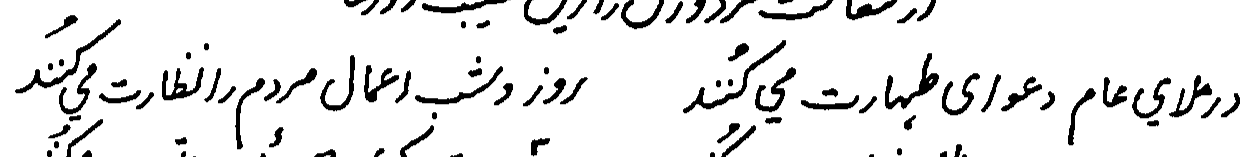

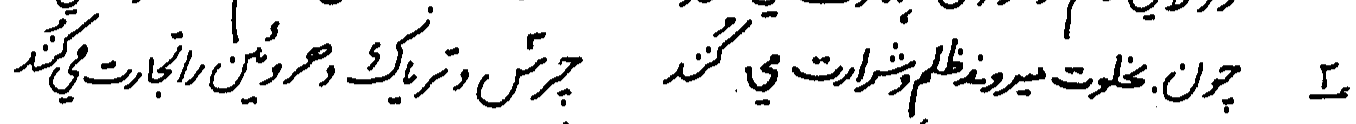

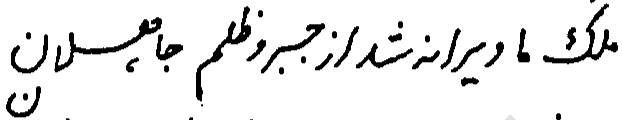
ال

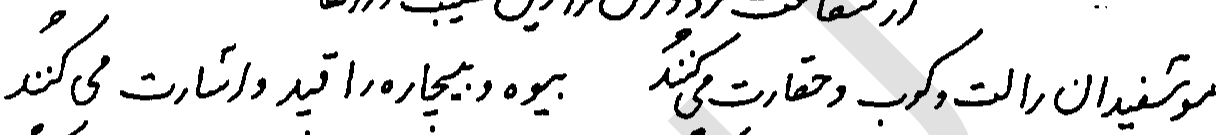

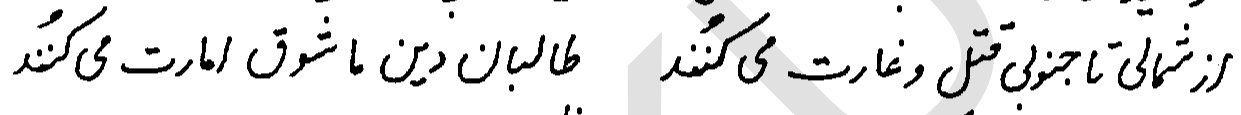

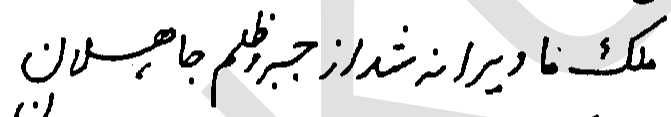

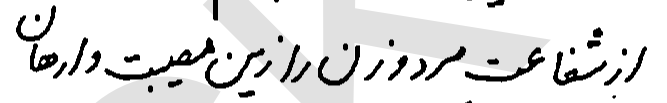

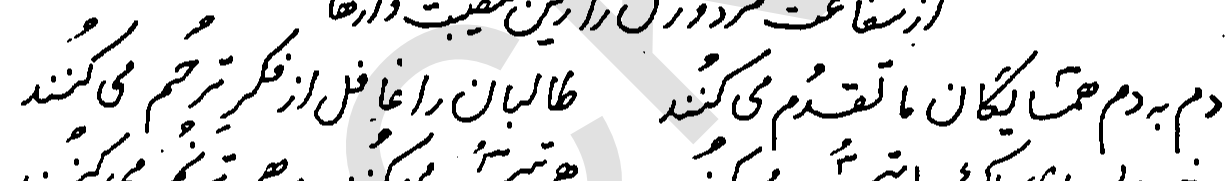

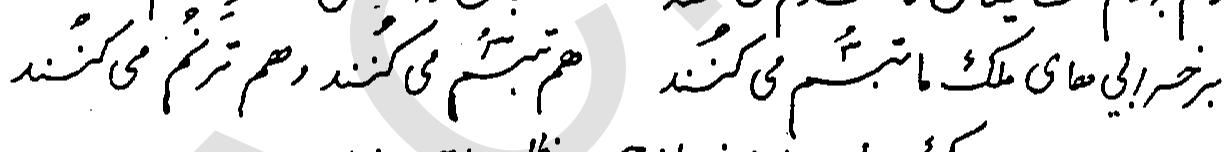

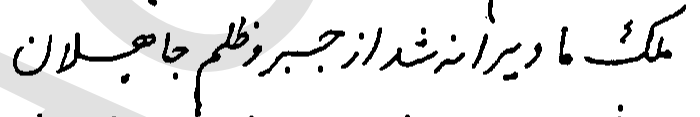

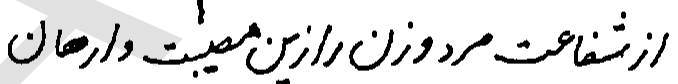

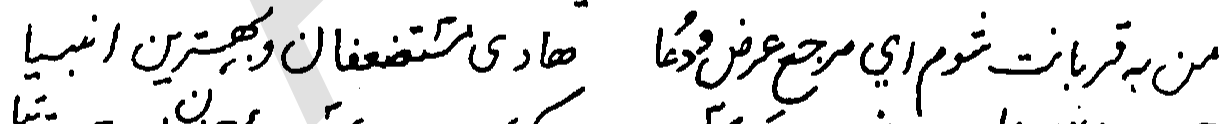

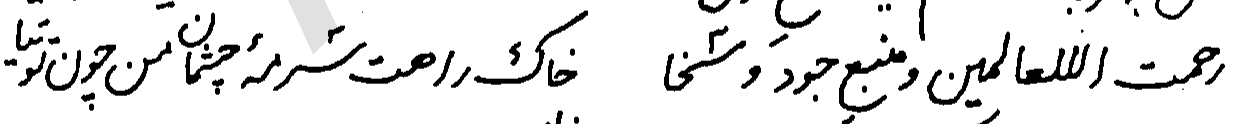

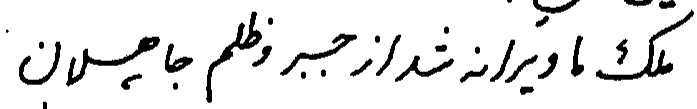

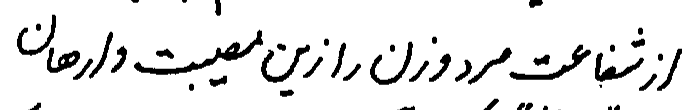

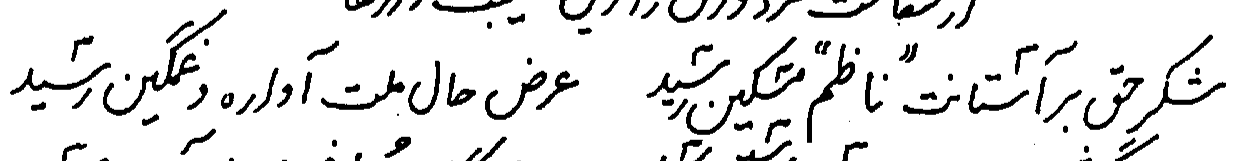

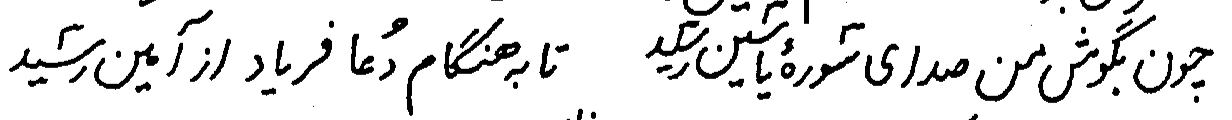

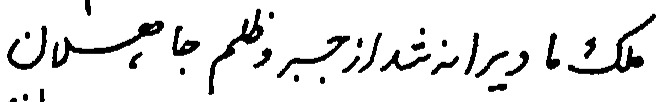

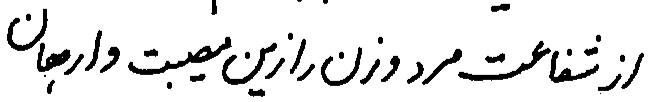

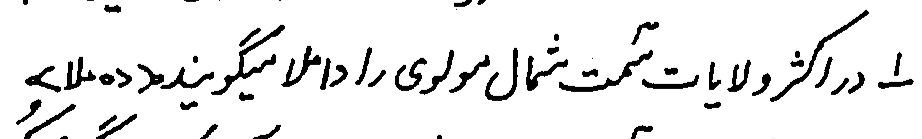

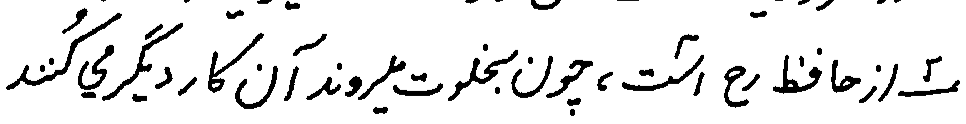




\section{5

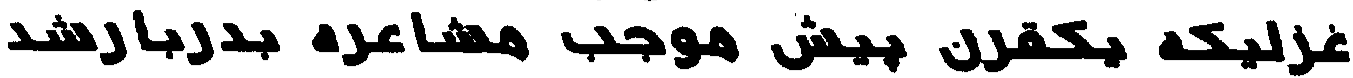

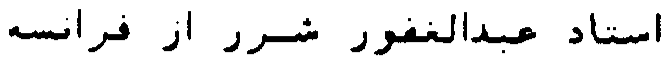

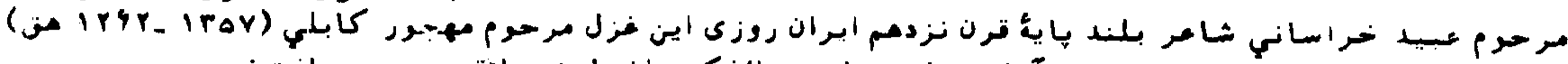

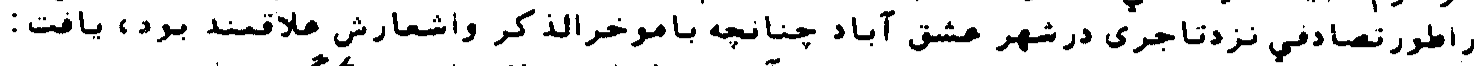

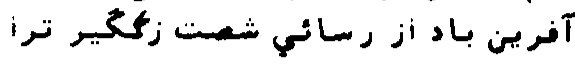

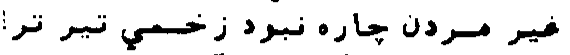

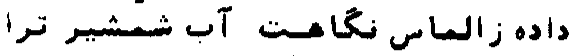

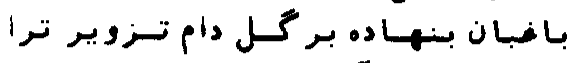

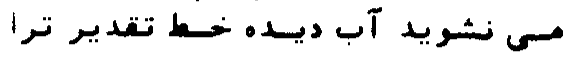

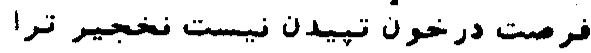

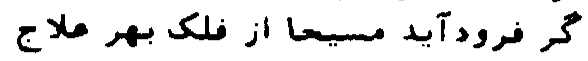

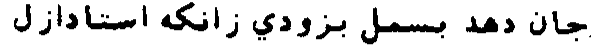

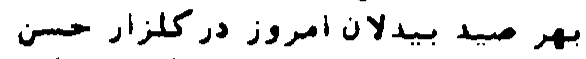

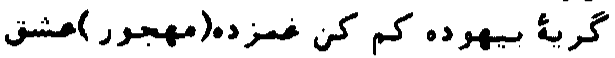

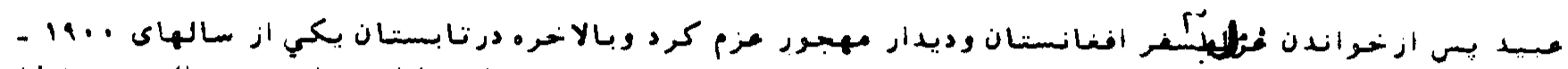

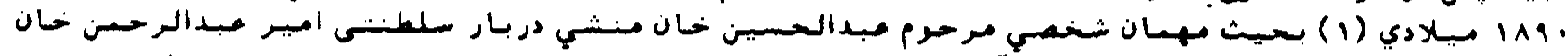

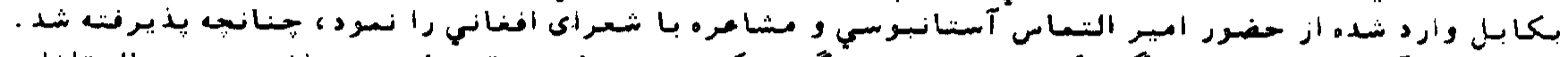

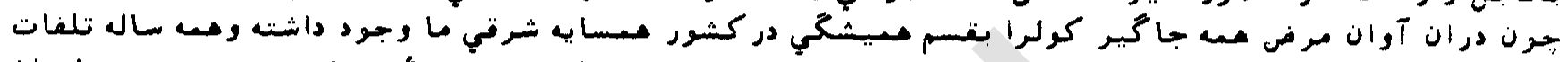

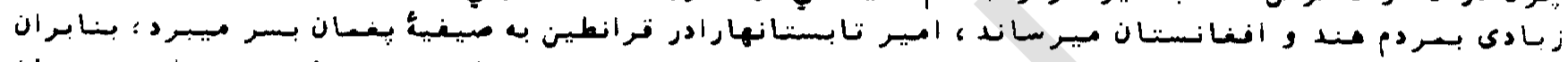

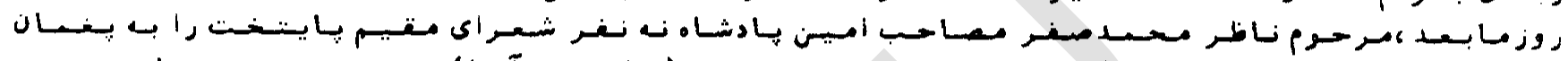

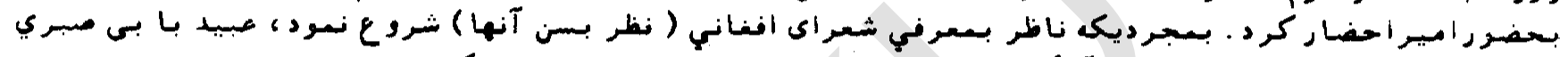

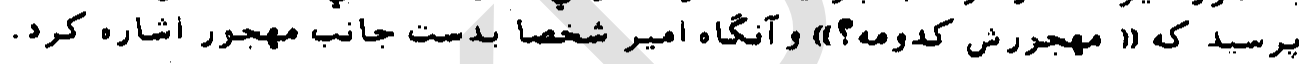

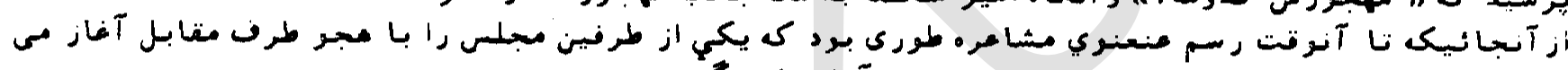

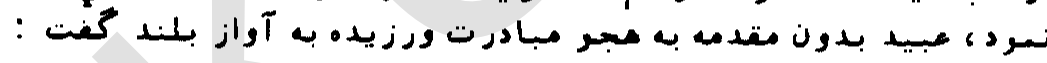

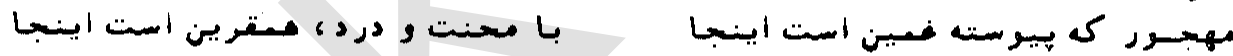

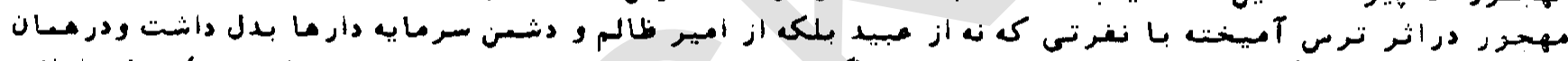

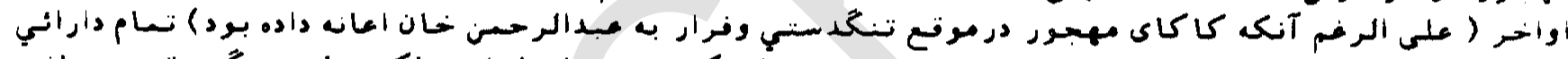

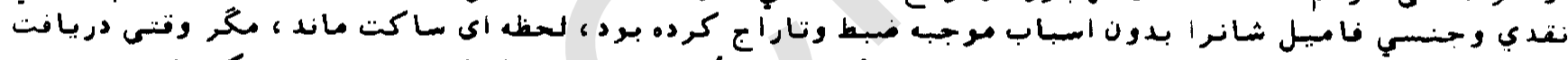

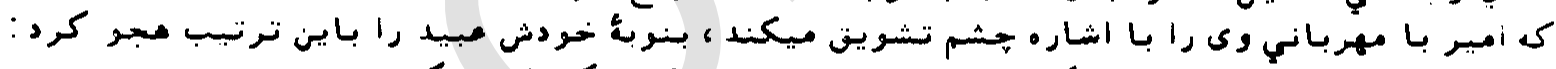

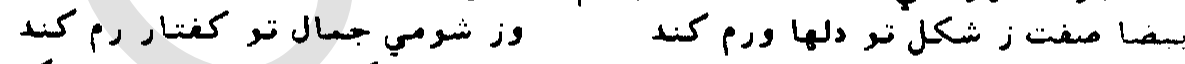

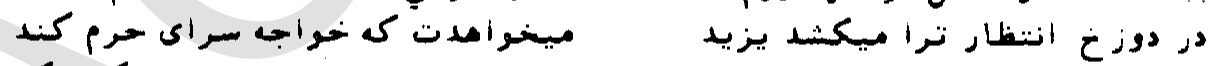

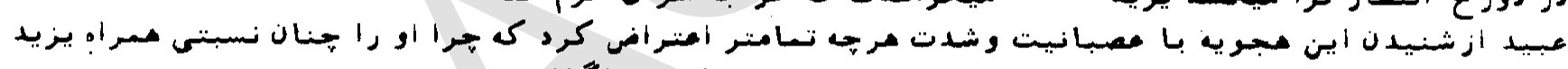

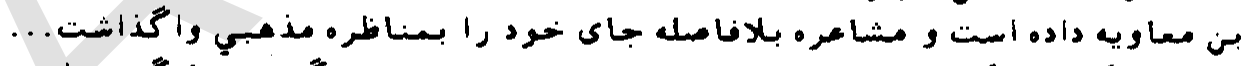

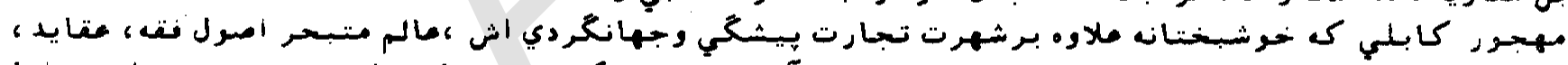

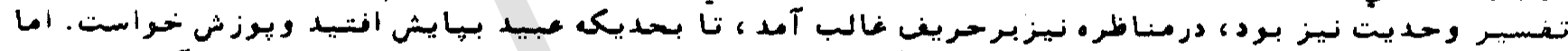

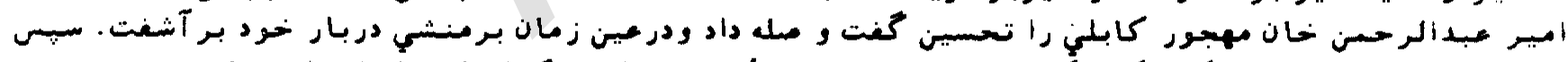

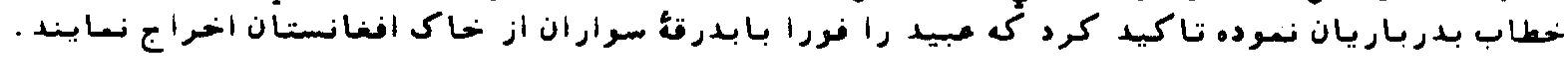

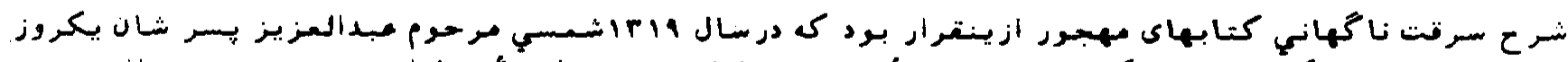

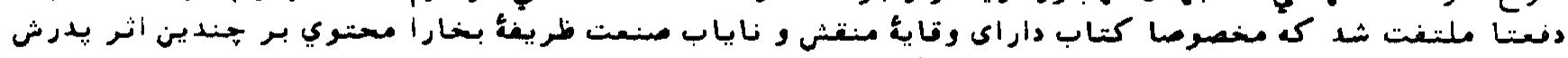

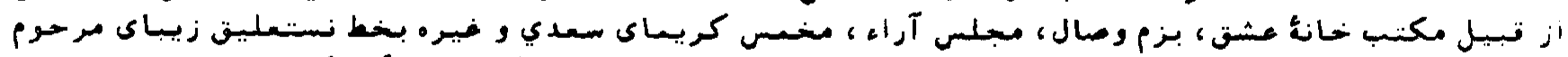

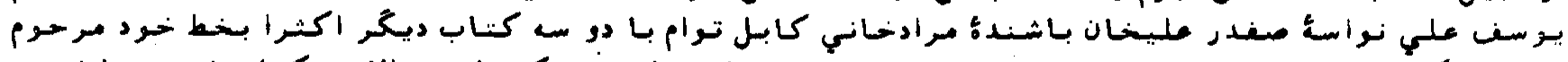

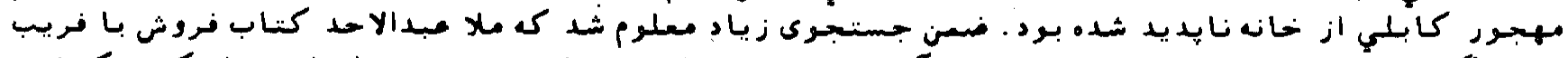

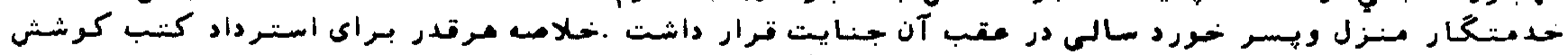

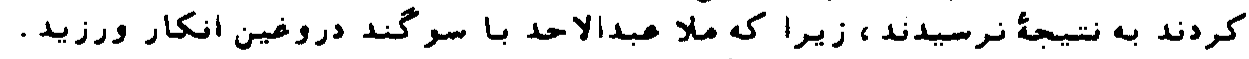

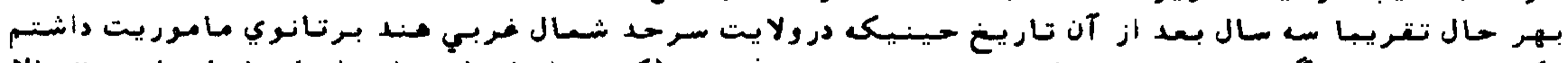

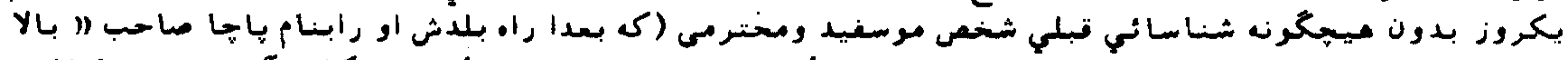

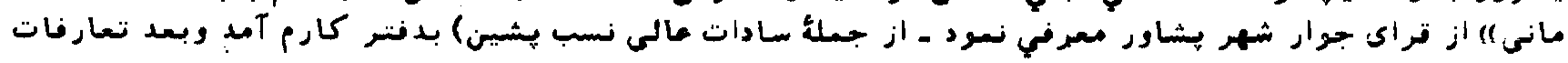

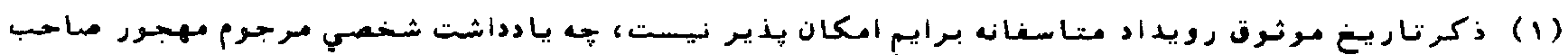

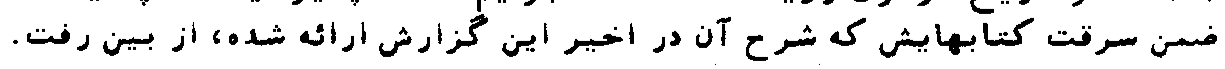




\section{6}

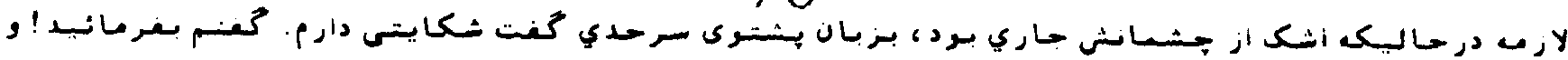

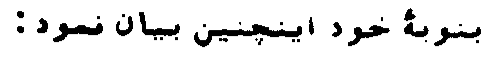

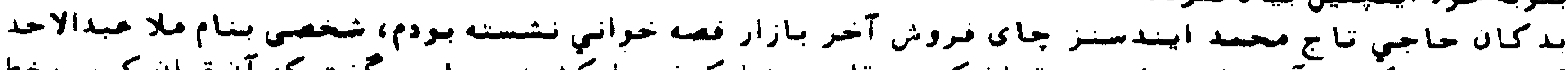

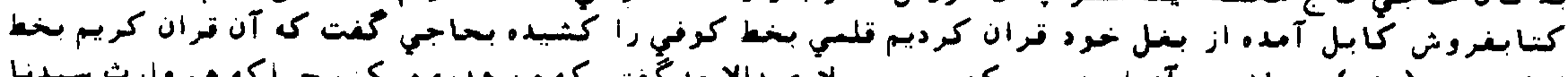

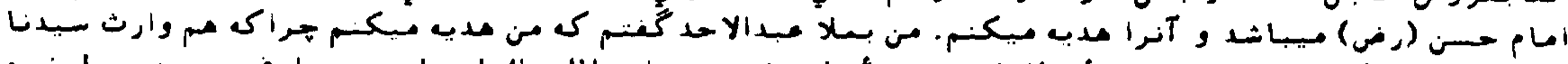

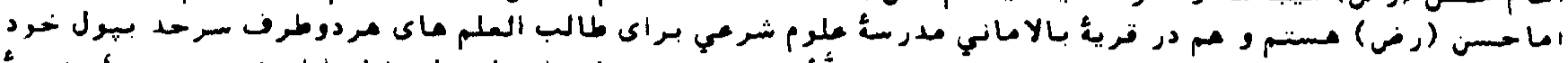

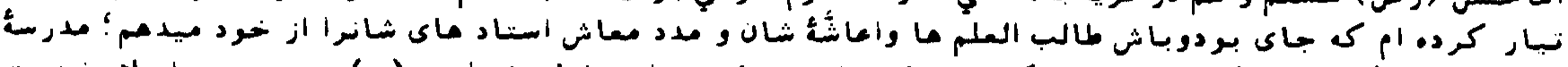

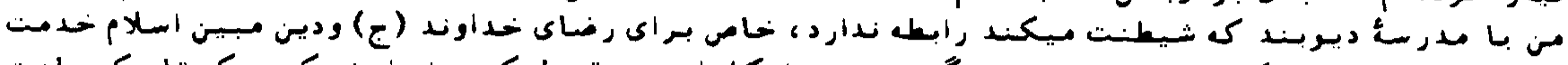

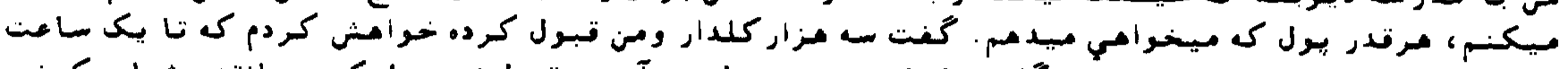

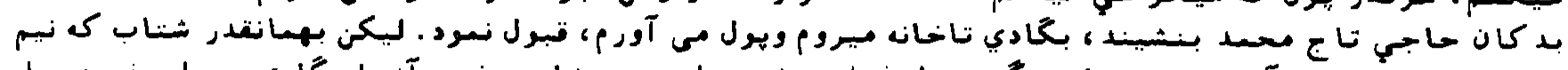

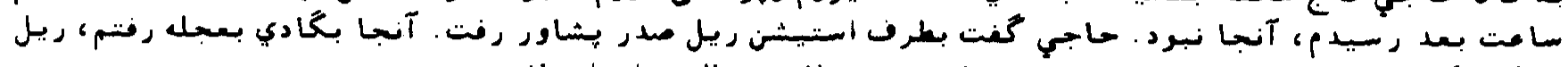

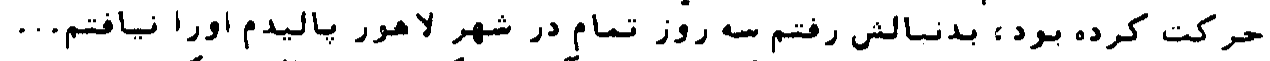

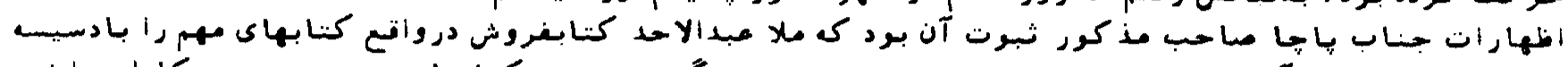

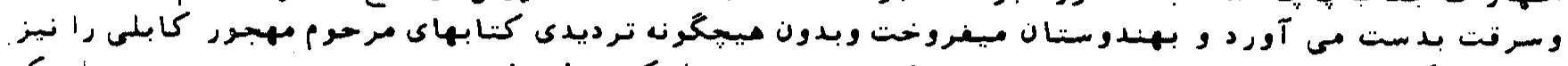

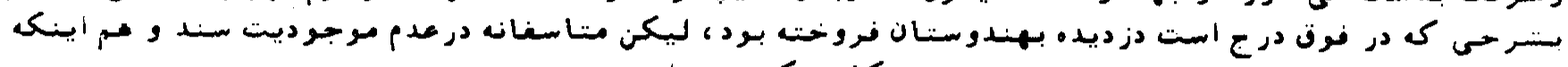

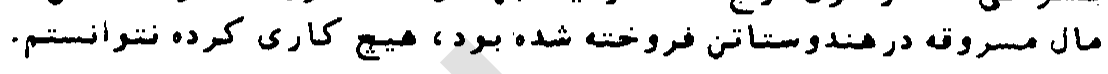

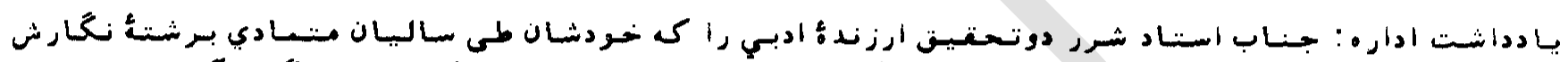

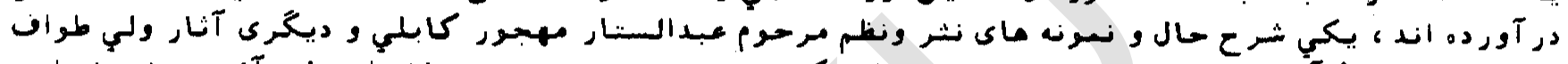

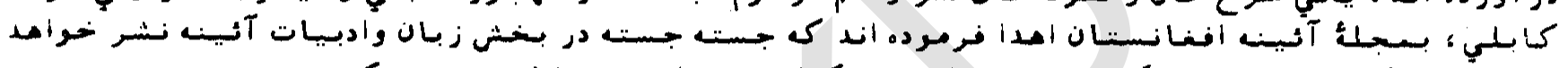

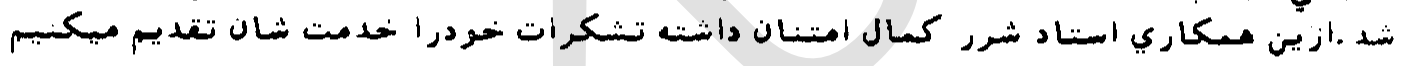

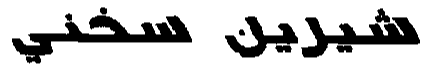

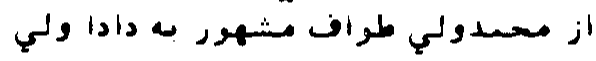

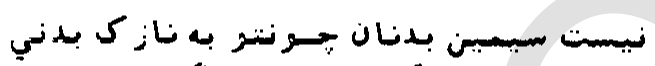

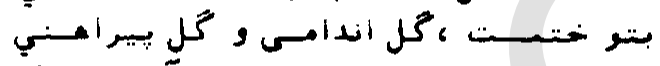

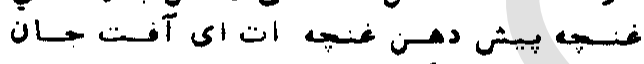

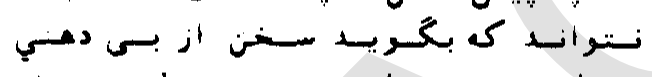

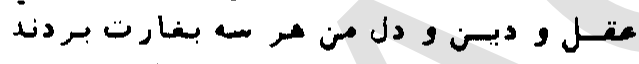

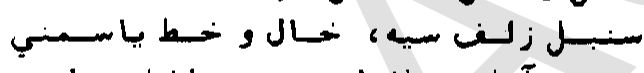

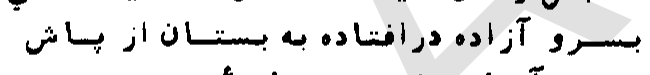

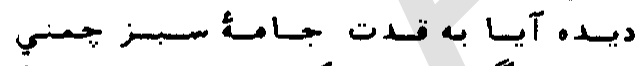

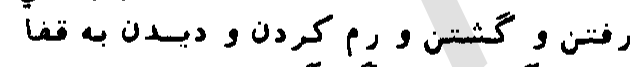

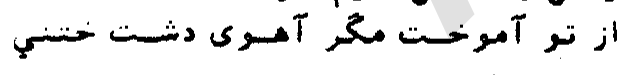

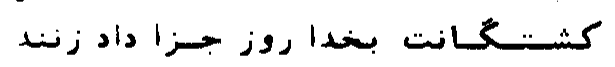

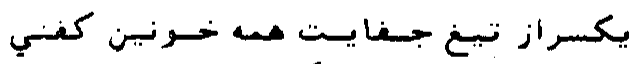

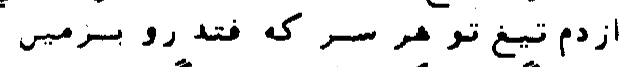

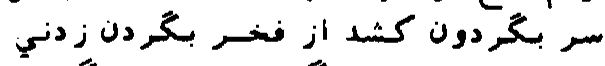

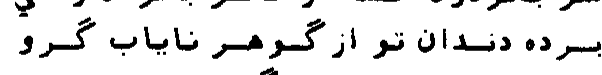

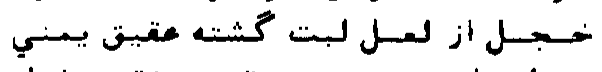

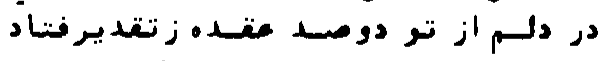

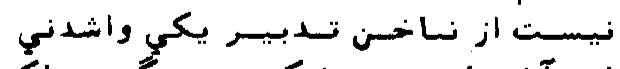

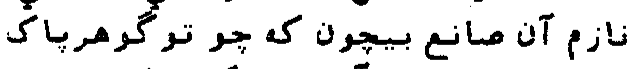

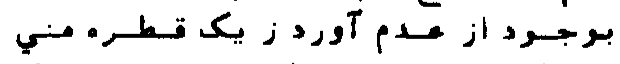

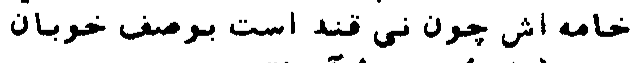

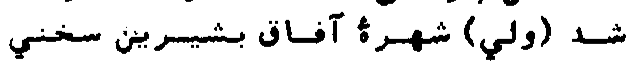

از عبدالستار مهجيور كابلمي

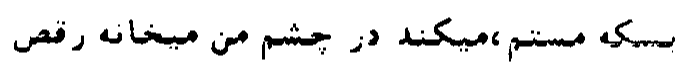

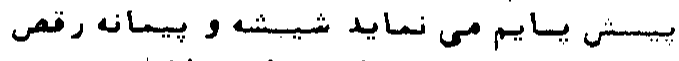

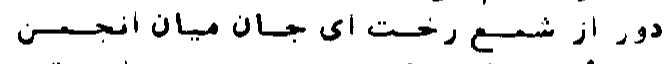

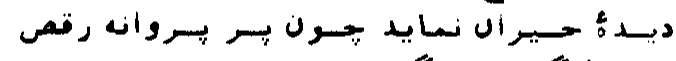

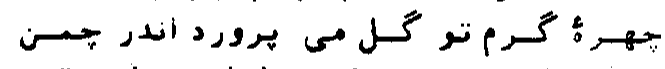

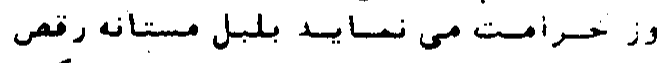

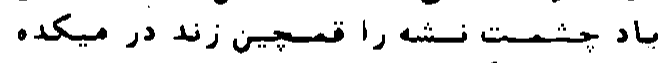

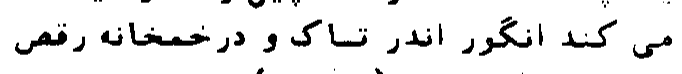

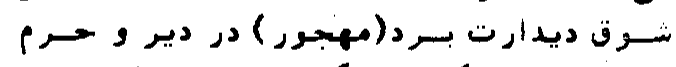

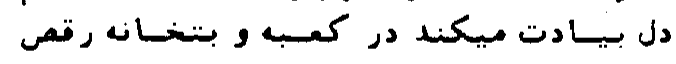

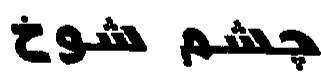

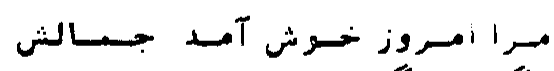

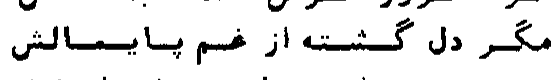

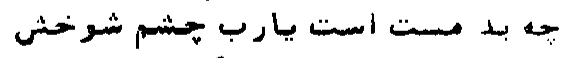

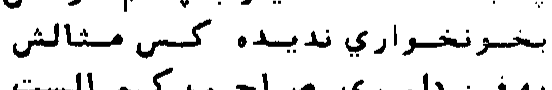

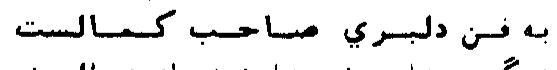

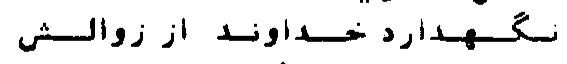

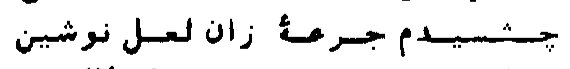

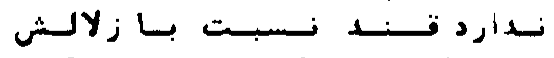

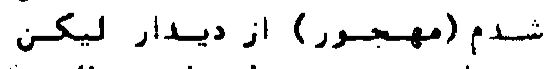

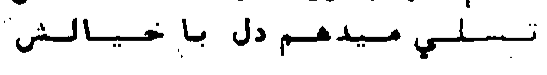


 \\ .

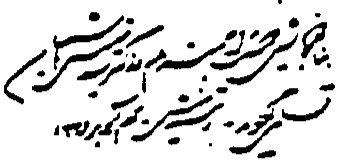 \\ $1: 1$ \\ DORROR \\ RO. BOX 408 \\ MONTCLAIR, CA 91763 \\ April 21, 1999

\author{
FA: $(909$ ' $625-6751$ \\ (909) 626-83It
} \\ EDITORIAL \\ COMMENTS ON THE INCORRECT, PARTIAL AND EXAGGERATED CLAIMS CONTAINED IN THE LETTER OF FEMINIST MAJORITY FOUNDATION ADDRESSED TO PRESIDENT CLINTON}

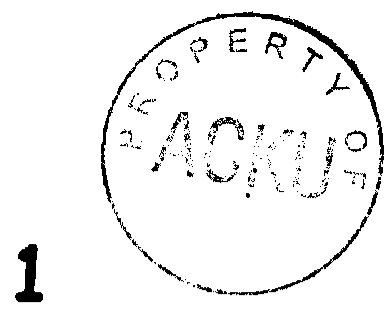

The Editor of Afghanistan Mirror contends that the efforts made by the Feminist Majority Foundation for human rights and women.s rights in Afghanistan should be impartial and its reports on the events and situations in Afghanistan should be correct and free from exaggeration. This was not observed in the letter of the Feminist Majority addressed to President Clinton. To convince you, please spare half an he $r$ to read the following:

1) In the first parargraph of the lettter, we read:" Girls are not allowed to go to school- not even elementary school."

this claim concerning elementary school is completely false and baseless. This editor saw in 1997 small girls going to elementary schools in Kabul; however, secondary schools for girls were closed and continue to be so.

2) In the third paragraph of the letter, we read:"Before the Taliban's control, women in Afghanistan were educated and employed." This false claim seems to be included in the letter by supporters of Rabbani -Massoud regime who are signatories of the letter. For your information, before Taliban, the regime of Rabbani - Massoud was in control for five years and in the second year a decree was issued by Rabbani to ban women from working in the government civil service, including radio and television, and ordering women to sit at home. Then, the Supreme Court of Rabbani issued a judicial judgement; certain articles of the judgement pertaining to womentweil and covering and education are as follows:-

The title of the judgement is "Judiciary Judgement for Veil and Covering" which was issued in response to an of ficial inquiry by President Rabbani,m by his Supreme Court, dated 5 Sunbulla 1372 (September 1993), signed by Mawlavi Abdul Ghafour Sanai, President of Supreme Court, and it ordred that " the Judgement be implemented in all government units of the Islamic republic of Afghanistan." The said Judgement was published in its original text in Afghanistan Mirror, issue No.34, dated October 1993,pp.74-77; it was also published in the Afghan Press in Peshawar - Pakistan.

Important points of the Judgement pertaining to Veil and Covering are as follows:

---"Woman is private and naked, when she goes out of the house, Satan instigates his followers of suspicion, sedition and libidinousness towards that woman...therefore women better stay 
indoor- No insistence or recommendations are necessary concerning outgoing of women from the house, and those who do so are foolish, ignorant or followers of lust."

--- " Woman is prohibited from raising her voice (loudspeaking) which may be heard by aliens, because from hearing the voice of her jeweleries and anklet bells seductive acts may arise..." (Note by Editor: prohibition of wearing high heel shoes in public which is considered noise producing and attractive, pertains to this Judgement).

--- "The entire body of women should be covered from men, and women must not show themselves to men, muslim women should even cover themselves from adulterous women because adulterous women would describe them to men; a virtuous woman therefore should keep herself covered in the presence of an adulterous woman. Muslim women are not allowed even to see a blind man, if he is alien to them."

--- "When women have to go out of their houses for the needs of life, they must cover their faces and bodies with big veils, and clothings should be covering, because the prupose of covering is avoiding exposure, clothings which are not covering are not considered "Hijab" a means to avoid body exposure; clothings used for "Hijab" covering the body, should not be thin, ornamented or colorful so as to attract attention of the public."

--- " Clothings for "Hijab" avoiding exposure should not be tight and figure- showing, it should be wide and free so as not to show the body or naked parts or seductive and attractive parts of. Clothings should not be perfumed so as to attract public attention; a woman wearing perfumes and passing by a gathering of men is considered adulterous; women should not wear clothings like those of men, men wearing clothings special to women and women wearing clothings special to men, have been cursed."

and

On Women/Education:-

--- ". Acquiring knowledge and inquiry about knowledge is an obligation for the Muslim men and women, the knowledge being that of the sciences of religion, such as Beliefs and Faith, Qoran (the Book), Sunna (deeds of the Prophet and Islamic customs \& traditions), and Islamic Jurisprudence...Muslim women are obligated to acquire the primary and necessary knowledge of the religion, observing all the religious obligations and prohibitions, from their close relatives and non-prohibited persons, because Islamic Jurisprudence limits the place of education and schools for women only in their houses or the houses of their non-prohibited relatives." ( Note by Editor: According to this Judgement, education ( or cquisition of knowledge) for men is limited only to matters of religion, and for women the basic and primary knwledge of the religion.)

--- "For women to go out of the house for the acquisition of religious knowledge is ridiculous and null, and construction of school for them, and allowing them to go out by groups to schools, is prohibited, even if their teacher is a Muslim, and even if there is preparation of covering and non-exposure in those schools, because experience has shown that schools have left bad and ugly effects on the morality of women, and continue to do so, bringing bad and undesired results, contrary to Islamic principles, and defecting their morality."

--- " Acquisition of modern sciences in schools by Muslim children, girls in particular, is prohibited, before the acquisition of Islamic knowledge, and experience has shown that such a practice has resulted in abandoning and quitting the acquisition of the knowledge of Islam. It is 
ordered "Not to teach girls writing." therefore, in all judgements passed by authorised scholars of Islam education and teaching of composition to women is prohibited. Ibn Massoud says:" Teaching composition to women has, in most cases, resulted in seductive and evil acts, because they write to aliens and prohibited ones restricted and desirous matters..."

The above are excerpted from the main text of the Judgement issued during Rabbani government, and the Judgement concludes with the following lines:

:... Since 16 months muslim women of our Islamic State have been working as before in the units of the Islamic government and they have been active in schools and in radio and television, also they walk freely in the bazaars without veil, and no change has taken place from the past, however we hear everyday from Kabul Radio that God's Orders are being implemented, especially in respect to Veil ( $\mathrm{Hijab}$ ) and Covering (Satr). It is seriously and recommendably required from the authorities of the Islamic State and Government to implement all God's Orders, especially those of the Veil and Covering immediately, and to expeli women from the government offices, and to close women schools, which are in facts centers of adultery and prostitution, also to expell women from Radio and television, hence proving that God's Orders are truly implemented. The Judgement of the Supreme Court hereby submitted so requires."

In accordance with another report published by (WUFA) in Peshawar in March 1993, which was excerpted by Afghanistan Mirror issue No. 32, July 1993, President Rabbani has declared schools and hotels as centers of corruption.

Submitting an English translation of the excerpted text of the Jurisprudence Judgement during Rabbani's government in 1993 for the information of the American public, wo would like to draw the attention of the Feminist Majority Foundation to the influence exerted by the followers and supporters of Rabbani-Massoud among the leaders of Feminist Majority, injecting and submitting incorrect and false reports. In other words, what is considered in the letter of Feminist Majority as "Before the Taliban's control...", that period was the shameful ruling of Rabbani-Massoud government, when women,s rights and respect for human dignity were much worse than the current period of Taliban. In Rabbani's ruling period (1992-1996), thousands women, including married ones, were abducted and a good number of those are still alive in Panchir, included in the harem of Massoud's commandants. However, during the reign of King Zahir Shah until 1973, also during the pseudo republic of Mohammad Daoud until 1978 human dignity of women as well as women rghts for education and employment were observed to a good extent. Continuation of such a policy by Feminist Majority, i.e outdoor influence and incorrect reports, would damage its prestige among the Afghan intellectuals and public.

3) Statistics published in the letter of Feminist Majority on the employment of women-- that half of Kabul University population was female, that $60 \%$ of University teachers were women, that $50 \%$ of school teachers and $40 \%$ of Afghan doctors were women-- are incorrect and exaggerated. Such exaggerated figures given to you by your Afghan friends- those who always exaggerate facts two to three folds, and you published them without rechecking, damages the prestige of the Feminist Majority Foundation. Reliable statistics on Afghanistan until 1978 may be obtained from the United Nations records.

4) Among your three demands from the government of the USA and United Nations, your first two demands are very unjust and partial and you seem to be influenced. Feminist Majority should prove to all Afghans, or at least to the intellectuals, that it is an impartial Foundation, striving only for women rights in Afghanistan, and does not take side in the internal politics of Afghansitan. Your demand "not recognize any government that violates women's rights, as a 
legitimate government in Afghanistan; and not allow any government that violates women's rights in Afghansitan, access to a United Nations seat;" would bo considered impartial and non-aligned when both the US and UN rebuff their recognition from the non-existing Rabbani regime, leaving the Afghanistan seat currenlty occuppied by an illegitimate representative of a zovernment in exile suspended until a legitimate government is established in Afghanistan.

From the above cited Judgements passed and implemented during Rabbani's government in Af ghanistan, you are now in a position to realise that violations of women,s rights and invasion of women dignity and privacy began during Rabbani's government and peaked to maximum. A huge women demonstration in Kabul in August 1992,in fron of President Rabbani's office, was reported by B.B.C., VOA and Af ghansitan Free Radio in Germany, which was also published in Af ghanistan Mirror, issue No.27 of August 1992,p.31; over 1500 women were shouting the following slogans for four hours:

"We want the right to live, the right to work and the right to seek education. Why they cut women,s breasts, ears and noses? Don't we have the right to live in our own country? Is there a government in our country and in this city? If you cannot defend women inside their houses, then resign!"

Unfortunately, still to this date, a representative of the same government represents Af ghanistan in the United Nations!!! Neutrality and good will of the US government and the Feminist Majority Foundation would be proven to the Afghan people when they see that you do not recognize both guilty regimes, Taliban and Rabbani regimes both. As long as you extend your hands of friendship and cooperation to a guilty, corrupt and ousted regime, considering the other guilty regime illigitimate and unworthy of your trust, your good will, neutrality and non-aligned position would be questioned by the majority of Afghan people!

Your other demand from the US and UN to exert pressure on "Pakistan and Saudi Arabia to stop funding the Taliban," is again unjust and extremely partial; many Afghan intellectuals have expressed a some what similar opinion to this editor that this demand may have been dictated by Dr. Rawan Farhadi to Feminist Majority, because it has appeared repeatedly in weekly Omaid and other Rabbani publications. For the F.M.Foundation which embracess learned individual of both sexes, such an uncalculated demand is childish and damaging, because:

(a) Saudi Arabia has been displeased with Taliban for over a year, suspending its diplomatic relations, and it is a common knowledge that it has not given any financial assitance to Taliban for two years. However, the Saudi government cannot stop pro Bin Laden Arab millionaires from their intended contributions.

(b) Pakistan continues to support Taliban, but for reasons unknown to many, US and UN are not willing to exert enough pressure to stop Pakistan from what it is doing.

(c) A genuine and general criticism on your partial judgement and position stems from the fact, as you know, that Iran, Russia and India have been assisting Rabbani-Massoud ousted regime by giving it destructive weapons, among other things. Why as a humanity loving and neutral Foundation, the Feminist Majority did not ask the US and UN also to exert similar pressure on these governments???

Respected Leaders and Authorities of Feminist Majority Foundation!

This editor is 70 years old, a former Professor of Kabul University who earned his degrees in America and has also taught here, he is also a naturalized Afghan-American. Permit me as a fellow-American to tell you that your policy of not inviting a number of Afghan journalists residing in California, such as Mr.Omar Khetab, Editor of Radio Payam Afghan for 5 years, Mrs. 
Farida Anwari, Editor of Radio 24 hours at San Franciso for 5 years and Mrs. Mina Karbassi, Editor of Radio 24 Hours in Los Angles for 2 years, and the writer of this letter, Editor of Afghanistan Mirror for 10 years, in your event of March 29th, 1999 in Hollywood, has been interpreted by many Afghans residing in California as a non-American and un-democratic gesture. We, the Afghan-Americans are naturally much more interested in and informed on Afghanistan, than you native Americans, and we can never hide the facts and truth. I visited Af ghanistan in 1997 for four months and I observed the situations closely; I published my observations and documentary filming of Kabul in a three hours video cassette, over two thousand copies distributed to Afghan circles in US and Europe, Australia, etc.

I avail this opportunity to declare candidly that from the contents of your letter, with the exception of the points discussed and criticised above, duly documented, other critics of the abuses of human and women,s rights contained in your letter, are factual and correct.

Also, it is my observation that the only quality and priority in connection with women,s rights and human dignity of women that Taliban may be credited for, compared to Rabbani's ousted regime, is that Taliban have truly respected the honor and chastity of women, but Taliban's record in all other aspects of human rights and women,s rights is bad, whilst during Rabbani's regime women's honor and chastity were of ten violated by government commandants.

You, the Feminist Majority, always raise the issue of women's rights, but there is no one to raise the issue of men's rights in Afghanistan, for violation of men's rights is worse than that of women,s at the present time: Employemnt and Retirement laws, with equal opportunity, which prevailed before, are abandoned; in a poor country where the government is the only employer, thousands of civil and military personnel have been ousted, adding to unemployment and poverty; education and foreign degrees are devaluated; from the leadership of the Taliban to the care taker cabinet to the low level personnel at the rank of branch directors are all Talibans; the nation has no control over the state revenues, no such inquiry is allowed and the Finance Ministry is not accountable; free press does not exist and the rights of free speech and criticism are abolished; the majority of Kabul,s residents can neither afford coffin and funeral expenses nor have the right to burry their deads; clothings and facial appearance are imposed on men, and all entertainment means and occupations are declared illegal.

And to the Talibans, I say the following:-

Oh brothers," What you cannot hear or see, you cannot say!" Yes, there are things that we see, hear and know very well, but you are following an "ostrich policy" which penetrates its head under sand after running a long distance and then sees the world in the dark. Concerning women's rights, with the exception of women's honor and chastity which have been observed honorably by your government, in all other aspects women are treated like animals: to eat, to give birth, to serve in the house and to die. Unfortunately, the practice of multi marriage which had decreased in Af ghanistan, has been renewed and accelerated in your period. All the noises and voices raised around the world, and the disrespects addressed to you by the international institutions concerning women's rights, to cite an Afghan proverb "Is this dead worth this mourning?"

On the one hand you claim that security has been established throuhgout the territories under your control, and it is so a fact; on the other hand the closure of schools and university for women is cited as a matter of security concern, thus creating a logical controversy ! If security 
exists, and it is a known fact that it does, then the closure of schools and university for women is an excuse tied with your Talibism conditions and orientation, which should not be imposed on the Afghan deserving nation by bayonets at this dawn of the 21 st Century !

Brothers! You cannot hide facts from the world, also you cannot fight with the world! Humanity's speed for progress and civilization does not stop because of your regressive desires, the world humanity in the 21 st Century is proceeding towards accurate computerized calculations to dominate the space, and you cannot violate men,s and women,s rights in Af ghanistan by gun and bayonet because your stratum policies so desire- a stratum which does not exceed fifty thousands in number; the Afghan nation is alert and alive and is a history making nation, it is obliged to go along with the caravan of humanity, because humanity caravan too does not allow for one of its parts to decay. You should shake yourcelf and make 2 self evaluation and critics of yourself, the Afghan nation has not yet given you its political power and destiny by its own free will and choice, you do not enjoy a political legitimacy and you rule by means of ceased powers like Rabbani's ousted regime. If you do not return sooner the peoples powers, with which you are entrusted temporarily, peoples of Afghanistan and those of the world would join hands together to retake the entrusted powers from you, and you will have no more trust. The assumption that you have collected weapons from the public to arm the Taliban stratum, so there may not be any public rise against you, is completely raw and empty. During the occupation of about 200 thousands Soviet troops, Afghans were empty handed, they did not have any weapons, but gradually they became organized , they found weapons with which they defeated the famous Red Army; you should fear that day ! Islam, thanks God, has been in Afghanistan before and will remain to eternity, and God Almighty Himself protects Islam; you have the right to and can implement Talibic Islam to your own stratum, but you do not have the right to turn the entire Afghan nation Talib. Communists pursued a similar policy, but they failed and became disreputed.

Dear Talib Brothers! Your treatment of the cultural aspects of Afghan people also deserves criticism for it seems that you want to establish Arabic culture in Afghanistan; however, Semitic culture differs from Arian culture. Afghans have accepted Islam willingly and treasures it dearly, but they have not accepted Arabic culture nor Talibic culture; beard and turban, for instance, are not obligated by Islam, but you have made both obligatory on Afghan people. In Af ghan culture and traditions men in majority have worn beard willingly, but it has never been imposed and Afghans in the course of history have had a choice. Afghan men have worn four types of head covers: turban, karaqul hat, embroidered taqin and pakol, why then you have imposed turban alone on people? A point noteworthy in this connection is that the majority of muslim men perform the Haj pilgrimage in Holy Mecca with bare heads!

Your treatment of the New Year's Day, Nauroz, is also critical. The celebration of Nauroz to welcome the new year on March 21 st is an ancient tradition, as well as an aspect of Arian identity of Afghan people. Your abolishing the Nauroz celebration, which has no diversion with Islam, not only has deprived the people from a good, useful and ancient Arian tradition, but has also inadvertently damaged the high interests of Islam and Afghanistan. The interests of Islam in the traditional celebration of Nauroz in Balkh, northern Afghanistan, is embodied in the belief that the majority of muslims in the countries of Tajikistan, Uzbekistan, Turkmanistan and others consider Balkh as a great Islamic center, where the tomb of Ali, the Fourth Khalif of Islam, is believed to exist, and even during the Soviet and communist domination, every year on the New Year's Day thousands of peoples took the risk of crossing the Oxus River by swimming to attend the religious and cultural ceremony in Mazare Sharif, also people living along the Oxus 


\section{7}

River borders in the Soviet territory prayed on the sound of Azan that crossed the Oxus by waves. Now that those countries are free and independent, their citizens may freely come to Balkh to attend the tradtional celebration of the New Year, which has economic advantages for Afghanistan, as well as political advantages, such as the creation of brotherly relations and coordination of Islamic togetherness between the muslims of the region. However, your unreasonable decision to abolish the historic and traditional celebration, would send the Muslims of the region, especially the Shiite population, to Meshad-Iran, instead of Mazare SharifAfghanistan!

This unreasonable policy of yours, in my opinion, is a service to the policy of Iran, because Iran has never recognized Mazare Sharif as the tomb of the Fourth Khalif (something that the majority of the peoples of Afghanistan and northern independent countries believe in); Iran is now striving to make Meshad more sacred to the eyes of those peoples. As a result of your unreasonable policy, peoples of Afghanistan from norther provinces and from central provinces, instead of being led towards Mazare Sharif, they are ridden to Meshad-Iran and you cannot stop the pilgrimage!

From the economic view point, New Year Nauroz brings about a great economic movement throughout the country, from tree plantation, plowing, shoveling competition to animals show, wrestling, national dancing and provision of recreation and entertertainment for children, varieties of candies, sweets cookies and dried fruits, competition of poetry and celebration of orange blossom; the day has been known as a joyfull day for Afghans for thousands of years and has been very yielding and profit making for many people. In view of all the good aspects of Nauroz, you tell us and show us which aspect of Nauroz is controvercial to. Islamic jurisprudence? Your government continually engaged in war during the past three years, not only failed to provide work, salary and food for the people, but also led the people to poverty and disease, instead of creating additional joyfull and celebration days, good for economy, sports and recreation, you have abolished a good economic and cultural day, Nauroz, why?

Dear Taliban Brothers! Your greatest service to Afghanistan, which will be remembered always, with great respect and attribution, by future generations and history, is the salvation of Afghansitan from disintegration and division; nothing else is better than satisf ying yourself with this great achievement and honor and to transfer the political powers to the people, so that people could elect a government by their own free will, so that it always praises you and your services and sacrifices and strives for the establishment of peace and security in Afghanistan with your consultation and cooperation. If you are truly Talib "i.e.,students of religion", as you claim to be, then the art and science of governing is not your profession and duty; it seems that your failure in domestic and foreign policies has stemmed from this lacking. Those with vision would believe hitherto!

Your benevolent brother

Sayed Khalilollah Hashemeyan (Ph.D.)

Editor of Afghanistan Mirror

Former Professor of Kabul University

Copies sent to:

Eleanor Smeal, President of Feminist Majority Foundation 
(NOTE: This same letter will also be sent to Secretary of State Madeleine Abright and U.N. Secretury-General Ko6 Annm)

To: Prexident Bill Clintor

The White House

Washingtion, DC 20500

\section{PLEASE DO EVERYTHING IN YOUR POWER TO RESTORE WOMENS EUMAN RIGETS IN AFGEANISTAN AND END GENDER APARTEIGID}

\section{Dear Prosident Clintom,}

We the undersigned, over 120 U.S. women's rights groups, human rights organizations, and women's stodies progrems in U.S. colleges and universities, are outraged by the gender apartheid in Afghmistan. With the thbeover of most of Afghanistan by the Taliban armed militia, wowen have been forbidden to wort, 80 to university, or leave their homes without a close malo relative. Girls are not allowed to go to school - not even elementary school. Women are forced to wear the bead-to-toe "burga" in public, with only a mesh opening to see and breathe through.

Hundrods of women have been beaten for not being "properly" dressed. In late March 1997, a womas was stoned to death for attempting to leave the country with a man who was not her relative. Homes with womea ere required to have their windows painted opaque, so the women cannox be seen. Women are noc even allowed to wear shoes that make noise when they wall Wo have beard from a journalist that at the stute orphanage in Kabul, the girls have not been allowod outside since September of 1996.

This is troly a bectelesh apainst women. Before the Taliban's control, women in Afthnnistron were educated and exoployed. At Kabul University balf the students and $60 \%$ of the tenchers were womer. In addition, women made up $50 \%$ of civilian government workers, $70 \%$ of schoolteachers, and $40 \%$ of docton.

The ban on womea worting has throwa tens of thousends of families inte destitution. becanse many women in Afthenisten are widows and are the sole source of support for their familiea. Although a very few women aurses end doctors are permitted to continue worting to serve women patientr, they are harassed. Women bave aleo been shot at for leaving their homes without mele encort to receive medical care.

We ank that the United States government and the United Nations: 
- not recognize the Taliban, or any goverument that violates wowed's human righes, as a legitimate government in Afthniston;

- not allow the Taliben, or any government that violates women's bumnn tights in Af chrnistan, scceas to a United Nations seat;

- do everything in your power to restore women's buman rights to work, edveation, health care and safety in Afghanistan, including pressuring Pakistan and Saudi Arrbia to stop fonding the Taliben.

\section{Sincerely,}

Eleasor Smeal, Preaident

Feminiat Majority

Kim Gandy, Execurtive Vice President

National Orfanitation for Women

Gloria Feldt, Preaident

Planned Paranthood Fedaration of Amerlea

Marcia Ann Gillespie, Editor in Chief

Me Magadine

Susan Bianchi-Sand, Chair

Natbonal Counell of Women's Orounirations

Prema Mathaj-Devis, CEO

YWCA of the USA

Jumes Wagoner, Executive Ditector

Adrocotem for Youth

Cheryl A. Peterson, Ascociste Diruetor, Federal.

Government Relintion

Amertem Nurns Amodntion

Gloria Johneon, Provident

Contition of Labor Union Women

Ormar Samed, Prasident

Afghnictan Information Center/Asad Arthen

Radio

Roma Popal, Excecutive Director

Afghen Women's Amoctation

Bichop Imegeoe Stewart, Nutional Precident

Apricin Amertican Woonen's Cors Ascociation

Amy Inaces, Netional Dirvetoc

Amertemen for Democratle Action

Robert Herris, Administontive Astirtant

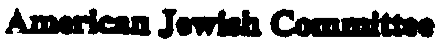

Eileen McGrath. Executive Director

American Medical Women's Associntion

Binnce Jagper, Member of the Executive Director's

Leadership Council

Amsecty Internutional

Kathleen Ferrer, Acting Director

Wornen's Sundies Progrm

Artoon State Und verstty

Arum Reo, Presideat

Areociatton for Women in Development

Nancy Cervetti, Director

Women's Studies

Avila Collewe

Emily Ferguson, Administrative Assistant

Belfor Centar for Amertona Plarallem

- Dr. Yromes Serugen-Letwich, Executive Director and C.O.O.

Blect Leadexhilp Forum, Inc.

Sudith Robbing Adininistrotor

Women's Sucties Progrm

Borrioth Collep:

Kethieen Murny Dixon, Director

Women's Smoties

Bowiting Grwen State Undrexdty

Joyes Morrisery

Callfornis Foderation of Butones and

Protendonel Women

Prences Xinding, President

Catbolies for a Irew Choles

Marthe Burk Presideat

Conter for Advancument of Public Pollog 
March 29, 1998

Greatings to all of you who have gethered for this important event. I regzet belng unable to be vith you in person but wast to ahase with you my strong aupport for efforts to help the women and gifls of Mfghnotsean.

Ior two decadea the Afohan people have ouffered--glest from invasion and then from civil strite. Mere than 300,000 persona have been internaliy dieplaceds wore than 2.6 militon are
refugees.

The Eactioas responalble for the Elghting have gystematically abused human rlghts and axe deserving of our
contempt in theis. treatment of women.

Curzently the most powerful faction 13 the raliban, which appears to believe that the only rights woun have are the righte to reanin silone, unerployod, uneducated and unseen. $A$ the $2^{\text {*t }}$ contury proparee 50 dam, the Tallban in leading Afghanistan back to the past. ous alatera deservo better; they deserve a real choles and a true chance at a ilfe of dignity and
opportunity.

Afghan women have asked for our help, and we have reaponded by Increasing our ald to feed, educate and traln refugees; by pressing both lactions to peantt nongovernmental orgarizations to operate freely and securelys by sooking a peaceful setelement to the was: and by anklag it clens to Talibar leadera that thoy will not gain the diplangte recognitlon they seek antil they accord Afghan otrle and women the righte they deaerve.

ance agnin. I grant you and congretulate you for your efforts. You may be aneured that as loag as I an secretary of state, Ameriean foralgn polley will strtve to advanea the cave of human dfonity in Rehurietan.

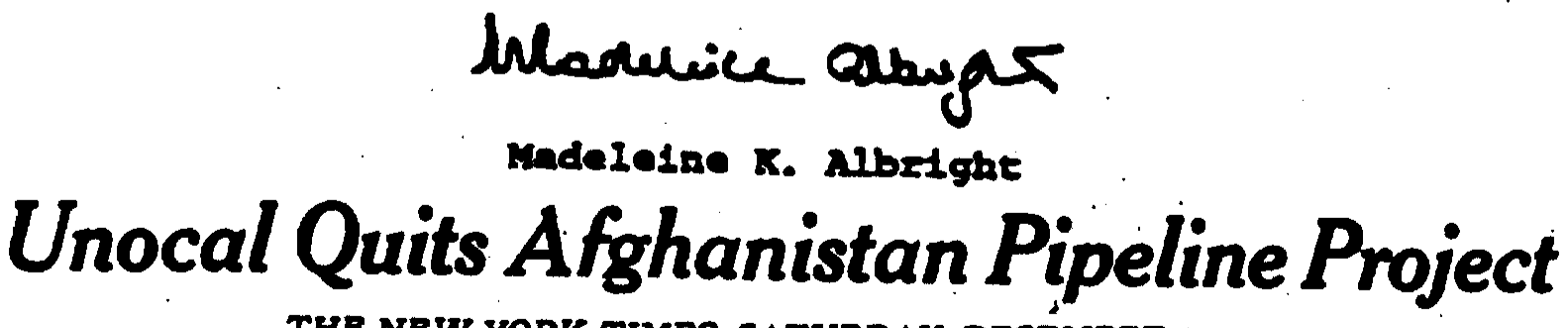

THB NBW YORK TIMBS, SATURDAY, DECEMBER \&, $19 \%$

The declsion wes mede under tin: preapure ol bow word ol pres, lom. inite froup that enelled Unocals contict whe the rallog th turas

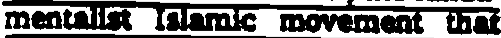
rues Armanten, and concarn about the presence or ta leched terrorix 0ame bia Laden ha the coildalas.
The other comertium members are the Government of Turmant. stan, the former Sovitet republic; the Delia Oll Company of Sund Aribis; the Itocin Corporation and Inpar of Japan; the Hyodal Endinepring and Construetion Company of South Ko res, and the Craneent Group of Pely$\operatorname{stan}$
The Poulnte Merts Fomds:

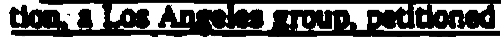
the State a Callormin to rovore Une

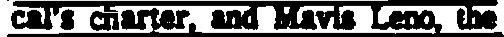

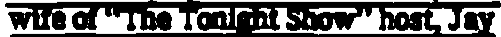
copo attencra e company anr hoter mexin that tom to comprin

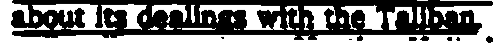




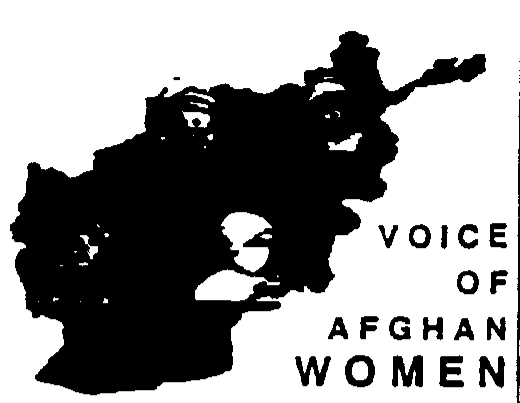

Board of Director:
Atifi, Hamida
Bimar. Fauzia
Ghausse. Amena
Gausse. Razia
Hamid, Najia
Haidari, Torpekay
Isaaczai. Latifa
Kochai, Malal
Mohsini, Fahima
Noorzoy, Farkhunda
Waiz, Tahira
World Wide

Represenrtatives:

Dadgar. Fatima

Akbar. Alia

Farhad, Qamar

Los Angeles

Mazhari Suraia

Canada

Waseal Malalai

Connecticut

Shakera Nadi

Germany

Laili Zikria

New York

Office \& Mailing Address

37240 St. Matthew Dr

Newark, CA 94560

Tel: (510) 793-1021

\section{1 \\ VOICEOF \\ AF GHAN WOMEN}

INDEPENDENT \& UNALIGNED

March 29, 1999

\section{DECLARATION}

We typical and proud women of Afghanistan have gathered here today to protest against the unfair and misleading campaign that the Feminist Majority and like-minded group have started under the name of Women's Rights in Afghanistan.

The Feminist Majority organization has been giving out incorrect information to the public, which is intended to create bias against Afghanistan and Islam. They spread propaganda and cause economic hardship in Afghanistan. They have a hidden agenda, which has nothing to do with Women's Rights in Afghanistan. They have no accurate knowledge of real conditions in our devastated homeland. Moreover, we question the credibility and reliability of their sources that provide them information as well as underlying motives of these sources.

To our best knowledge, these sources are paid agents who previously worked with the communist regime that destroyed our beloved country and want to now make fortunes out of Afghans' misfortune.

While we call your attention to the following issues we strongly request that The Feminist Majority organization and the likemined groups put an immediate end to their false claims and destructive campaign. We would like to make it very clear that they will be accountable and responsible for the severe hardships and damages that they are causing the Afghan women and children. Although their intention to help the Afghan women is laudable, their misleading and misdirected campaign is causing more harm than good.

\section{I}

Are you aware of the fact ?

that men, women and children of Afghanistan side by side took part in our glorious struggle against the Red Army and regained our independence.

(Page one of five) 


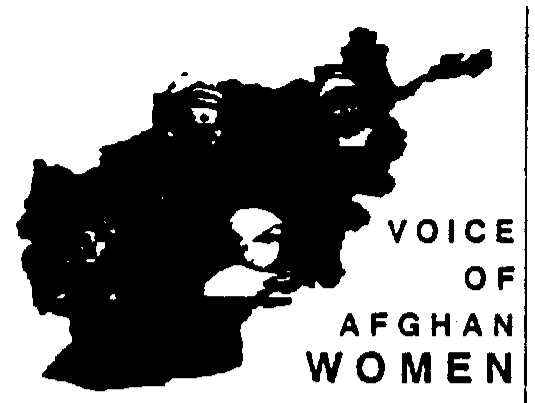

Board of Director:

Atifi, Hamida

Bimar, Fauzia

Ghausse, Amena

Gausse, Razia

Hamid, Najia

Haidari, Torpekay

Isaaczai, Latifa

Kochai, Malal

Mohsini, Fahima

Noorzoy, Farkhunda

Waiz, Tahira

World Wide

Represenrtatives:

Dadgar, Fatima

Akbar, Alia

Farhad, Qamar

Los Angeles

Mazhari Suraia

Canada

Waseal Malalai

Connecticut

Shakera Nadi

Germany

Laili Zikria

New York

Office \& Mailing Address

37240 St. Matthew Dr

Newark, CA 94560

Tel: (510) 793-1021

\section{2 \\ VOICEOF \\ AFGHAN WOMEN}

II

Do you realize?

that even approaching women's rights in Afghanistan without knowing about our glorious history, social, cultural, religious, and economic conditions and values is a crime that cannot be tolerated.

BEFORE PRESCRIBING MEDICATION ONE NEEDS TO DIAGNOSE THE PROBLEM AND WHAT HAS CAUSED IT. THE TREATMINT THEN SHOULD FIRST START WITH THE MOST CRITICAL AREAS SUCH AS HEART, LVER, BLOOD, ETC. AT THE SECOND STAGE COSMETIC PLASTIC SURGERY NEEDS TO BE RECOMMENDED AND IMPLEMENTED. IN ORDER TO REBUILD OUR DESTROYED COUNTRY, MEN AND WOMEN ARE NEEDED TO PARTICIPATE: TO DO SO, THEY HAVE TO BE EDUCATED AND SKILLED. IN ITS PRESENT CONDITION, AFGHANISTAN CAN BE COMPARED TO A PATIENT WHO IS IN INTENSIVE CARE.

\section{III}

Did you know ?

that Afghans have extreme respect for women that are recognized as part of honor and dignity. Their protection and security is cultural and religious duty of society.

\section{IV}

The women of Afghanistan acknowledge and highly appreciate what the Ruling Administration (Emarath-Islami Afghanistan) has done for Afghanistan. When Taleban took over the power and conquered $95 \%$ of the entire country, they inherited demolished cities, villages, and collapsed economy. Armed bands and robbers were everyday challenge for peace and unity. Rape and kidnapping women and girls was a common act. The Ruling Administration collected Arms and restored peace and security after a long deprivation of 20 years. They prevented the disintegration of our beloved country and saved territorial integrity as well as national unity, the values we Afghans cherish and die for. At present conditions, one can peacefully sleep at night and can travel with any amount of money without fear and risk. Once again we would like to emphasize the fact that Taleban came into

(Page two of five) 


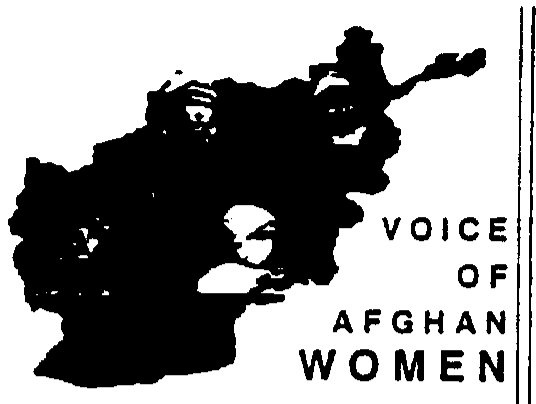

Board of Director:

Atifi, Hamida

Bimar, Fauzia

Ghausse, Amena

Gausse, Razia

Hamid, Najia

Haidari, Torpekay

Isaaczai, Latifa

Kochai, Malal

Mohsini, Fahima

Noorzoy, Farkhunda

Waiz, Tahira

World Wide

Represenrtatives:

Dadgar, Fatima

Akbar, Alia

Farhad, Qamar

Los Angeles

Mazhari Suraia

Canada

Waseal Malalai

Connecticut

Shakera Nadi

Germany

Laili Zikria

New York

Office \& Mailing Address

37240 St. Matthew Dr

Newark, CA 94560

Tel: (510) 793-1021

\section{3 \\ VOICE OF \\ AFGHAN WOMEN}

existence to combat the lawless and systematic human rights abuse by the warring factions with judicial structure destroyed and widespread anarchy.

\section{V}

Did you know?

that the protection of women's rights under Islamic laws are: right to live;

$$
\begin{aligned}
& \text { right to have edueation; } \\
& \text { right to have properties; } \\
& \text { right to marriage; } \\
& \text { right to do business. }
\end{aligned}
$$

Over 1426 years ago Islam directly identified the above rights.

Prophet Mohammed (PBUH) in regards to women's rights stated in his farewell speech: 'Do treat your women well and be kind to them, for they are your partmers and committed helpers." In Islam acquiring education is obligatory for men and women. Any Islamic government including Emarath - Islami Afghanistan can not ignore these Islamic orders.

\section{VI}

Those who raise women's issue in Afghanistan are using it for political games and power. The issue has became profitable source of making fortune out of people's misfortune at the hands of a limited selfish group. The Afghan women do not benefit from most of the fundraisings that has been done in their names.

\section{VII}

The international isolation and sanctions demanded by the Feminist Majority is starving the Afghan people: men, women and children. The Feminist Majority is badly misrepresenting the reality of life for women in Afghanistan. The have yet to check the credibility and the motivation of those who provide them with misleading information in order to isolate Afghanistan.

\section{VIII}

There are pitifully few hospitals or schools or even standing buildings in Afghanistan, but women go to them. The Afghan tradition requires that women must be modest in dress. The burqa (also known as

(Page three of five) 


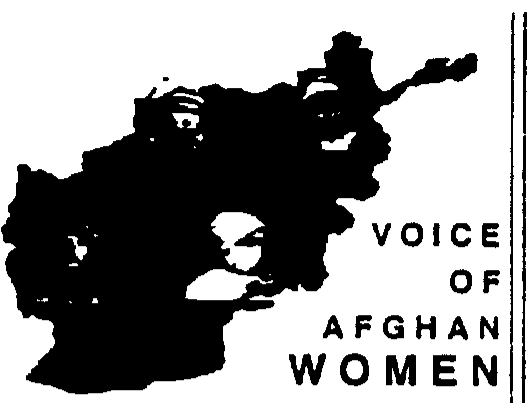

Board of Director:

Atifi, Hamida

Bimar, Fauzia

Ghausse, Amena

Gausse, Razia

Hamid, Najia

Haidari, Torpekay

Isaaczai, Latifa

Kochai, Malal

Mohsini, Fahima

Noorzoy, Farkhunda

Waiz, Tahira

World Wide

Represenrtatives:

Dadgar, Fatima

Akbar, Alia

Farhad, Qamar

Los Angeles

Mazhari Suraia

Canada

Waseal Malalai

Connecticut

Shakera Nadi

Germany

Laili Zikria

New York

Office \& Mailing Address

37240 St. Matthew Dr

Newark, CA 94560

Tel: (510) 793-1021

\section{4 \\ VOICE OF \\ AF G HAN WOMEN}

chadari) is not the law, but long held tradition. Women are free to leave their homes unaccompanied and do so. They were not able to do so when the warlords reigned and ruined Afghanistan, when rape and kidnapping women and girls were the orders of the day. Women do get treatment by the best medical personnel available, whatever the gender of the doctors. The policy of isolation imposed and promoted by the Feminist Majority has left women's medical facilities with a severe shortage of supplies. Over $80 \%$ of women patients suffer from Anemia due to lack of nutritious food and proper medication. Children suffer and die of respiratory diseases everyday. The rehabilitation programs for mine victims have closed. UNOCAL gave up its plans for building a pipeline through Afghanistan. If would have provided jobs for thousands of Afghans and fed their women and children.

\section{IX}

Emarath - Islami Afghanistan seeks help to open more schools for girls and awaits funding of women's universities. For the second year women have been tested and registered for a medical university in Kabul. Due to the isolation and sanctions promoted by the Feminist Majority and the like-minded groups funding have not been forthcoming. The right of widows to work is protected by religious edict. Other women may work and do work with foreign aid programs in professional projects and medical facilities. Women are very actively and freely engaged in agricultural and small industrial projects. They work in farms, shepherd animals, and participate in other community chores on a daily basis. They are allowed to dress in traditional clothes (everyday outfits and a long scarf).

\section{$\mathbf{X}$}

Afghanistan is getting on its feet. Finally, after 20 years of warfares, in $95 \%$ of the country peace and security which are the comerstone for progress and development, have been restored. Afghans, men and women are hard-working and are trying to rebuild their lives and their country. They need real help: support, love, respect, recognition, advice, understanding, medicine, food and funds to grow and prosper. 


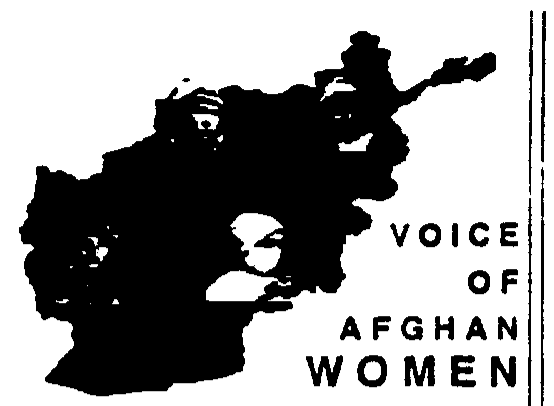

Board of Director:

Atifi, Hamida

Bimar, Fauzia

Ghausse, Amena

Gausse, Razia

Hamid, Najia

Haidari, Torpekay

Isaaczai, Latifa

Kochai, Malal

Mohsini, Fahima

Noorzoy, Farkhunda

Waiz, Tahira

World Wide

Represenrtatives:

Dadgar, Fatima

Akbar, Alia

Farhad, Qamar

Los Angeles

Mazhari Suraia

Canada

Waseal Malalai

Connecticut

Shakera Nadi Germany

Laili Zikria New York

Office \& Mailing Address

37240 St. Matthew Dr

Newark, CA 94560

Tel: (5i0) 793-1021

\section{5 \\ VOICE OF \\ AFGHAN WOMEN}

Please do not listen to one-sided non-representative allies of the Feminist Majority. We encourage you to hear the real voice of Afghan women from inside Afghanistan who plead with the international community for help to fund programs for health care, development and education. So that they will over come adversity and move towards a normal life.

The Ruling Administration has extended an invitation, to whomever is interested, to visit Afghanistan and gather first - hand information.

\section{XI}

Lastly, we suggest that you devote today's fundraising money to sponsor desperately needed medical facilities, rehabilitation programs and schools in Afghanistan. We also strongly recommend that you closely monitor these funds and see for yourselves where and how they are being spent.

We will be glad if we could be of any assistance to you in order to understand the current status of Afghanistan and together we could approach the most essential areas that Afghan women could benefit from. We know our people have many problems to resolved, but we need to deal with them in terms of priority.

\section{WITH BEST REGARDS}
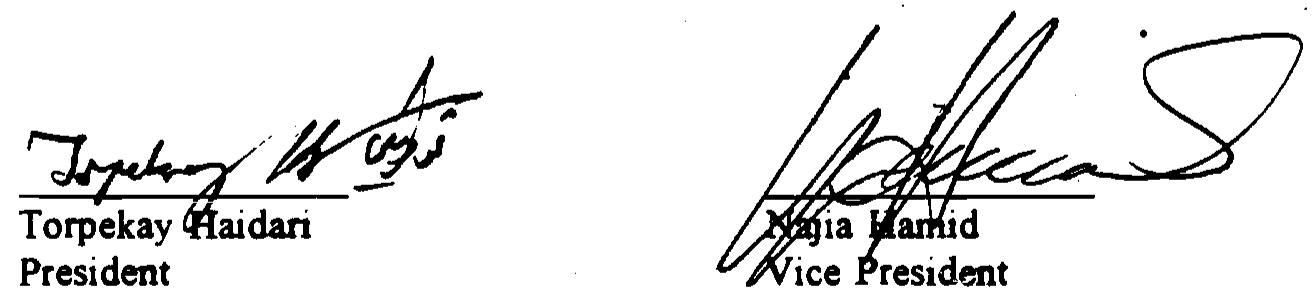

VOICE OF AFGHAN WOMEN SAN FRANCISCO BAY AREA

(Page five of five) 
Surely one of the darkest chapters in recent Afghanistan history, is the story of the infamous Pul-I-Charki Prison. Here, ordinary citizens: men, women, and children. regardless of age or circumstance were arbitrarily subjected to inhumane treatment and abuse that deties description. Physical and mental torture, summary execution; loss of property rights; protracted incarceration without formal charge; starvation; venality; violence; and degradation: uncivilized, assaults against the Afghan people, their millennium-old institutions of culture and religious convictions by the USSR and their Communist allies.

This insanity was neither accidental, nor the aberrant behavior of a minority of psychopathic individuals, these unspeakable crimes constituted institutionalized terror. terror fully-sanctioned by Moscow! This manifestation of horror and barbarity represented an instrument in which the Soviets prosecuted a war of genocide in their quest for econonic and strategic supremacy in Afghanistan!

For the sake of future generations of Afghans, and for the historical record this story must be told in the most minute and graphic detail. And though I am humbled by the solemnity and enormity of this project. I am confident that the support and assistance of the Afghan people will guide me in the rendering of a complete and truthful account.

Therefore, through this letter I seek the assistance of any and all Afghans that have personal experience of Pul-I-Charkhi or other satellite detention centers such as Sadarat, and have had family members that were incarcerated in any of these facilities and wish to share those experiences with me for the purpose of researching and producing a written history of the SovietCommunist Penal System in Afghanistan from 1978-1989. Those who wish to share those experiences, photographs, and/or documents, are encouraged to contact me at the above address or telephone. Submissions will be graciously accepted in English, Pashtu, and Dari.

As this work progresses, I anticipate traveling to Pakistan, Afghanistan, and Russia in search of material and will be conducting interviews at those locations.

It is my intention to dedicate this historical narrative to the menory of those courageous Afghans who suffered and perished within an evil, Soviet-Communist penal system.

The summary execution and torture of tens-of-thousands of innocent people must not and can not have been in vain, their story must be told, and their story must be recorded, cherished, and preserved for all time. It is my hope that one day this document may stand as evidence in a war crimes tribunal, convened to bring to justice those who participated in this unspeakable horror.

Please pass on a copy of this letter to anyone you know who has suffered from the Soviet-Communist penal system. Bruce Richardson

166 Haverhill Road, Topsfield, MA 01983-1027 (978) 887-8063
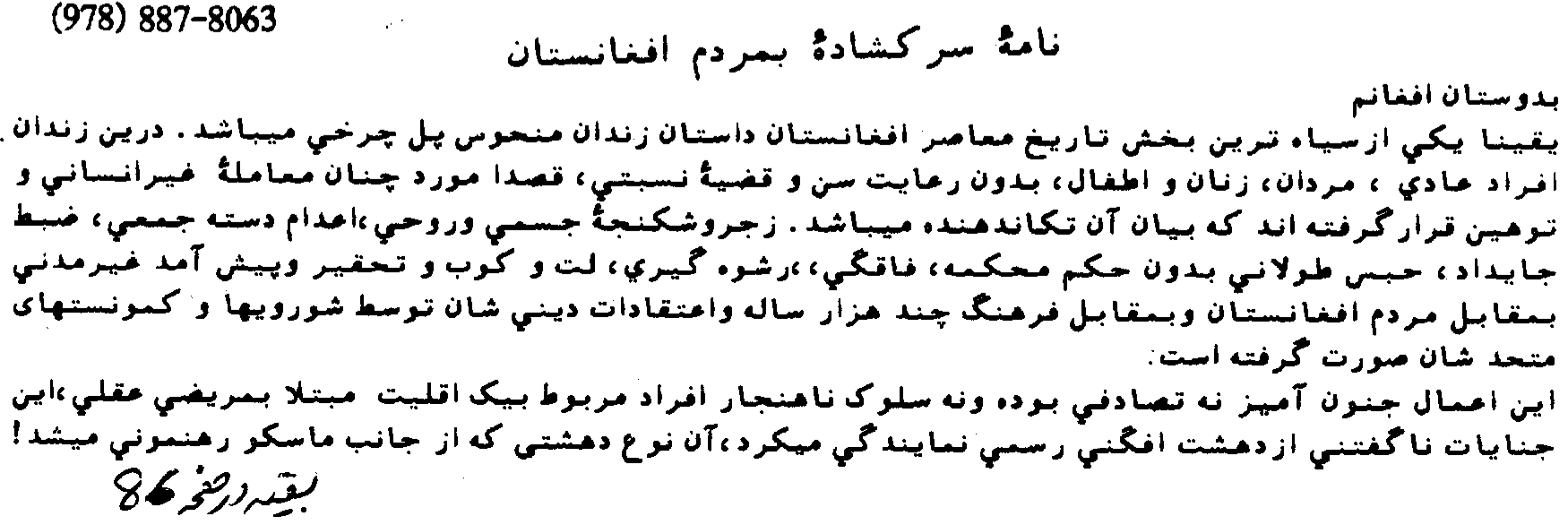
1.1
$D S$
371.3
991
$v v 2 i$
$v \cdot 10: 76$

$\begin{array}{cc}5 \\ 3 \\ 3 & 3 \\ 3 & 3 \\ 3 & 3 \\ 3 & 3\end{array}$

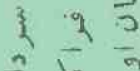

$\begin{cases}5 & 5 \\ 3 & 3\end{cases}$

(5). . .

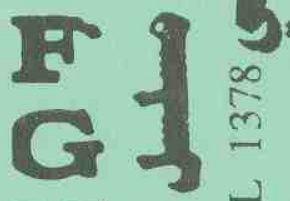

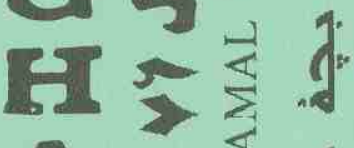

A

$\mathbf{I}$ ?

I $3 \frac{9}{y}$

No

$\sigma \hat{1}$

$\mathbf{M} \div \frac{2}{2}$

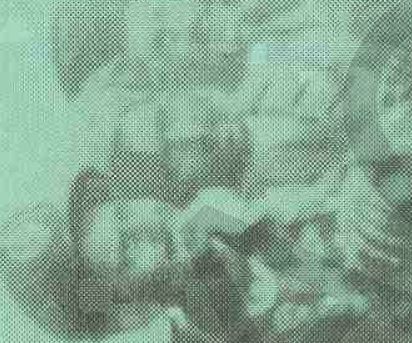

I

R 1 睡?

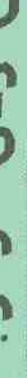

R âे

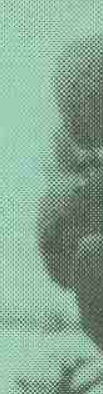

(b)

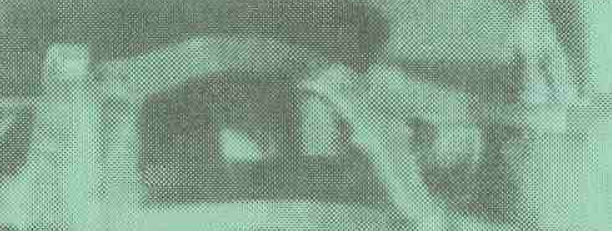

a d

I 3

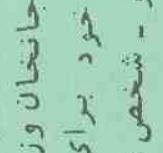

?

3

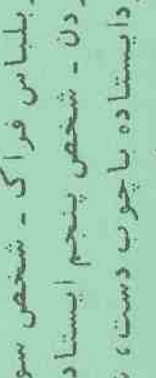

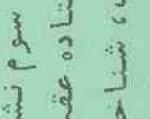

3)

? 3

3 :

30

के की.

? 3

$\frac{1}{3}, \frac{9}{9}$

3 y
3

R:

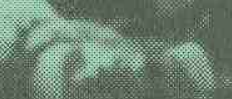

. 3

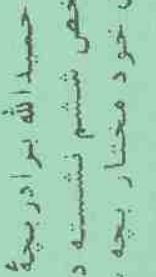

के

, उy

3.

? 